\title{
Kombinierte Programmevaluierung der Christian Doppler Labors und Josef Ressel Zentren 2016
}

Alt R., Berrer H., Borrmann J., Brunner Ph., Dolle B., Helmenstein C., Jöchle J., Pirker J., Pohl, P., Popko, J., Schmidl M., Schneider H. 


\section{Inhalt}

1 Einleitung 1

1.1 Ausgangslage und Zielsetzung 1

1.2 Durchführungs- und Umsetzungskonzept 3

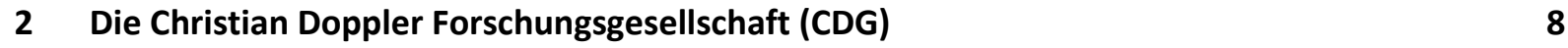

2.1 Organisation und Ziele der CDG 9

2.2 Die Christian Doppler Labors (CD-Labors) 14

2.3 Die Josef Ressel Zentren (JR-Zentren) 16

3 Kenndatenerhebung $\quad 19$

3.1 Statistische Analyse der CD-Labors $\quad 21$

3.1.1 Basisanalyse der CDG-Abschlussevaluierungen 22

3.1.2 Detailanalyse der CDG-Abschlussevaluierungen 27

$\begin{array}{lll}3.2 & \text { Zusammenfassung der CDG-Kenndaten } & 47\end{array}$

3.2.1 Korrelationsanalyse der Outputwerte zu den Budgetmitteln 48

3.2.2 Übersicht der normierten Outputwerte 49

3.2.3 Vergleich der CDG-Evaluierungen 2011 und 2016

3.3 Statistische Analyse der JR-Zentren 54

$\begin{array}{lll}3.4 & \text { Schlussfolgerungen } & 60\end{array}$

$4 \quad$ Programm- und Systemevaluierung $\quad 63$

$\begin{array}{lll}4.1 & \text { Evaluierung der Programmziele } & 63\end{array}$

$\begin{array}{lll}4.2 & \text { Abschlussfragebögen } & 70\end{array}$

4.2.1 Abschlussfragebögen der Laborleiter 70

4.2.2 Abschlussfragebögen der Unternehmen 76

4.3 Tiefeninterviews Unternehmen $\quad 81$

$\begin{array}{lll}4.4 & \text { Online Fragebogen } & 95\end{array}$

4.4.1 Teilgruppenbetrachtung (Befragungsgruppe CD-Laborleiter) 97

4.4.2 Teilgruppenbetrachtung (Befragungsgruppe JR-Zentrumsleiter) 119

4.4.3 Teilgruppenbetrachtung (Befragungsgruppe CD-Labor Unternehmenspartner) 132

4.4.4 Teilgruppenbetrachtung (Befragungsgruppe JR-Zentrum Unternehmenspartner) 153 
4.5 Schlussfolgerungen

5 Erweiterte Systemevaluierung $\quad 173$

5.1 Analyse der Beteiligungen an CD-Labors und JR-Zentren 173

$\begin{array}{lll}\text { 5.1.1 Analyse der Unternehmenspartner } & 173\end{array}$

5.2 Finanzierung der Grundlagenforschung in Österreich 185

5.2.1 Öffentliche Forschungsförderung in Österreich 188

$\begin{array}{ll}\text { 5.2.2 Internationaler Vergleich } & 190\end{array}$

5.2.3 Impakt der Grundlagenforschung 193

$\begin{array}{lll}5.3 & \text { Patentanalyse } & 197\end{array}$

5.3.1 Identifikation der Technologiestärkefelder Österreichs 197

5.3.2 Weltweite Wachstumsthemen 204

5.3.3 Verknüpfung der Patentanalyse mit CD-Labors bzw. JR-Zentren 208

$\begin{array}{lll}5.4 & \text { Schlussfolgerungen } & 210\end{array}$

6 Synopse 213

$\begin{array}{lll}7 & \text { Tabellenverzeichnis } & 216\end{array}$

$\begin{array}{lll}8 & \text { Abbildungsverzeichnis } & 218\end{array}$

9 Quellen $\quad 225$

$\begin{array}{llr}10 & \text { ANHANG } & 228\end{array}$

Fragebogen CD-LABOR: LABORLEITERIN/-LEITER 228

Fragebogen CD-LABOR: UNTERNEHMENSPARTNER 232

Fragebogen JR-ZENTRUM: ZENTRUMSLEITERIN/-LEITER 236

Fragebogen JR-ZENTRUM: UNTERNEHMENSPARTNER 240

Patentanalyse: Beschreibung der Technologiestärkefelder Österreich 244

Technologiestärkefeld „Aufzüge“ 244

$\begin{array}{ll}\text { Technologiestärkefeld "Cellulose“ } & 247\end{array}$

Technologiestärkefeld „Eisenbahn“ 251

Technologiestärkefeld „Lebensmittel“ 256

$\begin{array}{ll}\text { Technologiestärkefeld "Metallbearbeitung" } & 259\end{array}$

Technologiestärkefeld „Möbel“ 264 
$\begin{array}{ll}\text { Technologiestärkefeld „Papier“ } & 268\end{array}$

Technologiestärkefeld „Stahl“ 271

Technologiestärkefeld „Stempel“ 275

$\begin{array}{ll}\text { Technologiestärkefeld „Werkzeug“ } & 279\end{array}$

Patentanalyse: Beschreibung der weltweiten Wachstumsthemen 282

Weltweite Wachstumsthemen im Bereich Additive Fertigungsverfahren 282

Weltweite Wachstumsthemen im Bereich Verteilung elektrischer Leistung 283

Weltweite Wachstumsthemen im Bereich nicht-schaltbare Kupplungen 284

Weltweite Wachstumsthemen im Bereich Licht 285

Weltweite Wachstumsthemen im Bereich Chirurgie/anwendungsspezifische Robotik 286

Weltweite Wachstumsthemen im Bereich Futter- oder Lebensmittel 287 


\section{Einleitung}

Die Christian Doppler Forschungsgesellschaft (CDG) gilt in Österreich als Wegbereiterin für eine erfolgreiche Kooperation zwischen Wissenschaft und Wirtschaft. Sie ermöglicht Unternehmen einen effektiven Zugang zu neuem Wissen und fördert anwendungsorientierte Grundlagenforschung an Universitäten in den sogenannten Christian Doppler Labors (CD-Labors), seit dem Jahr 2012 auch an Fachhochschulen in Josef Ressel Zentren (JR-Zentren). ${ }^{1}$

Zu den besonderen Strukturmerkmalen der CDG zählen insbesondere: ${ }^{2}$

- die Trägerschaft durch forschende Unternehmen,

- die Autonomie in allen wissenschaftlichen Angelegenheiten,

- die flexible, anpassungsfähige Struktur

- und die langjährige Erfahrung in der Kooperationskultur zwischen Wissenschaft und Wirtschaft.

Die vorliegende Evaluierung wird im Auftrag des Bundesministeriums für Wissenschaft, Forschung und Wirtschaft (BMWFW) durchgeführt, deren Förderprogramme „Förderung der Einrichtung und des Betriebs von Christian Doppler Labors" und „Förderung und Einrichtung des Betriebs von Josef Ressel Zentren" von der CDG abgewickelt werden. Die gesamte Evaluierung orientiert sich am Vorbild der Nutzen-, Programm- und Systemevaluierung aus dem Jahr 2011 (vgl. Economica/IWI 2012). Zusätzlich werden in der aktuellen Evaluierung die Josef Ressel Zentren sowie ergänzende Module (Finanzierung der Grundlagenforschung, Patentanalyse) einbezogen.

\subsection{Ausgangslage und Zielsetzung}

Sowohl im „Programmdokument 2014 zur Förderung der Einrichtung und des Betriebs von Christian Doppler Labors“ als auch im „Programmdokument 2014 zur Förderung der Einrichtung und des Betriebs von Josef Ressel Zentren" ist eine Evaluierung des Förderprogramms auf zwei Ebenen vorgesehen. Während sich die Projektebene auf die begleitende Kontrolle der geförderten Vorhaben (CDLabors, JR-Zentren) bezieht, ist auf einer darüber liegenden Stufe (Programmebene) die Erreichung von Zielen laut Programmdokument des Förderprogramms ebenfalls zu evaluieren.

\footnotetext{
${ }^{1}$ Vgl. https://www.cdg.ac.at/ueber-uns/

2 BMWFJ/CDG (2013b)
} 
Zu beachten ist die Doppelrolle der CDG, einerseits als Förderagentur, die mit der Abwicklung der beiden zu evaluierenden Programme betraut ist und andererseits als gemeinnütziger Verein, zu deren Mitgliedern die in den CD-Labors bzw. JR-Zentren aktiven Unternehmen gehören.

Zielsetzung ist es, das Förderprogramm bzw. die beiden zu evaluierenden Programme hinsichtlich ihrer Wirkungsebenen Output (Kennzahlenebene), Outcome (Ebene der operationalisierbaren Ziele) und Impact (Ebene der wirtschafts- und gesellschaftspolitischen Ziele) zu untersuchen.

\section{Abbildung 1: Wirkungsebenen des Förderprogramms}

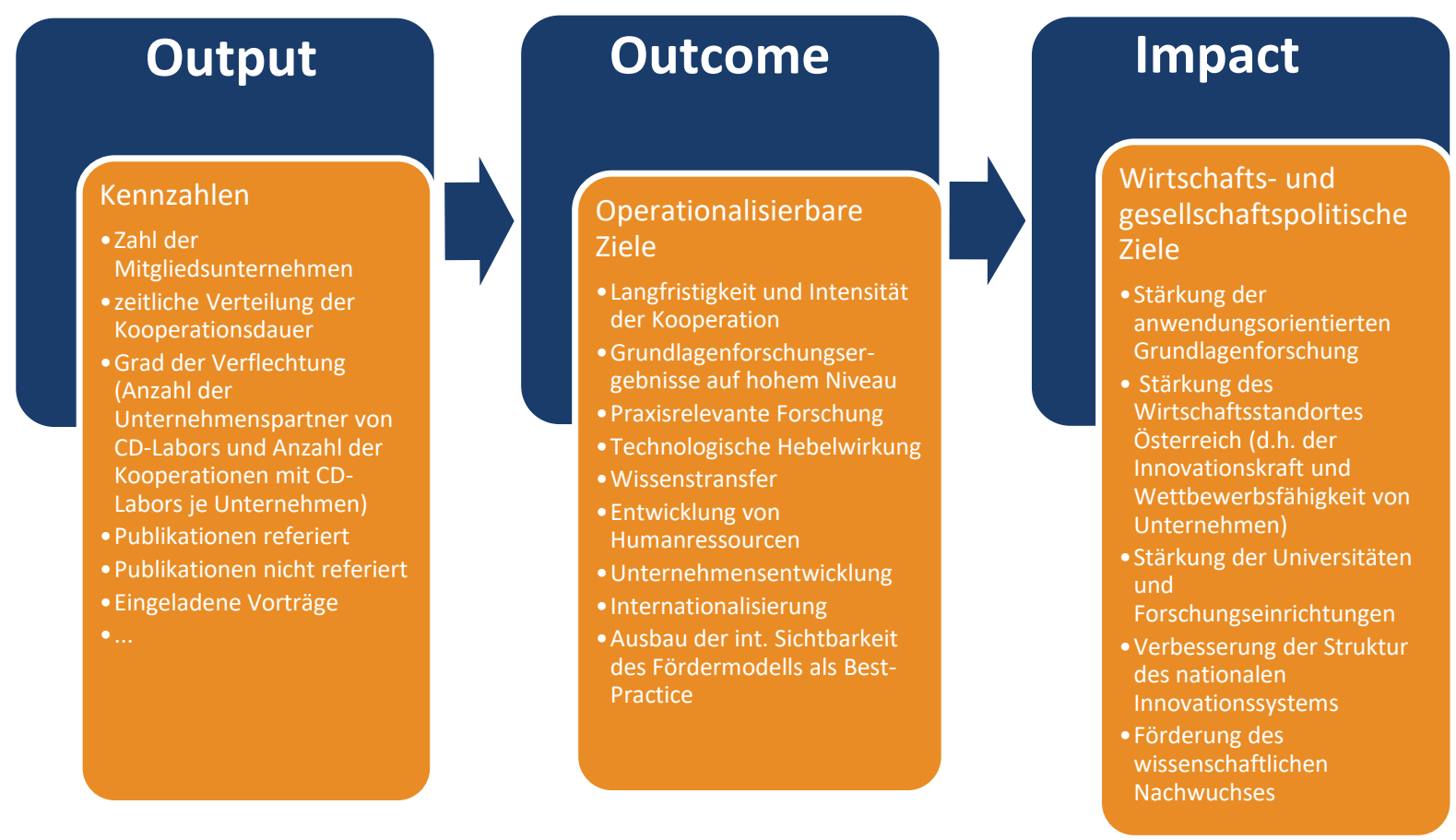

Quelle: CDG, Darstellung Economica/IWI. 


\subsection{Durchführungs- und Umsetzungskonzept}

Die Evaluierung besteht aus drei Teilen: In einem ersten Schritt wird eine Kenndatenerhebung über jene 42 CD-Labors ${ }^{3}$, die seit der letzten Evaluierung im Jahr 2011 ausgelaufen sind, und jene 6 JRZentren, die sich in der Verlängerungsphase befinden, vorgenommen.

\section{Abbildung 2: Umsetzungskonzept}

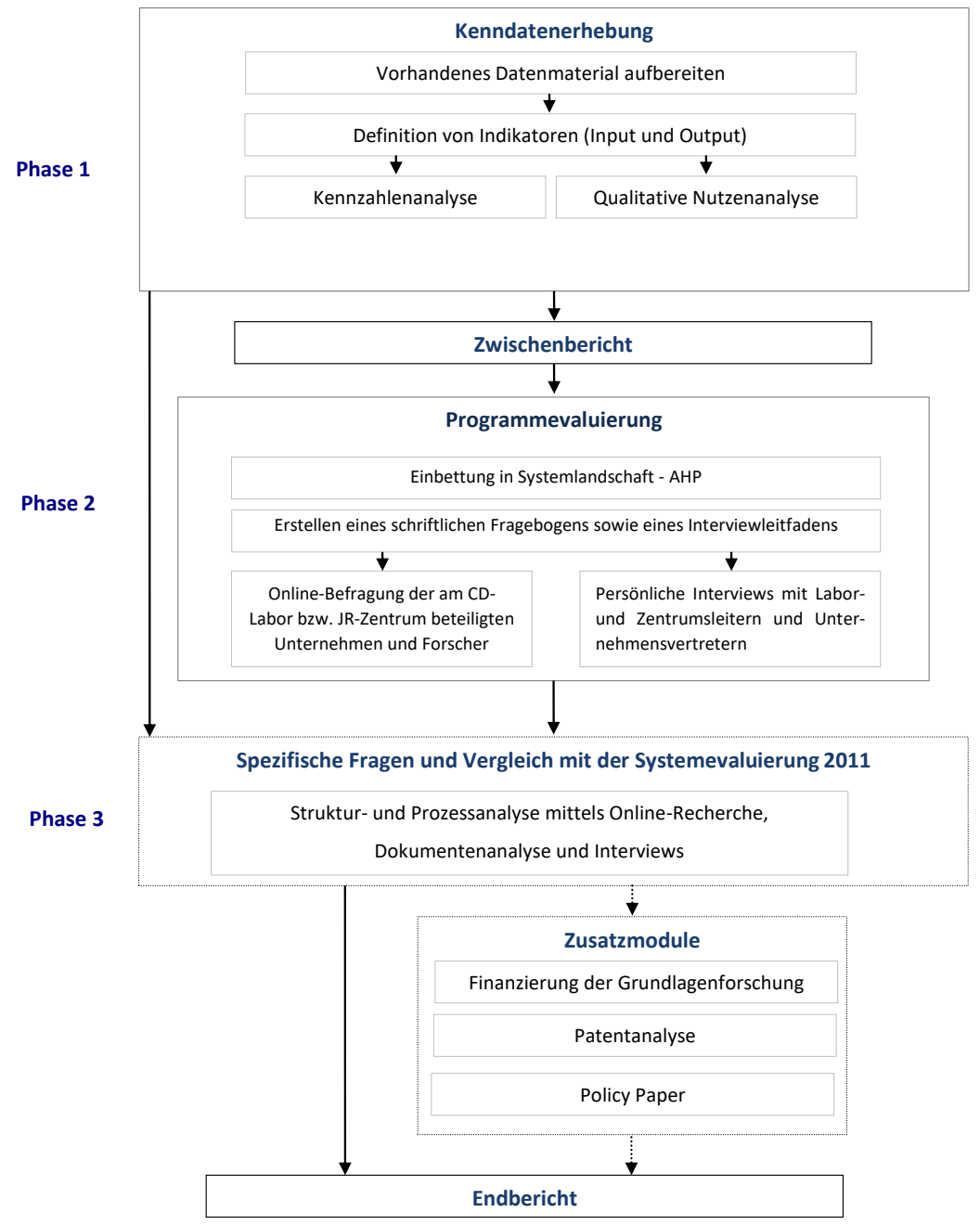

Quelle: Economica/IWI. Methodik

${ }^{3}$ Im Rahmen dieser Studie wurden insgesamt 45 CD-Labors betrachtet, wobei einige von ihnen einen untypischen Verlauf aufwiesen (Unterbrechungen, vorzeitiger Abbruch). Eines der CD-Labors musste aufgrund eines Auslandsaufenthaltes des CD-Labor Leiters pausieren. Zwei weitere CD-Labors wurden wegen Konkurs der Partnerunternehmen vorzeitig geschlossen, konnten jedoch mit anderen Unternehmen wieder aufgenommen werden. In den statistischen Aufzeichnungen werden diese drei CD-Labors daher vor und nach der Unterbrechung als eigener Datenpunkt angeführt. Da sie jedoch jeweils nur mit einer Abschlussevaluierung vertreten sind, reduziert sich die Anzahl der untersuchten CD-Labors auf 42. 
Aufbauend auf den Ergebnissen der Kenndatenerhebung erfolgt in einem zweiten Schritt die Untersuchung der Zielerreichung der Programme im Rahmen der Programmevaluierung.

Schließlich wird ein Vergleich der aktuellen Ergebnisse mit jenen aus 2011 vorgenommen. Die Evaluierung orientiert sich (mit gewissen Adaptierungen) an der Methodik der Nutzen-, Programmund Systemevaluierung aus dem Jahr 2011.

\section{Kenndatenerhebung}

Im Rahmen der Kenndatenerhebung wird der Output der Forschungseinrichtungen (CD-Labors, JRZentren) erhoben und dargestellt sowie in Relation zum Input gesetzt. Als Output wird etwa die Anzahl der Publikationen, Dissertationen, Konferenzteilnahmen, Patente, Prototypen und Produktinnovationen herangezogen. Als Inputgrößen werden u.a. die Höhe der Fördermittel, die Anzahl der Mitarbeiter und Mitarbeiterinnen verwendet.

Quotienten zwischen Output- und Inputgrößen stellen Maße für die Produktivität dar und ermöglichen Vergleiche im Zeitverlauf (nur bei CD-Labors) sowie zwischen Forschungseinrichtungen (CD-Labors und JR-Zentren). Um die Vergleichbarkeit der Datenuntersuchung für die Evaluierung der CD-Labors mit jener im Rahmen der Evaluierung im Jahre 2011 zu gewährleisten, wird eine analoge Methodik wie in der Programmevaluierung 2011 verwendet. Dies umfasst auch eine Korrelationsanalyse sowie die Berechnung von Indikatoren normiert mit den Gesamtmitteln. ${ }^{4}$

Für die Kenndatenerhebung wird in einem ersten Schritt auf bereits vorhandenes Datenmaterial zurückgegriffen. Basis hierfür sind der CDG vorliegende statistische Jahresberichte, Evaluierungsberichte und die jeweiligen Gutachten, Abschlussevaluierungen der CD-Labors, Ansuchen für CDLabors und die jeweiligen Gutachten.

Um die qualitative Dimension der Leistungen der Forschungseinrichtungen zu untersuchen, wird dieser Themenbereich in den im Rahmen der Programmevaluierung geführten Interviews mit den Laborleitern mitberücksichtigt.

\section{Programmevaluierung}

Der zweite Teil der Evaluierung bezieht sich auf alle bestehenden und auf alle seit 2011 ausgelaufenen CD-Labors, auf alle aktiven JR-Zentren sowie auf alle in den jeweiligen CD-Labors und JRZentren aktiven Unternehmenspartner der CDG. Aufbauend auf den Ergebnissen der Kenndaten-

\footnotetext{
${ }^{4}$ Siehe Programmevaluierung 2011, Abschnitt 3.3.
} 
erhebung werden die Programme in Bezug auf die Erreichung ihrer Programmziele untersucht. Folgende Aspekte werden berücksichtigt:

- Erreichung der Programmziele,

- Qualität und Eigenheit (uniqueness) der Forschung,

- Eignung der Fördermodelle für die beiden Programme,

- Anpassungs- bzw. Änderungsbedarf,

- aktuelle und zukünftige Rolle des Programms in der österreichischen Forschungs- und Innovationslandschaft (Konkurrenz, Synergien),

- aktuelle und zukünftige internationale Positionierung des Programms,

- Förderung des wissenschaftlichen Nachwuchses.

Im Rahmen der Programmevaluierung wird auch auf Daten der Kenndatenerhebung zurückgegriffen. Abgesehen von dem von der CDG zur Verfügung gestellten Datenmaterial, wird eine schriftliche Befragung aller an CD-Labors bzw. JR-Zentren beteiligten Unternehmen und Forschungsleiter durchgeführt. Die schriftliche Befragung, die mittels eines Online-Befragungstools erfolgt, bezieht sich vor allem auf die Erreichung der Programmziele.

Bei der Konzeption des Fragebogens wurde darauf geachtet, möglichst viele Fragen an die Erhebung aus dem Jahr 2005 bzw. 2011 anzupassen, um die Daten der beiden Zeiträume in möglichst vielen Punkten miteinander vergleichbar zu machen.

Im Rahmen von ergänzenden Tiefeninterviews soll der Nutzen aus Sicht beider Akteursgruppen dargestellt werden. Kernthemen der Interviews sind dabei u.a. folgende:

- Wie unterschieden sich die Forschungsaktivitäten im CD-Labor bzw. JR-Zentrum von jenen, die unternehmensintern / institutsintern durchgeführt werden?

- Wie haben sich die F\&E-Aktivitäten und (Forschungs-)Kooperationsmuster in den Unternehmen/Instituten verändert (Vorher-Nachher Vergleich)?

- Entsteht ein Nutzen für das Unternehmen/Institut (Signalwirkung, Folgeprojekte etc.)?

Die Ergebnisse der Programmevaluierung werden getrennt für die beiden Programme, CD-Labors und JR-Zentren, dargestellt, jedoch unter Berücksichtigung der zwischen ihnen bestehenden Synergien, etwa in der Abwicklung.

Um etwaige unterschiedliche Präferenzen hinsichtlich der operationalen Ziele identifizieren zu können, wurde ein Analytischer Hierarchischer Prozess (AHP) zur Bestimmung der relativen 
Bedeutung der einzelnen Kennzahlen und Ziele durchgeführt. In diesem Prozess wurden neben dem BMWFW und Repräsentanten der CDG auch ausgewählte Unternehmen und Labor- bzw. Zentrumsleiter eingebunden. Im Ergebnis konnten einerseits Differenzen der Priorisierung der verschiedenen Stakeholder aufgezeigt werden, aber es zeigt sich andererseits auch eine gewisse heterogene Sichtweise innerhalb der Wissenschaft und Wirtschaft.

\section{Spezifische Fragen und Vergleichbarkeit mit der Systemevaluierung 2011}

In weiterer Folge wurden spezifische Fragen der Programm- und Prozessevaluierung betrachtet sowie ein Vergleich mit der (System)Evaluierung 2011 ermöglicht.

In Bezug auf den Programmablauf wurde u.a. die Klarheit des Förderkonzepts aus Sicht der Antragsteller, LaborleiterInnen und Unternehmen untersucht, die Einfachheit und Effizienz der Antragstellung bzw. des Betriebs eines CD-Labors und JR-Zentrums, die Eignung und Transparenz der Prozesse (Evaluierungsprozess, Förderungsabwicklung gegenüber den CD-Labors und JR-Zentren bzw. den Betreibern und gegenüber dem programmbeauftragenden Ressort) sowie die Angemessenheit der Entscheidungsprozesse.

Außerdem wurde eine Analyse des Beteiligungsverhaltens an den CD-Labors bzw. JR-Zentren durchgeführt. Im Falle der CD-Labors konnte darüber hinaus sowohl auf Ebene der Unternehmen als auch auf Ebene der Universitäten dabei außerdem untersucht werden, in wie weit sie sich die Beteiligungsstrukturen im Zeitverlauf verändern. Bei den JR-Zentren wurde zusätzlich untersucht, ob der durch die CD-Labors bereits bestehende Unternehmenspool erweitert wird.

\section{Finanzierung der Grundlagenforschung in Österreich}

Um einen Überblick über die Größe des Beitrags der Grundlagenforschung der CDG zur gesamten Grundlagenforschung Österreichs zu bekommen, kann als Inputgröße berechnet werden, wie groß der Anteil der Budgetmittel ist, die für Förderprogramme der CDG (zur Grundlagenforschung) ausgeschüttet werden. Dieser kann dann mit jenen der gesamten österreichischen Grundlagenforschung (bzw. jener im technisch-naturwissenschaftlichen Bereich) verglichen werden.

Des Weiteren wurde untersucht, inwieweit die CDG einen Beitrag zur Grundlagenforschung in Österreich liefert.

\section{Patentanalyse}

Mittels einer Patentdatenbank (die nationale Patentanmeldungen aus über 90 Staaten sowie jene beim Europäischen Patentamt enthält) wurde untersucht, in welchen Technologiebereichen Erfinder 
aus Österreich bzw. am Standort Österreich besonders stark patentieren und mit welchen anderen Themenbereichen diese verbunden sind.

Weiters wurde überprüft, in welchen Technologiebereichen weltweit die stärkste Patentaktivität stattfindet. Die Top-Technologien laut dieser Betrachtung können nach Anzahl und Dynamik der Patentanmeldungen ermittelt werden. Dies ermöglichte es, technologische Trends zu erkennen, der österreichischen Patentaktivität gegenüberzustellen und Potenziale für Aktivitäten der CDG zu identifizieren. ${ }^{5}$

\section{Policy Paper}

Das Policy Paper ist darauf ausgerichtet, geeignete, implementierbare Ergebnisse zu liefern. Die Suche nach solchen Ergebnissen erfordert nicht nur die in den vorangegangenen Arbeitspaketen des Endberichts ausgearbeiteten Analysen, sondern auch eine wertgetriebene Beurteilung bezüglich der geeignetsten Handlungsalternative. Die vorangegangenen Arbeitspakete dienen in diesem Sinne als Basis konkreter Handlungsempfehlungen.

Berücksichtigt werden dabei die Positionierung und Ausrichtung des CD-Programms, spezielle Programmspezifika, die administrative Effizienz und strategische Programmoptionen für die Zukunft. Ein weiterer bedeutender Punkt behandelt die Einbettung der Fachhochschulen bzw. des JR-Programms (bzw. der JR-Zentren) in die Christian Doppler Forschungsgesellschaft (CDG) bzw. das "System CDG“.

\footnotetext{
${ }^{5}$ Bei dieser Patentanalyse wurden alle Technologiebereiche gleich behandelt, unabhängig von der Patentneigung in den jeweiligen Branchen.
} 


\section{Die Christian Doppler Forschungsgesellschaft (CDG)}

Die Christian Doppler Forschungsgesellschaft (CDG) besteht seit 1988 und wurde, ursprünglich unter dem Namen Christian Doppler Gesellschaft, als Forschungseinrichtung im Rahmen der ÖIAG gegründet.

Die 1993 durchgeführte Umgestaltung der ÖIAG vom Industriekonzern in eine "Beteiligungs- und Privatisierungsagentur" bedingte auch eine Reform der CDG. Im Jahr 1995 wurde deshalb eine neue Finanzierungsgrundlage geschaffen und der Verein strukturell neu organisiert; gleichzeitig erfolgte die Übernahme in die Zuständigkeit des damaligen Bundesministeriums für wirtschaftliche Angelegenheiten. Als gesetzliche Grundlage für die Förderung diente von 1995 bis 2007 das Forschungsorganisationsgesetz (FOG).

Mit 1. 1. 2008 wurde die CDG dem Forschungs- und Technologieförderungsgesetz (FTFG) sowie den Richtlinien zur Förderung der wirtschaftlich-technischen Forschung und Technologieentwicklung gemäß § 15 FTFG (FTE-Richtlinien) vom 30. 11. 2007 unterstellt. $^{6}$

Die aktuell geltenden Rechtsgrundlagen sind:

- das Forschungs- und Technologieförderungsgesetz (FTFG), ${ }^{7}$

- die Richtlinie zur Förderung der wirtschaftlich-technischen Forschung, Technologieentwicklung und Innovation (FTI-Richtlinie 2015): Struktur-FTI-RL (Nachfolgerichtlinie zur FTE-RL 2007),

- der Unionsrahmen für staatliche Beihilfen zur Förderung von Forschung, Entwicklung und Innovation (2014/C 198/01) vom 27. 6. 2014, sowie

- die Programmdokumente 2014.

Die Laufzeit des Programms erstreckt sich vom 01.01.2013 bis zum 31.12.2017 für die CD-Labors und vom 01.01.2012 bis zum 31.12.2017 für die JR-Zentren. ${ }^{8}$

\footnotetext{
${ }^{6}$ BMWFJ/CDG (2013b)

${ }^{7}$ Bundesgesetz zur Förderung der Forschung und Technologieentwicklung (Forschungs- und Technologieförderungsgesetz FTFG) StF: BGBI. Nr. 434/1982 (WV)

${ }^{8}$ BMWFJ/CDG (2013b)
} 


\subsection{Organisation und Ziele der CDG}

Die CDG ist Fördereinrichtung (Abwicklungsstelle) und als gemeinnütziger Verein für die Einrichtung und den Betrieb von Forschungseinheiten (Christian Doppler Labors bzw. Josef Ressel Zentren) verantwortlich. Diese sind an Universitäten bzw. außeruniversitären Forschungseinrichtungen (CDLabors) und Fachhochschulen (JR-Zentren) eingerichtet, die auch formelle Förderungsnehmer sind. ${ }^{9}$

Die Mitglieder sind kleine oder große Unternehmen, die in CD-Labors und/oder JR-Zentren aktiv sind. Im Rahmen einer öffentlich-privaten Partnerschaft (PPP - Public Private Partnership) finanzieren die Unternehmen gemeinsam mit dem Bundesministerium für Wissenschaft, Forschung und Wirtschaft und der Nationalstiftung für Forschung, Technologie und Entwicklung die Forschung der CD-Labors und JR-Zentren. Damit bietet die CDG zusätzlich zu ihrer Rolle als Förderungseinrichtung auch eine Kooperationsplattform, in der sich die Stakeholder selbst organisieren und die Fördermodelle der CDG gemeinsam weiterentwickeln. ${ }^{10}$

Die zentralen Organe der CDG sind gemäß Statuten:

- Die Generalversammlung,

- das Kuratorium,

- der Senat $^{11}$

In der Generalversammlung sind alle Unternehmenspartner als Mitgliedsunternehmen der CDG vertreten. Die Generalversammlung bestellt das Kuratorium und trifft Beschlüsse zu den Statuten und zum Prüfungsabschluss. Das Kuratorium trifft alle Entscheidungen in Grundsatz- und Strukturfragen, die nicht der Generalversammlung vorbehalten sind, und ist mit der Führung der Forschungsgesellschaft beauftragt. Es besteht aus bis zu 20 Mitgliedern aus der Wirtschaft sowie Vertreterlnnen des Bundesministeriums für Wissenschaft, Forschung und Wirtschaft und von Schwesterinstitutionen. Das Kuratorium bestellt den Senat und trifft sämtliche Beschlüsse in Bezug auf Forschungseinheiten sowie die Mitgliedschaft von Unternehmen.

Der Senat ist das wissenschaftliche Beratungsgremium der CDG und sichert die Qualität der Forschung. Er gestaltet die wissenschaftlichen Rahmenbedingungen der Fördermodelle, prüft Anträge auf Einrichtung von CD-Labors und JR-Zentren sowie Anträge auf Änderungen in bestehenden For-

\footnotetext{
${ }^{9}$ BMWFJ/CDG (2013b), CDG (2015)

${ }^{10}$ https://www.cdg.ac.at/ueber-uns/organisation/

${ }^{11}$ CDG (2015)
} 
schungseinheiten. Er bewertet auch den Forschungsfortschritt im Rahmen von Zwischenevaluierungen. Der Senat setzt sich aus rund 40 hochqualifizierten Persönlichkeiten aus Wissenschaft und Wirtschaft zusammen und ist in zwei Kurien gegliedert: Für CD-Labors ist der CD-Senat zuständig, für JRZentren der JR-Senat. Das Generalsekretariat der CDG ist eine Serviceeinrichtung für ForscherInnen, Unternehmen, öffentliche FörderungsgeberInnen, Gremien und FunktionärInnen. Es beantwortet alle Fragen zu den Fördermodellen und begleitet die Forschungseinheiten während des gesamten Lebenszyklus. ${ }^{12}$

\title{
Abbildung 3: Organisationsstruktur der CDG
}

\author{
Generalversammlung \\ Mitgliederversammmlung \\ - gestaltet die Vereinsstatuten \\ - beschließt den Prüfungsabschluss \\ - bestellt das Kuratorium
}

\author{
Kuratorium \\ Strategie- und Entscheidungsgremium \\ - leitet die Forschungsgesellschaft \\ - trifft alle Beschlüsse zu Forschungseinheiten \\ und Unternehmen \\ - bestellt den Senat
}

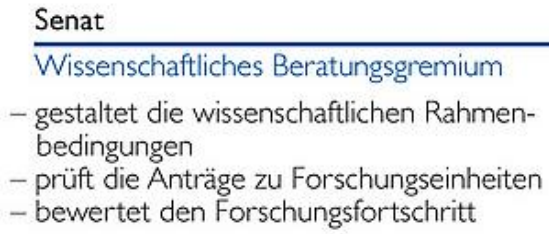

Senat

Wissenschaftliches Beratungsgremium

- gestaltet die wissenschaftlichen Rahmenbedingungen

- prüft die Anträge zu Forschungseinheiten

- bewertet den Forschungsfortschritt

\author{
Generalsekretariat \\ Serviceeinrichtung \\ - unterstützt ForscherInnen, Unternehmen \\ und Gremien \\ - beantwortet Fragen zu den Fördermodellen \\ - begleitet Forschungseinheiten während \\ des gesamten Lebenszyklus
}

Quelle: https://www.cdg.ac.at/ueber-uns/organisation/

Die österreichische Bundesregierung hat sich das Ziel gesetzt, Österreich in die Gruppe der Europäischen Innovationsleader zu führen. ${ }^{13}$ Die FTI-Richtlinie 2015 - basierend auf dem FTFG - soll dazu beitragen, durch Förderungsmaßnahmen und -programme den Wissenschafts-, Forschungs- und Wirtschaftsstandort Österreich im internationalen Wettbewerb vorteilhaft zu positionieren. Die geförderten Vorhaben sollen den dafür notwendigen Strukturwandel in Wissenschaft, Forschung und

\footnotetext{
${ }^{12}$ https://www.cdg.ac.at/ueber-uns/organisation/

${ }^{13}$ Vgl. dazu „Strategie der Bundesregierung für Forschung, Technologie und Innovation - Der Weg zum Innovation Leader“ (https://www.bka.gv.at/DocView.axd?Cobld=42655)
} 
Wirtschaft voranzutreiben und dadurch die Innovationskraft der österreichischen Wirtschaft nachhaltig stärken. ${ }^{14}$

Die Förderprogramme „CD-Labors“ und „JR-Zentren“ verstehen sich auch als Beitrag zur Umsetzung der Zielsetzung der FTI-Strategie der Bundesregierung, nach der die Zusammenarbeit und eine arbeitsteilige Profilbildung von Universitäten und Fachhochschulen einerseits und Unternehmen andererseits intensiviert werden soll. ${ }^{15}$

Die Ziele der CDG (und der von ihr für das BMWFW abgewickelten Programme) lassen sich wie folgt zusammenfassen:

- Stärkung der anwendungsorientierten Grundlagenforschung

- Stärkung von Universitäten, Fachhochschulen und Forschungseinrichtungen

- Stärkung des Forschungs- und Wirtschaftsstandortes Österreich mit Innovationskraft und Wettbewerbsfähigkeit

- Unterstützung des Wissens- und Technologietransfers zwischen den wissenschaftlichen Partnern und Unternehmen

- Verbesserung der Struktur des nationalen Innovationssystems

- Förderung des wissenschaftlichen Nachwuchses (junger Forscherinnen und Forscher)

Eine detaillierte Darstellung zu den einzelnen Zielen von CD-Labors und JR-Zentren findet sich in Punkt 1.1 Wirtschafts- und gesellschaftspolitische Ziele der jeweiligen Programmdokumente. ${ }^{16}$ Zur näheren Konkretisierung der übergeordneten Ziele dienen folgende operationalisierbare Ziele.

\footnotetext{
${ }^{14} \mathrm{Vgl.} \mathrm{BMVIT/BMWFW} \mathrm{(2015)}$

${ }^{15} \mathrm{Vgl.} \mathrm{BMWFJ/CDG} \mathrm{(2014)}$

${ }^{16}$ Vgl. BMWFJ/CDG (2013b), BMWFJ/CDG (2014)
} 
Tabelle 1: Operationalisierbare Ziele

\begin{tabular}{|c|c|}
\hline Programm „Christian Doppler Labors“ & Programm „Josef Ressel Zentren“ \\
\hline \multicolumn{2}{|c|}{ Langfristigkeit und Intensität der Kooperation } \\
\hline $\begin{array}{l}\text { Erzielung von Grundlagenforschungsergebnissen } \\
\text { auf hohem Niveau }\end{array}$ & $\begin{array}{l}\text { Erzielung von Forschungsergebnissen in der } \\
\text { angewandten Forschung auf hohem Niveau }\end{array}$ \\
\hline \multicolumn{2}{|c|}{ Praxisrelevante Forschung } \\
\hline Technologische Hebelwirkung & Hebelwirkung in den Unternehmen \\
\hline \multicolumn{2}{|c|}{ Wissenstransfer } \\
\hline \multicolumn{2}{|c|}{ Entwicklung von Humanressourcen } \\
\hline Internationalisierung & - \\
\hline
\end{tabular}

Quelle: BMWFJ/CDG (2013b), BMWFJ/CDG (2014)

Diese sind mit entsprechenden Indikatoren zur Überprüfung der Zielerreichung verbunden, wobei zwischen quantitativen und qualitativen Indikatoren unterschieden werden kann. Die Indikatoren dienen primär der Evaluierung des Programms sowie mittelbar auch der Evaluierung einzelner CDLabors bzw. einzelner JR-Zentren.

Als Informationsquellen für die Erhebung der Indikatorwerte dienen insb. die Berichte der CD-Labors und JR-Zentren, die Prozess- und Programmdatenbank sowie Fragebögen.

Tabelle 2: Indikatoren zur Prüfung der Zielerreichung

\begin{tabular}{|c|c|c|c|}
\hline \multicolumn{2}{|c|}{ Programm „Christian Doppler Labors“ } & \multicolumn{2}{|c|}{ Programm „Josef Ressel Zentren“ } \\
\hline Programmziel & Indikatoren & Programmziel & Indikatoren \\
\hline $\begin{array}{l}\text { Langfristigkeit und Intensität der } \\
\text { Kooperation }\end{array}$ & $\begin{array}{l}\text { Zahl der Unternehmen } \\
\text { Kooperationsdauer } \\
\text { Grad der Verflechtung } \\
\text { Zahl und Gründe von } \\
\text { vorzeitigen Ausstiegen } \\
\text { Neue Module, Erweiterung von } \\
\text { Modulen } \\
\text { Kooperation mit anderen CD- } \\
\text { Labors und JR-Zentren } \\
\text { Kooperation mit COMET } \\
\text { Sonstige Kooperationen }\end{array}$ & $\begin{array}{l}\text { Langfristigkeit und Intensität } \\
\text { der Kooperation }\end{array}$ & $\begin{array}{l}\text { Zahl der Unternehmen } \\
\text { zeitliche Verteilung der } \\
\text { Kooperationsdauer } \\
\text { Grad der Verflechtung } \\
\text { Zahl und Gründe von } \\
\text { vorzeitigen Ausstiegen } \\
\text { neue Module } \\
\text { Kooperation mit anderen JR- } \\
\text { Zentren und CD-Labors } \\
\text { Kooperation mit COMET } \\
\text { sonstige Kooperationen }\end{array}$ \\
\hline
\end{tabular}




\begin{tabular}{|c|c|c|c|}
\hline & $\begin{array}{l}\text { Fluktuation innerhalb der } \\
\text { Forschungsgruppe }\end{array}$ & & $\begin{array}{l}\text { Fluktuation der } \\
\text { Forschungsgruppe }\end{array}$ \\
\hline $\begin{array}{l}\text { Grundlagenforschungsergebnisse } \\
\text { auf hohem Niveau }\end{array}$ & $\begin{array}{l}\text { Publikationen referiert } \\
\text { Publikationen nicht referiert } \\
\text { Konferenzen } \\
\text { Wissenschaftliche Preise und } \\
\text { Auszeichnungen }\end{array}$ & $\begin{array}{l}\text { Forschungsergebnisse auf } \\
\text { hohem Niveau }\end{array}$ & $\begin{array}{l}\text { Publikationen referiert } \\
\text { Publikationen nicht referiert } \\
\text { Konferenzen } \\
\text { wissenschaftliche Preise und } \\
\text { Auszeichnungen }\end{array}$ \\
\hline Praxisrelevante Forschung & $\begin{array}{l}\text { Praxisnähe der Themenstellung } \\
\text { Art und Intensität der } \\
\text { Kooperation zwischen } \\
\text { Forschungs- und } \\
\text { Unternehmenspartnern }\end{array}$ & Praxisrelevante Forschung & $\begin{array}{l}\text { Praxisnähe der Themenstellung } \\
\text { Art und Intensität der } \\
\text { Kooperation zwischen } \\
\text { Forschungs- und } \\
\text { Unternehmenspartnern }\end{array}$ \\
\hline Technologische Hebelwirkung & $\begin{array}{l}\text { Erfindungen } \\
\text { Patente } \\
\text { Umsetzungs-Folgeaktivitäten } \\
\text { Induzierte weitere } \\
\text { Forschungsprojekte }\end{array}$ & Hebelwirkung & $\begin{array}{l}\text { Erfindungen } \\
\text { Patente } \\
\text { Umsetzungs-Folgeaktivitäten } \\
\text { induzierte weitere } \\
\text { Forschungsprojekte }\end{array}$ \\
\hline Wissenstransfer & $\begin{array}{l}\text { Prozessinnovationen } \\
\text { Produktinnovationen } \\
\text { Entwicklung der } \\
\text { Forschungsauftragslage beim } \\
\text { Forschungspartner }\end{array}$ & Wissenstransfer & $\begin{array}{l}\text { Prozessinnovationen } \\
\text { Produktinnovationen } \\
\text { Entwicklung der } \\
\text { Forschungsauftragslage beim } \\
\text { Forschungspartner }\end{array}$ \\
\hline $\begin{array}{l}\text { Entwicklung von } \\
\text { Humanressourcen }\end{array}$ & $\begin{array}{l}\text { Master-/Diplomarbeiten } \\
\text { Dissertationen } \\
\text { Habilitationen } \\
\text { Berufungen } \\
\text { Wissenschaftliche Preise und } \\
\text { Auszeichnungen } \\
\text { Wechsel von Laborpersonal in } \\
\text { Unternehmen }\end{array}$ & $\begin{array}{l}\text { Entwicklung von } \\
\text { Humanressourcen }\end{array}$ & $\begin{array}{l}\text { Masterarbeiten } \\
\text { Diplomarbeiten } \\
\text { Dissertationen } \\
\text { wissenschaftliche Preise und } \\
\text { Auszeichnungen } \\
\text { Wechsel von Zentrumspersonal } \\
\text { in Unternehmen }\end{array}$ \\
\hline Unternehmensentwicklung & $\begin{array}{l}\text { Umsatzentwicklung } \\
\text { Entwicklung der } \\
\text { Beschäftigtenzahl } \\
\text { Zahl der } \\
\text { Forschungsarbeitsplätze } \\
\text { Entwicklung der Sparte, der das } \\
\text { CD-Labor zuzurechnen ist, } \\
\text { innerhalb des Unternehmens }\end{array}$ & - & - \\
\hline Internationalisierung & $\begin{array}{l}\text { Zahl ausländischer } \\
\text { Unternehmenspartner } \\
\text { Internationale CD-Labors } \\
\text { Internationale Module } \\
\text { Internationale Sichtbarkeit der } \\
\text { CDG }\end{array}$ & - & - \\
\hline- & - & Lehre & $\begin{array}{l}\text { Berücksichtigung der } \\
\text { Forschungsergebnisse im } \\
\text { Ausbildungsangebot der } \\
\text { Fachhochschule } \\
\text { Verknüpfung des } \\
\text { Forschungsangebotes mit der } \\
\text { Lehre, insbesondere durch die } \\
\text { Mitarbeit von Studierenden } \\
\text { „Upgrading“ bzw. } \\
\text { „Realitätsnähe“ der Ausbildung }\end{array}$ \\
\hline
\end{tabular}

Quelle: BMWFJ/CDG (2013b), BMWFJ/CDG (2014) 


\subsection{Die Christian Doppler Labors (CD-Labors)}

CD-Labors stellen den regulären Haupttypus der geförderten Einzelvorhaben dar. Weitere Projektarten (förderbare Einzelvorhaben) sind: Christian Doppler Pilotlabor, internationales CD-Labor sowie ein internationales Modul eines CD-Labors. CD-Labors bestehen aus kleinen bis mittelgroßen Forschungsgruppen unter der Leitung von hochqualifizierten Wissenschaftlerinnen/Wissenschaftlern, die zu Fragestellungen des kooperierenden Unternehmenspartners Forschungsergebnisse an einer Universität bzw. außeruniversitären Forschungseinrichtung erarbeiten. Der Fokus liegt auf anwendungsorientierter Grundlagenforschung, wobei die Laufzeit eines CD-Labors sieben Jahre beträgt. ${ }^{17}$ Wesentliche Charakteristika von CD-Labors sind: ${ }^{18}$

- Anwendungsorientierte Grundlagenforschung auf hohem Niveau

- Einbettung in das wissenschaftliche Umfeld von Universitäten und außeruniversitären Forschungseinrichtungen

- Maximale Laufzeit von sieben Jahren

- Strenge wissenschaftliche Qualitätskontrolle

- Bottom-up-Orientierung

- Kompakte Forschungsgruppen (5-15 Personen)

- Zentrale Stellung der Laborleitung

- Garantierter wissenschaftlicher Freiraum für die Wissenschaftlerinnen/Wissenschaftler

- Gemeinsame Finanzierung durch die öffentliche Hand und Unternehmen

- Flexibilität und relativ geringer Organisationsaufwand

Die wissenschaftliche Qualität eines Antrags und die Qualifikation der vorgesehenen Laborleiterin bzw. des vorgesehenen Laborleiters werden im Rahmen eines wissenschaftlichen Begutachtungsverfahrens unter Hinzuziehung mindestens dreier Gutachten externer internationaler Expertinnen und Experten (Peer Review Verfahren) geprüft. ${ }^{19}$

\footnotetext{
${ }^{17}$ BMWFJ/CDG (2013b)

${ }^{18}$ BMWFW/CDG (2015), BMWFW/CDG (2015b)

${ }^{19}$ BMWFW/CDG (2015)
} 
Die Bewertung der Förderungswürdigkeit des Antrags erfolgt im Hinblick auf zwei wesentliche Kriterien: ${ }^{20}$

- wissenschaftliche Qualität des Antrags

- wissenschaftliche Qualifikation der vorgesehenen Laborleiterin/des vorgesehenen Laborleiters und ihre/seine Befähigung, eine Forschungsgruppe zu leiten

Die Entscheidungskompetenzen sind in der CDG dem Kuratorium zugeordnet. Die Vorschläge für die Entscheidungen des Kuratoriums werden von einem durch das Kuratorium bestellten wissenschaftlichen CD-Senat erarbeitet. Der CD-Senat setzt sich aus VertreterInnen aus Wissenschaft und Wirtschaft zusammen, wobei alle relevanten wissenschaftlichen Disziplinen vertreten sowie alle Aspekte und Zielsetzungen der CDG entsprechend berücksichtigt sind. Das Bewertungsverfahren sieht zuerst eine formale Prüfung des Antrags durch das Generalsekretariat der CDG vor. Sind die formalen Kriterien erfüllt, wird der Antrag im CD-Senat behandelt. Um die Entscheidung der CDG nachvollziehbar zu machen, wird im Falle eines Ablehnungsschreibens eine Begründung angeführt. Im Sinne einer Verfahrenstransparenz werden bei einer Ablehnung des Antrags die anonymisierten Gutachten weitgehend vollständig an die Antragstellerin/den Antragsteller weitergeleitet; bei einer Genehmigung werden Empfehlungen der GutachterInnen an die Laborleiterin/den Laborleiter weitergeleitet, überdies erhält sie/er Einsicht in die anonymisierten Gutachten (ein den GutachterInnen als vertraulich zugesagter Teil wird nicht weitergeleitet und nicht zur Einsicht gegeben). ${ }^{21}$

Nach der Genehmigung des Antrags auf Einrichtung eines CD-Labors durch das Kuratorium kann das CD-Labor gegründet werden.

\footnotetext{
${ }^{20}$ BMWFW/CDG (2015)

${ }^{21}$ BMWFW/CDG (2015)
} 
Abbildung 4: Verfahren zur Prüfung und Beurteilung von Anträgen zur Einreichung eines CD-Labors

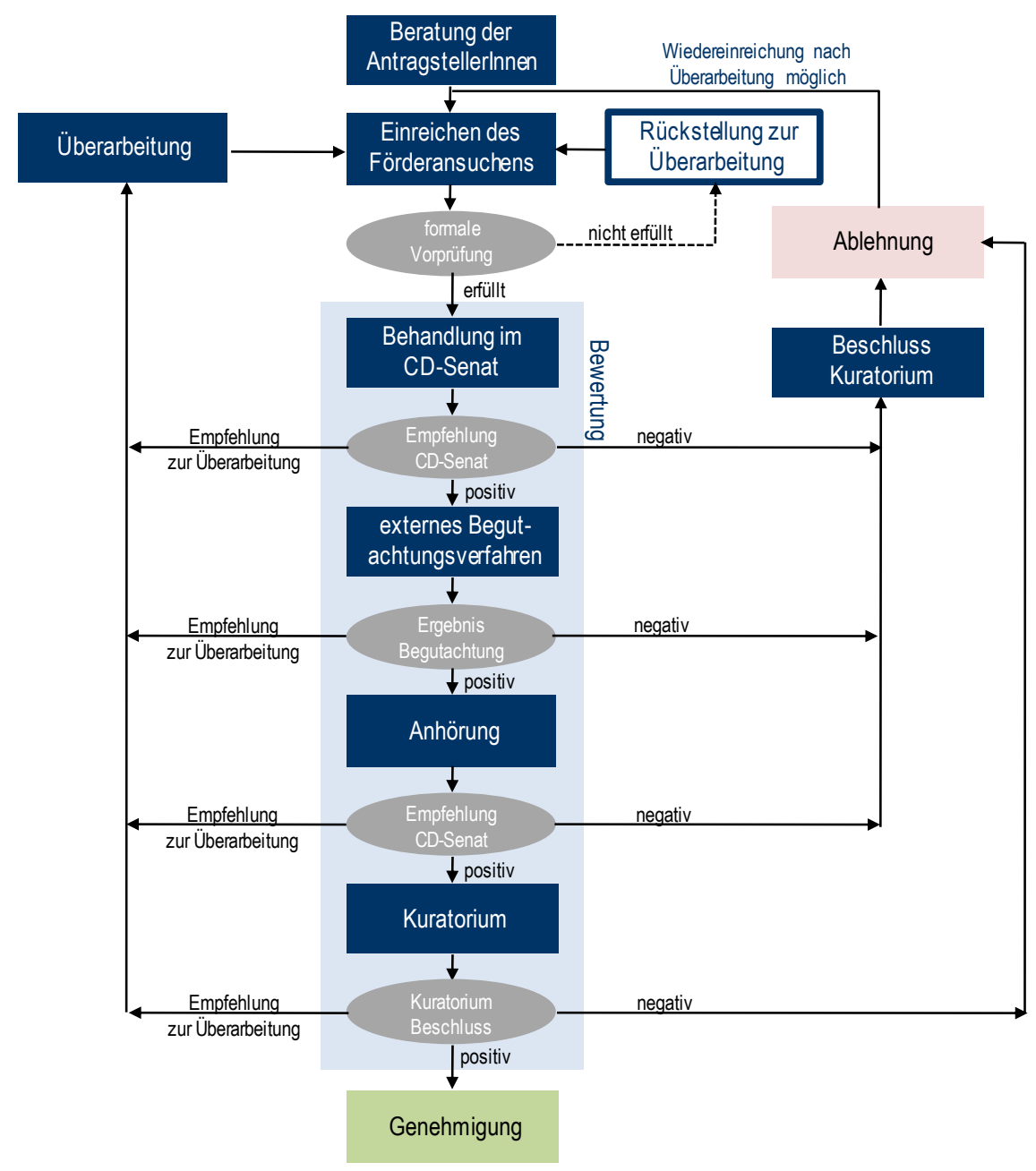

Quelle: BMWFW/CDG (2015), Eigene Darstellung

\subsection{Die Josef Ressel Zentren (JR-Zentren)}

Im Jahr 2012 wurde die CDG mit der Abwicklung eines zweiten, neuen Förderprogramms für JR-Zentren betraut, welches sich speziell an forschungserfahrene Fachhochschulen richtet und sich in Konzeption und Abwicklung weitgehend am Fördermodell für CD-Labors orientiert.

Mit den JR-Zentren sollen sich Fachhochschulen, die schon F\&E-Erfahrung haben, über stabile, längerfristige Kooperationsbeziehungen als regionale F\&E-Partner für die Wirtschaft etablieren. Die (regionalen) Wirtschaftspartner wiederum sollen Zugang zu fundierter Expertise erhalten und dabei ihre Produkte und Prozesse innovieren können. Mit dem Programm soll die Forschungskompetenz 
ausgebaut sowie eine hohe Qualität in der angewandten Forschung und Entwicklung erreicht werden. Beides soll der Ausbildung und Lehre dieser Fachhochschulen zu Gute kommen. ${ }^{22}$

Es ist möglich, dass sich in einem JR-Zentrum auch ausländische Unternehmenspartner beteiligen bzw. JR-Zentren haben unter besonderen Umständen auch die Möglichkeit, eines ihrer Module an einem ausländischen Standort zu betreiben. Diese Varianten stellen jedoch keine eigenständige Projektart dar, sondern ergänzen JR-Zentren um spezifische Elemente mit Auslandsbezug. ${ }^{23}$

Wesentliche Charakteristika von JR-Zentren lassen sich wie folgt zusammenfassen: ${ }^{24}$

- Anwendungsorientierte Forschung auf hohem Niveau

- Einbettung in das wissenschaftliche Umfeld von Fachhochschulen

- Maximale Laufzeit von fünf Jahren

- Strenge wissenschaftliche Qualitätskontrolle

- Bottom-up-Orientierung

- Kompakte Forschungsgruppen (3-10 Personen)

- Zentrale Stellung der Zentrumsleitung

- Gemeinsame Finanzierung durch die öffentliche Hand und Unternehmen

- Flexibilität und relativ geringer Organisationsaufwand

Die Bewertung der Förderungswürdigkeit des Antrags erfolgt analog zu den CD-Labors im Hinblick auf die zwei wesentlichen Kriterien: Qualität des im Antrag beschriebenen Forschungsvorhabens einschließlich der wirtschaftlichen Relevanz und Umsetzungsnähe sowie Qualifikation der vorgesehenen Zentrumsleiterin / des vorgesehenen Zentrumsleiters und ihrer / seiner Befähigung, eine Forschungsgruppe zu leiten. ${ }^{25}$

Die Vorschläge für die Entscheidungen des Kuratoriums werden von einem durch das Kuratorium bestellten wissenschaftlichen JR-Senat erarbeitet. Das Verfahren zur Prüfung und Beurteilung von Anträgen zur Einreichung eines JR-Zentrums entspricht jenem der Prüfung und Beurteilung von Anträgen zur Einreichung eines CD-Labors (siehe folgende Abbildung).

\footnotetext{
${ }^{22}$ Vgl. BMWFJ/CDG (2014)

${ }^{23}$ Vgl. BMWFJ/CDG (2014)

${ }^{24}$ BMWFW/CDG (2015d)

${ }^{25}$ Vgl. BMWFW/CDG (2015c)
} 
Abbildung 5: Verfahren zur Prüfung und Beurteilung von Anträgen zur Einreichung eines JRZentrums

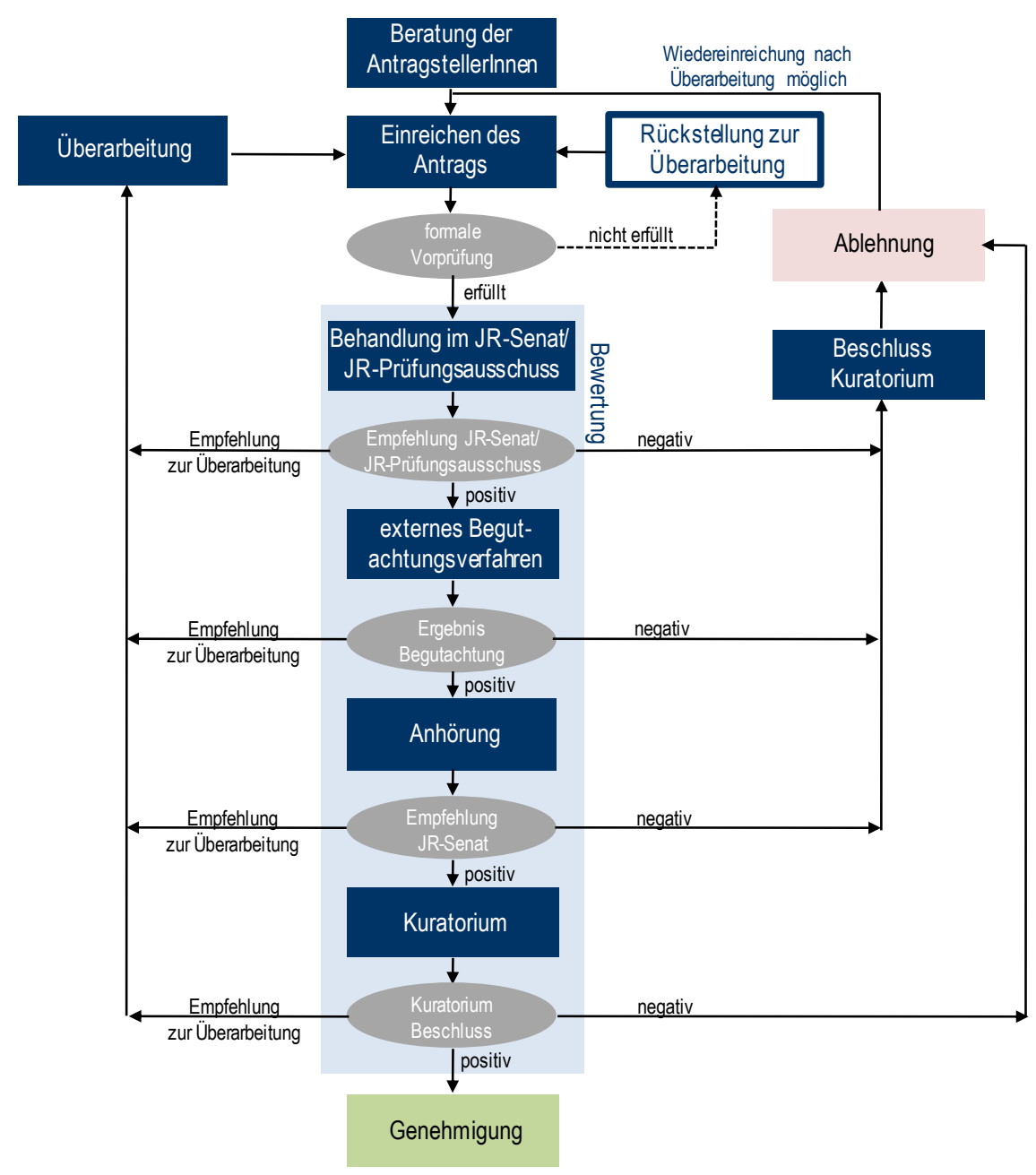

Quelle: BMWFW/CDG (2015c), Eigene Darstellung 


\section{Kenndatenerhebung}

Die Kenndatenerhebung (in der Evaluierung 2011: „Nutzenevaluierung“) dient der Erfassung des Zielerreichungsgrads auf der Ebene der CD-Labors bzw. JR-Zentren. Im Rahmen der Kenndatenerhebung wird der Output der Forschungseinrichtungen (CD-Labors bzw. JR-Zentren) erhoben und dargestellt sowie in Relation zum Input gesetzt.

Gegenstand der Kenndatenerhebung sind 42 CD-Labors, die seit der letzten Evaluierung im Jahr 2011 ausgelaufen sind, sowie sechs aktive JR-Zentren in der Verlängerungsphase ${ }^{26}$.

\section{Thematische Zuordnung der CD-Labors und JR-Zentren}

CD-Labors und JR-Zentren können zu allen Themen, in denen Unternehmen Forschungsbedarf haben, eingerichtet werden. Die Fördermodelle der CDG machen keinerlei thematische Vorgaben und vermeiden Einschränkungen. Dennoch hat sich im Lauf der Jahre eine gewisse thematische Clusterbildung ergeben. Eine enge Zusammenarbeit von Forschungseinheiten innerhalb eines Clusters wird von der CDG ermutigt, aber nicht verlangt. Dabei spielen auch wettbewerbliche Überlegungen der jeweiligen Unternehmenspartner eine Rolle.

Die Forschungsthemen können acht Clustern zugeordnet werden: ${ }^{27}$

- Chemie

- Life Sciences und Umwelt

- Maschinen- und Instrumentenbau

- Mathematik, Informatik, Elektronik

- Medizin

- Metalle und Legierungen

- Nichtmetallische Werkstoffe

- Wirtschafts-, Sozial- und Rechtswissenschaften

Tabelle 3 weist den verschiedenen CD-Labors ihre Forschungsthemen zu. Dabei wird unterschieden, ob die Labors vor 2011 oder nach 2011 ausgelaufen sind, beziehungsweise noch aktiv sind. In der

\footnotetext{
${ }^{26}$ Stand Datenübergabe Juli 2016.

${ }^{27}$ https://www.cdg.ac.at/forschungseinheiten/thematische-cluster/
} 
Kategorie "Ausgelaufen vor 2011“ ist das Forschungsthema Mathematik, Informatik, Elektronik mit 15 Labors am stärksten vertreten, gefolgt vom Bereich der Metalle und Legierungen (11 Labors). Mit jeweils 10 Labors waren die Themen Chemie und Maschinen- und Instrumentenbau nur unwesentlich stärker vertreten als der Bereich der nichtmetallischen Werkstoffe mit 9 Labors. Ein kleinerer Anteil der Labors, die vor 2011 ausgelaufen sind, beschäftigte sich mit Medizin (2 Labors) und Life Science und Umwelt (1 Labor).

Tabelle 3: Übersicht Forschungsthemen (Themencluster) CD-Labors

\begin{tabular}{|c|c|c|c|c|c|c|c|c|}
\hline \multirow{2}{*}{$\begin{array}{l}\text { CD-Labors } \\
\text { Forschungsthema }\end{array}$} & \multicolumn{2}{|c|}{$\begin{array}{c}\text { Ausgelaufen vor } \\
2011\end{array}$} & \multicolumn{2}{|c|}{$\begin{array}{c}\text { Ausgelaufen nach } \\
2011\end{array}$} & \multicolumn{2}{|c|}{ Aktiv } & \multicolumn{2}{|c|}{ Gesamt } \\
\hline & Anzahl & in $\%$ & Anzahl & in $\%$ & Anzahl & in \% & Anzahl & in \% \\
\hline Chemie & 10 & $17 \%$ & 5 & $11 \%$ & 9 & $14 \%$ & 24 & $14 \%$ \\
\hline Life Sciences und Umwelt & 1 & $2 \%$ & 9 & $20 \%$ & 13 & $20 \%$ & 23 & $14 \%$ \\
\hline Maschinen- und Instrumentenbau & 10 & $17 \%$ & 6 & $13 \%$ & 4 & $6 \%$ & 20 & $12 \%$ \\
\hline Mathematik, Informatik, Elektronik & 15 & $26 \%$ & 6 & $13 \%$ & 16 & $24 \%$ & 37 & $22 \%$ \\
\hline Medizin & 2 & $3 \%$ & 10 & $22 \%$ & 11 & $17 \%$ & 23 & $14 \%$ \\
\hline Nichtmetallische Werkstoffe & 9 & $16 \%$ & 1 & $2 \%$ & 4 & $6 \%$ & 14 & $8 \%$ \\
\hline Metalle und Legierungen & 11 & $19 \%$ & 8 & $18 \%$ & 7 & $11 \%$ & 26 & $15 \%$ \\
\hline Wirtschafts-, Sozial- und Rechtswissenschaften & 0 & $0 \%$ & 0 & $0 \%$ & 2 & $3 \%$ & 2 & $1 \%$ \\
\hline Gesamt & 58 & $100 \%$ & 45 & $100 \%$ & 66 & $100 \%$ & 169 & $100 \%$ \\
\hline
\end{tabular}

Quelle: https://www.cdg.ac.at/Stand Juli 2016

Bei den Labors, die nach 2011 ausgelaufen sind und auf denen der Fokus dieses Berichts liegt, zeigt sich eine ganz andere Struktur. Hier waren die meisten Labors in den Bereichen Medizin und Life Science tätig, also genau in den Bereichen, die in den vor 2011 ausgelaufenen Labors am schwächsten vertreten waren. Metalle und Legierungen blieben mit 8 Labors relativ wichtig. Jeweils 6 Labors stellten der Maschinen- und Instrumentenbau und der Bereich Mathematik, Informatik, Elektronik. 5 Labors untersuchten Fragestellungen zum Thema Chemie und nur noch ein Labor, das nach 2011 ausgelaufen war, beschäftigte sich mit nichtmetallischen Werkstoffen.

Betrachtet man die 66 noch aktiven Labors, dann fällt auf, dass ein neuer thematischer Cluster hinzugekommen ist, nämlich die Wirtschafts-, Sozial- und Rechtswissenschaften, welches 2 Labors für sich beansprucht. Der am stärksten vertretene Bereich ist Mathematik, Informatik, Elektronik (16 Labors), wie schon bei den vor 2011 ausgelaufenen Labors, gefolgt von Life Science und Umwelt (13 Labors) sowie Medizin (11 Labors). Viele Forschungen sind im Bereich der Chemie, dem 9 Labors zugeteilt sind sowie im Bereich der Metalle und Legierungen mit 7 Labors, angesiedelt. Weniger stark vertreten sind der Maschinen- und Instrumentenbau und die nichtmetallischen Legierungen mit 
jeweils 4 Labors. Hinsichtlich der Themenzuordnung der JR-Zentren dominiert derzeit eindeutig der Bereich Mathematik, Informatik, Elektronik.

Tabelle 4: Übersicht Forschungsthemen (Themencluster) JR-Zentren

\begin{tabular}{|c|c|c|c|c|c|c|}
\hline \multirow{3}{*}{$\begin{array}{l}\text { JR-Zentren } \\
\text { Forschungsthema } \\
\text { Chemie }\end{array}$} & \multicolumn{2}{|c|}{$\begin{array}{c}\text { Aktiv in der } \\
\text { Verlängerungsphase }\end{array}$} & \multicolumn{2}{|c|}{ Aktiv } & \multicolumn{2}{|c|}{ Gesamt } \\
\hline & Anzahl & in $\%$ & Anzahl & in $\%$ & Anzahl & in $\%$ \\
\hline & \multicolumn{6}{|c|}{ Chemie } \\
\hline \multicolumn{7}{|l|}{ Life Sciences und Umwelt } \\
\hline \multicolumn{7}{|l|}{ Maschinen- und Instrumentenbau } \\
\hline Mathematik, Informatik, Elektronik & 5 & $83 \%$ & 1 & $100 \%$ & 6 & $86 \%$ \\
\hline \multicolumn{7}{|l|}{ Medizin } \\
\hline Nichtmetallische Werkstoffe & 1 & $17 \%$ & & $0 \%$ & 1 & $14 \%$ \\
\hline \multicolumn{7}{|l|}{ Metalle und Legierungen } \\
\hline \multicolumn{7}{|l|}{ Wirtschafts-, Sozial- und Rechtswissenschaften } \\
\hline Gesamt & 6 & $100 \%$ & 1 & $100 \%$ & 7 & $100 \%$ \\
\hline
\end{tabular}

Quelle: https://www.cdg.ac.at/Stand Juli 2016

\subsection{Statistische Analyse der CD-Labors}

\section{Anzahl und Verlauf der CD-Labors}

Im Rahmen dieser Studie wurden insgesamt 45 CD-Labors betrachtet, wobei einige von ihnen einen untypischen Verlauf aufwiesen (Unterbrechungen, vorzeitiger Abbruch). Eines der CD-Labors musste aufgrund eines Auslandsaufenthaltes des CD-Labor Leiters pausieren. Zwei weitere CD-Labors wurden wegen Konkurs der Partnerunternehmen vorzeitig geschlossen, konnten jedoch mit anderen Unternehmen wieder aufgenommen werden. In den statistischen Aufzeichnungen werden diese drei CD-Labors daher vor und nach der Unterbrechung als eigener Datenpunkt angeführt. Da sie jedoch jeweils nur mit einer Abschlussevaluierung vertreten sind, reduziert sich die Anzahl der untersuchten CD-Labors auf 42.

Außerdem wurde ein CD-Labor nach der 5-Jahresevaluierung vorzeitig geschlossen, ein anderes wurde aufgrund eines Konkurses des Partnerunternehmens ebenfalls vorzeitig geschlossen. Bei dem zuletzt erwähnten Labor wurde ein Bericht zur 2-Jahresevaluierung geliefert, allerdings fand keine 2Jahresevaluierung mehr statt. Darüber hinaus gab es drei Fälle vorzeitiger Schließungen, die durch den Ausstieg von Unternehmen bedingt waren. 
Insgesamt wiesen daher nur acht von zehn CD-Labors ${ }^{28}$ einen regulären Verlauf auf. Besonders positiv ist anzumerken, dass es in zwei Fällen gelungen ist, alternative Unternehmen nach Konkurs des ursprünglichen Partners aus der Wirtschaft zu finden. Obwohl es für die betroffenen CDLaborleiter nicht erfreulich ist, wenn ein CD-Labor geschlossen wird, so zeigt dies (exemplarisch) auf, dass sowohl die wissenschaftliche Kontrolle durch die Evaluierungen als auch die wirtschaftliche Prüfung durch die Unternehmen funktioniert, und ein CD-Labor den jeweiligen Standards entsprechen muss, um eine Weiterführung gewährt zu bekommen.

Tabelle 5: Verlauf der CD-Labors

\begin{tabular}{lrr} 
Vollständige Laufzeit & 37 & $88,1 \%$ \\
Regulärer Verlauf & 34 & \\
$\begin{array}{l}\text { Pausierung } \\
\text { Konkurs Unternehmen / Wiederaufnahme }\end{array}$ & 1 & \\
& 2 & \\
Vorzeitige Beendigung & 5 & $11,9 \%$ \\
Negative Evaluierung & 1 & \\
Konkurs Unternehmen & 1 & \\
Ausstieg Unternehmen & 3 & \\
& & \\
Summe & 42 & $100,0 \%$ \\
\hline
\end{tabular}

Quelle: $C D G$, Economica/IWI.

Im Durchschnitt wurden für 37 CD-Labors mit voller Laufzeit Auslaufphasen zwischen fünf und sechs Monaten gewährt. Bezogen auf die 25 von 37 CD-Labors mit voller Laufzeit, die eine Auslauffinanzierung besaßen, wurde diese durchschnittlich für eine Auslaufphase des CD-Labors zwischen sieben bis acht Monate verwendet.

\subsubsection{Basisanalyse der CDG-Abschlussevaluierungen}

Zum Zeitpunkt der Datenauswertung (Oktober 2016) lagen dem Evaluatoren-Team von den insgesamt 45 bzw. den 42 verschiedenen, seit 2011 ausgelaufenen und in Modul 1 zu evaluierenden CD-Labors, 41 Abschlussfragebögen von Laborleiterinnen und Laborleitern vor, sodass lediglich ein Abschlussfragebogen eines CD-Labors nicht vorhanden war. ${ }^{29}$

\footnotetext{
${ }^{28} 34 / 42=81 \%$

${ }^{29}$ Die Gesamtzahl N liegt somit bei 42. Für einige Fragen konnten auch die Angaben des 42. CD-Labors erhoben werden, sodass hier $\mathrm{N}=\mathrm{n}=42$ gilt.
} 
Tabelle 6: Übersicht Abschlussfragebögen CD-Labors

\begin{tabular}{|l|r|r|r|r|}
\hline CD-Labors & Gesamt & Vorhanden & NZ & Fehlend \\
\hline Abschlussfragebogen & 45 & 41 & 3 & 1 \\
\hline
\end{tabular}

Quelle: https://www.cdg.ac.at/ ; NZ nicht zutreffend

Der Fragebogen gliedert sich in drei Abschnitte, und zwar in Aspekte betreffend den akademischen Bereich, die Unternehmenspartner sowie die Interessenslage der öffentlichen Hand und der CDG.

Insgesamt waren 928 wissenschaftliche Mitarbeiter während der Laufzeit in den untersuchten CDLabors tätig. Dabei waren DiplomandInnen und DissertantInnen (je 40\%) am stärksten vertreten. Die übrigen 20\% entfielen auf Postdocs. Somit reduzierte sich der Anteil der DissertantInnen im Vergleich zur Vorgängerevaluierung 2011 geringfügig. ${ }^{30}$

Tabelle 7: Anzahl der wissenschaftlichen Mitarbeiter, wissenschaftlichen Arbeiten

\begin{tabular}{|l|r|r|r|}
\hline \multicolumn{1}{|c}{ Wissenschaftliche Mitarbeiter während der Laufzeit des CD-Labors } \\
\hline DiplomandInnen & 371 & 9,0 & $40 \%$ \\
\hline DissertantInnen & 369 & 9,0 & $40 \%$ \\
\hline Postdocs & 188 & 4,6 & $\mathbf{2 0 \%}$ \\
\hline Gesamt & $\mathbf{9 2 8}$ & $\mathbf{2 2 , 9}$ & $\mathbf{1 0 0 \%}$ \\
\hline \multicolumn{3}{|c|}{ Abgeschlossene wissenschaftliche Arbeiten im Rahmen des CD-Labors } \\
\hline Diplomarbeiten & 383 & 9,3 & $60 \%$ \\
\hline Dissertationen & 235 & 5,7 & $37 \%$ \\
\hline Habilitationen & 20 & 0,5 & $3 \%$ \\
\hline Gesamt & $\mathbf{6 3 8}$ & $\mathbf{1 5 , 8}$ & $\mathbf{1 0 0 \%}$ \\
\hline
\end{tabular}

Quelle: Abschlussfragebögen der CDG (n=41).

Während der Laufzeit des CD-Labors wurde eine Reihe von wissenschaftlichen Arbeiten abgeschlossen. Mehr als die Hälfte, nämlich 60\%, dieser insgesamt 638 abgeschlossenen Arbeiten waren Diplomarbeiten. Einen weiteren wesentlichen Anteil von 37\% machten die Dissertationen aus. Weniger häufig wurden hingegen Habilitationen (3\%) abgeschlossen. Bemerkenswert ist auch der durchschnittliche Output pro Labor. Dieser beläuft sich auf über neun Diplomarbeiten, sowie beinahe sechs Dissertationen pro untersuchtem Labor. Wie der Unterschied zwischen DiplomandInnen und

\footnotetext{
${ }^{30}$ Die Gründe für die etwas veränderte Mitarbeiterstruktur in den CD-Labors bedarf einer weiterführenden Analyse, die die allgemeine Entwicklung der Anzahl und Struktur der Abschlüsse an den Universitäten berücksichtigt.
} 
abgeschlossenen Diplomarbeiten zeigt, beteiligt sich ein weiter Personenkreis an der Forschungstätigkeit der CD-Labors, welcher sich nicht „nur" aus den aus Fördermitteln der CDG finanzierten MitarbeiterInnen der CD-Labors zusammensetzt.

Die nachstehende Tabelle zeigt die Anzahl der referierten, der nicht referierten sowie der sonstigen Publikationen. Den größten Teil machten hier die referierten Publikationen aus. Insgesamt waren es 2.487, beziehungsweise durchschnittlich 59 Publikationen pro untersuchtem Labor, die sich aus Journalen, Bucheinträgen und Konferenzpublikationen zusammensetzen.

Mit 1.879 Journalen wurden mehr als dreimal so viele Journale wie Konferenzpublikationen (552) referiert. Einen nochmals wesentlich geringeren Anteil machten die 56 referierten Buchbeiträge aus.

Tabelle 8: Publikationen im Rahmen des CD-Labors

\begin{tabular}{|c|c|c|}
\hline & Anzahl & $\varnothing$ pro Labor \\
\hline \multicolumn{3}{|c|}{ Referierte Publikationen } \\
\hline Journale & 1.879 & 44,7 \\
\hline Buchbeiträge & 56 & 1,3 \\
\hline Konferenzpublikationen & 552 & 13,1 \\
\hline Gesamt & 2.487 & 59,2 \\
\hline \multicolumn{3}{|c|}{ Nicht referierte Publikationen } \\
\hline Fachzeitschriften & 304 & 7,2 \\
\hline Proceedings & 39 & 0,9 \\
\hline Konferenzpublikationen & 108 & 2,5 \\
\hline Buchbeiträge & 4 & 0,1 \\
\hline Gesamt & 455 & 10,7 \\
\hline \multicolumn{3}{|c|}{ Weitere Publikationen } \\
\hline Publikationen für die breite Öffentlichkeit & 352 & 8,4 \\
\hline Monographien & 76 & 1,8 \\
\hline Lehrbücher & 9 & 0,2 \\
\hline Gesamt & 437 & 10,4 \\
\hline
\end{tabular}

Quelle: Abschlussfragebögen der CDG (n=42).

Unter der Überschrift „Nicht referierte Publikationen“ werden Fachzeitschriften, Proceedings, Konferenzpublikationen und Buchbeiträge ausgewiesen. Der größte Anteil, nämlich 304 der insgesamt 455 nicht referierten Publikationen, entfiel auf die Fachzeitschriften, gefolgt von 108 Konferenzpublikationen. Außerdem wurden 39 Proceedings und vier Buchbeiträge in den untersuchten CDLabors erstellt. Zur Kategorie „Weitere Publikationen“ gehörten Publikationen für die breite Öffentlichkeit (352), Monographien (76) und Lehrbücher (9). 
Wie aus Tabelle 9 ersichtlich ist, wurden insgesamt 3.474 Konferenzbeiträge erfasst, d.h. im Durchschnitt 86,8 pro Labor. Diese Beiträge lassen sich in drei Kategorien („Geladener Vortrag“, "Vortrag" und „Poster") unterteilen. Insbesondere hebt sich die Kategorie „Vortrag“ mit einer Anzahl von 1.554 weit von den beiden anderen Kategorien (jeweils 932 bzw. 988) ab. Über die gesamte Laufzeit eines durchschnittlichen CD-Labors entspricht dies über 12 Konferenzbeiträge pro Jahr.

Tabelle 9: Konferenzteilnahmen

\begin{tabular}{|l|r|r|}
\hline & Anzahl & $\varnothing$ pro Labor \\
\hline \multicolumn{3}{|c|}{ Konferenzbeitrag } \\
\hline Geladener Vortrag & 932 & 23,3 \\
\hline Vortrag & 1.554 & 38,8 \\
\hline Poster & 988 & 24,7 \\
\hline Gesamt & 3.474 & 86,8 \\
\hline
\end{tabular}

Quelle: Abschlussfragebögen der CDG $(n=40)$.

Insgesamt wurden 146 Preise und 10 Rufe an MitarbeiterInnen des CD-Labors während und nach der Laufzeit des CD-Labors vergeben. 33 der PreisträgerInnen waren Laborleiter/-leiterin eines CDLabors, mit einer etwas größeren Anzahl von 49 Preisen wurden Postdocs prämiert. Die meisten Auszeichnungen gingen an die Gruppe der Dissertanten und Dissertantinnen, die mit 70 Preisen etwa $45 \%$ aller Preise verliehen bekamen. Sehr gering blieb die Menge der Verleihungen an Diplomanden / Diplomandinnen (4). Im Mittel erhielten Mitarbeiter eines durchschnittlichen CD-Labors 3,8 Auszeichnungen, wovon 3,6 auf Preise und 0,2 auf Rufe zurückzuführen sind.

Tabelle 10: Anzahl der Preise/Rufe im Rahmen des CD-Labors

\begin{tabular}{|c|c|c|}
\hline & Anzahl & $\varnothing$ pro Labor \\
\hline \multicolumn{3}{|c|}{ Preise / Rufe } \\
\hline Laborleiterln & 33 & 0,8 \\
\hline Postdoc & 49 & 1,2 \\
\hline Dissertantln & 70 & 1,7 \\
\hline Diplomandln & 4 & 0,1 \\
\hline Gesamt & 156 & 3,8 \\
\hline
\end{tabular}

Quelle: Abschlussfragebögen der CDG ( $n=41)$.

Die Frage „Für wie viele Fachzeitschriften waren Sie während der Laufzeit des CD-Labors als Gutachterln tätig? Wie viele Artikel haben Sie durchschnittlich pro Jahr und Fachzeitschrift begut- 
achtet?" der Abschlussevaluierung weist bei den Antworten eine hohe Variation auf. Es gibt bei der Anzahl der Fachzeitschriften Werte bis zu 61, und auch die maximale Anzahl von 35 Artikeln (bei 46 Fachzeitschriften) scheint sehr hoch. Aus diesem Grund wird hier der Median der Rückmeldungen mit acht Zeitschriften und drei Artikel pro Jahr und Fachzeitschrift als statistische Kenngröße angeführt.

Von den insgesamt 476 Kooperationen mit anderen Forschergruppen im Rahmen des CD-Labors waren etwa 243 Neuzugänge dabei. In etwa gleicher Höhe (233) waren bereits vor Beginn des CDLabors kooperierende Forschergruppen vorhanden.

Im Schnitt wurden beinahe 13 Kooperationen im Rahmen des CD-Labors ${ }^{31}$ mit anderen Forschergruppen durchgeführt, wobei etwa die Hälfte der Kooperationen schon vor der Gründung des CDLabors bestand.

Tabelle 11: Kooperationen mit anderen Forschergruppen im Rahmen des CD-Labors

\begin{tabular}{|c|c|c|c|c|c|}
\hline Forschergruppe & Neu & $\begin{array}{l}\text { Vor- } \\
\text { handen }\end{array}$ & Gesamt & $\begin{array}{l}\varnothing \text { pro } \\
\text { Labor }\end{array}$ & $\frac{\%-}{\text { Anteil }}$ \\
\hline univ. Institut in Österreich & 45 & 58 & 103 & 2,8 & $22 \%$ \\
\hline univ. Institut im Ausland & 97 & 99 & 196 & 5,3 & $41 \%$ \\
\hline Kompetenzzentrum Kind/Knet/Kplus & 12 & 7 & 19 & 0,5 & $4 \%$ \\
\hline CD-Labor & 10 & 10 & 20 & 0,5 & $4 \%$ \\
\hline außeruniv. Forschungseinrichtung Inland & 17 & 14 & 31 & 0,8 & $6 \%$ \\
\hline außeruniv. Forschungseinrichtung Ausland & 46 & 28 & 74 & 2,0 & $16 \%$ \\
\hline Sonstige & 16 & 17 & 33 & 0,9 & $7 \%$ \\
\hline Gesamt & & & 476 & 12,9 & $100 \%$ \\
\hline & 243 & & & 6,6 & $51 \%$ \\
\hline & & 233 & & 6,3 & $49 \%$ \\
\hline
\end{tabular}

Quelle: Abschlussfragebögen der CDG. (n=37)

Die meisten Kooperationen, nämlich 41\% aller Kooperationen mit anderen Forschungsgruppen im Rahmen des CD-Labors, kamen mit universitären Instituten im Ausland zustande. In absoluten Zahlen waren es 196 Kooperationen. Da mit 103 universitären Instituten in Österreich zusammengearbeitet wurde, ist auch diese Forschungsgruppe von großer Bedeutung. Vergleichsweise wenige Kooperationen, nämlich 19 und 20, wurden mit Kompetenzzentren und anderen CD-Labors geschlossen. 16\% aller Kooperationen wurden mit außeruniversitären Forschungseinrichtungen im Ausland

${ }^{31}$ Diese Frage haben 37 CD-Labors detailliert beantwortet. 
geschlossen und $6 \%$ mit außeruniversitären FE-Einrichtungen im Inland. Die verbleibenden 33 Kooperationen beziehen sich auf Forschungseinrichtungen, die keiner der erwähnten Gruppe zuzuordnen sind.

Bemerkenswert ist darüber hinaus, dass mehr neue Kooperationen geschlossen wurden, als bereits bestanden haben. Auch bei den neuen Kooperationen beziehen sich die meisten auf universitäre Institute im Ausland (97), gefolgt von außeruniversitären FE-Einrichtungen im Ausland (46) und universitären Einrichtungen im Inland (45).

\subsubsection{Detailanalyse der CDG-Abschlussevaluierungen}

Im vorangegangen Abschnitt wurden statistische Auswertungen aller Fragebögen der Abschlussevaluierung durchgeführt, die wir in diesem Abschnitt um Detailbetrachtungen der einzelnen CDLabors erweitern. Es werden dabei alle 41 CD-Labors ${ }^{32}$ mit vorhandener Abschlussevaluierung in die Analyse einbezogen, die nun jedoch, im Unterschied zu den vorhergehenden Betrachtungen, auf Fragestellungen mit quantitativem Charakter eingeschränkt werden.

\subsubsection{Budget der CD-Labors}

Zu Beginn dieses Unterkapitels wird darauf Bezug genommen, wie hoch die zur Verfügung gestellten Gesamtmittel (=gesamte Fördermittel von öffentlicher und privater Seite) der einzelnen CD-Labors waren. Diese Kenngröße wird in weiterer Folge auch als Inputfaktor für die geförderten Vorhaben herangezogen. Die verschiedenen Outputs eines Labors können dadurch normiert werden, um Größenunterschiede zwischen den verschiedenen Förderprojekten entsprechend berücksichtigen zu können.

Insgesamt standen den in der Evaluierungsperiode abgelaufenen CD-Labors Gesamtmittel von etwas über 100 Mio. Euro zur Verfügung, woraus sich ein durchschnittliches Budget von 2,38 Mio. Euro pro CD-Labor ergeben würde. Berücksichtigt man jedoch, dass 5 der 42 CD-Labors nicht die volle Laufzeit aufwiesen, und dadurch auch nicht den vollen Budgetumfang bei regulärem Verlauf zeitigten ${ }^{33}$, erhöht sich das durchschnittliche Budget der verbleibenden 37 CD-Labors auf 2,48 Mio. Euro. Im

\footnotetext{
32 Die Budgetzahlen liegen für alle 42 CD-Labors vor, sodass bei dieser Kenngröße alle ausgelaufenen CD-Labors berücksichtigt werden können.

${ }^{33}$ Das Gesagte gilt vor allem für die CD-Labors 7 und 12, die nach 2 bzw. 3 Jahren abgebrochen wurden.
} 
Vergleich dazu waren die mittleren Gesamtmittel der CD-Labors der letzten Evaluierung mit 2,16 bzw. 2,28 (nur volle Laufzeit) Mio. Euro etwas geringer.

Die tatsächlichen Gesamtmittel zeigen, wie auch schon bei der Evaluierung der letzten Förderperiode, eine relativ große Bandbreite. Die gesamten Fördermittel von öffentlicher und privater Seite schwanken je nach Labor zwischen 0,81 und 3,79 Mio. Euro.

\section{Abbildung 6: Fördermittel der CD-Labors in Mio. Euro}

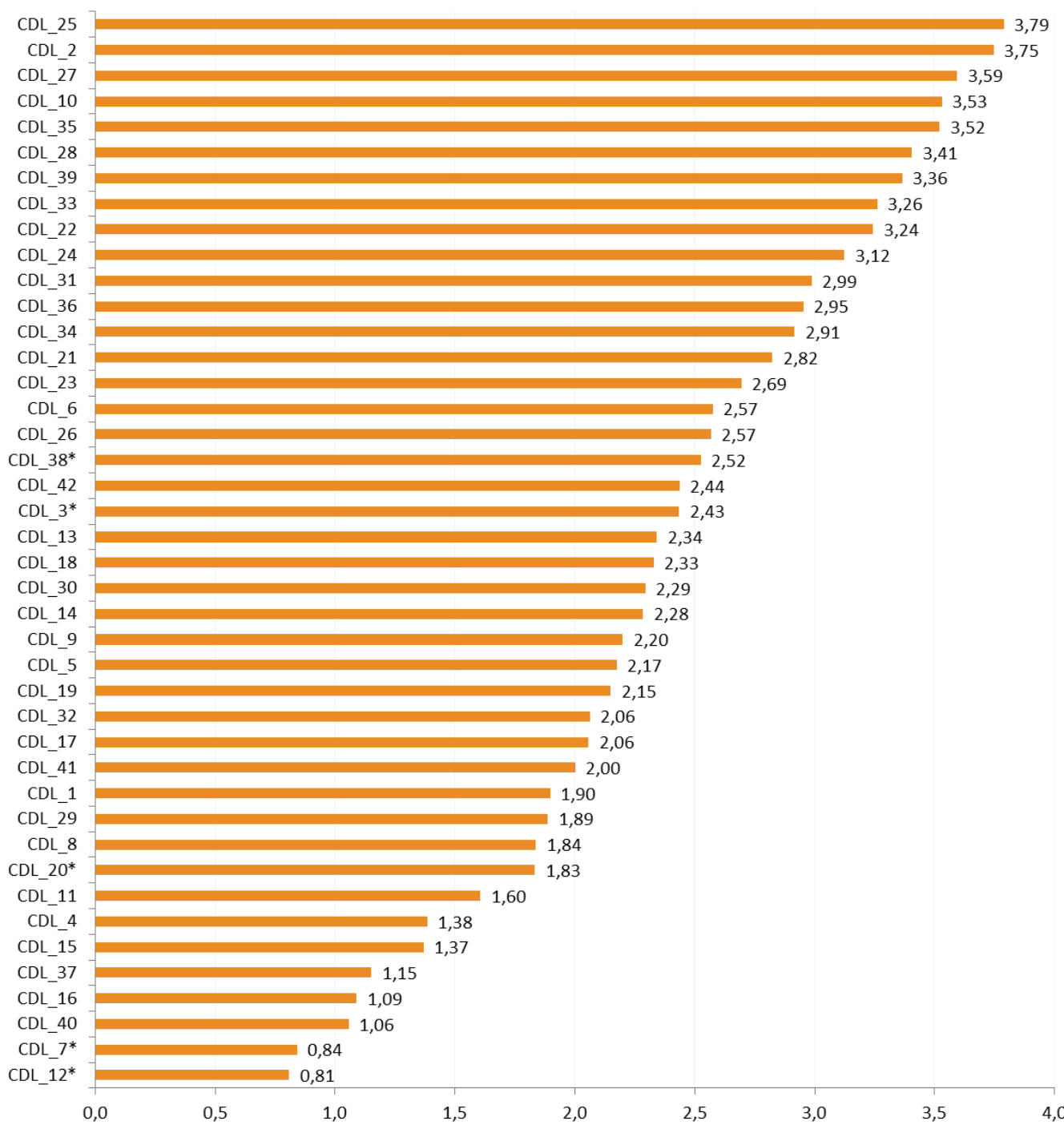

Quelle: Finanzdaten der CDG; Anmerkung *Vorzeitige Beendigung eines CD-Labors (CDL)

Betrachtet man die Entwicklung der Budgetvolumina der CD-Labors zwischen 2010 und 2016 nach dem Jahr des regulären Auslaufens (also ohne Auslaufphase), so lässt sich keine tendenzielle 
Entwicklung erkennen. Die Schwankungen zwischen den einzelnen Jahren waren auch schon in der vorangegangenen Evaluierung vorhanden. Es konnte jedoch festgestellt werden, dass das durchschnittliche Volumen der CD-Labors zwischen 2005 und 2007 von ca. 2 Mio. Euro auf 2,4 Mio. Euro zwischen 2008 und 2010 angestiegen ist. Bildet man den Durchschnittswert der Periode 2011 bis 2013, so erhöht sich das mittlere Gesamtbudget auf 2,56 Mio. Euro. Obwohl eines der beiden 2016 ausgelaufenen CD-Labors lediglich ein Volumen von 1 Million Euro aufwies ${ }^{34}$, sanken die durchschnittlichen Gesamtmittel aller zwischen 2014 und 2016 ausgelaufenen CD-Labors nur geringfügig auf 2,37 Mio. Euro.

Abbildung 7: Mittlere Gesamtmittel der CD-Labors, nach Endjahr

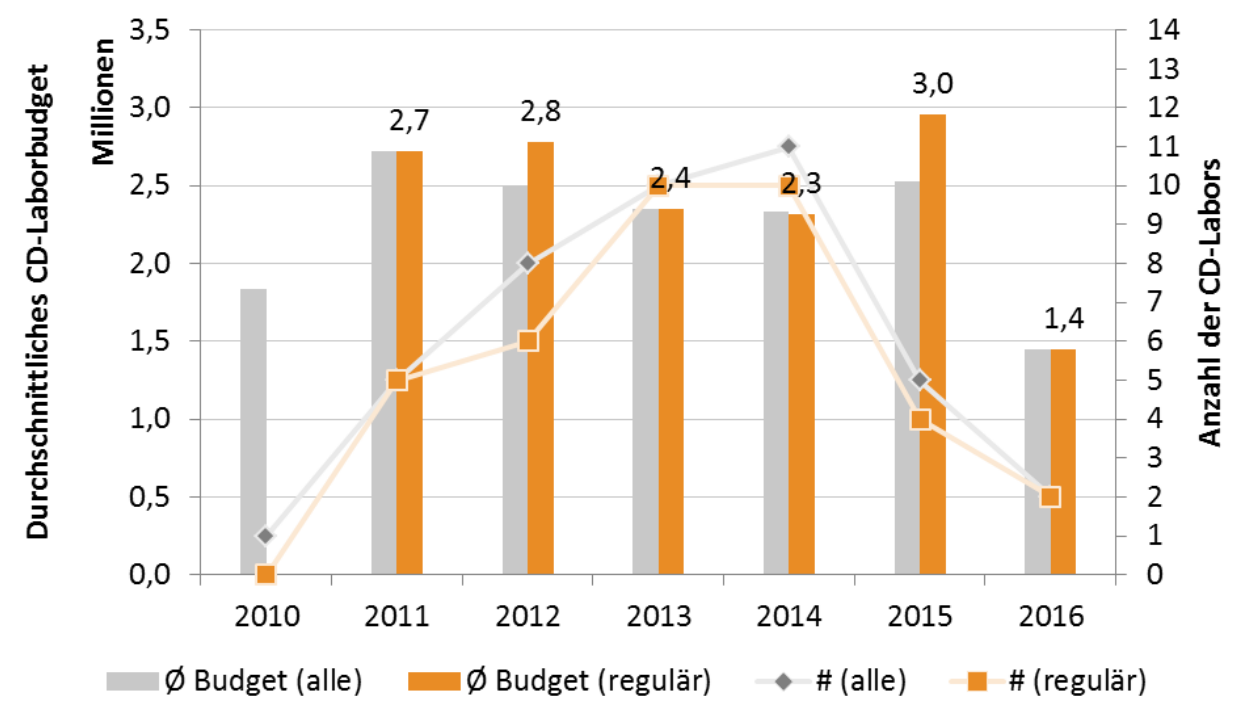

Quelle: Finanzdaten der CDG;

\subsubsection{Aspekte betreffend den akademischen Bereich}

Die Bandbreite an wissenschaftlichen Mitarbeitern ist wie in der vorangegangenen Evaluation sehr groß. Durchschnittlich waren neben dem CD-Laborleiter 22,6 wissenschaftliche Mitarbeiter tätig, die sich auf die Kategorien Diplomanden, Dissertanten und Postdocs verteilen.

Das Programmziel „Entwicklung von Humanressourcen“ kann durch im Rahmen des CD-Labors verfasste Abschlussarbeiten und gewonnene wissenschaftliche Preise, aber auch durch den Wechsel

\footnotetext{
${ }^{34}$ Die Ursache der Reduktion könnte auch in der allgemeinen wirtschaftlichen Lage beim Start der beiden CD-Labors 2009 gelegen haben, was sich eben auch an der geringeren Anzahl von CD-Labors manifestiert.
} 
von CD-Labormitarbeitern in ein Unternehmen erreicht werden. Die Anstellung von Mitarbeitern kann diesen Prozess positiv beeinflussen, denn mit einer Affiliation bei einem CD-Labor geht auch eine gewisse (soziale) Absicherung für (angehende) Wissenschaftler einher. Darüber hinaus kann der dadurch bedingte verstärkte Kontakt mit den Unternehmenspartnern auch für einen Wechsel in diese Unternehmen förderlich sein.

Abbildung 8: Anzahl der wissenschaftlichen Mitarbeiterinnen und Mitarbeiter

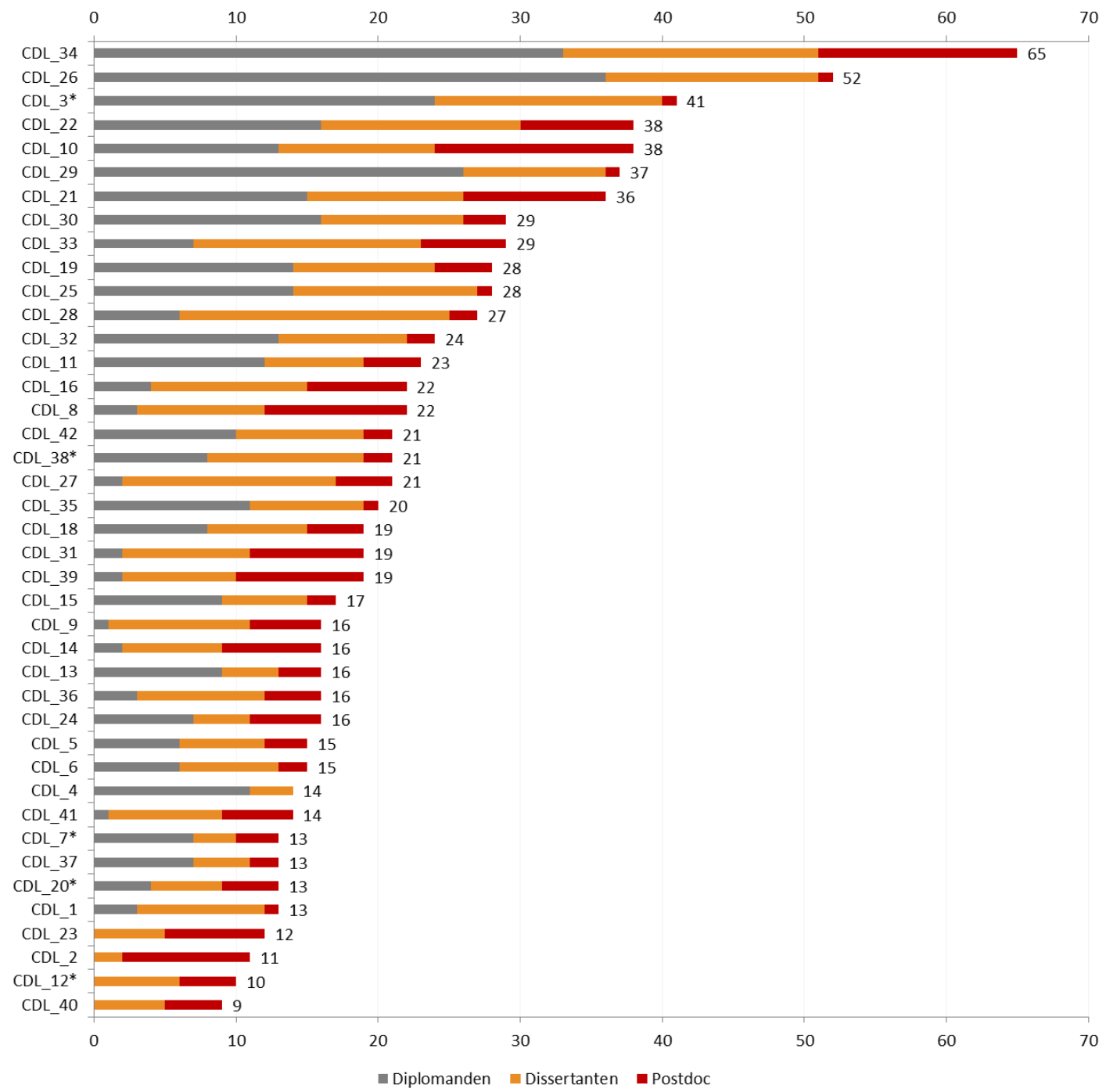

Quelle: Abschlussfragebögen der CDG

Grundsätzlich existiert zwischen der Höhe der Fördermittel und der Anzahl der wissenschaftlichen Mitarbeiterinnen und Mitarbeiter zwar ein gewisser Zusammenhang, jedoch ist dieser sehr schwach ausgeprägt. Der ressourceneffiziente Einsatz der Fördermittel obliegt den CD-Laborleitern, die 
entsprechend der folgenden Abbildungen anscheinend unterschiedliche Unterstützung in Form von wissenschaftlichen Mitarbeitern benötigen. Aus diesem Grunde und um die Vergleichbarkeit mit den Ergebnissen der Vorgängerevaluierung herstellen zu können, werden die einzelnen Outputs absolut und in Relation zu den eingesetzten Fördermitteln dargestellt.

Abbildung 9: Zusammenhang Fördermittel und Anzahl der wissenschaftlichen Mitarbeiterinnen und Mitarbeiter

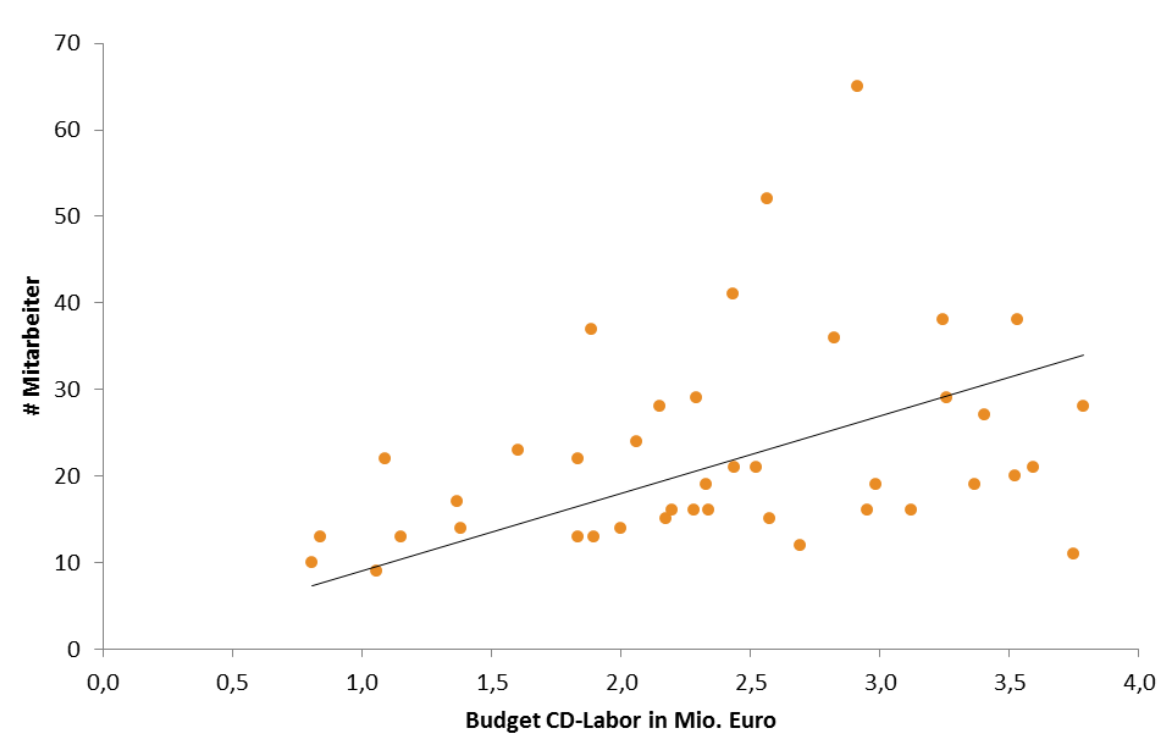

Quelle: Abschlussfragebögen und Finanzdaten der CDG

\section{Akademische Abschlüsse}

Die Anzahl der akademischen Abschlüsse von Diplomarbeiten liegt etwas über der Anzahl von Diplomanden als Mitarbeiter, während die Anzahl von Dissertationen und Habilitationen eines CDLabors teilweise beträchtlich unter der Anzahl der wissenschaftlichen Beschäftigten in der jeweiligen Kategorie liegt (64 \% = \# Dissertationen / \# Dissertanten bzw. 11 \% = \# Abgeschlossene Habilitationen / \# Postdoc). Dies ist besonders bei den Habilitationen nicht überraschend, aber aufgrund der Laufzeit eines CD-Labors erscheint der relative Anteil von Dissertationen gering.

Als normierende Kenngröße werden im vorliegenden Fall die Gesamtmittel (in Mio. Euro) des CDLabors herangezogen. Aus der allgemeinen Grundgesamtheit der 42 CD-Labors liegen Daten zu 41 CD-Labors hinsichtlich der Anzahl abgeschlossener Diplomarbeiten vor. Im Mittel wurden im Rahmen dieser 41 CD-Labors 3,95 Diplomarbeiten pro Fördermillion abgeschlossen, wobei jedoch vier CD- 
Labors keine Diplomarbeit vorweisen konnten. Dies hängt jedoch auch damit zusammen, dass in drei der vier CD-Labors kein Diplomand als wissenschaftlicher Mitarbeiter angestellt war.

Abbildung 10: Anzahl der Diplomarbeiten, absolut und in Relation zu den Gesamtmitteln in Mio. Euro

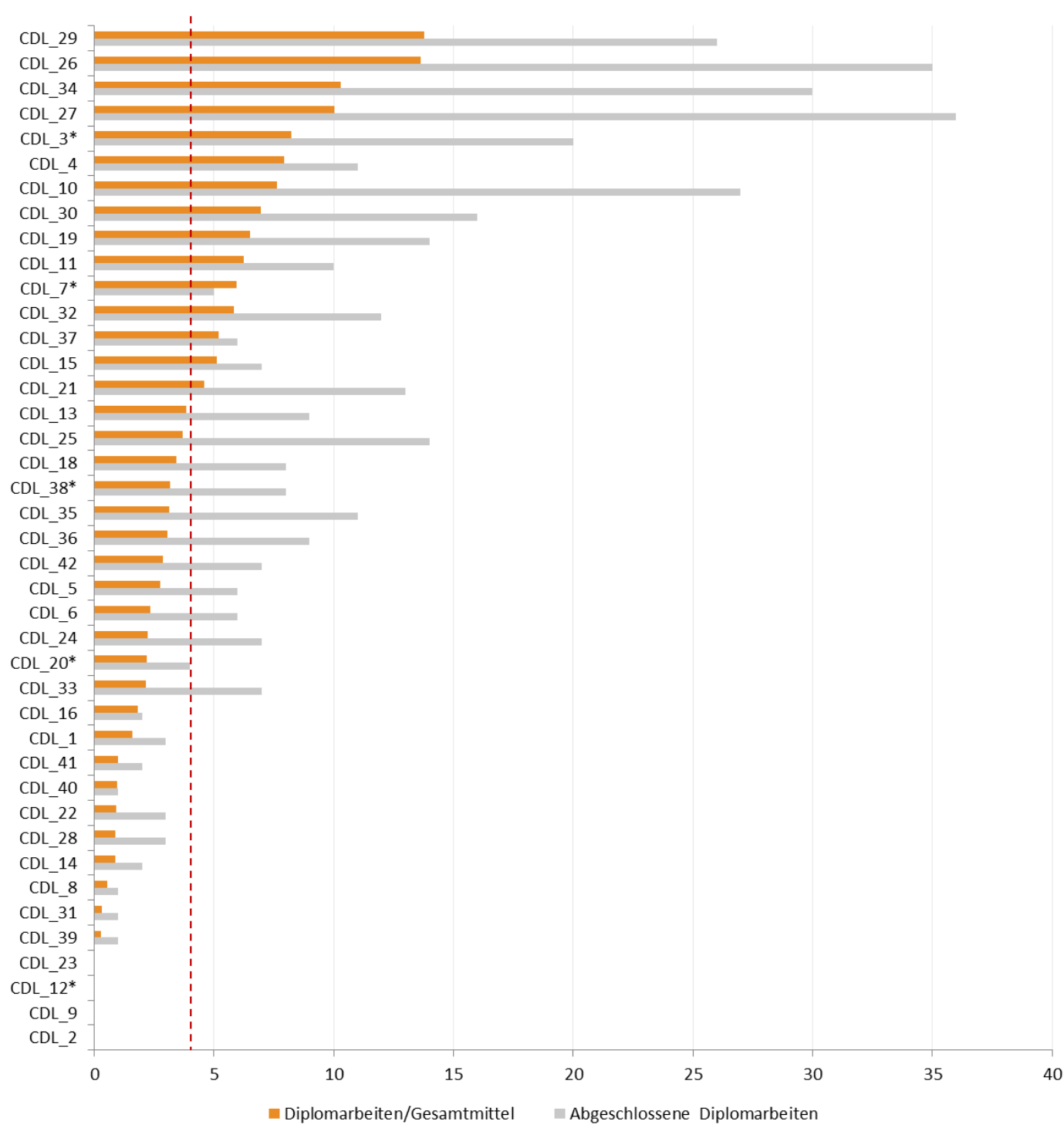

Quelle: Abschlussfragebögen und Finanzdaten der CDG

Bezüglich der Anzahl abgeschlossener Dissertationen liegen abermals Daten von 41 der insgesamt 42 CD-Labors vor, im Rahmen jener es im Durchschnitt 2,52 Dissertationen je Fördermillion gab. Außerdem ist zu erwähnen, dass zumindest eine Dissertation im Rahmen jeder der 41 CD-Labors entstanden ist. 
Abbildung 11: Anzahl der Dissertationen, absolut und in Relation zu den Gesamtmitteln in Mio. Euro

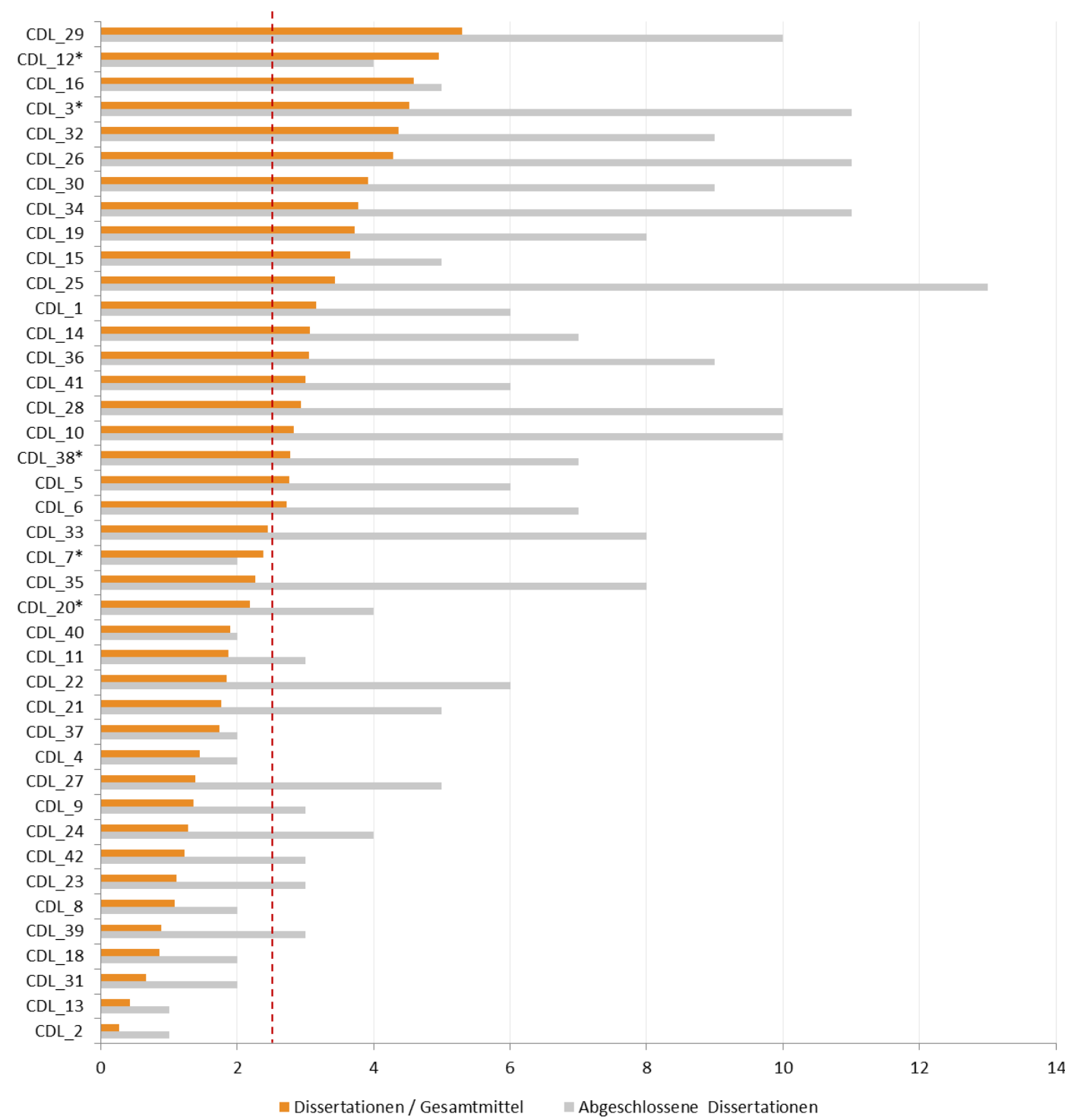

Quelle: Abschlussfragebögen und Finanzdaten der CDG

Abgeschlossene Habilitationen, welche im Zusammenhang mit einem CD-Labor stehen, können zwar nur 13 der 41 betrachteten CD-Labors aufweisen, umso bemerkenswerter aber ist es, dass es vereinzelten CD-Labors sogar gelang, zwei oder mehr Habilitationen hervorzubringen. Durchschnittlich ergibt sich ein Mittelwert von 0,21 Habilitationen pro Fördermillion. 
Abbildung 12: Anzahl der Habilitationen, absolut und in Relation zu den Gesamtmitteln in Mio. Euro

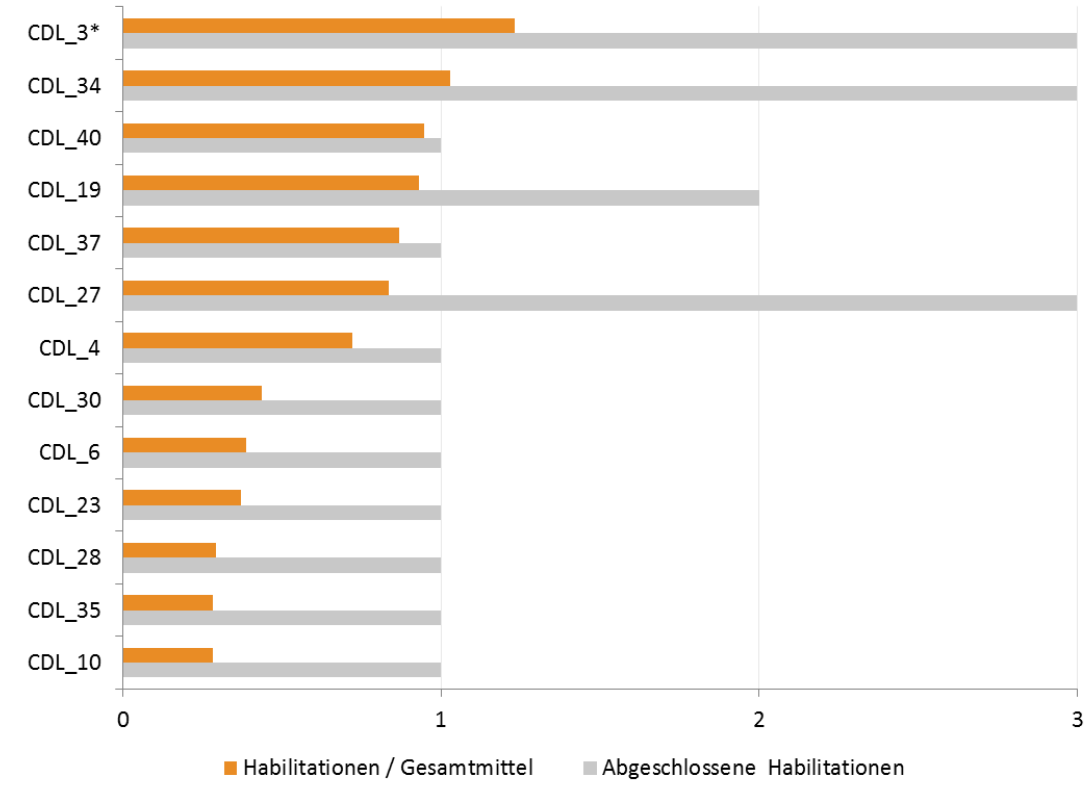

Quelle: Abschlussfragebögen und Finanzdaten der CDG

\section{Beiträge für internationale wissenschaftliche Konferenzen bzw. Zeitschriften}

Im folgenden Abschnitt soll die Aktivität der CD-Labors im Rahmen wissenschaftlicher Konferenzen, sowie deren Beiträge in wissenschaftlichen Zeitschriften analysiert werden. Hierzu liegen wiederum die Daten von 41 CD-Labors vor. Die Auswertung und Zuordnung der diversen Veröffentlichungen wurde anhand der den Schlussevaluierungen beigelegten Referenzlisten durchgeführt.

Wie die nachstehende Abbildung zeigt, wurden pro eingesetzter Fördermillion im Durchschnitt 15 Vorträge bei wissenschaftlichen Konferenzen gehalten. CD-Labor 9 ist bei dieser Betrachtung ein negativer Ausreißer, da kein Vortrag bei internationalen, wissenschaftlichen Konferenzen gehalten wurde. 
Abbildung 13: Vorträge, absolut und in Relation zu den Gesamtmitteln in Mio. Euro

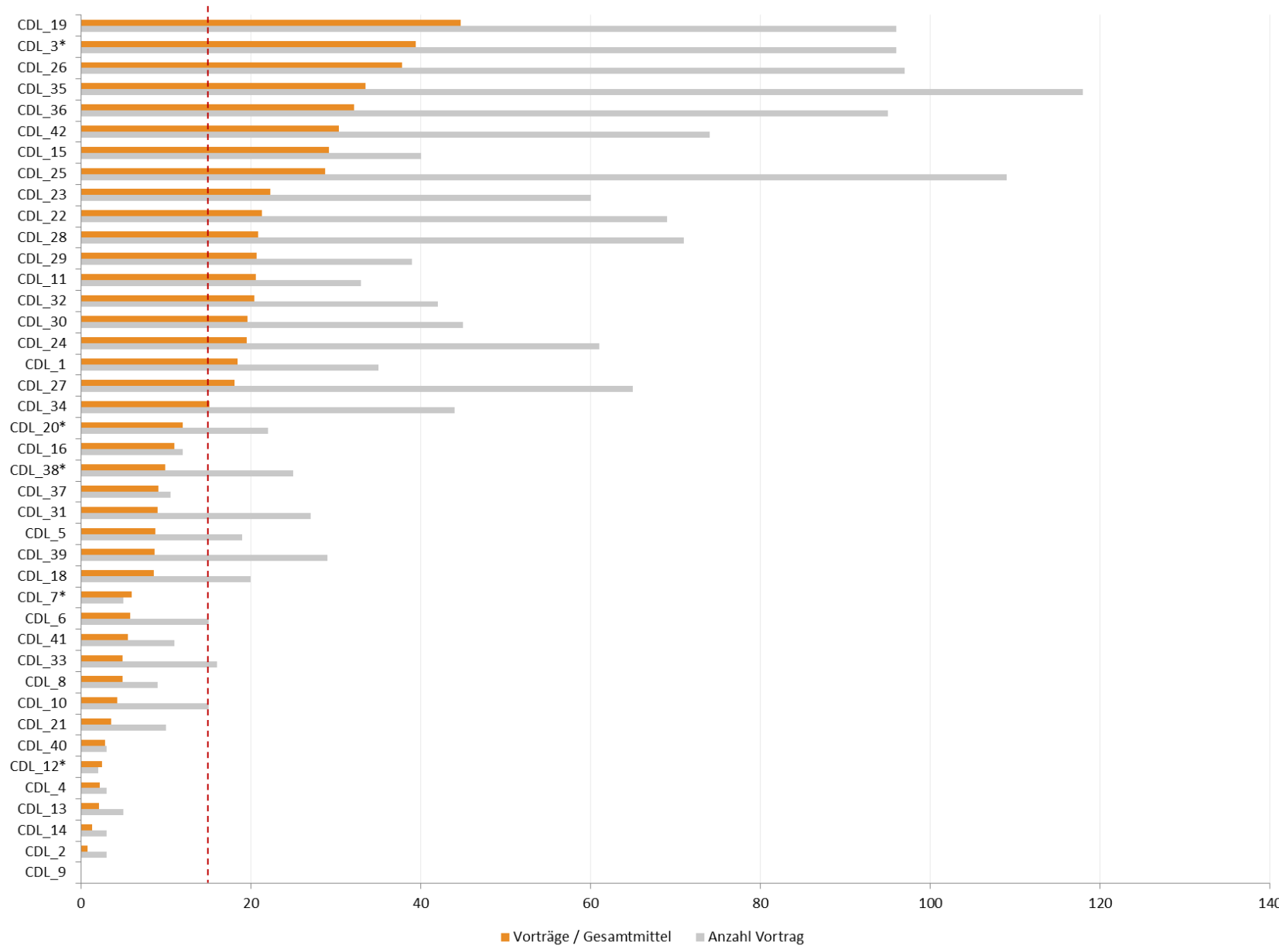

Quelle: Abschlussfragebögen und Finanzdaten der CDG

Die durchschnittliche Anzahl geladener Vorträge bei wissenschaftlichen Konferenzen liegt bei 9,76 je Fördermillion, wobei zu erwähnen ist, dass die Mitarbeiter von vier der 41 betrachteten CD-Labors $(1,9,20$ und 37) im Betrachtungszeitraum keinen geladenen Vortrag gehalten haben. 
Abbildung 14: Geladene Vorträge, absolut und in Relation zu den Gesamtmitteln in Mio. Euro

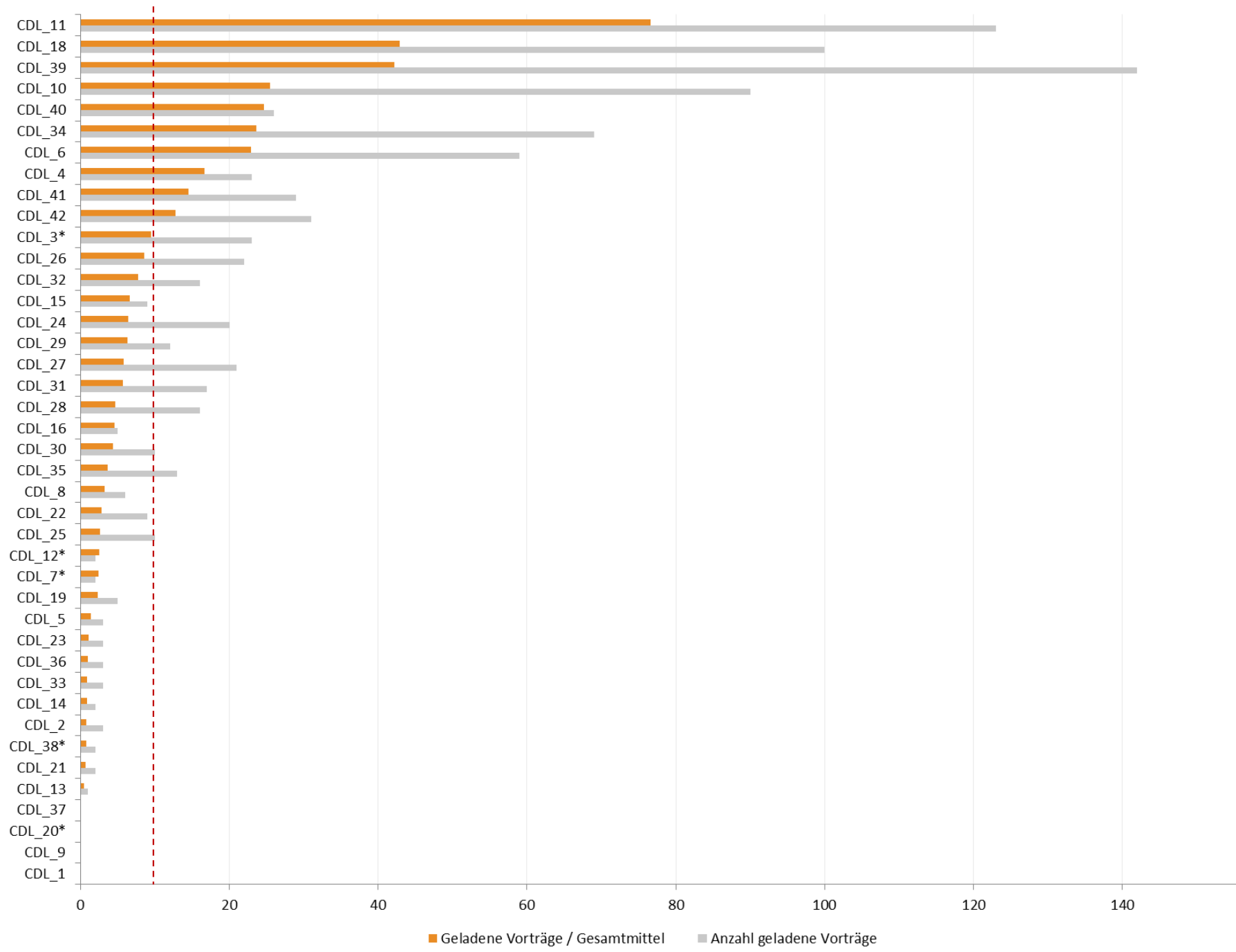

Quelle: Abschlussfragebögen und Finanzdaten der CDG

Hinsichtlich der Platzierung von Postern bei wissenschaftlichen Konferenzen, ergibt sich für die 41 CD-Labors ein Mittelwert von 10,42 Postern pro eingesetzter Fördermillion. Dabei verteilte sich die Gesamtzahl von 988 Postern auf insgesamt 36 CD-Labors. Folgende CD-Labors haben im Betrachtungszeitraum keine Poster im Rahmen wissenschaftlicher Konferenzen präsentiert: 1, 9, 20, 28 und 32 . 
Abbildung 15: Poster, absolut und in Relation zu den Gesamtmitteln in Mio. Euro

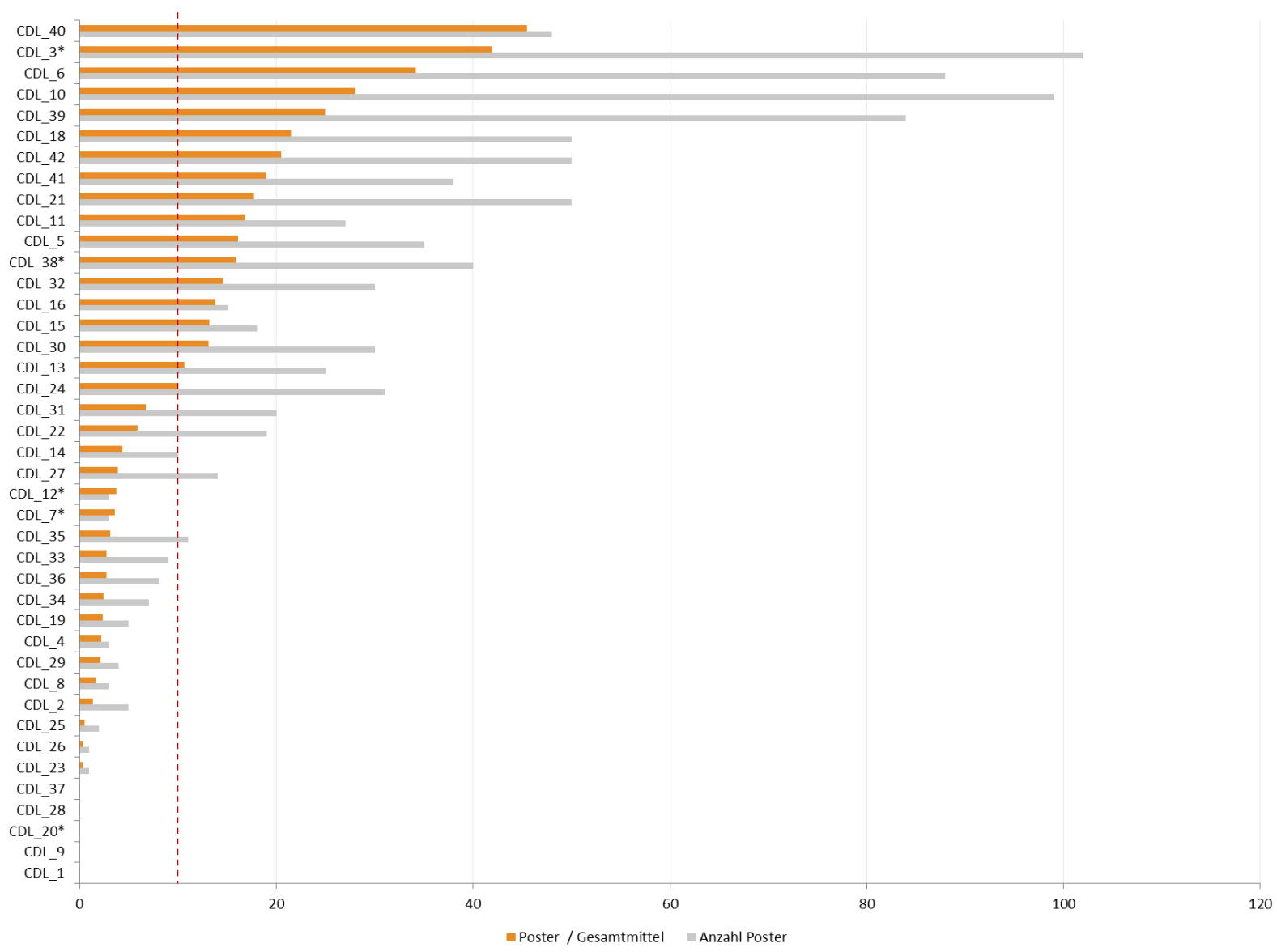

Quelle: Abschlussfragebögen und Finanzdaten der CDG

Vor der Auswertung der wissenschaftlichen Publikationen der CD-Labors sei darauf hingewiesen, dass im Zuge der vorliegenden Untersuchung eine Unterscheidung zwischen referierten und nicht referierten Artikeln vorgenommen werden konnte. Diese Unterscheidung war bei der zuletzt vorgenommenen Untersuchung im Jahr 2012 aufgrund unvollständiger Angaben in den Jahresberichten vor 2006 nicht möglich.

Über alle 42 CD-Labors hinweg wurde ein Mittelwert von 24,16 referierten Artikeln in wissenschaftlichen Zeitschriften pro eingesetzter Fördermillion ermittelt. Wie die nachstehende Abbildung veranschaulicht, wurden von jedem CD-Labor mindestens sieben referierte Artikel veröffentlicht. Spitzenreiter im Hinblick auf die absolute Zahl veröffentlichter, referierter Artikel ist das CD-Labor 3 mit 214 referierten Artikeln, gefolgt vom CD-Labor 26 mit 171 und dem CD-Labor 10 mit 158 referierten Artikeln. Hingegen liegen die CD-Labors 13, 4 und 31 mit jeweils 7, 8 bzw. 19 referierten Artikeln an 
den letzten Positionen. An dieser Stelle sei angemerkt, dass der Impakt-Faktor einer Zeitschrift derzeit nicht erhoben wird ${ }^{35}$.

\section{Abbildung 16: Referierte Artikel, absolut und in Relation zu den Gesamtmitteln in Mio. Euro}

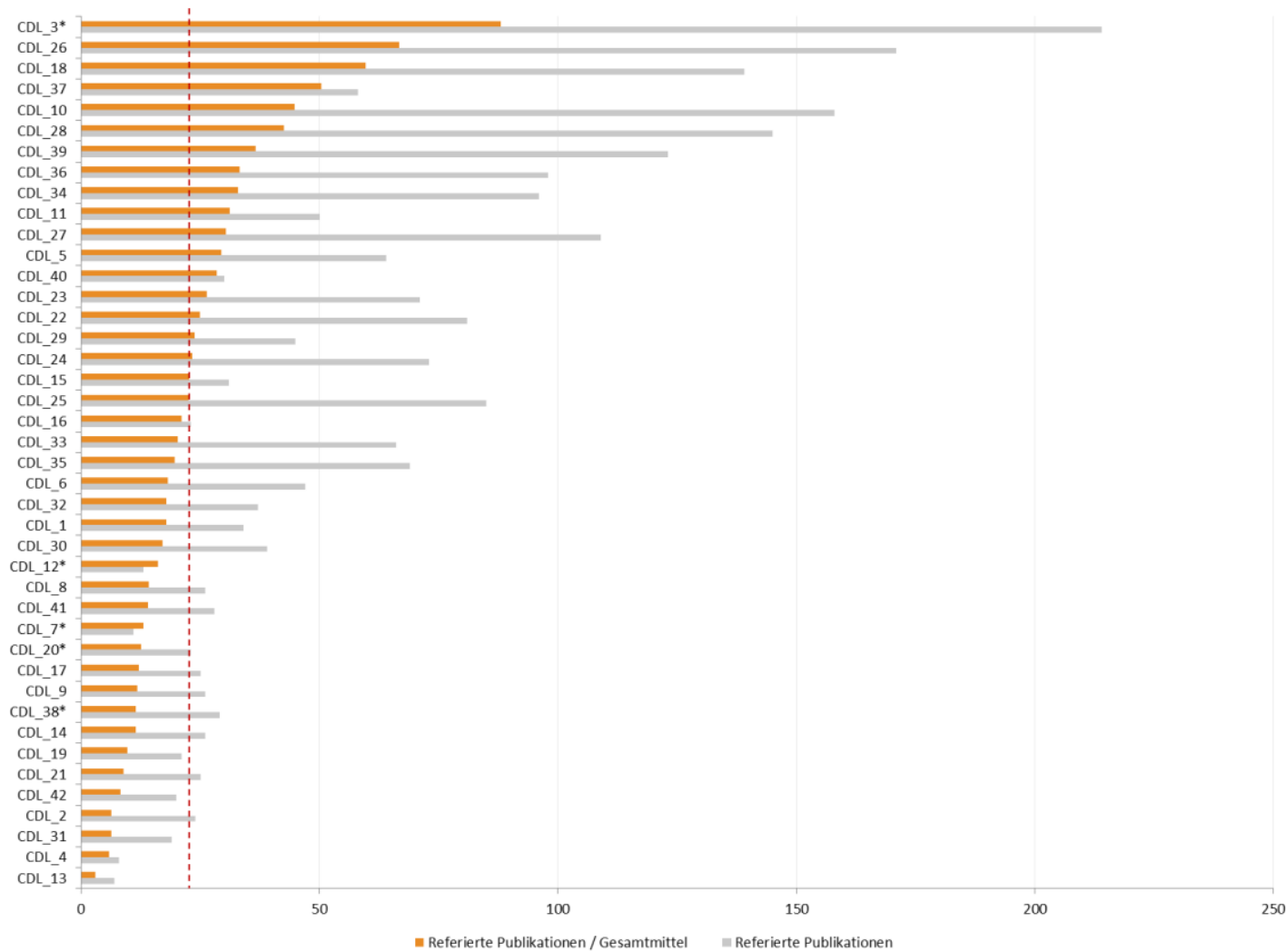

Quelle: Abschlussfragebögen und Finanzdaten der CDG

Der berechnete Mittelwert der veröffentlichten, nicht referierten Artikel aller 42 CD-Labors liegt bei 4,07 je eingesetzter Fördermillion. Dabei verteilt sich die Gesamtzahl von 451 nicht referierten Artikeln auf insgesamt 35 CD-Labors. Demnach gab es bei den CD-Labors 2, 7, 13, 16, 37, 40 und 41 im Betrachtungszeitraum keine Veröffentlichung eines nicht referierten Artikels in einer wissenschaftlichen Zeitschrift.

${ }^{35}$ Für zukünftige Erhebungen sollte daher angedacht werden, auch diese Information aufzunehmen, da erhebliche (Qualitäts-)Unterschiede zwischen den Fachzeitschriften bestehen können. Dies wurde bereits in der Vergangenheit vorgeschlagen, allerdings mit dem Argument, dass es große Unterschiede zwischen den verschiedenen wissenschaftlichen Bereichen und damit den zugeordneten Zeitschriften gibt, nicht umgesetzt. 
Abbildung 17: Nicht referierte Artikel, absolut und in Relation zu den Gesamtmitteln in Mio. EUR

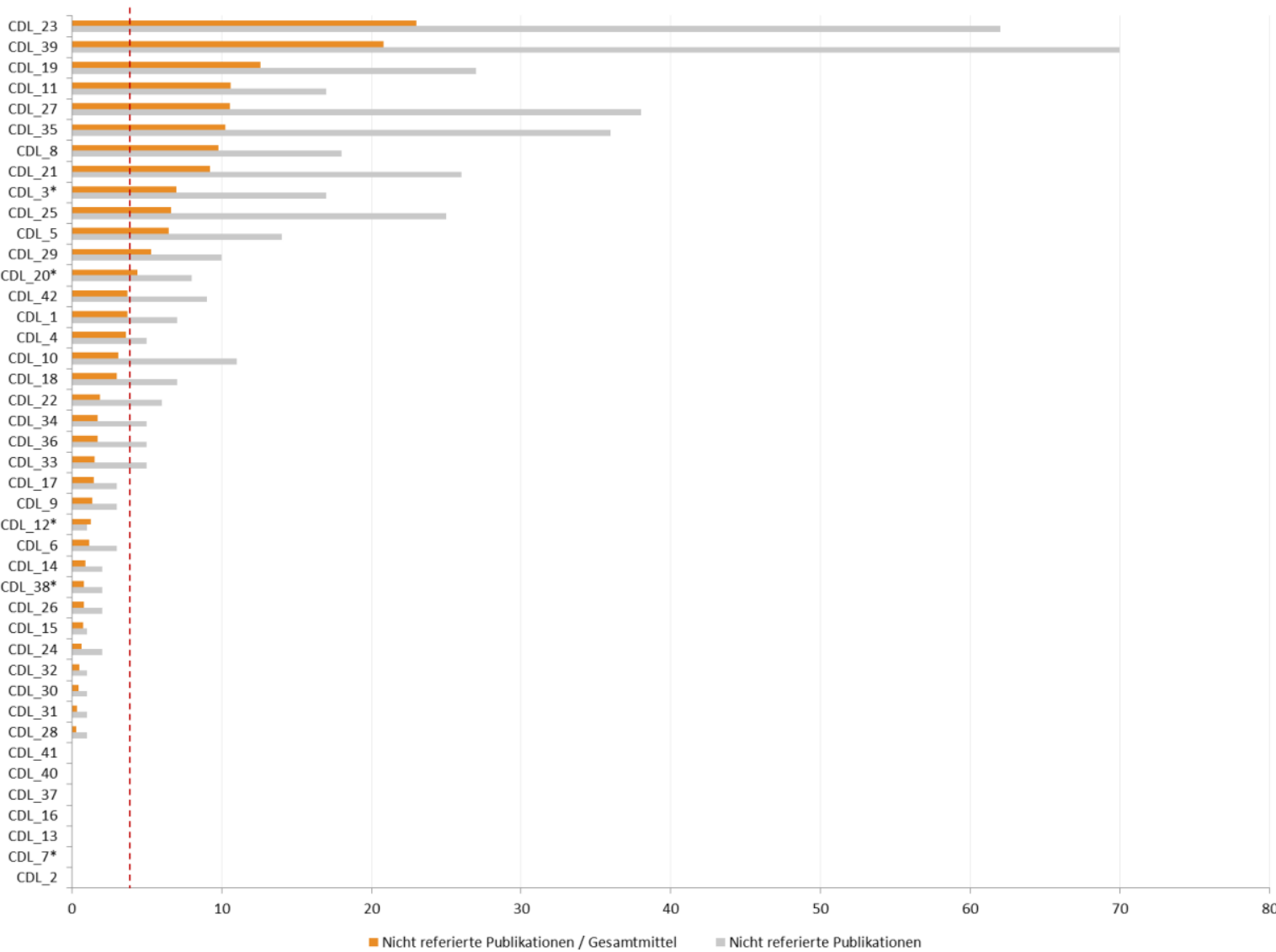

Quelle: Abschlussfragebögen und Finanzdaten der CDG

Da es bei den Jahresberichten teilweise keine Unterscheidung allfälliger Publikationen gab (Monographien, Publikationen in Sammelbänden etc.), wurden jeweils die gesamten Veröffentlichungen in den nachstehenden Vergleich der sonstigen Publikationen pro CD-Labor einbezogen. Im Durchschnitt erfolgten demnach 4,38 sonstige Publikationen je eingesetzter Fördermillion. Wobei sich die Gesamtzahl von 437 allfälligen Publikationen wiederum auf 35 der insgesamt 42 CD-Labors verteilt. 
Abbildung 18: Sonstige Publikationen, absolut und in Relation zu den Gesamtmitteln in Mio. Euro

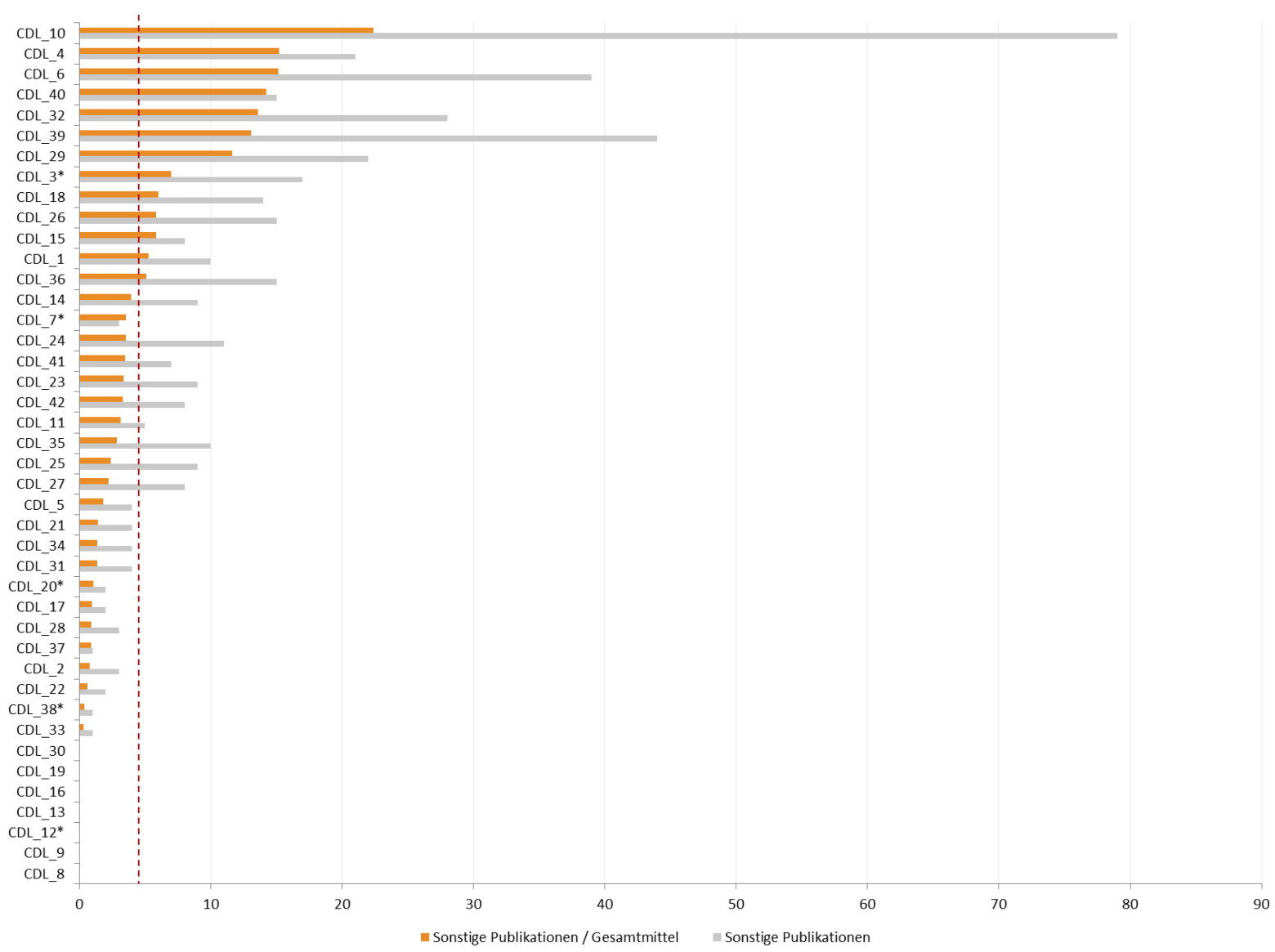

Quelle: Abschlussfragebögen und Finanzdaten der CDG

\section{Weitere Förderungen}

Die weiteren Förderungen anderer Förderstellen wie FWF, FFG, Jubiläumsfonds der Nationalbank u.a., die ohne die Arbeiten im CD-Labor nicht ausgeschüttet worden wären, beliefen sich während der Laufzeit des CD-Labors im Mittel auf 1,32 Mio. Euro. Dieser Wert bezieht sich auf 41 der insgesamt 42 CD-Labors, da vom CD-Labor 17 keine Angaben betreffend Fördervolumen gemacht wurden. Die Gesamtmittel aller CD-Labors konnten somit insgesamt um 54\% erhöht werden, und dies, obwohl neun der 41 CD-Labors keine weitere Förderung lukrieren konnten. 


\section{Abbildung 19: Zusätzliche Fördermittel in Relation zu den Gesamtmitteln}

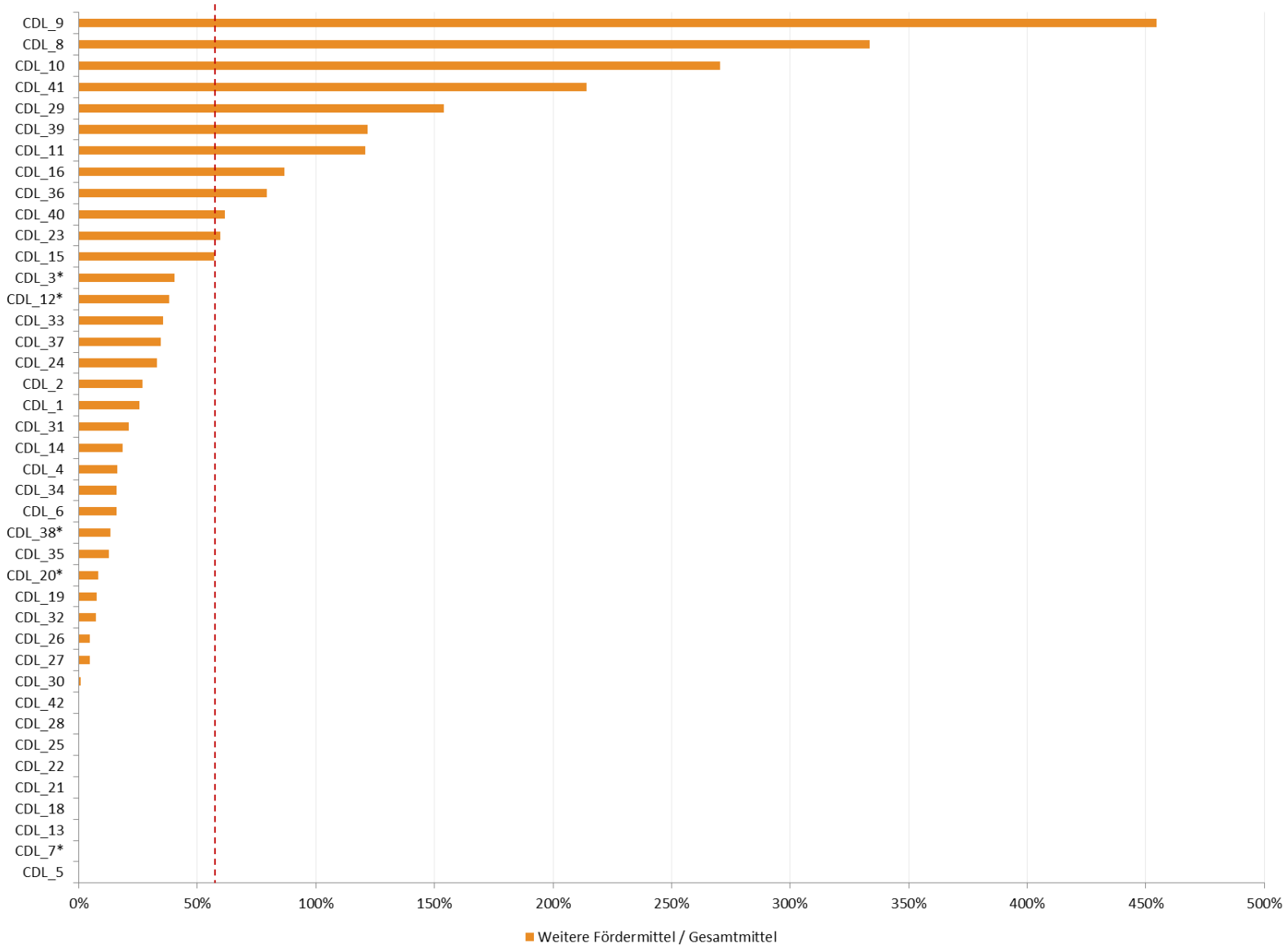

Quelle: Abschlussfragebögen und Finanzdaten der CDG

\section{Preise und Rufe}

In der Gesamtbetrachtung der 41 CD-Labors, von denen Daten über die wissenschaftlichen Auszeichnungen vorliegen, gab es insgesamt 1,62 wissenschaftliche Preise bzw. 0,12 wissenschaftliche Rufe also in Summe also 1,74 wissenschaftliche Auszeichnungen pro Fördermillion. Zu erwähnen ist, dass sieben der 41 betrachteten CD-Labors keine Auszeichnung erhielten, mit anderen Worten, die Gesamtzahl an 156 Auszeichnungen (146 wissenschaftlichen Preise bzw. 10 Rufe) verteilt sich auf 34 CD-Labors. 
Abbildung 20: Preise und Rufe, absolut und in Relation zu den Gesamtmitteln in Mio. Euro

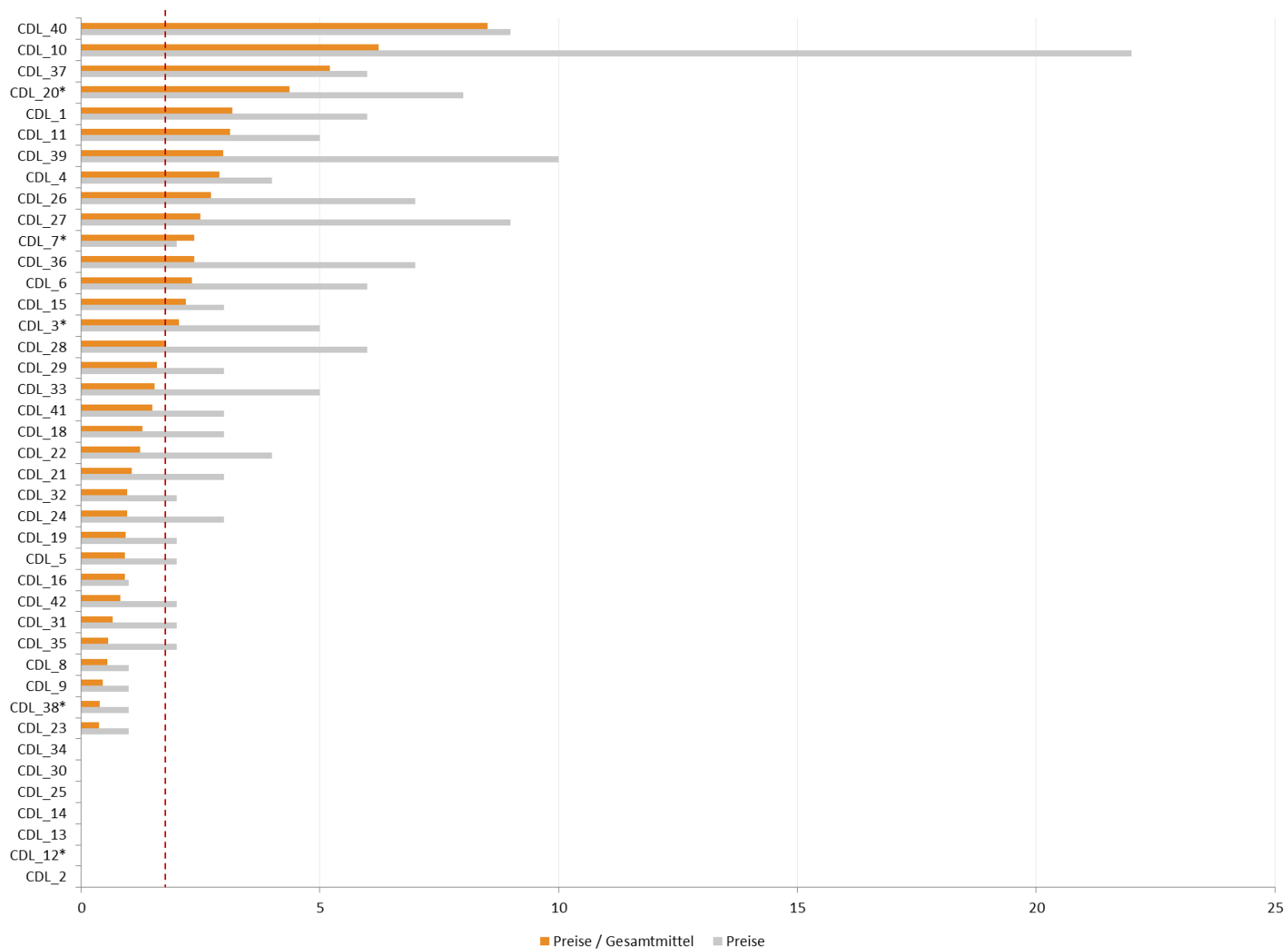

Quelle: Abschlussfragebögen und Finanzdaten der CDG

\section{Gutachtertätigkeit}

Im Hinblick auf die Verfassung von Gutachten der in den jeweiligen CD-Labors angestellten Mitarbeiter, ergab sich eine Gutachtertätigkeit für im Mittel 6,68 Zeitschriften je eingesetzter Fördermillion. Zur Berechnung dieses Wertes wurden die Angaben von 41 CD-Labors herangezogen. Lediglich das CD-Labor 3 verzeichnete während des Betrachtungszeitraumes keine Gutachtertätigkeiten. 
Abbildung 21: Gutachtertätigkeit: Absolute Anzahl der Zeitschriften und in Relation zu den Gesamtmitteln in Mio. Euro

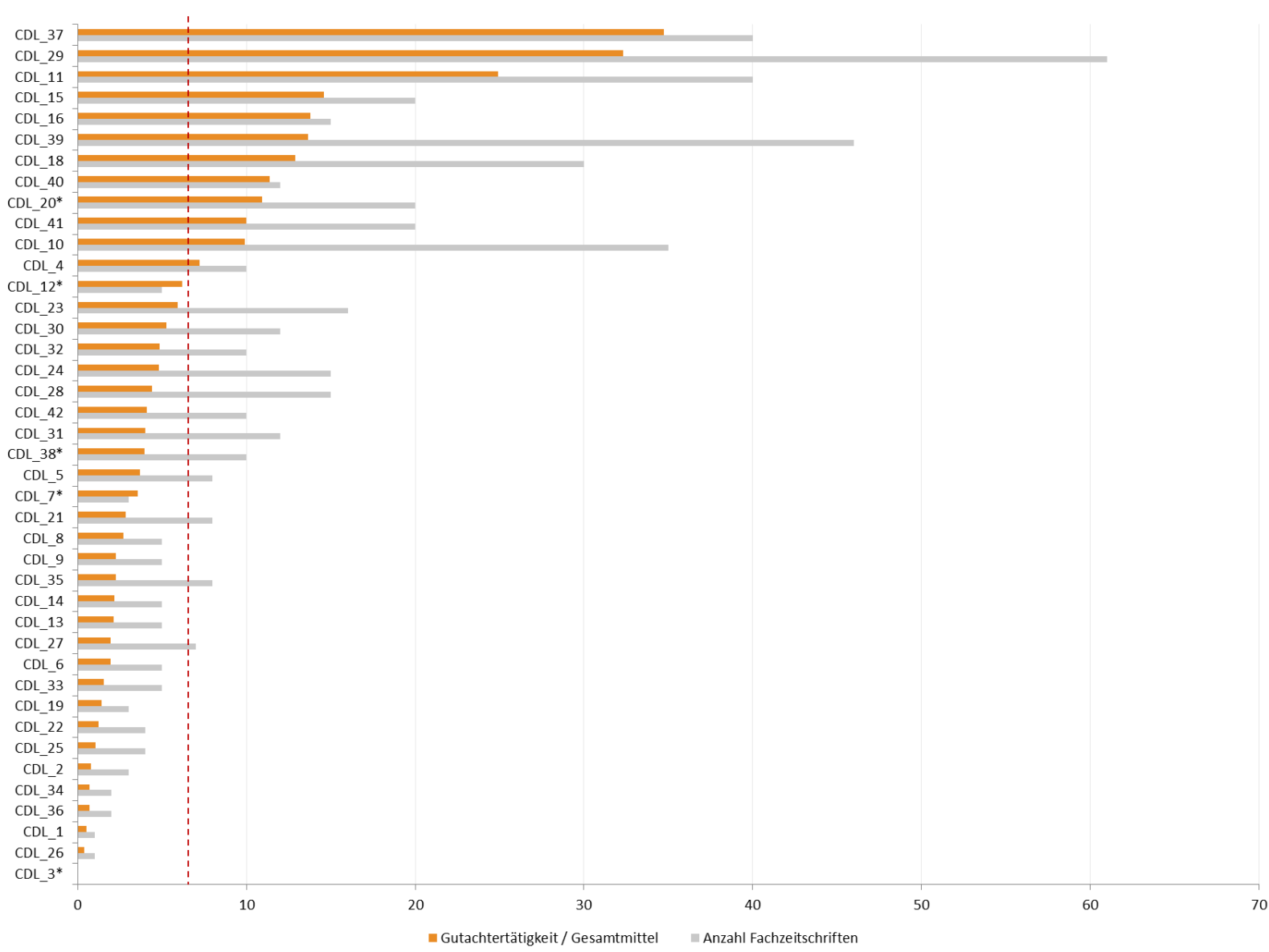

Quelle: Abschlussfragebögen und Finanzdaten der CDG

\section{Kooperationen}

In diesem Abschnitt werden die Kooperationen untersucht, die im Rahmen der CD-Labors mit anderen Forschergruppen durchgeführt wurden.

Die nachstehende Abbildung zeigt das Ausmaß an neuen Kooperationen, die während des Betrachtungszeitraumes durch die CD-Labors aufgebaut wurden. Über die 39 CD-Labors hinweg, von denen Daten zur Bildung neuer wissenschaftlicher Kooperationen vorliegen, konnten die bereits bestehenden Kooperationen um 2,89 je eingesetzter Fördermillion erhöht werden. Lediglich bei zwei CD-Labors wurden während der Laufzeit des Förderprogramms keine neuen Kooperationen gebildet. 
Abbildung 22: Kooperationen neu, absolut und in Relation zu den Gesamtmitteln in Mio. Euro

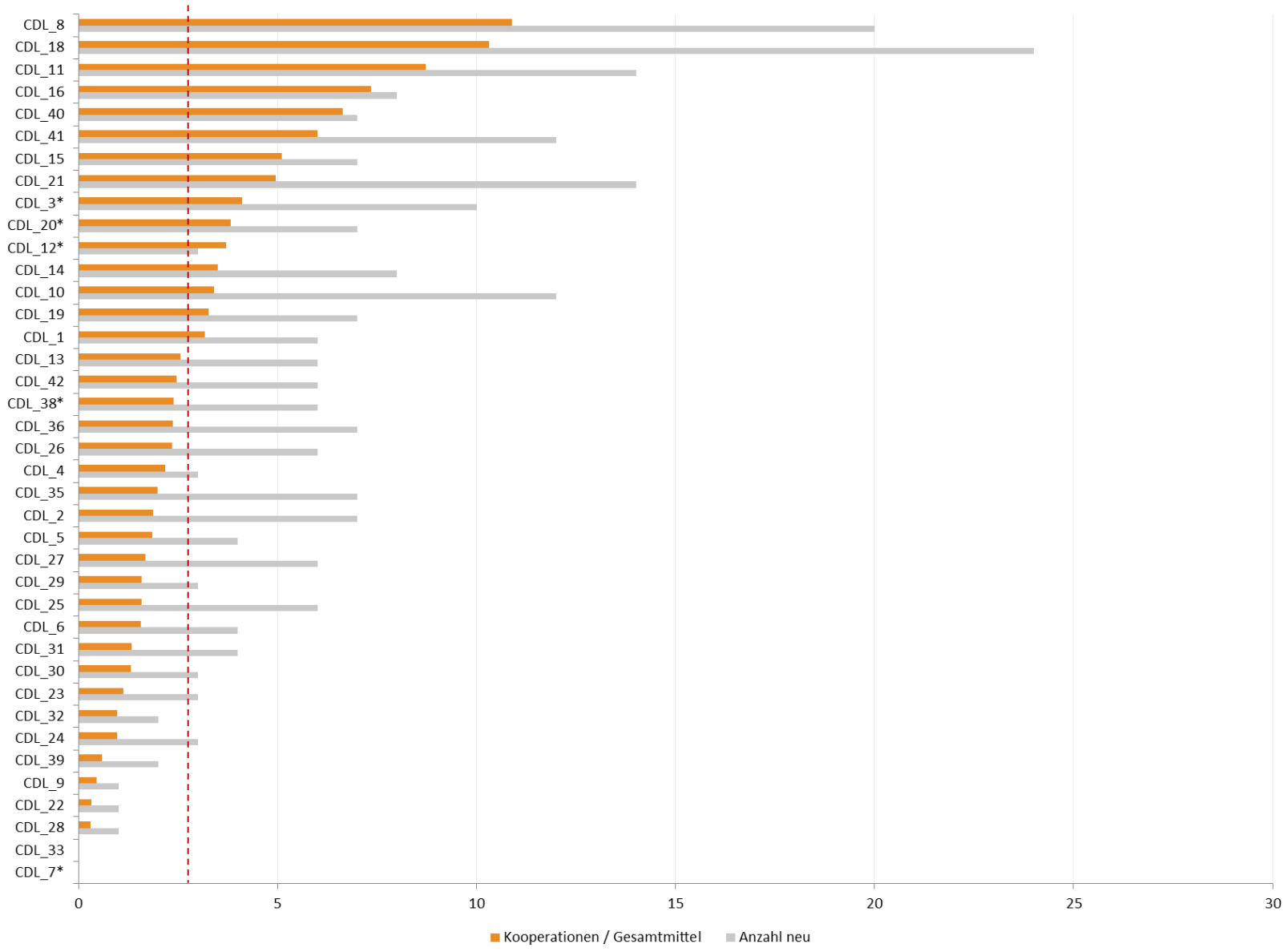

Quelle: Abschlussfragebögen und Finanzdaten der CDG

Betrachtet man die bereits bestehenden Kooperationen der 39 CD-Labors mit anderen Forschergruppen, so ergibt sich ein Mittelwert von 2,55 Kooperationen je eingesetzter Fördermillion. Zu erwähnen ist, dass 10 der 39 CD-Labors über keine bestehenden Kooperationen verfügten, mit anderen Worten, die Gesamtzahl von 239 bereits bestehenden Kooperationen verteilt sich auf 29 CD-Labors. 
Abbildung 23: Kooperationen vorhanden, absolut und in Relation zu den Gesamtmitteln in Mio. Euro

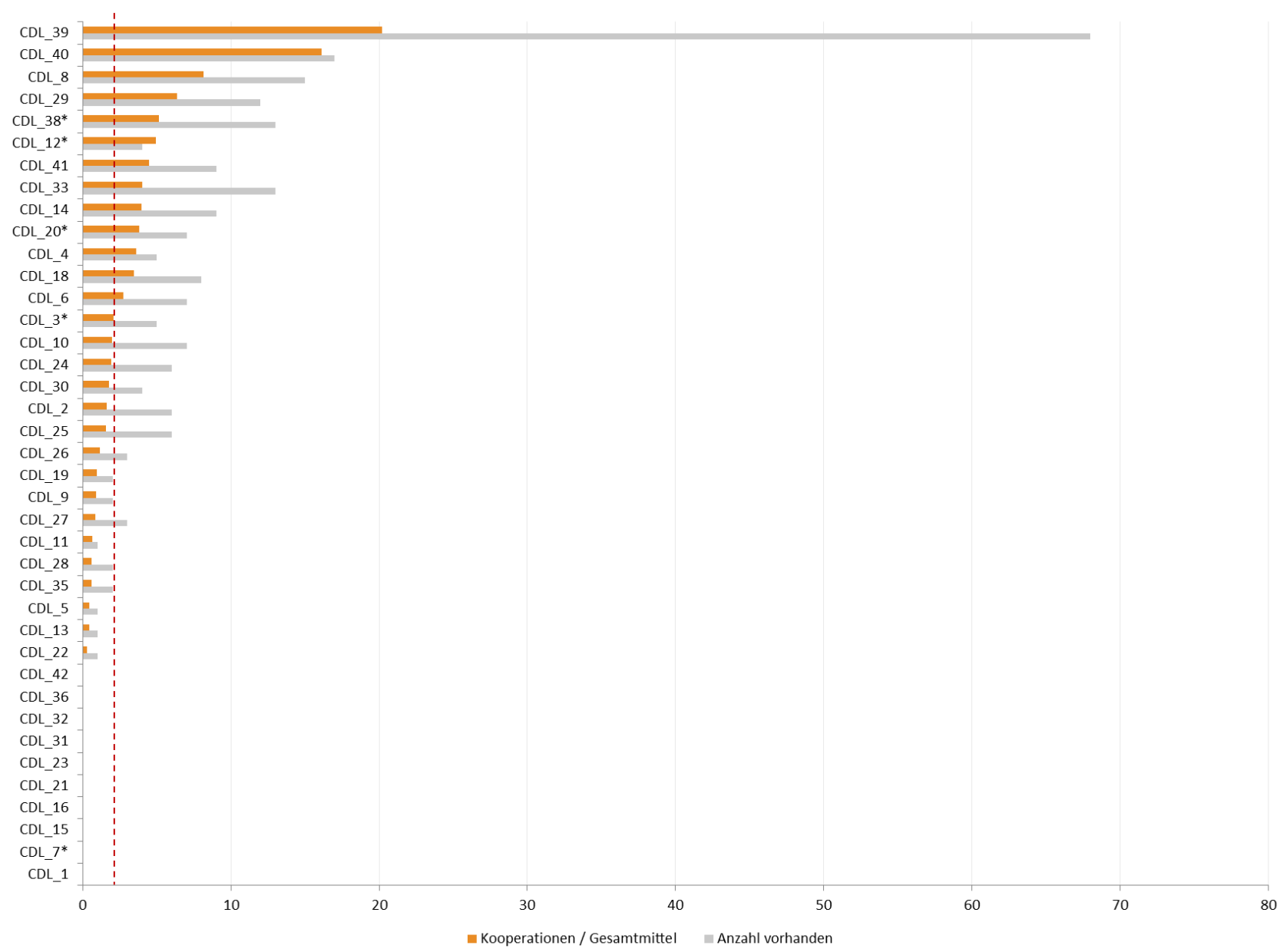

Quelle: Abschlussfragebögen und Finanzdaten der CDG

\subsubsection{Aspekte betreffend die Unternehmenspartner}

\section{Wechsel von Mitarbeitern in die Wirtschaft}

Hinsichtlich der Wechsel von Mitarbeitern der CD-Labors zu den Unternehmen zeigt sich, dass es lediglich drei CD-Labors gab, bei denen kein Wechsel von Mitarbeitern in die Wirtschaft stattfand. In Relation zu den gesamten Fördermitteln aller 41 CD-Labors, welche Angaben betreffend Mitarbeiterwechsel gemacht haben, gab es im Durchschnitt 1,64 Wechsel von Mitarbeitern zu Unternehmen je Fördermillion. 
Abbildung 24: Mitarbeiterwechsel in die Wirtschaft, absolut und in Relation zu den Gesamtmitteln in Mio. Euro

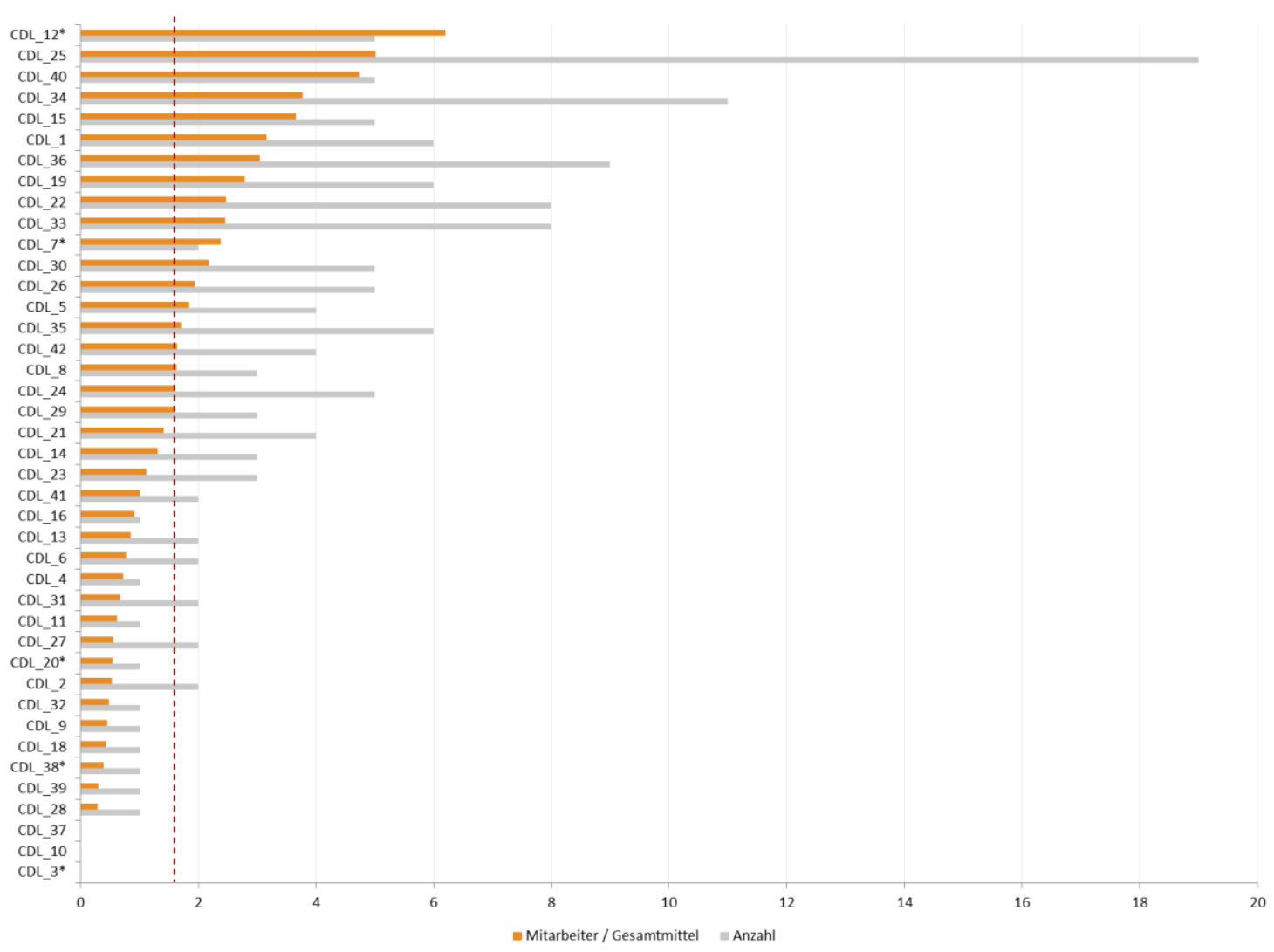

Quelle: Abschlussfragebögen und Finanzdaten der CDG

\section{Patente}

Die Anzahl der Patente, die von den am CD-Labor beteiligten Partnern eingereicht wurden, liegt im Durchschnitt bei 0,98 je Fördermillion. Die absoluten Spitzenreiter sind die CD-Labors 33 und 39, die für 26 bzw. 25 Patente und somit zusammen beinahe für die Hälfte aller eingereichten Patente verantwortlich zeichnen. Außerdem ist zu erwähnen, dass zumindest jedes zweite CD-Labor während des Betrachtungszeitraumes Patente eingereicht hat. Demnach verteilt sich die Gesamtzahl von 109 eingereichten Patenten auf 21 von insgesamt 41 CD-Labors. 
Abbildung 25: Patente, absolut und in Relation zu den Gesamtmitteln in Mio. Euro

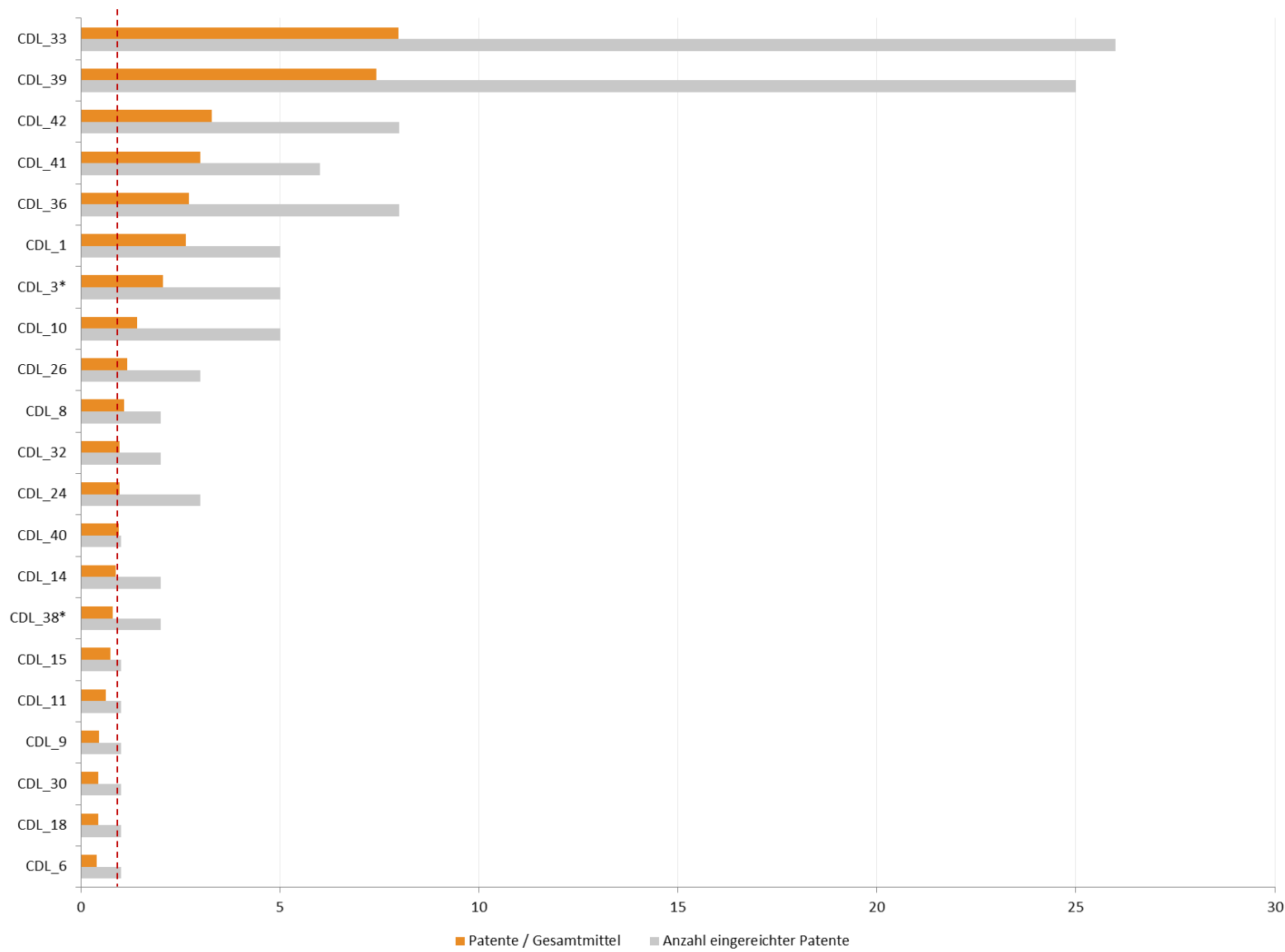

Quelle: Abschlussfragebögen und Finanzdaten der CDG

\subsection{Zusammenfassung der CDG-Kenndaten}

Die Kenndatenerhebung wird mit einer quantitativen Betrachtung abgeschlossen, die schon einen ersten Schritt zur Programmevaluierung darstellt. Sowohl bei der Korrelationsanalyse als auch bei der normierten Darstellung der Outputs wird dabei ausschließlich auf das Gesamtbudget eines CD-Labors als relevante Inputgröße abgestimmt. Dies erfolgt in Übereinstimmung mit der Vorgängerevaluation, da aus Sicht des Fördergebers dieser Input die maßgebliche Kenngröße darstellt, und eine effiziente Verwendung öffentlicher Mittel im unmittelbaren Vergleich zu anderen Fördermaßnahmen durchgeführt werden kann. Auf diese Weise erhält man aber auch ein Instrumentarium, mit dessen Hilfe die einzelnen CD-Labors untereinander auf einheitlicher Basis verglichen werden können. 


\subsubsection{Korrelationsanalyse der Outputwerte zu den Budgetmitteln}

Die Korrelationsanalyse der eingesetzten Mittel mit den weiteren Kenndaten zeigt den generellen positiven Zusammenhang zwischen Input und Output auf. Beinahe alle untersuchten (Output-) Indikatoren ${ }^{36}$ sind in ihrer Originalausprägung (d.h. bevor sie mit den Gesamtmitteln in Beziehung gesetzt wurden) positiv mit den gewährten CD-Labor-Budgets korreliert. Dieses Ergebnis bestätigt nochmals das Resultat der Vorgängerevaluation, bei dem ebenfalls ein positiver Zusammenhang gefunden wurde. Signifikant korrelieren demnach die Gesamtmittel mit der Anzahl der Mitarbeiter mit Qualifikation Dissertant, Postdoc, mit der Anzahl der Dissertationen, der Anzahl der Vorträge bei Konferenzen und der Anzahl der referierten und nicht referierten Artikel und somit auch die Gesamtanzahl der Artikel.

\section{Tabelle 12: Korrelationskoeffizienten}

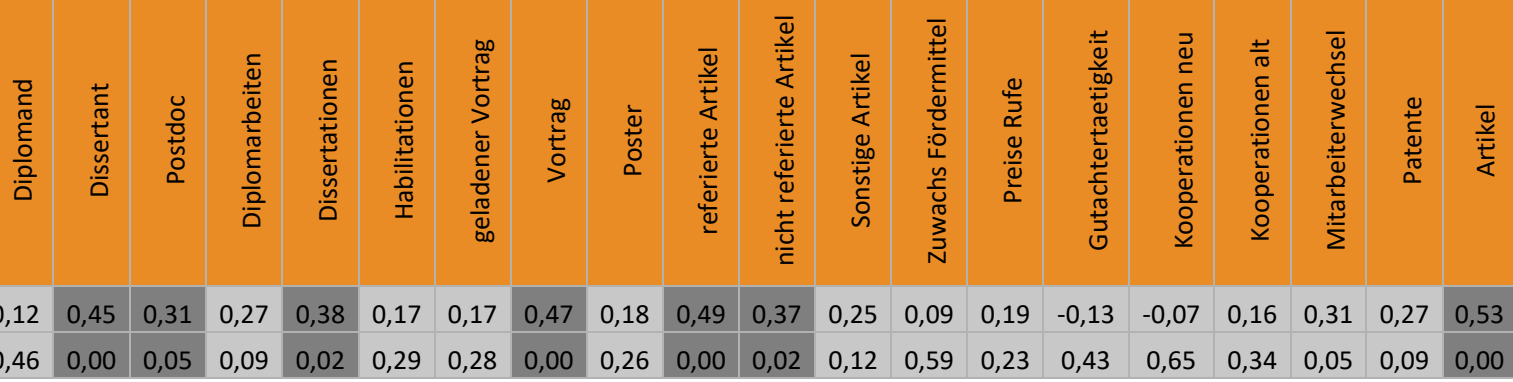

Quelle: https://www.cdg.ac.at/ (R...Korrelationskoeffizient, P...Signifikanzniveau)

Grundsätzlich spricht dieser Befund für das Förderprogramm, da ein positiver (linearer) Zusammenhang von vornherein nicht als gegeben angenommen werden kann. Für das Förderprogramm CDG bedeutet dies, dass eine positive Wechselwirkung zwischen der Höhe des Outputs und der Höhe des Inputs existiert. Die Stärke des linearen Zusammenhangs, basierend auf den empirischen Daten, ist jedoch nicht für alle Outputs signifikant. Im Vergleich zur Evaluierung 2011 und der aktuellen Evaluierung zeigt sich, dass die Anzahl der Artikel in beiden Fällen einen signifikant positiven Zusammenhang zu den eingesetzten Fördermitteln aufweist.

\footnotetext{
${ }^{36}$ Nur die Anzahl der Kooperationen und die Gutachtertätigkeit weisen einen negativen Zusammenhang mit den Gesamtmitteln auf, wobei laut Korrelationsanalyse dieser nicht signifikant ist.
} 


\subsection{2 Übersicht der normierten Outputwerte}

Die Tabelle 13 gibt für jedes CD-Labor die auf die Gesamtmittel normierten Outputwerte für ausgewählte Kennzahlen wieder. Dabei wurde der jeweilige Absolutwert auf das Gesamtbudget des CD-Labors in Mio. Euro in Bezug gesetzt. Die farbliche Markierung der einzelnen Zellen ergibt sich aus einer Über- (grün) bzw. Unterperformance (rot) des betrachteten CD-Labors, bezogen jeweils auf die durchschnittliche Performance eines Indikators über alle CD-Labors hinweg.

Zeilenweise betrachtet, gibt diese Tabelle also Aufschluss darüber, wie ein CD-Labor über alle betrachteten Erfolgsdimensionen hinweg im Vergleich zur Gesamtheit abschneidet. Spaltenweise kann abgelesen werden, welche CD-Labors hinsichtlich eines Outputs überdurchschnittlich und welche unterdurchschnittlich waren.

\begin{tabular}{|c|c|c|c|c|c|c|c|c|c|c|c|c|c|c|c|c|c|c|c|}
\hline 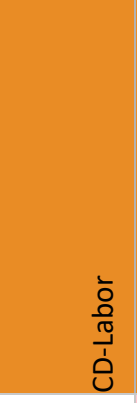 & $\begin{array}{l}\frac{}{0} \\
\frac{0}{0} \\
\frac{1}{0} \\
\frac{1}{0} \\
\frac{0}{0} \\
\frac{0}{0}\end{array}$ & 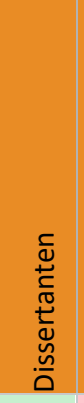 & $\begin{array}{l}\tilde{O} \\
\stackrel{0}{0} \\
\tilde{O} \\
\stackrel{0}{0}\end{array}$ & 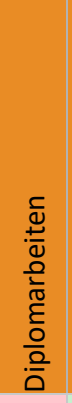 & 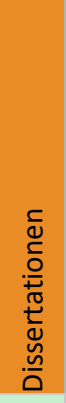 & 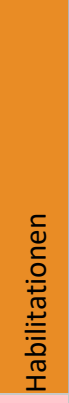 & 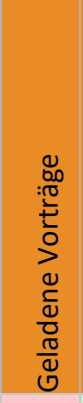 & 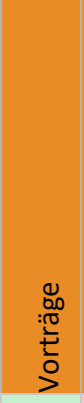 & 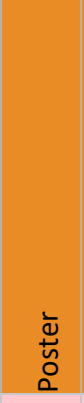 & 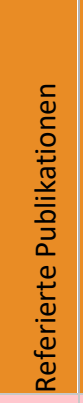 & 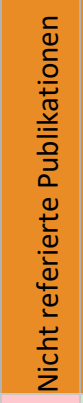 & 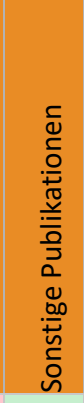 & 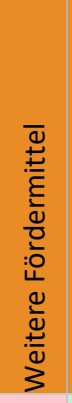 & 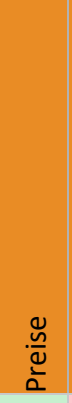 & 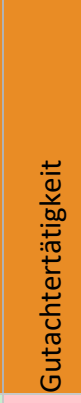 & 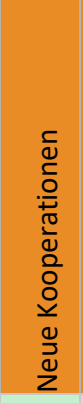 & 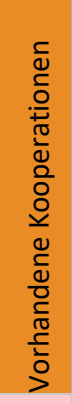 & 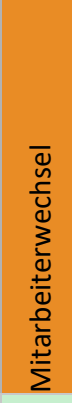 & \\
\hline CDL_1 & 1,6 & 4,7 & 0,5 & 1,6 & 3,2 & 0,0 & 0,0 & 18,4 & 0,0 & 17,9 & 3,7 & 5,3 & 0,3 & 3,2 & 0,5 & 3,2 & 0,0 & 3,2 & 2,6 \\
\hline CDL_2 & 0,0 & 0,5 & 2,4 & 0,0 & 0,3 & 0,0 & 0,8 & 0,8 & 1,3 & 6,4 & 0,0 & 0,8 & 0,3 & 0,0 & 0,8 & 1,9 & 1,6 & 0,5 & 0,0 \\
\hline CDL_3* & 9,9 & 6,6 & 0,4 & 8,2 & 4,5 & 1,2 & 9,4 & 39,4 & 41,9 & 87,9 & 7,0 & 7,0 & 0,4 & 2,1 & 0,0 & 4,1 & 2,1 & 0,0 & 2,1 \\
\hline CDL_4 & 7,9 & 2,2 & 0,0 & 7,9 & 1,4 & 0,7 & 16,6 & 2,2 & 2,2 & 5,8 & 3,6 & 15,2 & 0,2 & 2,9 & 7,2 & 2,2 & 3,6 & 0,7 & 0,0 \\
\hline CDL_5 & 2,8 & 2,8 & 1, & 2 & 2,8 & 0,0 & 1,4 & 8,7 & 16,1 & 29,4 & 6,4 & 1,8 & 0,0 & 0,9 & 3,7 & 1,8 & 0,5 & 1,8 & 0,0 \\
\hline CDL_6 & 2,3 & 2,7 & 0,8 & 2,3 & 2,7 & 0,4 & 22,9 & 5,8 & 34,2 & 18,3 & 1,2 & 15,2 & 0,2 & 2,3 & 1,9 & 1,6 & 2,7 & 0,8 & 0,4 \\
\hline CDL_7* & 8,3 & 3,6 & 3,6 & 5,9 & 2,4 & 0,0 & 2,4 & 5,9 & 3,6 & 13,1 & 0,0 & 3,6 & 0,0 & 2,4 & 3,6 & 0,0 & 0,0 & 2,4 & 0,0 \\
\hline CDL_8 & 1,6 & 4,9 & 5,4 & 0,5 & 1,1 & 0,0 & 3,3 & 4,9 & 1,6 & 14,2 & 9,8 & 0,0 & 3,3 & 0,5 & 2,7 & 10,9 & 8,2 & 1,6 & 1,1 \\
\hline CDL_9 & 0,5 & 4,5 & 2,3 & مח & 11 & 0,0 & 0,0 & 0,0 & 0,0 & 11,8 & 1,4 & 0,0 & 4,5 & 0,5 & 23 & 0 & 0,9 & 0,5 & 0,5 \\
\hline CDL_10 & 3,7 & 3,1 & 4,0 & 7,6 & 2,8 & 0,3 & 25,5 & 4,2 & 28,0 & 44,8 & 3,1 & 22,4 & 2,7 & 6,2 & 9,9 & 3,4 & 2,0 & 0,0 & 1,4 \\
\hline CDL_11 & 7,5 & 4,4 & 2,5 & 6,2 & 1,9 & 0,0 & 76,6 & 20,6 & 16,8 & 31,2 & 10,6 & 3,1 & 1,2 & 3,1 & 24 , & 8 & 0,6 & 0,6 & 0,6 \\
\hline CDL_12* & 0,0 & 7,4 & 5,0 & 0,0 & 5,0 & 0,0 & 2,5 & 2,5 & 3,7 & 16,1 & 1,2 & 0,0 & 0,4 & 0,0 & 6,2 & 3,7 & 5,0 & 6,2 & 0,0 \\
\hline CDL_13 & 3,8 & 1,7 & 1,3 & 3,8 & 0,4 & 0,0 & 0,4 & 2,1 & 10,7 & 3,0 & 0,0 & 0,0 & 0,0 & 0,0 & 2,1 & 2,6 & 0,4 & 0,9 & 0,0 \\
\hline CDL_14 & 0,9 & 3,1 & 3,1 & 0,9 & $J, 1$ & 0,0 & 0,9 & 1,3 & 4,4 & 11,4 & 0,9 & 3,9 & 0,2 & 0,0 & 2,2 & 3,3 & 3,9 & 1,3 & 0,9 \\
\hline CDL_15 & 6,6 & 4,4 & 1,5 & 5,1 & 3,7 & 0,0 & 6,6 & 29,2 & 13,1 & 22,6 & 0,7 & 5,8 & 0,6 & 2,2 & 14,6 & 5,1 & 0,0 & 3,7 & 0,7 \\
\hline CDL_16 & 3,7 & 10,1 & 6,4 & 1,8 & 4,6 & 0,0 & 4,6 & 11,0 & 13,8 & 21,1 & 0,0 & 0,0 & 0,9 & 0,9 & 13,8 & 7,3 & 0,0 & 0,9 & 0,0 \\
\hline
\end{tabular}




\begin{tabular}{|c|c|c|c|c|c|c|c|c|c|c|c|c|c|c|c|c|c|c|c|}
\hline $\begin{array}{l}\grave{0} \\
\frac{0}{\pi} \\
\overline{1} \\
0\end{array}$ & $\begin{array}{l}\frac{c}{d} \\
\frac{d}{0} \\
\frac{c}{\pi} \\
\varepsilon \\
\frac{0}{0} \\
\frac{0}{0}\end{array}$ & 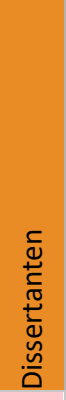 & $\begin{array}{l}\tilde{O} \\
\stackrel{0}{0} \\
\tilde{H} \\
0\end{array}$ & 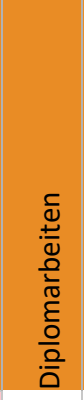 & 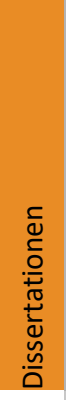 & 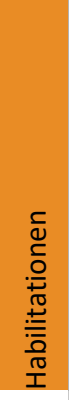 & $\begin{array}{l}0 \\
0 \\
: 00 \\
: 0 \\
0 \\
0 \\
0 \\
0 \\
\frac{1}{0} \\
\frac{0}{0} \\
\frac{\pi}{0} \\
0\end{array}$ & 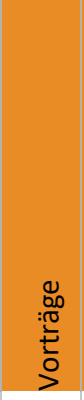 & 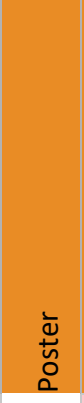 & 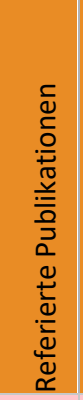 & 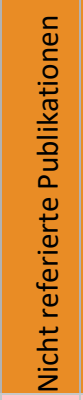 & 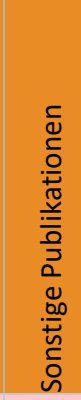 & 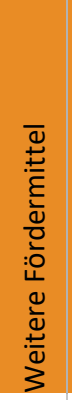 & 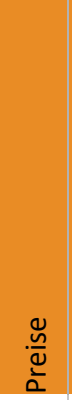 & 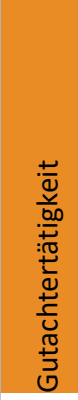 & 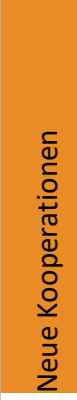 & $\begin{array}{l}\frac{1}{0} \\
\frac{0}{0} \\
.0 \\
\frac{1}{0} \\
\frac{0}{0} \\
\frac{0}{0} \\
0 \\
\frac{0}{0} \\
\frac{0}{0} \\
\frac{0}{0} \\
\frac{c}{0} \\
\frac{c}{0}\end{array}$ & 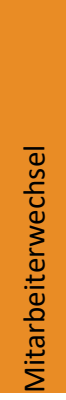 & 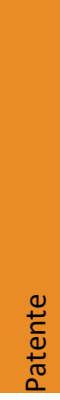 \\
\hline CDL_17 & 0,0 & 0,0 & 0,0 & & & & & & & 12,2 & 1,5 & 1,0 & & & & & & & \\
\hline CDL_18 & 3,4 & 3,0 & 1,7 & 3,4 & 0,9 & 0,0 & 42,9 & 8,6 & 21,5 & 59,7 & 3,0 & 6,0 & 0,0 & 1,3 & 12,9 & 10,3 & 3,4 & 0,4 & 0,4 \\
\hline CDL_19 & 6,5 & 4,7 & 1,9 & 6,5 & 3,7 & 0,9 & 2,3 & 44,7 & 2,3 & 9,8 & 12,6 & 0,0 & 0,1 & 0,9 & 1,4 & 3,3 & 0,9 & 2,8 & 0,0 \\
\hline CDL_20* & 2,2 & 2,7 & 2,2 & 2,2 & 2,2 & 0,0 & 0,0 & 12,0 & 0,0 & 12,5 & 4,4 & 1,1 & 0,1 & 4,4 & 10,9 & 3,8 & 3,8 & 0,5 & 0,0 \\
\hline CDL_21 & 5,3 & 3,9 & 3,5 & 4,6 & 1,8 & 0,0 & 0,7 & 3,5 & 17,7 & 8,9 & 9,2 & 1,4 & 0,0 & 1,1 & 2,8 & 5,0 & 0,0 & 1,4 & 0,0 \\
\hline CDL_22 & 4,9 & 4,3 & 2,5 & 0,9 & 1,9 & 0,0 & 2,8 & 21,3 & 5,9 & 25,0 & 1,9 & 0,6 & 0,0 & 1,2 & 1,2 & 0,3 & 0,3 & 2,5 & 0,0 \\
\hline CDL_23 & 0,0 & 1,9 & 2,6 & 0,0 & 1,1 & 0,4 & 1,1 & 22,3 & 0,4 & 26,4 & 23,0 & 3,3 & 0,6 & 0,4 & 5,9 & 1,1 & 0,0 & 1,1 & 0,0 \\
\hline CDL_24 & 2,2 & 1,3 & 1,6 & 2,2 & 1,3 & 0,0 & 6,4 & 19,5 & 9,9 & 23,4 & 0,6 & 3,5 & 0,3 & 1,0 & 4,8 & 1,0 & 1,9 & 1,6 & 1,0 \\
\hline CDL_25 & 3,7 & 3,4 & 0,3 & 3,7 & 3,4 & 0,0 & 2,6 & 28,8 & 0,5 & 22,4 & 6,6 & 2,4 & 0,0 & 0,0 & 1,1 & 1,6 & 1,6 & 5,0 & 0,0 \\
\hline CDL_26 & 14,0 & 5,8 & 0,4 & 13,6 & 4,3 & 0,0 & 8,6 & 37,8 & 0,4 & 66,6 & 0,8 & 5,8 & 0,0 & 2,7 & 0,4 & 2,3 & 1,2 & 1,9 & 1,2 \\
\hline CDL_27 & 0,6 & 4,2 & 1,1 & 10,0 & 1,4 & 0,8 & 5,8 & 18,1 & 3,9 & 30,3 & 10,6 & 2,2 & 0,0 & 2,5 & 1,9 & 1,7 & 0,8 & 0,6 & 0,0 \\
\hline CDL_28 & 1,8 & 5,6 & 0,6 & 0,9 & 2,9 & 0,3 & 4,7 & 20,8 & 0,0 & 42,6 & 0,3 & 0,9 & 0,0 & 1,8 & 4,4 & 0,3 & 0,6 & 0,3 & 0,0 \\
\hline CDL_29 & 13,8 & 5,3 & 0,5 & 13,8 & 5,3 & 0,0 & 6,4 & 20,7 & 2,1 & 23,8 & 5,3 & 11,7 & 1,5 & 1,6 & 32,3 & 1,6 & 6,4 & 1,6 & 0,0 \\
\hline CDL_30 & 7,0 & 4,4 & 1,3 & 7,0 & 3,9 & 0,4 & 4,4 & 19,6 & 13,1 & 17,0 & 0,4 & 0,0 & 0,0 & 0,0 & 5,2 & 1,3 & 1,7 & 2,2 & 0,4 \\
\hline CDL_31 & 0,7 & 3,0 & 2,7 & 0,3 & 0,7 & 0,0 & 5,7 & 9,0 & 6,7 & 6,4 & 0,3 & 1,3 & 0,2 & 0,7 & 4, & 1,3 & 0,0 & 0,7 & 0,0 \\
\hline CDL_32 & 6,3 & 4,4 & 1,0 & 5,8 & 4,4 & 0,0 & 7,8 & 20,4 & 14,5 & 17,9 & 0,5 & 13,6 & 0,1 & 1,0 & 4,8 & 1,0 & 0,0 & 0,5 & 1,0 \\
\hline CDL_33 & 2,1 & 4,9 & 1,8 & 2,1 & 2,5 & 0,0 & 0,9 & 4,9 & 2,8 & 20,2 & 1,5 & 0,3 & 0,4 & 1,5 & 1,3 & 0,0 & 4,0 & 2,5 & 8,0 \\
\hline CDL_34 & 11,3 & 6,2 & 4,8 & 10,3 & 3,8 & 1,0 & 23,7 & 15,1 & 2,4 & 32,9 & 1,7 & 1,4 & 0,2 & 0,0 & 0,7 & 0,0 & 0,0 & 3,8 & 0,0 \\
\hline CDL_35 & 3,1 & 2,3 & 0,3 & 3,1 & 2,3 & 0,3 & 3,7 & 33,5 & 3,1 & 19,6 & 10,2 & 2,8 & 0,1 & 0,6 & 2,3 & 2,0 & 0,6 & 1,7 & 0,0 \\
\hline CDL_36 & 1,0 & 3,0 & 1,4 & 3,0 & 3,0 & 0,0 & 1,0 & 32,2 & 2,7 & 33,2 & 1,7 & 5,7 & 0,8 & 2,4 & 0,7 & 2,4 & 0,0 & 3,0 & 2,7 \\
\hline CDL_37 & 6,1 & 3,5 & 1,7 & 5,2 & 1,7 & 0,9 & 0,0 & 9,1 & 0,0 & 50,4 & 0,0 & 0,9 & 0,3 & 5,2 & 34,8 & 0,0 & 0,0 & 0,0 & 0,0 \\
\hline CDL_38* & 3,2 & 4,4 & 0,8 & 3,2 & 2,8 & 0,0 & 0,8 & 9,9 & 15,8 & 11,5 & 0,8 & 0,4 & 0,1 & 0,4 & 4,0 & 2,4 & 5,1 & 0,4 & 0,8 \\
\hline CDL_39 & 0,6 & 2,4 & 2,7 & 0,3 & 0,9 & 0,0 & 42,2 & 8,6 & 25,0 & 36,6 & 20,8 & 13,1 & 1,2 & 3,0 & 13,7 & 0,6 & 20,2 & 0,3 & 7,4 \\
\hline CDL_40 & 0,0 & 4,7 & 3,8 & 0,9 & 1,9 & 0,9 & 24,6 & 2,8 & 45,5 & 28,4 & 0,0 & 14,2 & 0,6 & 8,5 & 11,4 & 6,6 & 16,1 & 4,7 & 0,9 \\
\hline CDL_41 & 0,5 & 4,0 & 2,5 & 1,0 & 3,0 & 0,0 & 14,5 & 5,5 & 19,0 & 14,0 & 0,0 & 3,5 & 2,1 & 1,5 & 10,0 & 6,0 & 4,5 & 1,0 & 3,0 \\
\hline CDL_42 & 4,1 & 3,7 & 0,8 & 2,9 & 1,2 & 0,0 & 12,7 & 30,4 & 20,5 & 8,2 & 3,7 & 3,3 & 0,0 & 0,8 & 4,1 & 2,5 & 0,0 & 1,6 & 3,3 \\
\hline$\varnothing$ & 3,9 & 3,9 & 2,0 & 4,0 & 2,5 & 0,2 & 9,8 & 15,0 & 10,4 & 24,2 & 4,1 & 4,4 & 0,6 & 1,7 & 6,7 & 2,9 & 2,6 & 1,6 & 1,0 \\
\hline
\end{tabular}

Quelle: https://www.cdg.ac.at/

In Abbildung 26 sind die Indikatoren, welche vorher in Relation zu den Gesamtmitteln gesetzt wurden, ihrer Größe nach aufgereiht. Dazu wurden z.B. die Anzahl der Diplomanden in allen CDLabors zur Summe der Gesamtmittel in den CD-Labors in Beziehung gesetzt. Die Werte entsprechen 
somit der letzten Zeile der Tabelle 13. Dies spiegelt demnach den durchschnittlichen Nutzen eines CD-Labors wider, gemessen an diesen speziellen Kenngrößen. Diese Abbildung soll noch einmal optisch veranschaulichen, welcher Output in Relation zu den gewährten Fördermitteln von den untersuchten CD-Labors erbracht wurde. Hier gilt es anzumerken, dass die Normierung anhand der Gesamtmitteln zu laufenden Preisen stattgefunden hat, was bei einem intertemporalen Vergleich zu berücksichtigen ist.

Abbildung 26: Indikatoren in Relation zu den Gesamtmitteln (in Mio. Euro)

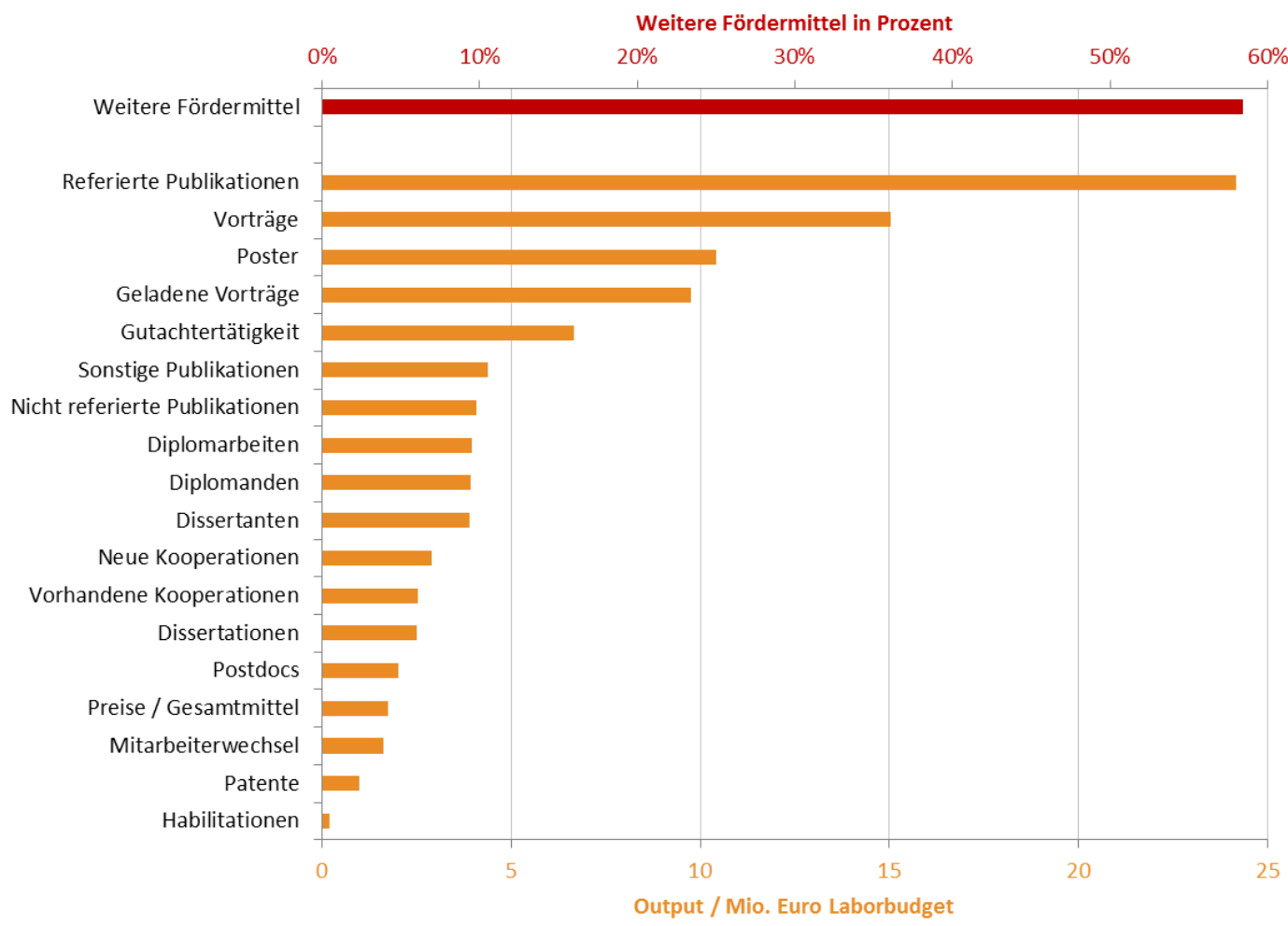

Quelle: Abschlussfragebögen und Finanzdaten der CDG

\subsubsection{Vergleich der CDG-Evaluierungen 2011 und 2016}

Beim Vergleich der beiden Evaluierungen können bereits Aussagen über die Entwicklung des Förderprogramms getätigt werden. Die durchschnittlichen Budgetmittel (inklusive der Finanzierung der Auslaufphase) je CD-Labor sind von der letzten Evaluierung auf die aktuelle Auswertung von 2,16 auf 2,38 Mio. Euro ( $\varnothing$ Budget) angestiegen. Bereinigt um die CD-Labors ohne regulären Verlauf war der Zuwachs von 2,28 auf 2,48 Mio. Euro ( $\varnothing$ Budget*). Hier gilt es jedoch zu beachten, dass es sich 
dabei um nominelle Werte ohne Berücksichtigung der allgemeinen Preisentwicklung handelt. Zieht man die Inflation laut Verbraucherpreisindex zwischen den beiden Evaluierungen als Vergleichsmaßstab heran, so ist die nominelle Veränderung der Preise in etwa gleich groß wie jene der mittleren Gesamtmittel. ${ }^{37}$ Daraus ist zu folgern, dass das durchschnittliche Volumen eines CD-Labors (zu festen Preisen also real) sich beinahe nicht verändert hat (Siehe Tabelle 14 Werte für 2011 und 2016*).

Tabelle 14: Vergleich Evaluierung 2011 und 2016

\begin{tabular}{|c|c|c|c|}
\hline \multirow[b]{2}{*}{ Ouput / Gesamtmittel } & \multicolumn{3}{|c|}{ Evaluierung } \\
\hline & 2011 & 2016 & $2016 *$ \\
\hline \multicolumn{4}{|l|}{ Fördermittel } \\
\hline$\varnothing$ Budget & 2,16 & 2,38 & 2,20 \\
\hline$\varnothing$ Budget* & 2,28 & 2,48 & 2,29 \\
\hline Zusätzliche Fördermittel & $53,6 \%$ & $58 \%$ & \\
\hline \multicolumn{4}{|l|}{ Wissenschaftliche Mitarbeiter } \\
\hline Diplomanden & 3,4 & 3,9 & 4,3 \\
\hline Dissertanten & 4,2 & 3,9 & 4,2 \\
\hline Postdoc & 1,7 & 2,0 & 2,2 \\
\hline \multicolumn{4}{|l|}{ Abschlüsse } \\
\hline Diplomarbeiten & 4,1 & 4,0 & 4,3 \\
\hline Dissertationen & 3,2 & 2,5 & 2,7 \\
\hline Habilitationen & 0,2 & 0,2 & 0,2 \\
\hline \multicolumn{4}{|l|}{ Konferenzen } \\
\hline Geladene Vorträge & 6,0 & 9,8 & 10,6 \\
\hline Vorträge & 18,0 & 15,0 & 16,4 \\
\hline Poster & 8,2 & 10,4 & 11,3 \\
\hline \multicolumn{4}{|l|}{ Publikationen } \\
\hline Referierte Publikationen & & 24,2 & 26,3 \\
\hline Nicht referierte Publikationen & 19,4 & 4,1 & 4,4 \\
\hline \multirow[t]{2}{*}{ Sonstige Publikationen } & & 4,4 & 4,8 \\
\hline & & 32,6 & 35,5 \\
\hline Gutachtertätigkeit & 3,4 & 6,7 & 7,3 \\
\hline \multicolumn{4}{|l|}{ Kooperationen } \\
\hline Neue Kooperationen & 2,5 & 2,9 & 3,1 \\
\hline Vorhandene Kooperationen & 0,6 & 2,6 & 2,8 \\
\hline \multicolumn{4}{|l|}{ Weitere Erfolge } \\
\hline Preise und Rufe & 2,2 & 1,7 & 1,9 \\
\hline Mitarbeiterwechsel & 1,8 & 1,6 & 1,8 \\
\hline Patente & 1,3 & 1,0 & 1,1 \\
\hline
\end{tabular}

Quelle: Economica, IWI. 2016* Werte unter Berücksichtigung der Inflation zwischen 2011 und 2016.

\footnotetext{
${ }^{37}$ Der Anstieg von ca. $10 \%$ entspricht dabei in etwa dem Preisanstieg zwischen den beiden Evaluierungen.
} 
Bei dem Vergleich der normierten Outputwerte zwischen den beiden Evaluierungen ist dieser Umstand dementsprechend auch zu berücksichtigen. Jede Steigerung eines normierten Outputs berechnet mit nominellen CD-Laborbudgets (Spalte 2011 und 2016) ist daher besonders beachtlich. Der preisbereinigte Vergleich erfolgt zwischen den Spalten 2011 und 2016* in Tabelle 14.

Die Entwicklung der normierten Mitarbeiterzahlen ist auch vor dem Hintergrund der allgemeinen Entwicklung der Studenten, Dissertanten und Post-Docs an den betroffenen Universitäten zu sehen. Isoliert auf die CD-Labors betrachtet, ist sowohl bei den Diplomanden als auch bei den Post-Docs die normierte Größe zwischen den beiden Evaluierungszeitpunkten angestiegen (2011, 2016*). Dieser Zuwachs kann jedoch vielerlei Ursachen haben, u.a. können nun auch CD-Labors mit höherem Personaleinsatz in der aktuellen Evaluierung enthalten sein. Für eine detaillierte Ursachenforschung müsste hierzu eine weiterführende Analyse durchgeführt werden. Diese Untersuchung müsste die allgemeine Entwicklung der Studentenzahlen als auch des Personaleinsatzes in den CD-Labors enthalten. Bei den Abschlüssen zeigt sich, dass bei nomineller Betrachtung zwar generell ein leichter Rückgang zu verzeichnen war, dies wird aber bei der Betrachtung der normierten Outputwerte mit preisbereinigten Gesamtmitteln mehr als relativiert. So gab es demnach bei den Diplomarbeiten einen Zuwachs, nicht jedoch bei den Dissertationen.

Die Summe aus geladenen Vorträgen und Vorträgen von 2011 auf 2016 ist angestiegen, und darüber hinaus gab es auch eine Verschiebung hin zu geladenen Vorträgen, was wiederum ein Beweis für eine weitere Qualitätsverbesserung darstellt. Beachtlich ist der Anstieg der Publikationen je eingesetzter Fördermillion. Der Rückgang der Preise, der Rufe und der Patente ist ebenfalls nicht auf die nominelle Erhöhung der Gesamtmittel zurückzuführen, da die relative Abweichung höher als die Veränderung der Preisentwicklung ist. 


\subsection{Statistische Analyse der JR-Zentren}

Zum Zeitpunkt der Datenübergabe befanden sich insgesamt 6 Josef-Ressel-Zentren in der Verlängerungsphase, wobei durch die unterschiedlichen Startzeitpunkte dieser JR-Zentren auch eine unterschiedliche Anzahl an statistischen Jahresberichten vorliegt. Dies gilt es bei den folgenden Auswertungen zu berücksichtigen, da besonders jene JR-Zentren mit einer zum Zeitpunkt der Evaluierung geringen Laufzeit sich meist noch in einer Einarbeitungsphase befinden, und daher der Output im Allgemeinen noch nicht so hoch sein wird, wie bei einem Zentrum, das kurz vor dem Abschluss steht.

Die gesamten Budgetmittel laut Antrag betragen im Mittel der betrachteten JR-Zentren 1,27 Mio. Euro. Die bis 2015 erzielten Outputs werden jedoch den tatsächlich verbrauchten und nicht den geplanten Mitteln gegenübergesetzt, wobei ebenfalls nur jene Budgetgrößen betrachtet werden, die in der Periode 2013-15 angefallen sind, da in Übereinstimmung damit die statistischen Jahresberichte auch für dieses Zeitspanne vorhanden sind. Auf diese Weise ist gewährleistet, dass der jeweilige kumulierte Mitteleinsatz eines JR-Zentrum mit dem gesamten Output im Beobachtungszeitraum in Relation gesetzt wird, und somit letztendlich auch ein Vergleich zwischen den JR-Zentren - mit den bereits genannten Einschränkungen - vorgenommen werden kann.

\section{Abbildung 27: Fördermittel laut Budgetplan der JR-Zentren in Mio. Euro}

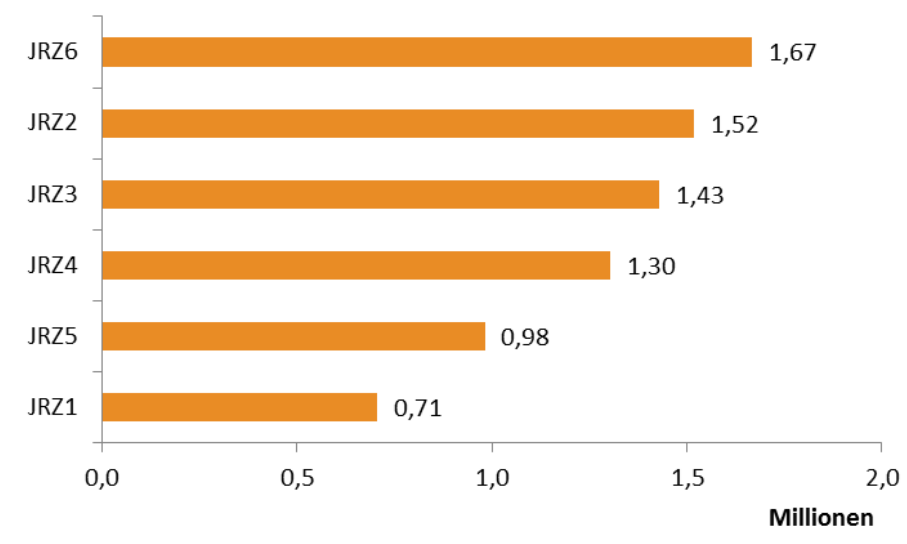

Quelle: Finanzdaten der CDG 
Abbildung 28: Kumulierte Fördermittel 2013-15 der JR-Zentren in Mio. Euro

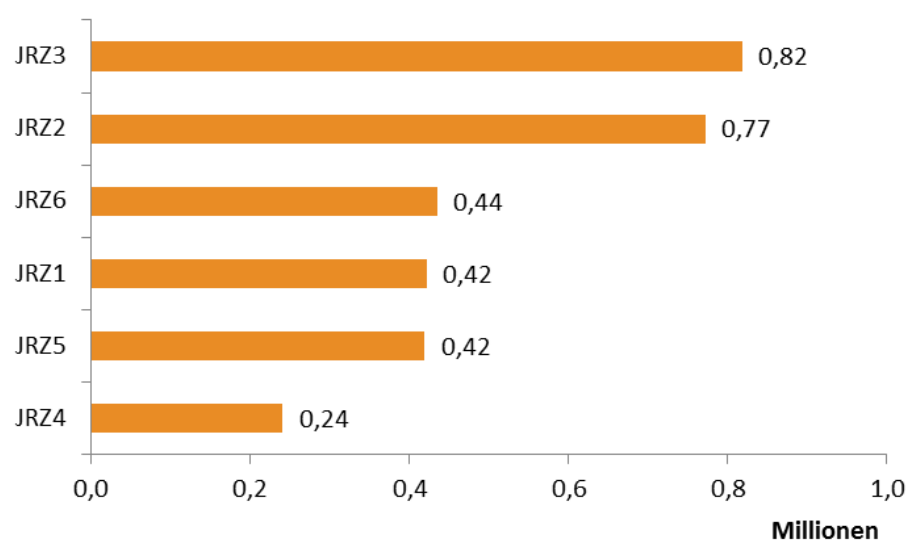

Quelle: Finanzdaten der CDG

Die Unternehmensbeteiligung der JR-Zentren zeigen, dass die Forschungseinheiten bis zum Jahr 2015 fast ausschließlich auf österreichische Partner der Wirtschaft (22 von 23) zurückgreifen. Etwas mehr als jedes dritte Unternehmen ist dabei ein KMU aus Österreich.

Abbildung 29: Unternehmensbeteiligungen der JR-Zentren (2013-15)

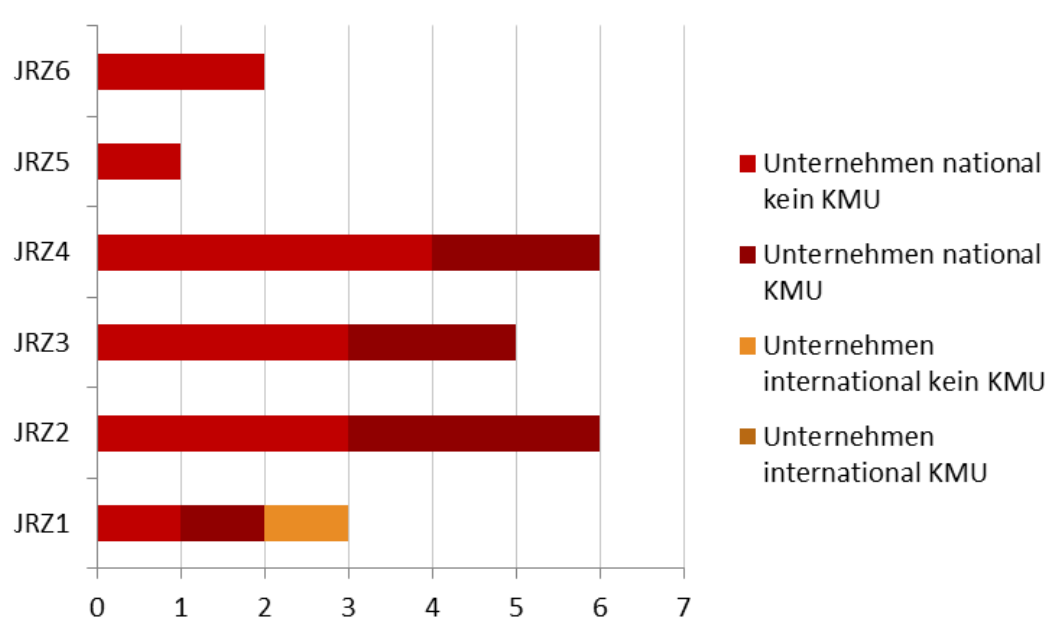

Quelle: Jahresberichte der CDG

Das Personal finanziert durch die CDG ausgedrückt in Jahresarbeitsplätzen in Köpfen hängt grundsätzlich mit den jeweilig eingesetzten Mitteln zusammen. So weisen die JR-Zentren JRZ2 und JRZ3 bei beiden Kennzahlen auch den höchsten Wert auf. Auf der anderen Seite gibt es aber im Fall 
der JR-Zentren JRZ1, JRZ6 zu JRZ5 auch bei annähernd gleich hohen verrechneten Kosten, eine sehr unterschiedliche Anzahl von Anstellungen.

Abbildung 30: Personal finanziert durch die CDG in den JR-Zentren versus Budgetmittel (kumulierte Werte 2013-15)

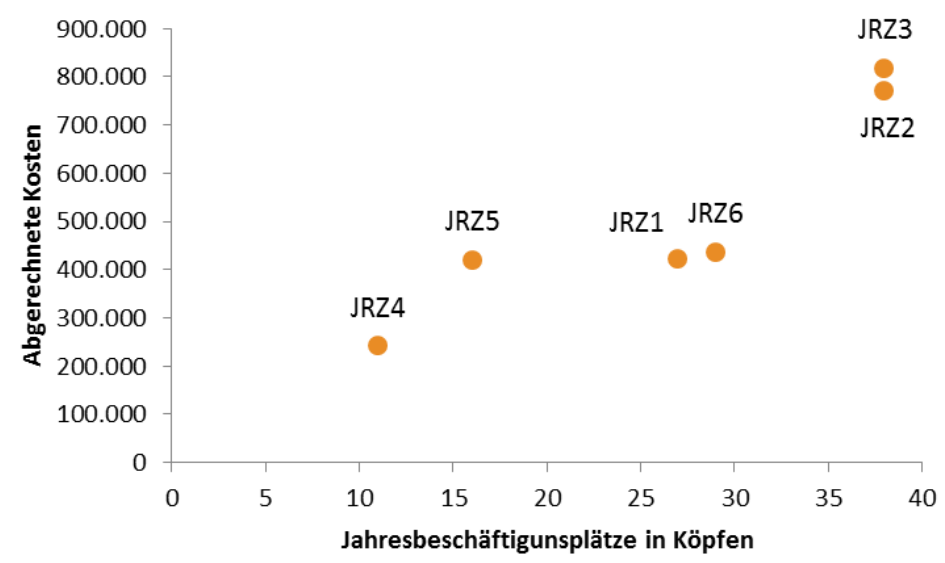

Quelle: Finanzdaten bzw. Jahresberichte der CDG

Aber nicht nur die Anzahl unterscheidet sich, sondern auch die jeweilige Mitarbeiterstruktur. Während in einigen JR-Zentren etliche technische Fachkräfte und Assistenten angestellt sind, kommen diese Mitarbeiter in anderen JR-Zentren nur vereinzelt vor.

Abbildung 31: Personal finanziert durch die CDG in den JR-Zentren (in Köpfen, 2013-15)

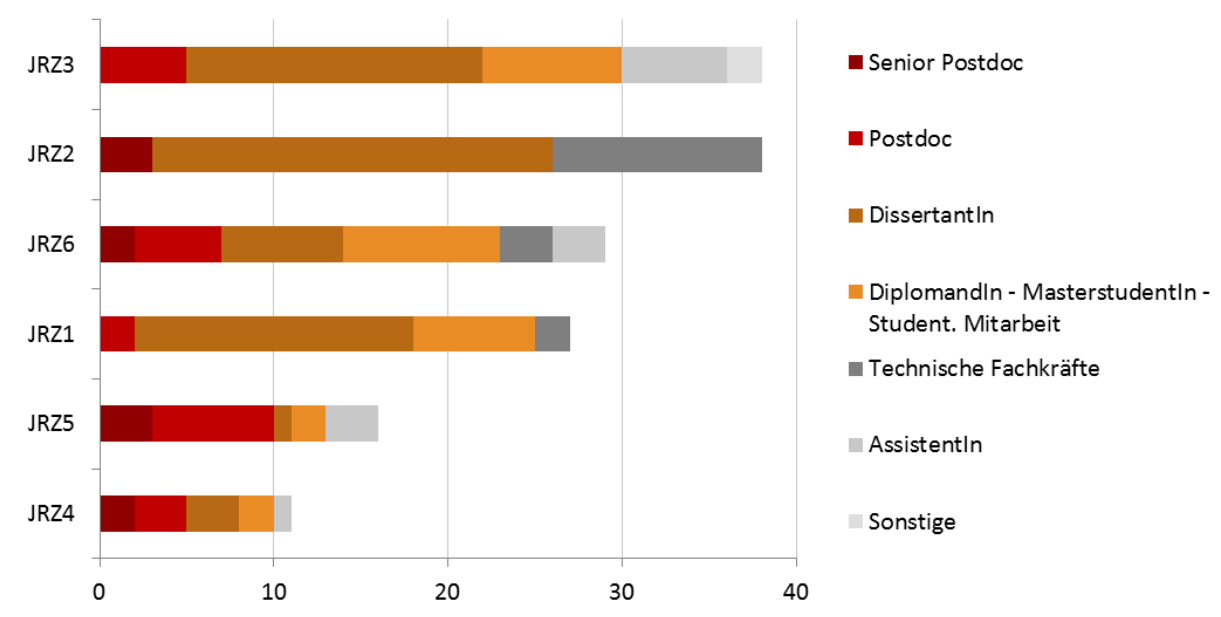

Quelle: Jahresberichte der CDG 
Da nur drei der sechs JR-Zentren zum Zeitpunkt der Evaluierung eine Laufzeit von über drei Jahren aufwiesen, kann dementsprechend auch der Output der restlichen Zentren noch nicht so hoch ausfallen. Wie schon zu Beginn angemerkt ist daher auch bei der Normierung der bisherig verwendeten Gesamtmittel dieser Umstand zu bedenken.

Zeitschriftenpublikationen fanden bis auf eine Ausnahme ausschließlich in Journals mit Peer Review statt. Bemerkenswert ist, dass es bereits eine Monographie gab. Daneben wurden auch noch in drei Publikationen in Sammelbänden veröffentlicht. Die Aufsätze für die breite Öffentlichkeit entstammen zu beinahe drei Viertel aus einem auch in diesem Kontext sehr aktiven JR-Zentrum (JRZ3), das auch genau die Hälfte aller Zeitschriftenpublikationen (10 von 20$)$ veröffentlicht hat.

Normiert man den Output an Publikationen mit den bisher verwendeten Mitteln, erhält man eine durchschnittliche Anzahl von 0,6 Zeitschriftenpublikationen mit Peer Review je 100 Tausend Euro Gesamtmittel. Publikationen für die breite Öffentlichkeit erreichen exakt den gleichen Wert.

Tabelle 15: Publikationen der JR-Zentren (2013-2015, absolut und normiert)

\begin{tabular}{|c|c|c|c|c|c|}
\hline & \multicolumn{2}{|c|}{ Zeitschriftenpublikationen } & \multicolumn{3}{|c|}{ Publikationen } \\
\hline & $\begin{array}{c}\text { mit Peer } \\
\text { Review }\end{array}$ & $\begin{array}{c}\text { ohne Peer } \\
\text { Review }\end{array}$ & Monographien & Sammelbänden & $\begin{array}{c}\text { breite } \\
\text { Öffentlichkeit }\end{array}$ \\
\hline JRZ1 & 3 & 1 & 0 & 3 & 0 \\
\hline JRZ2 & 3 & 0 & 0 & 0 & 0 \\
\hline JRZ3 & 10 & 0 & 1 & 0 & 14 \\
\hline JRZ4 & 0 & 0 & 0 & 0 & 0 \\
\hline JRZ5 & 1 & 0 & 0 & 0 & 2 \\
\hline JRZ6 & 2 & 0 & 0 & 0 & 3 \\
\hline Summe & 19 & 1 & 1 & 3 & 19 \\
\hline \multicolumn{6}{|c|}{ Normiert mit Gesamtmittel (100 Tsd. Euro) } \\
\hline JRZ1 & 0,7 & 0,2 & - & 0,7 & - \\
\hline JRZ2 & 0,4 & - & - & - & - \\
\hline JRZ3 & 1,2 & - & 0,1 & - & 1,7 \\
\hline JRZ4 & - & - & - & - & - \\
\hline JRZ5 & 0,2 & - & - & - & 0,5 \\
\hline JRZ6 & 0,5 & - & - & - & 0,7 \\
\hline Mittel & 0,6 & 0,0 & 0,0 & 0,1 & 0,6 \\
\hline
\end{tabular}

Quelle: CDG, Economica/IWI. 
Bei den Konferenzaktivitäten (Teilnahmen und Publikationen) ist der Unterschied zwischen den JRZentren mit einer geringeren Laufzeit zu den bereits länger laufenden besonders deutlich zu sehen. Wiederum ist auch hier das JR-Zentrum JRZ3 sehr aktiv, und wird nur bei den eingeladenen Vorträgen von JRZ1 übertroffen.

Bei der Normierung mit den bisher eingesetzten Gesamtmitteln liegen die durchschnittlichen Konferenzpublikationen (mit/ohne Peer Review, Poster) mit 2,8 je 100 Tsd. Euro Gesamtmittel etwas unter dem Wert der Konferenzteilnahmen (mit /mit eingeladenen Vorträgen, nur Teilnahme) von 3,5/100.000 Euro Zentrumsbudget.

Die JR-Zentren leisten am überwiegenden Teil der teilgenommenen Konferenzen bereits einen Beitrag, so findet nur jede fünfte Konferenzteilnahme ohne einen Vortrag statt (23 / 110).

Tabelle 16: Konferenzaktivitäten der JR-Zentren (2013-15, absolut und normiert)

\begin{tabular}{lcccccc} 
& $\begin{array}{c}\text { Konferenzteilnahmen } \\
\text { mit } \\
\text { eingeladenen } \\
\text { Vorträgen }\end{array}$ & $\begin{array}{c}\text { mit } \\
\text { Vorträgen }\end{array}$ & $\begin{array}{c}\text { Konferenzpublikationen } \\
\text { mit Peer } \\
\text { Review }\end{array}$ & $\begin{array}{c}\text { ohne Peer } \\
\text { Review }\end{array}$ & $\begin{array}{c}\text { Poster } \\
\text { Nur } \\
\text { Konferenz- } \\
\text { teilnahmen }\end{array}$ \\
\hline JRZ1 & 11 & 18 & 20 & 2 & 4 & 1 \\
JRZ2 & 0 & 12 & 12 & 0 & 0 & 0 \\
JRZ3 & 5 & 24 & 30 & 1 & 5 & 14 \\
JRZ4 & 0 & 1 & 0 & 0 & 0 & 0 \\
JRZ5 & 0 & 16 & 1 & 7 & 1 & 0 \\
JRZ6 & 0 & 0 & 2 & 0 & 1 & 8 \\
\hline Summe & $\mathbf{1 6}$ & $\mathbf{7 1}$ & $\mathbf{6 5}$ & $\mathbf{1 0}$ & $\mathbf{1 1}$ & $\mathbf{2 3}$ \\
& & & & & & \\
\hline JRZ1 & 2,6 & $\mathbf{2 0}$ & 4,7 & 0,5 & 0,9 & 0,2 \\
JRZ2 & - & 1,6 & 1,6 & - & - & - \\
JRZ3 & 0,6 & 2,9 & 3,7 & 0,1 & 0,6 & 1,7 \\
JRZ4 & - & 0,4 & - & - & - & - \\
JRZ5 & - & 3,8 & 0,2 & 1,7 & 0,2 & - \\
JRZ6 & - & - & 0,5 & - & 0,2 & 1,8 \\
\hline Mittel & $\mathbf{0 , 5}$ & $\mathbf{2 , 3}$ & $\mathbf{2 , 1}$ & $\mathbf{0 , 3}$ & $\mathbf{0 , 4}$ & $\mathbf{0 , 7}$ \\
& & & & & & \\
\hline
\end{tabular}

Quelle: CDG, Economica/IWI.

Die sechs untersuchten JR-Zentren hatten vor Beginn des JR-Zentrums keine Kooperation mit anderen Einrichtungen bzw. Unternehmen. Dies blieb auch für die CD-Labors und COMET-Zentren in den Jahren 2013 bis 2015 so, während es 2015 eine Kooperation zwischen zwei JR-Zentren gab. 
Die neuen nationalen Kooperationen lagen insgesamt gesehen (14) erstaunlicherweise sogar unter dem Wert der neuen internationalen Kooperationen (22). Ein JR-Zentrum hat dabei mit zwei heimischen Unternehmen eine Kooperation aufgebaut. Auch hier ist es nicht verwunderlich, dass bereits länger laufende JR-Zentren eine höhere Anzahl von Kooperationen aufweisen.

Tabelle 17: Kooperationen mit Forschungseinrichtungen und Unternehmen der JRZ (2013-15)

\begin{tabular}{|c|c|c|}
\hline & \multicolumn{2}{|c|}{ Kooperation } \\
\hline & national neu & international neu \\
\hline JRZ1 & 7 & 6 \\
\hline JRZ2 & 0 & 2 \\
\hline JRZ3 & 2 & 7 \\
\hline JRZ4 & 1 & 3 \\
\hline JRZ5 & 3 & 3 \\
\hline JRZ6 & 1 & 1 \\
\hline
\end{tabular}

Quelle: $C D G$, Economica/IWI.

Auch bei den weiterreichenden Effekten gibt es hauptsächlich bei den bereits länger laufenden JRZentren Einträge. Insgesamt konnten bereits 16 wissenschaftliche Auszeichnungen und Preise von den sechs untersuchten JR-Zentren gewonnen werden. Erfreulich ist auch, dass es schon zwei Berufungen gab. Daneben wurde auch sieben Mal die Organisation von wissenschaftlichen Events übernommen. Die wissenschaftliche Leistung wird weiters durch sechs genehmigte Anträge für Folgeprojekte bzw. Förderungen dokumentiert.

Tabelle 18: Weiterreichende Effekte

\begin{tabular}{lcccc} 
& $\begin{array}{c}\text { Wissenschaftliche } \\
\text { Auszeichnungen } \\
\text { und Preise }\end{array}$ & Berufungen & $\begin{array}{c}\text { Genehmigte } \\
\text { Wissenschaftlicher } \\
\text { Events }\end{array}$ & $\begin{array}{c}\text { Gention } \\
\text { Anträge für } \\
\text { Folgeprojekte } \\
\text { \& }\end{array}$ \\
\hline JRZ1 & 11 & 0 & 2 & 3 \\
JRZ2 & 1 & 0 & 2 & 3 \\
JRZ3 & 3 & 1 & 3 & 0 \\
JRZ4 & 0 & 1 & 0 & 0 \\
JRZ5 & 1 & 0 & 0 & 0 \\
JRZ6 & 0 & 0 & 0 & 0 \\
\hline Gesamt & $\mathbf{1 6}$ & $\mathbf{2}$ & $\mathbf{7}$ & $\mathbf{6}$ \\
\hline \hline
\end{tabular}

Quelle: CDG, Economica/IWI. 


\subsection{Schlussfolgerungen}

Im Vergleich zur letzten Evaluierung fällt auf, dass es nun mehr CD-Labors mit einem irregulären Verlauf gab. Unter den im Jahr 2011 betrachteten CD-Labors gab es nur zwei vorzeitige Beendigungen. Diese Zahl erhöhte sich jetzt nicht nur absolut auf fünf, sondern bei insgesamt 42 betrachteten CDLabors stieg auch der relative Anteil an. Grundsätzlich ist die Abhängigkeit eines CD-Labors von den handelnden Akteuren zwar hauptsächlich durch den Laborleiter gegeben, aber auch Entwicklungen rund um den Unternehmenspartner spielten bei dieser Evaluierung eine bedeutendere Rolle. So gab es insgesamt drei Fälle von Konkursen, die zu einer (zumindest kurzfristigen) Beendigung des CDLabors führten. Für die Flexibilität des Programms spricht, dass in zwei Fällen ein alternativer Unternehmenspartner gefunden wurde, mit dem das CD-Labor abgeschlossen werden konnte. Aber nicht nur wirtschaftliche Probleme des Unternehmenspartners führten zu einer vorzeitigen Beendigung ${ }^{38}$, denn in weiteren drei Fällen gab es einen Ausstieg des Unternehmens. Es ist hier zu überprüfen inwieweit die Einbindung ${ }^{39}$ der Unternehmenspartner bei den Zwischenevaluierungen als ausreichend empfunden wird. Anzudenken wäre in etwa eine verpflichtende Teilnahme der Unternehmenspartner an den Evaluierungsveranstaltungen.

Betrachtet man alle abgeschlossenen und laufenden CD-Labors, so kann man erkennen, dass sie relativ gleichmäßig auf die schon länger bestehenden Forschungsthemen verteilt sind, wobei ein gewisser Ausgleich durch die vor und nach 2011 ausgelaufenen CD-Labors vorgenommen wurde. Etwas überproportional ist der Bereich „Mathematik, Informatik, Elektronik“ vertreten, während die „Nichtmetallischen Werkstoffe“ etwas unterrepräsentiert sind. Im neuen Bereich „Wirtschafts-, Sozial- und Rechtswissenschaften“ laufen bereits zwei CD-Labors.

\footnotetext{
${ }^{38}$ In den „Informationen zur Mitgliedschaft in der Christian Doppler Forschungsgesellschaft“ werden im Kapitel „3. Mitgliedschaft in der CDG" Abschnitt „3.1 Relevante Unterlagen“ Angaben für den wirtschaftlichen Nachweis der Unternehmen spezifiziert: „Antrag auf ordentliche Mitgliedschaft in der Christian Doppler Forschungsgesellschaft (einschließlich des Geschäftsberichts des letzten Geschäftsjahres).“

39 „Handbuch zum Betrieb eines Christian Doppler Labors: 5. Vertragswerk und zentrale Arbeitsdokumente bzw. instrumente > 5.9. Zentrale Arbeitsdokumente und -instrumente für ein CD-Labor > 5.9.2. Jährliche Arbeits- und Budgetpläne: Die Eingabe des Budgets erfolgt über das Webtool der CDG. Nach firmenmäßiger Zeichnung des Budgets durch die jeweiligen Unternehmenspartner ist dieses der CDG zeitgerecht zu übermitteln. Auf Wunsch der Unternehmenspartner sind jährlich wissenschaftliche Arbeitspläne von der Laborleiterin/vom Laborleiter an diese abzuführen."
}

„11. Wissenschaftliche Begleitung des CD-Labors > 11.2. Wissenschaftliche Zweijahresevaluierung > 11.2.1. Evaluierungsbericht (Evaluation Report): Die Unternehmenspartner müssen dem Evaluierungsbericht zustimmen. Die entsprechenden Zustimmungserklärungen sind dem Evaluierungsbericht beizulegen. Alternativ ist es auch möglich, dass die Unternehmenspartner den Evaluierungsbericht direkt unterzeichnen.“ 
Die durchschnittliche Mitarbeiteranzahl je CD-Labor erhöhte sich seit der letzten Evaluierung nur marginal von 21 auf 23 Mitarbeiter, während die wissenschaftlichen Arbeiten (Diplomarbeiten, Dissertationen und Habilitationen) im Rahmen des CD-Labors von 16,7 auf 15,8 pro CD-Labor sanken. Bei der relativen Betrachtung durch Normierung der Gesamtmittel ist dieser Rückgang ebenfalls ersichtlich, wobei er hauptsächlich durch die verringerte Anzahl von Dissertationen begründet ist. In der aktuellen Evaluierung gab es beinahe 60 referierte Publikationen je CD-Labor, hinzu kommen noch 11 nicht referierte Publikationen und 10 weitere Publikationen. Der deutliche Anstieg der Publikationen ist auch bei der Normierung mit den Gesamtmitteln markant.

Die Anzahl der geladenen Vorträge bei Konferenzen hat sich von 13,6 auf 23,3 je CD-Labor bzw. von 6 auf 9,8 pro Budgetmillion erhöht, was wiederum für die Qualität der Forschung spricht. Etwas relativiert wird diese Aussage durch den Rückgang der Preise und Auszeichnungen von 5 auf 3,8 pro CD-Labor (2,2 auf 1,7 je Fördermillion). Die Kooperationshäufigkeit nimmt zu, wobei verstärkt Partner aus dem Ausland gesucht und gefunden werden.

Im Zeitablauf ist eine Erhöhung der durchschnittlichen budgetären Gesamtmittel eines CD-Labors zu erkennen, wobei es natürlich aufgrund der teilweise geringen Anzahl von abgeschlossenen CD-Labors in einem Jahr zu Schwankungen kommt. Die eingesetzten Mittel weisen einen positiven Zusammenhang mit den weiteren Kenndaten auf. Beinahe alle untersuchten (Output-) Indikatoren steigen mit den gewährten CD-Labor-Budgets an.

Die sechs betrachteten JR-Zentren waren zum Zeitpunkt der Evaluierung zwar bereits in der Verlängerungsphase, aber aufgrund von diverser Vorlaufzeiten und Bearbeitungsdauern ist ihre volle Wirkungsentfaltung nur teilweise messbar. Um gesicherte Aussagen über das JR-Programm tätigen zu können, bedarf es daher einer weiteren Evaluierung nach Abschluss von mehreren JR-Zentren. Diese Zwischenevaluierung kann daher hauptsächlich dazu genützt werden, derzeit laufende JRZentren miteinander in Bezug zu setzen, und etwaige unterdurchschnittliche Entwicklungen einzelner JR-Zentren früh möglichst zu erkennen, wobei auch hier auf die unterschiedlichen Laufzeiten Rücksicht genommen werden muss.

Sowohl die budgetierten Gesamtkosten als auch die kumulierten tatsächlichen angefallenen Kosten zwischen 2013 und 2015 weisen eine hohe Schwankungsbreite auf. Die Unternehmenspartner entstammen fast ausschließlich aus Österreich. Der Zusammenhang zwischen angefallenen Kosten und Anstellungen in Jahresbeschäftigungsplätzen in Köpfen ist zwar generell bei den JR-Zentren etwas ausgeprägter als bei den CD-Labors, aber auch hier gibt es große Schwankungen. Dissertanten 
stellen die bedeutendste Mitarbeitergruppe dar, gefolgt von Senior Postdocs plus Postdocs. Dies ist als Anzeichen für den hohen wissenschaftlichen Anspruch zu verstehen.

Der Output an Publikationen in Zeitschriften ist aufgrund der noch relativ kurzen Laufzeit von einigen JR-Zentren und unter Berücksichtigung der Bearbeitungsdauer beim Einreichprozess im Vergleich zu den CD-Labors noch relativ gering. Bei den normierten Werten der Konferenzteilnahmen mit eingeladenen Vorträgen und Vorträgen liegen die JR-Zentren im Mittel sogar etwas über dem Wert der CD-Labors (JRZ: 2,8 / 100.000 Euro versus CDL: 24,8 / Mio. Euro). Für die Fachhochschulen stellen die JR-Zentren eine besondere Bedeutung hinsichtlich Kooperationen dar, die eine Vernetzung mit internationalen und nationalen Partner ermöglichen, die vorher nicht bestanden.

In Anbetracht der bis 2015 Gesamtkosten des JR-Programms von ca. 3,1 Mio. Euro stellt die relativ hohe Anzahl an wissenschaftlichen Auszeichnungen und Preise einen beachtlichen Wert dar, der auch im Vergleich der normierten Werte zu den CD-Labors um einiges höher ausfällt. Ebenfalls positiv zu sehen sind die Organisation von wissenschaftlichen Events und die genehmigten Anträge für Folgeprojekte und Förderungen, während es auf dem Gebiet der Patente und Erfindungen noch keinen Erfolg zu vermelden gab. 


\section{Programm- und Systemevaluierung}

Da die einzelnen Stakeholder des CDG-Programms (BMWFW, CDG, Wirtschaft, Wissenschaft) unterschiedliche Sichtweisen bzw. Prioritäten hinsichtlich des Förderprogramms haben, wurde in einem ersten Schritt versucht, diese anhand eines speziellen Verfahrens offenzulegen.

Danach werden die Erfahrungen der Unternehmenspartner von bereits abgeschlossenen CD-Labors dargestellt. Die Auswertung der Abschlussfragebögen seitens der Unternehmen zeigt, wie weit die Wirtschaft mit dem Förderprogramm zufrieden ist.

Der letzte Teil der Programmevaluierung bezieht sich auf alle bestehenden und alle seit 2011 ausgelaufenen CD-Labors, auf alle aktiven JR-Zentren sowie auf alle in den jeweiligen CD-Labors und JRZentren aktiven Unternehmenspartner der CDG. Aufbauend auf den Ergebnissen der Kenndatenerhebung werden die Programme in Bezug auf die Erreichung ihrer Programmziele untersucht. Dabei werden folgende Aspekte berücksichtigt:

- Erreichung der Programmziele,

- Qualität und Eigenheit (uniqueness) der Forschung,

- Eignung der Fördermodelle für die beiden Programme,

- Anpassungs- bzw. Änderungsbedarf,

- aktuelle und zukünftige Rolle des Programms in der österreichischen Forschungs- und Innovationslandschaft (Konkurrenz, Synergien),

- aktuelle und zukünftige internationale Positionierung des Programms,

- Förderung des wissenschaftlichen Nachwuchses.

\subsection{Evaluierung der Programmziele}

Um etwaige unterschiedliche Präferenzen hinsichtlich der operationalen Ziele identifizieren zu können, wird ein Analytischer Hierarchischer Prozess (AHP) zur Bestimmung der relativen Bedeutung der Ziele durchgeführt. In diesem Prozess wurden neben dem BMWFW und dem Präsidium der CDG auch ausgewählte Unternehmen sowie Labor- bzw. Zentrumsleiter eingebunden. Im Ergebnis konnten unterschiedliche Priorisierungen der verschiedenen Stakeholder festgestellt bzw. aufgezeigt werden.

Um die Prioritäten hinsichtlich der Programmziele zu erhalten, wurden diese mit den jeweils unterlegten Indikatoren beschrieben. 
Tabelle 19: Programmziele und Indikatoren

\begin{tabular}{|c|c|}
\hline Programmziel & Indikatoren \\
\hline \multicolumn{2}{|c|}{ Langfristigkeit und Intensität der Kooperation } \\
\hline & Zahl der Unternehmen \\
\hline & Kooperationsdauer \\
\hline & Zahl und Gründe von vorzeitigen Ausstiegen (von Unternehmen) \\
\hline & Grad der Verflechtung (mit Unternehmen) \\
\hline & Kooperation mit anderen CD-Labors und JR-Zentren \\
\hline & Kooperation mit COMET \\
\hline & Sonstige Kooperationen \\
\hline & Neue Module, Erweiterungen von Modulen \\
\hline & Fluktuation innerhalb der Forschungsgruppe \\
\hline \multicolumn{2}{|c|}{ Grundlagenforschungsergebnisse auf hohem Niveau } \\
\hline & Publikationen referiert \\
\hline & Publikationen nicht referiert \\
\hline & Konferenzen \\
\hline & Wissenschaftliche Preise und Auszeichnungen \\
\hline \multicolumn{2}{|c|}{ Praxisrelevante Forschung } \\
\hline & Praxisnähe der Themenstellung \\
\hline & Art und Intensität der Kooperation zwischen Forschungs- und Unternehmenspartnern \\
\hline \multicolumn{2}{|c|}{ Technologische Hebelwirkung } \\
\hline & Erfindungen \\
\hline & Patente \\
\hline & Umsetzungs-Folgeaktivitäten \\
\hline & Induzierte weitere Forschungsprojekte \\
\hline \multicolumn{2}{|c|}{ Wissenstransfer } \\
\hline & Prozessinnovationen \\
\hline & Produktinnovationen \\
\hline & Entwicklung der Forschungsauftragslage beim Forschungspartner der Betreiberorganisation \\
\hline \multicolumn{2}{|c|}{ Entwicklung von Humanressourcen } \\
\hline & Master-/Diplomarbeiten \\
\hline & Dissertationen \\
\hline & Habilitationen \\
\hline & Berufungen \\
\hline & Wissenschaftliche Preise und Auszeichnungen \\
\hline & Wechsel von Laborpersonal in Unternehmen \\
\hline \multicolumn{2}{|c|}{ Unternehmensentwicklung } \\
\hline & Umsatzentwicklung \\
\hline & Entwicklung der Beschäftigtenzahl \\
\hline & Zahl der Forschungsarbeitsplätze \\
\hline & Entwicklung der Sparte, der das CD-Labor zuzurechnen ist, innerhalb des Unternehmens \\
\hline \multicolumn{2}{|c|}{ Internationalisierung } \\
\hline & Zahl ausländischer Unternehmenspartner \\
\hline & Internationale CD-Labors \\
\hline & Internationale Module \\
\hline & internationale Sichtbarkeit der CDG \\
\hline
\end{tabular}

Quelle: $C D G$ 
Anhand einer vorgegebenen Bewertungsskala erfolgte ein paarweiser Vergleich der Programmziele, welcher in einer Bewertungsmatrix mündete.

Tabelle 20: Beispiel einer Bewertungsmatrix

\begin{tabular}{|c|c|c|c|c|c|c|c|c|}
\hline Programmziele & A: & B: & C: & D: & $\mathrm{E}:$ & $\mathrm{F}:$ & G: & $\mathrm{H}:$ \\
\hline A: Langfristigkeit und Intensität der Kooperation & 1 & $1 / 4$ & $1 / 2$ & 3 & 2 & 4 & 1 & 7 \\
\hline B: Grundlagenforschungsergebnisse auf hohem Niveau & 4 & 1 & 3 & 7 & 6 & 7 & 3 & 8 \\
\hline C: Praxisrelevante Forschung & 2 & $1 / 3$ & 1 & 4 & 3 & 4 & 1 & 7 \\
\hline D: Technologische Hebelwirkung & $1 / 3$ & $1 / 7$ & $1 / 4$ & 1 & $1 / 2$ & $1 / 2$ & $1 / 5$ & 3 \\
\hline E: Wissenstransfer & $1 / 2$ & $1 / 6$ & $1 / 3$ & 2 & 1 & 1 & $1 / 3$ & 6 \\
\hline F: Entwicklung von Humanressourcen & $1 / 4$ & $1 / 7$ & $1 / 4$ & 2 & 1 & 1 & $1 / 3$ & 6 \\
\hline G: Unternehmensentwicklung & 1 & $1 / 3$ & 1 & 5 & 3 & 3 & 1 & 8 \\
\hline $\mathrm{H}$ : Internationalisierung & $1 / 7$ & $1 / 8$ & $1 / 7$ & $1 / 3$ & $1 / 6$ & $1 / 6$ & $1 / 8$ & 1 \\
\hline
\end{tabular}

Quelle: Economica/IWI.

Tabelle 21: Bewertungsskala des paarweisen Vergleichs

\begin{tabular}{c|l|l|l|l} 
Wert & Definition & $\begin{array}{l}\text { Interpretation } \\
\text { absolut dominierend }\end{array}$ & $\begin{array}{l}\text { Es handelt sich um den größtmöglichen Bedeutungsunterschied (dominierend) } \\
\text { zwischen zwei Elementen }\end{array}$ \\
\hline $\mathbf{7}$ & $\begin{array}{l}\text { sehr viel größere } \\
\text { Bedeutung }\end{array}$ & $\begin{array}{l}\text { Die sehr viel größere Bedeutung eines Elements hat sich in der Vergangenheit } \\
\text { klar gezeigt. }\end{array}$ \\
\hline $\mathbf{5}$ & $\begin{array}{l}\text { erheblich größere } \\
\text { Bedeutung }\end{array}$ & $\begin{array}{l}\text { Erfahrung und Einschätzung sprechen für eine erheblich größere Bedeutung } \\
\text { eines Elements im Vergleich zu einem anderen. }\end{array}$ \\
\hline $\mathbf{3}$ & $\begin{array}{l}\text { etwas größere } \\
\text { Bedeutung }\end{array}$ & $\begin{array}{l}\text { Erfahrung und Einschätzung sprechen für eine etwas größere Bedeutung eines } \\
\text { Elements im Vergleich zu einem anderen. }\end{array}$ \\
\hline $\mathbf{1}$ & gleiche Bedeutung & $\begin{array}{l}\text { Beide verglichenen Elemente haben die gleiche Bedeutung für das } \\
\text { nächsthöhere Element. }\end{array}$ \\
\hline $\mathbf{1 / 3}$ & $\begin{array}{l}\text { etwas geringere } \\
\text { Bedeutung }\end{array}$ & $\begin{array}{l}\text { Erfahrung und Einschätzung sprechen für eine etwas geringere Bedeutung } \\
\text { eines Elements im Vergleich zu einem anderen. }\end{array}$ \\
\hline $\mathbf{1 / 5}$ & $\begin{array}{l}\text { erheblich geringere } \\
\text { Bedeutung }\end{array}$ & $\begin{array}{l}\text { Erfahrung und Einschätzung sprechen für eine erheblich geringere Bedeutung } \\
\text { eines Elements im Vergleich zu einem anderen. }\end{array}$ \\
\hline $\mathbf{1 / 7}$ & $\begin{array}{l}\text { sehr viel geringere } \\
\text { Bedeutung }\end{array}$ & $\begin{array}{l}\text { Die sehr viel geringere Bedeutung eines Elements hat sich in der Vergangenheit } \\
\text { klar gezeigt. } \\
\text { Es handelt sich um den größtmöglichen Bedeutungsunterschied (unterlegen) }\end{array}$ \\
\hline $\mathbf{1 / 9}$ & absolut unterlegen & \begin{tabular}{l} 
zwischen zwei Elementen \\
\hline
\end{tabular}
\end{tabular}

Quelle: AHP, Saaty.

Für jede Bewertungsmatrix kann nun ein Gewichtungsvektor (bestimmt aus dem Eigenvektor des höchsten Eigenwerts der Bewertungsmatrix) angegeben werden. Darüber hinaus bietet die Methode 
auch noch die Möglichkeit, die Konsistenz der verschiedenen paarweisen Vergleiche zu untersuchen. Eine extreme Verletzung der Konsistenz wäre zum Beispiel, wenn die Transitivität der Bewertung nicht eingehalten würde (z.B. $A>B$ \& $B>C$ aber $A<C$ ).

Im Ergebnis zeigen sich sowohl innerhalb der Stakeholder-Gruppen als auch zwischen innen markante Unterschiede, die auf eine heterogene Erwartungshaltung gegenüber den Programmzielen deuten.

Im Vergleich zwischen den Bewertungen der zuständigen Abteilung innerhalb des Ministeriums und des Präsidiums der CDG liegen besonders bei den Programmzielen „Grundlagenforschungsergebnisse auf hohem Niveau“ und „Entwicklung von Humanressourcen“ größere Unterschiede in der Bewertung vor. Während von Seiten des Programträgers die überwiegende Bedeutung auf die Grundlagenforschung gelegt wird, liegt dieses Programmziel laut Präsidium nur etwas über der Entwicklung von Humanressourcen. Auch beim Wissenstransfer und der Unternehmensentwicklung sind die Prioritäten gegensätzlich gerichtet. Da bei den restlichen vier Programmzielen die Prioritäten sehr eng beieinander liegen, ist der positive Zusammenhang, bestimmt durch einen Korrelationswert von 0,56 (nicht signifikant), noch (einigermaßen) gegeben.

\section{Abbildung 32: Bewertung der Programmziele: BMWFW, CDG}

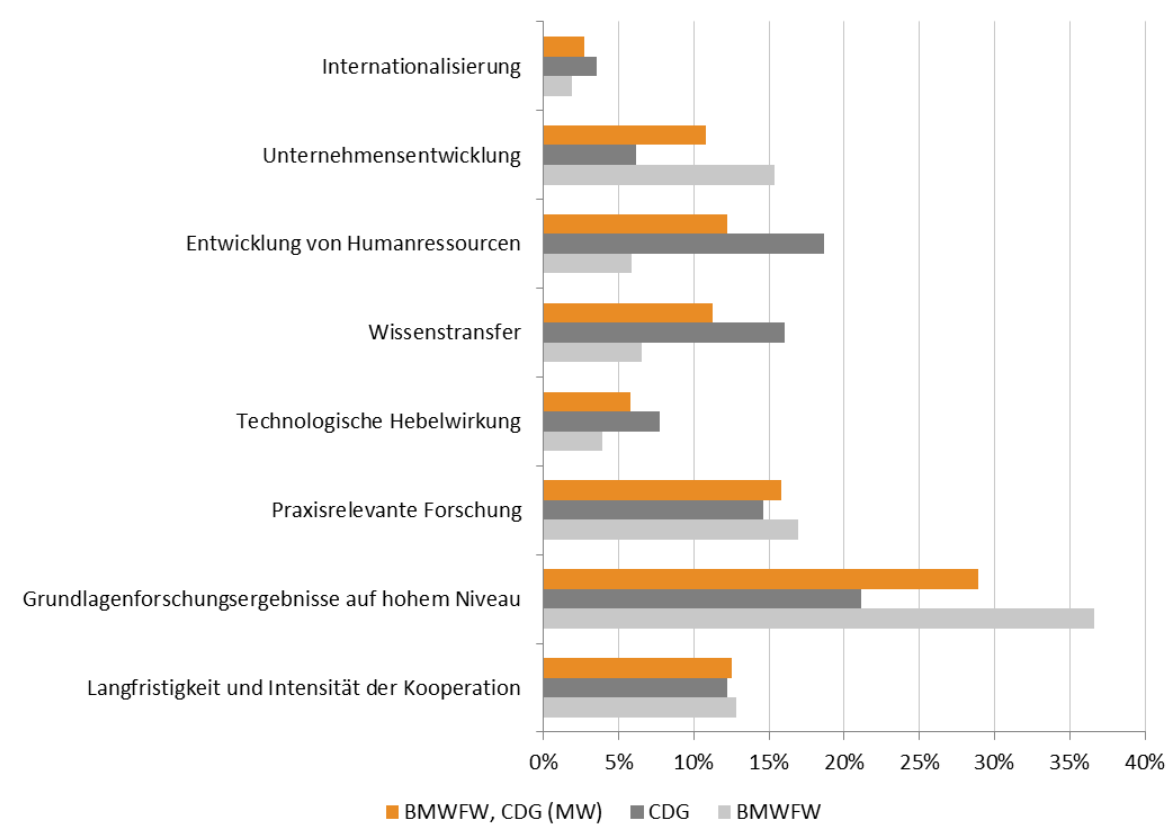

Quelle: Einzelinterviews, Economica, IWI. 
Die beiden Bewertungen des Labor- bzw. Zentrumsleiter waren zwar im Vergleich aller StakeholderGruppen am ähnlichsten, aber auch hier gab es zwischen den Programmzielen „Langfristigkeit und Intensität der Kooperation", „Technologische Hebelwirkung“ und „Entwicklung von Humanressourcen“ größere Abweichungen. Insgesamt war der Korrelationskoeffizient der beiden Gewichtungen mit einem Wert von 0,64 etwas höher, und dies bei einem (schwach) signifikanten PWert von 0,09 vor.

Abbildung 33: Bewertung der Programmziele: Labor- und Zentrumsleiter

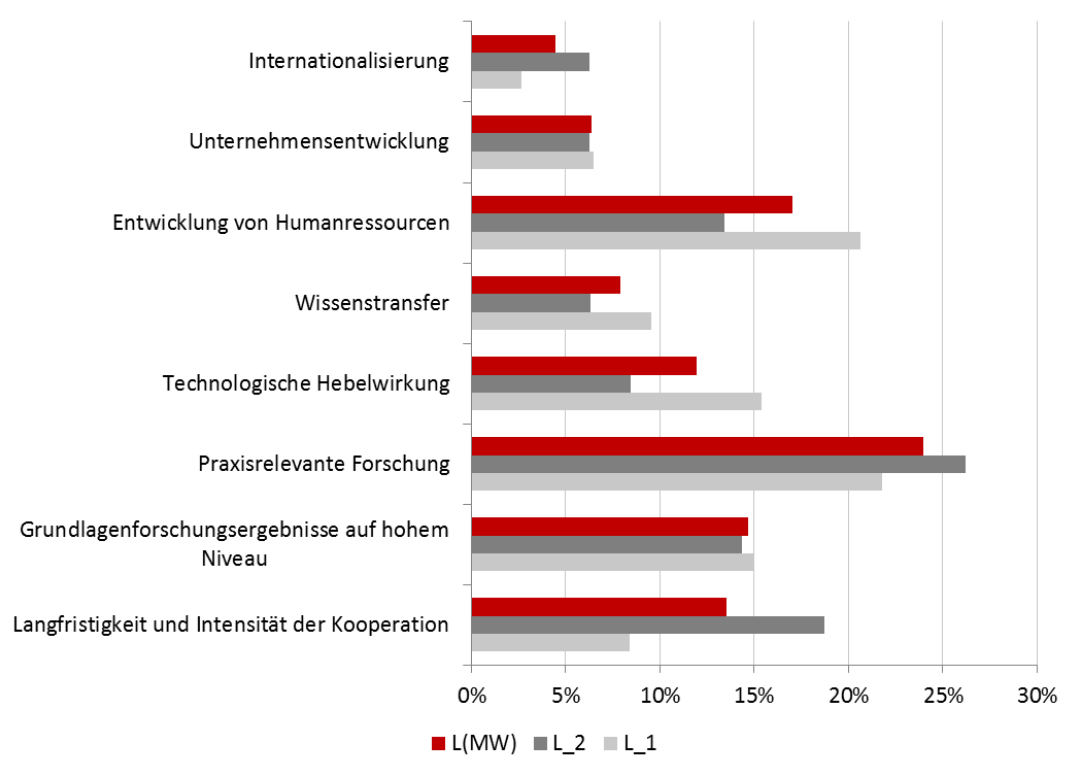

Quelle: Einzelinterviews, Economica, IWI. L(MW) Mittelwert der Gewichte der beiden Leiter L_n

Am heterogensten waren die Bewertungen der beiden Unternehmenspartner, was auch durch einen Korrelationskoeffizient von 0,45 (Irrtumswahrscheinlichkeit ${ }^{40} \mathrm{P}=0,26$ ) der Gewichtungsvektoren zum Ausdruck kommt. Die einzelnen Bewertungen der Unternehmenspartner, für sich genommen, waren aber sehr wohl konsistent. Somit erscheint es speziell von Seiten der Wirtschaft eine heterogenere Erwartungshaltung gegenüber dem Forschungsprogramm zu geben.

Besonders die Programmziele "Grundlagenforschungsergebnisse auf hohem Niveau" und „Unternehmensentwicklung" wurden konträr beurteilt.

\footnotetext{
40 Die Wahrscheinlichkeit eines systematischen Zusammenhangs zwischen zwei Variablen wird mit der Signifikanz überprüft, wobei diese Kennzahl überprüft, ob ein Zusammenhang rein zufälliger Natur sein könnte oder mit hoher Wahrscheinlichkeit tatsächlich vorliegt. Entsprechend ist auch die Kennzahl Irrtumswahrscheinlichkeiten zu interpretieren.
} 
Abbildung 34: Bewertung der Programmziele: Unternehmen

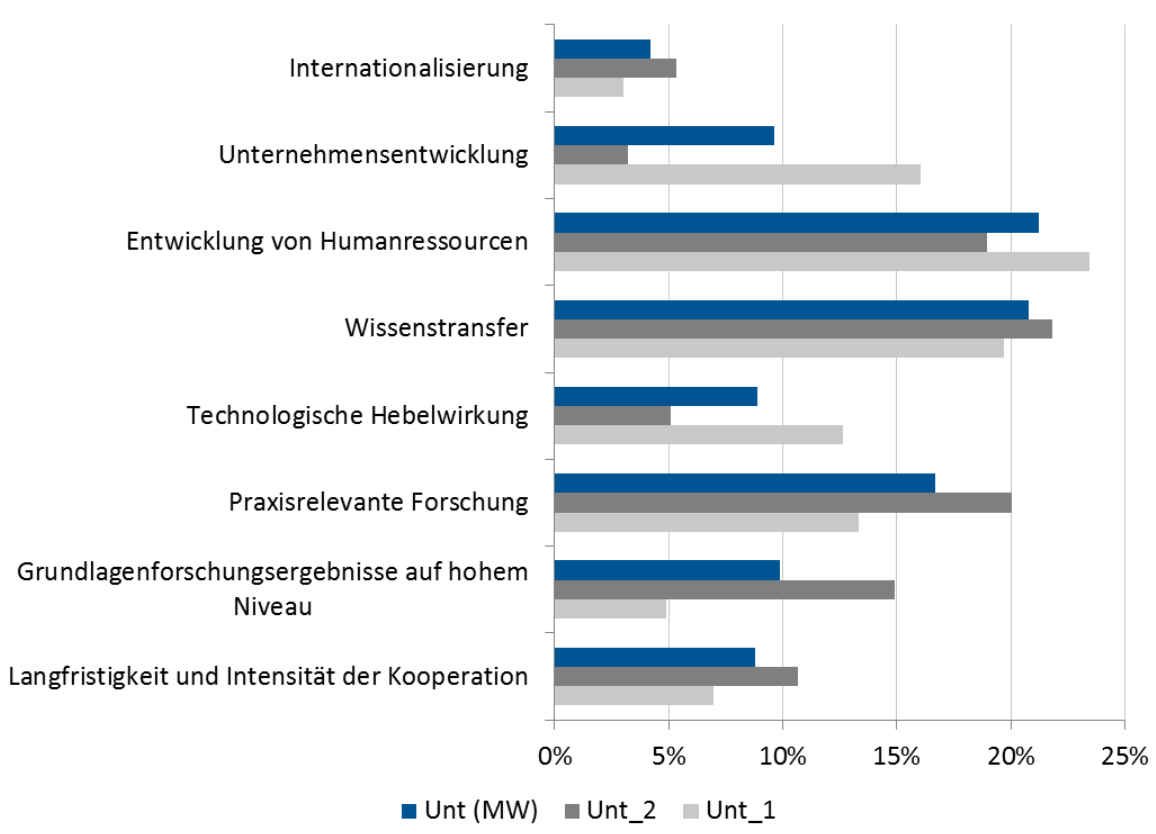

Quelle: Einzelinterviews, Economica, IWI. Unt(MW) Mittelwert der Gewichte der beiden Unternehmen Unt_n

Vergleicht man die Korrelationskoeffizienten der Gewichtungen der einzelnen Stakeholder, so gibt es zwischen der Bewertung der CDG und des Laborleiters 1 bzw. des Unternehmenspartners 2 einen signifikant positiven Zusammenhang ( $R=0,65$ bzw. $R=0,83 ; P=0,08$ bzw. $P=0,01)$.

Fasst man die einzelnen Bewertungen innerhalb und zwischen den Stakeholder-Gruppen in Form des arithmetischen Mittels zusammen, so erhält man ein Gewichtungsschema über alle Interessen hinaus, das einen gewissen Ausgleich der unterschiedlichen Erwartungshaltungen an das CDGProgramm bildet. Das Programmziel Grundlagenforschung weist die zweithöchste Gewichtung auf, wobei dies einzig auf die überdurchschnittliche Bewertung des Programmträgers zurückzuführen ist. Ein weiterer markanter Unterschied liegt beim Programmziel Wissenstransfer vor, das von den Unternehmenspartnern eine höhere Bedeutung zugewiesen bekommen hat. Die Unternehmensentwicklung ist den Wirtschaftsvertretern, wenig überraschend, wichtiger als den Laborleitern. Im Gegensatz dazu wird die Langfristigkeit und Intensität der Kooperation für die Unternehmen im Vergleich zur Wissenschaft als weniger wichtig beurteilt.

Insgesamt gesehen liegt der Fokus eindeutig auf dem Bereich Forschung, wobei diese zwar grundlagenorientiert, aber gleichzeitig auch eine praxisrelevante Dimension aufweisen sollte. Einen zweiten bedeutenden Bereich stellt die Umsetzung der gewonnenen Erkenntnisse in Form von 
Humanressourcen und Wissenstransfer dar. Die weiteren Programmziele werden anscheinend teilweise als Resultat der ersten beiden Blöcke angesehen, und erhalten daher ein geringeres Gewicht.

Abbildung 35: Bewertung der Programmziele: Stakeholder

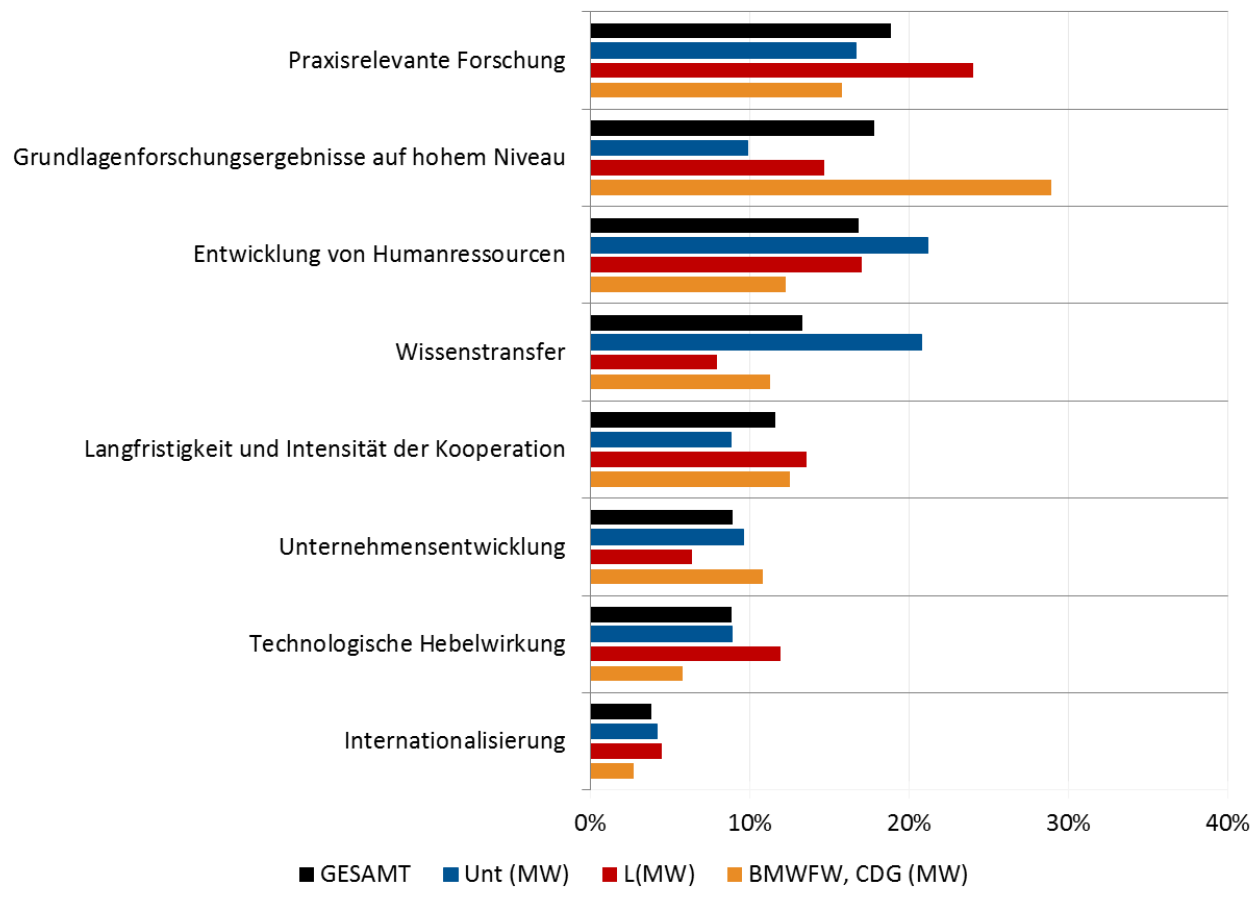

Quelle: Einzelinterviews, Economica, IWI. 


\subsection{Abschlussfragebögen}

Im Rahmen der Evaluierung konnte nur auf Abschlussfragebögen der CD-Labors zurückgegriffen werden, da bis dato noch kein JR-Zentrum abgeschlossen wurde.

\subsubsection{Abschlussfragebögen der Laborleiter}

Erfahrungen aus den industrierelevanten Projekten sind im akademischen Betrieb des Heimat gebenden Instituts vor allem in den Bereich der Forschung eingeflossen. 63\% der CD-Laborleiter attestieren einen „in großem Ausmaß“ bestehenden Einfluss auf die Forschung, 95\% (=63\% + 32\%) einen Einfluss zumindest „im mittleren Ausmaß“. Bei der Untersuchung der Lehre reduzieren sich diese Werte auf $17 \%$ bzw. 66\% (= 17\% + 49\%).

Abbildung 36: Ausmaß, in denen Erfahrungen in der Forschung bzw. Lehre wirksam geworden sind (falls ein Einfluss besteht)

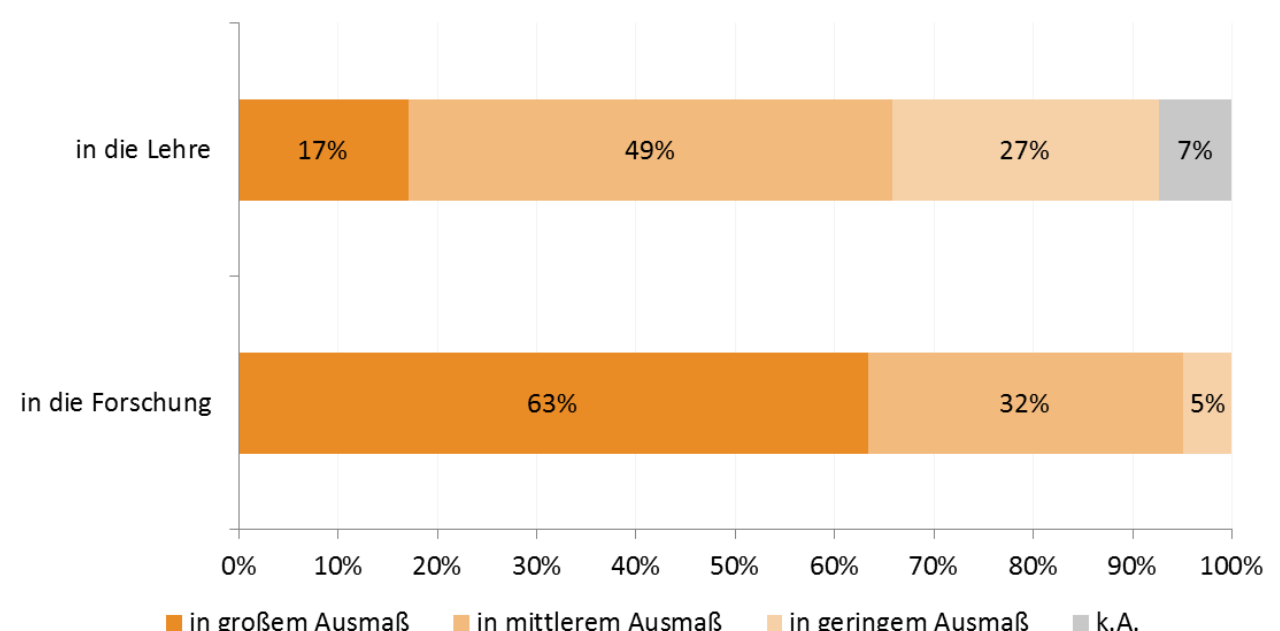

Quelle: Abschlussfragebögen der CDG ( $n=41)$.

Der Gesamteindruck von der Qualität der Zusammenarbeit mit der Industrie wird von den CDLaborleitern je nach betrachtetem Aspekt (unter anderem intellektueller und materieller Input, Ausmaß an wissenschaftlichen Freiraum) etwas unterschiedlich gesehen, aber grundsätzlich wird von wissenschaftlicher Seite aus die Kooperation mit der Industrie als sehr positiv angesehen. 


\section{Abbildung 37: Gesamteindruck von der Qualität der Zusammenarbeit mit der Industrie}

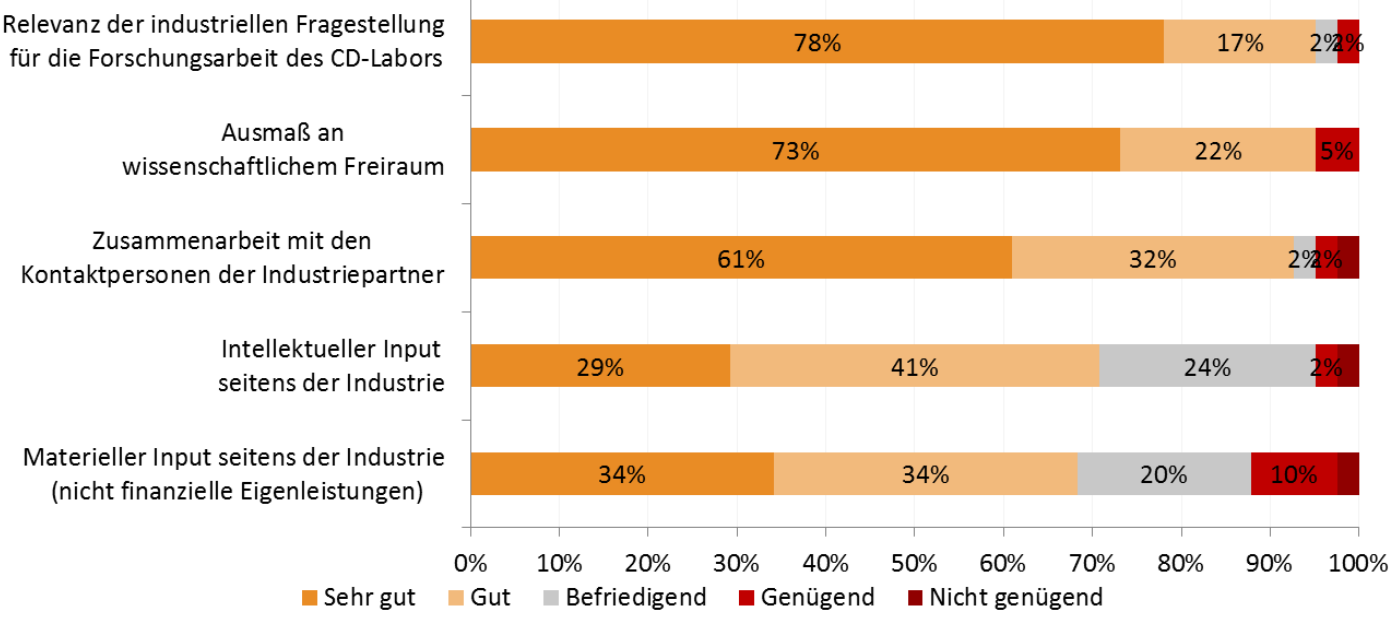

Quelle: Abschlussfragebögen der CDG (n=41).

Besonders gute Noten erzielten die Bereiche „Relevanz der industriellen Fragestellung für die Forschungsarbeit des CD-Labors" (sehr gut: 78\%) und "Ausmaß an wissenschaftlichem Freiraum" (sehr gut: 73\%). Im Vergleich mit diesen relativ hohen (sehr guten) Werten, besteht bei den Aspekten intellektueller und materieller Input seitens der Industrie noch Verbesserungsbedarf.

Die Laborleiterinnen und Laborleiter gaben bis auf eine Ausnahme $(1 / 41=2,4 \%)$ an, dass für das Themengebiet des CD-Labors eine Folgeexpertise herangebildet wurde, die weitere Forschungsarbeiten in diesem Bereich, nach dem Auslaufen des CD-Labors, zur Folge hatte. Bezugnehmend auf das Ausmaß der Forschungsarbeiten im Vergleich zum CD-Labor fallen die Ergebnisse sehr heterogen aus. In 31,7\% der Fälle wurden die thematischen Arbeiten des CD-Labors in der Zeit danach in größerem Ausmaß weitergeführt, in 29,3\% der Fälle hingegen in geringerem Umfang. Zusammenfassend kann gesagt werden, dass rund bei einem Drittel eine Folgeexpertise für das Themengebiet des CD-Labors herangebildet wurde, die Forschungsarbeiten in diesem Bereich nach dem Auslaufen des CD-Labors ermöglichte. 
Abbildung 38: Ausmaß der Forschungsarbeiten nach dem Auslaufen des CD-Labors (Folgeexpertise)

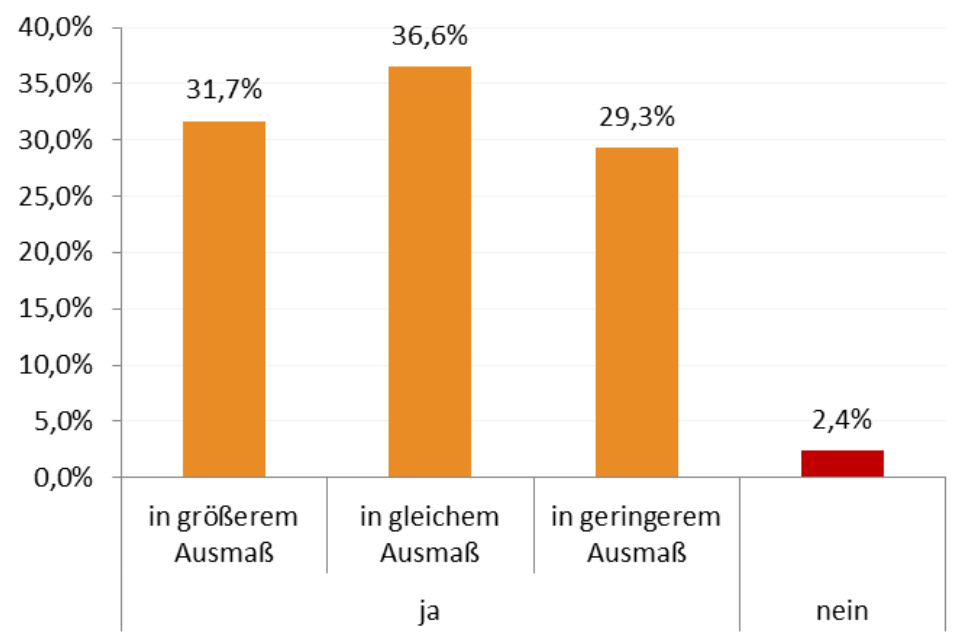

Quelle: Abschlussfragebögen der CDG (n=41).

Aus etwas mehr als der Hälfte der untersuchten CD-Labors (52\%, 22 von 42) gingen Wissenschaftlerinnen und Wissenschaftler hervor, die danach ihre eigenen Forschergruppen leiten oder geleitet haben. Da dies teilweise für zwei oder mehr Personen aus einem CD-Labor der Fall war, sind insgesamt 54 Personen angeführt worden. Umgerechnet auf alle 42 CD-Labors leiten oder leiteten somit im Mittel 1,3 ehemalige CD-Labor Wissenschaftler eigene Forschungsgruppen.

Abbildung 39: Gingen aus dem CD-Labor Wissenschaftler hervor, die eigene Forschungsgruppen leiten bzw. geleitet haben?

Quelle: Abschlussfragebögen der CDG (n=42). 


\subsubsection{Aspekte betreffend Unternehmenspartner}

Bei den untersuchten CD-Labors ( $n=42)$ sind 151 Mitarbeiterinnen und/oder Mitarbeiter während der Laufzeit des Labors in die relevante Industrie gewechselt, was einem Schnitt von 3,6 Abgängen in die Wirtschaft pro CD-Labor entspricht.

Schulungen für Unternehmenspartner fanden lediglich bei 13 der 42 untersuchten CD-Labors statt, abgesehen von einigen Schulungen mit einer sehr großen Anzahl von Teilnehmern, waren dies meist Kleingruppen mit fünf Personen.

Bei den 42 teilnehmenden CD-Labors waren 31 Labors (74\%) zumindest an einer gemeinsamen PRAktivität der Unternehmenspartner (Mehrfachnennung möglich) beteiligt. Mitunter bestand diese Zusammenarbeit aus der gemeinsamen Präsenz und/oder Beteiligung an Vorträgen (59,5\% =25/42) bzw. der Nennung des CD-Labors im Werbematerial des Unternehmens (50\% $=21 / 42)$. Nennenswert scheinen auch die gemeinsamen Publikationen in praxisorientierten Zeitschriften (31\%=13/42). Die gemeinsame Präsenz von Unternehmenspartnern und CD-Labormitarbeitern beim Kunden wurde hingegen seltener praktiziert $(19 \%=8 / 42)$.

\section{Abbildung 40: Gemeinsame PR-Aktivitäten des CD-Labors und der Unternehmenspartner}

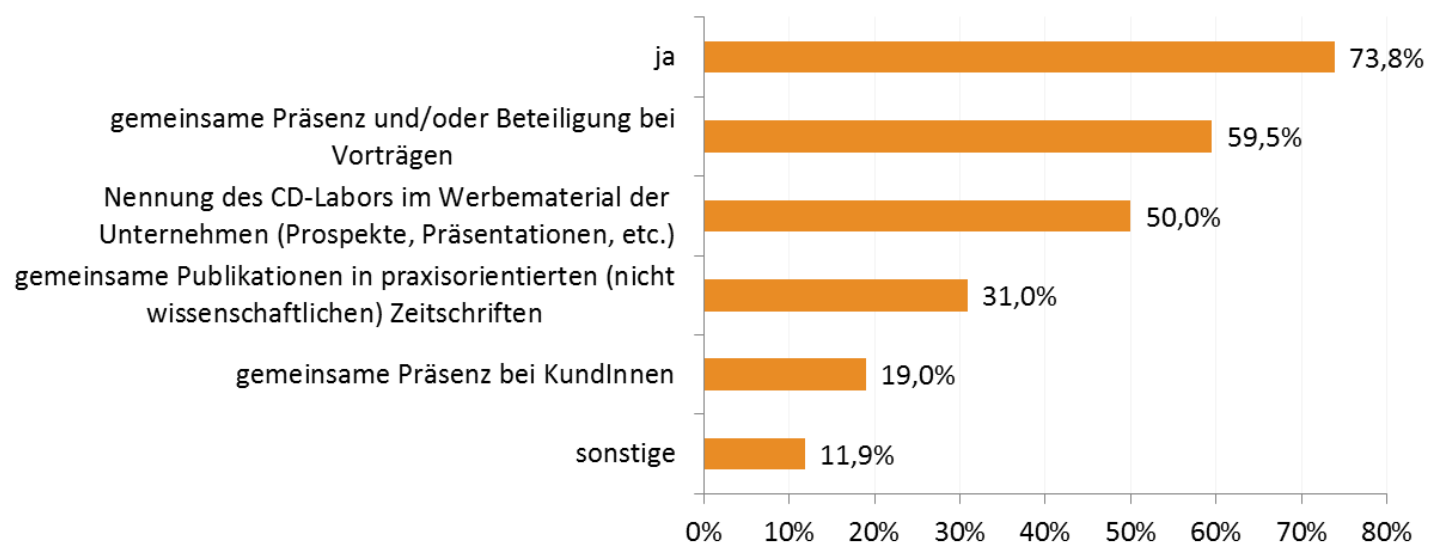

Quelle: Abschlussfragebögen der CDG

Von den 42 befragten CD-Laborleitern gaben 12 an, dass von den am CD-Labor beteiligten Partnern (Labor-Mitarbeiter, Institut, Unternehmen) Patente eingereicht wurden. Insgesamt reichten diese CD-Labors 109 Patente ein, was einem Schnitt von 2,6 Patenteinreichungen pro Labor bezogen auf alle 42 untersuchten CD-Labors entspricht. 


\subsubsection{Aspekte betreffend die Interessenslage der öffentlichen Hand und der CDG}

Der dritte Abschnitt der Schlussevaluierung widmete sich verschiedenen Aspekten, welche die Interessen der öffentlichen Hand sowie der CDG betreffen.

Ein Großteil (38 von 42) der befragten Laborleiter $(90,5 \%)$ sehen durch die Forschungsaktivitäten des Heimat gebenden Instituts eine nachhaltige Verstärkung im Themenbereich des CD-Labors (über den Förderzeitraum hinaus). 71,4 \% (30 von 42) nennen eine Erhöhung des Mitarbeiterstandes und $66,7 \%$ (28 von 42) eine Verbesserung der Drittmittelsituation in diesem Themenbereich. Einen Anteil von $35,7 \%$ (15 von 42) nimmt die Einrichtung neuer Forschungsgruppen zu diesem Themenbereich ein.

Unter Sonstiges wurden folgende Aspekte angeführt: Verbreiterung des Forschungsspektrums, Ankauf von Geräten, Etablierung von internationalen Kooperationen sowie Internationale Wahrnehmung durch multiple einschlägige Publikationen im Fachbereich.

Abbildung 41: Wurden aufgrund der Tätigkeiten des CD-Labors die Forschungsaktivitäten des Heimat gebenden Instituts im Themenbereich des CD-Labors nachhaltig (über den Förderzeitraum hinaus) verstärkt?

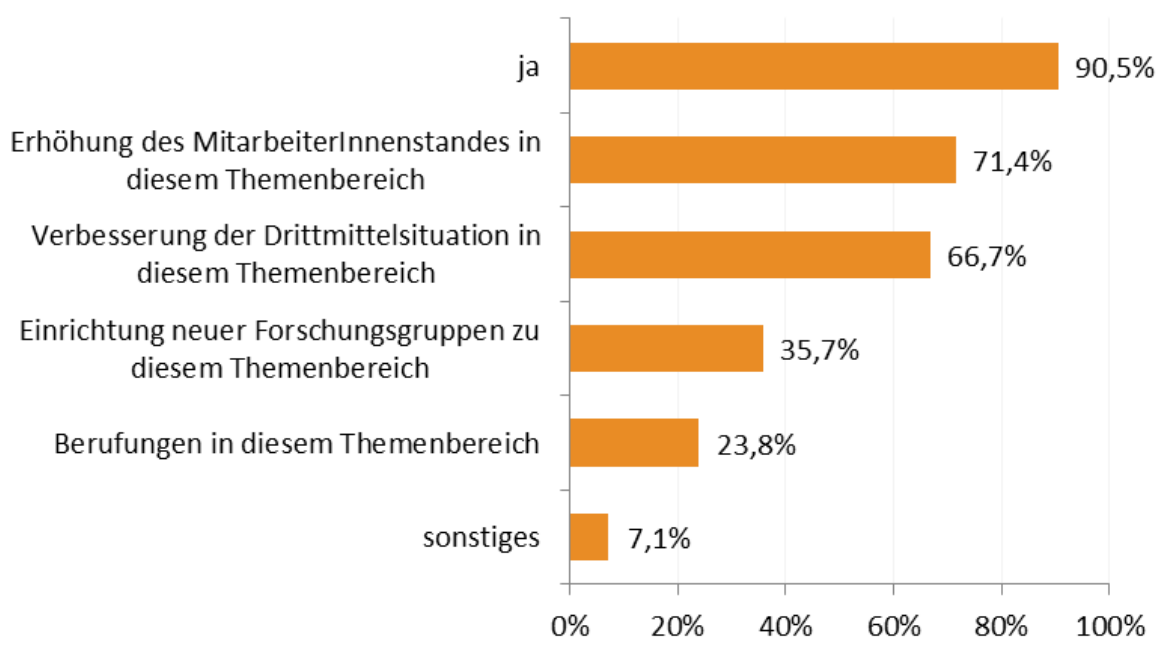

Quelle: Abschlussfragebögen der CDG $(n=42)$.

Die CD-Labors haben einen nachhaltigen Einfluss auf die universitäre Lehre. Dieser Einfluss wird am stärksten in einer inhaltlichen Erweiterung bzw. Änderung bestehender Lehrveranstaltungen spürbar (81\%). Fast ein Viertel der Befragten gibt außerdem an, dass das CD-Labor auch zur Einführung neuer Lehrveranstaltungen beitragen konnte. Unter der Kategorie „andere“ wurde genannt: Erhöhung der 
Praxisnähe, Erhöhung der Praxisnähe der Ausbildung, Verknüpfung Forschung mit Lehre, Diplomarbeiten.

\section{Abbildung 42: Einfluss des CD-Labors auf die Universitäre Lehre}

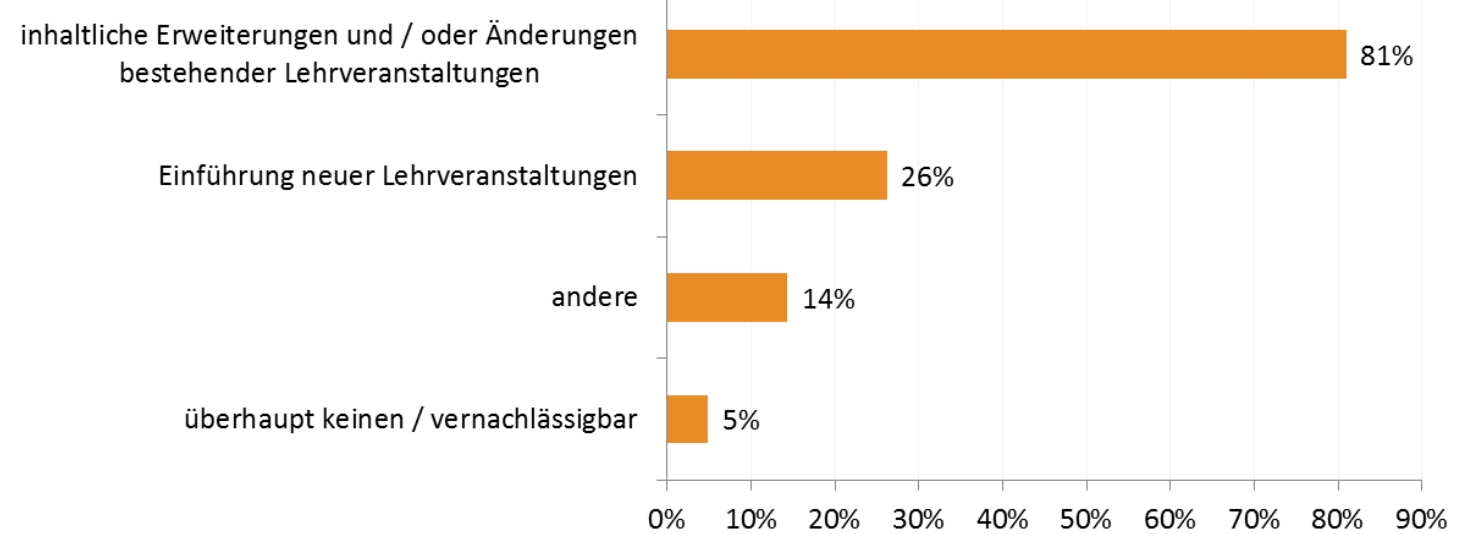

Quelle: Abschlussfragebögen der CDG $(n=42)$.

Wie aus Abbildung 43 ersichtlich, ist der positive Einfluss auf das Drittmittelvolumen des Heimat gebenden Instituts im Themenkreis des CD-Labors sehr hoch, wobei sich die Wirkung nicht nur auf den Themenbereich des CD-Labors beschränkt. Auf die Frage, wie stark sich dieser Einfluss sowohl inner- als auch außerhalb des Themenbereichs des CD-Labors entwickelt hat, antworteten 24\%, dass dieser sich stark zunehmend bzw. zunehmend (60\%) entwickelt hat.

Abbildung 43: Generelle Auswirkungen des CD-Labors auf das Drittmittelvolumen des Heimat gebenden Instituts/Departments (auch außerhalb des Themenbereichs des CDLabors)

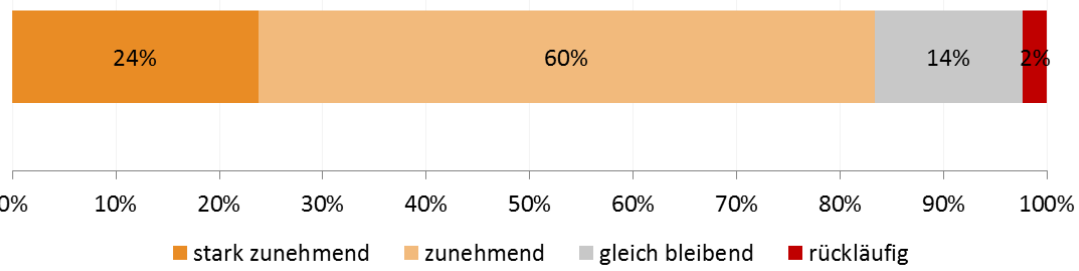

Quelle: Abschlussfragebögen der CDG $(n=42)$. 
Die Unternehmenskooperation des Heimat gebenden Instituts bzw. Departments sehen $12 \%$ der Befragten als stark zunehmend bzw. zunehmend (57\%) an, 29\% bewerten den Einfluss des CD-Labors auf die Kooperationsstruktur mit Unternehmen zumindest gleichbleibend.

Abbildung 44: Generelle Auswirkungen des CD-Labors auf die Unternehmenskooperationen

\begin{tabular}{ccccccccccc}
\hline $12 \%$ & & & & $29 \%$ & & $2 \%$ \\
\hline
\end{tabular}

Quelle: Abschlussfragebögen der CDG (n=42).

Zumindest drei von 42 der befragten CD-Labors gaben an, Initiativen entwickelt zu haben, um das CD-Labor nach dem Förderungszeitraum als eigenständiges Unternehmen weiterzuführen. Von den 42 befragten CD-Labors wurden während des Förderungszeitraumes allerdings keine Unternehmen aus dem CD-Labor ausgegründet. Während der Laufzeit des CD-Labors konnten von den untersuchten CD-Labors weitere Förderungen in Höhe von 54,32 Mio. Euro eingeworben werden. Der durchschnittliche zusätzliche Förderbetrag pro CD-Labor lag bei 1,29 Mio. Euro.

\subsubsection{Abschlussfragebögen der Unternehmen}

In diesem Abschnitt werden die Abschlussfragebögen der Unternehmen statistisch ausgewertet, diese Analysen stellen somit das Pendant zu den qualitativen Fragen der Abschlussevaluierung der CD-Laborleiter dar.

Die Unternehmen beurteilen die Zusammenarbeit im CD-Labor/JR-Zentrum bis auf zwei Ausnahmen zumindest als gut, wobei dreiviertel sogar der Meinung waren, sie war sehr gut. 
Abbildung 45: Zusammenarbeit im CD-Labor

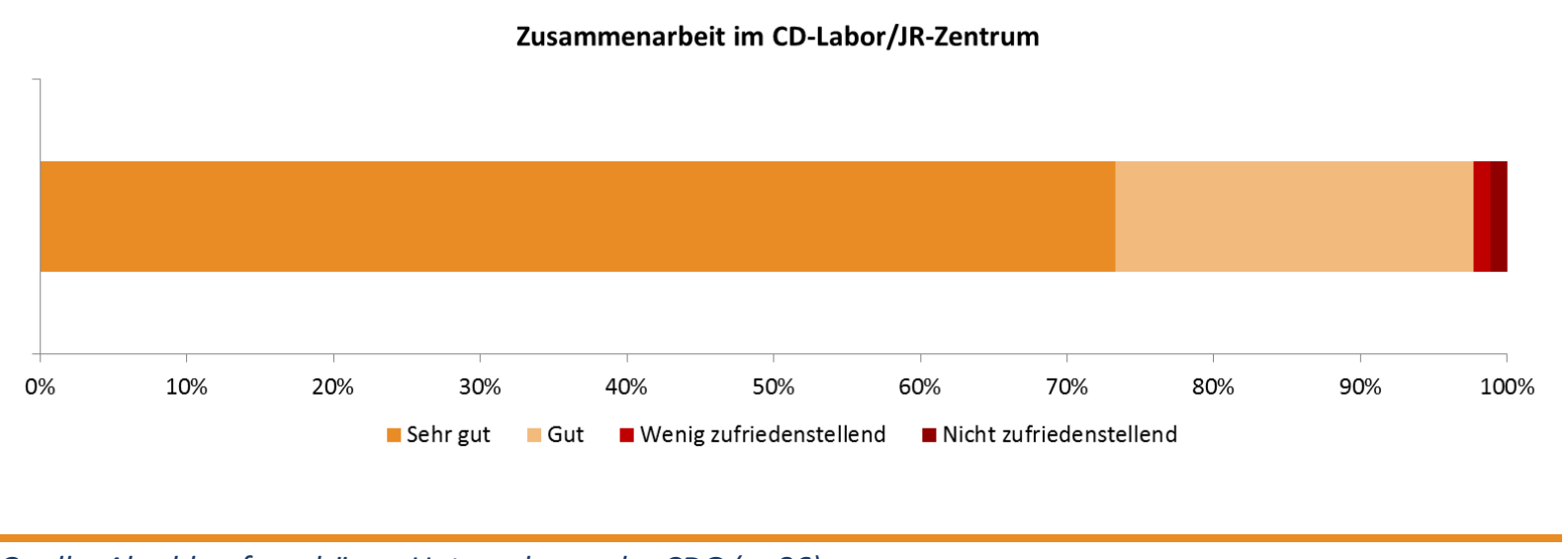

Quelle: Abschlussfragebögen Unternehmen der CDG (n=86).

Die Forschungsarbeit des CD-Labors/JR-Zentrums wird ebenfalls als praxisnah beurteilt. Hier gab es nur ein Unternehmen von insgesamt 85 Respondenten, das die Forschungsarbeit als nur wenig praxisnah bezeichnete.

Abbildung 46: Praxisnähe der Forschungsarbeit

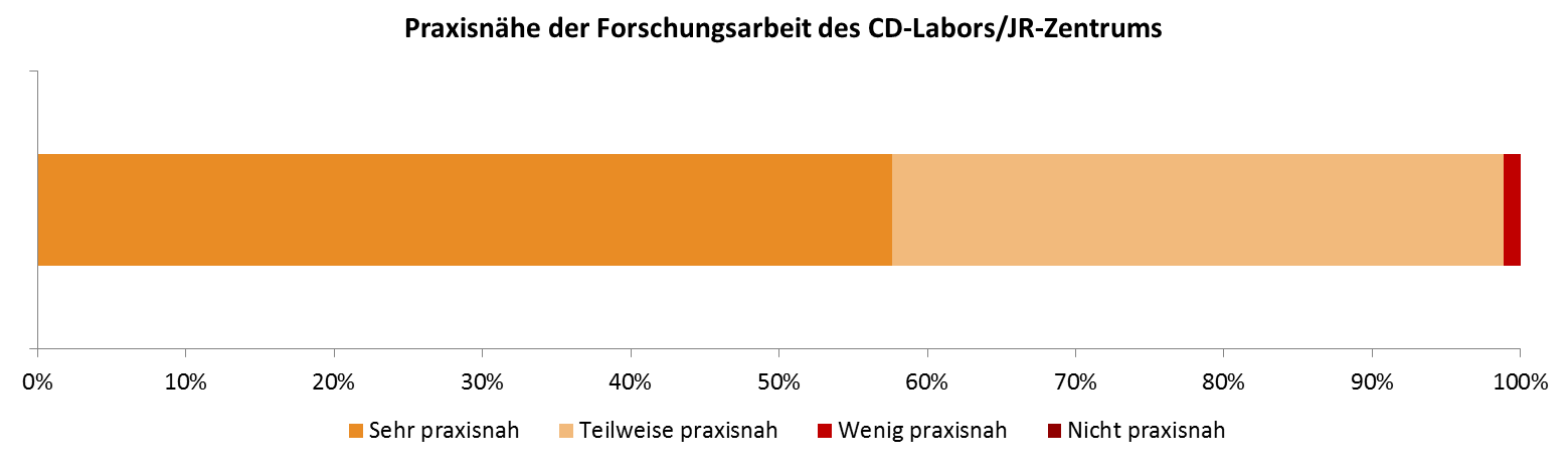

Quelle: Abschlussfragebögen Unternehmen der CDG ( $n=85)$.

Die Ergebnisse des CD-Labors/JR-Zentrums lieferten vielfach die Basis für Produkt- oder Prozessinnovationen. 22 Unternehmen gaben an, dass die Innovationen in großem Ausmaß stattgefunden haben, weitere 27 attestieren zumindest ein geringes Ausmaß. Obwohl bei 24 Unternehmen noch keine Innovationen auf Basis der Ergebnisse des CD-Labors eingetreten sind, werden sie zumindest noch erwartet. Bei sieben Unternehmen kam es zu keinen neuen Produkten bzw. konnten die Prozesse nicht verbessert werden. 
Abbildung 47: Produkt- oder Prozessinnovationen

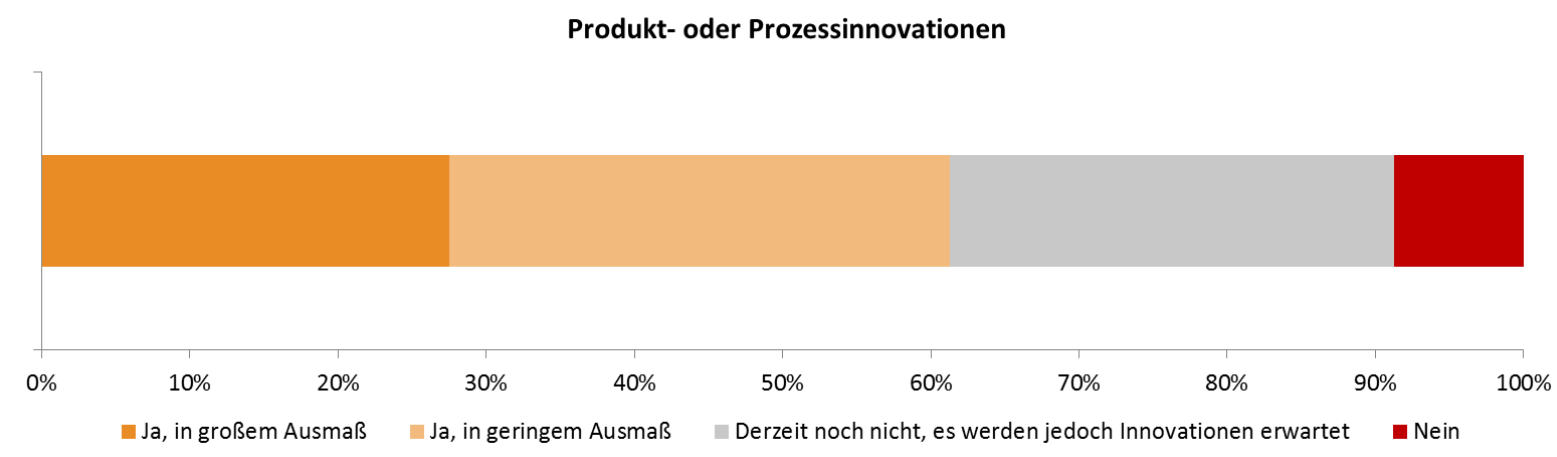

Quelle: Abschlussfragebögen Unternehmen der CDG $(n=80)$.

In rund dreiviertel der Fälle gibt es weiterführende innerbetriebliche Forschungs- und Entwicklungsaktivitäten aufgrund des CD-Labors. Lediglich acht Unternehmen verfolgen das behandelte Thema des CD-Labors nicht weiter.

Abbildung 48: Weiterführende innerbetriebliche Forschungs- und Entwicklungsaktivitäten

Weiterführende innerbetriebliche Forschungs- und Entwicklungsaktivitäten

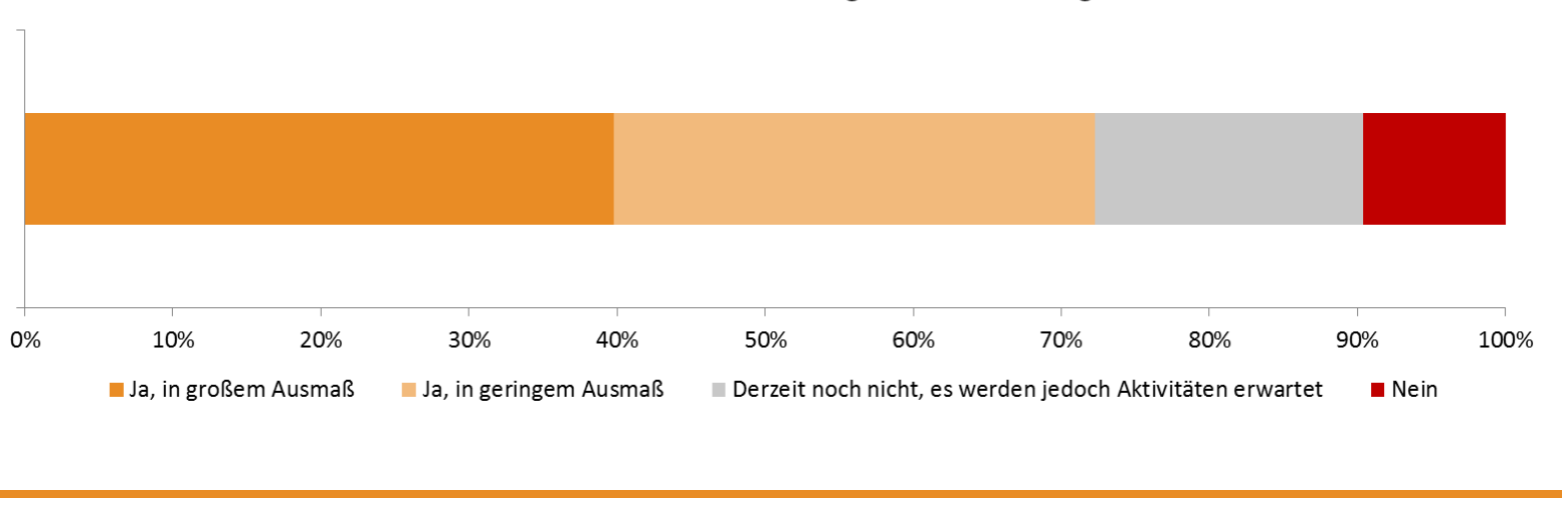

Quelle: Abschlussfragebögen Unternehmen der CDG (n=83).

In 60 Prozent der Fälle werden Folgeprojekte mit der universitären Forschungseinrichtung durchgeführt, von denen rund die Hälfte sogar in großem Ausmaß anfallen wird. 
Abbildung 49: Folgeprojekte mit der Universität Forschungseinrichtung/Fachhochschule

Folgeprojekte mit der Universität Forschungseinrichtung/Fachhochschule

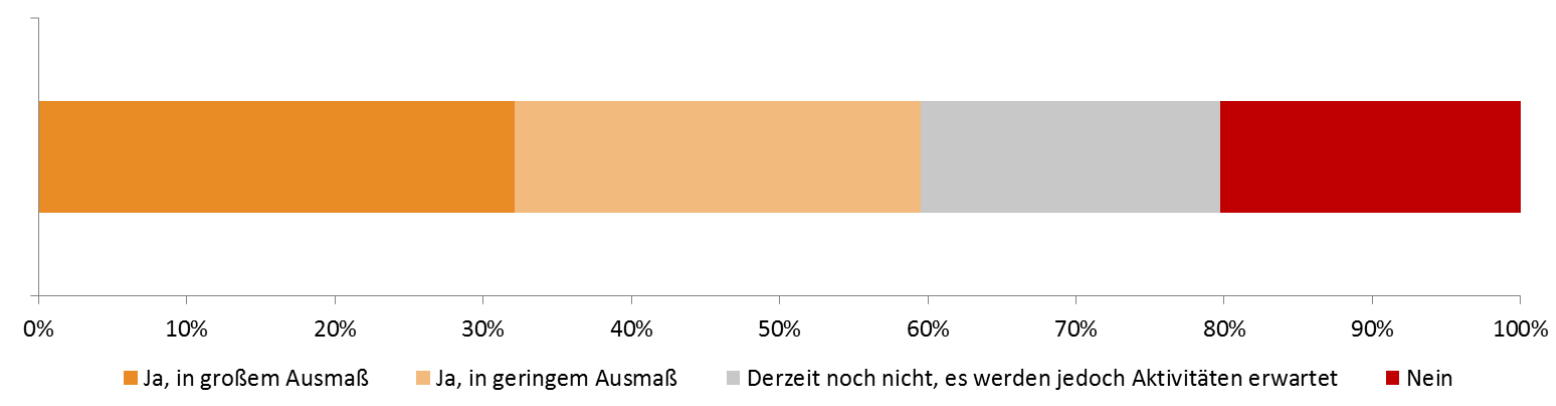

Quelle: Abschlussfragebögen Unternehmen der CDG ( $n=84)$.

Die Bearbeitung eines Forschungsthemas führte bei fast jedem dritten Unternehmen dazu, dass verstärkt Förderungen (z.B. bei der EU) eingeworben werden konnten. Der jedoch relativ geringe Anteil lässt darauf schließen, dass das CD-Förderprogramm ein (thematisches) Alleinstellungsmerkmal aufweist, da alternative Förderungen der Unternehmen mehrheitlich nicht vorkommen.

\section{Abbildung 50: Zusätzliche Förderungen}

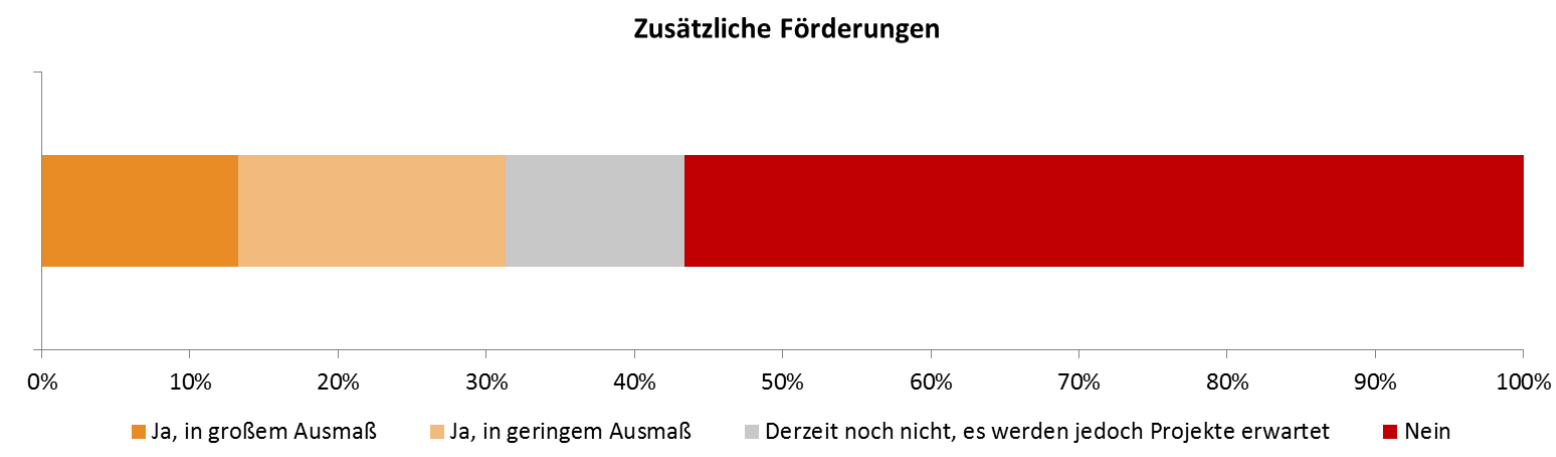

Quelle: Abschlussfragebögen Unternehmen der CDG (n=83).

Die Aktivitäten des CD-Labors wirkten sich auf die verschiedenen Bereiche des Unternehmens sehr heterogen aus. Der Wissenstransfer in Form von erhöhtem fachlichen Know-how der Mitarbeiter und einem erhöhten technologischem Niveau funktioniert gut. Die unmittelbare Unternehmensentwicklung hingegen, ausgedrückt durch Umsatz und Beschäftigte, kann (erwartungsgemäß) durch das Förderprogramm nur in vereinzelten Teilbereichen verbessert werden. Generell scheinen strukturelle innerbetriebliche Veränderungen, wie z.B. die Anzahl der Forschungsarbeitsplätze nur in sehr geringem Ausmaß von einem CD-Labor beeinflussbar zu sein. Positiv ist anzumerken, dass, 
verbunden mit dem Wissenstransfer, eine Erhöhung der Wettbewerbsfähigkeit gegenüber Konkurrenten einhergeht, verbunden mit einer Stärkung des Unternehmensstandorts.

\section{Abbildung 51: Auswirkungen des CD-Labors auf das Unternehmen}

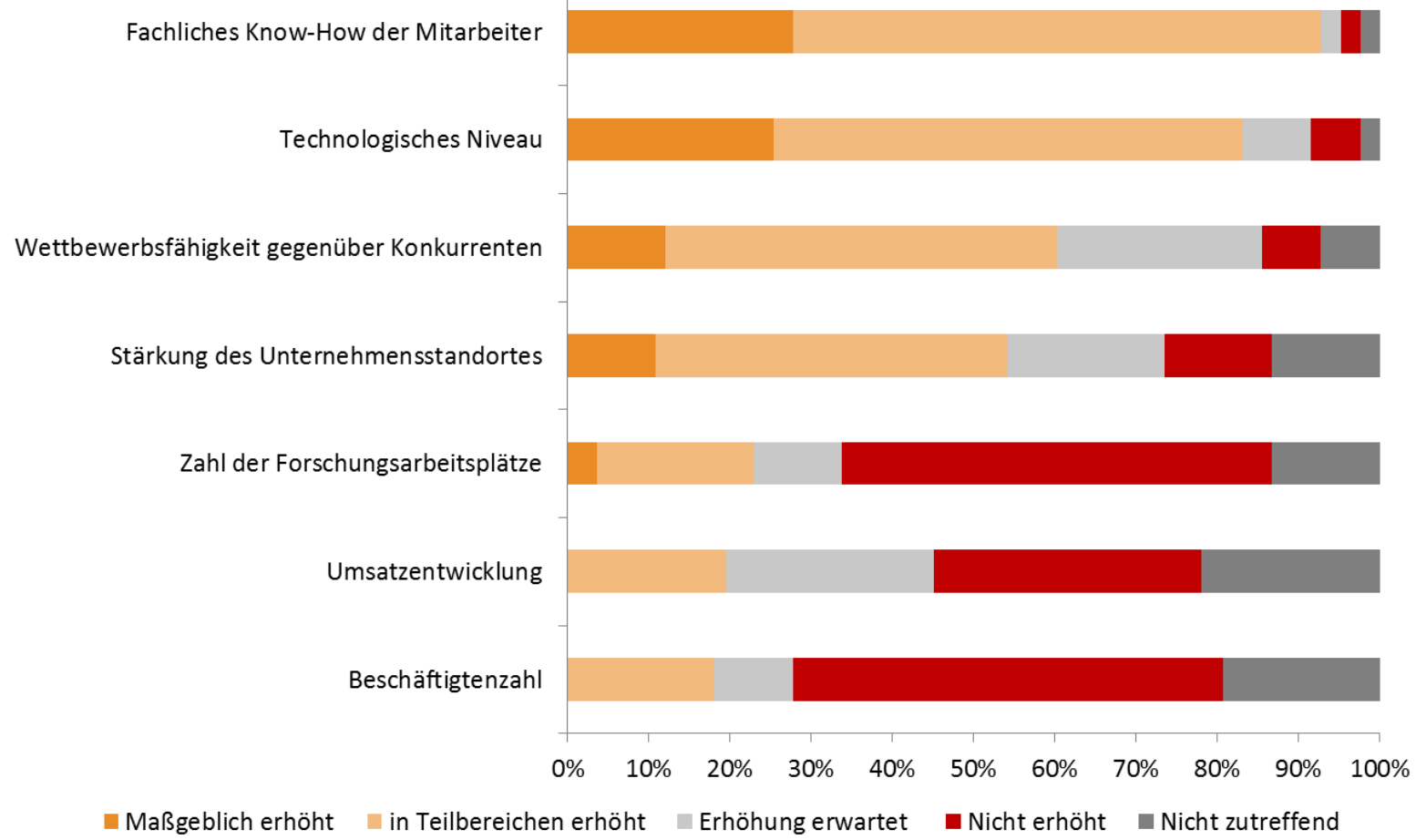

Quelle: Abschlussfragebögen Unternehmen der CDG ( $n=)$. 


\subsection{Tiefeninterviews Unternehmen}

\section{Unterschiede zur firmeninternen F\&E}

Das primäre Motiv, sich an CD-Labors bzw. JR-Zentren zu beteiligen, besteht darin, Wissen und Know-how aus der Grundlagenforschung zu Themen, die für die Unternehmen von Interesse sind, zu erwerben und für die Unternehmen nutzbar umzusetzen. Die F\&E-Aktivitäten, die im Labor bzw. Zentrum gemacht werden, sind bei den befragten Unternehmenspartnern deutlich stärker grundlagenorientiert als die firmeninterne F\&E. Es werden Themen bearbeitet, die unternehmensintern nicht bearbeitet werden können bzw. sollen, stattdessen wird externes Wissen von spezialisierten Forschern in das Unternehmen hereingeholt.

\section{Alleinstellungsmerkmal des Programms}

\section{Langfristige Grundlagenforschung mit thematischer Flexibilität als USP}

Als Alleinstellungsmerkmal des CDG-Programms wird der Fokus auf die Grundlagenforschung über eine lange Laufzeit, gepaart mit einer thematisch offenen Ausrichtung und bilateralen Kooperationsmöglichkeit, angesehen. Ein Gesprächspartner fasst die Besonderheit des Programms wie folgt zusammen: „Das CDG-Programm ermöglicht eine intensive Befassung mit der Grundlagenforschung, indem langfristige Themen in der Laborstruktur, in kleineren Netzwerken abgearbeitet werden. Man hat sieben Jahre lang Zeit, sich bilateral eines Themas anzunehmen, ohne große Netzwerke. Am Ende der sieben Jahre muss es Ergebnisse geben“.

Das CDG-Programm ist „das Programm“ für Wirtschafts-Wissenschafts-Kooperationen, wenn es darum geht, „Fragestellungen aus der Wirtschaft in der Wissenschaft zu behandeln und die Ergebnisse dann wiederum in eine Form gebracht werden sollen, die für die Wirtschaft verdaubar und verwendbar sind".

Ein Unternehmen stellt die Ausrichtung des CDG-Programms dem COMET-Programm und den FFGBasisprogrammen gegenüber: „Wenn man tief in die Grundlagenforschung eintauchen möchte und inhaltlich noch viel zu lösen hat, dann ist man im CDG-Programm gut aufgehoben. Für Kooperationsprojekte zu fortgeschrittenen Themenstellungen, bei denen eventuell sogar ein Kunde des Unternehmens an Bord ist, ist man bei COMET gut aufgehoben“. „Die FFG-Basisprogramme sind ein tolles Tool, um Produktentwicklung zu machen, bei denen es sogar möglich ist, universitäre Partner hineinzunehmen, allerdings kann man nicht so tief in ein Gebiet eintauchen“. 
Es wird auch erwähnt, dass es für Wirtschafts-Wissenschafts-Kooperationen alternativ das FFGBridge-Programm gäbe, dieses jedoch in der Größe begrenzt und dadurch nur für kleinere Forschungsvorhaben geeignet ist. Thematische Förderprogramme gibt es auch, jedoch sind diese nur dann relevant, wenn das vom Unternehmen angestrebte Forschungsvorhaben mit dem geförderten Thema übereinstimmt. EU-Projekte stehen theoretisch auch zur Verfügung, werden jedoch sehr kritisch gesehen, da mit der Antragstellung ein hoher Aufwand verbunden ist und die Förderwahrscheinlichkeit niedrig ist („,liegt nur bei rund 10 Prozent"), außerdem müssen mehrere internationale Partner ins Boot geholt werden. Man ist „froh, dass es so ein Programm wie die CDG gibt“.

\section{Zugang zu Know-how und hochkarätiger technischer Infrastruktur}

Als primäres Motiv für die Kooperation wird angesehen, dass das Labor Zugang zu Personen mit dem entsprechenden Grundlagenforschungswissen bietet. Ein Unternehmenspartner merkte an, dass die Kooperation mit den wissenschaftlichen Partnern (Universitäten) außerdem wichtig ist, um Zugang bzw. Zugriff auf Geräte und Anlagen zu bekommen, die das Unternehmen selbst nicht besitzt, die aber erforderlich sind, um die Grundlagenforschung zu betreiben.

\section{Anpassungsfähigkeit während der Labor- bzw. Zentrumslaufzeit}

Als sehr positives Merkmal des Programms wird die Möglichkeit angesehen, Module ergänzen zu können, wenn sich neue Fragestellungen ergeben. Das Forschungsthema ist zwar fixiert durch den Labor- bzw. Zentrumsleiter, aber im Rahmen dieses Themas kann auf die Bedürfnisse der Unternehmen und Änderungen derselben eingegangen werden. Dies ist von besonderer Relevanz in Hinblick auf die sich rasch ändernden Bedürfnisse der Unternehmen.

\section{Bilaterale Kooperationsmöglichkeit}

Ein Vorteil des CDG-Programms, etwa im Vergleich zu EU- oder COMET-Programmen liegt darin, dass man bei der CDG auch bilaterale Kooperationen eingehen kann. Wenn mehrere Unternehmenspartner in einem CD-Labor dabei sind, sind diese durch Module getrennt. Beim COMET-Programm gibt es immer mehrere Unternehmenspartner, wodurch sich Probleme in Hinblick auf das Geistige Eigentum ergeben können. Dadurch kann das COMET-Programm aufwändig bzw. nervenaufreibend sein. Beim CDG-Programm hat man etwa nur mit dem wissenschaftlichen Partner (der Universität) eine Diskussion in Hinblick auf das Geistige Eigentum. Das ist wesentlich einfacher, als die IP-Rechte mit anderen Unternehmen auszuverhandeln. 


\section{Starke Bindung an den Laborleiter}

Als strukturelle Besonderheit der CD-Labors bzw. JR-Zentren wird gesehen, dass das Programm stark an die Person des Labor- bzw. Zentrumsleiters gebunden ist. Es gibt kein anderes Förderprogramm, das die Person (den wissenschaftlichen Leiter) so stark in den Mittelpunkt stellt. Es entsteht eine intensive Beziehung mit dem wissenschaftlichen Partner, die in Programmen, die große Netzwerke vorsehen, nicht gegeben wäre. „Im CD-Labor ist man in einer kleineren Zelle" und nicht in einem großen Netzwerk.

\section{Administrative Schlankheit}

Ein weiteres Alleinstellungsmerkmal des CDG-Programms ist der - im Vergleich zu anderen Förderprogrammen - geringe administrative Aufwand, der für das Unternehmen anfällt. Die Abwicklung bzw. die administrative Komponente liegt bei den Laborleitern. Es prasseln keine immensen Overheadkosten auf die Unternehmen ein.

"Das CDG-Programm ist schlank, streamlined [gut organisiert, stromlinienförmig] und effizient". Ein Unternehmen meint, dass beim CDG-Programm für das Unternehmen rund fünf Prozent Overheadkosten anfallen, während es bei anderen Förderprogrammen viel höhere Overheadkosten wären, die nicht abrechenbar sind. Typischerweise sind Overheadkosten nur für den wissenschaftlichen Partner abrechenbar. Durch die schlanke Administration werden die Ressourcen für das inhaltliche Arbeiten verwendet, „es kommt [beim CDG-Programm] viel beim Inhalt an“. Es geht bei der CDG nicht um das „I'art pour l'art“, sondern man könne sich auf den Inhalt des Forschungsvorhabens konzentrieren.

\section{Hohe Förderrate}

Eine weitere Besonderheit ist, aus Sicht eines Unternehmens, die "hohe Förderrate". Die Unternehmen erhalten zwar keine direkte Förderung, aber die zur Hälfte geförderte Kooperation mit den CD-Labors liefert Ergebnisse, die wiederum in die Entwicklung neuer Produkte und Verfahren eingehen.

\section{Keine Wertschöpfungsstrategie erforderlich}

Als positiv wird von einem Unternehmen außerdem angesehen, dass der Wertschöpfungsstrategie nicht ganz so große Bedeutung beigemessen wird, wie etwa bei EU-Projektanträgen, in denen bereits bei der Antragstellung der wirtschaftliche Nutzen der Forschung demonstriert bzw. gezeigt werden muss, obwohl es sich um unreife Themen bzw. um Grundlagenforschung handelt. Diese unrealistische Vorgehensweise wurde von der CDG nicht gewählt. 


\section{„Es ist ein schönes Modell, das man in andere Länder exportieren könnte“ (Interview-Zitat)}

Ein Unternehmenspartner merkt noch an, dass das CD-Modell „ein schönes Modell [sei], das man in andere Länder exportieren könnte". Der Gesprächspartner findet das Programm sehr attraktiv und ihm ist nicht bekannt, dass es ein derartiges Modell in anderen Ländern geben würde.

\section{Erwartungen und deren Erfüllung}

\section{Erfüllte Erwartungen in Hinblick auf den Know-how-Erwerb}

Die primäre Erwartung an die Beteiligung am CD-Labor oder JR-Zentrum ist, vertiefendes Wissen in ausgewählten Bereichen der Grundlagenforschung zu erwerben, die für die Unternehmen von Interesse sind. Diese Erwartung hat sich bei allen befragten Unternehmenspartnern erfüllt. Wie gut der Wissenstransfer funktioniert, hänge aber stark vom Labor- bzw. Zentrumsleiter ab. Man muss sich daher gut überlegen, mit wem man kooperiert; man braucht einen Labor- bzw. Zentrumsleiter, der sich auf die Industrie einlässt.

Da bei der Forschung immer ein Restrisiko bestehen bleibt, kann es technische oder menschliche Probleme geben, wodurch die Erwartungen bei manchen Labors und Zentren stärker und bei anderen weniger erfüllt wurden. „Manchmal ist ein inhaltliches Ergebnis nicht, wie man es sich gewünscht hätte, aber die Erkenntnis, dass ein Forschungsvorhaben nicht die inhaltlich erhofften Ergebnisse liefert, ist auch eine Erkenntnis“. Angestrebt wird aber, dass sich aus dem Wissenszuwachs ein Nutzen für die Produkt- und Prozessentwicklung bzw. Ausrichtung der Dienstleistungen bei den Industriepartnern ergeben.

Die überwiegende Zahl der interviewten Unternehmenspartner betrachtet die Labors und Zentren als eine Möglichkeit, qualifizierte Forscher/innen kennenzulernen und diesen gegebenenfalls Jobs im Unternehmen anzubieten bzw. zu rekrutieren. Die Labors bzw. Zentren bieten die Möglichkeit zu beobachten, wie sich die Mitarbeiter des wissenschaftlichen Partners (vor allem Dissertanten und Post-Docs) über einen längeren Zeitraum bewähren und diese für das Unternehmen zu interessieren. Für einen interviewten Unternehmenspartner hat sich die Erwartung, Mitarbeiter zu übernehmen jedoch nicht erfüllt, da die Attraktivität des Unternehmenspartners aufgrund der großen örtlichen Distanz zum wissenschaftlichen Partner als zu wenig hoch eingeschätzt wurde, im Vergleich zu den guten Arbeitsplatzmöglichkeiten rund um den Standort des wissenschaftlichen Partners. 
Bei jenem Unternehmenspartner, bei dem außerdem der Wunsch nach einem Zugang zu geeigneten Anlagen und Geräten für die Umsetzung der Grundlagenforschung im Fokus stand, hat sich auch diese Erwartung erfüllt.

Ein anderer Unternehmenspartner hat angemerkt, dass man die Ressourcen unterschätzt hat, die benötigt werden, um die Ergebnisse aus der Grundlagenforschung wieder in Wissen umzuformulieren, das für die Geschäftstätigkeit des Unternehmens genutzt werden kann.

\section{Zusatznutzen, abgesehen vom Wissenstransfer}

\section{Aufmerksamkeitsgewinn für Unternehmen innerhalb der wissenschaftlichen Community}

Abgesehen von den für die Unternehmen wichtigsten Nutzenkomponenten aus einer Labor- bzw. Zentrumskooperation - dem Wissenszuwachs und dem frühzeitigen Kennenlernen von zukünftigen Mitarbeitern - kann Zusatznutzen in Form eines höheren Bekanntheitsgrades bzw. durch einen Aufmerksamkeitsgewinn entstehen.

Das Labor bzw. das Zentrum trägt dazu bei, die Bekanntheit des Unternehmens in der wissenschaftlichen Community zu erhöhen, etwa indem ein Unternehmen als potenzieller Forschungspartner oder Auftraggeber von Forschungsprojekten bei Universitäten bekannt wird. Ein Unternehmen berichtet, dass Universitäten (außerhalb des Labors), die man vorher nicht kannte, auf das Unternehmen aufmerksam wurden, da durch die Bekanntgabe der CD-Labor-Gründung öffentlich bekannt wurde, dass man bestimmte Forschungsinteressen hat.

Die Beteiligung am CD-Programm bringt eine Erleichterung bei der Akquisition zusätzlicher Forschungsprojekte und -mittel. Aus der Kooperation im CD-Labor ergeben sich außerdem neue Projektanträge. Wissenschaftliche Partner wenden sich an Unternehmen und fragen an, ob man sich an einer Einreichung (etwa einem EU-Projekt) beteiligen möchte. Da EU-Projekte mit einem großen Aufwand verbunden sind, ist man froh, wenn ein wissenschaftlicher Partner einen „ins Boot" holt, wenn das Thema für das Unternehmen interessant ist. Es entsteht eine „Kontaktbündelung“. International wurde aus Sicht des Unternehmens jedoch kein erhöhter Bekanntheitsgrad konstatiert, da die CD-Labors etwa in Deutschland wenig bekannt sind.

Ein Prestigegewinn entstehe tendenziell nicht beim Unternehmen, beim wissenschaftlichen Partner aber im Allgemeinen schon. Ein Unternehmen merkte jedoch an, dass das CD-Programm sehr wohl einen Prestigegewinn brächte, da man bei aktuellen Technologieentwicklungen vorne dabei sei. Ein Gesprächspartner meint, dass die Sichtbarkeit der CDG nicht groß genug sei für einen Prestigegewinn beim Unternehmen. Ein breiterer Nutzen wäre nur möglich, wenn man ein sehr großes Projekt- 
budget hätte. Andererseits seien größere Unternehmen bereits bekannt und bräuchten daher auch nicht notwendigerweise einen Prestigegewinn.

\section{Einfluss auf die unternehmensinterne F\&E}

\section{Unternehmensinterne F\&E-Aktivitäten tendenziell unbeeinflusst}

Auf den thematischen Fokus hat die Beteiligung am CD-Programm keine Auswirkung, da sich die Themen aus den firmeninternen Bedürfnissen ergeben; der Markt legt fest, welche Themen von Interesse sind.

Die Grundlagenarbeit liefert jedoch bei manchen Unternehmen Impulse für das tägliche Arbeiten; das Wissen aus der Grundlagenforschung im Labor fließt etwa in die Arbeit der unternehmensinternen F\&E-Abteilung ein. Die Teilnahme an CD-Labors führt zu einer stärkeren oder auch intensiven Befassung mit der Grundlagenforschung. Dies hat die Ausrichtung bei einem Unternehmen stärker in Richtung Grundlagenforschung verändert.

Ein Unternehmen berichtet, dass die Anzahl der Kooperationen durch die Beteiligung an den CDLabors laufend gewachsen sei, vor allem, weil sich die wissenschaftlichen Partner an das Unternehmen wenden und anfragen, ob man sich an Projektkooperationen beteiligen möchte („, das wäre doch etwas für euch?“). Dadurch steigt die Anzahl der Kooperationen fast automatisch, solange die unternehmensinternen Mittel dafür freigegeben werden.

Der Zugang zu anderen Förderprogrammen, wie etwa COMET und K-Plus war durch die Erfahrungen in den CD-Labors teilweise leichter. Ein befragtes Unternehmen berichtet, dass das Labor Auslöser für eine Dissertation war, die vom Unternehmen extern vergeben wurde (und die nicht innerhalb des Labors, sondern vollständig vom Unternehmen finanziert wird), wobei die Dissertantin bei allen Treffen im Rahmen des CD-Labors dabei ist und dazu beiträgt, das Wissen, welches im Labor generiert wird, in die unternehmensinterne F\&E-Abteilung zu übertragen.

\section{Wissenschaftlicher Freiraum}

\section{Akzeptanz des wissenschaftlichen Freiraums abhängig vom Laborleiter}

Die Nutzung des wissenschaftlichen Freiraums steht im Allgemeinen sehr gut im Einklang mit den Vorstellungen des Unternehmens. Dies ist jedoch stark vom Laborleiter abhängig. Ein Gesprächspartner meint: „Ein gewisser Freiraum ist notwendig bei einer so langen Kooperation und bringe im Endeffekt wieder Nutzen für das Unternehmen“. Bei der Auswahl des Laborleiters muss aber bereits darauf geachtet werden, dass der Laborleiter auf die Interessen des Unternehmens eingeht. Der 
Freiraum der Laborleiter ist kein Problem, solange die Industrie Antworten und Ergebnisse auf ihre Forschungsfragen erhält. Man würde kein CD-Labor mit einem wissenschaftlichen Partner beantragen, mit dem man noch nie zusammengearbeitet hat, „,von dem man nicht weiß, wie er tickt“.

Prinzipiell könnte der wissenschaftliche Freiraum zu einer Entzweiung führen, derartige Erfahrungen wurden aber selten gemacht. Es handelt sich um einen Spagat zwischen der wissenschaftlichen Freiheit und der Finanzierung durch das Unternehmen. Es wird geraten, diesen Punkt „im Auge zu behalten".

\section{Folgeaktivitäten}

\section{Kooperationen werden fortgesetzt}

Vor Beginn des Labors bestand meist schon Erfahrung bei der Zusammenarbeit mit den wissenschaftlichen Partnern (Universitäten, Instituten und Wissenschaftlern); im Labor institutionalisiert man die Kooperation, die vorher schon bestand. Es gab mit vielen Partnern Folgeaktivitäten. Wenn die Zusammenarbeit gut funktioniert, dann bleibt die Beziehung zum Laborleiter und zum Institut (den Forschern) bestehen. Das Thema wird allerdings nur dann weiter behandelt, wenn die Thematik weiterhin interessant für das Unternehmen ist.

Ob man geförderte oder nicht geförderte Folgeaktivitäten eingeht, hängt davon ab, mit wie viel Aufwand das Einwerben der Förderung verbunden ist und wie hoch die Wahrscheinlichkeit ist, die Förderung zu erhalten. Bei EU-Projekten beispielsweise ist der Aufwand sehr hoch und die Förderwahrscheinlichkeit sehr niedrig. Abgesehen von Förderprogrammen ist die Forschungsprämie bei förderungswürdigen Vorhaben attraktiv, da 12 Prozent des Aufwands gefördert wird.

\section{$\underline{\text { Strukturelle Möglichkeiten zum Wissenstransfer }}$}

\section{Wissenstransfer findet statt}

Der Wissenstransfer funktioniert in den CD-Labors und ist sehr wichtig. Die Art des Wissenstransfers ist von Labor zu Labor verschieden und hängt von den beteiligten Personen ab, vor allem vom Laborleiter und der im Unternehmen verantwortlichen Person. Tendenziell ist man zufriedener, wenn sich der wissenschaftliche Partner in der Nähe des Standorts vom Unternehmenspartner befindet. Bei großen örtlichen Distanzen werden unterschiedliche Erfahrungen gemacht. Für manche Unternehmen ist es wichtig, dass sich der wissenschaftliche Partner die Forschungserfordernisse beim Unternehmenspartner ansieht, dazu ist jedoch nicht jeder Laborleiter bereit. 
Ein Unternehmen, das mit dem Wissenstransfer zufrieden war, hat unter anderem einen Studierenden-Austausch initiiert, bei dem Dissertanten und Diplomanden aus der F\&E-Gruppe des Unternehmen das Labor kennenlernen konnten, und auch umgekehrt. Ein anderes Unternehmen organisierte ein paar Mal im Jahr Workshops, an welchen ein erweiterter, aber thematisch qualifizierter, Kreis von Personen aus dem Unternehmen teilnahm.

\section{Internationale Kooperationen}

\section{Zusammenarbeit mit wissenschaftlichen Partnern aus dem Ausland attraktiv}

Man ist zufrieden mit den bestehenden Möglichkeiten für eine Zusammenarbeit mit einem wissenschaftlichen Partner aus dem Ausland. Es ist sinnvoll, dass auch ausländische Unternehmen eingebunden werden können, dies ist aber, seitens des Unternehmens, aus Konkurrenzgründen nicht unbedingt gewollt. Viel wichtiger sind die Möglichkeiten, ausländische Universitäten einzubinden; die bestehenden Möglichkeiten sind hier aber gut. „Es gibt bereits viele Möglichkeiten im CDG-Programm, wie man Internationalität leben kann".

Wichtig ist, dass die Partner die Richtigen sind, egal wo sie ihren Sitz haben. Wenn sich ein internationaler wissenschaftlicher Partner "aufdrängt im positiven Sinne“, d.h. wegen der fachlichen Kompetenz, dann soll es möglich sein, ihn ins Labor hineinzunehmen. Manche Unternehmen, die bereits Erfahrung mit einem wissenschaftlichen Partner aus dem Ausland haben, haben sich für einen ausländischen Partner entschieden, weil es etwa keine ausreichende Kompetenz im Inland gab.

Man hofft, dass die Internationalität des Konsortiums kein Muss-Kriterium im CDG-Programm wird, sondern dass das Programm offen ist für internationale Partner, aber diese nicht zwingend erforderlich sind, wie etwa bei EU-Projekten. Die Anforderung in EU-Programmen, mindestens drei Partner aus mindestens zwei Ländern zu haben, ist „mühsam“.

Bei einem internationalen Partner bzw. einem Partner, der weiter entfernt ist, entstehen meist höhere Reisekosten. Bislang wurden seitens der Gesprächspartner aber keine Schwierigkeiten bei der Genehmigung höherer Reisebudgets wahrgenommen. Generell ist die Kommunikation leichter, wenn der Partner in örtlicher Nähe ist, es können Kooperationen aber auch über große Distanzen funktionieren.

EU-Projekte werden von keinem Gesprächspartner als attraktive Möglichkeiten zur internationalen Kooperation angesehen. Dies läge am schlechten Kosten-Nutzen-Verhältnis, da die Wahrscheinlichkeit niedrig ist, eine Förderung zu erhalten. Außerdem ergeben sich in EU-Projekten Probleme mit 
den Geistigen Eigentumsrechten, da mehrere Unternehmenspartner beteiligt sind und viele Fragestellungen aus Konkurrenzgründen nicht in einem Konsortium bearbeitet werden können. Außerdem benötigt man den richtigen Partner-Mix, was wiederum aufwändig ist.

\section{Administration und Zwischenevaluierungen}

Überschaubarer administrativer Aufwand für die Unternehmen, aber möglicherweise weniger Forschungs-Output wegen hohem Zeitaufwand beim Laborleiter

Der administrative Aufwand beim Unternehmen wird als überschaubar empfunden, jedoch nehmen die Unternehmen wahr, dass der Laborleiter einen hohen administrativen Aufwand trägt. Da der Laborleiter sich mit gewissen administrativen Anfragen wiederum an das Unternehmen wendet, ist auch dieses davon betroffen, wenngleich nur wenig. Wird berücksichtigt, dass der administrative Aufwand des Laborleiters negative Rückkopplungseffekte auf den Forschungsoutput hat, sodass die für Forschung zur Verfügung stehenden zeitlichen Ressourcen reduziert werden, ist der administrative Aufwand laut einem Interviewpartner „in Summe ein bisschen höher als erwartet“.

Alle Unternehmenspartner merken an, dass der administrative Aufwand bei EU-Projekten deutlich höher ist. „Es gibt nichts Schlimmeres [als ein EU-Projekt]“.

„Der administrative Aufwand bei einer Beteiligung von Unternehmen am COMET-Programm ist deutlich höher als beim CDG-Programm. Die GmbH-Konstruktion (im COMET-Programm) kann etwa zu erheblichen Overheadkosten führen. Beim COMET-Programm kann man allerdings als Unternehmen In-Kind-Leistungen monetarisieren. Wenn das kein wichtiges Ziel des Unternehmens ist, dann gibt es keinen Vorteil im COMET-Programm, im Vergleich zum CDG-Programm, jedoch einige Nachteile“. Das befragte Unternehmen hat in Summe wesentlich bessere Erfahrungen mit dem CDG-Programm als mit dem COMET-Programm gemacht.

\section{Zeitpunkte für Zwischenevaluierungen sind passend, Nutzen teils kritisch gesehen}

Der Zeitpunkt für die erste Zwischenevaluierung wird als geeignet angesehen. „Wenn Korrekturen erforderlich wären, wäre dies nach drei Jahren erst sehr spät“. „Prinzipiell ist es gut so, wie es derzeit ist, nach zwei Jahren und nach fünf Jahren“. „Wenn es Anlaufschwierigkeiten gibt, kann eine ein bisschen spätere Zwischenevaluierung sinnvoll sein. Beim Vorliegen eines Staffing-Problems oder falls der Set-up des Labors bzw. das „Hochfahren“ des Labors oder Zentrums länger dauert, wäre es aber sinnvoll, die Möglichkeit zu bieten, die erste Zwischenevaluierung erst nach 2,5 Jahren oder am 
Anfang des dritten Jahres zu machen". "Grundsätzlich ist es aber besser, die erste Zwischenevaluierung lieber früher als später zu machen“.

Der Nutzen der 2- und 5-Jahres-Evaluierung für die Unternehmen wurde von einem Unternehmen in Frage gestellt. In diesem Fall wurde darauf hingewiesen, dass es einen erheblichen Aufwand für den Laborleiter darstellt, den wissenschaftlichen Bericht $\mathrm{zu}$ erstellen und die Evaluierungen vorzubereiten. Angemerkt wurde in diesem Zusammenhang auch, dass die Unternehmen bei fehlender Zufriedenheit mit dem Labor sowieso eine Ausstiegsmöglichkeit (Kündigung) hätten. Somit werde die Qualitätssicherung aus Sicht des Unternehmens bereits durch die Kündigungsmöglichkeit sichergestellt. Es wird vom entsprechenden Unternehmen aber auch konzediert, dass die Evaluierung in Hinblick auf die ordnungsgemäße Verwendung von öffentlichen Mitteln notwendig sein mag.

Im Gegensatz dazu wurde von einigen Unternehmen positiv angemerkt, dass die Evaluierung den wissenschaftlichen Partner dazu motiviert, eine Zusammenstellung der bisherigen Ergebnisse vorzunehmen. Dadurch hat man eine Übersicht, statt nur eine Aneinanderreihung von Einzelergebnissen.

\section{Anfangsevaluierung nützlich}

Ein Unternehmen merkte als eine Besonderheit des Programms an, dass die Anfangsevaluierung eine Absicherung hinsichtlich der wissenschaftlichen Qualität des Laborleiters wäre. Wenn die Evaluierung zur Person des Laborleiters positiv ausfällt, hat man die qualitative Gewähr, dass der Laborleiter die fachlichen, wissenschaftlichen Ansprüche erfüllt. Obwohl man im ersten Moment nicht erfreut ist, wenn ein Laborleiter von den Evaluatoren abgelehnt wird, sieht man die Evaluierung als sehr nützlich an, um „sich leere Kilometer zu ersparen“, da man sieben Jahre aneinander gebunden ist und als Unternehmenspartner die wissenschaftliche Qualität nicht so gut beurteilen könne („glücklich über die Evaluierung, weil man die wissenschaftliche Qualität des Laborleiters als Unternehmen gar nicht so gut beurteilen kann").

\section{Qualitätsunterschiede zwischen den Labors}

Das Gelingen eines CD-Labors und JR-Zentrums hängt sehr stark vom Laborleiter bzw. vom Zentrumsleiter ab. „Dieser benötigt Führungskompetenz und technisches Knowhow; dies paart sich aber leider nicht immer".

Es gibt Qualitätsunterschiede zwischen den Laborleitern und den Instituten. Wichtig ist, wen man auf der anderen Seite hat. Das beeinflusst, ob das Forschungsvorhaben "fliegt“ oder nicht. Es geht vor 
allem um die Person des Laborleiters und um die Unterstützung durch den Institutsvorstand, bei dessen Institut der Laborleiter beschäftigt ist.

Die Rahmenbedingungen des Laborleiters hängen u.a. von den universitären Rahmenbedingungen und der Unterstützung durch das Institut ab. Das Institut muss einen Prozentsatz der eingeworbenen Forschungsgelder an die Universität abführen. Es ist daher wichtig, dass der Institutsvorstand hinter dem Labor steht und eine gute Verhandlungsposition bei der Verhandlung mit der Universität hat. „Ein junger Laborleiter kann das nicht, das muss der Institutsvorstand machen“. Außerdem ist der Laborleiter bei der Universität (oder Fachhochschule) beschäftigt. Falls der Laborleiter keine Laufbahnstelle hat, ist die Rückendeckung durch den Institutsvorstand umso wichtiger und dessen Einsatz dafür, dass die Beschäftigung des Labor- oder Zentrumsleiters für die gesamte Laborlaufzeit sichergestellt ist.

„Die administrative Qualität ist durch die CDG sichergestellt, die wissenschaftliche Qualität durch die Evaluierungen der CDG. Die Erfüllung der Anforderungen der Industrie stellt die Industrie selber sicher. Die CDG hat aber keinen Einfluss darauf, ob man einen guten Kooperationspartner findet".

\section{$\underline{\text { JR-Zentren / Zusammenarbeit mit Fachhochschulen }}$}

Jene Unternehmenspartner, die bislang noch keine Zusammenarbeit mit Fachhochschulen eingegangen sind, meinten, dass eine Kooperation mit FHs prinzipiell denkbar sei. Zwei Unternehmen wiesen darauf hin, dass FHs oft (noch) nicht die erforderliche Ausstattung bzw. Forschungsinfrastruktur haben, die oft auch sehr teuer ist. „Bei den FHs ist die Forschung oft erst im Aufbau, daher hat es sich bislang noch nicht ergeben".

Es bestehen Unterschiede zwischen den CD-Labors und JR-Zentren, die sich aus den Unterschieden zwischen Universitäten und FHs ergeben. Die FHs haben etwa keinen so großen Personalstock und einen anderen Zeithorizont bei der Forschung. Man gehe mit anderen Zielsetzungen zur FH. Tendenziell gehe man mit Fragestellungen zur FH, die weniger wissenschaftlich sind und ein kleineres Volumen haben. Vor allem wählt man aber die Person des Labor- oder Zentrumsleiter bzw. das Institut nach den jeweiligen Kompetenzen aus, an denen man interessiert ist.

\section{Veränderungen über die Zeit im CD-Programm}

Ein Unternehmen merkt an: „Das Fördertool ist ursprünglich geblieben und bietet einen flexiblen Umgang mit den Bedürfnissen der Partner." 


\section{Höhere Flexibilität im Programmzeitablauf}

Bei den CD-Labors habe sich im Zeitablauf verändert, dass die CDG nicht mehr so stringent in Hinblick auf die Mittelzuordnung ist. Früher musste man sich über die gesamte Laborlaufzeit hinweg sehr eng an die ursprünglich in der Antragstellung vorgesehene Zuordnung der Mittel für Sachausgaben oder Personalausgaben halten. Jetzt wird dies viel flexibler gehandhabt, was positiv in Hinblick auf Veränderungen ist, die sich im Zeitablauf ergeben.

\section{Bessere Kommunikation}

Die Ergebnisorientierung und die unternehmensorientierte bzw. unternehmensgerechte Kommunikation der Ergebnisse werden immer wichtiger. Es ist heute viel stärker erforderlich, dass sich beide Partner darauf einlassen, wie der andere arbeitet und man eine geeignete Form der Kommunikation findet, damit die Inhalte verstanden werden und man nicht aneinander vorbeiredet. Die Problemstellung aus der Wirtschaft muss erklärt, dann in die Wissenschaftswelt übersetzt und schließlich wieder rückübersetzt werden in die Sprache des Unternehmens.

\section{Besonders positive Aspekte und Verbesserungsvorschläge}

Als besonders positiv ist anzumerken, dass das Programm eine Lücke schließt als langfristiges Grundlagenforschungsprogramm im Rahmen einer Unternehmens-Wissenschaftskooperation. Das Modell wird als sehr attraktiv empfunden und als Exportmodell vorgeschlagen. Auch die Gestaltung des Programms ist bereits gut. Es bietet viel Flexibilität, wobei dieser Punkt auch in Zukunft immer wichtiger werden wird. Ein Gesprächspartner merkt an: „Das Programm ist sehr flexibel. Es besteht kein Änderungsbedarf und soll möglichst so fortgeführt werden, wie es jetzt ist“.

An den Förderprogrammen der CDG ist generell positiv, dass die Unternehmen sich die Wissenschaftler aussuchen können und mit ihnen langfristig kooperieren können, ohne alle paar Jahre wieder neue Anträge schreiben zu müssen. Die Kontinuität hat immense Vorteile. Allerdings hat die Langfristigkeit der Labors wiederum den Nachteil, dass es schwierig ist, vom Management eine Zusage (ein "Commitment") über sieben Jahre zu bekommen, weil sich das Management alle paar Jahre ändert. Aus Sicht eines Unternehmens wäre die Möglichkeit attraktiv, das Labor auch über eine kürzere Programmlaufzeit laufen zu lassen, etwa eine Laufzeit von vier bis sieben Jahren. Bei kurzen Labors (d.h. etwa mit einer Dauer von vier Jahren) könnte dann eine Evaluierung stattfinden, ob man weitermachen möchte oder nicht. „Das Programm solle sich jedoch nicht in Richtung COMET verändern". 
Sehr positiv ist, dass das CDG-Programm geradlinig und einfach in der Handhabung („,straight forward") ist, sobald man sich mit dem Laborleiter auf das Thema und das Budget geeinigt hat. Ab diesem Moment ist auch der administrative Aufwand überschaubar, was wiederum attraktiv ist. Außerdem muss man sich als Unternehmenspartner nicht mit einer sinnvollen Mittelverwendung auseinandersetzen, weil die CDG das prüft. Dies ist bei anderen Förderprogrammen nicht immer der Fall.

Die CDG bietet außerdem eine sehr gute Betreuung und beantwortet Fragen prompt, „das ist Spitze“. Die FFG biete auch eine sehr gute Betreuung, ist allerdings viel bürokratischer, etwa in Hinblick auf die Stundenaufzeichnung und beim „in die Bücher gehen“, wodurch bei FFG-Programmen mehr Administration für die Unternehmen entsteht.

Der hohe wissenschaftliche Freiraum könnte laut Aussage eines Unternehmens potenziell problematisch sein. Dieses Unternehmen regt an, den Forschungsfreiraum enger zu fassen, etwa durch eine stärkere Bindung an das Forschungsthema. Ohne diese Bindung sei es schwierig, eine Arbeit am Forschungsthema einzufordern, falls der Laborleiter den Freiraum überstrapaziert.

Ein Gesprächspartner merkte in Hinblick auf den administrativen Aufwand an, dass es „bei der Abwicklung des Budgets einen erhöhten Aufwand gab, weil die CDG den Gemeinschaftsbeitrag nicht auf die Labors aufteilt, sondern eine Summe verrechnet". Das Unternehmen muss diesen Betrag dann auseinanderteilen und auf die Labors aufteilen.

\section{Zukünftige Ausrichtung}

„Es ist toll, dass es ein derartiges Bottom-up-Programm gibt, in dem man mit dem Forschungsthema wirklich weiterkommt". „Solange es Personen (Wissenschaftler) in Österreich gibt, die sich auf ein Thema draufwerfen und in die Grundlagenforschung investieren (tiefgehendes Wissen erwerben) und die Unternehmen außerdem genügend Geld für die grundlagenorientierte Forschung haben, hält das Modell“.

„Das Umfeld hat sich verändert und ist schneller geworden. Der Druck in der kurzfristigen Forschung ist groß und im Zeitverlauf gestiegen. Die Budgets sind knapper geworden. Aus Sicht der Finanzverantwortlichen in Unternehmen sind kürzere Forschungsprogramme besser, jedoch kann man in einem 3-Jahres-Programm keine Grundlagenforschung machen. Nur bei Forschungskooperationen über einen längeren Zeitraum wird in die Grundlagenforschung investiert und dann kommt auch 
etwas [Neuartiges] heraus". Insbesondere jene Unternehmen, bei denen die bestehenden State-ofthe-art Technologien bereits ausgereizt sind, kommen nur in der Grundlagenforschung weiter.

Eventuell wäre eine Laborlaufzeit von sechs Jahren bzw. im Rahmen von vier bis sieben Jahren leichter für die Unternehmen bzw. unternehmensintern besser argumentierbar. Es ist gut, dass die Labors nach sieben Jahren zu Ende sind und es ein abgegrenztes Forschungsvorhaben ist, denn innerhalb der sieben Jahre verändert sich viel in einem Unternehmen. Danach kann es einen „clean start" mit einem neuen Labor geben.

Flexibilität ist bereits jetzt wichtig und wird in Zukunft noch wichtiger werden. Alles, was über den 4Jahres-Business-Plan hinausgeht, ist eine Herausforderung für die Unternehmen. Daher ist es wichtig, dass das Labor auch nach Projektbeginn wachsen kann und Module später beantragt werden können, um weitere Partner an Bord zu holen und damit auch das Thema auszuweiten. Die Möglichkeit, Partner noch während der Laufzeit des Labors hereinzuholen und damit eine thematische Erweiterung zuzulassen, soll jedenfalls bestehen bleiben und der damit verbundene administrative Aufwand soll überschaubar sein. Die Flexibilität ist wichtig, weil sich die Ausrichtung der Unternehmen und ihre Forschungsinteressen im Zeitablauf ändern.

Da der zeitliche Horizont der CD-Labors und JR-Zentren „,jenseits der Business-Plan-Planungsperiode“ liegt, ist es gut, dass es eine Ausstiegsmöglichkeit als Not-Reißleine gibt, sonst könnten die Unternehmen sich nicht daran beteiligen. Aus Sicht der Industrie ist eine kurze Kündigungsfrist [für die Beteiligung am Labor oder Zentrum] attraktiv, falls sich die Forschungsinteressen des Unternehmens ändern. Dies würde aber eine langfristige Kooperation unmöglich machen, da der wissenschaftliche Partner Kontinuität seitens des Industriepartners benötigt. Mit der aktuellen Regelung „kann man leben".

Ein problematischer Aspekt kann sich ergeben, wenn Laborleiter ihren Arbeitsplatz wechseln (einen neuen Dienstgeber haben / ein Sabbatical nehmen / in Vaterkarenz gehen). Was passiert etwa, wenn ein Zentrumsleiter (eines JR-Zentrums) von einer FH an eine Uni wechselt? Verbleibt das Zentrum an der FH oder wandert es mit zur Uni und ist es dann noch förderungswürdig als JR-Zentrum, obwohl keine FH beteiligt ist? Es sollte Regelungen für allfällige Änderungen im Karrierepfad des Laborleiters geben, z.B. das Labor oder Zentrum bestehen lassen, aber einen neuen Labor- oder Zentrumsleiter finden. 


\subsection{Online Fragebogen}

Im Rahmen des zweiten Moduls, der Programmevaluierung, wurde eine schriftliche Befragung in Form eines Online-Fragebogens aller bestehenden sowie aller seit dem Jahr 2011 ausgelaufenen CDLabors und aller bestehenden JR-Zentren durchgeführt. Befragt wurden CD-Laborleiter und leiterinnen, JR-Zentrumsleiter und -leiterinnen, sowie deren Unternehmenspartner. Die Befragung fokussierte dabei stark auf die Erreichung der Programmziele und orientiert sich gemäß der Leistungsbeschreibung der Evaluierung inhaltlich am Frageprogramm der Evaluierungen aus dem Jahre 2011 und 2005 um Vergleiche über die Zeit zu ermöglichen.

Im Oktober 2016 wurden die Fragebögen konzipiert und überarbeitet sowie parallel dazu deren Programmierung umgesetzt. Die Erhebung selbst wurde im November 2016 durchgeführt.

In Summe wurden vier Online-Fragebogen-Versionen für folgende Befragungsgruppen umgesetzt: ${ }^{41}$

- CD-Laborleiter

- CD-Labor Unternehmenspartner

- JR-Zentrumsleiter

- JR-Zentrum Unternehmenspartner

Für Unternehmenspartner im nicht-deutschsprachigen Ausland wurde zusätzlich eine englische Version des Frageprogramms umgesetzt.

Insgesamt wurden 114 Laborleiter und 8 Zentrumsleiter kontaktiert. Die Unternehmensliste (Unternehmenspartner eines CD-Labors und/oder eines JR-Zentrums) umfasste 198 Dateneinträge. ${ }^{42}$ Die Kontaktdaten bzw. die Adresslisten wurden dem Evaluierungsteam von der CDG zur Verfügung gestellt. Die CDG unterstützt die Erhebung auch durch eine Vorinformation aller Kontaktpersonen der Befragungsgruppe. Dadurch sollte die Informationsqualität und auch der Rücklauf der Erhebung erhöht werden.

Von den 114 kontaktierten Laborleitern konnten 81 vollständig ausgefüllte Fragebögen eingeholt werden. Dies entspricht einer Rücklaufquote von 71\%. Von den acht angeschriebenen JR-Zentrums-

\footnotetext{
${ }^{41}$ Die Fragebögen befinden sich im Anhang des Berichts.

42177 Unternehmenskontakte waren ausschließlich einem oder mehreren CD-Labor(s) zuzuordnen, 15 Unternehmenskontakte einem JR-Zentrum und 6 Unternehmenskontakte waren einem oder mehreren CD-Labor(s) und einem JR-Zentrum zuzuordnen. An jene 6 Unternehmenskontakte wurde sowohl ein Fragebogen betreffend das/die CD-Labor(s) als auch ein Fragebogen betreffend das JR-Zentrum verschickt.
} 
leiterinnen und -leitern lagen am Ende der Erhebung sieben vollständig beantwortete Fragebögen vor (Rücklaufquote 88\%).

Von den $177^{43}$ kontaktierten CD-Labor-Partnerunternehmen haben insgesamt 90 einen vollständig ausgefüllten Fragebogen rückübermittelt. Dies entspricht einer Rücklaufquote von 51\%. Von den 21 JR-Zentrums-Unternehmenspartnern haben insgesamt 12 einen Fragebogen ausgefüllt, 10 vollständig und 2 teilweise. Dies entspricht ebenfalls einer Rücklaufquote von 57\% bzw. 48\%.

Folgende Tabelle zeigt den Rücklauf nochmals in einer tabellarischen Übersicht:

Tabelle 22: Rücklauf der Onlineerhebung (Übersicht)

\begin{tabular}{|l|r|r|r|r|r|r|}
\hline Befragungsgruppe & $\mathbf{N}$ & $\mathbf{n}(\mathbf{v})$ & $\mathbf{n}(\mathbf{v})$ in \% & $\mathbf{n}(\mathbf{t})$ & $\mathbf{n}(\mathbf{g})$ & $\mathbf{n}(\mathbf{g})$ in \% \\
\hline CD-Laborleiter & 114 & 81 & $71 \%$ & & 81 & $\mathbf{7 1 \%}$ \\
\hline JR-Zentrumsleiter & 8 & 7 & $88 \%$ & & 7 & $\mathbf{8 8 \%}$ \\
\hline CDL-Unternehmenspartner & 177 & 90 & $51 \%$ & & 90 & $\mathbf{5 1 \%}$ \\
\hline JRZ-Unternehmenspartner & 21 & 10 & $48 \%$ & 2 & 12 & $\mathbf{5 7 \%}$ \\
\hline
\end{tabular}

Anm.: $n(v)=v o l l s t a ̈ n d i g$ ausgefüllter Fragebogen, $n(t)=$ teilweise ausgefüllter Fragebogen, $n(g)=i n s g e s a m t$ ausgefüllte Fragebögen

\section{Quelle: Economica/IWI (2016)}

Im Folgenden werden die Ergebnisse der Online-Erhebung dargestellt. Die Darstellung erfolgt gegliedert nach den einzelnen Befragungsgruppen. In der Teilgruppe CD-Laborleiter sowie CD-Labor Unternehmenspartner ist ein Vergleich mit den bisherigen Evaluierungen $(2005,2011)$ möglich. Der Fokus liegt dabei auf einem Vergleich der Daten der aktuellen Evaluierung mit jenen der Evaluierung von 2011. Hier ist es auch aufgrund des gleichen Evaluierungsteams möglich auf einen vollständigen Rohdatensatz des Jahres 2011 zurückzugreifen. Betreffend die Evaluierung von 2005 werden die Daten - so weit möglich - aus dem veröffentlichten Endbericht entnommen bzw. rekonstruiert.

Zusätzlich und in Ergänzung zur Online-Erhebung wurden mit drei CD-Laborleiterinnen und -leitern bzw. einem JR-Zentrumsleiter Tiefeninterviews geführt. Die Ergebnisse dieser Gespräche fließen zusätzlich in die Ausführungen der folgenden Abschnitte mit ein.

\footnotetext{
${ }^{43}$ Anm.: 177 gültige Adressen von insgesamt 183.
} 


\subsubsection{Teilgruppenbetrachtung (Befragungsgruppe CD-Laborleiter)}

Zu Beginn der Befragung wurden die Laborleiter gebeten ihre Zufriedenheit mit einzelnen Aspekten des (Förder-) Programms bzw. der Programmabwicklung zu bewerten. Was die Klarheit des Förderkonzepts und des Förderziels betrifft, zeigen sich die Befragten überaus zufrieden. Alle Laborleiterinnen und Laborleiter bekunden diesbezüglich ihre Zufriedenheit (85\% zeigen sich sehr zufrieden, 15\% zufrieden). Auch die Zufriedenheit mit der Angemessenheit der Evaluierungskriterien sowie der Bearbeitungsdauer der eingereichten Anträge ist (sehr) hoch, in beiden Fällen liegt sie über 95\%.

\section{Abbildung 52: Zufriedenheit mit Aspekten des Programms bzw. der Programmabwicklung}

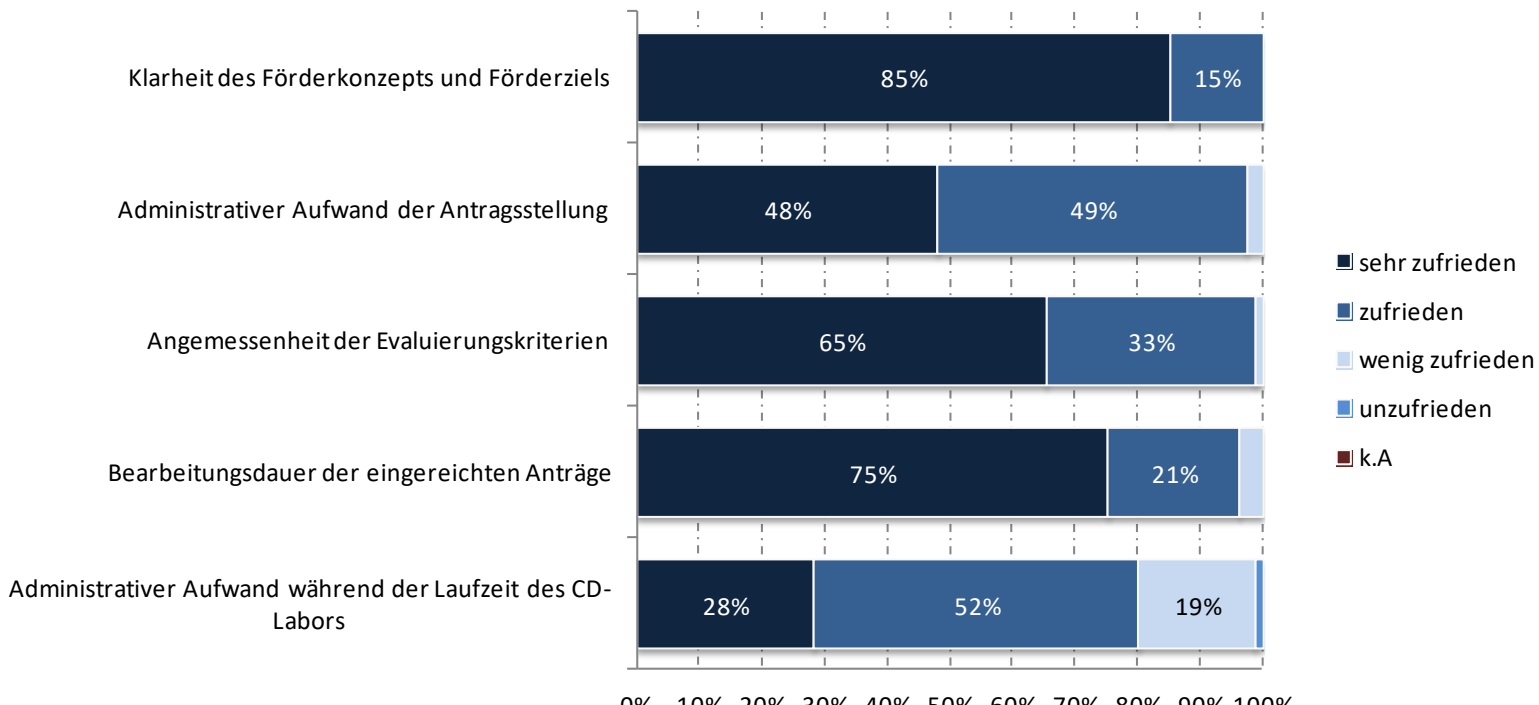

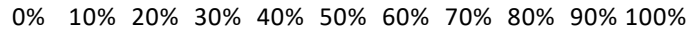

Anm.: Prozentwerte $<4 \%$ werden aus Platzgründen in den Abbildungen nicht dargestellt. ${ }^{44}$ Quelle: Economica/IWI (2016); Online-Erhebung: $n=81$

Was den administrativen Aufwand betrifft, so wird dieser - bezogen auf die Antragstellung - fast einheitlich als (sehr) zufriedenstellend bewertet (97\%). Bei der Bewertung des administrativen Aufwands während der Laufzeit des CD-Labors verschieben sich die Zufriedenheitswerte etwas. Rund $20 \%$ bewerten diesen als wenig zufriedenstellend, jedoch nur einer der Befragten als gar nicht zufriedenstellend.

\footnotetext{
${ }^{44}$ Aufgrund von Rundungen können sich im vorliegenden Bericht bei Summenbildungen und bei der Berechnung von Prozentangaben geringfügige Abweichungen ergeben.
} 
Ein Vergleich mit der Evaluierung 2011 zeigt beim administrativen Aufwand im Rahmen der Antragstellung kaum Verschiebungen der Zufriedenheitswerte. Diese waren im Jahr 2011 sehr hoch und sind es nach wie vor. Was den administrativen Aufwand während der Laufzeit betrifft, konnten sich die Werte in Summe leicht verbessern. Die Gruppe der "sehr Zufriedenen“ konnte gegenüber 2011 deutlich erhöht, die Gruppe der „wenig sowie Unzufriedenen“ hingegen verringert werden. Dies kann als ein erstes Anzeichen dafür gewertet werden, dass die von der CDG gesetzten Maßnahmen hinsichtlich administrativer Vereinfachungen bereits erste Wirkung zeigen.

Abbildung 53: Zufriedenheit mit Aspekten des Programms bzw. der Programmabwicklung im Zeitverlauf $(2005,2011,2016)$

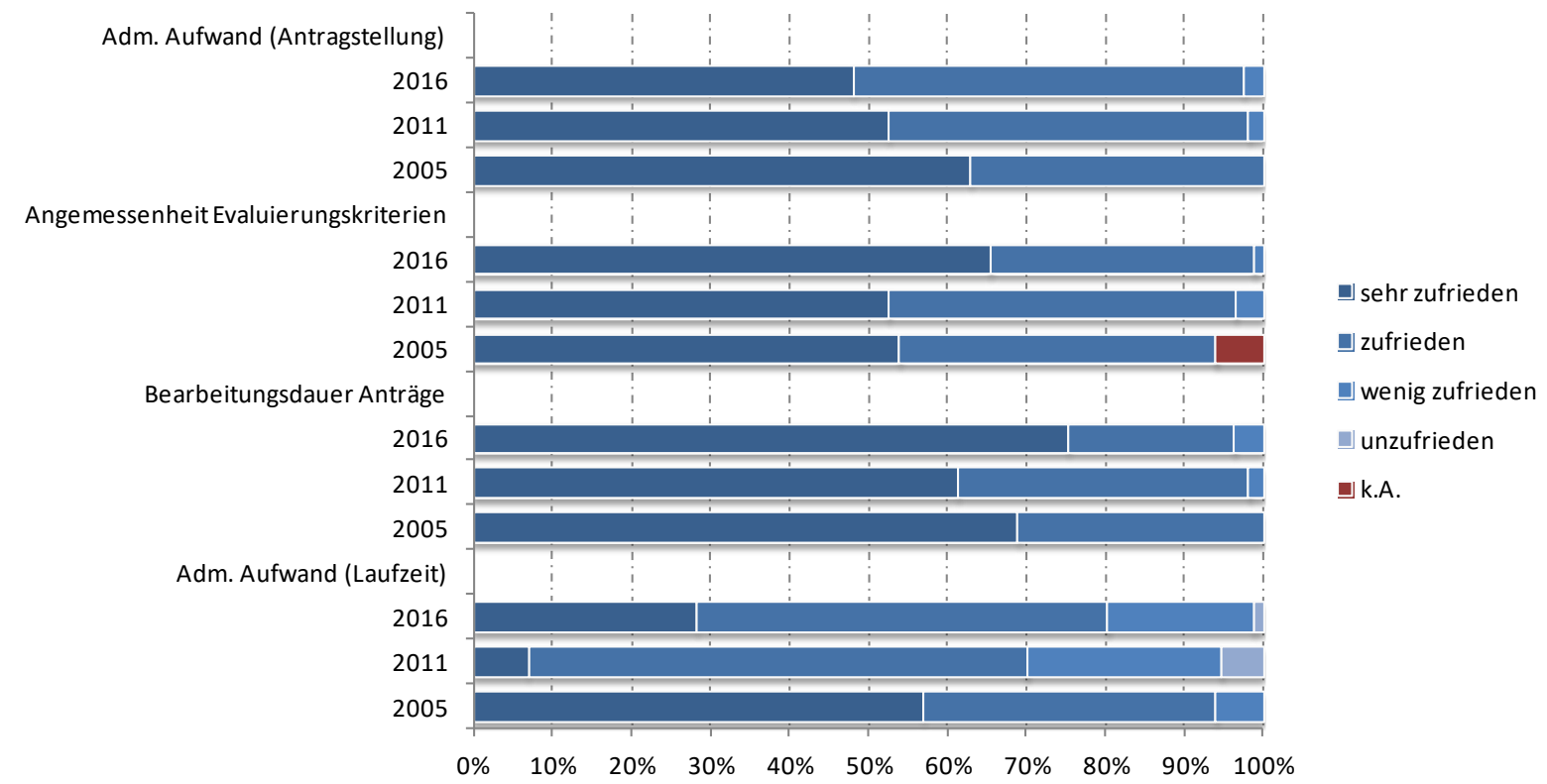

Anm.: Der Aspekt "Klarheit des Förderkonzepts und Förderziels" wurde in den Jahren 2011 und 2005 nicht abgefragt.

Quelle: Economica/IWI (2016, 2011), Joanneum Research/Technopolis/KMU Forschung Austria/Fraunhofer - ISI (2005) ; $n=81$ (2016), $n=57$ (2011), $n=35$ (2005)

In den Bereichen „Angemessenheit der Evaluierungskriterien“, sowie „Bearbeitungsdauer der eingereichten Anträge" gab es lediglich leichte Verschiebungen. Die Angemessenheit der Evaluierungskriterien wurde ausgehend von einem bereits sehr hohen Zufriedenheitsniveau 2011 heuer in Summe noch eine Spur positiver bewertet. Die Bearbeitungsdauer wird von einer (auch gegenüber 2011 größer gewordenen) Mehrheit als sehr zufriedenstellend bewertet. 


\section{Exkursbox: Potential für administrative Vereinfachungen}

Die CDG hat in den vergangenen Jahren, auch in Bezugnahme auf die Evaluierung 2011, einige administrative Vereinfachungen umgesetzt (vgl. dazu CDG, „Kurzinfo zu administrativen Vereinfachungen in der CDG in den Jahren 2012-2015). Diese, das zeigen sowohl die Befragungsdaten als auch einzelne Statements von Laborleiterinnen und Laborleitern, haben bereits (positive) Wirkung gezeigt und einiges an Vereinfachungen und Flexibilität gebracht.

„Seit meiner Antragstellung vor ca. 8 Jahren hat sich schon viel verbessert, insbesondere die Budget- und die Report-Regeln. Besonders aufwändig waren die Budgetüberträge in das nächste Jahr, die quasi Monate im Voraus bekannt sein mussten. Ich glaube, die aktuellen Regelungen mit weniger Budget-Kategorien und pauschalem Übertrag bieten ausreichend Flexibilität, so dass ich nur auf die letzten 2 Jahre bezogen bei Administration "zufrieden" ankreuzen würde." (abgeschlossenes Labor)

„Der administrative Aufwand hat sich in den letzten Jahren deutlich verbessert, nimmt aber immer noch sehr viel Zeit in Anspruch." (aktives Labor)

„Zwischen 2006 und 2014 haben sich die Regeln für die Finanzabrechnungen leider mehrmals geändert (zuerst wurden die Modalitäten wesentlich komplizierter, später wieder etwas benutzerfreundlicher). Diese führte dazu, dass der CD Laborleiter zunehmend Zeit für die komplexer werdende Buchhaltung aufwenden musste (anstatt Wissenschaft zu betreiben). Die CDG Mitarbeiter waren in dieser Angelegenheit immer sehr engagiert und hilfsbereit." (abgeschlossenes Labor)

Im Folgenden sollen einige Anmerkungen und Verbesserungsvorschläge dargestellt werden, wobei der Schwerpunkt bei jenen liegt, die von Laborleiterinnen und Laborleitern eingebracht wurden, deren Labor derzeit aktiv ist. Einige der Anmerkungen von älteren Labors, die bereits seit einigen Jahren ausgelaufen sind, betreffen Verbesserungen, die teils schon umgesetzt wurden.

„Webportal könnte verbessert werden“. (aktives Labor)

„Das Webtool ist unendlich kompliziert. Die Jahresabrechnung hat dann in einem unabhängigen Excel File zu erfolgen, dann gibt es den Finanzbericht - alles scheint doppelt und dreifach, ist nicht durchsichtig und sehr zeitraubend". (aktives Labor)

„Im Budgetbereich sollte es zu einer Vereinfachung kommen. Das Unterschreiben durch die Firmen mit Stempel ist ein im angloamerikanischen Raum nicht bekanntes Konzept. Ein Online-Tool, das die Firmen online zeichnen können, wäre notwendig und zeitgemäß". (aktives Labor)

„Eine Kategorisierung von Moduländerungsanträgen wäre hilfreich, und zwar gestaffelt nach dem Ausmaß der beantragten Änderungen. Nur solche Änderungsanträge, die eine massive Änderung betreffen, sollen in dem 


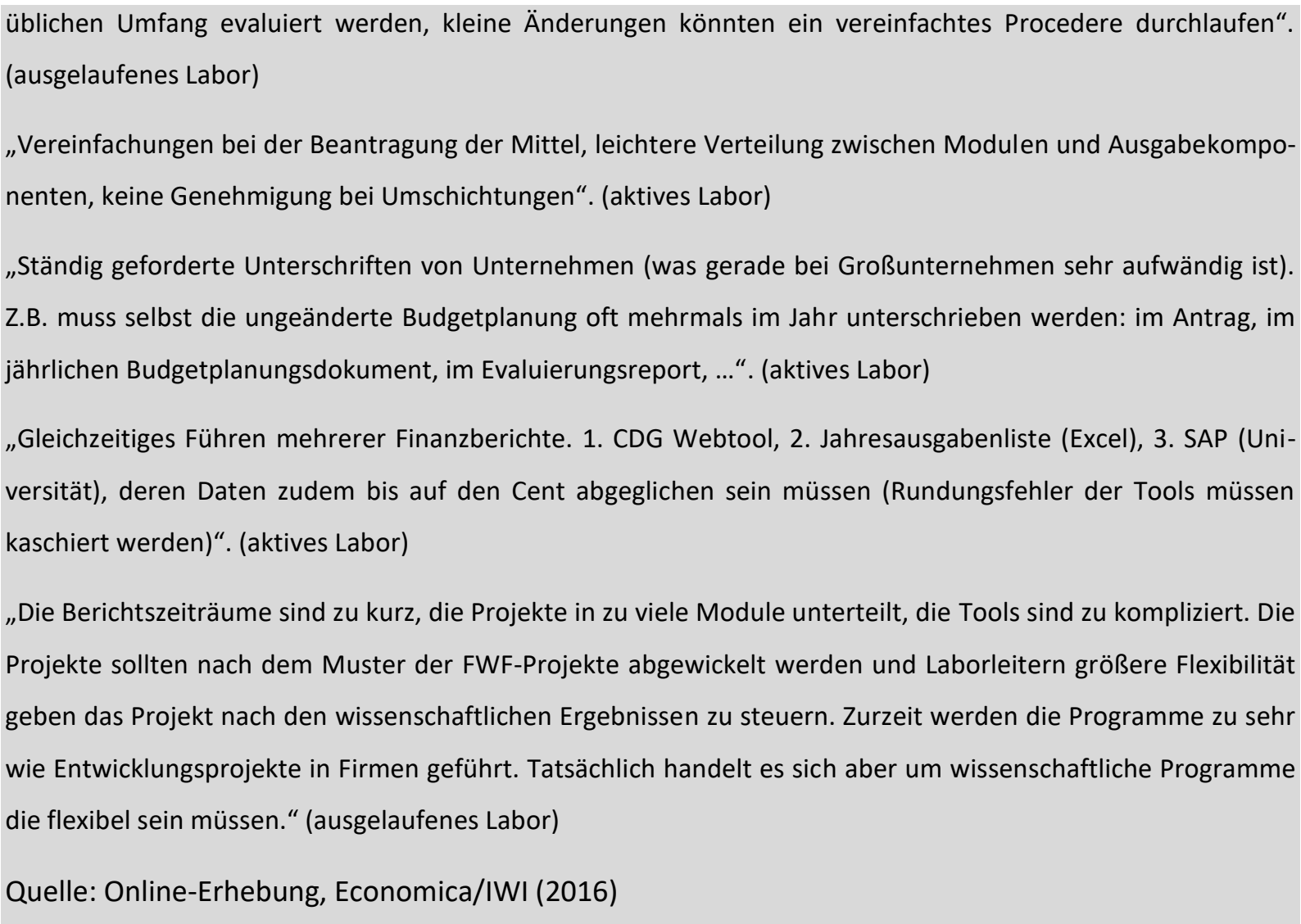

Was die Laufzeit des Programms von sieben Jahren betrifft so beurteilen die große Mehrheit der CDLaborleiter und Laborleiterinnen (84\%) diese als gerade richtig. 16\% der Befragten sind der Ansicht sie sei zu kurz, als zu lang empfindet keiner der Laborleiter und Laborleiterinnen die Laufzeit von sieben Jahren.

Die begleitende wissenschaftliche Kontrolle wird von den CD-Laborleitern und -Leiterinnen mehrheitlich als (sehr) hilfreich angesehen. 
Abbildung 54: Einschätzung der begleitenden wissenschaftlichen Kontrolle

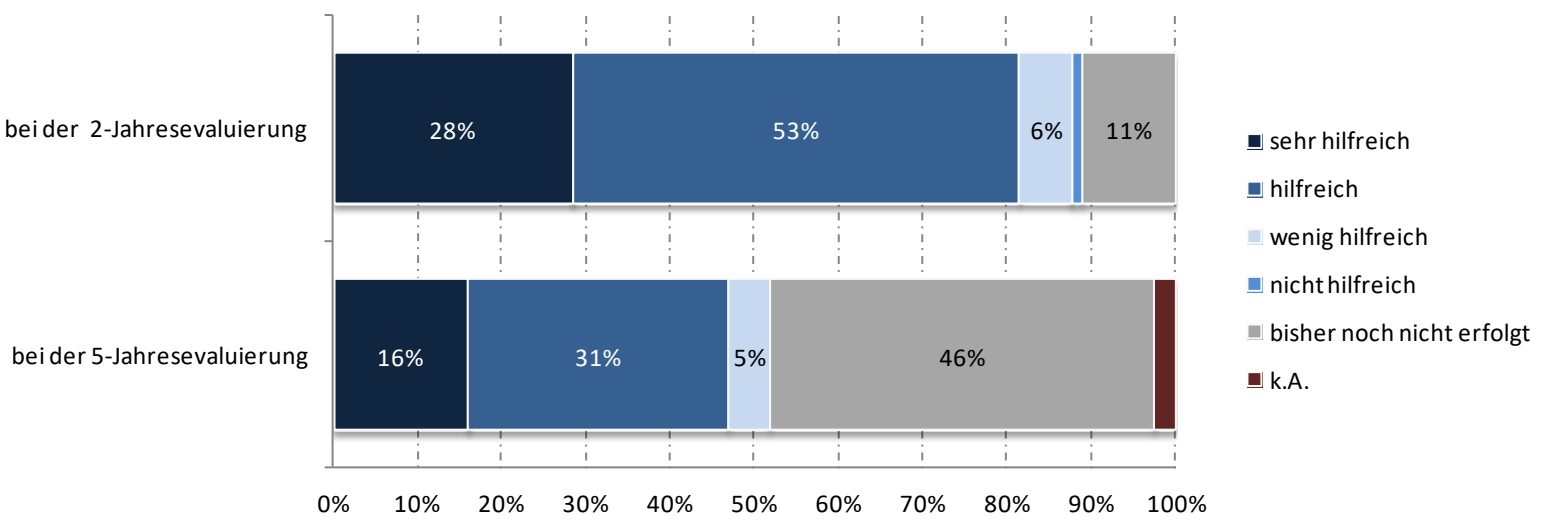

Quelle: Economica/IWI (2016); Online-Erhebung: $n=81$

Betrachtet man nur jene Gruppe an Laborleitern und Laborleiterinnen bei denen eine 2- bzw. 5Jahresevaluierung bereits stattgefunden hat bzw. die hierzu Angaben gemacht haben, so zeigt sich, dass jeweils rund $90 \%$ der Befragten diese begleitende Maßnahme als (sehr) hilfreich ansehen. Ein Vergleich mit der Bewertung 2011 zeigt nur geringe Unterschiede.

Abbildung 55: Einschätzung der begleitenden wissenschaftlichen Kontrolle im Zeitverlauf (2011, 2016)

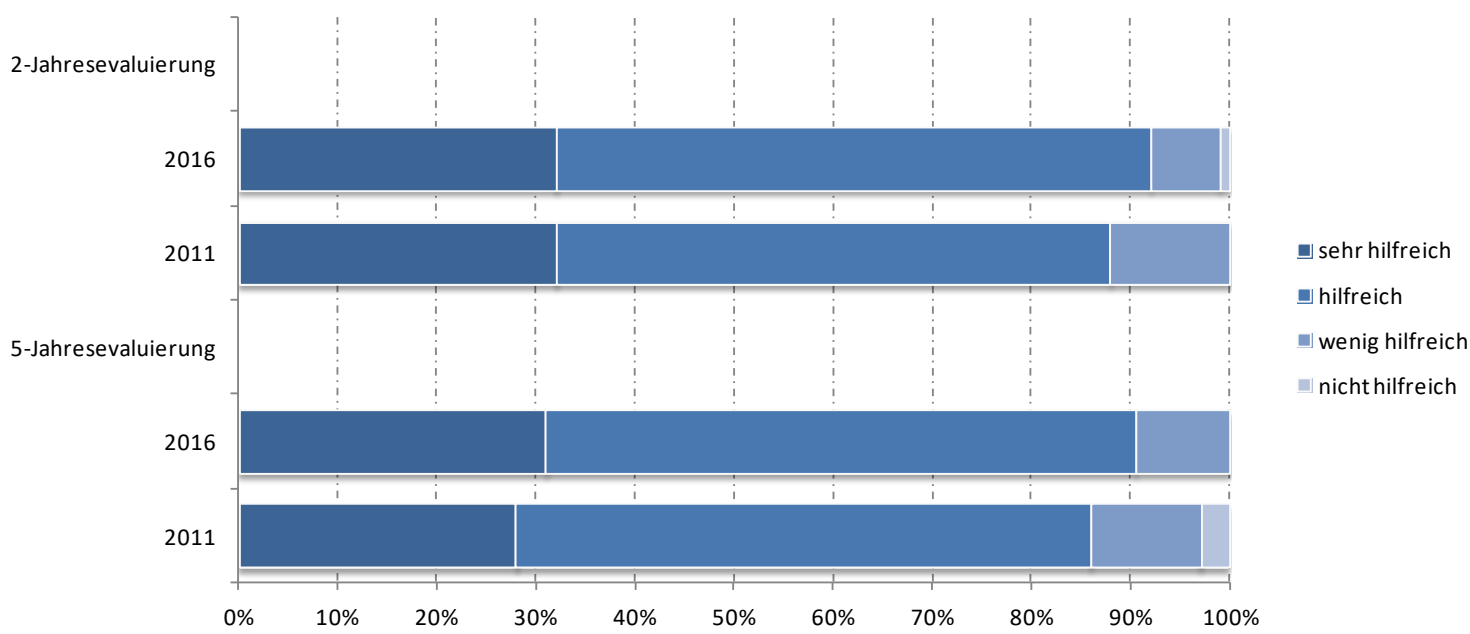

Quelle: Economica/IWI (2016, 2011); $n=81$ (2016), $n=57$ (2011)

Des Weiteren wurden die Befragten gebeten Anzahl und Zeitpunkt der Zwischenevaluierungen zu bewerten. Was die Anzahl der Zwischenevaluierungen betrifft wird diese von der großen Mehrheit 
der CD-Laborleiter und -Laborleiterinnen als gerade richtig empfunden (88\%). Mehr Zwischenevaluierungen findet keiner der Befragten wünschenswert.

\section{Abbildung 56: Bewertung von Anzahl und Zeitpunkt der Zwischenevaluierungen}

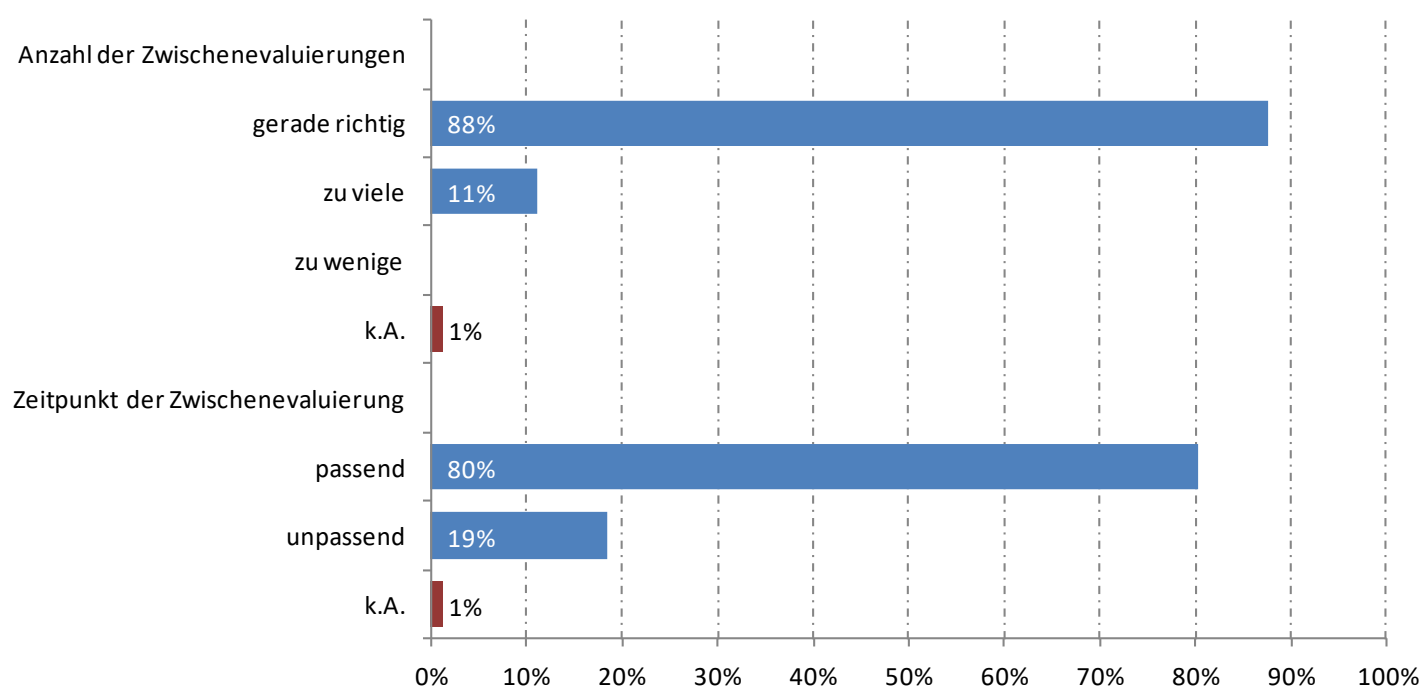

Quelle: Economica/IWI (2016); Online-Erhebung: $n=81$

Auch der Zeitpunkt der Zwischenevaluierungen wird von der großen Mehrheit (80\%) als passend empfunden. All jene die den Zeitpunkt als unpassend bewertet haben (19\%), hatten im Rahmen einer offenen Frage die Möglichkeit genauer auf die Thematik einzugehen und u.a. Gründe und/oder Verbesserungsvorschläge zu nennen. Nahezu alle Befragten dieser Teilgruppe sehen insbesondere die 2-Jahresevaluierung kritisch bzw. verfrüht. Viele dieser Befragten würden sich eine etwas spätere erste Zwischenevaluierung wünschen (nach 2,5 bzw. 3 Jahren).

\section{Auszug aus den Fragebögen (Zitate):}

„Die 2-Jahresevaluierung findet tatsächlich ja schon nach 1,5 Jahren statt - das ist sehr früh. 2,5 Jahre wären ein besserer Zeitraum".

„Je nach Startdatum ist die erste Evaluierung eher eine 1,25-Jahres Evaluierung, was mir deutlich zu kurz erscheint".

„Bei Evaluierung vor Ablauf des zweiten Jahres ist eine Berichtslegung bereits nach ca. 1,5 Jahren notwendig. Wenn das CDL nicht unmittelbar von Beginn weg vollumfänglich startet (Mitarbeiter müssen ja erst rekrutiert werden) wird nur rund ein Jahr Aktivität die Basis der Evaluierung liefern. Das ist doch sehr kurz!“.

„Die erste Evaluierung könnte etwas später stattfinden, um den Forschern mehr Zeit zu geben erste signifikante Beiträge zu leisten“. 


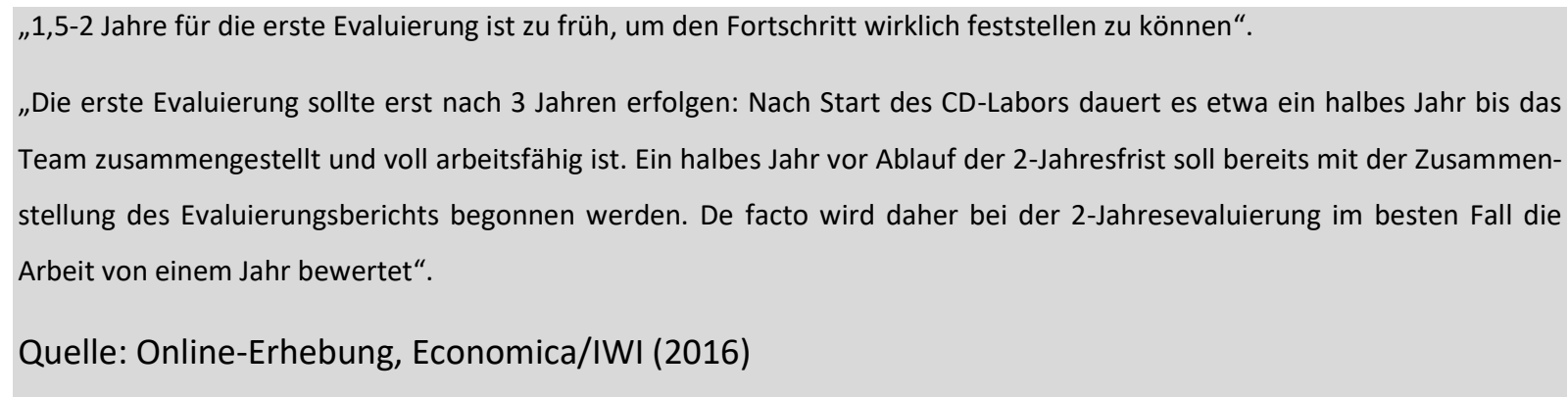

Von jenen, die auch die Anzahl der Zwischenevaluierungen als zu hoch empfinden, wird meist der Wunsch nach einer einzigen Zwischenevaluierung zur Halbzeit des CD-Labors genannt.

Mit den Betreuungsleistungen und der Öffentlichkeitsarbeit der CDG zeigen sich die Befragten CDLaborleiter und -Laborleiterinnen (sehr) zufrieden. Sowohl die Betreuung während der Antragstellung durch die CDG als auch die Betreuung des CD-Labors während der Laufzeit erzieht hohe Zufriedenheitswerte.

\section{Abbildung 57: Zufriedenheit mit den Leistungen der CDG}

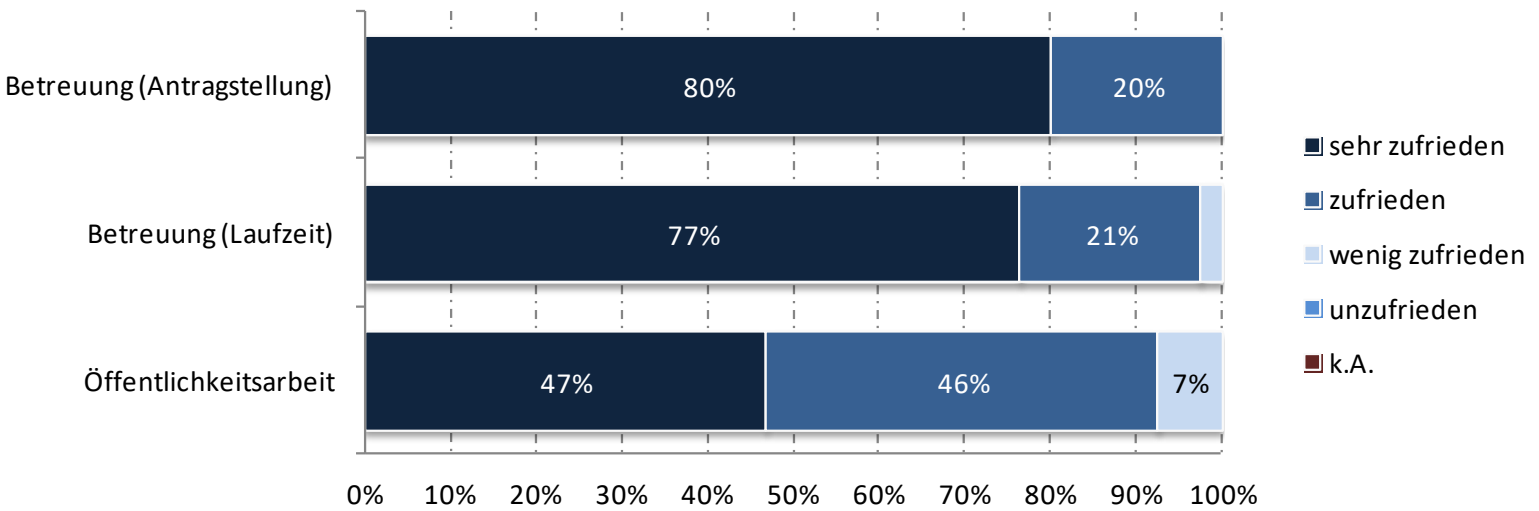

Quelle: Economica/IWI (2016); Online-Erhebung: $n=81$

Ein Verglich über die Zeit zeigt, ausgehend von sehr hohen Zufriedenheitswerten mit der Betreuung und Öffentlichkeitsarbeit, noch einen weiteren leichten Anstieg im Rahmen der aktuellen Evaluierung. Im Bereich der Betreuung während der Antragstellung, konnte insbesondere die Gruppe der „sehr Zufriedenen“ stark erhöht werden und sowohl im Vergleich zur Evaluierung im Jahr 2011, als auch gegenüber dem Jahr 2005 deutlich gesteigert werden. Auch hinsichtlich der Betreuung während der Laufzeit des CD-Labors konnte die Teilgruppe der „sehr Zufriedenen“ gegenüber der Vergangenheit erhöht werden. 
Auch die Öffentlichkeitsarbeit verbesserte sich gegenüber den Vergleichsjahren. Die Gruppe der „sehr Zufriedenen“ konnte erhöht, die an sich schon kleine Teilgruppe der „wenig Zufriedenen“ weiter verringert werden.

Abbildung 58: Zufriedenheit mit den Leistungen der CDG im Zeitverlauf $(2005,2011,2016)$

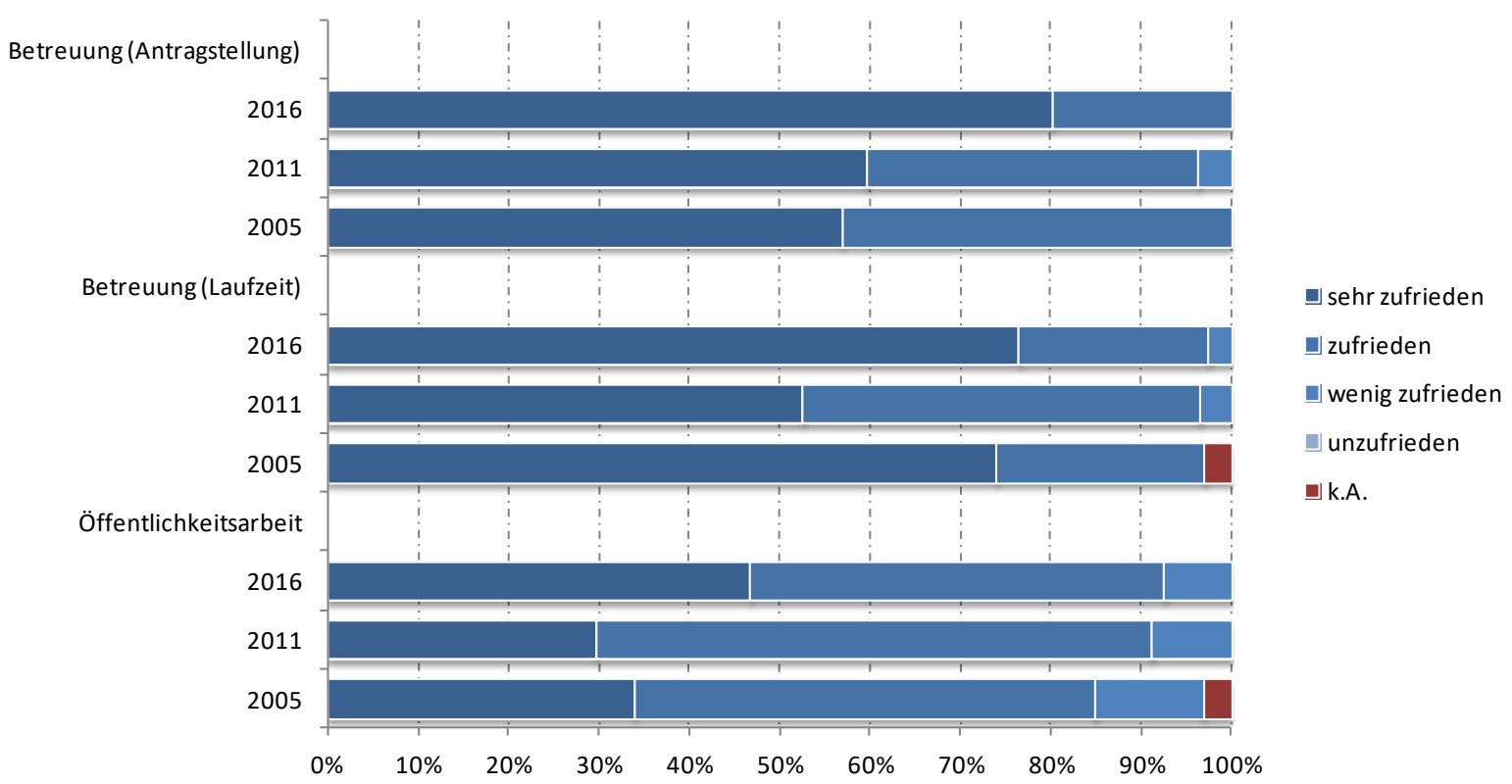

Quelle: Economica/IWI (2016, 2011), Joanneum Research/Technopolis/KMU Forschung Austria/Fraunhofer - ISI (2005) ; $n=81$ (2016), $n=57$ (2011), $n=35$ (2005)

Die Initiative zur CD-Laborgründung ging bei knapp der Hälfte der Labors (46\%) von beiden Partnern aus, bei rund $40 \%$ kam die Motivation vom wissenschaftlichen Partner. Eine Betrachtung im Zeitverlauf zeigt hier starke Verschiebungen. Während 2005 die Initiative noch sehr stark vom wissenschaftlichen Partner oder vom Unternehmenspartner ausging, zeigte sich bereits 2011 eine starke Verschiebung hin zu einer "gemeinsamen Initiative“ beider Partner. Dieser Trend verstärkt sich bei der aktuellen Befragung nochmals leicht. Interessant zu beobachten ist ein stetiger Rückgang hinsichtlich einer Initiative zur Laborgründung von Seiten der Unternehmenspartner.

Bei den meisten Laborgründungen bestand von Seiten des Laborleiters/der Laborleiterin bereits vorher Kontakt zum jeweiligen Unternehmenspartner. Rund 72\% der Befragten hatten mit dem Unternehmenspartner u.a. bereits im Rahmen gemeinsamer Forschungsprojekte Kontakt, rund ein Viertel hatte (auch) Kontakt über Absolventen und Absolventinnen und/oder lose Kontakte. 
Abbildung 59: Motivation zur CD-Laborgründung im Zeitverlauf $(2005,2011,2016)$

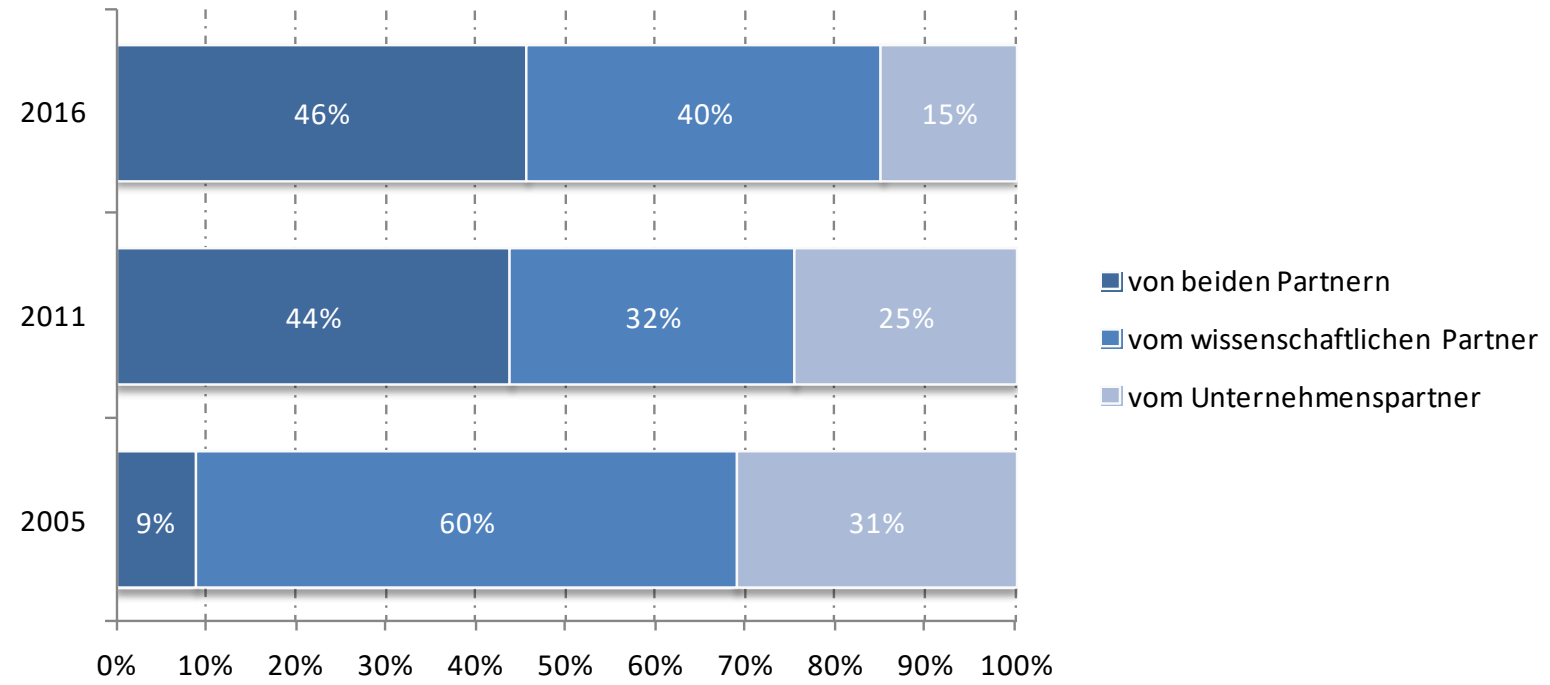

Quelle: Economica/IWI (2016, 2011), Joanneum Research/Technopolis/KMU Forschung Austria/Fraunhofer - ISI (2005) ; $n=81$ (2016), $n=57$ (2011), $n=35$ (2005)

Abbildung 60: Kontakt zum Unternehmenspartner vor der CD-Laborgründung

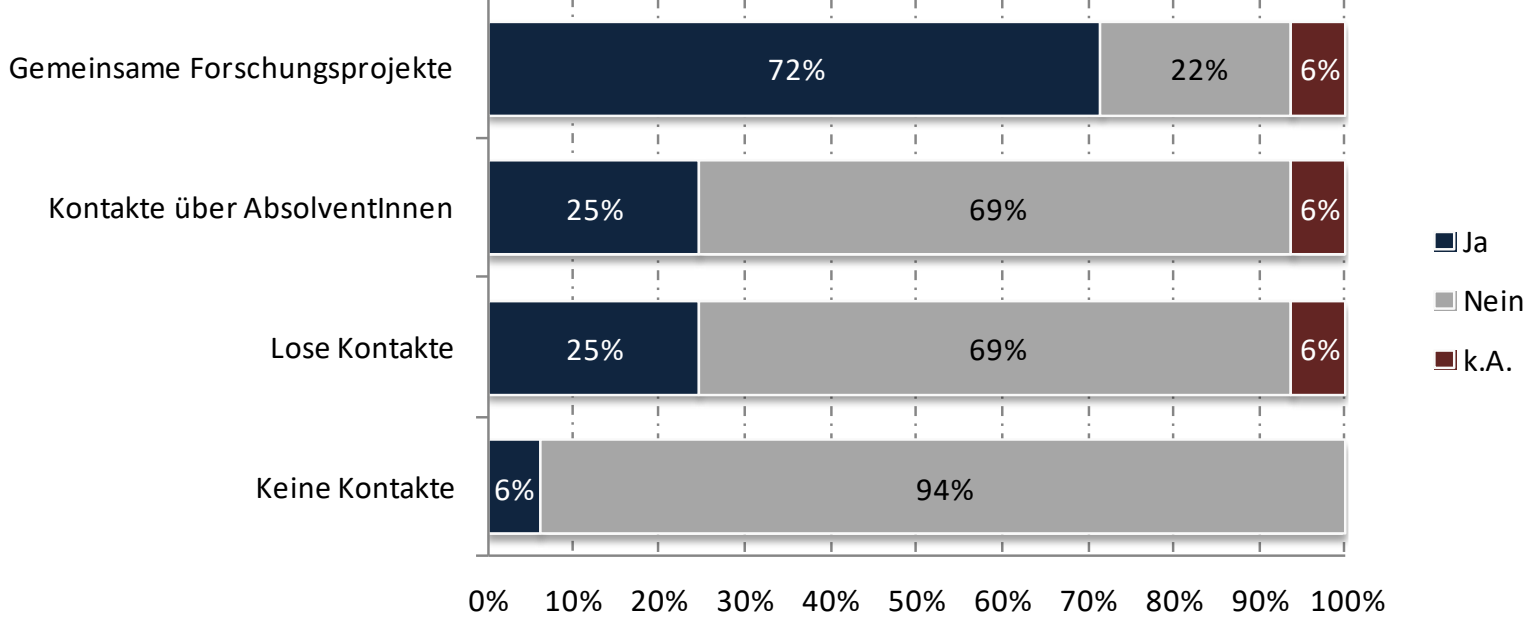

Quelle: Economica/IWI (2016); Online-Erhebung: $n=81$

Auch hier zeigen sich kaum Abweichungen zu jener „Kontaktstruktur“ aus dem Jahr 2011.

Bei den Erwartungen und Zielen, die im Zusammenhang mit der Gründung eines CD-Labors stehen, ist vor allem ein langfristig abgesichertes Arbeiten an einem Themenkreis von zentraler Bedeutung 
für die Laborleiter und Laborleiterinnen. Alle Befragten bewerten diesen Aspekt als wichtig, 90\% davon als sehr wichtig. Dieser Punkt - also das Thema Langfristigkeit und Intensität der Zusammenarbeit - wurde auch in den begleitend geführten Gesprächen immer wieder betont und teils auch als „Alleinstellungsmerkmal“ des CDG-Programms hervorgehoben.

Auch die finanzielle Absicherung der Forschungsarbeit, sowie der Aufbau eines eigenen Forschungsteams werden als (sehr) bedeutend angesehen. Ein Vergleich zur Evaluierung im Jahr 2011 zeigt hier so gut wie keine Verschiebungen im Antwortverhalten. Etwas weniger bedeutsam (im Vergleich zu 2011) ist für die Befragten die Intensivierung der Unternehmenskontakte. Dies mag daran liegen, dass diese Kontakte schon im Vorfeld, unter anderem durch weitere gemeinsame Forschungsprojekte, intensiv waren.

\section{Abbildung 61: Erwartungen und Ziele bei der CD-Laborgründung und deren Bedeutung}

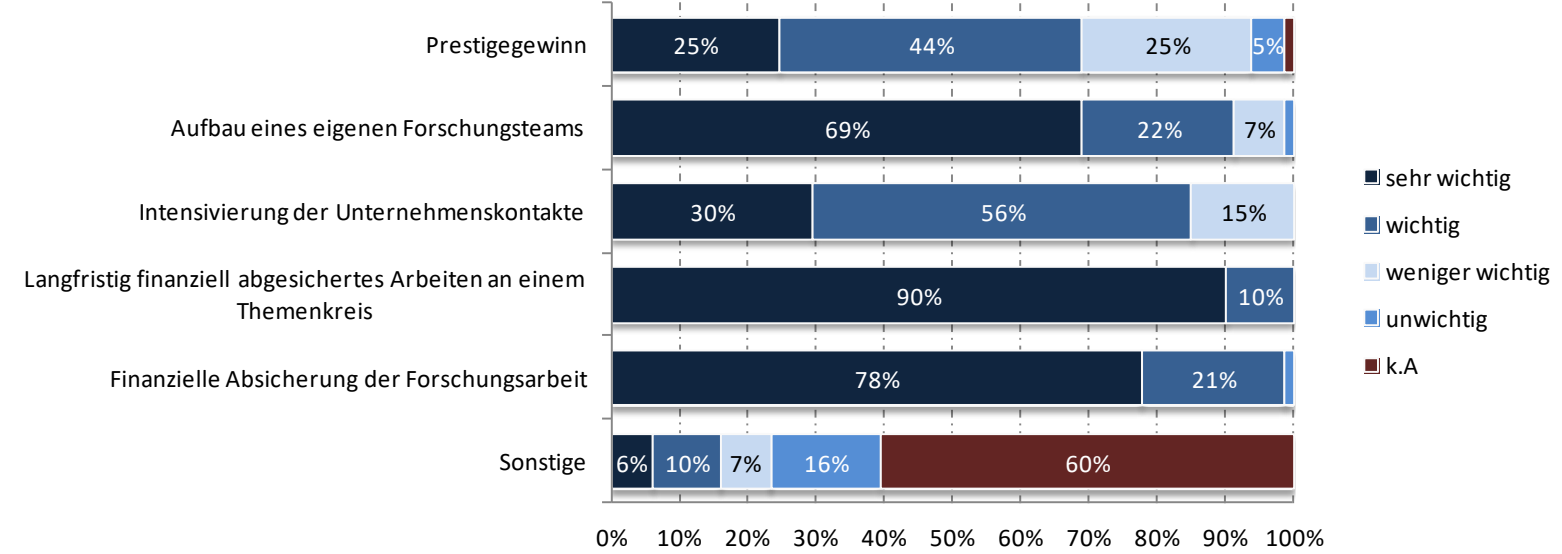

Quelle: Economica/IWI (2016); Online-Erhebung: $n=81$

\section{Sonstige Erwartungen und Ziele - Auszug aus den Fragebögen (Zitate):}

Sehr wichtig:

„Durchführung multilateraler Kooperationen mit mehreren Unternehmenspartnern“.

„Es war für mich klar, dass mein Karriereweg noch einem richtigen Schub brauchte, um international kompetitiv zu sein! CD Labors sind dafür perfekt, weil eine Semistruktur gegründet wird und der Laborleiter enorm an Sichtbarkeit gewinnt! Das CD Labor führte dazu, dass die Uni ihre Berufungs-Strategie änderte!“.

„Nebeneinkünfte durch Laborleiterhonorar".

„Erweiterung des Forschungsgebietes“. 


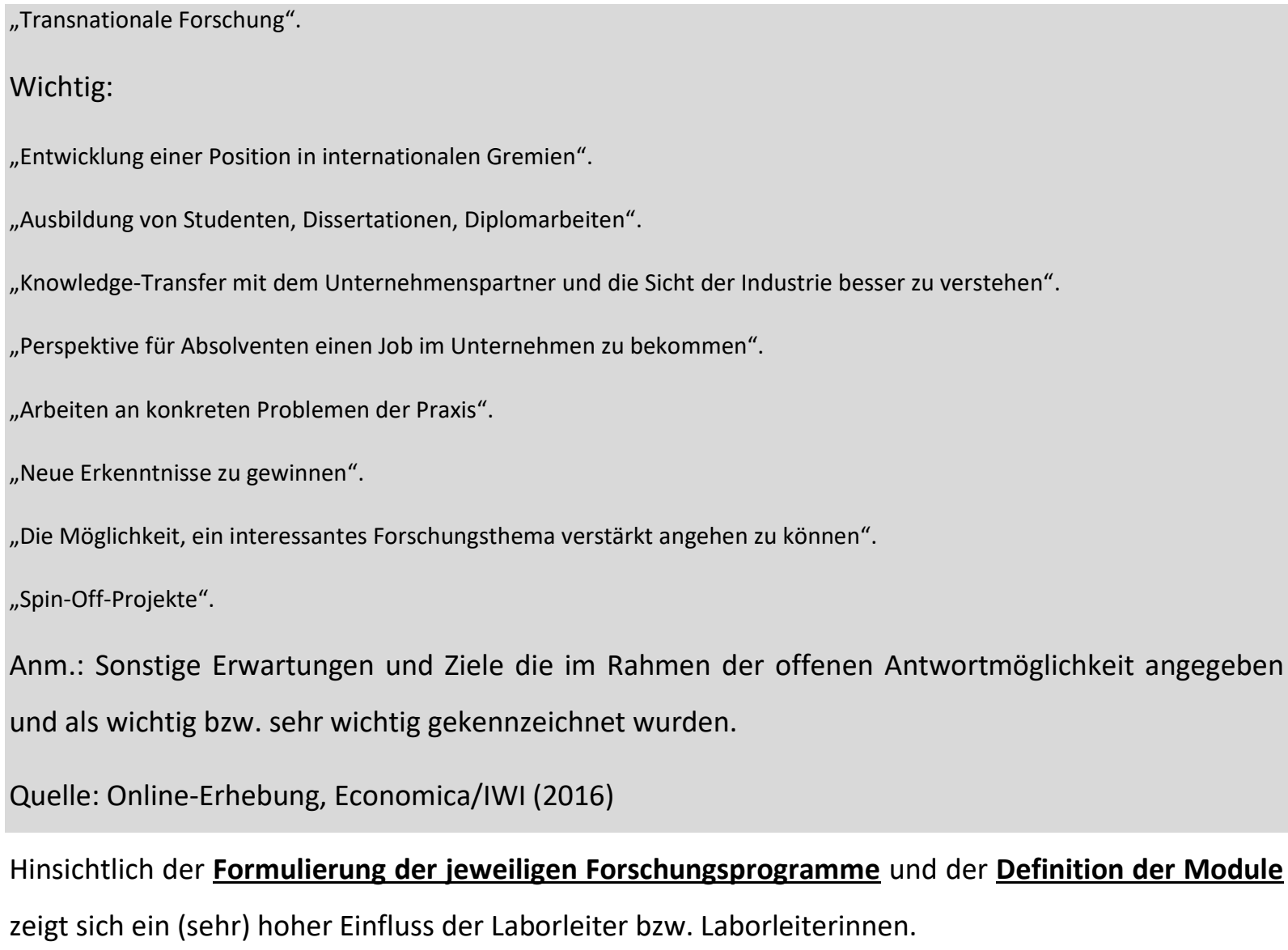

Abbildung 62: Einfluss der Laborleiter/Laborleiterinnen auf die Forschungsprogrammformulierung und auf die Moduldefinition

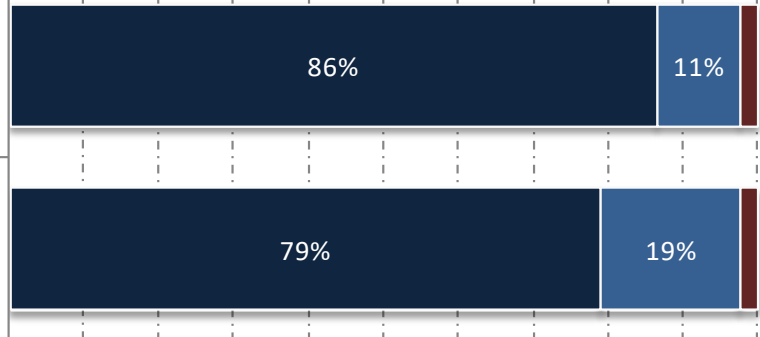

- sehr hohen Einfluss

$\square$ hohen Einfluss

wenig Einfluss

Moduldefinition

Quelle: Economica/IWI (2016); Online-Erhebung: $n=81$

Bemerkenswert ist die zu beobachtende Veränderung betreffend dieses Aspekts im Laufe der Zeit. Bei der Evaluierung im Jahre 2005 gaben noch 68\% der Laborleiter und Laborleiterinnen an hinsicht- 
lich der Forschungsprogrammformulierung nur wenig Einfluss zu haben. Auch bezüglich der Moduldefinition gaben nahezu die Hälfte (47\%) an, wenig Einfluss zu haben.

Bereits bei der Evaluierung 2011 änderte sich dieses Bild dramatisch. Lediglich knapp 4\% (Forschungsprogrammformulierung) bzw. rund 7\% (Moduldefinition) gaben hier einen geringen Einfluss an. Im Zuge der aktuellen Erhebung findet sich kein Befragter mehr der hinsichtlich dieser beiden Punkte einen geringen Einfluss angibt. Deutlich im Steigen ist analog dazu auch die Gruppe jener, die hier einen sehr hohen Einfluss sieht.

Abbildung 63: Einfluss der Laborleiter/Laborleiterinnen auf die Forschungsprogrammformulierung und auf die Moduldefinition im Zeitverlauf $(2005,2011,2016)$

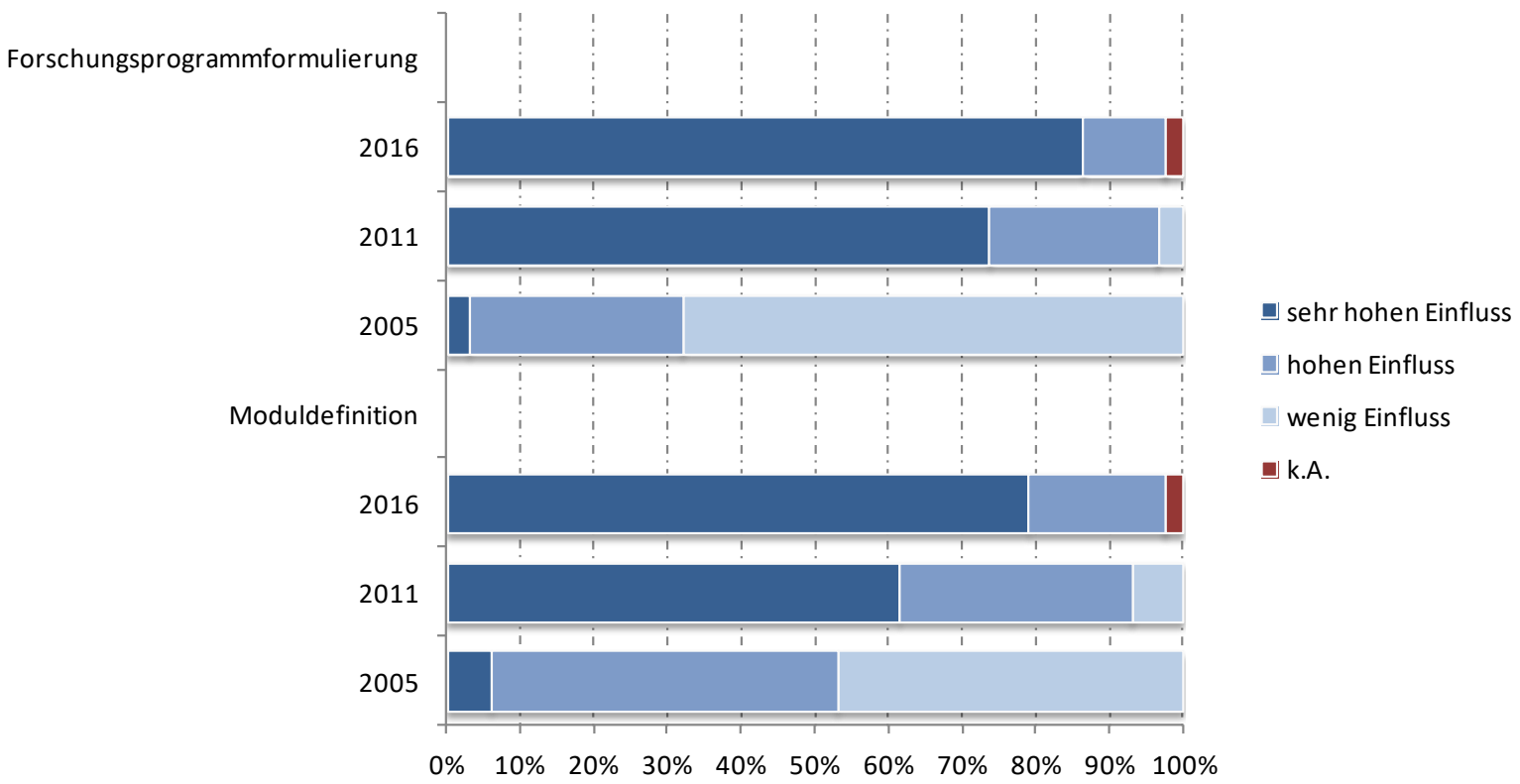

Quelle: Economica/IWI (2016, 2011), Joanneum Research/Technopolis/KMU Forschung Austria/Fraunhofer - ISI (2005) ; $n=81$ (2016), $n=57$ (2011), $n=35$ (2005)

Was die Inputs der Unternehmenspartner betrifft, so betraf dieser vor allem das Feedback zu den Forschungsaktivitäten. Auch hinsichtlich der Mitarbeit an Forschungsarbeiten, der Formulierung des Forschungsprogramms sowie der Moduldefinition und der Vermittlung von Kontakten sahen mehr als die Hälfte einen Input in (sehr) hohem Ausmaß.

Auch das Antwortverhalten dieser Frage weicht nur wenig von jenem im Jahr 2011 ab. Man kann diesbezüglich von einer gleichbleibenden Input-Struktur durch die Unternehmenspartner sprechen 
bzw. von einer gleichbleibenden Wahrnehmung des Input-Verhaltens durch die Laborleiter und Laborleiterinnen.

Abbildung 64: Inputs des Unternehmenspartners

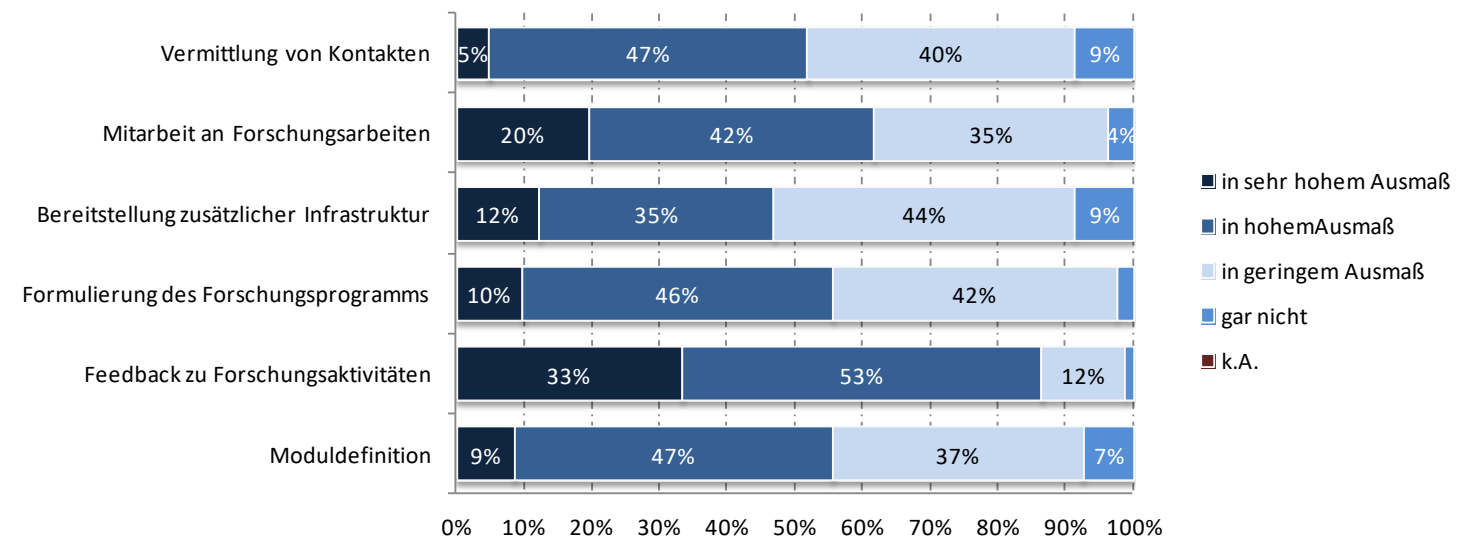

Quelle: Economica/IWI (2016); Online-Erhebung: $n=81$

Die folgende Abbildung zeigt eine Übersicht über den Anteil an der Gesamtarbeitszeit an der Universität, der dem CD-Labor gewidmet wird bzw. wurde. Während der durchschnittliche prozentuelle Anteil im Jahr 2011 etwa 40\% betrug liegt er 2016 bei rund 45\%. Der Anteil der Gesamtarbeitszeit an der Universität, der dem CD-Labor gewidmet wird/wurde ist also etwas im Steigen begriffen.

Abbildung 65: Prozent der Gesamtarbeitszeit an der Universität, die dem CD-Labor gewidmet wird/wurde

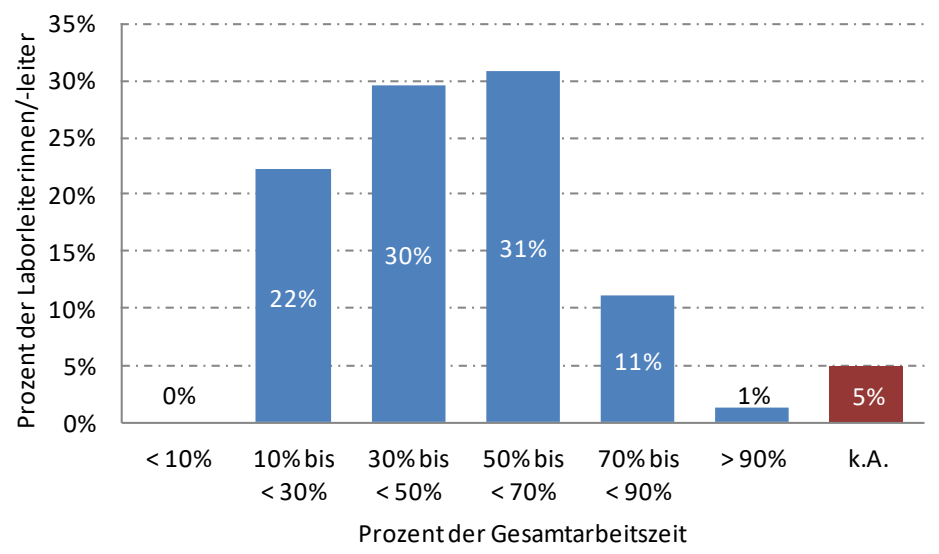

Quelle: Economica/IWI (2016); Online-Erhebung: n=81; Anmerkung: Zu berücksichtigen ist, dass das Vertragsverhältnis der CD-Laborleiter eine unterschiedliche Wochenstundenzahl beinhalten kann. 
Der 30\%ige wissenschaftliche Forschungsfreiraum im CD-Labor wird für unterschiedliche Zwecke genutzt. An erster Stelle steht, wie auch im Jahr 2011, die Publikationstätigkeit bzw. die Arbeit an Veröffentlichungen. Auch das Betreiben eigenständiger Forschung, aber auch die Teilnahme an Konferenzen und/oder die Arbeit an Master-, Diplomarbeiten, Dissertationen und Habilitationen wird häufig genannt. Eine geringe Bedeutung (wie auch schon 2011) weist hingegen die Lehre und auch die Weiterbildung auf. Letzterer Punkt hat gegenüber 2011 (und vor allem auch gegenüber 2005) nochmals an Bedeutung verloren.

Abbildung 66: Nutzung des 30\%igen wissenschaftlichen Forschungsfreiraums im Zeitverlauf (2005, 2011, 2016)

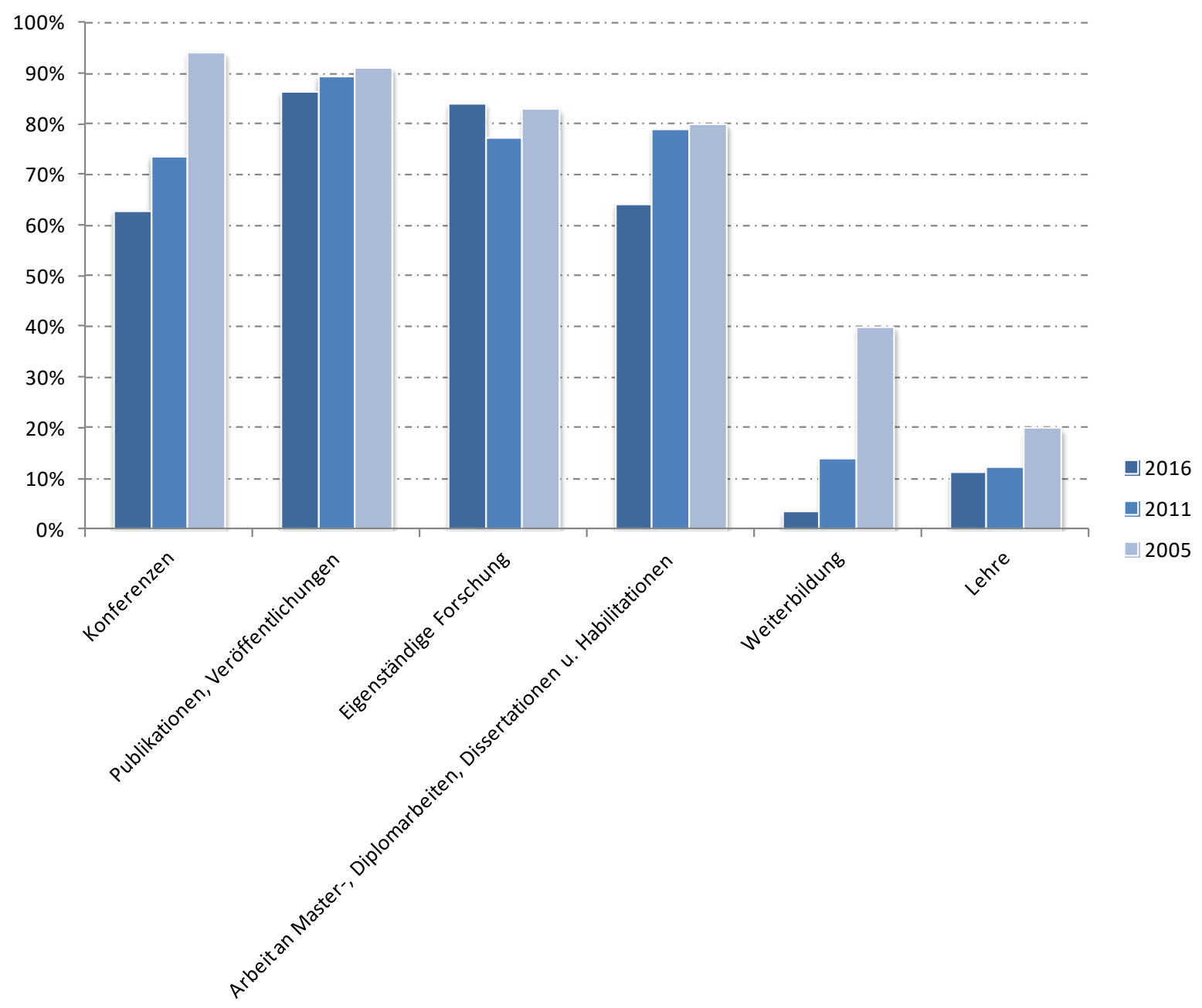

Quelle: Economica/IWI (2016, 2011), Joanneum Research/Technopolis/KMU Forschung Austria/Fraunhofer - ISI (2005) ; $n=81$ (2016), $n=57$ (2011), $n=35$ (2005) 
Hinsichtlich des Nutzes eines CD-Labors für das Universitäts- bzw. Forschungsinstitut wird der Prestigegewinn am bedeutsamsten erachtet. Dieser Punkt wurde auch in der Befragung 2011 als der Bedeutendste ausgewiesen. $\mathrm{Zu}$ beachten ist, dass sich dieser Nutzen meist schon mit Bewilligung/Gründung des CD-Labors einstellt. Andere Punkte, wie bspw. die Erhöhung des Praxisbezugs der Lehre, werden deutlich geringer vom Nutzen eingeschätzt.

Abbildung 67: Nutzen für das Universitäts-/Forschungsinstitut aus dem CD-Labor

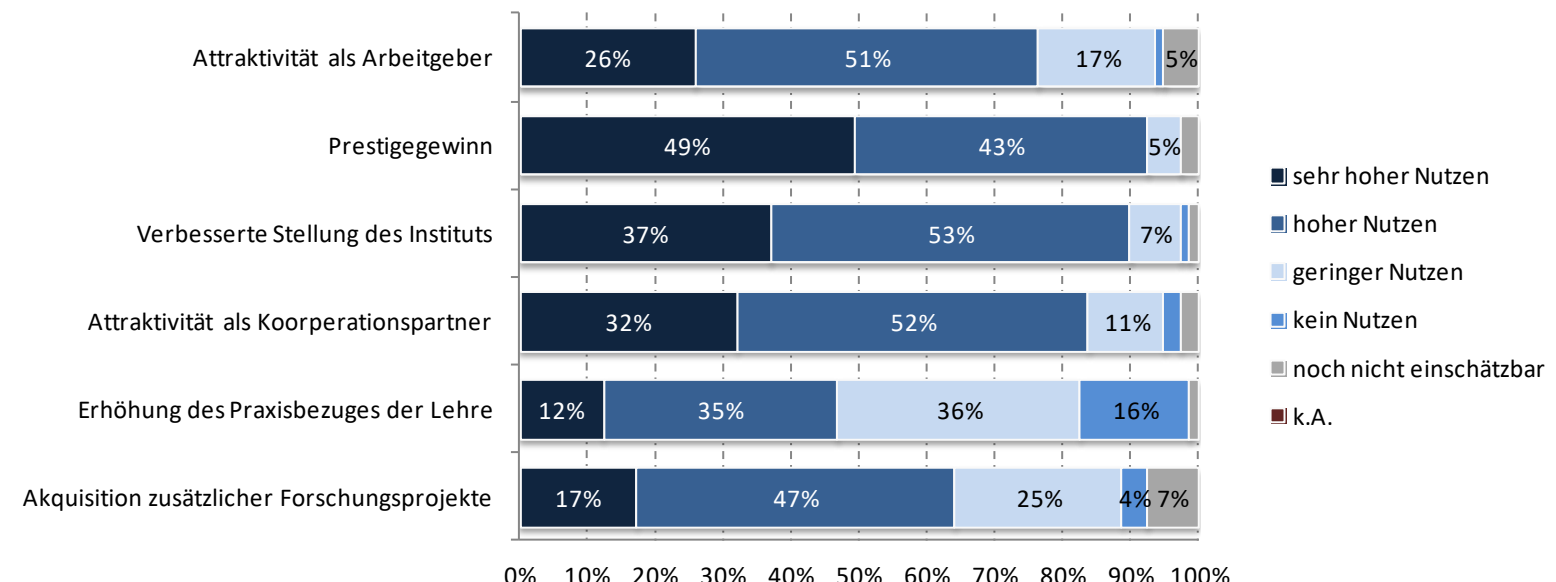

Quelle: Economica/IWI (2016); Online-Erhebung: $n=81$

31 der 81 vollständig ausgefüllten Fragebögen (38,3\%) stammen von bereits abgeschlossenen CDLabors. Für diese Subgruppe wurden im Rahmen der Online-Befragung zwei zusätzliche Fragebogenteile freigeschalten, die sich thematisch auf die Zeit nach dem CD-Labor beziehen.

Rund die Hälfte der Institute ist nunmehr wissenschaftlicher Partner bei einem COMET-Zentrum oder einem anderen Kompetenzzentrum bzw. einer Forschungseinrichtung. Bei etwas mehr als $16 \%$ haben die wissenschaftlichen Aktivitäten des CD-Labors anschließend zur Gründung eines COMET-Zentrums oder eines anderen Kompetenzzentrums beigetragen und bei knapp 13\% hat sich aus dem ehemaligen CD-Labor ein Spin-off entwickelt. 


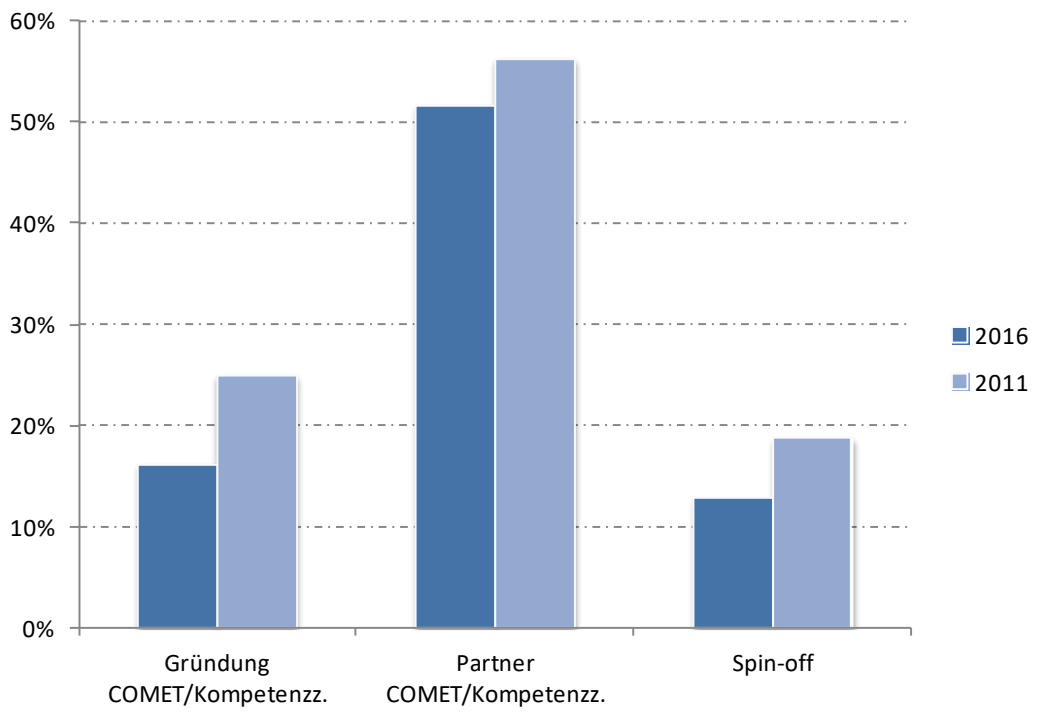

Quelle: Economica/IWI (2016, 2011); Online-Erhebung: $n=31$ (2016), n=16 (2011)

Diese Struktur entspricht in etwa jener, die auch 2011 beobachtet werden konnte, jedoch mit einem kleinen Rückgang in allen Bereichen. Ob es sich dabei um eine langfristige Entwicklung handelt, das Forschungsthema nach Beendigung des CD-Labors in einem anderen Zusammenhang weiter zu entwickeln, oder um ein Phänomen der Stichprobenauswahl der beiden Evaluierungen handelt, werden zukünftige Evaluierungen zeigen.

Im Folgenden wurden die Befragten Laborleiterinnen und Laborleiter von bereits abgeschlossenen CD-Labors gebeten eine Einschätzung zum Werdegang ihrer CD-Labormitarbeiterinnen und -mitarbeiter abzugeben. ${ }^{45}$ Für 29 der 31 abgeschlossenen CD-Labors konnten entsprechende Daten eingeholt werden. Durchschnittlich wechselten 56\% der ehemaligen Labormitarbeiterinnen und Mitarbeiter in die Industrie, ein kleinerer Teil davon (15\%) wechselte zu einem Unternehmenspartner aus dem entsprechenden CD-Labor, 41\% in ein anderes (Industrie-)Unternehmen. Durchschnittlich $30 \%$ verblieben an der Universität bzw. in der Forschungseinrichtung oder arbeiten nunmehr an einer anderen Universität/Forschungseinrichtung. Jeweils nur kleine Teile des Forschungsteams arbeiten nunmehr in einem anderen CD-Labor, einem COMET-Zentrum oder machten sich selbstständig.

\footnotetext{
${ }^{45}$ Die Befragten hatten die Möglichkeit eine prozentuelle Zuordnung zu sieben Kategorien vorzunehmen (siehe Fragebogen im Anhang)
} 


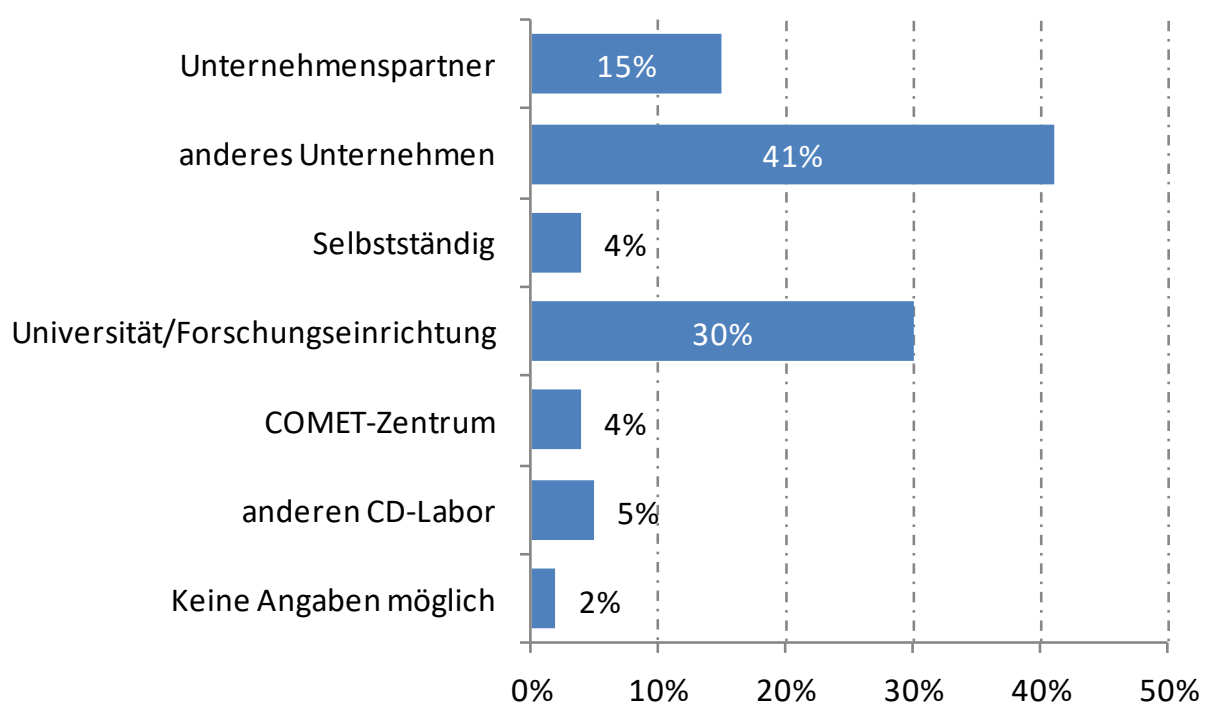

Quelle: Economica/IWI (2016); Online-Erhebung: $n=29$

Ein Vergleich mit den 2011er Daten zum Werdegang der ehemaligen Mitarbeiterinnen und Mitarbeiter zeigt eine ganz ähnliche Struktur. Bereits 2011 wechselte ein Großteil (knapp zwei Drittel) der ehemaligen Mitarbeiter zu einem (Industrie-)Unternehmen, ungefähr ein Drittel verblieb im Forschungsumfeld der Universität bzw. einer anderen Universität oder Forschungseinrichtung.

An die CD-Laborleiter von noch laufenden CD-Labors $(n=50)$ richtete sich die Frage, inwieweit das Labor nach dem Förderzeitraum als eigenständiges Unternehmen bzw. als eigenständige Einrichtung weiter geführt werden soll. Rund zwei Drittel der Befragten gab an, dies aus derzeitiger Sicht noch nicht abschätzen zu können. Von jenen, die hier schon eine Einschätzung vornehmen konnten, gaben rund $20 \%$ an, das Labor nach Auslaufen als eigenständige Einrichtung bzw. als Unternehmen weiterführen zu wollen.

Wieder an alle Befragten richtete sich die Frage inwieweit die Forschungsprojekte, die im Rahmen des CD-Labors durchgeführt werden bzw. wurden, auch ohne CD-Labor stattgefunden hätten und wenn ja, in welchem Ausmaß. Wie auch im Rahmen der Evaluierung im Jahr 2011 hätte keiner der Befragten das im Rahmen des CD-Labors durchgeführte Forschungsprojekt ohne CD-Labor im selben Umfang durchführen können. Interessant ist, dass eine deutlich größere Zahl der Befragten (rund 25\%) im Vergleich zur letztmaligen Erhebung angab, das Forschungsprojekt gar nicht hätten umsetzen zu können, auch nicht in geringerem Umfang. 
Abbildung 70: Durchführung der Forschungsprojekte, auch ohne CD-Labor

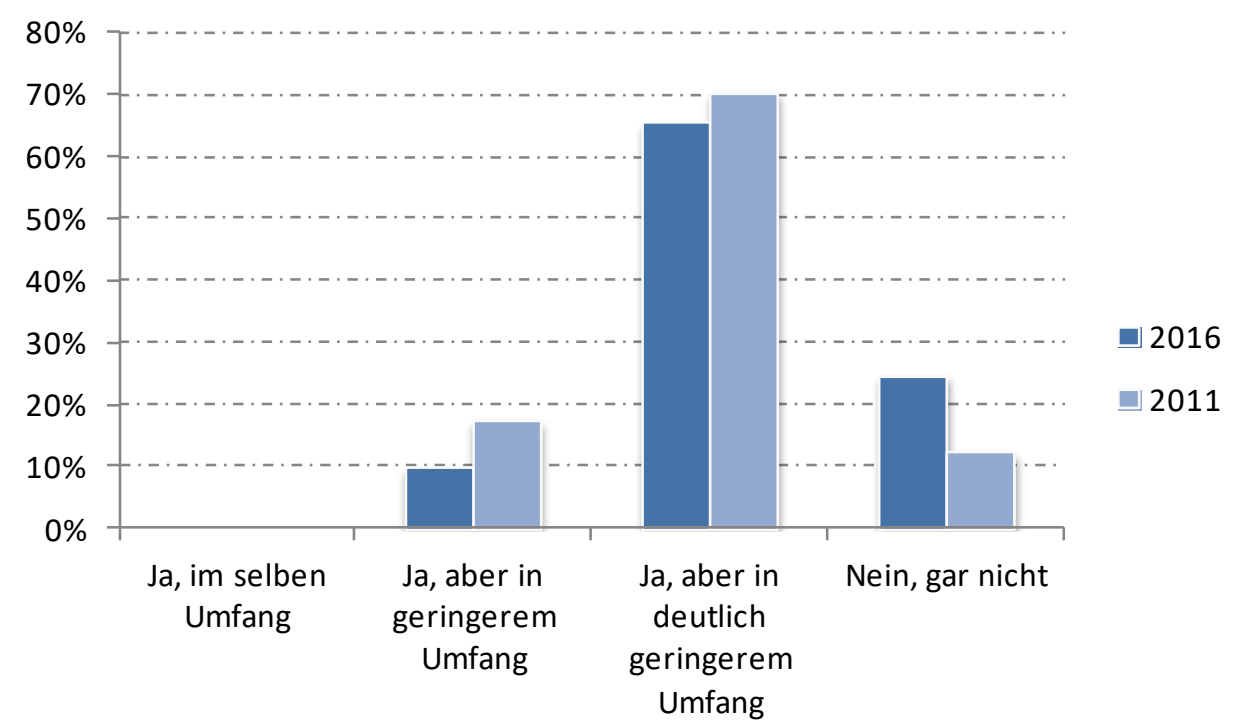

Quelle: Economica/IWI (2016, 2011); Online-Erhebung: $n=81$ (2016), n=51 (2011)

37 der 81 CD-Labors (45,7\%) haben/hatten einen internationalen Partner im Rahmen des CD-Labors, 10 von $81(12,4 \%)$ betreiben ein internationales Modul an einem ausländischen Standort. ${ }^{46}$

Zusammenfassend besteht bei $40 \mathrm{CD}$-Labors, also rund die Hälfte der Befragten, eine Form der internationalen Kooperation in Form eines internationalen Partners und/oder eines internationalen Moduls.

Gründe für einen internationalen Partner lagen vor allem im dadurch verfügbaren Know-how bzw. der Kompetenz und der führenden Stellung des internationalen Partners. Teils ist die benötigte Expertise auch in Österreich nicht verfügbar. Weiters wurden gemeinsame Themen in der Forschung bzw. eine bereits vorher bestehende Zusammenarbeit ins Treffen geführt.

\section{Gründe einer internationalen Kooperation - Auszug aus den Fragebögen (Zitate):}

„Expertise, Interesse“.

„Kompetenz".

„Weltweit führender Hersteller von Analyseinstrumenten“.

\footnotetext{
${ }^{46} 2011$ gaben 36,8\% der Befragten an, einen internationalen Partner im Rahmen des CD-Labors zu haben, 10,5\% gaben an ein internationales Modul zu betreiben.
} 


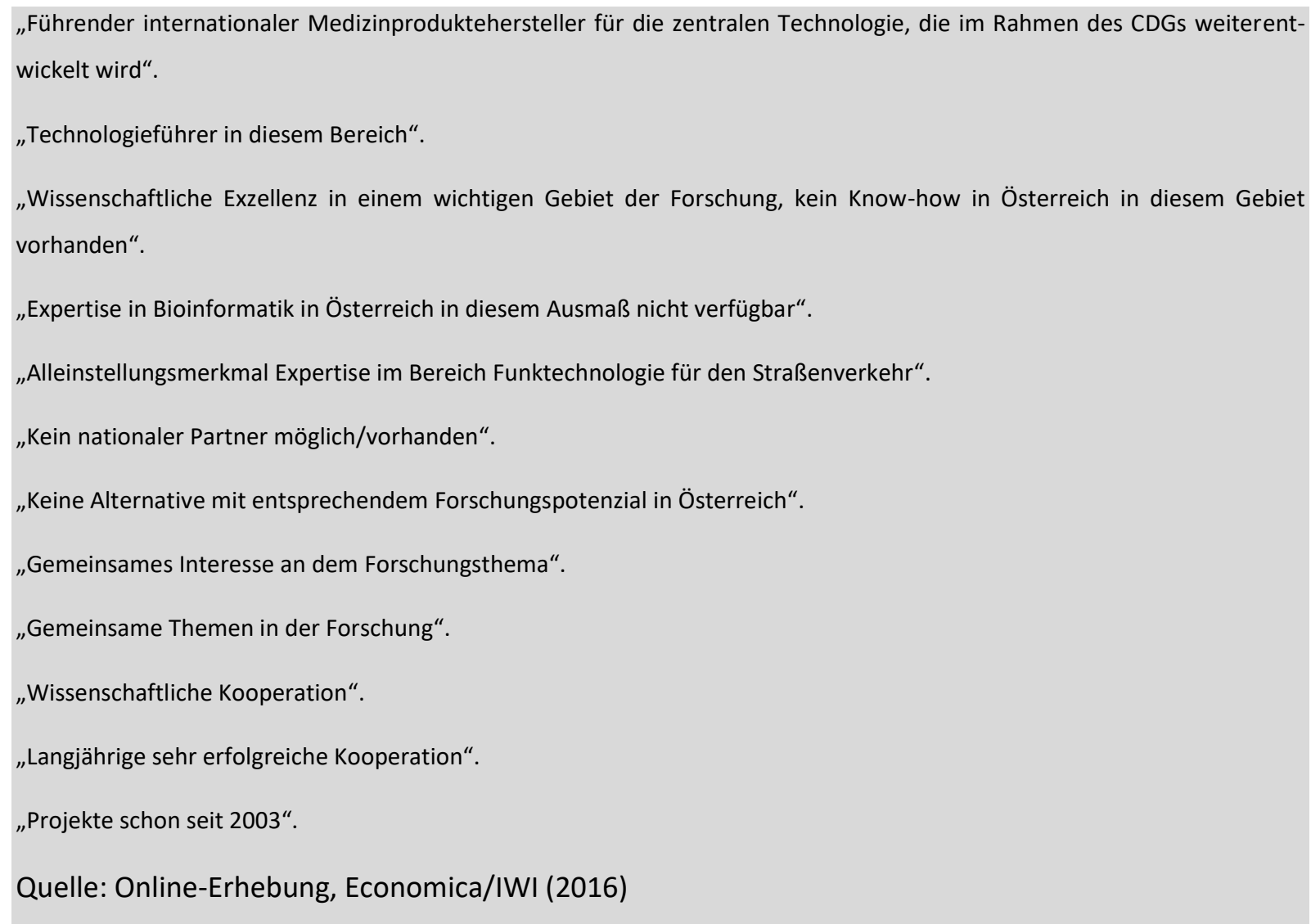

Als Gründe für ein internationales Modul wurde auch genannt, dass das Know-how in Österreich nicht verfügbar ist, oder zusätzliches Know-how beim internationalen Partner besteht. Auch Verfügbarkeit von Infrastruktur, der Wechsel von Mitarbeitern oder auch bestehende Partnerschaften waren Gründe.

\section{Gründe für ein internationales Modul - Auszug aus den Fragebögen (Zitate):}

„Kontakte waren bereits vorher vorhanden, kein Know-how in Österreich in diesem Gebiet vorhanden“.

„Know-how in Österreich nicht vorhanden“.

„Messtechnik in Österreich nicht vorhanden; Kontakte zum Forschungspartner waren bereits vorhanden“.

„Zusätzliches Know-how“.

„Verfügbarkeit von sehr teurer Infrastruktur (Messinstrumente) der neuesten Generation“.

„Wechsel eines Mitarbeiters an die Sheffield Hallam University“.

„Akademischer Partner".

„Forschungskooperation“.

Quelle: Online-Erhebung, Economica/IWI (2016) 
Damit setzt sich die bereits im Rahmen der Evaluierung 2011 sichtbare Tendenz einer zunehmenden Internationalisierung im Rahmen des CD-Programms weiter fort, wenn auch in moderater Weise.

Der Nutzen der internationalen Kooperation liegt (wie auch im Jahr 2011) nach Ansicht der Befragten vor allem im Erwerb von neuem Know-how durch den ausländischen Partner bzw. im Rahmen des ausländischen Moduls. Teilweise können so auch neue Unternehmenskontakte im Ausland erworben werden (rund die Hälfte der Befragten hat diesbezüglich profitiert). Ebenso analog zu 2011 wird die Forschungsleistung im Ausland nicht als kostengünstiger empfunden.

Abbildung 71: Wie haben Sie von der internationalen Kooperation profitiert bzw. profitieren Sie davon?

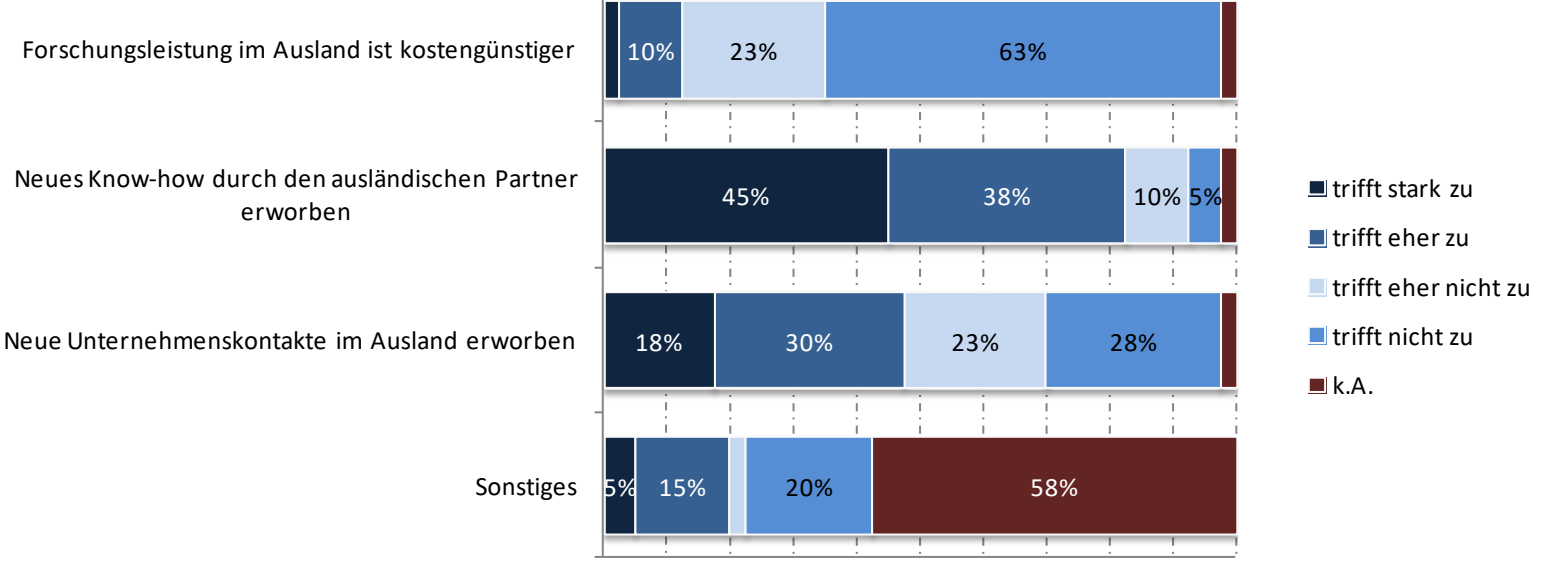

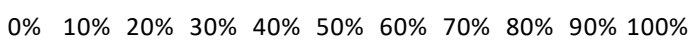

Quelle: Economica/IWI (2016); Online-Erhebung: $n=40$

Über die vorgegebenen Antwortkategorien hinausgehend nannten einige der Befragten weitere Punkte hinsichtlich eines Nutzens internationaler Kooperationen im Rahmen des CD-Labors.

\section{Sonstiger Nutzen einer internationalen Kooperation - Auszug aus den Fragebögen (Zitate):}

„Verfügbarkeit von Technologie, die für die Universität nicht leistbar ist, Kooperationspartner hat publizierbare Anwendungsbeispiele mit sehr starkem Praxisbezug“.

„Forschungskooperation; Wissenschaftlicher Ausbau des österreichischen Standortes".

„Komplementärer Input z.B. Patientenproben“.

„Mehr Publikationen waren dadurch möglich“.

„Gegenseitige Ergänzung des Know-hows führt zu besseren Projektergebnissen“. 


\begin{abstract}
„Partnerfirma hat Interesse an Expansion in Europa, insbesondere in Österreich. Büro wurde bereits angemietet (ausschlaggebend war insbesondere die Gründung des Labors); Großes Potenzial für zukünftige High-Tech-Arbeitsplätze in Wien; Chance für unsere Absolventen und für Schlüsselposition Wiens in unserem Forschungsbereichs".

„Verbesserte Sichtbarkeit im industriellen und wissenschaftlichen Umfeld“.

„Wir konnten mit dem ausländischen Partner ein schlagkräftiges Forschungskonzept (sowohl für Grundlagenforschung als auch für Industrieforschung) entwickeln, da er eine Kernkompetenz eingebracht hat, die in Österreich nicht vorhanden ist".

Quelle: Online-Erhebung, Economica/IWI (2016)
\end{abstract}

22 der 40 international agierenden Laborleitern und Laborleiterinnen (55\%) planen weitere Industry-

Science-Kooperationen im Ausland mit (ausländischen) Partnern, die sie im Rahmen des CD-Labors kennengelernt haben.

Abschließend wurde nochmals das Thema administrativer Aufwand adressiert und die Befragten gebeten den administrativen Aufwand eines CD-Labors im Vergleich zu anderen Förderprogrammen (FFG-Projekt, EU-Projekt, FWF-Projekt) einzuschätzen. Weiters wurden die CD-Laborleiter noch gebeten eine Schätzung des administrativen Aufwandes eines CD-Labors (Schätzung der Wochenstunden im Jahresdurchschnitt) abzugeben.

Bei einem Vergleich mit anderen Förderprogrammen lässt sich grob sagen: Der administrative Aufwand eines CD-Labors ist niedriger als der eines EU-Projekts, aber höher als der eines FWF-Projekts. Im Vergleich zu einem FFG-Projekt sind die Einschätzungen breit gestreut. 
Abbildung 72: Administrativer Aufwand eines CD-Labors im Vergleich zu anderen Förderprogrammen

...FFG-Projekt in Kooperation mit Unternehmen

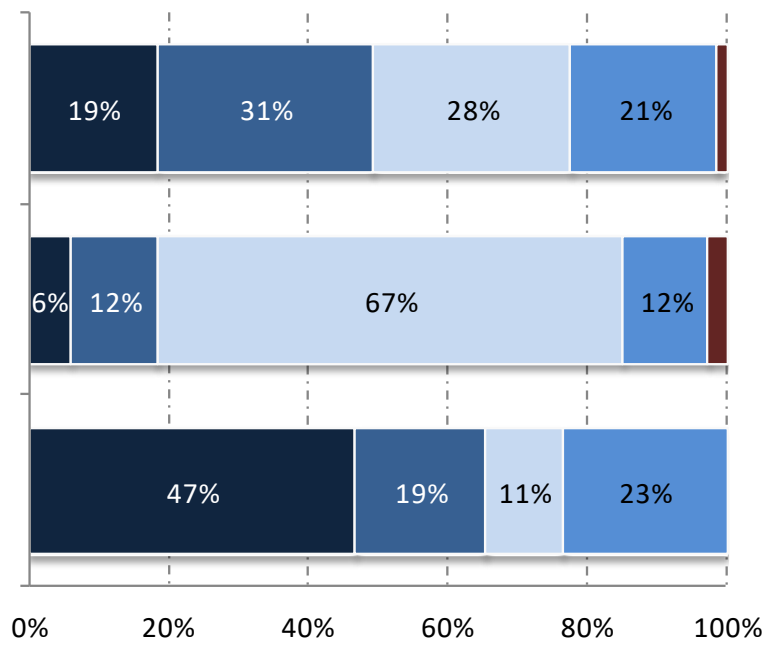

घhöher

...EU-Projekt in Kooperation mit Unternehmen

...FWF-Projekt

$\square$ gleich hoch

niedriger

$\square$ kein Vergleich möglich

$\square$ k.A.

Quelle: Economica/IWI (2016); Online-Erhebung: $n=81$

Was die Schätzung des administrativen Aufwandes eines CD-Labors hinsichtlich der Wochenstunden im Jahresschnitt betrifft variieren die Angaben der Befragten sehr stark. Von 72 Laborleiterinnen und Laborleitern konnten verwertbare Angaben eingeholt werden, welche zwischen den Polen „1 Wochenstunde“ und „25 Wochenstunden“ im Maximum streuen. Im Durchschnitt schätzen die Befragten den administrativen Aufwand (Wochenstunden im Jahresdurchschnitt) auf knapp 7 Wochenstunden ein.

Angemerkt werden soll, dass „administrativem Aufwand für das CD-Labor“ von den Respondenten im Rahmen dieser Frage natürlich subjektiv abgegrenzt wird und das die CDG zur Unterstützung von Laborleiterinnen und Laborleitern auch Assistenzen fördert. 


\subsubsection{Teilgruppenbetrachtung (Befragungsgruppe JR-Zentrumsleiter)}

Im Rahmen der Evaluierung konnten acht bestehende JR-Zentren berücksichtigt werden. Von diesen übermittelten im Rahmen der Online-Erhebung sieben Zentrumsleiterinnen und -leiter einen vollständig ausgefüllten Fragebogen. Zu Beginn wurden die Befragten gebeten, Ihre Zufriedenheit mit einzelnen Aspekten des Förderprogramms bzw. der Programmabwicklung zu bewerten.

Mit der Klarheit des Förderkonzepts und Förderziels ist der große Teil der Zentrumsleiterinnen und leiter (sehr) zufrieden. Auch wenn sich lediglich einer der Befragten unzufrieden zeigt, könnte dies ein Hinweis darauf sein, dass im Rahmen des Programmdesigns noch vereinzelt Nachjustierungen sinnvoll und notwendig wären. Dies ist in weiterer Folge mit zukünftigen Evaluierungen mit einer größeren Anzahl von JR-Zentrumsleitern nochmals zu verifizieren.

Durchgehend hohe Zufriedenheit herrscht bezüglich der Angemessenheit der Evaluierungskriterien, auch die Bearbeitungsdauer der eingereichten Anträge wird als (sehr) zufriedenstellend angesehen.

\section{Abbildung 73: Zufriedenheit mit Aspekten des Programms bzw. der Programmabwicklung}

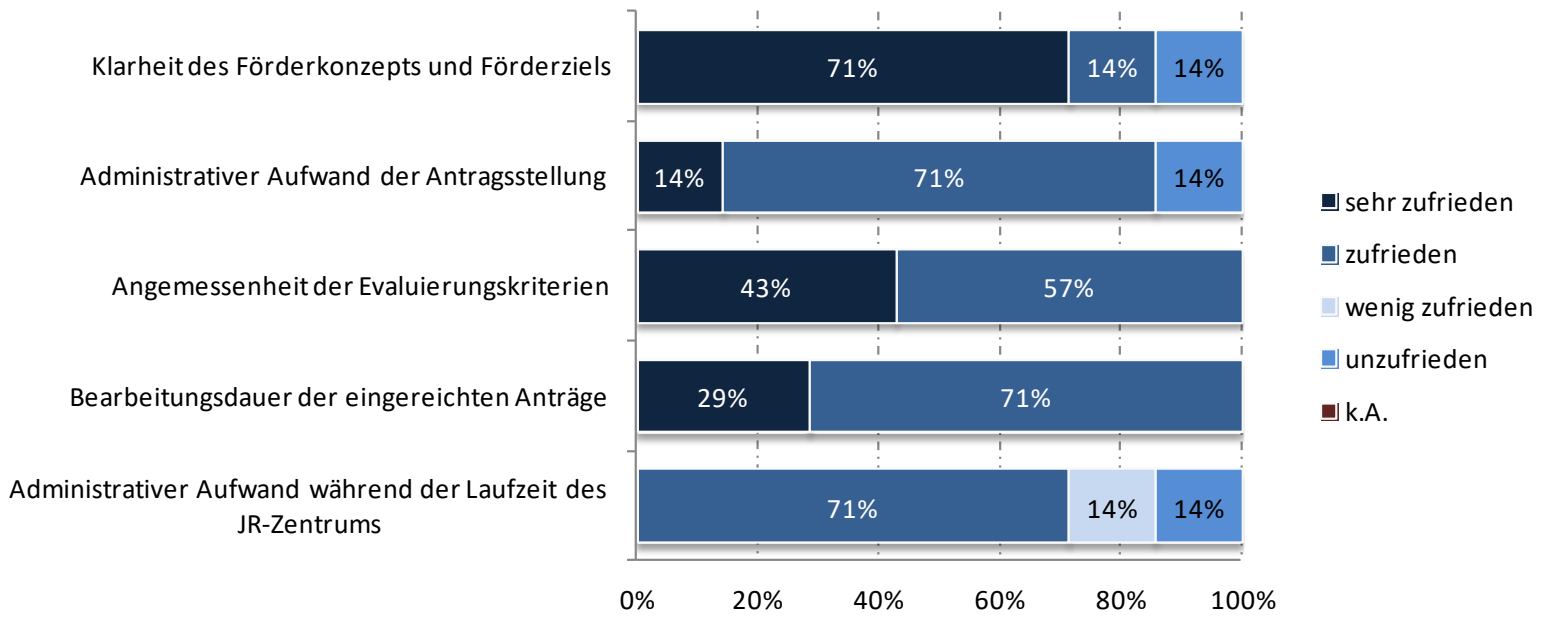

Quelle: Economica/IWI (2016); Online-Erhebung: $n=7$

Den administrativen Aufwand (Antragsstellung und Laufzeit) bewertet der Großteil generell zufriedenstellend, wobei der administrative Aufwand während der Laufzeit des JR-Zentrums vereinzelt etwas schlechter bewertet wird.

Nach Gründen befragt wird u.a. einerseits ein sehr starker Fokus auf Formalitäten von Seiten des Fördergebers genannt (auch moderate Änderungen im Projektverlauf müssen formal beantragt 
werden, Vorlaufzeit von Änderungsanträgen, für viele Änderungen sind Zustimmungen der Partnerfirmen einzuholen etc.), andererseits auch das CDG-Webtool (zu langsam, zu instabil und zu unflexibel). Als Verbesserungsvorschläge wurden u.a. folgende Punkte genannt:

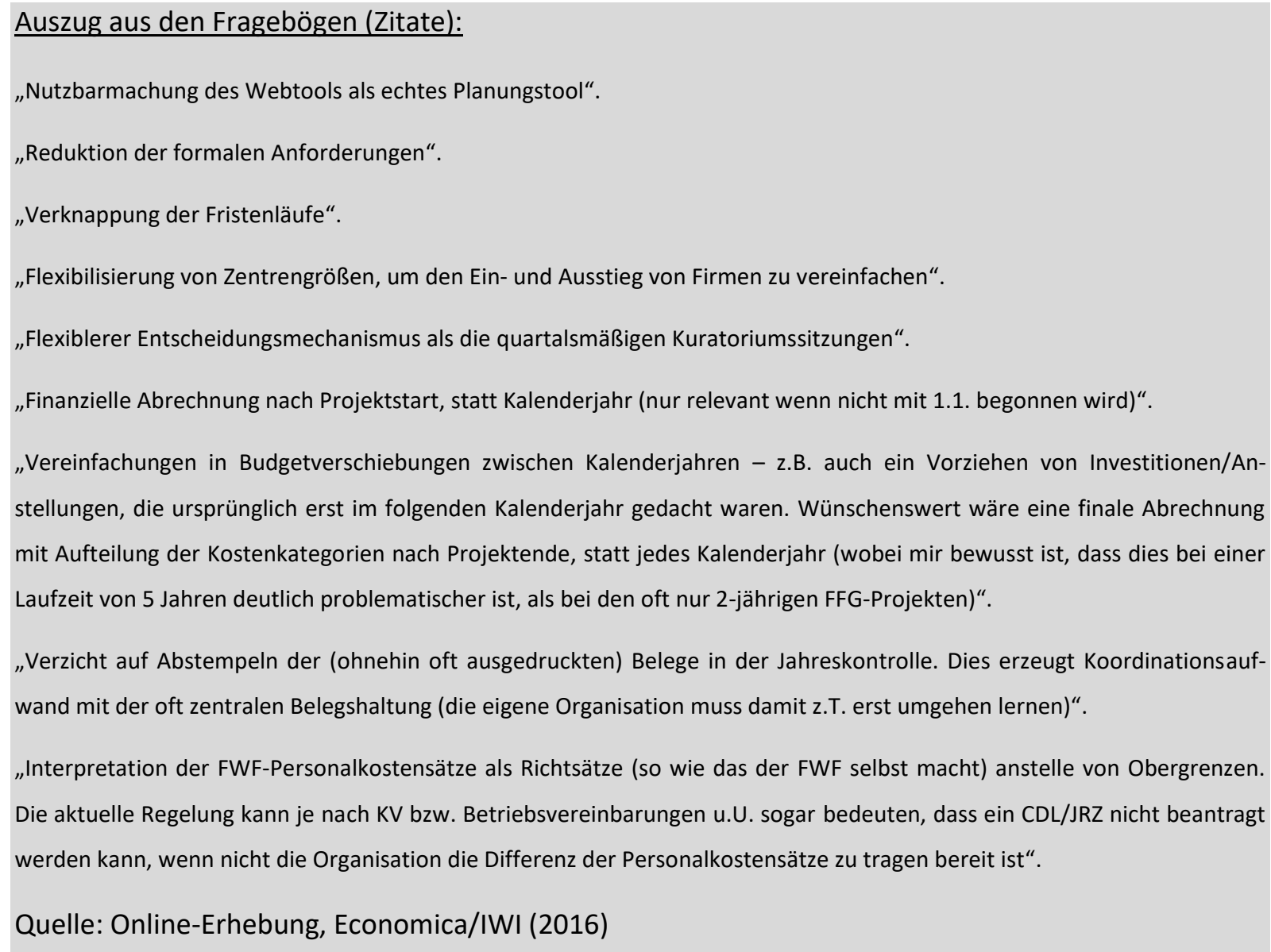

Die Laufzeit eines JR-Zentrums von 5 Jahren wird von einer knappen Mehrheit der Befragten (57\%) als gerade richtig gesehen, vielen (43\%) ist sie jedoch auch zu kurz. Als zu Lang empfindet (analog zu den CD-Laborleitern und Laborleiterinnen) keiner der Zentrumsleiter bzw. Zentrumsleiterinnen die Laufzeit des Programms.

Die begleitende wissenschaftliche Kontrolle wird von allen Zentrumsleiterinnen und -leitern, bei denen sie bereits erfolgte, als sehr hilfreich bzw. hilfreich angesehen. 
Abbildung 74: Einschätzung der begleitenden wissenschaftlichen Kontrolle

bei der 2-Jahresevaluierung

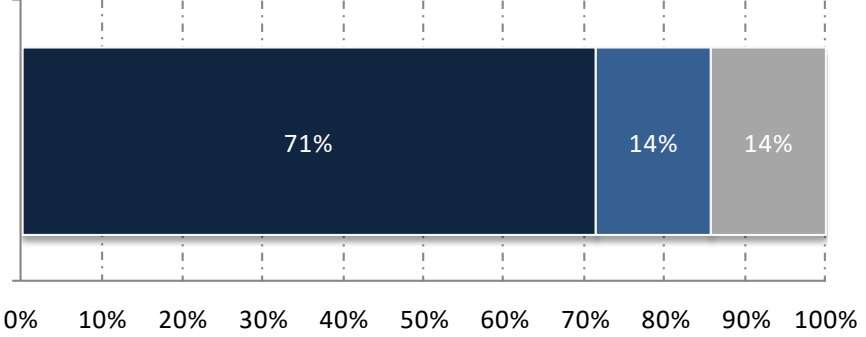

- sehr hilfreich

- hilfreich

wenig hilfreich

$\square$ nicht hilfreich

$\square$ bisher noch nicht erfolgt

- k.A.

Quelle: Economica/IWI (2016); Online-Erhebung: $n=7$

Auch der Zeitpunkt der Zwischenevaluierung wird mehrheitlich als passend empfunden.

Abbildung 75: Bewertung des Zeitpunktes der Zwischenevaluierung

Zeitpunkt der Zwischenevaluierung

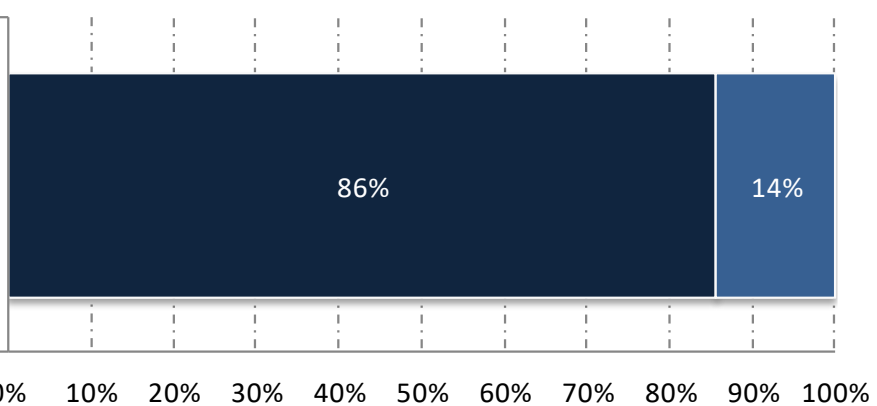

ㅁ passend

$\square$ unpassend

k.A.

Quelle: Economica/IWI (2016); Online-Erhebung: $n=7$

Lediglich ein Befragter empfindet den Zeitpunkt nach 2 Jahren als unpassend bzw. zu früh, und dieser hatte auch die Möglichkeit eine Anmerkung im Rahmen der Online-Befragung zu machen.

\section{Auszug aus den Fragebögen (Zitate):}

„Eine Evaluierung am Ende des zweiten Jahres ist eher früh - das wäre am Ende des dritten Jahres passend (evtl. kombiniert mit einer Verlängerung der Laufzeit auf 6 Jahre)“.

Quelle: Online-Erhebung, Economica/IWI (2016)

Mit der Betreuung der CDG während der Antragstellung sowie während der Laufzeit des JR-Zentrums sind mit einer Ausnahme alle Befragten sehr zufrieden. Auch die Öffentlichkeitsarbeit erzielt hohe Zufriedenheitswerte. 
Abbildung 76: Zufriedenheit mit den Leistungen der CDG

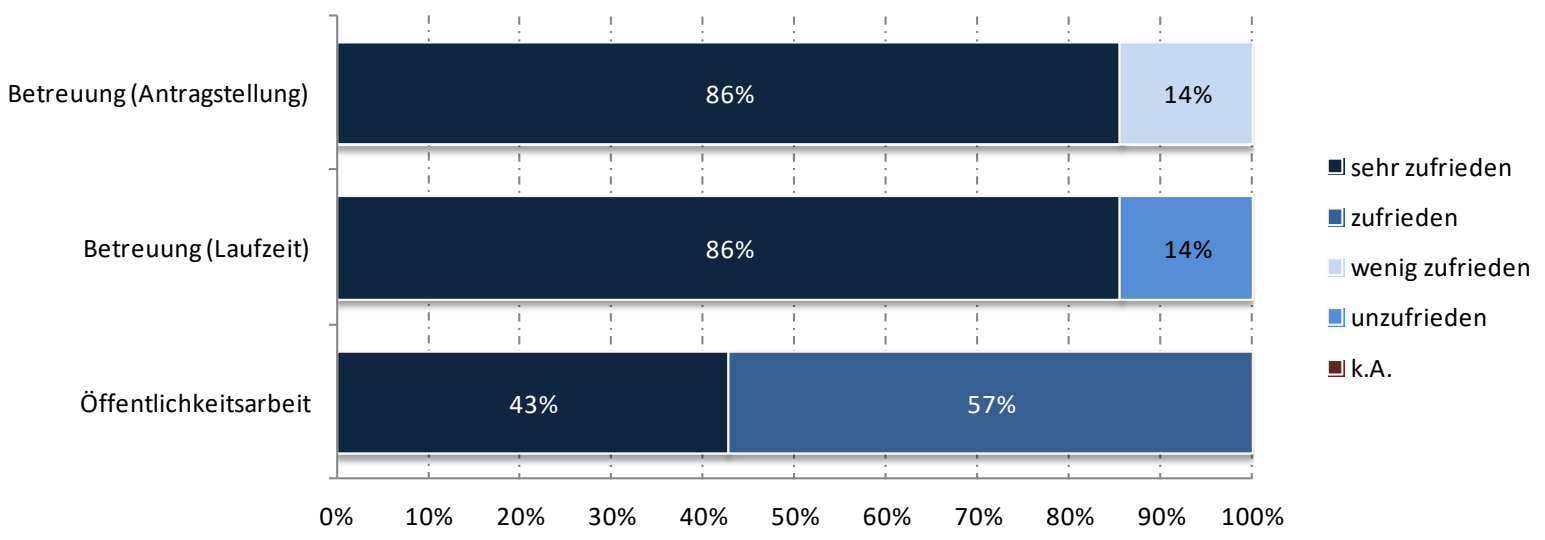

Quelle: Economica/IWI (2016); Online-Erhebung: $n=7$

Die Motivation zur Gründung eines JR-Zentrums ging mehrheitlich vom wissenschaftlichen Partner aus, sehr stark aber auch von beiden Partnern.

Abbildung 77: Motivation zur JR-Zentrumsgründung

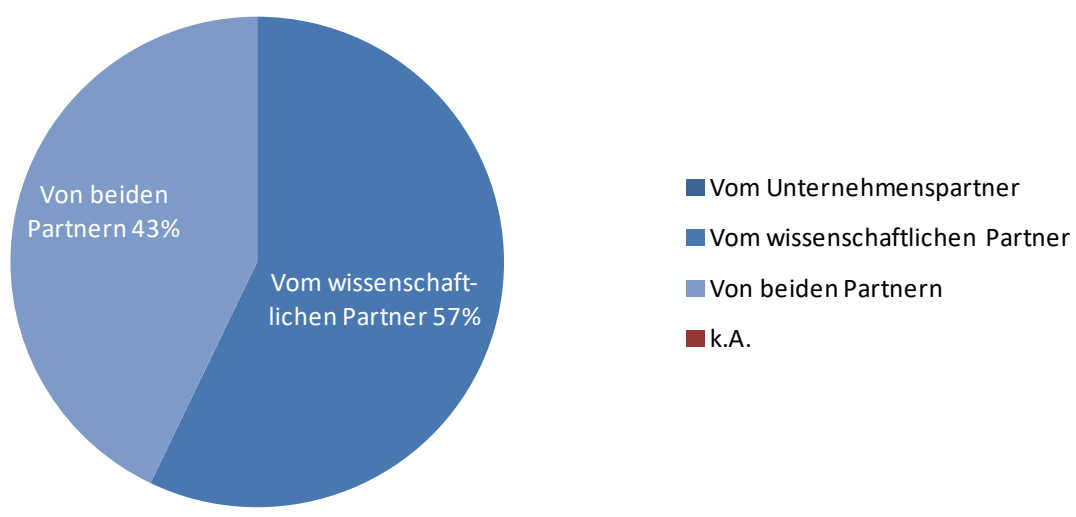

Quelle: Economica/IWI (2016); Online-Erhebung: $n=7$

Kontakt zum Unternehmenspartner vor der JR-Zentrumsgründung bestand bei allen Befragten, großteils über gemeinsame Forschungsprojekte, teils auch über Kontakte zu Absolventen und Absolventinnen bzw. lose Kontakte. 
Abbildung 78: Kontakt zum Unternehmenspartner vor der JR-Zentrumsgründung

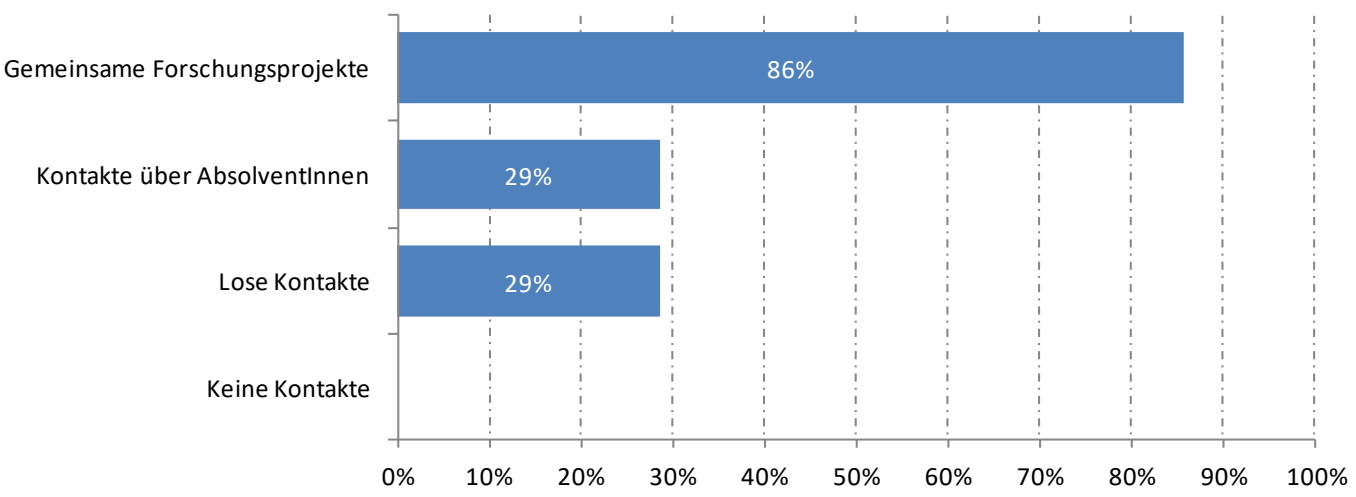

Anm.: Mehrfachantworten möglich

Quelle: Economica/IWI (2016); Online-Erhebung: $n=7$

Wie auch bei den CD-Laborleiterinnen und -leitern ist insbesondere das langfristig finanziell abgesicherte Arbeiten an einem Themenkreis und der Aufbau eines eigenen Forschungsteams von besonderer Wichtigkeit hinsichtlich Erwartungen und Ziele bei der Gründung eines JR-Zentrums. Diese Punkte wurden auch in den begleitenden Gesprächen immer wieder betont und hervorgehoben.

Abbildung 79: Erwartungen und Ziele bei der JR-Zentrumsgründung und deren Bedeutung

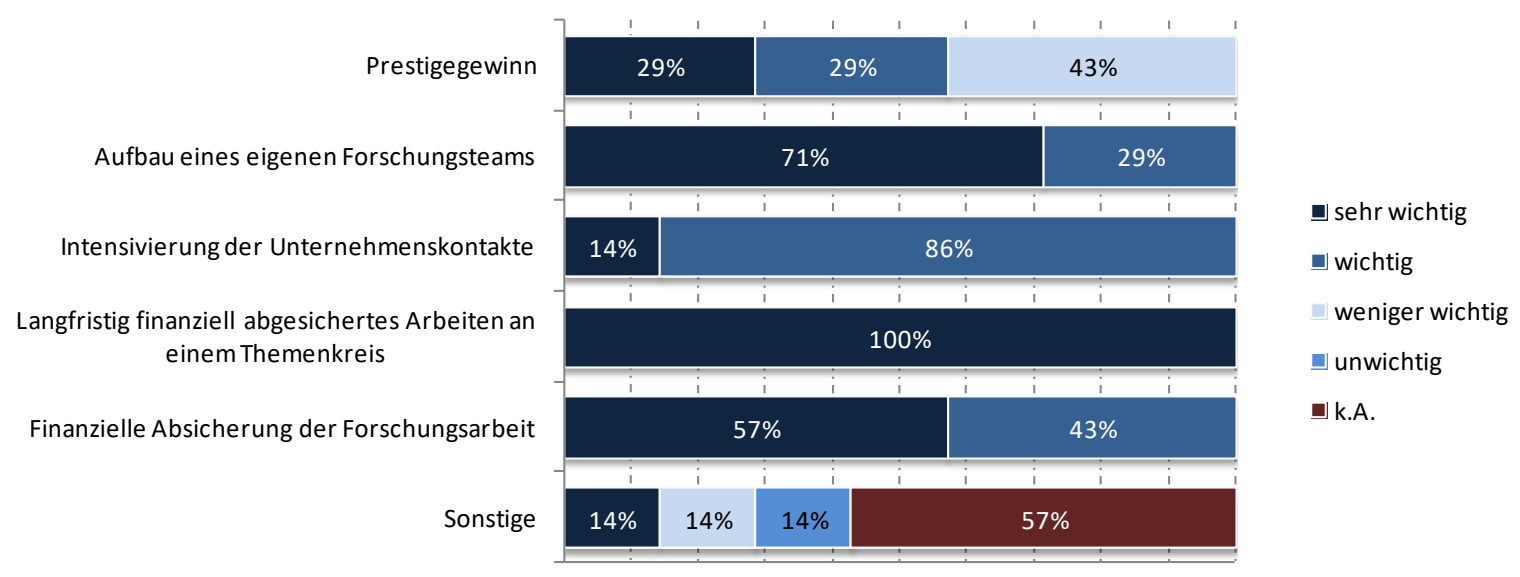

$\begin{array}{lllllllllllll}0 & 0 & 10 \% & 20 \% & 30 \% & 40 \% & 50 \% & 60 \% & 70 \% & 80 \% & 90 \% & 100 \%\end{array}$

Anm.: Sonstige Erwartungen/Ziele wurden zwar im Rahmen der Frage angegeben, jedoch im offenen Antwortbereich nicht näher ausgeführt.

Quelle: Economica/IWI (2016); Online-Erhebung: $n=7$ 
Auf die Forschungsprogrammformulierung hatten alle Zentrumsleiter/-innen sehr hohen Einfluss und auch der Einfluss auf die Moduldefinition wird als (sehr) hoch angesehen.

Abbildung 80: Einfluss des Zentrumsleiters/der Zentrumsleiterin auf die Forschungsprogrammformulierung und auf die Moduldefinition

Forschungsprogrammformulierung

Moduldefinition

$$
\text { (1) }
$$

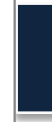

$\begin{array}{llllllllllllll}0 \% & 10 \% & 20 \% & 30 \% & 40 \% & 50 \% & 60 \% & 70 \% & 80 \% & 90 \% & 100 \%\end{array}$
- sehr hohen Einfluss

$\square$ hohen Einfluss

wenig Einfluss

$\square$ k.A.

Quelle: Economica/IWI (2016); Online-Erhebung: $n=7$

Die Inputs der Unternehmenspartner werden von den Zentrumsleitern bzw. Zentrumsleiterinnen sehr unterschiedlich bewertet. In (sehr) hohem Ausmaß lieferten die Unternehmenspartner vor allem Inputs hinsichtlich Bereitstellung zusätzlicher Infrastruktur und mehrheitlich auch zu den Forschungsaktivitäten. Gering war der Input der Unternehmenspartner nach Ansicht der Zentrumsleiterinnen und -leiter vor allem in Hinblick auf die Moduldefinition und die Vermittlung von Kontakten.

\section{Abbildung 81: Inputs des Unternehmenspartners}

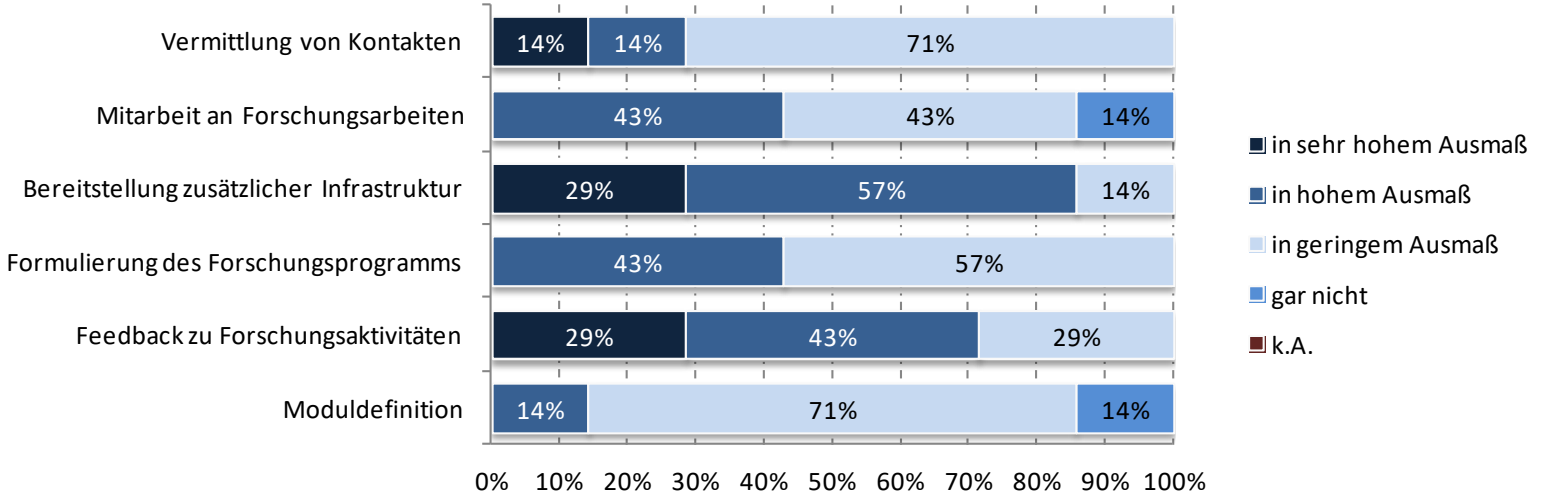

Quelle: Economica/IWI (2016); Online-Erhebung: $n=7$ 
Auch die Zentrumsleiterinnen und -leiter wurden gebeten abzuschätzen, wie viel Prozent der Gesamtarbeitszeit an der Fachhochschule, dem JR-Zentrum gewidmet wird. Der durchschnittliche prozentuelle Anteil der Gesamtarbeitszeit, der dem JR-Zentrum gewidmet wird liegt bei rund 53\% und damit leicht höher als bei den CD-Laborleitern.

Abbildung 82: Prozent der Gesamtarbeitszeit an der Fachhochschule, die dem JR-Zentrum gewidmet wird

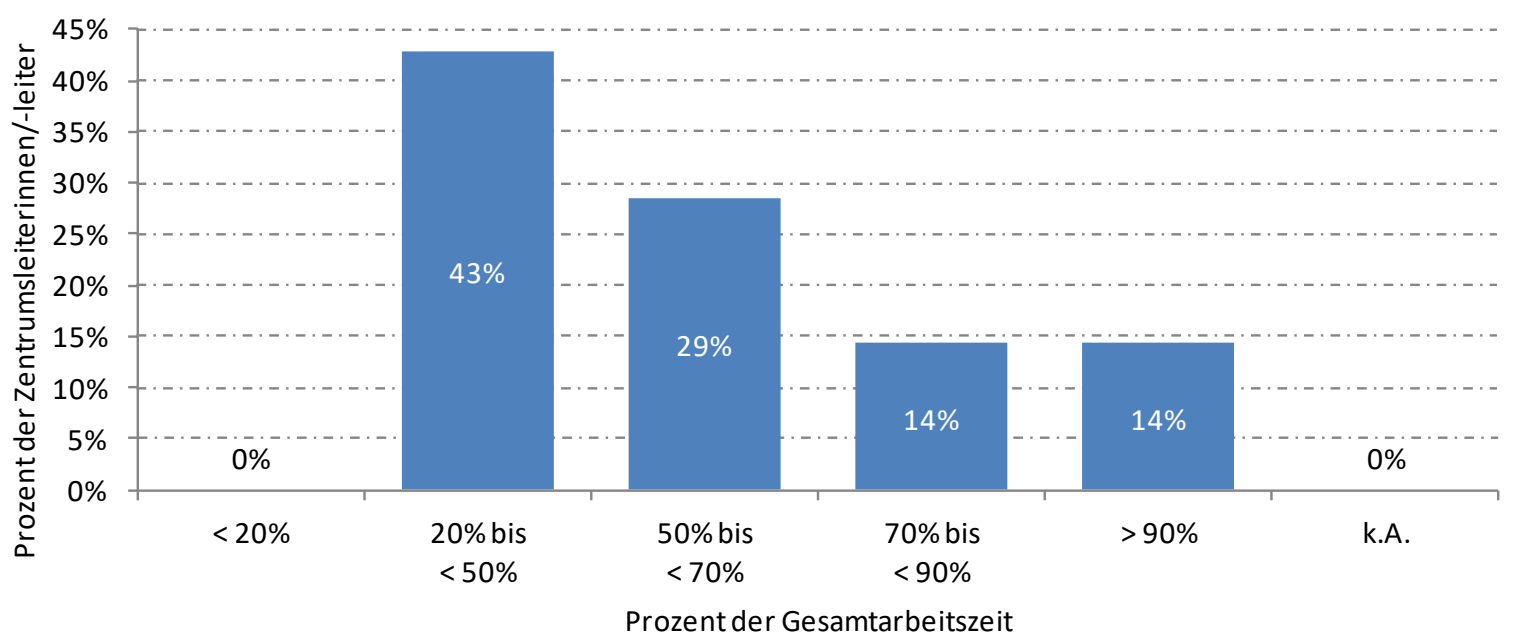

Quelle: Economica/IWI (2016); Online-Erhebung: n=7; Anmerkung: Zu berücksichtigen ist, dass das Vertragsverhältnis der JR-Zentrumsleiter eine unterschiedliche Wochenstundenzahl beinhalten kann.

Hinsichtlich des Nutzens für das FH-Institut ist das Antwortverhalten der Befragten teils sehr unterschiedlich. (Sehr) hohen Nutzen sieht die Mehrheit der Befragten vor allem im Prestigegewinn und der verbesserten Stellung des Instituts. Auch die höhere Attraktivität als Kooperationspartner wird mehrheitlich positiv als Nutzen hervorgehoben. 
Abbildung 83: Nutzen für das FH-Institut aus dem JR-Zentrum

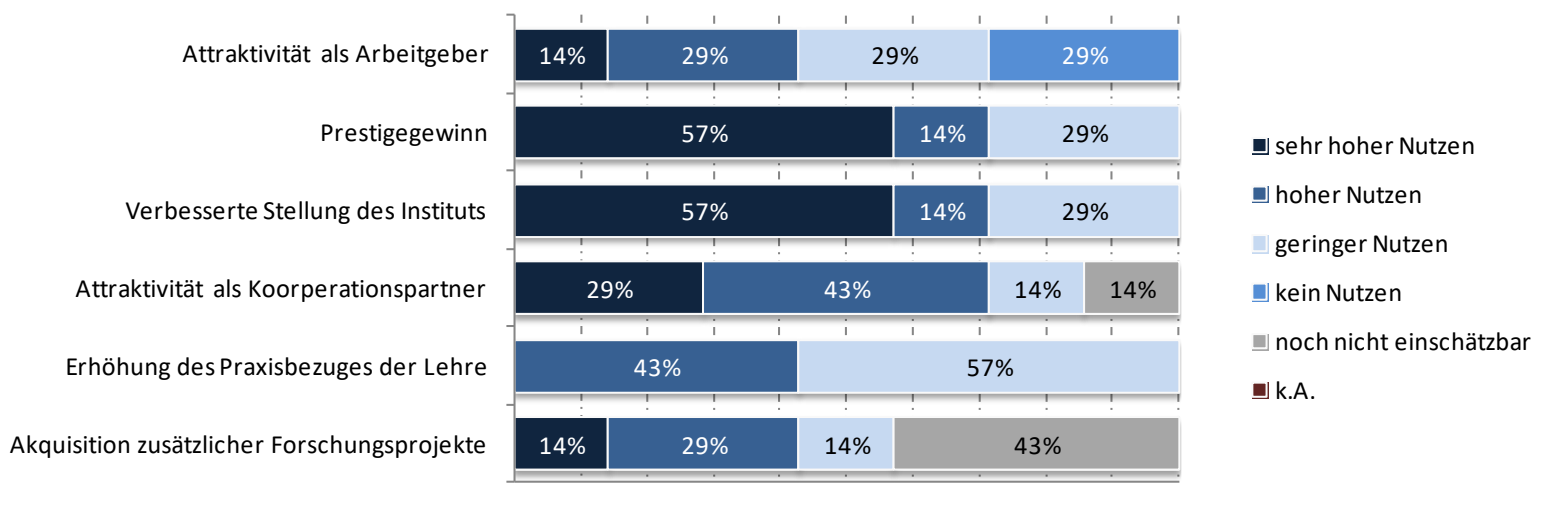

$\begin{array}{lllllllllll}0 \% & 10 \% & 20 \% & 30 \% & 40 \% & 50 \% & 60 \% & 70 \% & 80 \% & 90 \% & 100 \%\end{array}$

Quelle: Economica/IWI (2016); Online-Erhebung: $n=7$

Was die Themenbereiche „Attraktivität als Arbeitgeber" und „Erhöhung des Praxisbezugs der Lehre“ betrifft, gehen die Meinungen wiederum sehr stark auseinander.

Was die Durchführung des Forschungsprojekts, auch ohne JR-Zentrum betrifft, so hätten alle Befragten dieses nur in geringerem Umfang - mehrheitlich sogar nur in deutlich geringerem Umfang durchführen können.

\section{Abbildung 84: Durchführung der Forschungsprojekte, auch ohne JR-Zentrum}

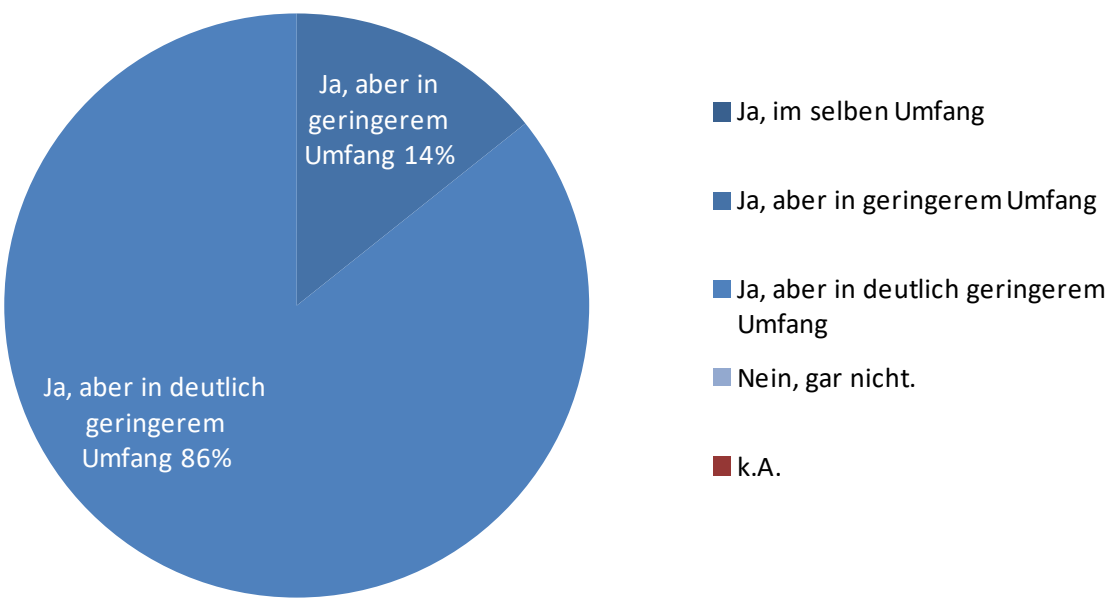

Quelle: Economica/IWI (2016); Online-Erhebung: $n=7$ 
Die „Internationalisierung" ist bei den JR-Zentren noch deutlich geringer ausgeprägt als bei den CDLabors. Lediglich eines der sieben JR-Zentren hat einen internationalen Partner (Unternehmenspartner aus Deutschland) im Rahmen des JR-Zentrums. Ein internationales Modul an einem ausländischen Standort wird derzeit von keinem Zentrum betrieben.

Im Folgenden wurden die JR-Zentrumsleiter bzw. -leiterinnen gebeten, einige Fragen zu beantworten, die sich inhaltlich an jenen orientieren, welche die CD-Laborleiterinnen und -leiter im Rahmen der von der CDG vorgelegten Abschlussbefragung nach Beendigung eines Labors vorgelegt bekommen. Da noch keines der JR-Zentren abgeschlossen ist, bestand die Überlegung darin, schon einige dieser Fragen "vorzuziehen", natürlich nur jene, wo davon ausgegangen werden konnte, dass diese auch schon während des noch laufenden Betriebs schon zu beantworten bzw. zumindest abzuschätzen sind.

Sowohl in die Lehre als auch in die Forschung sind Erfahrungen aus den industrierelevanten Projekten des JR-Zentrums bereits eingeflossen. Vor allem in die Forschung konnten Erfahrungen in sehr großem (43\%) und mittlerem Ausmaß (57\%) mitgenommen werden.

Abbildung 85: Sind Erfahrungen aus industrierelevanten Projekten bereits in den akademischen Betrieb des Heimat gebenden Instituts eingeflossen?

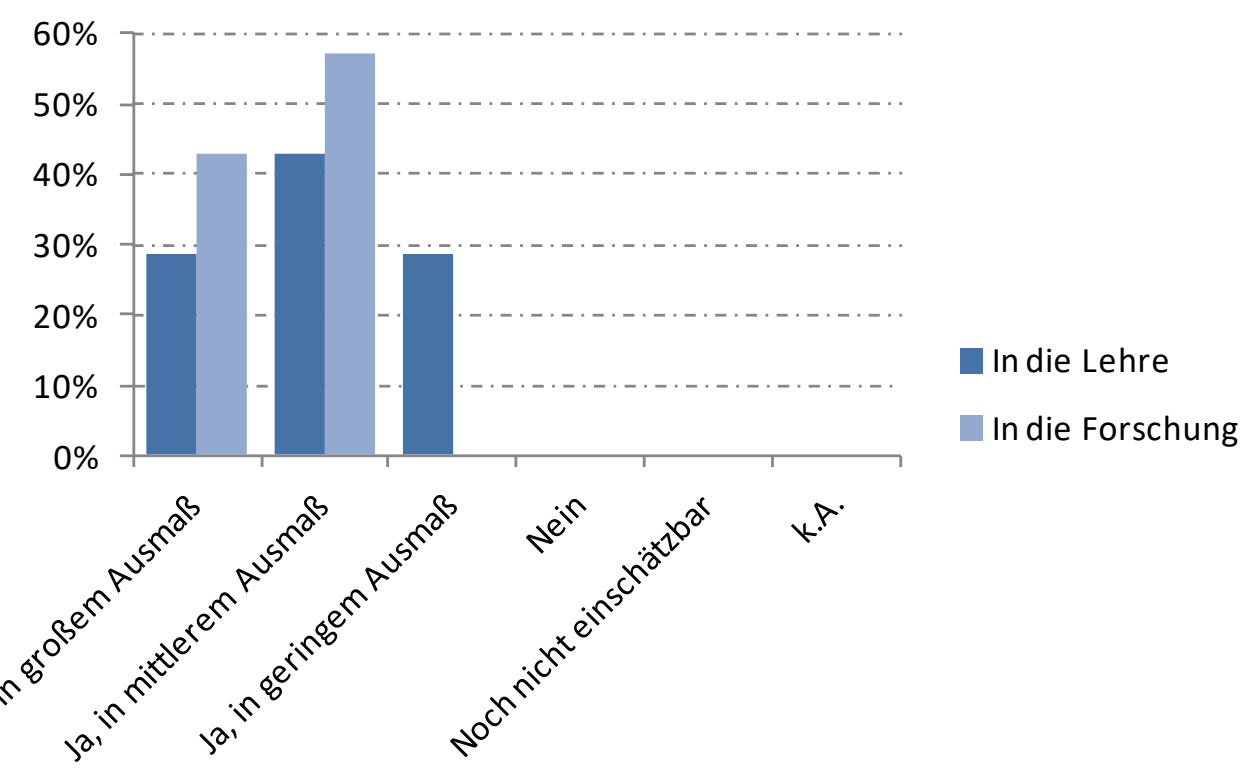

Quelle: Economica/IWI (2016); Online-Erhebung: $n=7$ 
Was die die bisherige Qualität der Zusammenarbeit mit der Industrie in den JR-Zentren betrifft, zeigt sich im Rahmen der Befragung ein weitgehend positives aber dennoch in Einzelfällen etwas abweichendes Bild. Die Relevanz der industriellen Fragestellung für die Forschungsarbeit des JRZentrums wird durchwegs und mit einer Ausnahme (sehr) gut bewertet. In der Zusammenarbeit mit den Kontaktpersonen der Industrie gibt es unterschiedliche Erfahrungen. Ein Großteil der Zentrumsleiterinnen und -leiter hat hier sehr gute Erfahrungen gemacht, in Einzelfällen werden hier aber auch negative Erfahrungen dargestellt.

Auch das Ausmaß des wissenschaftlichen Freiraums wird von den Befragten unterschiedlich wahrgenommen und streut zwischen sehr gut und genügend. Anzumerken ist hier, dass bei den JRZentren, anders als bei den CD-Labors, kein wissenschaftlicher Freiraum von 30\% in den Eckdaten des Programms festgelegt ist. Hier ist zu überlegen, ob es nicht sinnvoll wäre auch im JR-Programm einen gewissen Prozentsatz für kontinuierlichen Wissenszuwachs zu verankern.

\section{Abbildung 86: Bisheriger Gesamteindruck von der Qualität der Zusammenarbeit mit der Industrie}

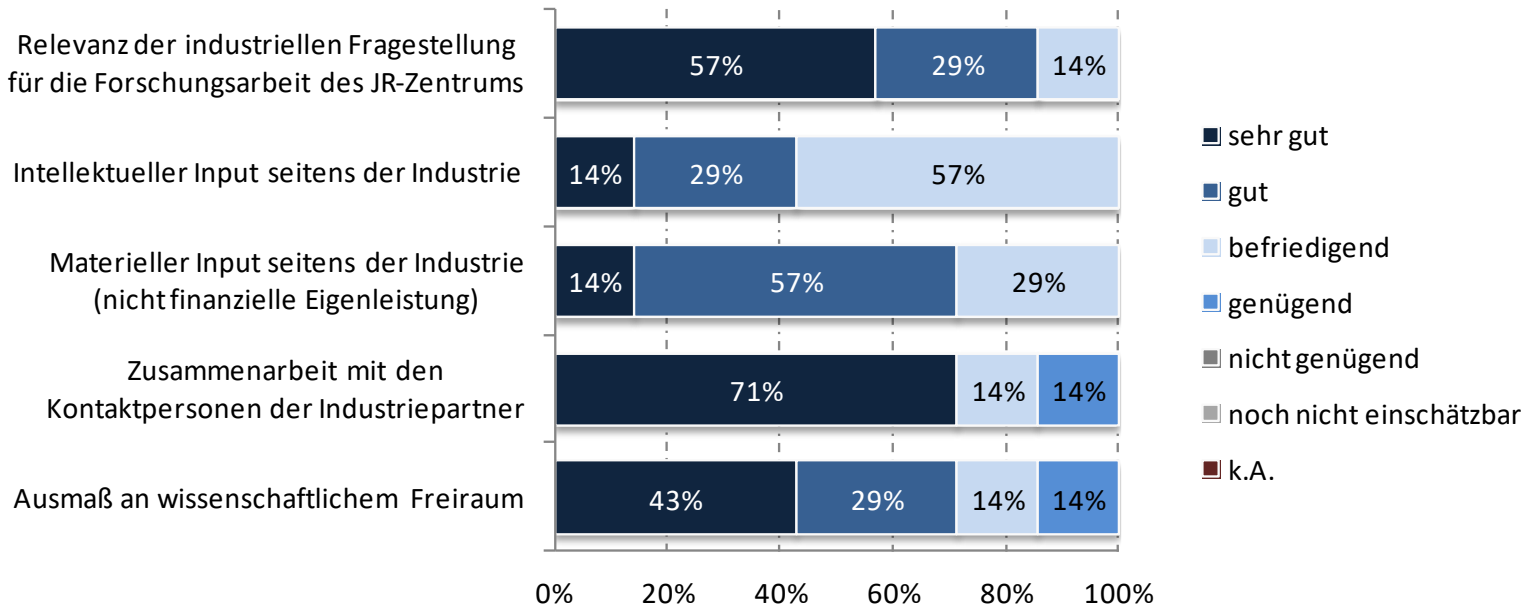

Quelle: Economica/IWI (2016); Online-Erhebung: $n=7$

Auch wenn die Auswirkungen des JR-Zentrums auf das Drittmittelvolumen des heimatgebenden Instituts noch nicht für alle Befragten einschätzbar sind, zeigt sich dennoch eine positive Tendenz. 
Abbildung 87: Auswirkung des JR-Zentrums auf das Drittmittelvolumen des Heimat gebenden Instituts/Departments

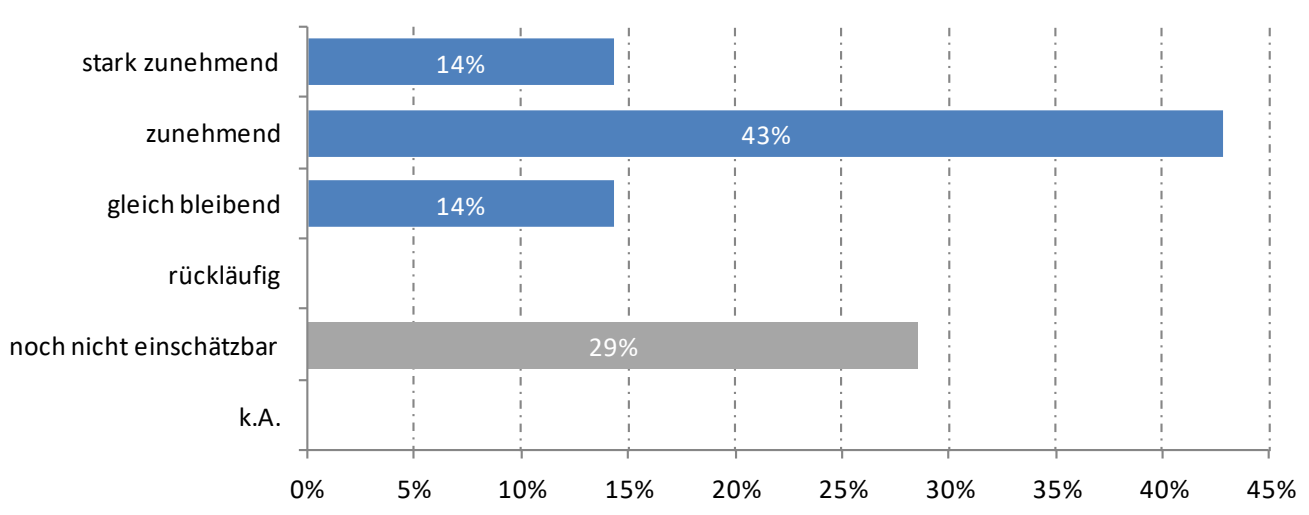

Quelle: Economica/IWI (2016); Online-Erhebung: $n=7$

Ähnliches gilt für die Auswirkungen des JR-Zentrums auf die Unternehmenskooperationen des Heimat gebenden Institutes.

Abbildung 88: Auswirkung des JR-Zentrums generell auf die Unternehmenskooperationen des Heimat gebenden Instituts/Departments

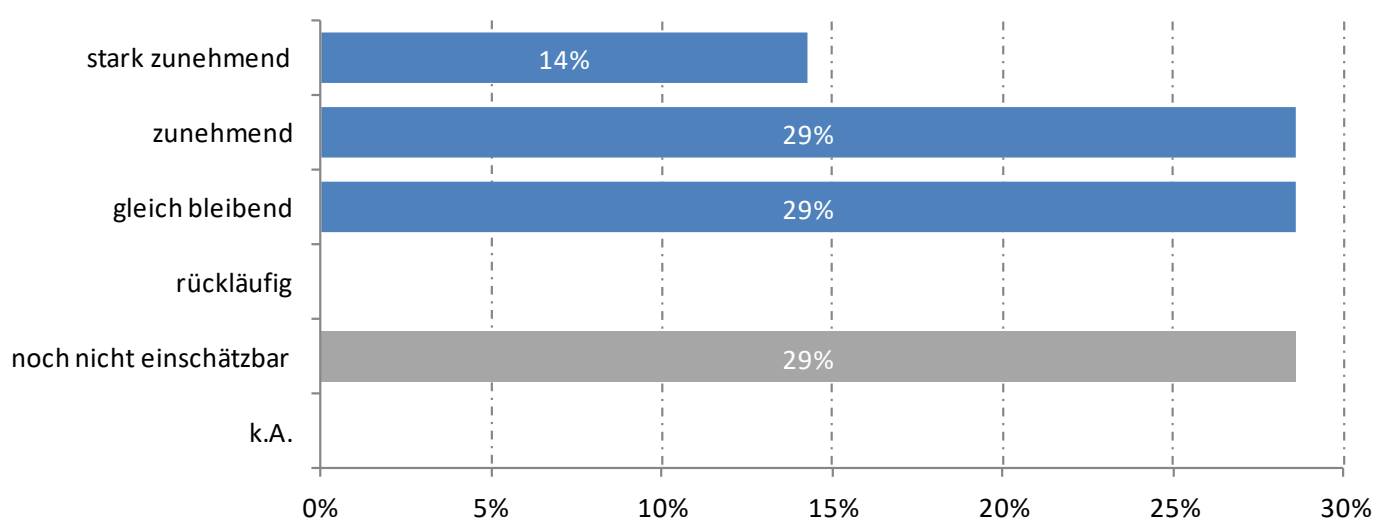

Quelle: Economica/IWI (2016); Online-Erhebung: $n=7$

Inwieweit das JR-Zentrum nach dem Förderzeitraum als eigenständiges Unternehmen bzw. eigene Einrichtung weitergeführt wird, ist für die Mehrheit der Befragten derzeit noch nicht einschätzbar. Zumindest bei einem JR-Zentrum gibt es jedoch schon jetzt die Absicht dies zu tun. 
Abbildung 89: Gibt es Initiativen das JR-Zentrum nach dem Förderzeitraum als eigenständiges Unternehmen/als eigene Einrichtung weiterzuführen?

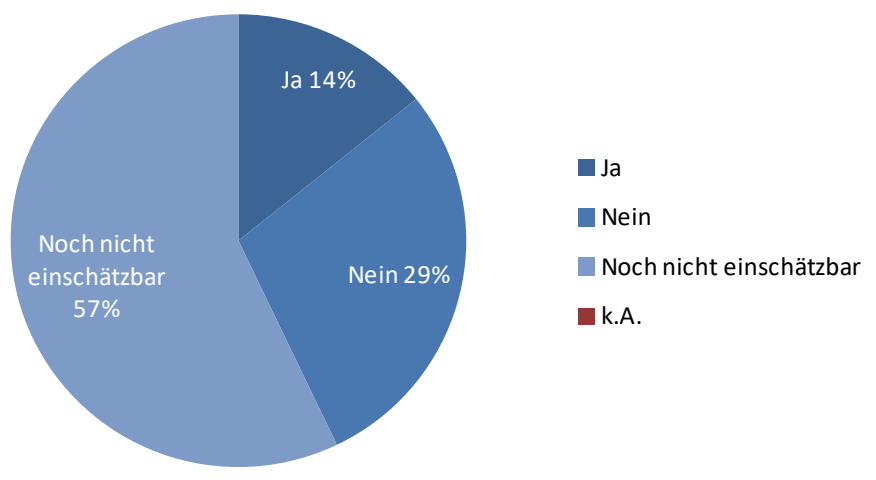

Quelle: Economica/IWI (2016); Online-Erhebung: $n=7$

Abschließend wurden auch die Befragten JR-Zentrumsleiter gebeten den administrativen Aufwand eines JR-Zentrums im Vergleich zu anderen Förderprogrammen (FFG-Projekt, EU-Projekt, FWFProjekt) einzuschätzen. Der Vergleich zu einem FWF-Projekt ist für die Mehrheit der Befragten nicht möglich. Jene die eine Beurteilung abgaben sehen den administrativen Aufwand eines JR-Zentrums als höher an. Unterschiedlich ist die Wahrnehmung im Vergleich zu einem FFG-Projekt, wo alle Befragten eine Einschätzung abgaben. Hier wird der Aufwand zwar tendenziell auch höher eingeschätzt, einige sehen ihn jedoch auch gleich hoch oder sogar niedriger. Im Vergleich zu einem EU-Projekt wird der administrative Aufwand eines JR-Zentrums mehrheitlich niedriger eingeschätzt, aber auch hier gehen die Meinungen auseinander. 
Abbildung 90: Administrativer Aufwand eines JR-Zentrums im Vergleich zu andern Förderprogrammen

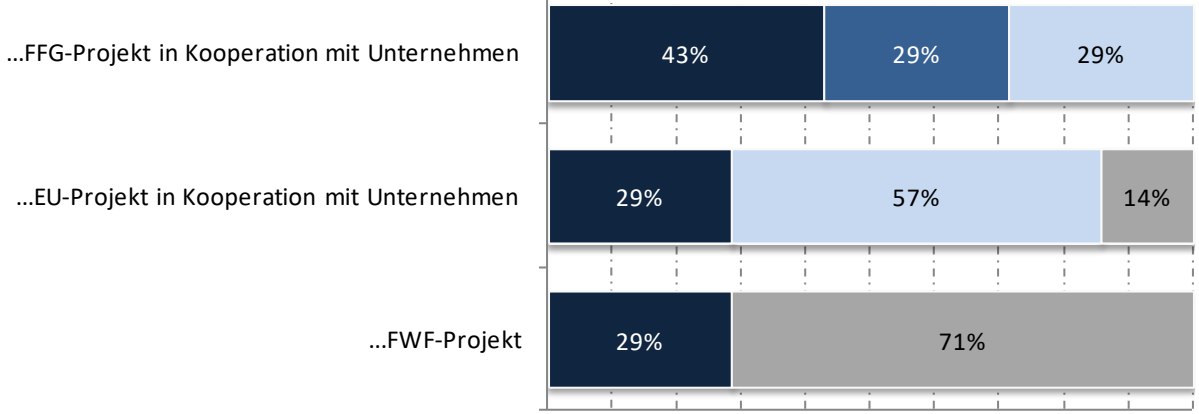

$\square$ höher

$\square$ gleich hoch

niedriger

$\square$ kein Vergleich möglich

$\square$ k.A.

$\begin{array}{llllllllllll}0 \% & 10 \% & 20 \% & 30 \% & 40 \% & 50 \% & 60 \% & 70 \% & 80 \% & 90 \% & 100 \%\end{array}$

Quelle: Economica/IWI (2016); Online-Erhebung: $n=7$

Zuletzt wurden die Befragten noch gebeten eine Schätzung des administrativen Aufwandes eines

JR-Zentrums (Schätzung der Wochenstunden im Jahresdurchschnitt) vorzunehmen. Diese fiel sehr unterschiedlich aus und zeigte eine starke Bandbreite, wie auch bereits bei den CD-Laborleiterinnen und -leitern. So wurden u.a. Schätzungen von 1 Stunde bis zu 20 Stunden pro Woche angegeben. 


\subsubsection{Teilgruppenbetrachtung (Befragungsgruppe CD-Labor Unternehmenspartner)}

Der Einstieg des Fragebogens betraf die Frage, in wie vielen CD-Labors die Unternehmen in Summe aktiv sind und/oder waren. Von den 90 Unternehmen, die rückgemeldet haben, sind bzw. waren 60 in einem CD-Labor, 30 bereits in mehreren CD-Labors aktiv.

71\% der Befragten beurteilten die Laufzeit des CD-Labors als adäquat. 22\% der befragten CD-LaborUnternehmenspartner bewerteten diese als zu lange und lediglich 4\% als zu kurz.

Abbildung 91: Beurteilung der Laufzeit des CD-Labors (von 7 Jahren) im Zeitverlauf (2005, 2011, 2016)

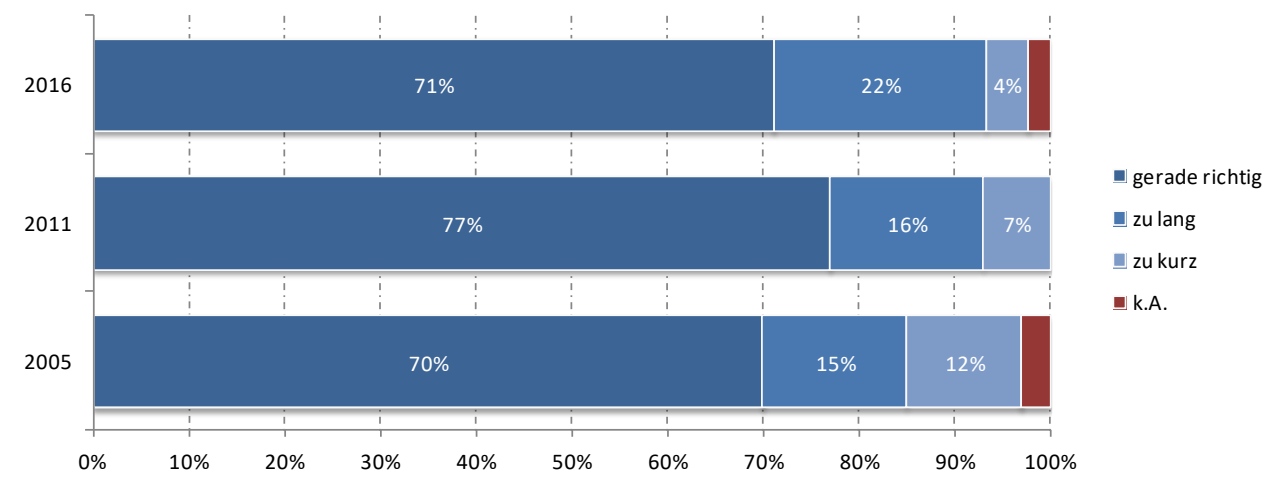

Quelle: Economica/IWI (2016, 2011), Joanneum Research/Technopolis/KMU Forschung Austria/Fraunhofer - ISI (2005) ; $n=90$ (2016), $n=61$ (2011), $n=74$ (2005)

Damit ist jener Anteil an Unternehmen, welche die Laufzeit als zu lange empfinden, im Vergleich zur Evaluierung 2011 (16\%) und 2005 (15\%) etwas angestiegen, und jener Anteil an Befragten, welche die Laufzeit als zu kurz empfinden, etwas gesunken (2011: 7\%, 2005: 12\%).

Trotz dieser leichten Verschiebungen kann mit einem Anteil von immer noch über 70\% darauf rückgeschlossen werden, dass eine 7-jährige Laufzeit auch für die Unternehmenspartner weiterhin mehrheitlich adäquat ist.

Auch die Unternehmenspartner wurden hinsichtlich ihrer Zufriedenheit mit einzelnen Aspekten des Programms bzw. der Programmabwicklung befragt. Es zeigt sich eine in allen Aspekten hohe Zufriedenheit. Keiner der Befragten zeigte sich unzufrieden, die wenig Zufriedenen bewegen sich bei einem Anteil von rund $10 \%$. Auch auf Seiten der Unternehmenspartner besteht insbesondere eine hohe Zufriedenheit mit der Klarheit des Förderkonzepts sowie des Förderziels. 92\% der Befragten zeigen sich bezüglich dieses Aspekts (sehr) zufrieden. 
Abbildung 92: Zufriedenheit mit Aspekten des Programms bzw. der Programmabwicklung

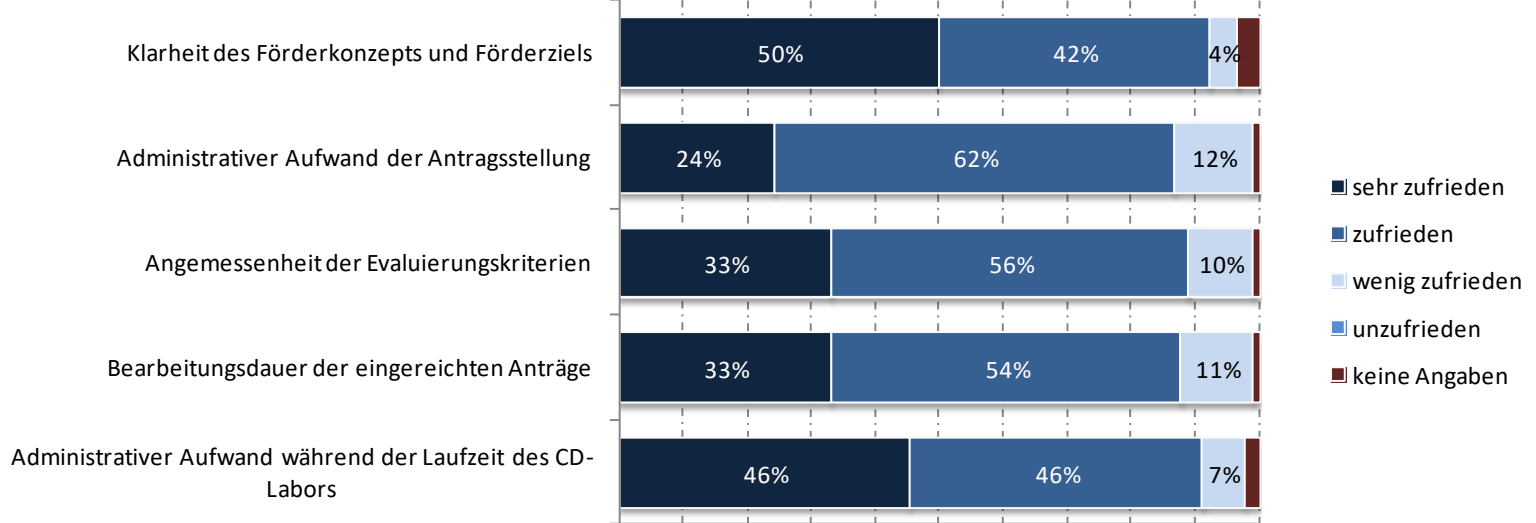

$\begin{array}{lllllllllll}0 \% & 10 \% & 20 \% & 30 \% & 40 \% & 50 \% & 60 \% & 70 \% & 80 \% & 90 \% & 100 \%\end{array}$

Quelle: Economica/IWI (2016); Online-Erhebung: $n=90$

Auch der administrative Aufwand wird von den Unternehmenspartnern tendenziell sehr zufriedenstellend eingestuft. Hervorzuheben ist, dass insbesondere der Aufwand während der Laufzeit eines CD-Labors von fast der Hälfte der Unternehmen als sehr zufriedenstellend bewertet wurde und die Bewertung etwas besser ausfällt, als auf Seiten der befragten CD-Laborleiterinnen und -Laborleiter. Dies stärkt die Annahme, dass der administrative Aufwand - vor allem während der Laufzeit - stark bei den Laborleiterinnen und Leitern liegt.

\section{Exkursbox: Potential für administrative Vereinfachungen}

Jene Unternehmenspartner, die mit dem administrativen Aufwand nicht zufrieden waren, hatten wie auch die Laborleiterinnen und Laborleiter - die Möglichkeit im Rahmen des Fragebogens Beispiele zu nennen bzw. anzugeben, wo sie Potential für administrative Vereinfachungen sehen. Aufgrund der Tatsache, dass die Unternehmenspartner hinsichtlich des administrativen Aufwands mehrheitlich sehr zufrieden sind und einige der Unternehmenspartner den administrativen Aufwand eher auf Seiten des wissenschaftlichen Partners (Laborleiterin/Laborleiter) sehen, gab es seitens der Befragten hier nur wenige Nennungen, die u.a. die Anzahl der Dokumente, die Unterschriftenregelung oder auch das Webtool betrafen:

„Aufwand eher auf Seiten der Wissenschaftler, nicht Industriepartner".

„Evaluierung während des CD-Labors".

„Weniger Unternehmensdokumente bei Antragstellung“. 
„Die Unterschriftenregelung bei der Antragstellung ist bei großen Unternehmen unverhältnismäßig (die Unterschrift des Finanzchefs am Standort ist für die verantwortete Geldsumme ausreichend, zwei Personen in ppa. kaum innerhalb eines Geschäftsbereichs zu finden)“.

„Verwenden Sie ein Tool ähnlich dem eCall der FFG...".

Quelle: Online-Erhebung, Economica/IWI (2016)

Ein Vergleich der Zufriedenheitswerte einzelner Aspekten des Programms bzw. der Programmabwicklung mit jenen aus dem Jahr 2011 zeigt eine minimale Verschlechterung, jedoch keine drastischen Verschiebungen der Werte.

Abbildung 93: Zufriedenheit mit Aspekten des Programms bzw. der Programmabwicklung im Zeitverlauf (2005, 2011, 2016)

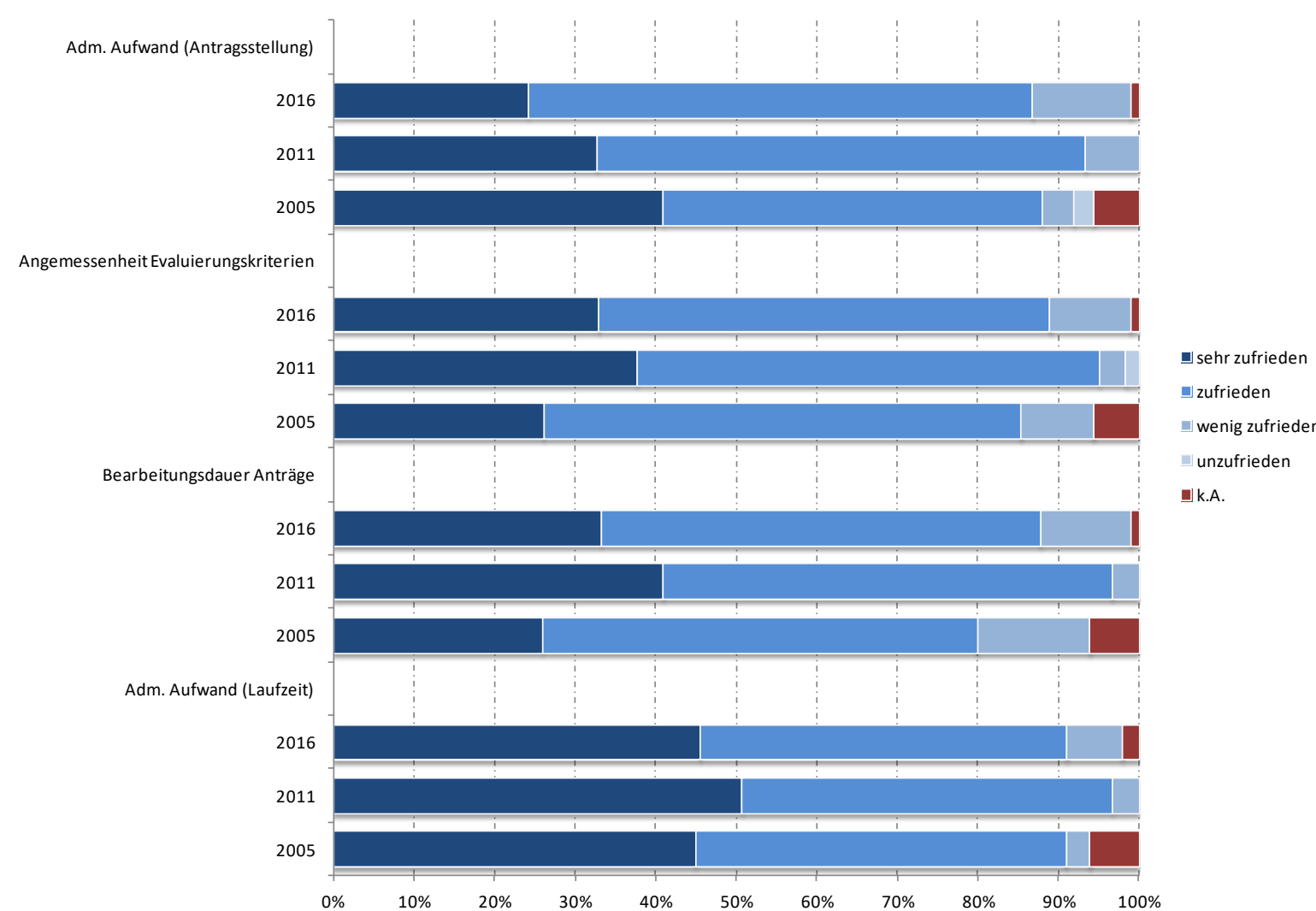

Anm.: Der Aspekt „Klarheit des Förderkonzepts und Förderziels" wurde in den Jahren 2011 und 2005 nicht abgefragt.

Quelle: Economica/IWI (2016, 2011), Joanneum Research/Technopolis/KMU Forschung Austria/Fraunhofer - ISI (2005) ; $n=90$ (2016), $n=61$ (2011), $n=74$ (2005) 
Die begleitende wissenschaftliche Kontrolle in Form der 2- und 5-Jahresevaluierungen wird von den Unternehmen mehrheitlich als (sehr) hilfreich angesehen. Dennoch bewerten 15\% (2-Jahresevaluierung) bzw. 17\% (5-Jahresevaluierung) der Befragten die entsprechenden Kontrollen als „wenig hilfreich“ oder auch „nicht hilfreich“.

\section{Abbildung 94: Einschätzung der begleitenden wissenschaftlichen Kontrolle}

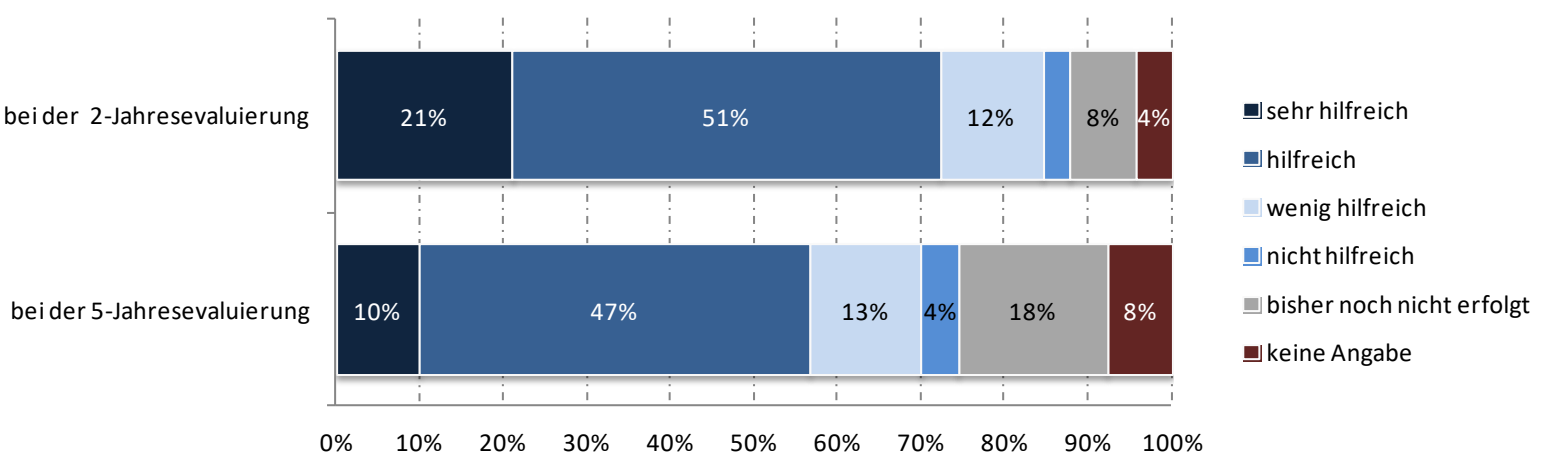

Quelle: Economica/IWI (2016); Online-Erhebung: $n=90$

Betrachtet man nur jene Gruppe an Unternehmen, bei denen eine Evaluierung bereits stattgefunden hat bzw. die zu dieser Frage Angaben machten, so zeigt sich ein zur Evaluierung 2011 sehr ähnliches Bild (auch wenn jene Gruppe, welche die begleitende wissenschaftliche Kontrolle als „sehr hilfreich" einschätzt, etwas kleiner geworden ist). Lediglich die 5-Jahresevaluierung wird von einigen Befragten etwas weniger hilfreich eingeschätzt als noch im Jahr 2011. 
Abbildung 95: Einschätzung der begleitenden wissenschaftlichen Kontrolle im Zeitverlauf (2011, 2016)

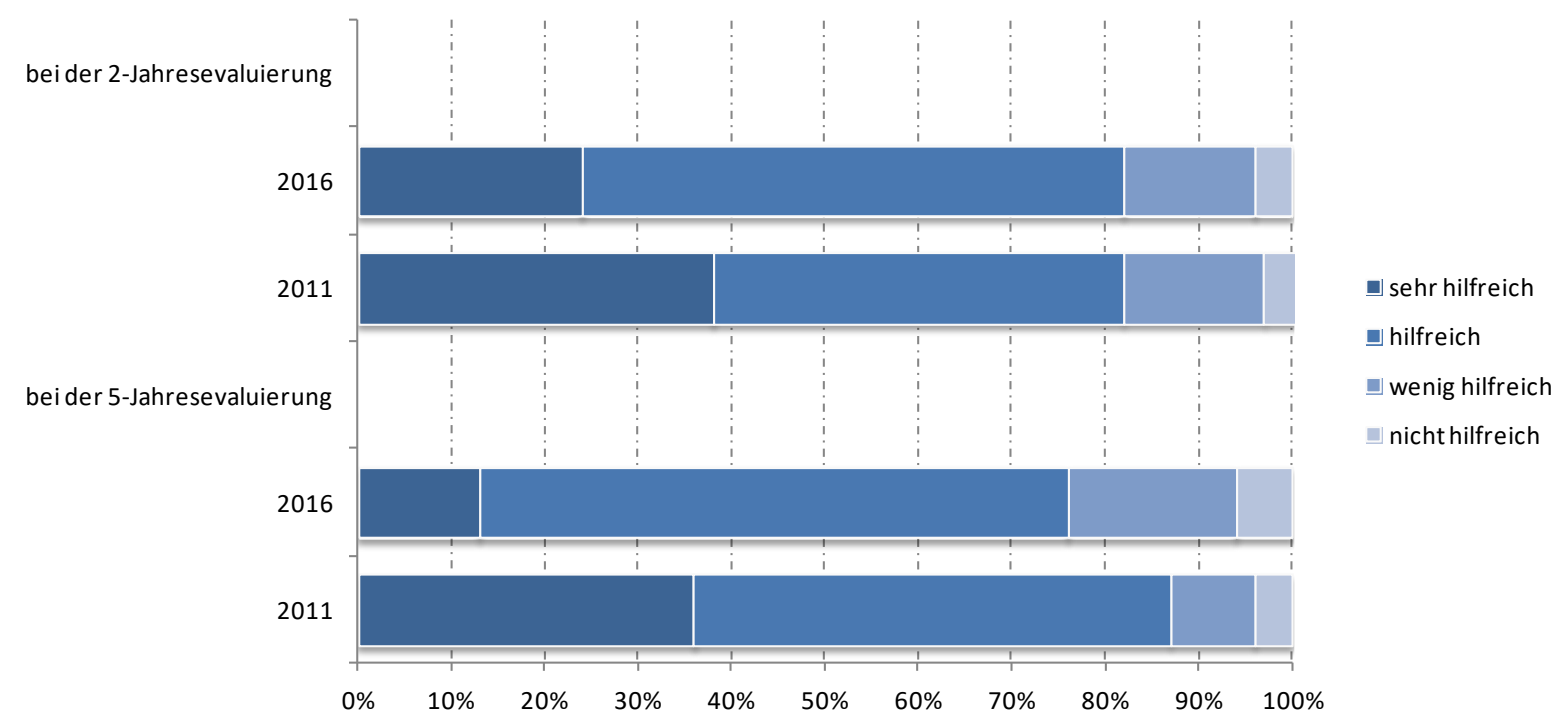

Quelle: Economica/IWI (2016, 2011); $n=90$ (2016), $n=61$ (2011)

Mit den Betreuungsleistungen und der Öffentlichkeitsarbeit der CDG zeigen sich auch die Unternehmenspartner überwiegend (sehr) zufrieden. Mit der Betreuung während der Antragstellung sind 92\% der Befragten (sehr) zufrieden, mit der Betreuung des CD-Labors während der Laufzeit 87\%. Mit der Öffentlichkeitsarbeit zeigen sich $82 \%$ (sehr) zufrieden, hier ist jedoch der Anteil der „sehr Zufriedenen" etwas geringer.

\section{Abbildung 96: Zufriedenheit mit den Leistungen der CDG}

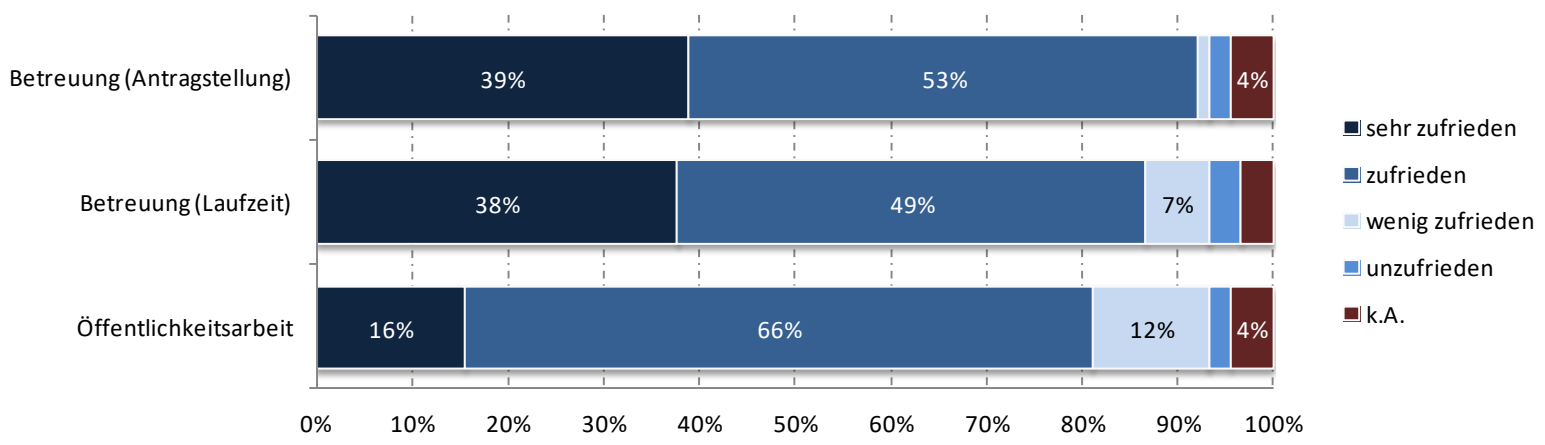

Quelle: Economica/IWI (2016); Online-Erhebung: $n=90$ 
Auch betreffend dieser Betreuungsaspekte bzw. der Bewertung der Öffentlichkeitsarbeit zeigen sich lediglich geringe Veränderungen bzw. Verschiebungen gegenüber 2011 (und auch gegenüber 2005).

\section{Abbildung 97: Zufriedenheit mit den Leistungen der CDG im Zeitverlauf $(2005,2011,2016)$}

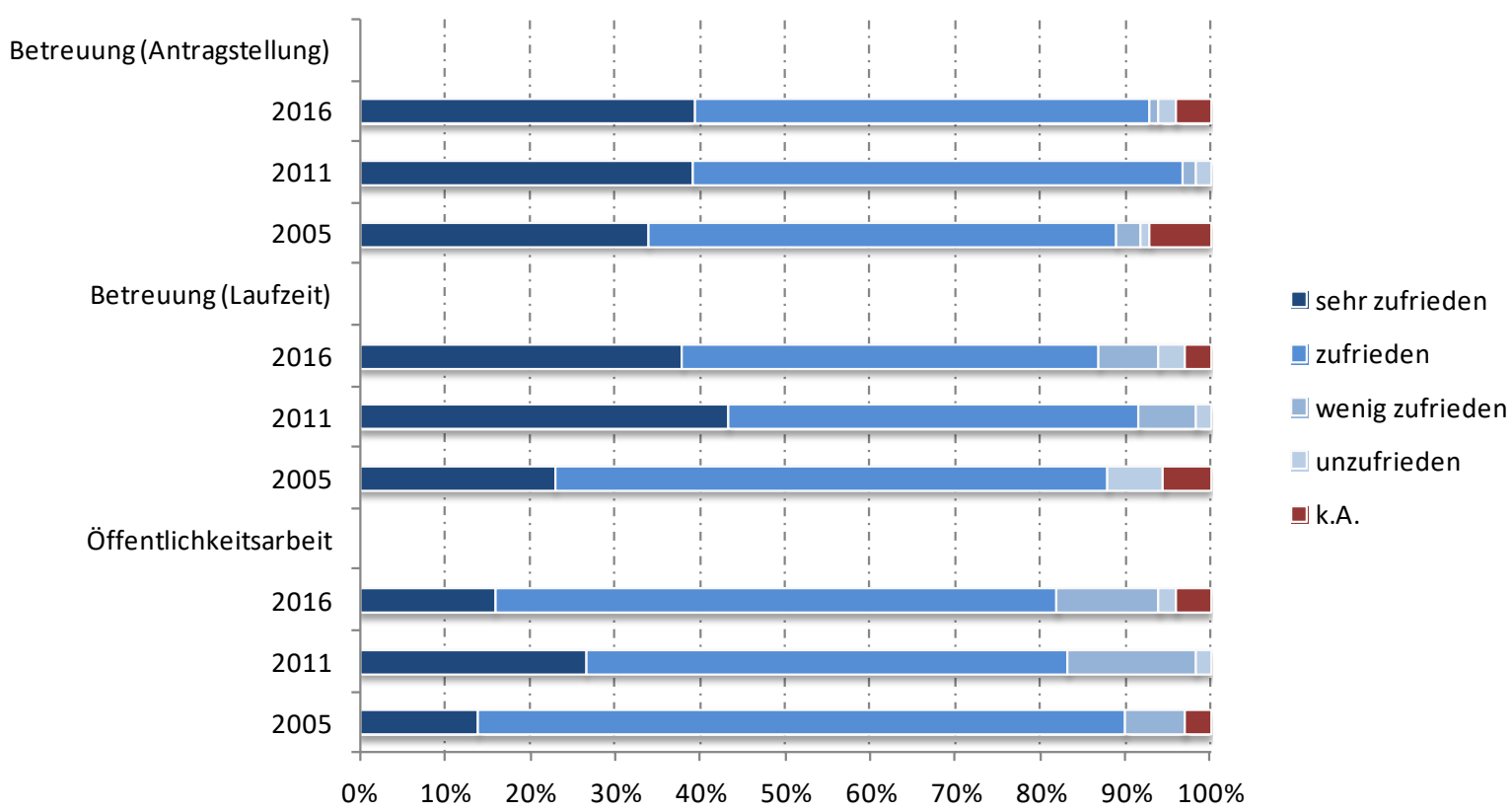

Quelle: Economica/IWI (2016, 2011), Joanneum Research/Technopolis/KMU Forschung Austria/Fraunhofer - ISI (2005) ; $n=90$ (2016), $n=60$ (2011), $n=74$ (2005)

Betrachtet man die Motivation der Unternehmenspartner zur CD-Laborgründung bzw. zur Beteiligung an einem CD-Labor, so ist den Befragten insbesondere die langfristige Sicherung des Zugangs zu wissenschaftlichem Know-how (94\% sehen diesen Punkt als „sehr wichtig“ bzw. als „wichtig“) und der Aufbau einer strategischen Allianz mit den Universitäten (88\%) bedeutsam. Auch der Einstieg in ein neues Forschungsthema (84\%) sowie die Tatsache, dass eigene anwendungsorientierte Grundlagenforschung zu teuer ist (83\%), ist eine für die Unternehmenspartner (sehr) wichtige Motivation. 
Abbildung 98: Motivation als Unternehmenspartner zur CD-Laborgründung bzw. zur Beteiligung am CD-Labor

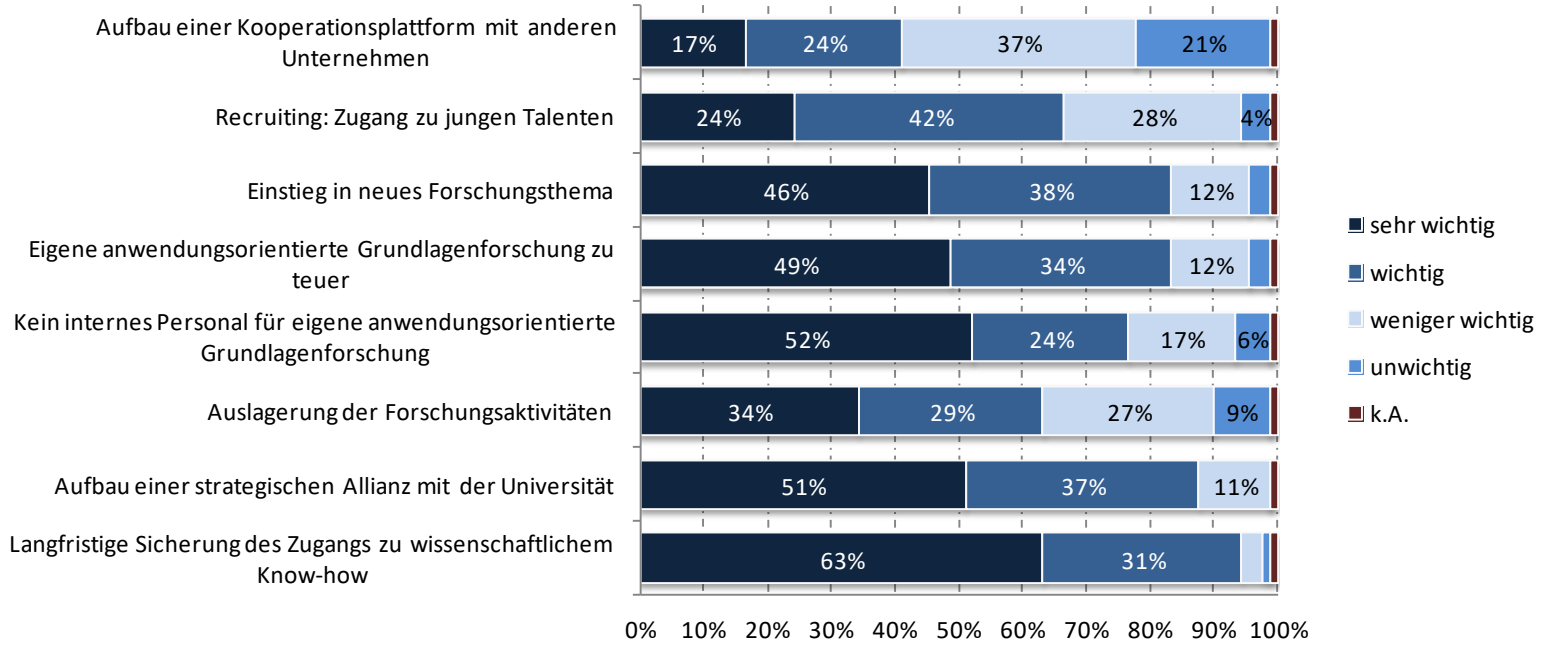

Quelle: Economica/IWI (2016); Online-Erhebung: $n=90$

Ein Vergleich mit der Evaluierung 2011 zeigt, dass sich diese drei wichtigsten Motive nicht verändert haben. Schon damals rangierten von ihrer Wichtigkeit her die langfristige Sicherung des Zugangs zu wissenschaftlichem Know-how, der Aufbau einer strategischen Allianz mit der Universität sowie der Einstieg in ein neues Forschungsthema auf den ersten drei Rängen.

Am unteren Ende der Motive rangierte 2011 der Aufbau einer Kooperationsplattform mit anderen Unternehmen, ein Punkt, dem auch in der aktuellen Befragung die geringste Wichtigkeit zugewiesen wurde.

Im Vergleich zu rein unternehmensintern durchgeführten F\&E-Tätigkeiten tendieren die Forschungsaktivitäten im CD-Labor zu einer höheren technologischen Komplexität und sind wissenschaftlich anspruchsvoller. 


\section{Abbildung 99: Art der Forschungsaktivitäten im CD-Labor}

\begin{tabular}{|l|l|l|l|l|l|l|}
\hline $\begin{array}{l}\text { außerhalb unseres technologischen } \\
\text { Kernbereichs (1) }\end{array}$ & & & & 3,3 & & $\begin{array}{l}\text { innerhalb unseres technologischen } \\
\text { Kernbereichs (5) }\end{array}$ \\
\hline geringes technologisches Risiko (1) & & & & & & \\
\hline \\
technologisch einfach (1)
\end{tabular}

Anm.: Die Unternehmen hatten die Möglichkeit, der Art ihrer Forschungsaktivitäten in den einzelnen Kategorien mit den Wert 1, 2, 3, 4 bzw. 5 zuzuordnen.

Quelle: Economica/IWI (2016); Online-Erhebung: $n=90$

Der Kontakt zu Wissenschaftern bzw. Wissenschafterinnen vor der CD-Laborgründung bestand größtenteils über gemeinsame Forschungsprojekte (70\%). Lose Kontakte bestanden bei $28 \%$ der Befragten. Lediglich bei $2 \%$ der Befragten bestand vorher überhaupt kein Kontakt.

Abbildung 100: Kontakt zu Wissenschaftlern/Wissenschaftlerinnen vor der CD-Laborgründung im Zeitverlauf $(2011,2016)$

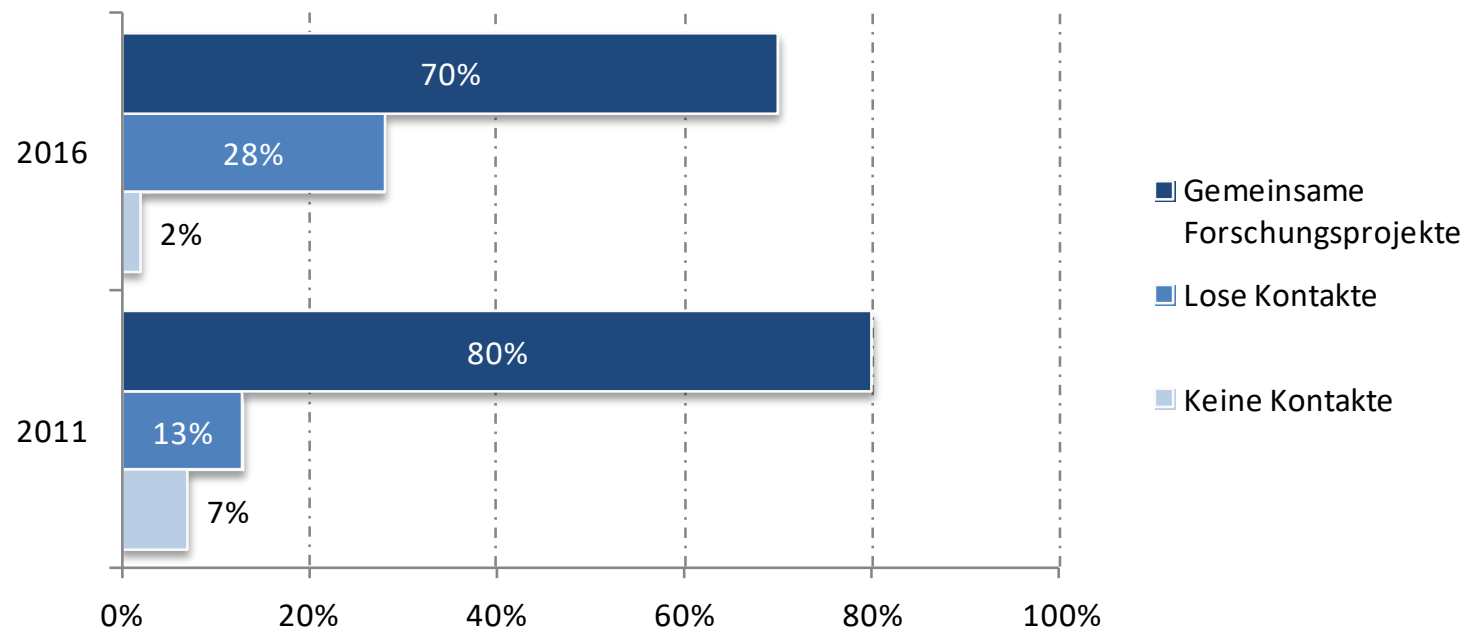

Anm.: Daten der Evaluierung 2005 (damals waren Mehrfachantworten möglich): Gemeinsame Forschungsprojekte (49\%), Lose Kontakte (41\%), Keine Kontakte (14\%) 
Quelle: Economica/IWI (2016, 2011); Online-Erhebung: $n=90$ (2016), $n=60$

Ein Vergleich zur letzten Evaluierung zeigt, dass im Gegensatz zu 2011 der Anteil jener, die sich bereits aus gemeinsamen Forschungsprojekten kennen, etwas zurückgegangen ist und dass es kaum mehr Unternehmen gibt, die vorher keine Kontakte zu Wissenschaftlern/Wissenschaftlerinnen hatten.

Der Einfluss der Unternehmen auf die Forschungsprogrammformulierung und die Moduldefinition wird von den Befragten unterschiedlich bewertet. Während rund $70 \%$ ihren Einfluss als hoch bzw. sehr hoch bewerten, sehen rund 30\% einen geringen oder teils keinen Einfluss.

Abbildung 101: Einfluss seitens des Unternehmens auf die Forschungsprogrammformulierung und auf die Moduldefinition

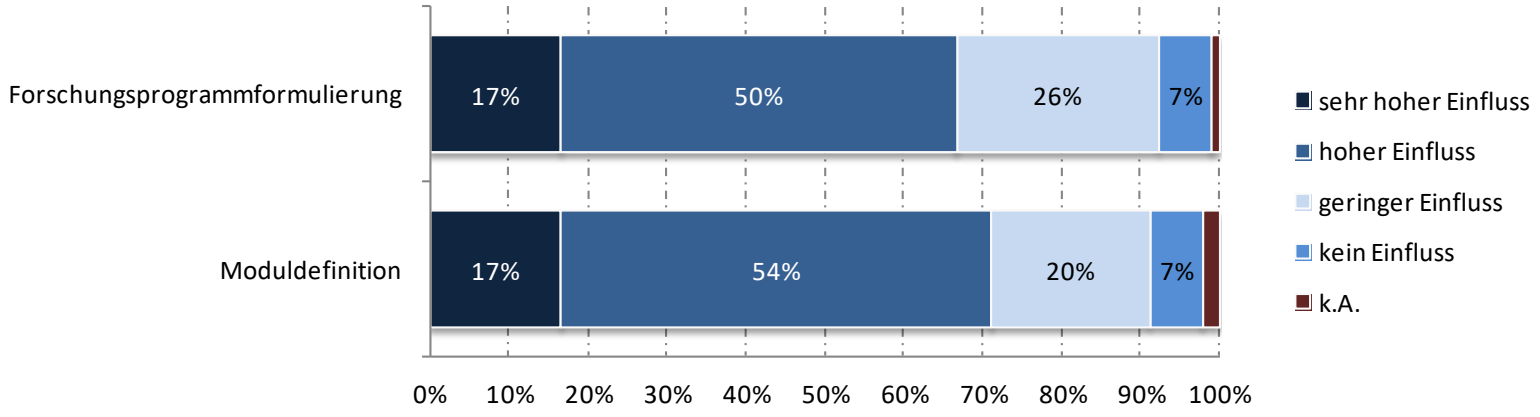

Quelle: Economica/IWI (2016); Online-Erhebung: $n=90$

Folgende Abbildung zeigt einen Vergleich mit den Evaluierungen der Jahre 2011 und 2005. Es zeigt sich ein leichter aber kontinuierlicher Rückgang des Einflusses der Unternehmen auf die Forschungsprogrammformulierung und auch ein leichter Rückgang des Einflusses hinsichtlich der Moduldefinition. 
Abbildung 102: Einfluss seitens des Unternehmens auf die Forschungsprogrammformulierung und auf die Moduldefinition im Zeitverlauf $(2005,2011,2016)$

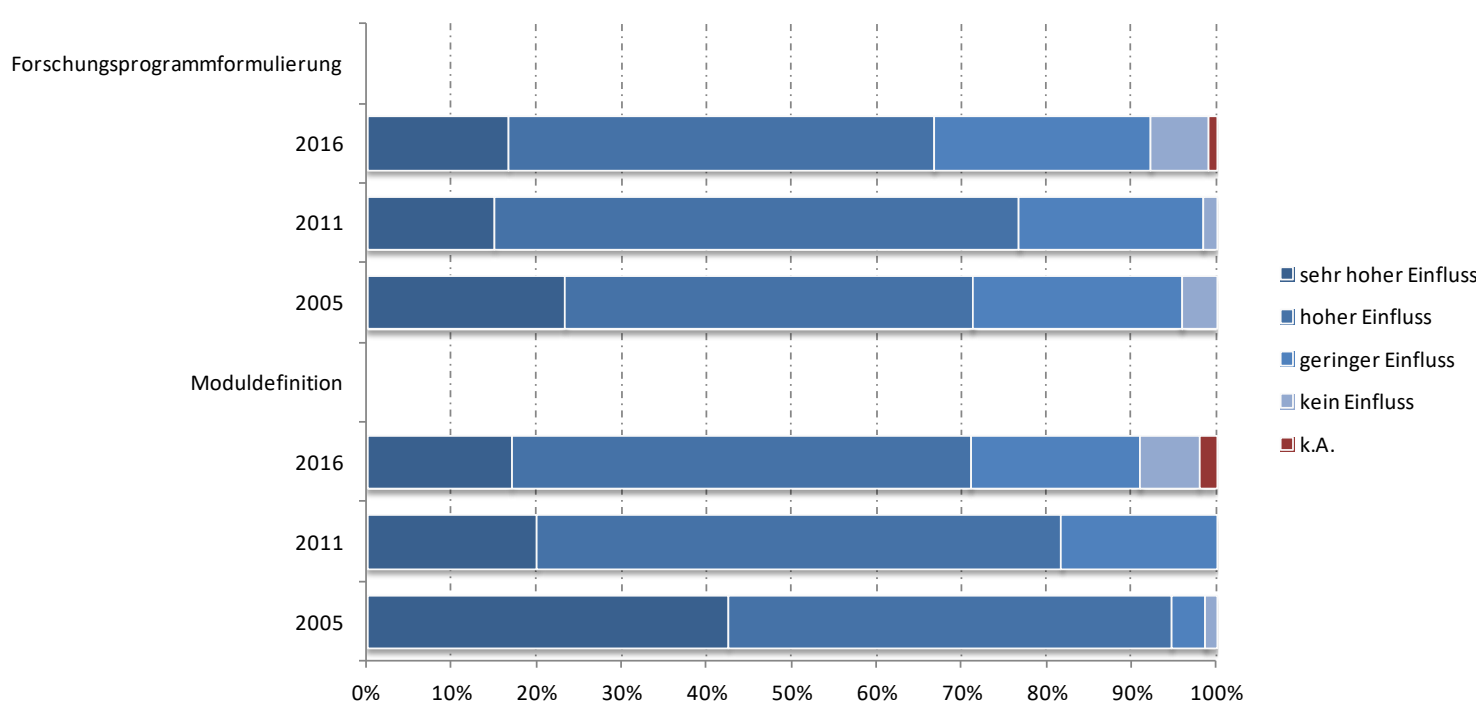

Quelle: Economica/IWI (2016, 2011), Joanneum Research/Technopolis/KMU Forschung Austria/Fraunhofer - ISI (2005) ; $n=90$ (2016), $n=60$ (2011), $n=74$ (2005)

Bei der Durchführung der Forschungskooperation wird nach Ansicht von rund 55\% der Befragten (eher) kooperativ zusammengearbeitet als (eher) eigenständig.

Abbildung 103: Durchführung der Forschungskooperation im CD-Labor im Zeitverlauf $(2005,2011$, 2016)

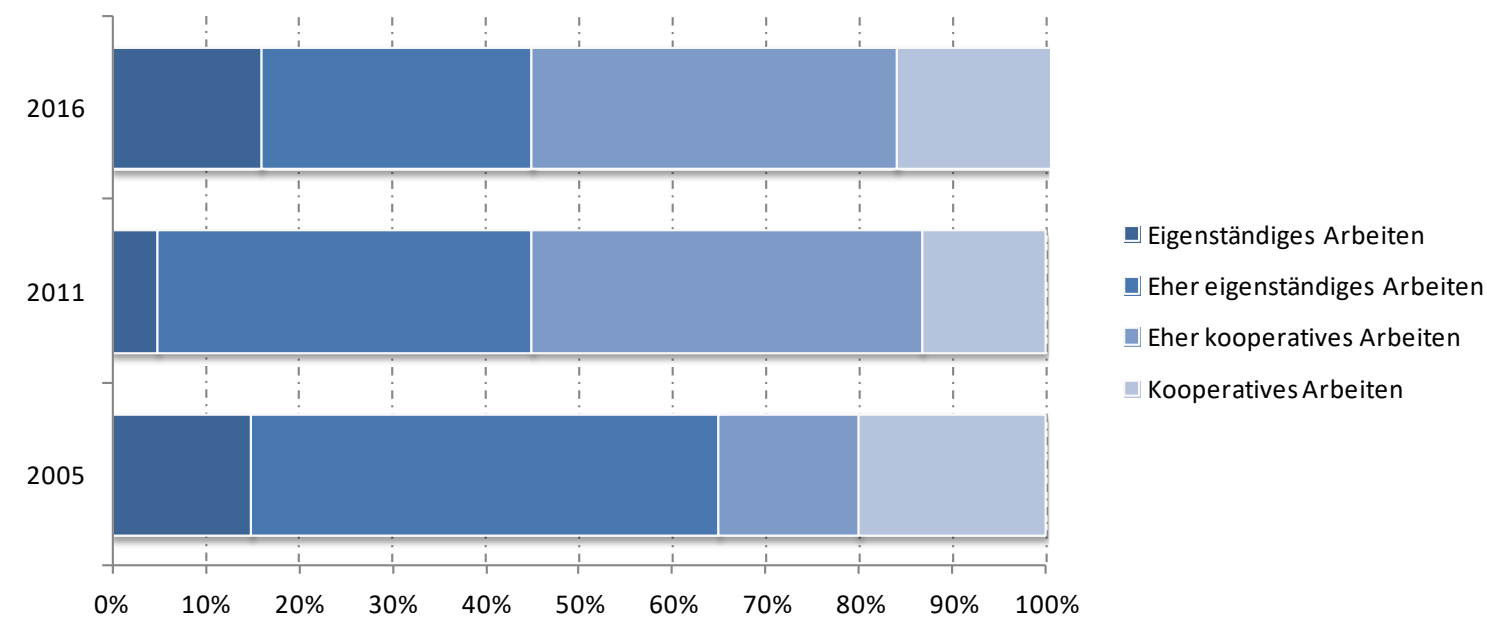

Quelle: Economica/IWI (2016, 2011), Joanneum Research/Technopolis/KMU Forschung Austria/Fraunhofer - ISI (2005) ; $n=90$ (2016), $n=60$ (2011), $n=74$ (2005) 
Damit entspricht die Struktur der Zusammenarbeit, laut Einschätzung der Befragten, nahezu exakt jener von 2011. Auch damals wurde bei über der Hälfte der Antworten „(eher) kooperatives Arbeiten" angegeben (55\%). Etwas stärker als noch bei der letzten Evaluierung gibt es bei der aktuellen Evaluierung eine Wahrnehmung in Richtung „stärkere Eigenständigkeit" der Arbeiten (16\%) in den CD-Labors.

Bei knapp 90\% der Unternehmen gab es regelmäßige Kontakte (zumindest mehrmals pro Quartal) mittels Email, Telefon etc. Bei rund der Hälfte der Befragten fanden diese Kontakte mehrmals pro Monat statt. Gemeinsame Arbeitssitzungen fanden bei mehr als der Hälfte der Unternehmen zumindest mehrmals im Quartal statt.

\section{Abbildung 104: Häufigkeit der Kontakte}

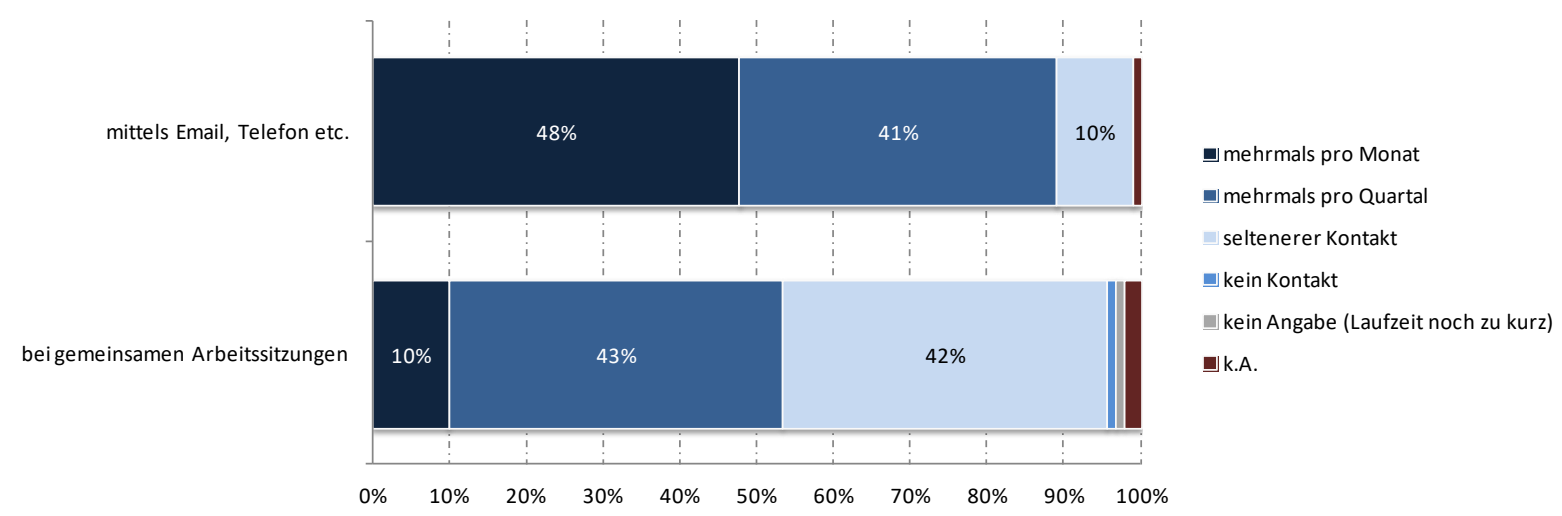

Quelle: Economica/IWI (2016); Online-Erhebung: $n=90$

Die Häufigkeit der Kontakte ist im Zeitverlauf bezogen auf 2011 leicht zurückgegangen. 
Abbildung 105: Häufigkeit der Kontakte im Zeitverlauf $(2005,2011,2016)$

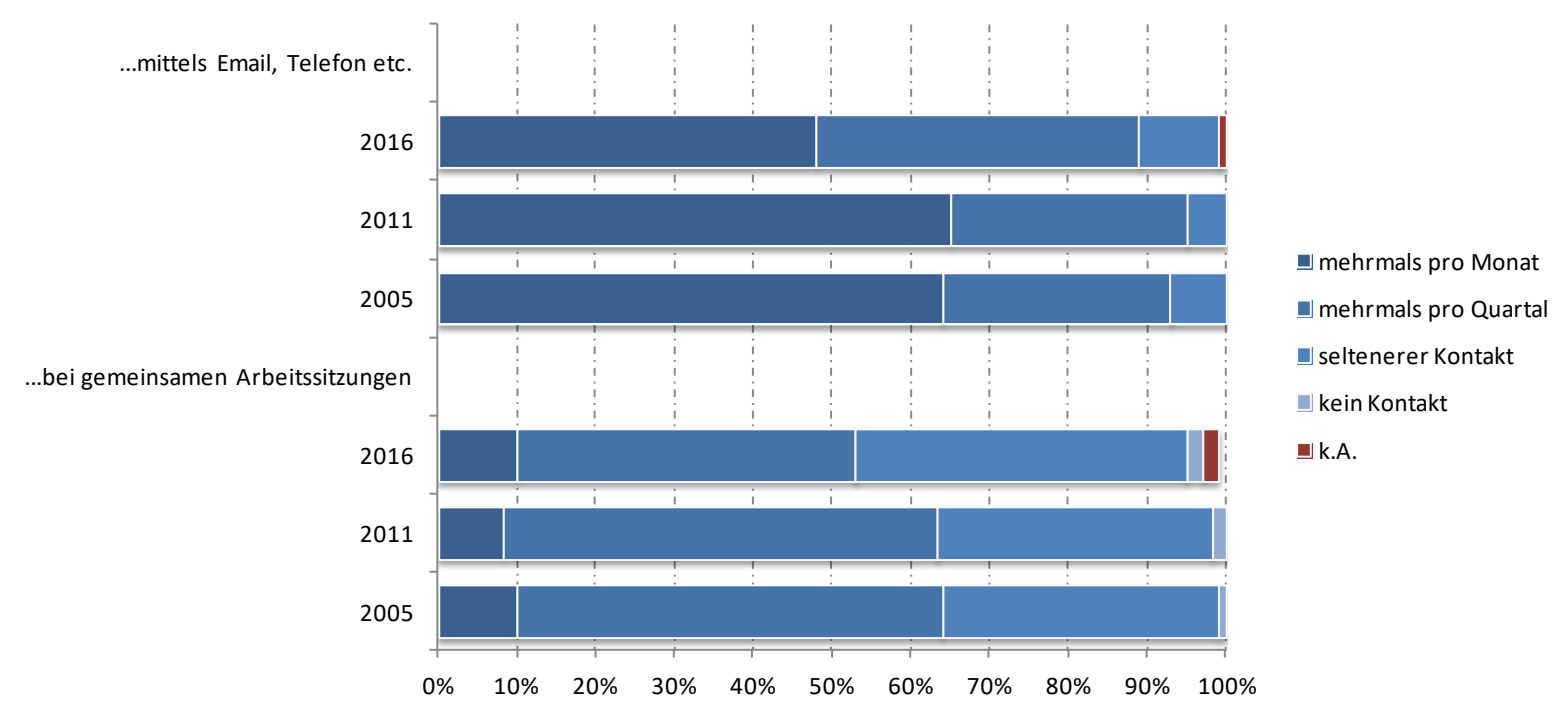

Quelle: Economica/IWI (2016, 2011), Joanneum Research/Technopolis/KMU Forschung Austria/Fraunhofer - ISI (2005) ; $n=90$ (2016), $n=60$ (2011), $n=74$ (2005)

Hinsichtlich der Bewertung von Aspekten der Zusammenarbeit im CD-Labor wurden vor allem die wissenschaftliche Qualität der Arbeiten (91\% bewerteten diesen Aspekt mit „sehr gut" oder "gut“), sowie das Verständnis des CD-Labors für die Problemstellung (85\%) positiv bewertet.

\section{Abbildung 106: Bewertung von Aspekten der Zusammenarbeit im CD-Labor}

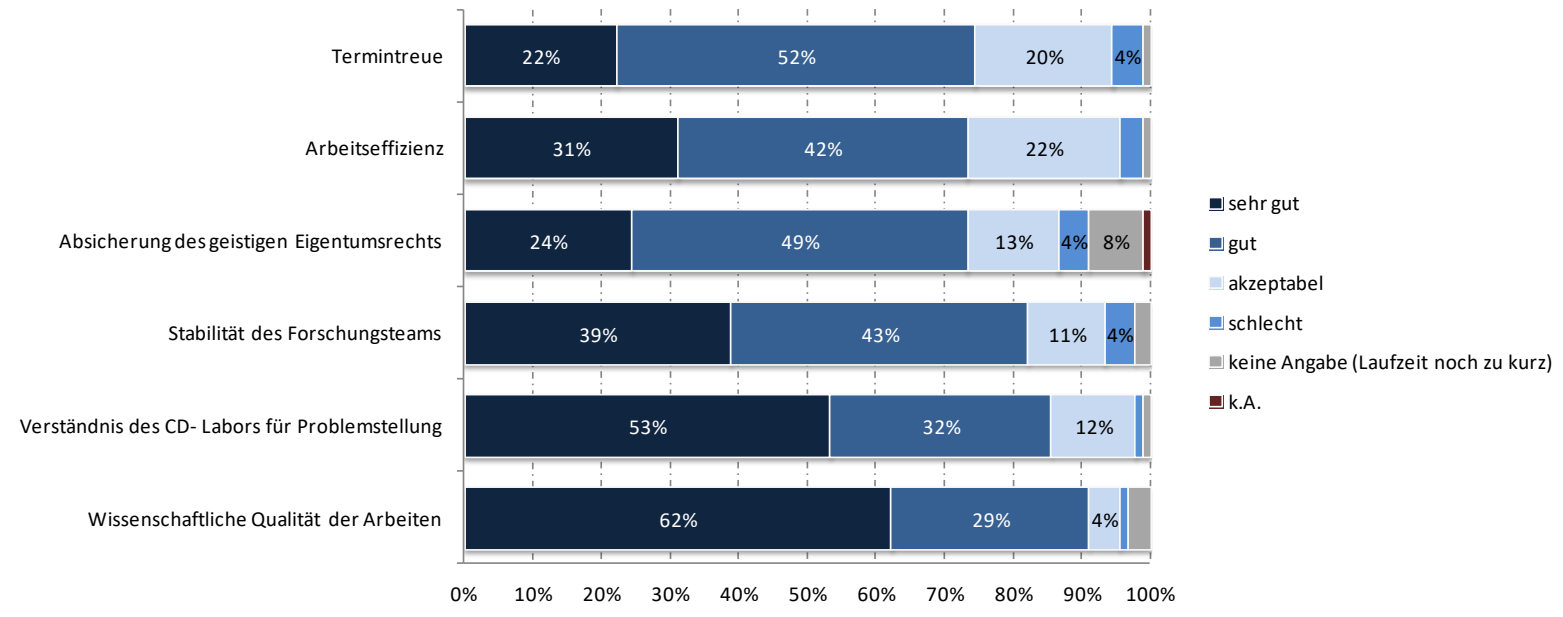

Quelle: Economica/IWI (2016); Online-Erhebung: $n=90$ 
Insgesamt 64 der 90 antwortenden Unternehmen (71\%) gaben an, dass zumindest ein weiteres Unternehmen am CD-Labor beteiligt war. Dies waren Partnerunternehmen aus der gleichen Branche, aus der Zulieferbranche sowie Partnerunternehmen auf der Kundenseite.

\section{Abbildung 107: Weitere beteiligte Unternehmen am CD-Labor}

Ja, Partnerunternehmen aus der gleichen Branche

Ja, Partnerunternehmen aus der Zulieferbranche

Ja, Partnerunternehmen auf der Kundenseite

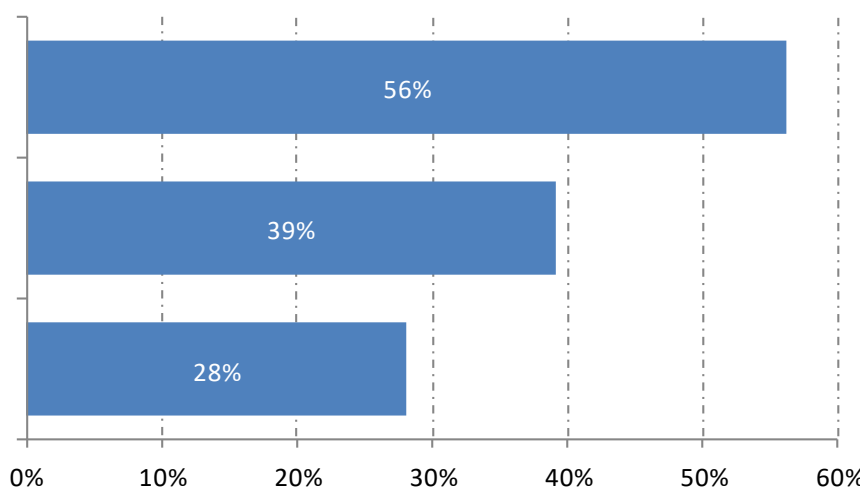

Anm.: Mehrfachnennungen möglich.

Quelle: Economica/IWI (2016); Online-Erhebung: $n=64$

Betrachtet man jene 36 Unternehmen, die mit Partnerunternehmen der gleichen Branche zusammenarbeiteten, so variiert die Anzahl der Partnerunternehmen zwischen einem Unternehmen und sieben Unternehmen. In den meisten Fällen stehen die Unternehmen mit diesen Partnerunternehmen gleicher Branche jedoch nicht in unmittelbarer Konkurrenz.

Tabelle 23: Konkurrenzsituation bei Partnerunternehmen gleicher Branche (Übersicht)

\begin{tabular}{|l|r|r|r|}
\hline $\begin{array}{l}\text { Anzahl } \\
\text { (Partnerunternehmen gleicher Branche) }\end{array}$ & Häufigkeit & \multicolumn{2}{|c|}{ Konkurrenzsituation } \\
\hline 1 & 17 & In unmittelbarer Konkurrenz & 4 \\
\hline 2 & 12 & Zum Teil in unmittelbarer Konkurrenz & 10 \\
\hline 3 & 2 & Keine unmittelbare Konkurrenz & 44 \\
\hline 4 & 0 & & \\
\hline 5 & 1 & & \\
\hline 6 & 1 & & \\
\hline 7 & 2 & & \\
\hline k.A. & 36 & & \\
\hline & & & \\
\hline
\end{tabular}

Quelle: Economica/IWI (2016) 
Die Forschungsergebnisse aus der Beteiligung am CD-Labor haben bzw. hatten für die Unternehmen vor allem Relevanz bei der Hilfe der Bewertung verschiedener Lösungsstrategien (Radarfunktion) sowie für die Entwicklung radikal neuer Produkte. Auch für die inkrementelle Verbesserung bestehender Produkte/Prozesse und die Entwicklung neuer Produktionsverfahren haben und hatten die Forschungsergebnisse Relevanz für die Unternehmen.

Gering ist der Anteil jener, die keinen Nutzen bzw. keine wirtschaftliche Relevanz in den Forschungsergebnissen sieht (7\%).

Abbildung 108: Haben bzw. hatten die Forschungsergebnisse aus der Beteiligung am CD-Labor wirtschaftliche Relevanz für folgende Aspekte?

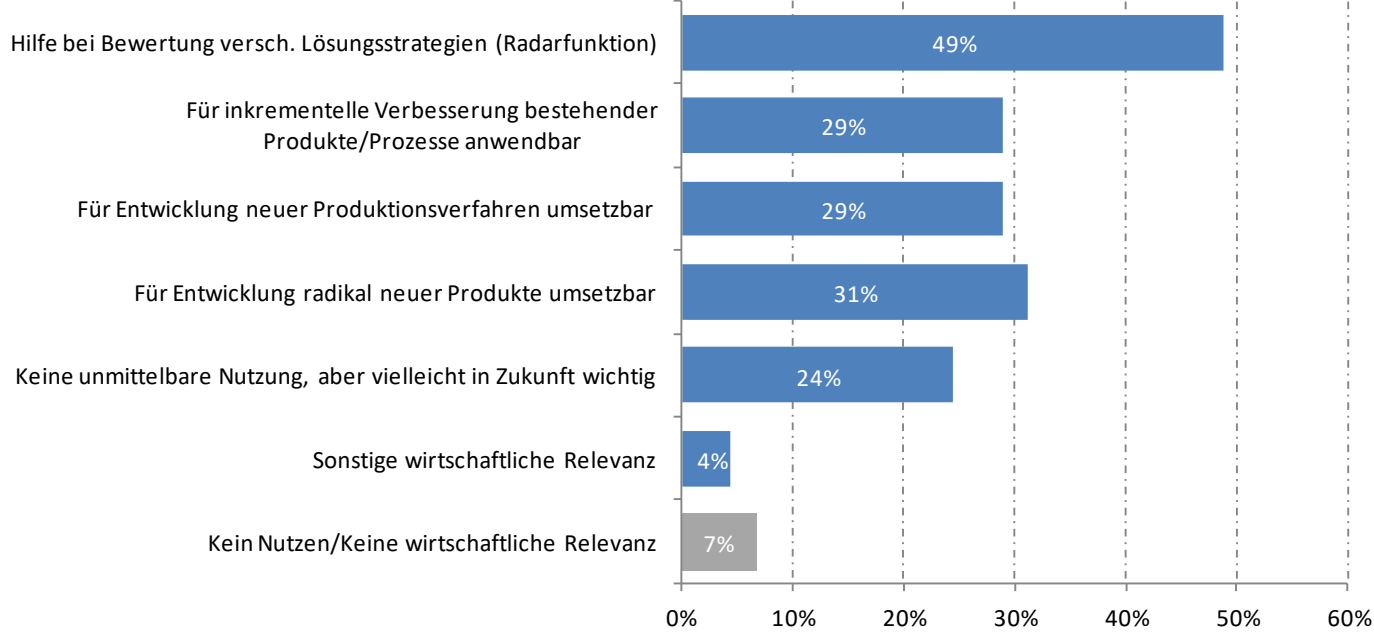

Anm.: Mehrfachantworten möglich.

Quelle: Economica/IWI (2016); Online-Erhebung: $n=90$

Einige Unternehmen nannten noch weitere für ihr Unternehmen relevante Aspekte.

\section{Sonstige wirtschaftliche Relevanz (offene Antworten von 3 Unternehmen):}

„Positionierung in diesem Thema in den Medien“.

„Entwicklung neuer Auslegungsprozesse/Methoden“.

„Eine maßgeschneiderte Lösung für spezielle Anforderungen“.

Quelle: Online-Erhebung, Economica/IWI (2016) 
Einen hohen Nutzen durch die CD-Laborgründung bzw. -beteiligung sehen die befragten Unternehmen vor allem im Bereich Kompetenzaufbau (88\% der Befragten sehen hier einen sehr hohen bzw. hohen Nutzen), dem Aufzeigen neuer technologischer Optionen (77\%) oder auch in der Stärkung der technologischen Problemlösungskompetenz (72\%).

Auch der Zugang zu qualifizierten Mitarbeitern (70\%) sowie der Aufbau von Technologieführerschaft (69\%) hat für die Mehrheit der befragten Unternehmen einen (sehr) hohen Nutzen.

Im Abbau der Zugangsbarrieren zu wissenschaftlicher Forschung sehen 40\% geringen bzw. keinen Nutzen. Geringer Nutzen wird von vielen Unternehmen auch im Prestigegewinn gesehen.

Abbildung 109: Nutzen für die Unternehmen durch die CD-Laborgründung bzw. -beteiligung

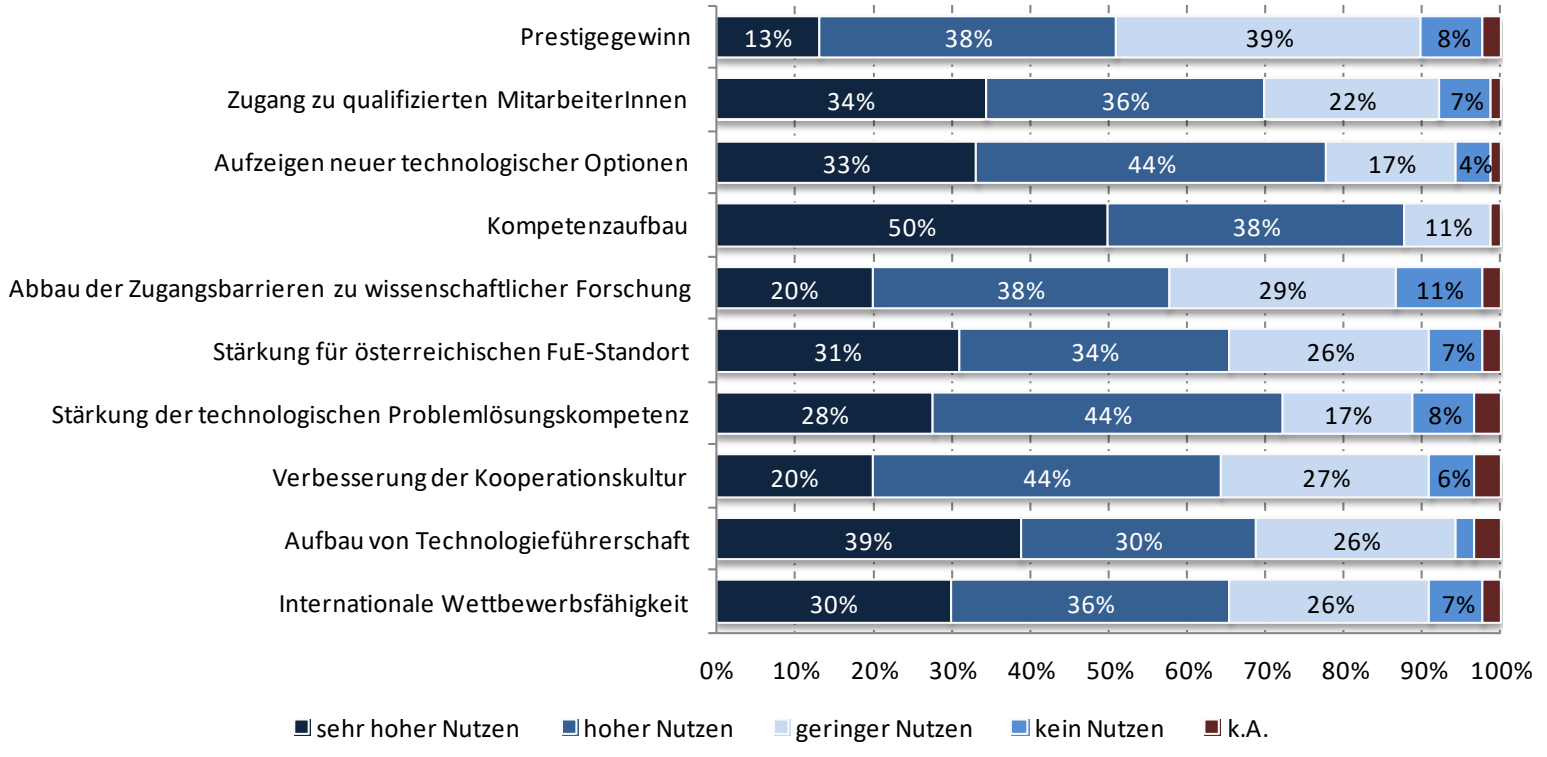

Quelle: Economica/IWI (2016); Online-Erhebung: $n=90$

Ein Vergleich mit der Evaluierung 2011 zeigt, dass sich der Nutzen für die beteiligten Unternehmen kaum verändert hat. Auch damals stand der Aufbau von Technologieführerschaft, die Stärkung der technischen Problemlösungskompetenz, der Kompetenzaufbau, das Aufzeigen neuer technologischer Optionen und der Zugang zu qualifizierten Mitarbeitern im Vordergrund.

Im Folgenden wurden die Auswirkungen einer CD-Laborgründung auf die F\&E-Aktivitäten der Unternehmen untersucht. 54\% der Befragten sahen eine bessere Umsetzung der F\&E-Aktivitäten, 51\% ein Eingehen von mit höherem Risiko behafteten F\&E-Aktivitäten. Auch eine gewonnene Kontinuität der F\&E-Aktivitäten (49\%) und eine nachhaltige Verbesserung des Kooperationsverhaltens 
(42\%) ist für zahlreiche Unternehmen feststellbar. Gestiegene F\&E-Ausgaben sehen $28 \%$ der Befragten.

Abbildung 110: Auswirkungen der CD-Laborgründung auf Unternehmen im Zeitverlauf $(2005,2011$, 2016)

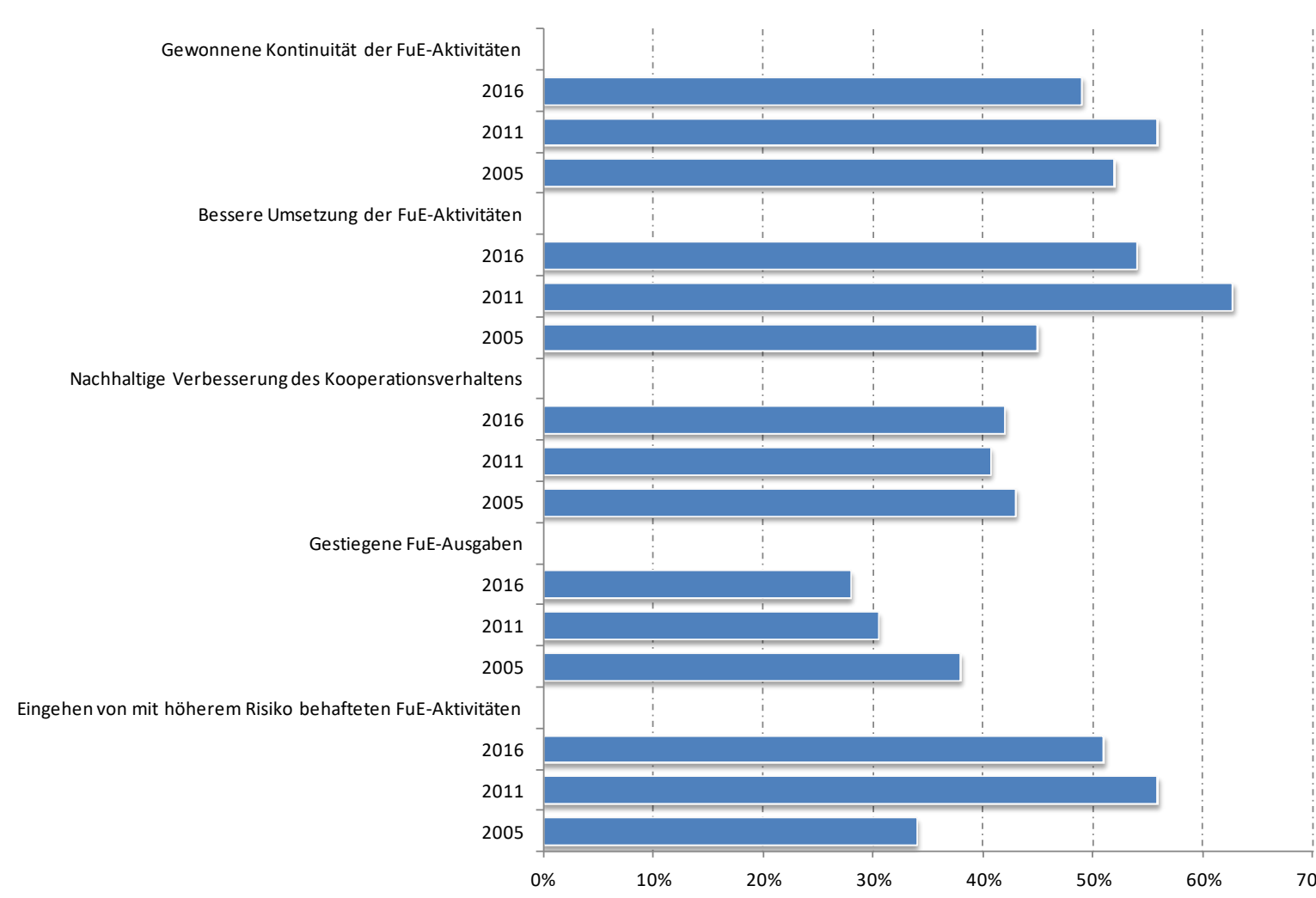

Quelle: Economica/IWI (2016, 2011), Joanneum Research/Technopolis/KMU Forschung Austria/Fraunhofer - ISI (2005) ; $n=90$ (2016), $n=59$ (2011), $n=74$ (2005)

Betrachtet man die Beurteilung der Kosten-Nutzen Relation des Engagements im CD-Labor aus Sicht der Unternehmen so sieht man im Zeitverlauf eine Verschiebung der Einschätzung. Im Zunehmen ist jene Gruppe, welche die Kosten etwas höher als den Nutzen einschätzt. Analog dazu ist die Gruppe, die den Nutzen etwas höher als die Kosten einschätzt geringer geworden. In den Extremkategorien (, Kosten deutlich höher als Nutzen“ und „Nutzen deutlich höher als Kosten“) sowie im Mittelbereich („Nutzen entspricht Kosten“) hat sich zwischen 2011 und der aktuellen Evaluierung kaum etwas verschoben. 
Abbildung 111: Kosten-Nutzen Relation im Zeitverlauf $(2005,2011,2016)$

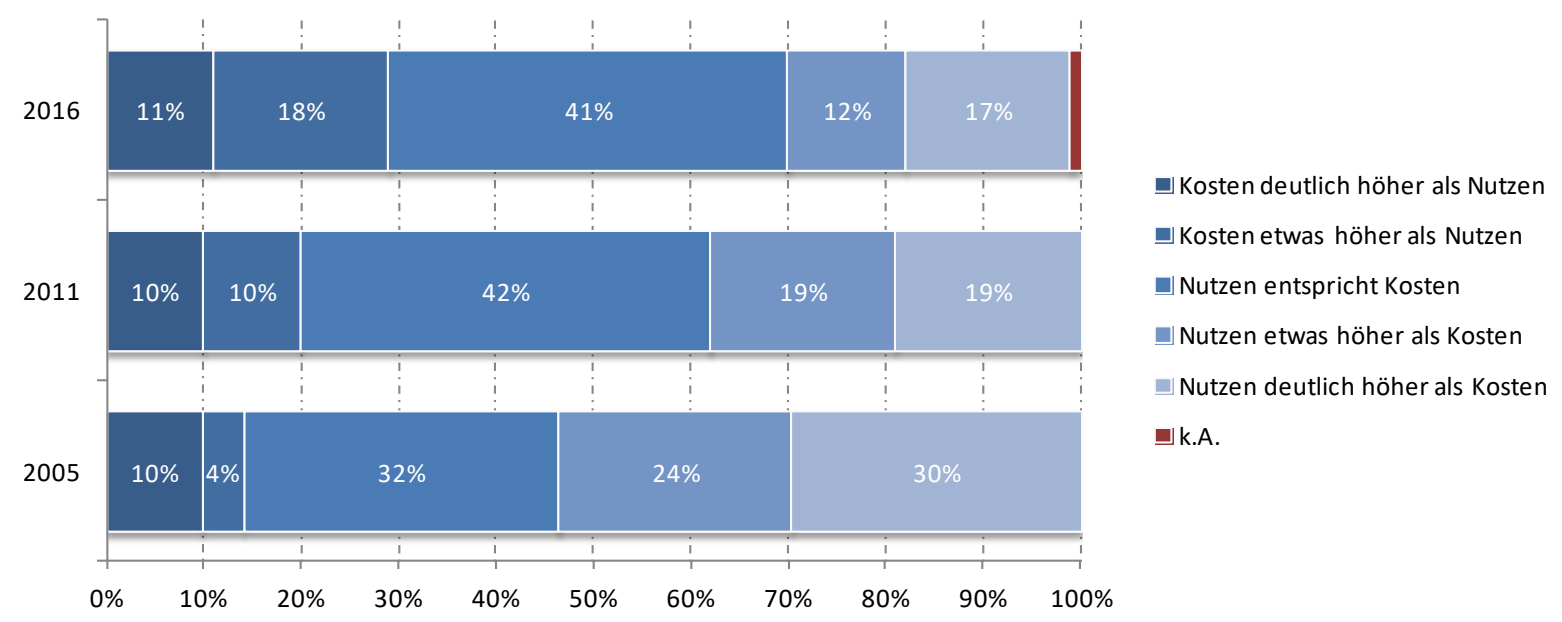

Quelle: Economica/IWI (2016, 2011), Joanneum Research/Technopolis/KMU Forschung Austria/Fraunhofer - ISI (2005) ; $n=90$ (2016), $n=59$ (2011), $n=74$ (2005)

72\% der befragten Unternehmen planen weitere Forschungsprojekte im Rahmen eines CD-Labors durchzuführen, $51 \%$ in gleichem und $9 \%$ in höherem Ausmaß.

Abbildung 112: Weitere Forschungsprojekte im Rahmen eines CD-Labors?

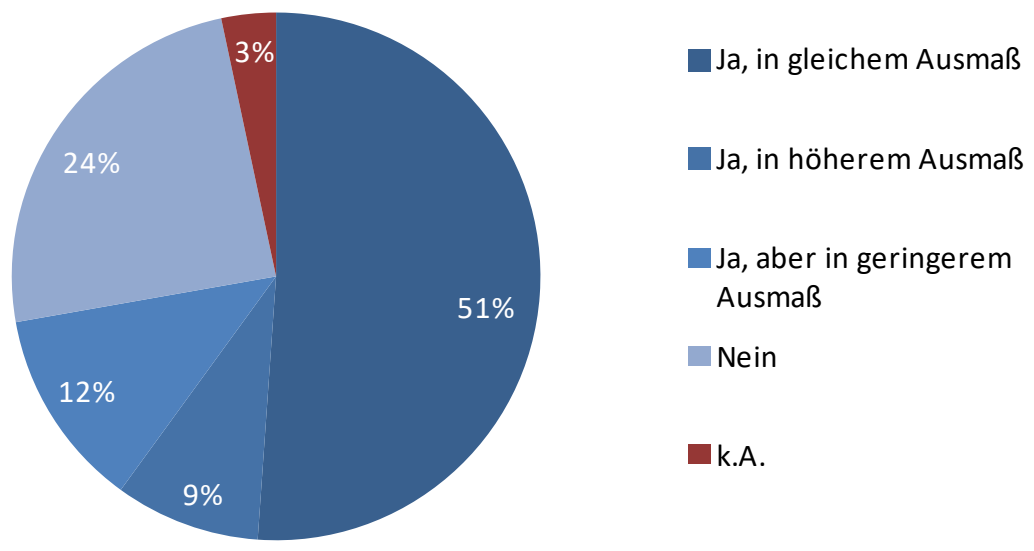

Quelle: Economica/IWI (2016); Online-Erhebung: $n=90$ 
24\% planen keine weiteren Projekte im Rahmen eines CD-Labors und nannten dafür teils auch Gründe im Rahmen der offenen Antwortkategorie. Dadurch ist auch ersichtlich, dass in jener Gruppe auch Unternehmen vertreten sind, die sich im jetzigen Stadium noch nicht entschieden haben.

$\underline{\text { Jene die "Nein" angaben, nannten u.a. folgende Gründe (Zitate): }}$

„Ergebnis und auch Umsetzung durch CD Labor und Uni in Summe nicht ausreichend!“.

„Ablehnung von Antrag“.

„Kosten/Nutzenverhältnis“.

„Keine passenden Themen“.

„Anderes Kollaborations-Model“.

„Noch nicht entschieden“.

"Weiss nicht ist eine bessere Antwort".

„Mangel an Betreuungskapazitäten“.

„Entscheidung fällt erst gegen Ende der Laufzeit des jetzigen CD-Labors“.

Quelle: Online-Erhebung, Economica/IWI (2016)

Im Folgenden wurden die Unternehmen gefragt, ob das entsprechende Forschungsprojekt auch ohne

CD-Labor durchgeführt worden wäre. 21\% hätten das Forschungsprojekt gar nicht durchgeführt, 74\%

in (deutlich) geringerem Umfang. Lediglich 4\% hätten das Projekt auch ohne CD-Labor im selben Umfang umgesetzt. Ein Vergleich mit den Daten der Evaluierung 2011 zeigt hier ein sehr ähnliches Bild. 
Abbildung 113: Durchführung auch ohne CD-Labor im Zeitverlauf $(2011,2016)$

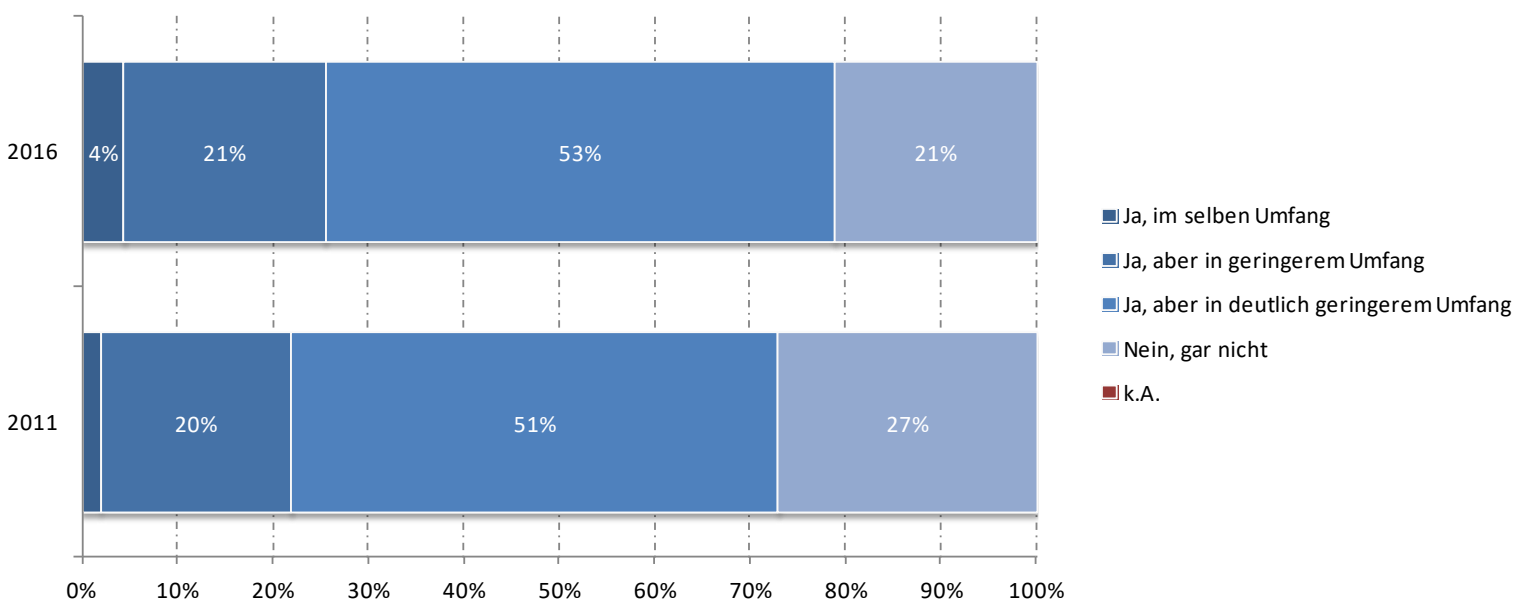

Quelle: Economica/IWI (2016, 2011); Online-Erhebung: $n=90$ (2016), $n=59$ (2011)

Von jenen 71 Unternehmen, die das Projekt dennoch in irgendeiner Form umgesetzt hätten (mehrheitlich in geringerem oder deutlich geringerem Umfang), nannten 70 eine voraussichtlich andere Finanzierungsform. 56\% der Unternehmen hätten eine firmeninterne Finanzierung angestrebt, 23\% eine Finanzierung teils firmenintern und über Förderprogramme. 21\% hätten eine Finanzierung über ein anderes Förderprogramm versucht.

Abbildung 114: Voraussichtliche Finanzierung des Forschungsprojekts ohne CD-Labor im Zeitverlauf (2011, 2016)

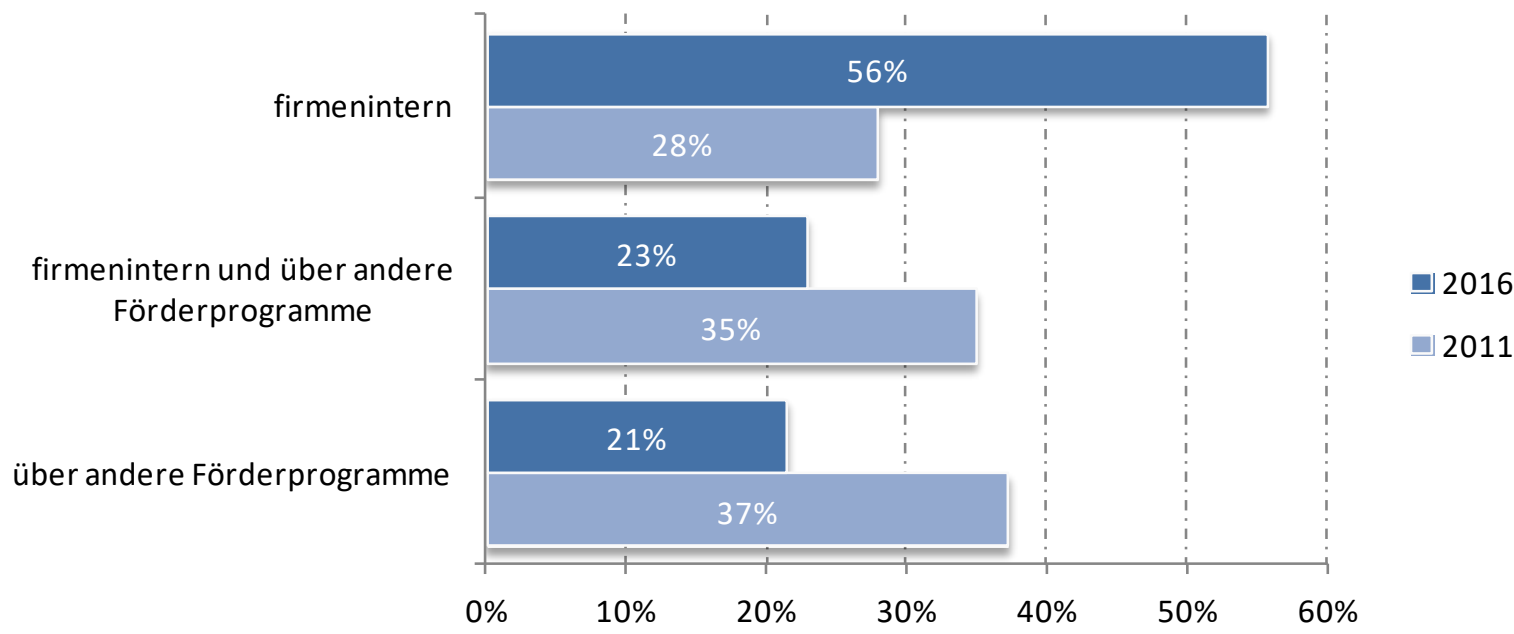

Quelle: Economica/IWI (2016, 2011); Online-Erhebung: $n=70$ (2016), n=43 (2011) 
Abschließend wurden die Unternehmensvertreter gebeten die zukünftige Nutzung direkter Forschungsförderprogramme in ihrem Unternehmen einzuschätzen. Mittelfristig (in den kommenden 5 Jahren) gehen $42 \%$ von einem Anstieg der Nutzung aus, $47 \%$ denken, dass die Nutzung gleich bleiben wird. Von einem Rückgang gehen lediglich $5 \%$ aus, von einem starken Zurückgehen keiner der Befragten. Langfristig (in den kommenden 10 Jahren) rechnen sogar 52\% mit einem Anstieg der Nutzung direkter Forschungsförderung. Auch in der langfristigen Perspektive rechnet keiner der Befragten mit einem starken zurückgehen.

\section{Abbildung 115: Nutzung direkter Forschungsförderprogramme in Zukunft im Unternehmen}

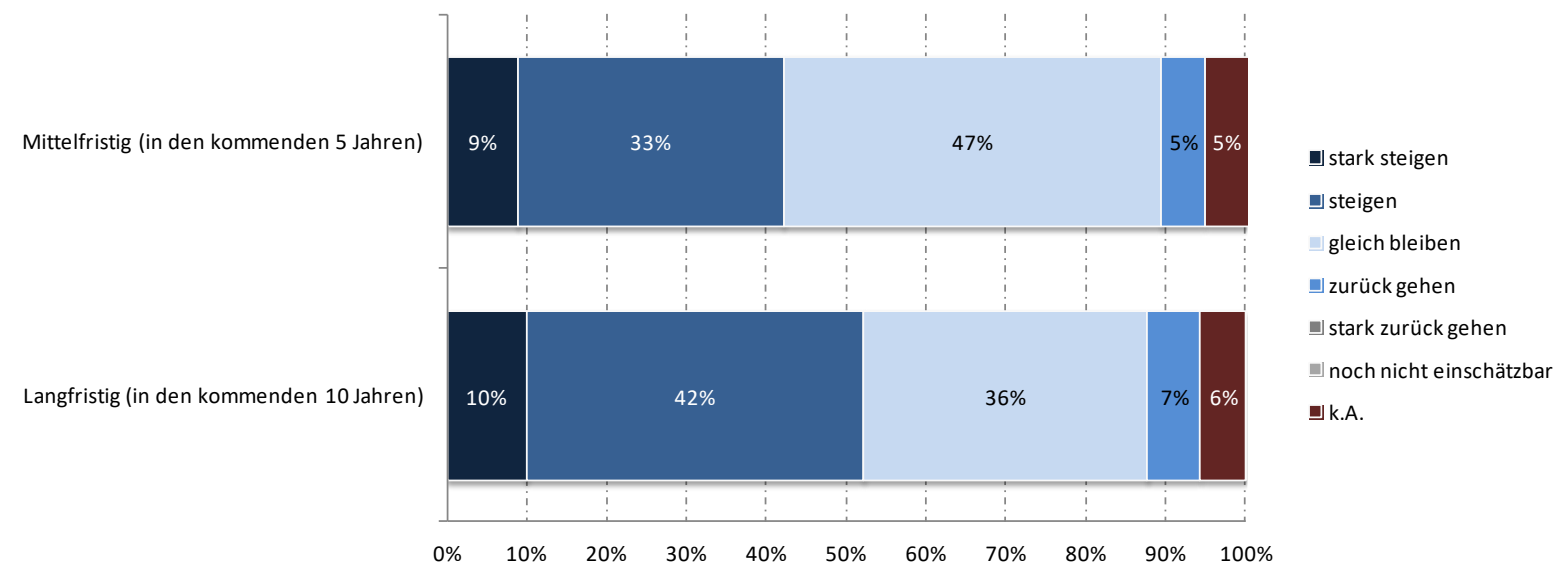

Quelle: Economica/IWI (2016); Online-Erhebung: $n=90$

Jenen Befragten, die mit einem Zurückgehen rechnen, wurden gebeten Gründe dafür zu nennen.

\section{Gründe jener, die angaben, „zurückgehen“ (Mittelfristig):}

„Der administrative Aufwand für die Abwicklung von Förderungen und die oft sehr niedrige Erfolgsquote machen es unrentabel“.

„Die CG Gesellschaft (sic!) bietet Grundlagenforschung. Wir sind ein pharmazeutisches Unternehmen und profitieren daher mehr von Anwendungsbeobachtungen oder Studien am Menschen".

„Langwierige Antragstellungen, bürokratische Abrechungsmodi, Dokumentationsaufwand, Interessenskonflikte mit publikationswütigen Professoren“.

„Bürokratie, Inkompetenz der Prüforganisation, fehlendes Industrie Know-how“.

Gründe jener, die angaben, „zurückgehen“ (Langfristig):

„Weil die administrativen Hürden bzw. Abwicklung für KMU schwer darstellbar sind. Im internationalen Vergleich die Relation von Programmmanagement zu Output deutlich schlechter ist. Unser Vergleich ist ein Programm mit Harvard durchge- 
führt in 2015. Der Kontakt Uni zur Wirtschaft sich auf das Feilschen für das Budget beschränkt. Die Uni jetzt tolle Geräte hat und ich ein mittelmäßiges Ergebnis. Deswegen bin ich vom Programm eigentlich massiv enttäuscht. Danke WR". „Administrativer Aufwand sehr hoch, kann nur mit qualifizierten Partnern vor sich gehen“.

„Der administrative Aufwand für die Abwicklung von Förderungen und die oft sehr niedrige Erfolgsquote machen es unrentabel“.

„Wie oben (Anm.: Langwierige Antragstellungen, bürokratische Abrechungsmodi, Dokumentationsaufwand, Interessenskonflikte mit publikationswütigen Professoren)“.

„Siehe oben (Anm.: Bürokratie, Inkompetenz der Prüforganisation, fehlendes Industrie Know-how)“.

Quelle: Online-Erhebung, Economica/IWI (2016) 


\subsubsection{Teilgruppenbetrachtung (Befragungsgruppe JR-Zentrum Unternehmenspartner)}

Von den 21 kontaktierten Unternehmenspartnern der JR-Zentren wurden 10 vollständig ausgefüllte Fragebögen rückübermittelt. Von zwei teilweise ausgefüllten Fragebögen wurden die Antworten bei Einzelfragen hinzugenommen.

Die Laufzeit eines JR-Zentrums von fünf Jahren wird von einem Großteil der Unternehmenspartner (83\%) als gerade richtig angesehen. Ein kleiner Teil empfindet die Laufzeit zu kurz (17\%). Als zu lange empfindet (wie auch bei den JR-Zentrumsleiterinnen und -leitern) keiner die Laufzeit des Programms.

Abbildung 116: Beurteilung der Laufzeit des JR-Zentrums (von 5 Jahren)

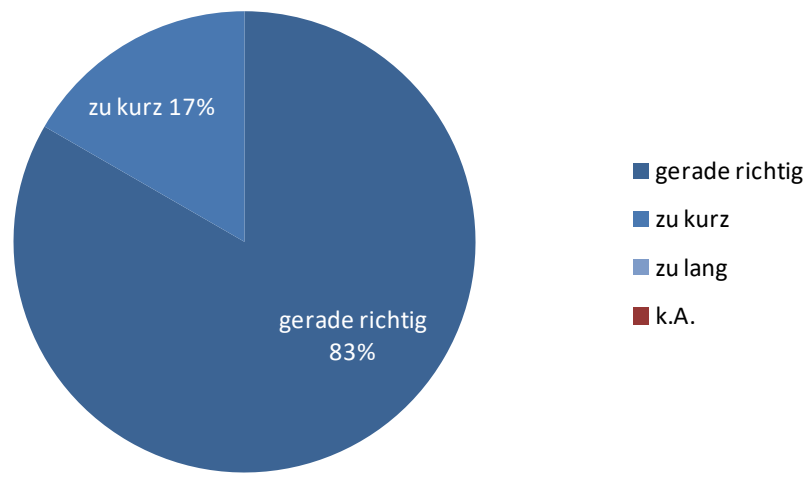

Quelle: Economica/IWI (2016); Online-Erhebung: $n=12$

Hinsichtlich einzelner Aspekten des Programms und der Programmabwicklung herrscht (hohe) Zufriedenheit mit der Klarheit des Förderkonzepts und des Förderziels. Auch mit der Angemessenheit der Evaluierungskriterien und der Bearbeitungsdauer der eingereichten Anträge zeigen sich die Befragten (sehr) zufrieden. 


\section{Abbildung 117: Zufriedenheit mit Aspekten des Programms bzw. der Programmabwicklung}

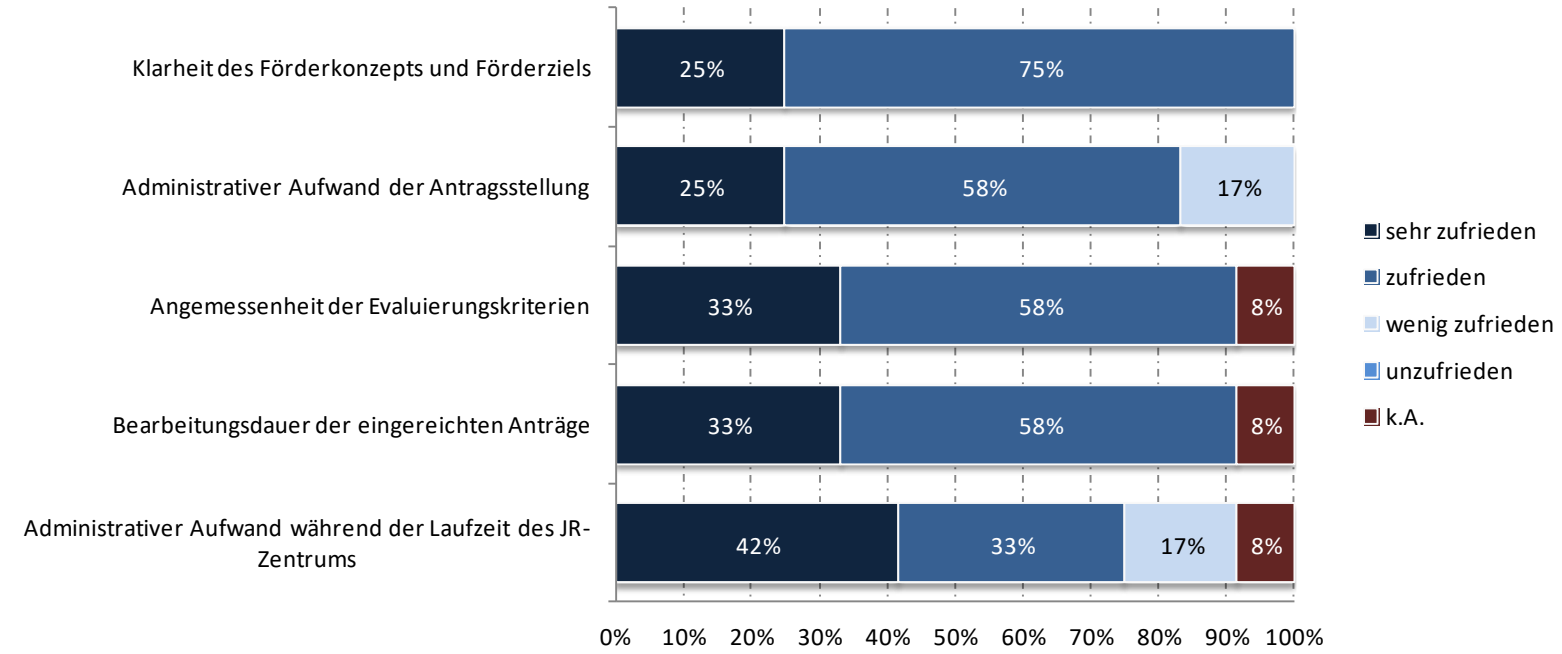

Quelle: Economica/IWI (2016); Online-Erhebung: $n=12$

Der administrative Aufwand der Antragstellung sowie der Aufwand während der Laufzeit des JRZentrums werden mehrheitlich positiv wahrgenommen, vereinzelt gibt es aber auch Unternehmen, die sich weniger zufrieden zeigen.

\section{Exkursbox: Potential für administrative Vereinfachungen}

Nach Gründen befragt, wurden von diesen Unternehmen u.a. die zahlreichen Einverständniserklärungen oder auch ein hoher Aufwand für Nachweise genannt.

„Zu viele Einverständniserklärungen zu allen möglichen Dingen (Beispiel: Mitwirkung eines neuen Partners)“.

„Weniger Aufwand für Nachweise, wobei die Nachweisverpflichtung verständlich ist“.

Quelle (Zitate): Online-Erhebung, Economica/IWI (2016)

Die begleitende wissenschaftliche Kontrolle wird von den Unternehmenspartnern als (sehr) hilfreich wahrgenommen. Hier zeigt sich eine einheitlich positive Bewertung. 
Abbildung 118: Einschätzung der begleitenden wissenschaftlichen Kontrolle

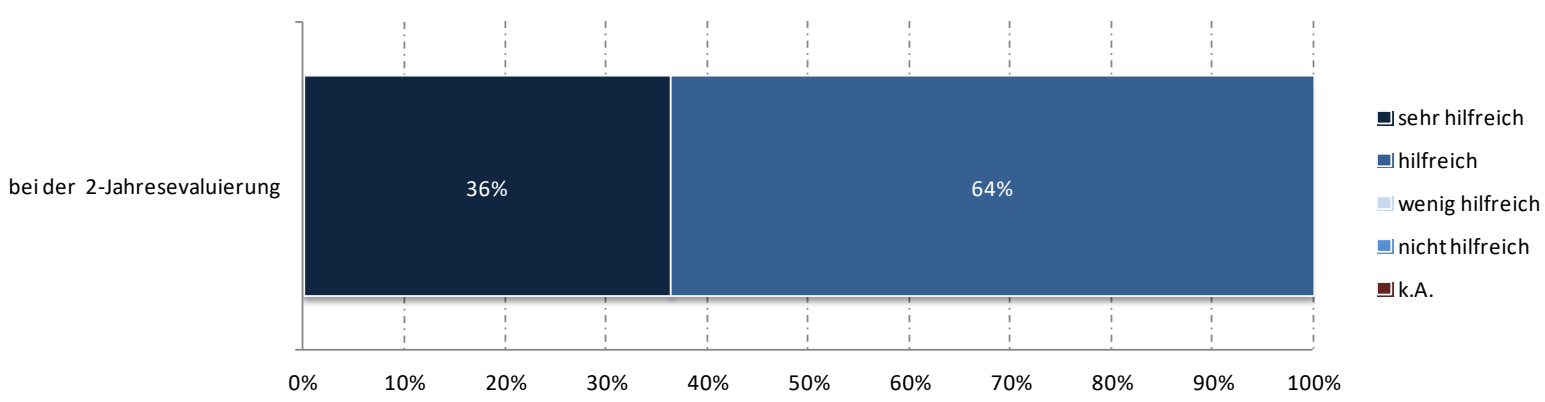

Quelle: Economica/IWI (2016); Online-Erhebung: $n=11$

Zufriedenheit besteht auch mit den Betreuungs-Leistungen der CDG während der Antragstellung und Laufzeit des JR-Zentrums. Was die Öffentlichkeitsarbeit der CDG betrifft sieht ein Drittel der Unternehmen noch Verbesserungsmöglichkeiten, zwei Drittel sind jedoch (sehr) zufrieden.

\section{Abbildung 119: Zufriedenheit mit den Leistungen der CDG}

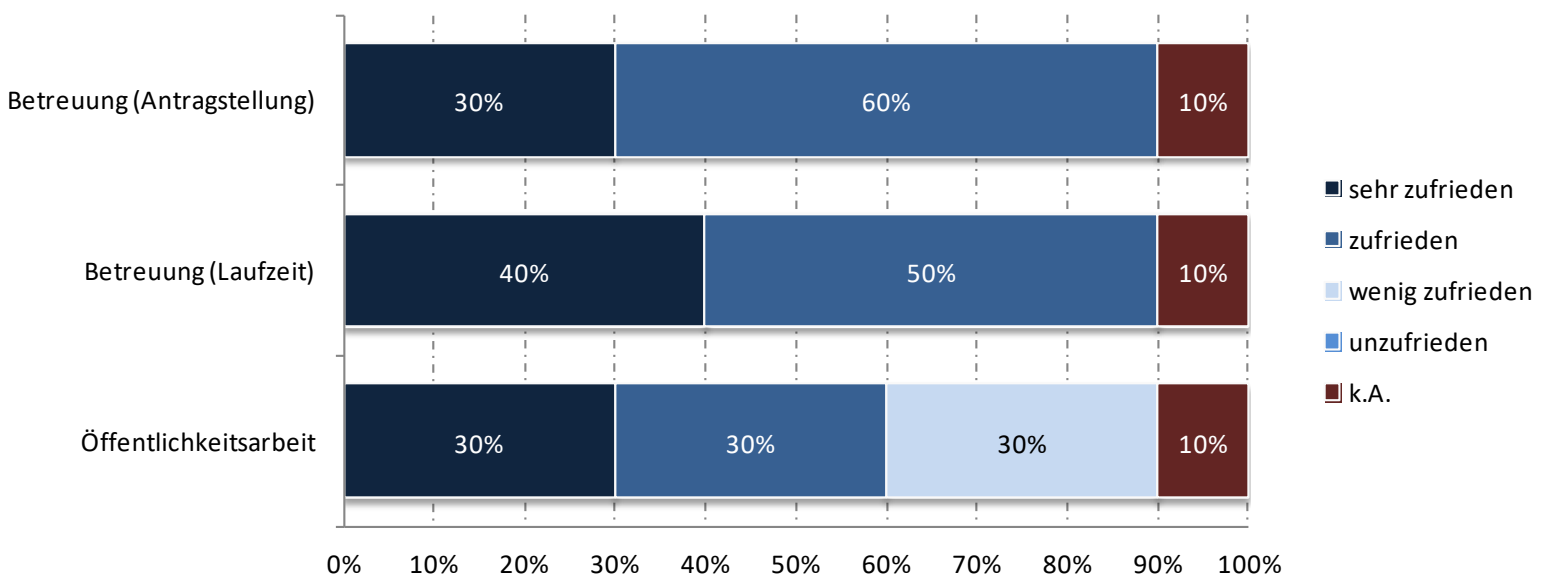

Quelle: Economica/IWI (2016); Online-Erhebung: $n=10$

Was die Motivation als Unternehmenspartner zur JR-Zentrumsgründung betrifft so ist den Befragten hier vor allem der Einstieg in ein neues Forschungsthema sowie die langfristige Sicherung des Zugangs zu wissenschaftlichem Know-how wichtig. Ebenso eine wichtige Motivation bildet der Aufbau einer strategischen Allianz mit der Fachhochschule und in diesem Rahmen auch der Zugang zu jungen Talenten. Geringere Bedeutung hat u.a. der Aufbau einer Kooperationsplattform mit anderen Unternehmen oder auch die Tatsache, dass eigene anwendungsorientierte Forschung zu teuer wäre. 
Abbildung 120: Motivation als Unternehmenspartner zur JR-Zentrumsgründung

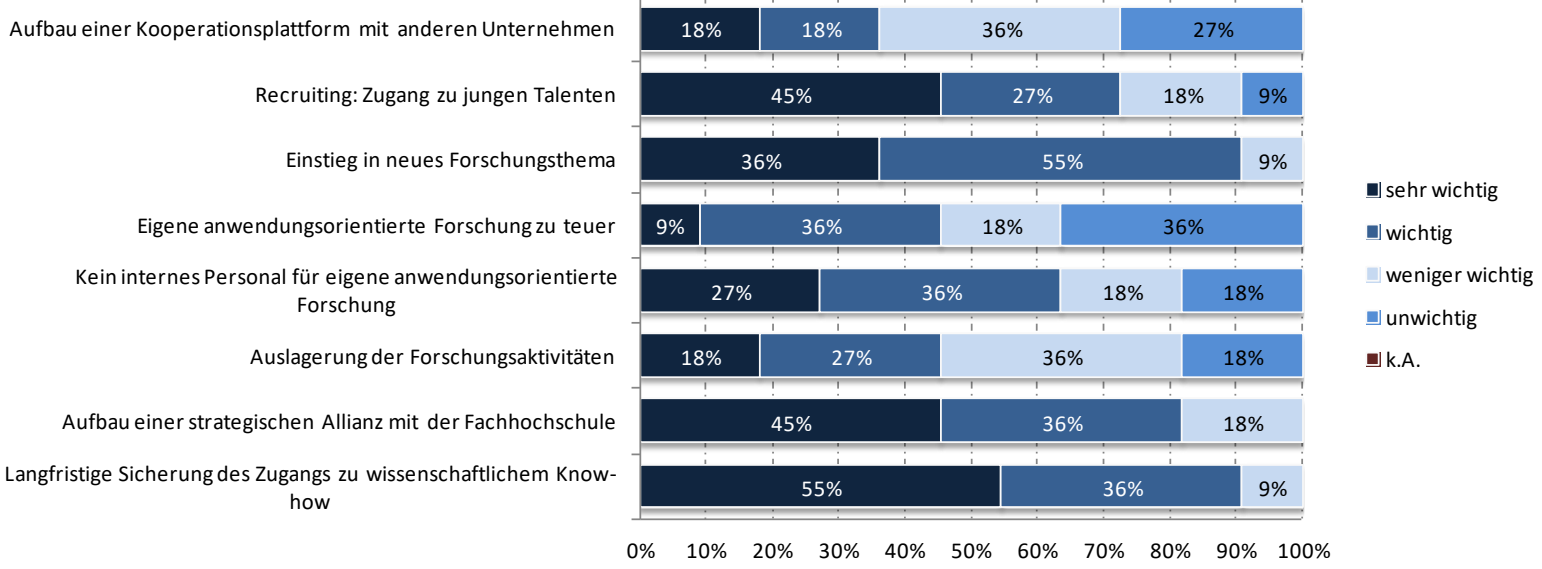

Quelle: Economica/IWI (2016); Online-Erhebung: $n=11$

Auch die Unternehmenspartner der JR-Zentren wurden um einen Vergleich zu rein unternehmensintern durchgeführten F\&E-Tätigkeiten und Forschungsaktivitäten im JR-Zentrum gebeten. Auch die Forschungsaktivitäten im JR-Zentrum tendieren (analog zu den Forschungsaktivitäten im CD-Labor) zu einer höheren technologischen Komplexität und sind wissenschaftlich anspruchsvoll.

Abbildung 121: Art der Forschungsaktivitäten im JR-Zentrum

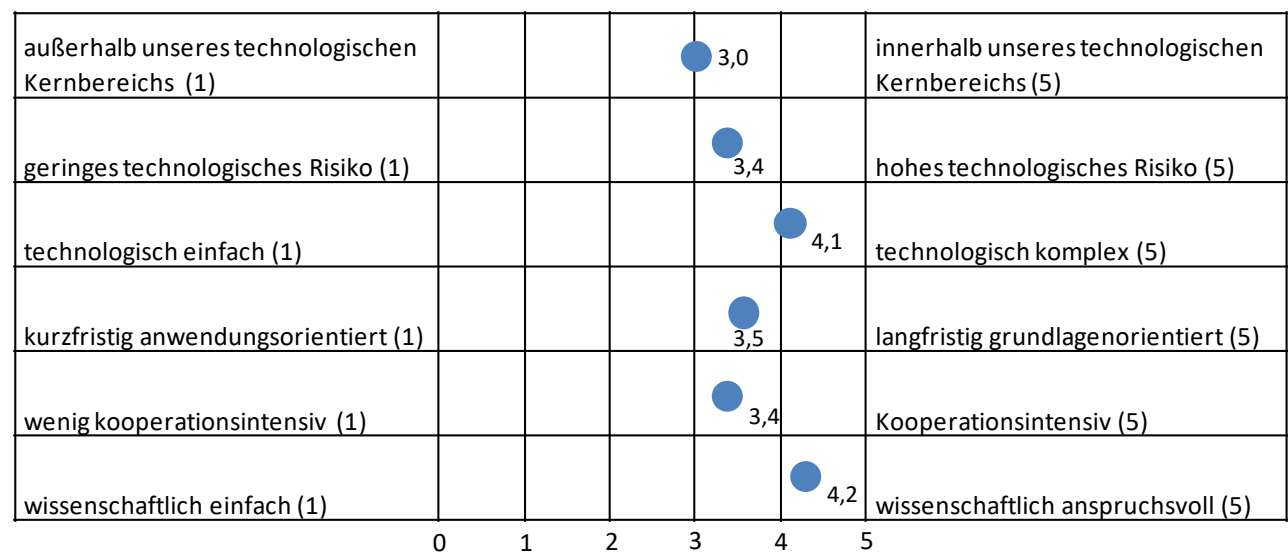

Anm.: Die Unternehmen hatten die Möglichkeit, der Art ihrer Forschungsaktivitäten in den einzelnen Kategorien mit den Wert 1, 2, 3, 4 bzw. 5 zuzuordnen.

Quelle: Economica/IWI (2016); Online-Erhebung: $n=10$ 
Kontakt zu den Wissenschaftlerinnen und Wissenschaftlern vor der JR-Zentrumsgründung bestand bei der Hälfte der Befragten über gemeinsame Forschungsprojekte. In den anderen Fällen bestanden meist, zumindest lose, Kontakte, in lediglich einem Fall gab es vor der Gründung des JR-Zentrums gar keinen Kontakt zur Leiterin bzw. dem Leiter.

Abbildung 122: Kontakt zu Wissenschaftlern/Wissenschaftlerinnen vor der JR-Zentrumsgründung

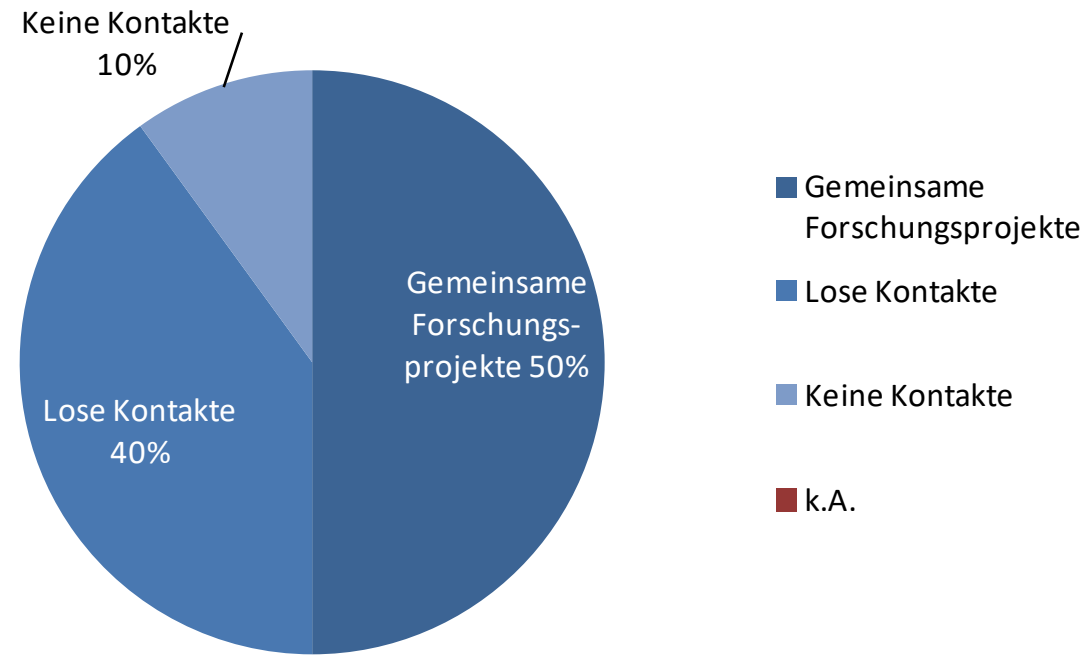

Quelle: Economica/IWI (2016); Online-Erhebung: $n=10$

Den Einfluss seitens des Unternehmens auf die Forschungsprogrammformulierung bewerten die Befragten unterschiedlich. Eine Hälfte sieht hier einen (sehr) hohen Einfluss, die andere Hälfte jedoch nur einen geringen. Ein etwas höherer Einfluss zeigt sich bei der Moduldefinition. Hier sehen $70 \%$ einen hohen Einfluss, kein Unternehmen jedoch einen sehr hohen. 
Abbildung 123: Einfluss seitens des Unternehmens auf die Forschungsprogrammformulierung und auf die Moduldefinition

die Forschungsprogrammformulierung

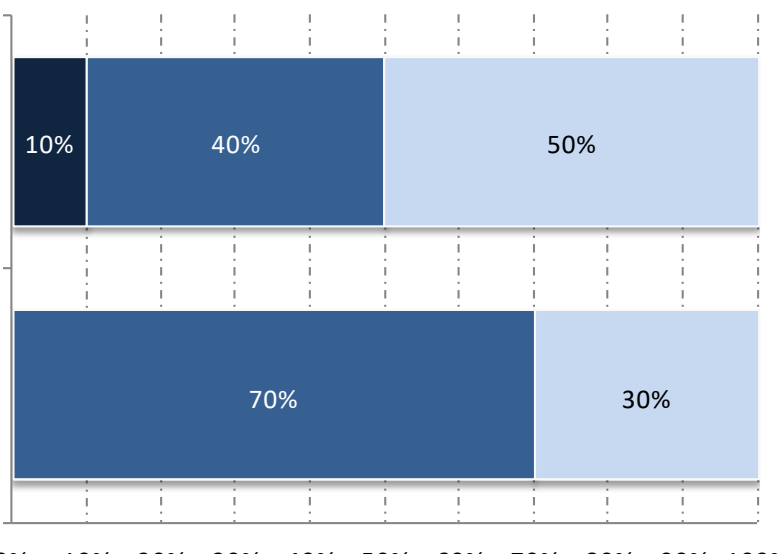

- sehr hoher Einfluss

$\square$ hoher Einfluss

geringer Einfluss

$\square$ kein Einfluss

die Moduldefinition

$\begin{array}{lllllllllllll}0 \% & 10 \% & 20 \% & 30 \% & 40 \% & 50 \% & 60 \% & 70 \% & 80 \% & 90 \% & 100 \%\end{array}$

$\square \mathrm{k} . \mathrm{A}$

Quelle: Economica/IWI (2016); Online-Erhebung: $n=10$

Inwieweit ein JR-Zentrum bei der Durchführung der Forschungskooperationen eher eigenständig oder kooperativ an den von den Unternehmen definierten Aufgaben arbeitet wird von den Befragten unterschiedlich gesehen. Eine Hälfte der Unternehmen bewertet die Arbeiten im JR-Zentrum (eher) eigenständig, die andere Hälfte (eher) kooperativ.

\section{Abbildung 124: JR-Zentrum eher eigenständig oder kooperativ}

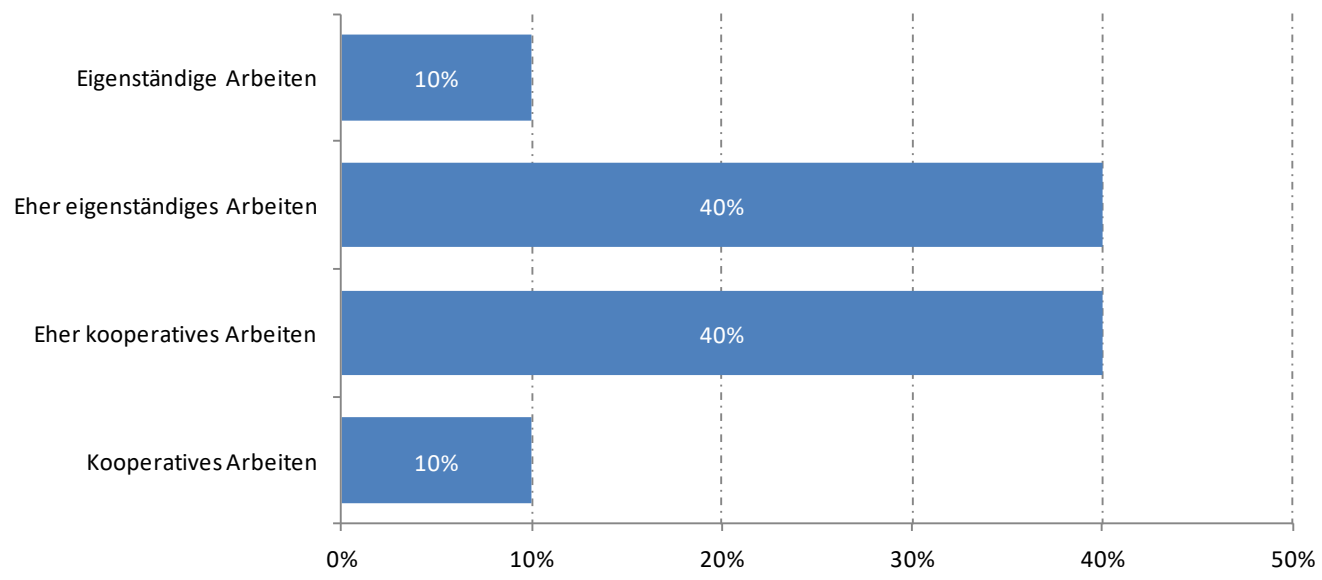

Quelle: Economica/IWI (2016); Online-Erhebung: $n=10$ 
Die Häufigkeit der Kontakte mittels Email, Telefon etc. findet bei $60 \%$ mehrmals pro Monat, bei den restlichen 40\% zumindest mehrmals pro Quartal statt. Auch der Kontakt bei gemeinsamen Arbeitssitzungen ist relativ hoch und findet bei $80 \%$ zumindest mehrmals pro Quartal statt.

\section{Abbildung 125: Häufigkeit der Kontakte}

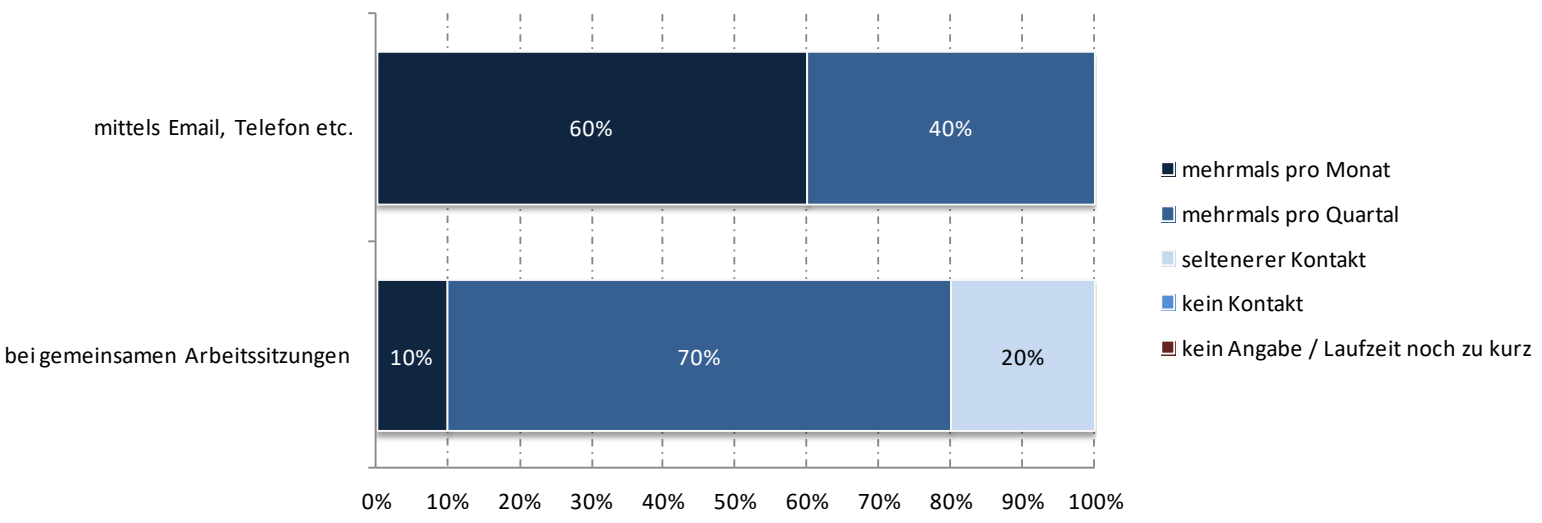

Quelle: Economica/IWI (2016); Online-Erhebung: $n=10$

Wenn es um die Bewertung von Aspekten der Zusammenarbeit im JR-Zentrum geht, so wird insbesondere das Verständnis des JR-Zentrums für die Problemstellung als auch die wissenschaftliche Qualität der Arbeiten (sehr) gut bewertet. Auch die Termintreue wird ausnahmslos positiv gesehen.

\section{Abbildung 126: Bewertung von Aspekten der Zusammenarbeit im JR-Zentrum}

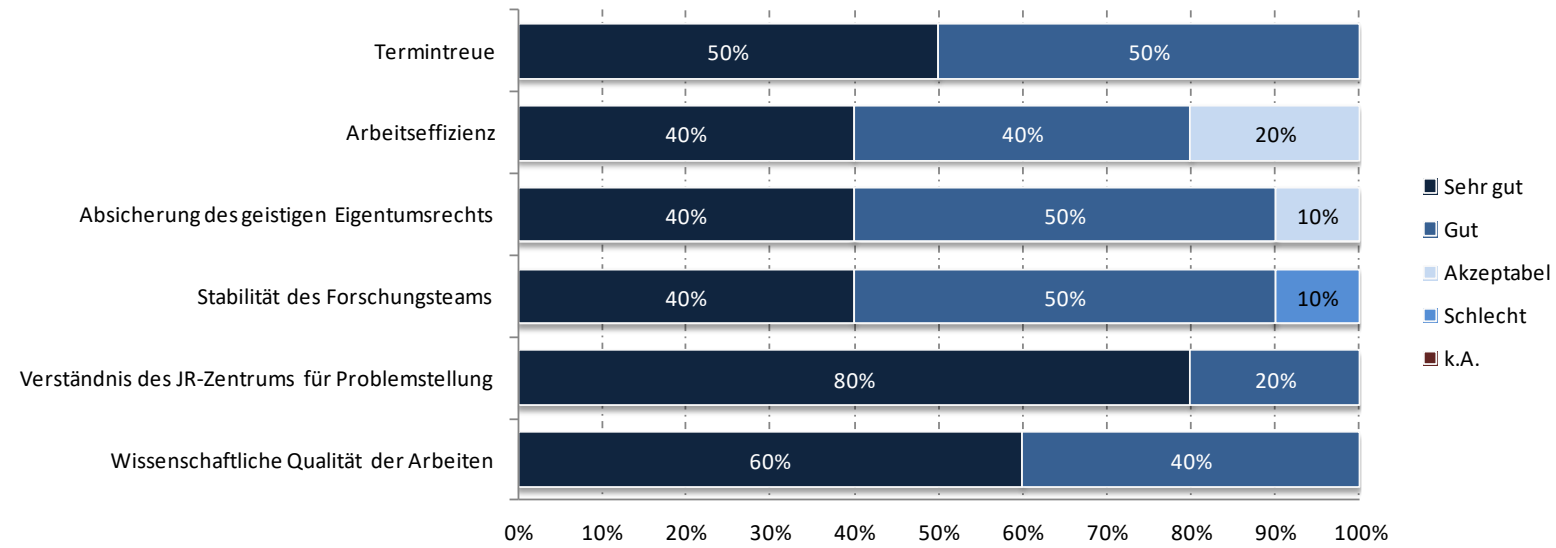

Quelle: Economica/IWI (2016); Online-Erhebung: $n=10$ 
Insgesamt 4 Unternehmen gaben an, dass zumindest ein weiteres Unternehmen am JR-Zentrum beteiligt war (Partnerunternehmen aus der gleichen Branche: 2 Nennungen, aus der Zulieferbranche: 2 Nennungen, auf Kundenseite: 1 Nennung).

Die Forschungsergebnisse aus der Beteiligung am JR-Zentrum haben bzw. hatten für die Unternehmen vor allem Relevanz bei der Hilfe der Bewertung verschiedener Lösungsstrategien (Radarfunktion) sowie für die inkrementelle Verbesserung bestehender Produkte/Prozesse und die Entwicklung radikal neuer Produkte. Vereinzelt war auch keine unmittelbare Nutzung im Vordergrund, aber die Möglichkeit die Forschungsergebnisse zukünftig zu nutzen bzw. einzusetzen.

Abbildung 127: Haben bzw. hatten die Forschungsergebnisse aus der Beteiligung am JR-Zentrum wirtschaftliche Relevanz für folgende Aspekte?

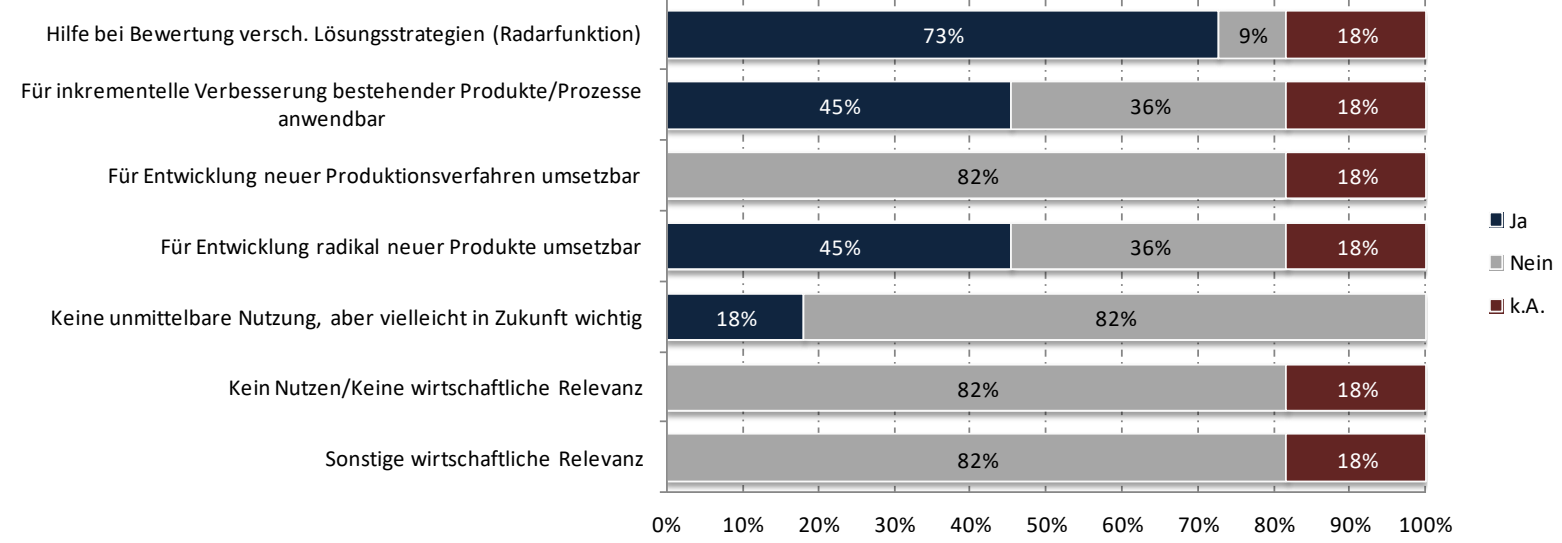

Quelle: Economica/IWI (2016); Online-Erhebung: $n=11$

Nach dem Nutzen durch die JR-Zentrumsgründung und -beteiligung gefragt, sehen die Unternehmen vor allem den Zugang zu qualifizierten Mitarbeitern und das Aufzeigen neuer technologischer Optionen als bedeutend an. Hier bewerten alle Befragten den Nutzen als sehr hoch bzw. hoch. Auch hinsichtlich einer Stärkung des österreichischen F\&E-Standorts und des Kompetenzaufbaus, insb. auch die Stärkung der technologischen Problemlösungskompetenz, bewerten nahezu alle Befragten (90\%) den Nutzen als (sehr) hoch.

Etwas geringer wird der Nutzen hinsichtlich eines Abbaus der Zugangsbarrieren zu wissenschaftlicher Forschung gesehen. Auch hinsichtlich eines Prestigegewinns bzw. der internationalen Wettbewerbsfähigkeit sehen einzelne Unternehmen keinen oder nur geringen Nutzen. 
Abbildung 128: Nutzen für die Unternehmen durch die JR-Zentrumsgründung bzw. -beteiligung

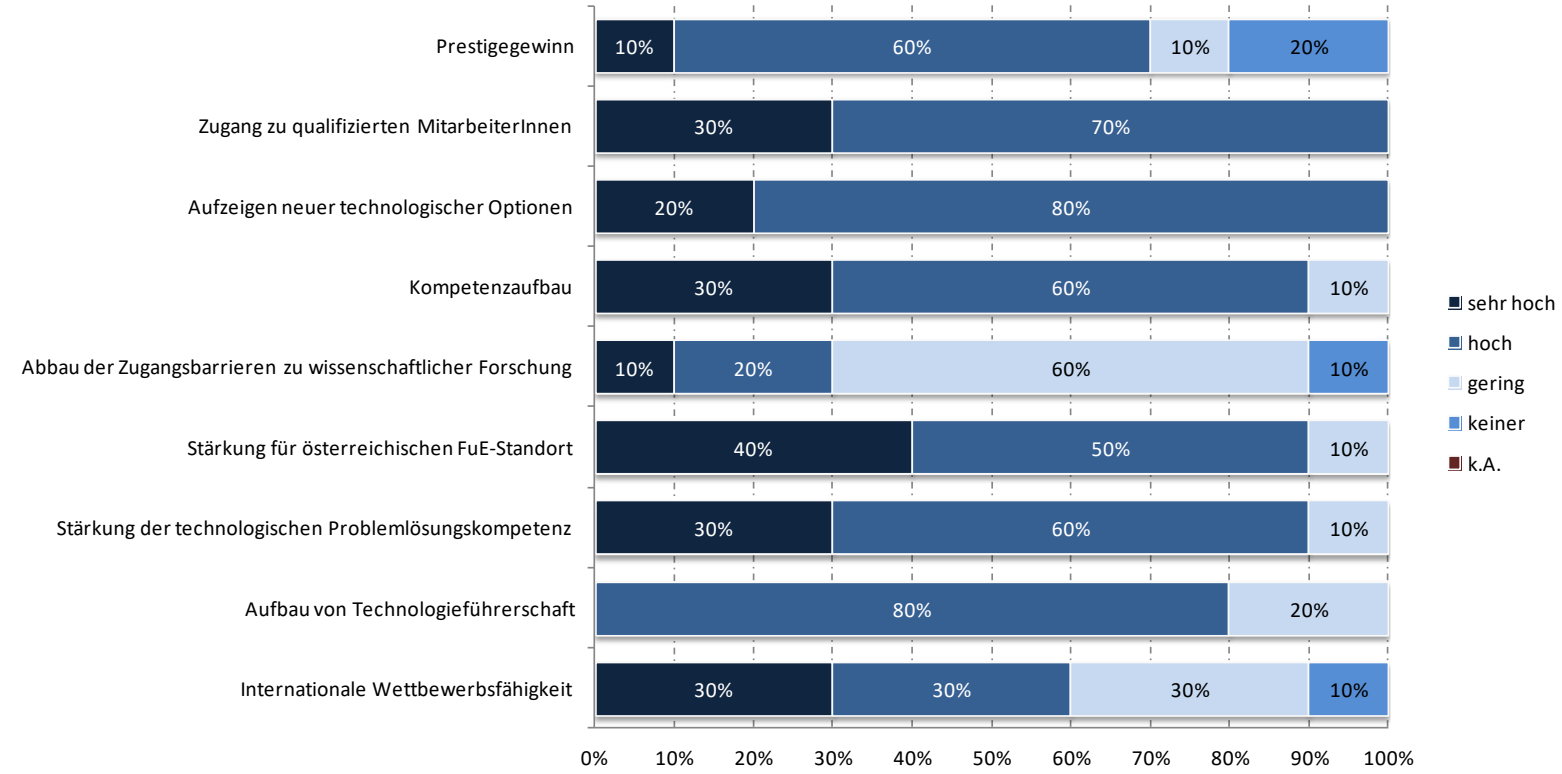

Quelle: Economica/IWI (2016); Online-Erhebung: $n=10$

Auf weitere Auswirkungen der JR-Zentrumsgründung auf das Unternehmen angesprochen, sehen die Befragten vor allem eine bessere Umsetzung sowie eine gewonnene Kontinuität der F\&E-Aktivitäten. Gestiegene F\&E-Ausgaben sehen die wenigsten der Befragten.

\section{Abbildung 129: Auswirkungen der JR-Zentrumsgründung auf Unternehmen}

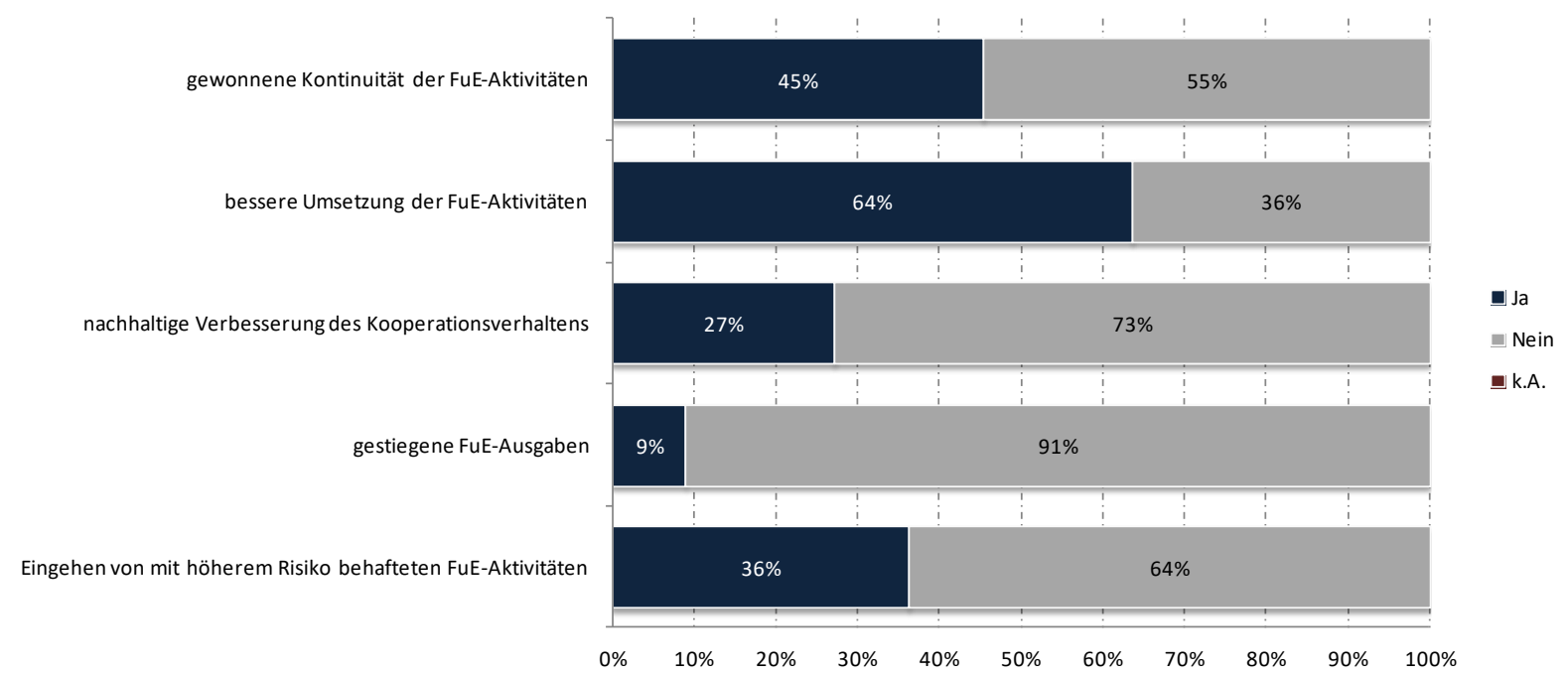

Quelle: Economica/IWI (2016); Online-Erhebung: $n=11$ 
Was eine Kosten-Nutzen Relation betrifft sieht die Hälfte der Befragten den Nutzen (deutlich) höher als die Kosten, 40\% sind der Ansicht der Nutzen entspricht den Kosten.

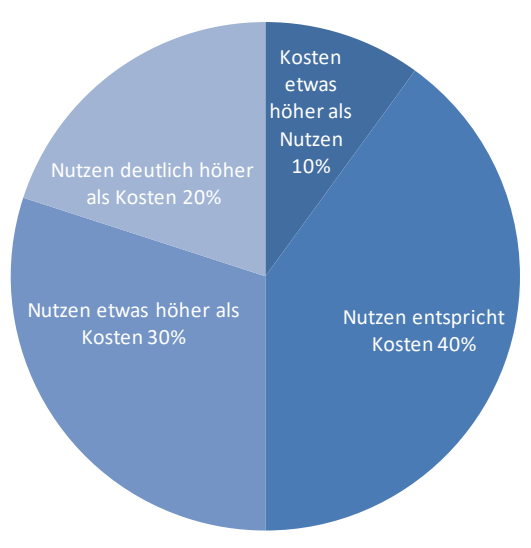

- Kosten deutlich höher als Nutzen

Kosten etwas höher als Nutzen

Nutzen entspricht Kosten

Nutzen etwas höher als Kosten

Nutzen deutlich höher als Kosten

a.A.

Quelle: Economica/IWI (2016); Online-Erhebung: $n=10$

$60 \%$ der Unternehmen planen weitere Forschungsprojekte im Rahmen eines JR-Zentrums in gleichem Ausmaß, ein Unternehmen sogar in höherem Ausmaß. Keines der Unternehmen gab an, kein Forschungsprojekt mehr im Rahmen des JR-Programms zu planen, was für eine grundsätzlich hohe Zufriedenheit der Unternehmenspartner spricht. 


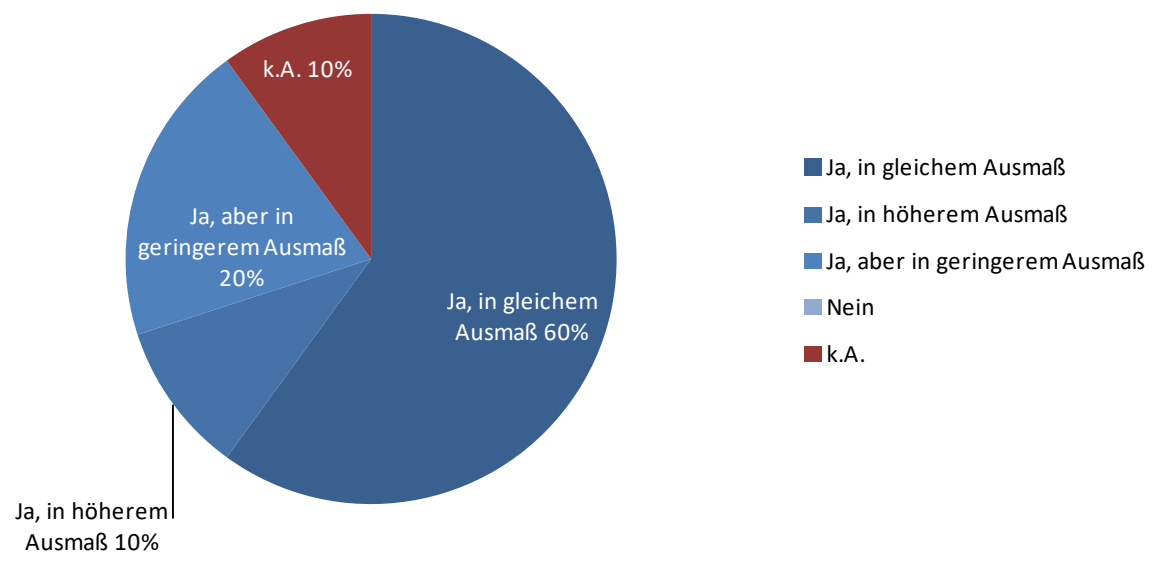

Quelle: Economica/IWI (2016); Online-Erhebung: $n=10$

Ein Großteil der Unternehmen hätte das Forschungsprojekt ohne JR-Zentrum lediglich in (deutlich) geringerem Umfang durchgeführt, ein Unternehmen gar nicht.

\section{Abbildung 132: Durchführung des Forschungsprojekts auch ohne JR-Zentrum?}

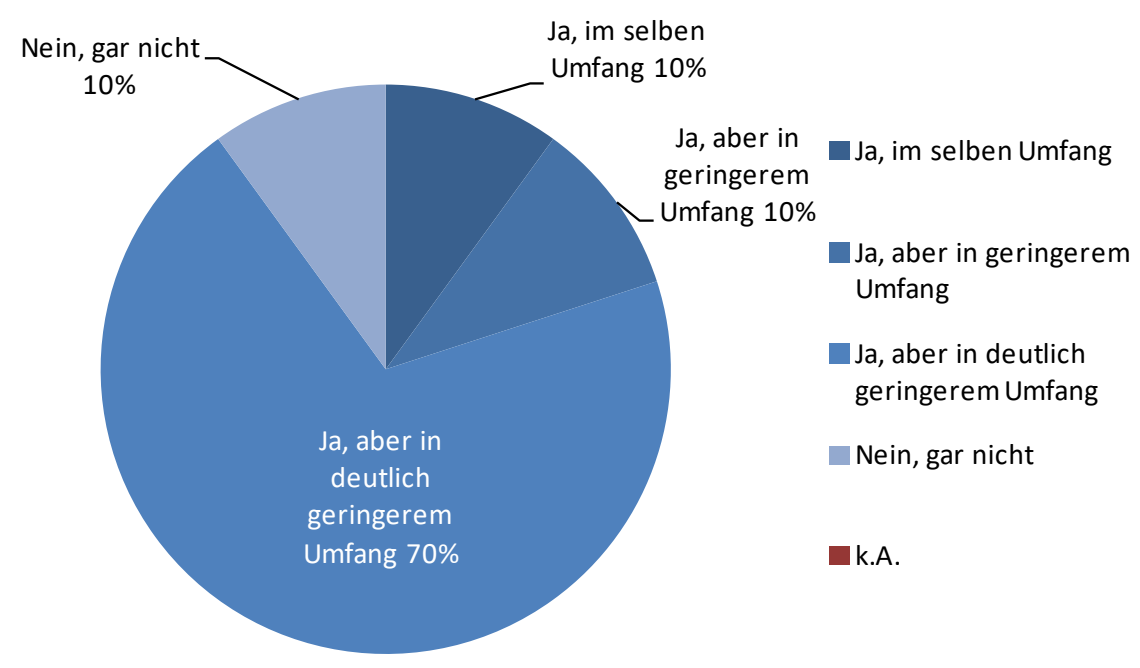

Quelle: Economica/IWI (2016); Online-Erhebung: $n=10$

Jene Unternehmen, die das Forschungsprojekt auch ohne JR-Zentrum durchgeführt hätten - wenn auch in geringerem Ausmaß - wurden gefragt, wie die Finanzierung ausgesehen hätte. Ein Großteil der Unternehmen hätte in diesem Fall eine firmeninterne Finanzierung angestrebt (78\%), zwei Unter- 
nehmen (22\%) hätten eine Finanzierung über andere Forschungsprogramme versucht. Genannt wurden in diesem Zusammenhang das österreichische Förderungsprogramm für Sicherheitsforschung - KIRAS (http://www.kiras.at/home/) sowie Programme der FFG bzw. CDG (CD-Labor).

Abschließend wurden auch die Partnerunternehmen der JR-Zentren gebeten einzuschätzen, wie sich die Nutzung direkter Forschungsförderprogramme in Zukunft im Unternehmen entwickeln wird. Mittel- und langfristig gehen $70 \%$ von einem Steigen bzw. Gleichbleiben der Nutzung aus.

Abbildung 133: Nutzung direkter Forschungsförderprogramme in Zukunft im Unternehmen

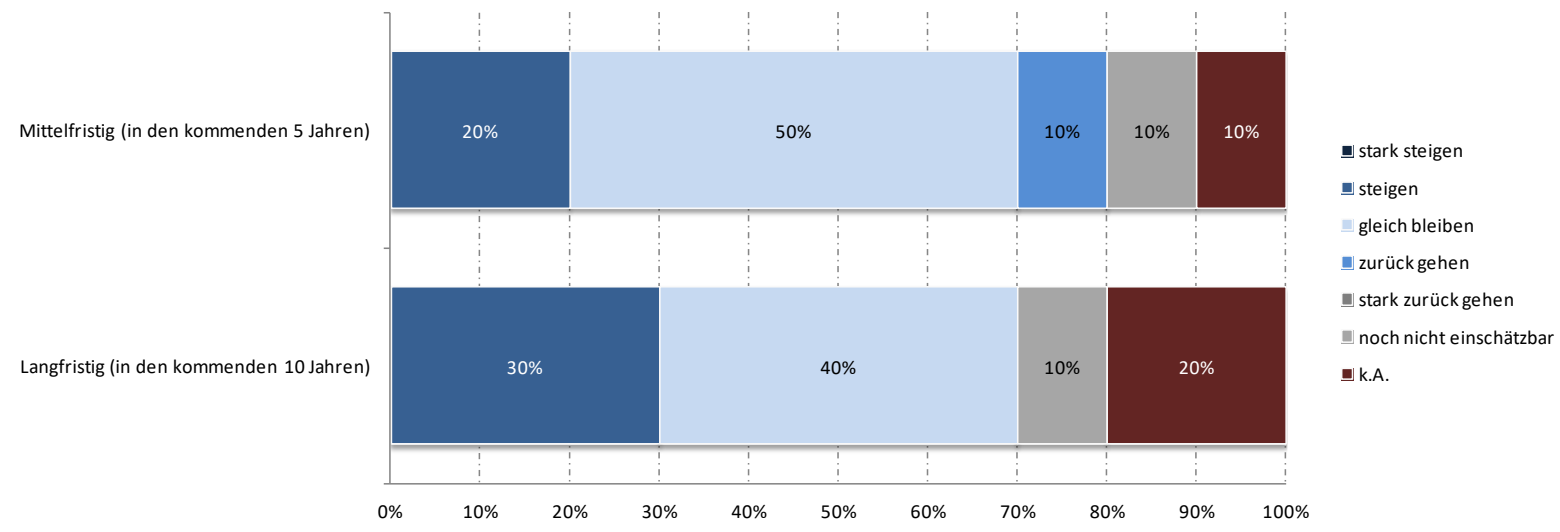

Quelle: Economica/IWI (2016); Online-Erhebung: $n=9$

Von einem (mittelfristigen) Rückgang geht lediglich eines der befragten Unternehmen aus und nannte dafür folgende Gründe:

\section{Auszug aus den Fragebögen (Zitate):}

„Kosten überwiegen; Kostendruck aufgrund EU-Regulierungen steigt derart an, dass für F\&E wenig übrigbleibt“.

Quelle (Zitate): Online-Erhebung, Economica/IWI (2016) 


\subsection{Schlussfolgerungen}

Die kumulierte Bewertung aller Stakeholder-Gruppen liefert ein Gewichtungsschema über alle Interessen hinweg, das einen gewissen Ausgleich der unterschiedlichen Erwartungshaltungen an das CDG-Programm bildet. Es ist ganz klar ersichtlich, dass es teilweise sehr unterschiedliche Erwartungshaltungen an das CDG-Programm gibt, was auf die heterogenen Bedürfnisse und Sichtweisen der Beteiligten zurückzuführen ist.

Insgesamt gesehen liegt der Fokus eindeutig auf dem Bereich Forschung, wobei diese zwar grundlagenorientiert, aber gleichzeitig auch eine praxisrelevante Dimension aufweisen sollte. Einen zweiten bedeutenden Bereich stellt die Umsetzung der gewonnenen Erkenntnisse in Form von Humanressourcen und Wissenstransfer dar. Die weiteren Programmziele werden anscheinend teilweise als Resultat der ersten beiden Blöcke angesehen, und erhalten daher ein geringeres Gewicht.

Laut Abschlussfragebögen der Laborleiter fließen die Erfahrungen des CD-Labors nun noch etwas schwächer in die Lehre ein, haben aber in der Forschung nach wie vor eine hohe Bedeutung. Die Zusammenarbeit mit der Industrie wird von den Laborleitern durchwegs positiv beurteilt, und sogar noch etwas besser als in der Vorgängerevaluierung bewertet.

Eine Folgeexpertise nach dem Auslaufen des CD-Labors gab es einerseits, als das Ausmaß der Forschungsarbeiten zumindest in zwei Drittel der Fälle gleich blieb, was konform zur letzten Untersuchung war, wobei der Anteil der Antwort „in größerem Ausmaß“ nun geringer ist. Andererseits gingen in mehr als der Hälfte der CD-Labors Wissenschaftler hervor, die eigene Forschungsgruppen leiten. Darüber hinaus wechselten in 90 \% (88 \% Evaluierung 2011) der CD-Labors Mitarbeiterinnen und/oder Mitarbeiter während der Laufzeit des Labors in die relevante Industrie, was einem Schnitt von 3,6 $(4,08)$ Abgängen in die Wirtschaft pro CD-Labor entspricht. Der Wechsel von Laborpersonal in Unternehmen als ein Indikator für das Programmziel „Entwicklung von Humanressourcen" ist somit relativ etwas geschrumpft, wobei die absolute Anzahl von 102 auf 151 Personen angestiegen ist. Gemeinsame PR-Aktivitäten des CD-Labors und der Unternehmenspartner verlieren an Bedeutung.

Ein Großteil der befragten Laborleiter sehen durch die Forschungsaktivitäten des Heimat gebenden Instituts eine nachhaltige Verstärkung im Themenbereich des CD-Labors, wobei die einzelnen Teilbereiche, wie Erhöhung des Mitarbeiterstandes, Verbesserung der Drittmittelsituation und Einrichtung an Bedeutung verlieren, während sich der Einfluss auf Berufungen in dem behandelten Themenbereich erhöht. Dies spiegelt sich in der universitären Lehre hauptsächlich in inhaltlichen Erwei- 
terungen von Lehrveranstaltungen wider, weniger in der Einführungen neuer Lehrveranstaltungen. Das CD-Labor hat generell eine positive Auswirkung auf das Drittmittelvolumen des Heimat gebenden Instituts, auch wenn die zusätzlichen Mittel außerhalb des Themenbereichs des CD-Labors zustande kamen.

Die Online-Erhebung zeigt hinsichtlich der CD-Labors, sowohl bei den CD-Laborleiterinnen/-Laborleitern, als auch bei den Unternehmenspartnern, eine hohe Zufriedenheit mit der Klarheit des Förderkonzepts und des Förderziels. Die Laufzeit des CD-Programms mit sieben Jahren wird mehrheitlich als gerade richtig erachtet. Bei den befragten CD-Laborleiterinnen/-Laborleitern beträgt die Zustimmung 84\%, bei den beteiligten Unternehmenspartnern $71 \%$. Bei den Unternehmenspartnern ist jene Gruppe, welche die Laufzeit als „zu lang“ empfindet - seit 2011 - allerdings etwas angewachsen (von $16 \%$ auf 22\%). Dies ist eine Entwicklung, die man in den kommenden Jahren beobachten sollte.

Anzahl und Zeitpunkt der Zwischenevaluierungen wird von der großen Mehrheit der Laborleiterinnen/Laborleiter als passend empfunden. Mehr Zwischenevaluierungen findet keiner der Befragten wünschenswert. Jene, die den Zeitpunkt der Zwischenevaluierungen als unpassend empfinden, sehen vor allem die 2-Jahresevaluierung kritisch bzw. verfrüht. Dies betrifft vor allem jene Labors, die einen „späteren Laborstart" haben und daher oft mit einer „1,25 bzw. 1,5-Jahresevaluierung" konfrontiert sind.

Mit der Betreuungsleistung der CDG im Rahmen der CD-Labors herrscht hohe Zufriedenheit, sowohl auf Seiten der Wissenschaft, als auch auf Unternehmensseite. Die CDG sei grundsätzlich immer bemüht eine Lösung zu finden, ist der allgemeine Tenor. Wenn Verbesserungsvorschläge und Wünsche genannt werden, gehen diese meist in Richtung flexibleres Vorgehen bzw. weniger Formalismen. In diesem Zusammenhang wurde auch das Aufeinanderprallen von zwei formalen und teils starren Systemen/Regelwerken - Universität(sverwaltung) und CDG - angesprochen, welches dann teils im Arbeitsalltag der Laborleiterin/des Laborleiters - im Rahmen administrativer „Kleinigkeiten“ - mit der Suche nach (Vermittlungs-)Lösungen oft unnötigen Aufwand verursacht.

Bei den Unternehmenspartnern ist auch die Tatsache, dass über $70 \%$ ein weiteres Forschungsprojekt im Rahmen eines CD-Labors planen, ein Indiz für eine hohe Zufriedenheit mit dem Programm und der Programmabwicklung.

Der Trend zur CD-Laborgründung als "gemeinsame Initiative“ von wissenschaftlichem Partner und Unternehmenspartner hält an, geht ansonsten jedoch stärker vom wissenschaftlichen Partner aus. Während 2005 die Initiative zur Laborgründung noch sehr stark entweder vom wissenschaftlichen 
Partner oder vom Unternehmenspartner ausging, zeigte sich bereits 2011 eine starke Verschiebung hin zu einer „gemeinsamen Initiative“. Dieser Trend verstärkt sich bei der aktuellen Befragung nochmals leicht. Interessant zu beobachten ist ein stetiger Rückgang hinsichtlich einer Initiative zur Laborgründung von Seiten der Unternehmenspartner.

Der hohe Anteil an Laborgründungen als „gemeinsame Initiative“ lässt sich auch auf die Tatsache zurückführen, dass fast alle Wissenschaftlerinnen/Wissenschaftler (ähnlich wie bereits 2011) schon vor CD-Laborgründung Kontakt zum Unternehmenspartner - meist über gemeinsame Forschungsprojekte - hatten, woraus sich dann häufig die gemeinsame Initiative eines (weiterführenden) Labors entwickelt.

Bei den Erwartungen und Zielen im Zusammenhang mit der Gründung eines CD-Labors ist nach wie vor ein langfristig abgesichertes Arbeiten an einem Themenkreis von zentraler Bedeutung für die Laborleiter und Laborleiterinnen. Alle Befragten bewerten diesen Aspekt als wichtig, 90\% davon als sehr wichtig. Dies stärkt auch die Bedeutung einer langen, in diesem Fall 7-Jährigen Laufzeit, die dies ermöglicht. Das Thema Langfristigkeit und Intensität der Zusammenarbeit wurde auch in den begleitend geführten Gesprächen immer wieder betont und teils auch als „Alleinstellungsmerkmal“ des CDG-Programms hervorgehoben. Auch die finanzielle Absicherung der Forschungsarbeit sowie der Aufbau eines eigenen Forschungsteam, die damit in Zusammenhang stehen, werden als (sehr) bedeutend angesehen.

Bei den Unternehmenspartnern sind (wie auch bereits 2011) insbesondere die langfristige Sicherung des Zugangs zu wissenschaftlichem Know-how und der Aufbau einer strategischen Allianz mit den Universitäten bedeutsam. Auch der Einstieg in ein neues Forschungsthema sowie die Tatsache, dass eigene anwendungsorientierte Grundlagenforschung zu teuer ist, sind (nach wie vor) wichtige Gründe, sich im Rahmen des CD-Programms zu engagieren.

Hohen Nutzen durch die CD-Laborgründung bzw. -beteiligung sehen die befragten Unternehmen vor allem im Bereich Kompetenzaufbau, dem Aufzeigen neuer technologischer Optionen und in der Stärkung der technologischen Problemlösungskompetenz. Auch der Zugang zu qualifizierten Mitarbeitern, sowie der Aufbau von Technologieführerschaft haben für die Mehrheit der befragten Unternehmen einen (sehr) hohen Nutzen.

Die wissenschaftlich Qualität der Arbeiten im Labor, sowie das Verständnis des CD-Labors für die Problemstellung werden von Unternehmensseite mehrheitlich sehr positiv bewertet. 
Die Forschungsergebnisse aus der Beteiligung am CD-Labor haben bzw. hatten für die Unternehmen vor allem Relevanz bei der Hilfe der Bewertung verschiedener Lösungsstrategien (Radarfunktion), sowie für die die Entwicklung radikal neuer Produkte. Auch für die inkrementelle Verbesserung bestehender Produkte/Prozesse und die Entwicklung neuer Produktionsverfahren haben und hatten die Forschungsergebnisse Relevanz für die Unternehmen. Dies bestätigt ein nach wie vor breites Nutzenspektrum des Programms für die Wirtschaft.

Hinsichtlich der Formulierung der jeweiligen Forschungsprogramme und der Definition der Module zeigt sich (mittlerweile) ein sehr hoher Einfluss der Laborleiterinnen und Laborleiter. Bemerkenswert ist die zu beobachtende Veränderung betreffend dieses Aspekts im Laufe der Zeit. Bei der Evaluierung im Jahre 2005 gaben noch rund zwei Drittel der Laborleiterinnen bzw. Laborleiter an, hinsichtlich der Forschungsprogrammformulierung nur wenig Einfluss zu haben. Auch bezüglich der Moduldefinition gab nahezu die Hälfte an, wenig Einfluss zu haben. Bereits bei der Evaluierung 2011 änderte sich dieses Bild jedoch dramatisch und im Zuge der aktuellen Erhebung findet sich kein Befragter mehr, der hinsichtlich dieser beiden Punkte einen geringen Einfluss angibt. Deutlich im Steigen ist analog dazu auch die Gruppe jener, die hier einen sehr hohen Einfluss sieht.

Der Prestigegewinn im Zusammenhang mit einem CD-Labor ist nach wie vor ein wichtiger Faktor für das Universitäts- bzw. Forschungsinstitut. Auch im (internationalen) Forschungsbetrieb ist ein CDLabor als „Marke“ nutzbar und stärkt die Sichtbarkeit, nicht nur des Instituts bzw. der Universität, sondern auch des Forschungsstandortes Österreich. Dies wird auch in den begleitenden Experteninterviews hervorgehoben. Das CD-Programm ist ein renommiertes Förderprogramm, welches (internationale) Sichtbarkeit erzeugt, v.a. auch Sichtbarkeit als Kooperationspartner.

Der „Werdegang“ ehemaliger Labormitarbeiterinnen und Mitarbeiter zeigt eine zur Evaluierung 2011 ähnliche Struktur. Bereits damals wechselte ein Großteil (knapp zwei Drittel) der ehemaligen Mitarbeiterinnen und Mitarbeiter zu einem (Industrie-)Unternehmen, ungefähr ein Drittel verblieb im Forschungsumfeld der Universität bzw. einer anderen Universität oder Forschungseinrichtung.

Der Trend einer zunehmenden Internationalisierung, der bereits 2011 sichtbar war, setzt sich - in moderater Art und Weise - weiter fort. Exzellente Forschung ist heutzutage international bzw. "grenzenlos" - ein Forschungsförderprogramm sollte diesem Umstand Rechnung tragen - das CDGProgramm tut dies den Programmzielen gemäß in gutem, für das Förderziel ausreichendem Masse. Gründe für eine internationale Partnerschaft liegen vor allem im dadurch verfügbaren Know-how 
(welches teils in Österreich nicht verfügbar ist) bzw. der Kompetenz und führenden Stellung des internationalen Partners.

Was den administrativen Aufwand des CD-Programms betrifft, so wird dieser - bezogen auf die Antragstellung - von den Laborleiterinnen und Laborleitern nahezu durchgehend als (sehr) zufriedenstellend bewertet (97\%). Bei der Bewertung des administrativen Aufwands während der Laufzeit des CD-Labors verschieben sich die Zufriedenheitswerte etwas. Rund $20 \%$ bewerten diesen wenig zufriedenstellend, jedoch nur einer der Befragten gar nicht zufriedenstellend.

Ein Vergleich mit der Evaluierung 2011 zeigt beim administrativen Aufwand im Rahmen der Antragstellung kaum Verschiebungen der Zufriedenheitswerte. Diese waren im Jahr 2011 sehr hoch und sind es nach wie vor. Was den administrativen Aufwand während der Laufzeit betrifft konnten sich die Werte in Summe leicht verbessern. Die Gruppe der "sehr Zufriedenen“ konnte gegenüber 2011 deutlich erhöht, die Gruppe der „wenig Zufriedenen und Unzufriedenen“ hingegen etwas verringert werden. Dies kann als ein erstes Anzeichen dafür gewertet werden, dass die von der CDG gesetzten Maßnahmen hinsichtlich administrativer Vereinfachungen bereits erste Wirkung zeigen. Betrachtet man nur die Subgruppe der derzeit aktiven Labors fällt auch die Gruppe der Unzufriedenen weg, ein weiteres Indiz für eine Verbesserung zu früher.

Auch die von zahlreichen Befragten abgegebenen Statements zum administrativen Aufwand zeigen, dass die umgesetzten administrativen Vereinfachungen (positiv) wahrgenommen wurden. Nach wie vor gibt es jedoch vereinzelt Verbesserungsvorschläge. Hier wurden vor allem die Themen „Webtool“ sowie weitere Vereinfachungen in den Abläufen bei z.B. Änderungsanträgen, Unterschriften von Unternehmenspartnern etc. genannt.

Auch von den Unternehmenspartnern wird der administrative Aufwand tendenziell sehr zufriedenstellend eingestuft, insbesondere auch der Aufwand während der Laufzeit eines CD-Labors, der von mehr als 90\% der Unternehmen als (sehr) zufriedenstellend bewertet wurde.

Den administrativen Aufwand des CD-Programms im Vergleich zu anderen Programmen sehen die Laborleiterinnen und Laborleiter unterschiedlich. Grob kann man sagen: Der administrative Aufwand eines CD-Labors wird von den Befragten tendenziell niedriger als bei einem EU-Projekt und tendenziell höher als bei einem FWF-Projekt bewertet. Im Vergleich zu einem FFG-Projekt sind die Beurteilungen breit gestreut.

Im Rahmen der Online-Erhebung konnten erstmals auch JR-Zentren berücksichtigt werden. Von den acht bestehenden übermittelten im Rahmen der Online-Erhebung sieben Zentrumsleiterinnen und - 
Leiter einen vollständig ausgefüllten Fragebogen. Auch wenn dies nahezu einer Vollerhebung entspricht, muss berücksichtigt werden, dass die befragte Gruppe eine kleine ist.

Mit der Klarheit des Förderkonzepts und Förderziels ist der große Teil der Zentrumsleiterinnen und leiter (sehr) zufrieden. Auch wenn sich lediglich einer der Befragten unzufrieden zeigt, könnte dies ein Hinweis darauf sein, dass im Rahmen des im Gegensatz zum CD-Programm noch jungen Programms der JR-Zentren vereinzelt noch Nachjustierungen des Programmdesigns sinnvoll und notwendig sind, es seinen "eigenen Charakter" noch finden muss. Dies ist in weiterer Folge mit zukünftigen Evaluierungen mit einer größeren Anzahl von JR-Zentrumsleitern nochmals zu verifizieren.

Durchgehend hohe Zufriedenheit herrscht bezüglich der Angemessenheit der Evaluierungskriterien, auch die Bearbeitungsdauer der eingereichten Anträge wird als (sehr) Zufriedenstellend angesehen.

Den administrativen Aufwand bewertet der Großteil zufriedenstellend, jener im Rahmen der Laufzeit des JR-Zentrums wird vereinzelt etwas schlechter bewertet. Nach Gründen befragt wird ähnlich wie bei den CD-Labors u.a. einerseits ein sehr starker Fokus auf Formalitäten von Seiten des Fördergebers genannt (auch moderate Änderungen im Projektverlauf müssen formal beantragt werden, Vorlaufzeit von Änderungsanträgen, für viele Änderungen sind Zustimmungen der Partnerfirmen einzuholen etc.), andererseits auch das CDG-Webtool (zu langsam, zu instabil und zu unflexibel) angesprochen.

Die Laufzeit eines JR-Zentrums von 5 Jahren wird von einer knappen Mehrheit der Befragten als gerade richtig angesehen, einigen ist sie jedoch zu kurz. Als zu Lang empfindet (analog zu den CDLaborleitern und Laborleiterinnen) keiner der Zentrumsleiter bzw. Zentrumsleiterinnen die Laufzeit des Programms. Die Unternehmenspartner bewerten die Laufzeit zu einem Großteil als gerade richtig, ein kleiner Teil empfindet sie als zu kurz. Als zu lange empfindet auch keiner der Unternehmenspartner das JR-Programm.

Die begleitende wissenschaftliche Kontrolle wird von allen Zentrumsleiterinnen und -leitern, bei denen sie bereits erfolgte, und auch von den Unternehmenspartnern als (sehr) hilfreich angesehen. Hier zeigt sich eine einheitlich positive Bewertung. Auch der Zeitpunkt der Zwischenevaluierung wird mehrheitlich als passend (wenn nicht, dann auch bei den JR-Zentren als zu früh) empfunden.

Zufriedenheit besteht (mit einer Ausnahme) auch mit den Betreuungsleistungen der CDG. Die Unternehmenspartner sehen teils bei der Öffentlichkeitsarbeit noch Verbesserungspotential. 
Die Motivation zur Gründung eines JR-Zentrums ging mehrheitlich vom wissenschaftlichen Partner aus, stark auch von beiden Partnern. Auch bei den JR-Zentrumspartnern bestand, wie auch bei den CD-Laborpartnern, großteils vorher schon Kontakt über gemeinsame Forschungsprojekte.

Wie auch bei den CD-Laborleiterinnen und -leitern ist insbesondere das langfristig finanziell abgesicherte Arbeiten an einem Themenkreis und der Aufbau eines eigenen Forschungsteams von besonderer Wichtigkeit, hinsichtlich Erwartungen und Ziele bei der Gründung eines JR-Zentrums. Diese Punkte wurden auch im Tiefeninterview betont und hervorgehoben.

Analog zu den CD-Labors sehen auch die JR-Zentrumsleiterinnen und -leiter sehr hohen Einfluss auf die Forschungsprogrammformulierung und die Moduldefinition.

Die Inputs der Unternehmenspartner werden von den Zentrumsleitern bzw. Zentrumsleiterinnen sehr unterschiedlich bewertet. In (sehr) hohem Ausmaß lieferten die Unternehmenspartner vor allem Inputs hinsichtlich Bereitstellung zusätzlicher Infrastruktur und mehrheitlich auch zu den Forschungsaktivitäten. Gering war der Input der Unternehmenspartner nach Ansicht der Zentrumsleiterinnen und -leiter vor allem in Hinblick auf die Moduldefinition und die Vermittlung von Kontakten.

Hinsichtlich des Nutzens für das FH-Institut sehen die Befragten vor allem den Prestigegewinn und die verbesserte Stellung des Instituts. Auch die höhere Attraktivität als Kooperationspartner wird mehrheitlich positiv als Nutzen hervorgehoben.

Was die Durchführung des Forschungsprojekts, auch ohne JR-Zentrum betrifft, so hätten alle Befragten dieses nur in geringerem Umfang - mehrheitlich sogar nur in deutlich geringerem Umfang durchführen können.

Die „Internationalisierung" ist bei den JR-Zentren deutlich geringer ausgeprägt als bei den CD-Labors. Lediglich eines der sieben JR-Zentren hat einen internationalen Partner (Unternehmenspartner aus Deutschland) im Rahmen des JR-Zentrums. Ein internationales Modul an einem ausländischen Standort wird derzeit von keinem Zentrum betrieben.

Obwohl noch keines der JR-Zentren abgeschlossen ist, sind sowohl in die Lehre als auch in die Forschung bereits Erfahrungen aus den industrierelevanten Projekten eingeflossen. Vor allem in die Forschung konnten nach Ansicht aller Befragten Erfahrungen in sehr großem und mittlerem Ausmaß mitgenommen werden. 
Was die bisherige Qualität der Zusammenarbeit mit der Industrie in den JR-Zentren betrifft, zeigt sich im Rahmen der Befragung ein weitgehend positives, aber dennoch in Einzelfällen etwas abweichendes Bild. Die Relevanz der industriellen Fragestellung für die Forschungsarbeit des JR-Zentrums wird durchwegs und mit einer Ausnahme (sehr) gut bewertet. In der Zusammenarbeit mit den Kontaktpersonen der Industrie gibt es unterschiedliche Erfahrungen. Ein Großteil der Zentrumsleiterinnen und -leiter hat hier sehr gute Erfahrungen gemacht, in Einzelfällen werden hier aber auch negative Erfahrungen dargestellt. Auch das Ausmaß des wissenschaftlichen Freiraums wird von den Befragten unterschiedlich wahrgenommen und streut zwischen sehr gut und genügend. Anzumerken ist hier, dass bei den JR-Zentren, anders als bei den CD-Labors, kein wissenschaftlicher Freiraum von 30\% in den Eckdaten des Programms festgelegt ist. Hier ist zu überlegen, ob es nicht Sinnvoll wäre auch im JR-Programm einen gewissen Prozentsatz für kontinuierlichen Wissenszuwachs zu verankern.

Auch wenn die Auswirkungen des JR-Zentrums auf das Drittmittelvolumen des Heimat gebenden Instituts noch nicht für alle Befragten einschätzbar sind, zeigt sich dennoch eine positive Tendenz. Ähnliches gilt für die Auswirkungen des JR-Zentrums auf die Unternehmenskooperationen des Heimat gebenden Institutes.

Abschließend wurden auch die Befragten JR-Zentrumsleiter gebeten den administrativen Aufwand eines JR-Zentrums im Vergleich zu anderen Förderprogrammen (FFG-Projekt, EU-Projekt, FWF-Projekt) einzuschätzen. Der Vergleich zu einem FWF-Projekt ist für die Mehrheit der Befragten nicht möglich. Jene, die eine Beurteilung abgaben, sehen den administrativen Aufwand eines JR-Zentrums als höher an. Unterschiedlich ist die Wahrnehmung im Vergleich zu einem FFG-Projekt, wo alle Befragten eine Einschätzung abgaben. Hier wird der Aufwand zwar tendenziell auch höher eingeschätzt, einige sehen ihn jedoch auch gleich hoch oder sogar niedriger. Im Vergleich zu einem EU-Projekt wird der administrative Aufwand eines JR-Zentrums mehrheitlich niedriger eingeschätzt, aber auch hier gehen die Meinungen auseinander. 


\section{Erweiterte Systemevaluierung}

In diesem Kapitel werden zunächst die Beteiligungen von Forschungseinheiten und Unternehmen an CD-Labors bzw. JR-Zentren untersucht. Von besonderem Interesse sind dabei zwei Fragen: (1) gelingt es, die wissenschaftliche Community in Österreich einzubinden, und (2) welche Unternehmen haben sich seit der Gründung des Forschungsprogramms an CD-Labors beteiligt. Der zweite Abschnitt beschäftigt sich mit der Grundlagenforschung in Österreich. Da sich die technologische Kompetenz der österreichischen Wirtschaft auch über Patente messen lässt, werden im dritten Abschnitt die Ergebnisse einer Patentanalyse präsentiert, bei der zuerst die nationalen Technologiestärkefelder identifiziert werden. Danach wird gezeigt welche Technologien sich besonders dynamisch entwickeln, und daher ein potenzielles Anwendungsgebiet für zukünftige CD-Labors bzw. JR-Zentren darstellen könnten.

Abgeschlossen wird diese Kapitel mit der Verschränkung zwischen der Auswertung der Unternehmensbeteiligungen und der Patentanalyse. Die zu untersuchende Fragestellung lautet hierbei, ob in der Vergangenheit besonders Unternehmen durch das Förderprogramm unterstützt wurden, die auf Grund der Förderung dazu beigetragen haben, die entwickelte Technologie als nationales Stärkefeld zu etablieren.

\subsection{Analyse der Beteiligungen an CD-Labors und JR-Zentren}

In den folgenden beiden Abschnitten werden die Beteiligungen der Forschungseinheiten und der Unternehmen während der gesamten Historie der CD-Gesellschaft dargestellt, also seit Herbst 1989. Mit Stichtag 8. Juli 2016 werden in der Statistik 169 CD-Labors mit 461 Unternehmenspartnern und 7 JR-Zentren mit 25 Unternehmenspartnern gezählt.

\subsubsection{Analyse der Unternehmenspartner}

Seit Herbst 1989 waren bei den 169 CD-Labors insgesamt 461 Unternehmenspartner beteiligt, einige allerdings an mehreren CD-Labors, sodass schließlich 257 verschiedene Unternehmen verbleiben. Fasst man die Unternehmen von Unternehmensgruppen, wie z.B. der voestalpine, ebenfalls zusammen, dann reduziert sich diese Liste weiter auf 235 verschiedene Unternehmensgruppen. 
Abbildung 134: Anzahl der Unternehmen, differenziert nach Anzahl der CD-Laborbeteiligungen

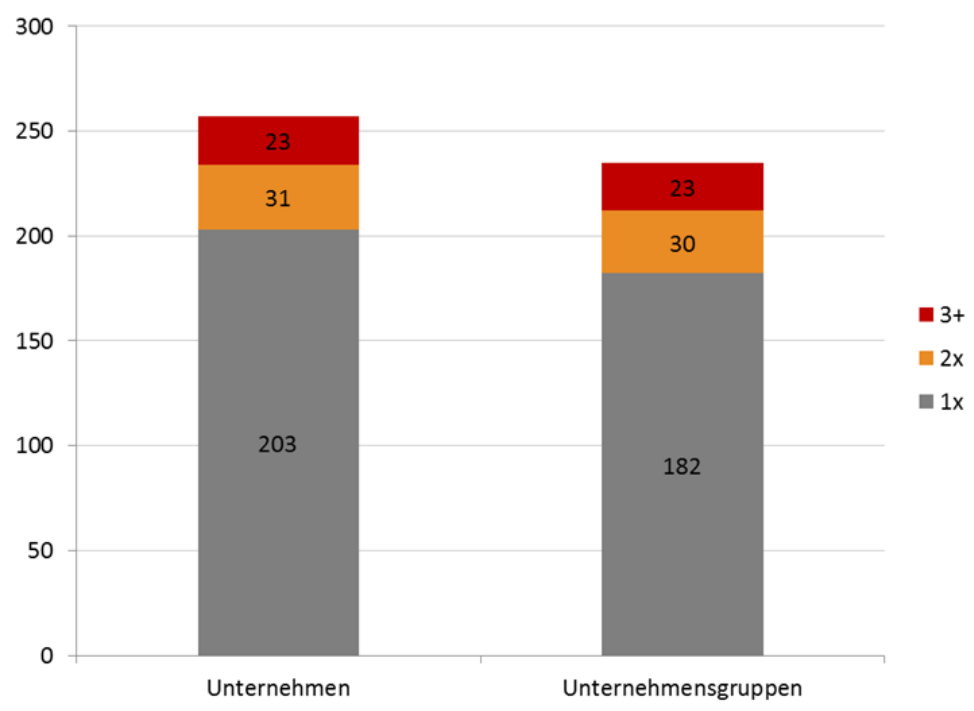

Quelle: Economica/IWI (2017).

Anmerkungen: 1x.... Anzahl der Unternehmen mit einmaliger Laborbeteiligung

$2 x \ldots .$. Anzahl der Unternehmen mit zweimaliger Laborbeteiligung

$3+\ldots .$. Anzahl der Unternehmen mit mehr als zweimaliger Laborbeteiligung

Abbildung 135: Anzahl der Unternehmensbeteiligungen, differenziert nach Anzahl der CD-Laborbeteiligungen

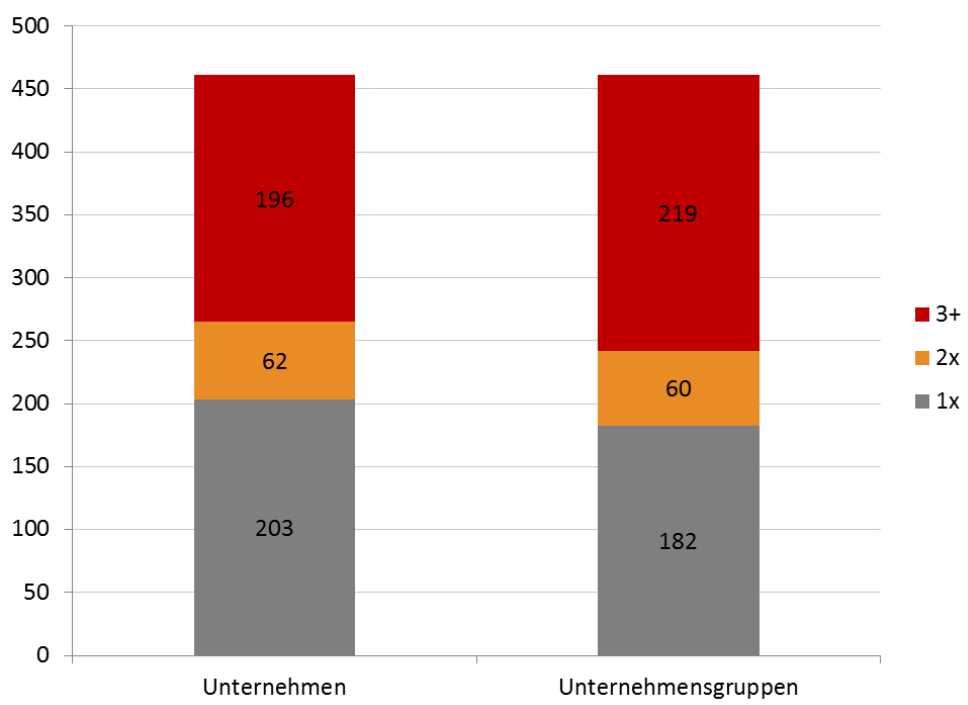

Quelle: Economica/IWI (2017).

Anmerkung: 1x ... Anzahl der Laborbeteiligungen von Unternehmen mit einmaliger Kooperation

$2 x$... Anzahl der Laborbeteiligungen von Unternehmen mit zweimaliger Kooperation

$3+\ldots$ Anzahl der Laborbeteiligungen von Unternehmen mit mehr als zweimaliger Kooperation 
Die Unternehmensbeteiligungen an den CD-Labors, differenziert nach Anzahl der CD-Laborbeteiligungen, geben wieder, in wieweit ein Unternehmenskern besteht, der bei den Aktivitäten des Förderprogramms verstärkt eingebunden ist. Die 461 Unternehmensbeteiligungen verteilen sich demnach auf 203 Unternehmenspartner, die nur bei einem CD-Labor involviert waren. 31 Unternehmen fungierten zweimal als wirtschaftlicher Counterpart, und die restlichen 23 Unternehmen wiesen im Schnitt 8,52 Beteiligungen auf. Bezogen auf die Unternehmensgruppen ist die Anzahl der Mehrfachbeteiligungen natürlich höher, wobei dann aber auch Beteiligungen von zwei Unternehmen einer Gruppe an einem CD-Labor als doppelte Beteiligung gezählt wurden, um die gleiche Basis beibehalten zu können.

Betrachtet man die Verteilung auf die drei Unternehmensgruppen, definiert über die Anzahl der Beteiligungen $(1 \mathrm{x}, 2 \mathrm{x}, 3+)$ an verschiedenen CD-Labors, so ist erkennbar, dass 10 Prozent der Unternehmen zusammengefasst in Unternehmensgruppen beinahe der Hälfte der Gesamtanzahl der CD-Laborpartner aus der Wirtschaft ausmachen (48\%).

\section{Abbildung 136: Verteilung der Unternehmen und CD-Laborbeteiligungen}

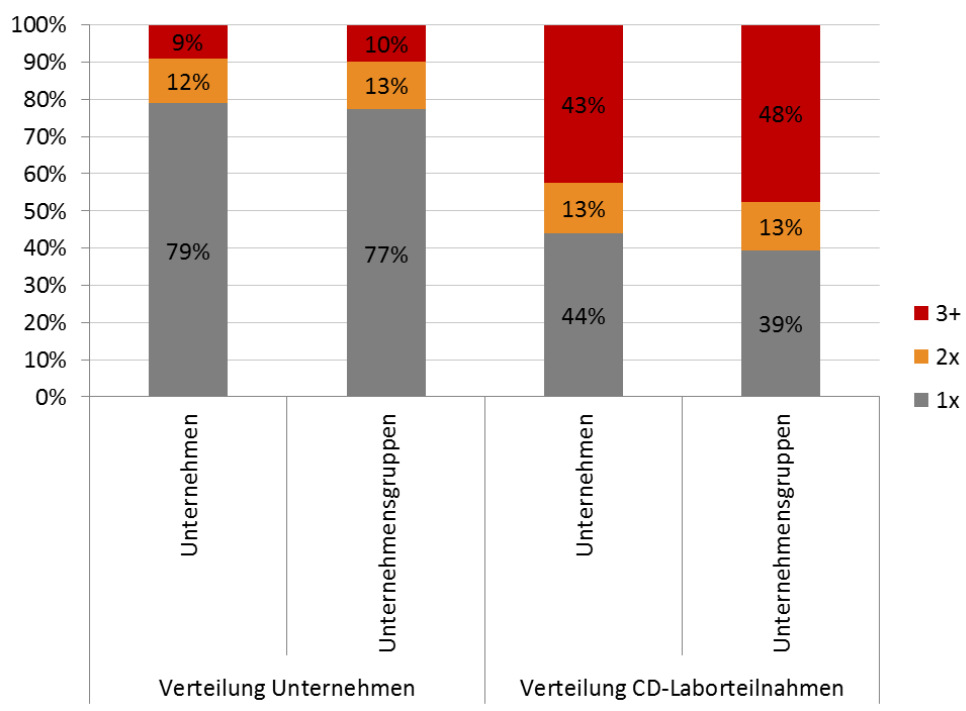

Quelle: Economica/IWI (2017).

In der folgenden Tabelle werden die Beteiligungen der Unternehmen nach Anzahl der CDLaborbeteiligungen detailliert dargestellt. Man kann dabei erkennen, dass es einige Unternehmensgruppen bzw. Konzerne gibt, die besonders häufig an CD-Labors beteiligt waren. 
Tabelle 24: Anzahl der Unternehmen, nach Anzahl der CD-Laborbeteiligungen

\begin{tabular}{lcccc} 
& \multicolumn{2}{c}{ Anzahl } & \multicolumn{2}{c}{ CD-Laborbeteiligungen } \\
\hline $\begin{array}{l}\text { \# CD-Labor } \\
\text {-beteiligungen }\end{array}$ & Unternehmen & $\begin{array}{c}\text { Unternehmens- } \\
\text { gruppen }\end{array}$ & $\begin{array}{c}\text { Unternehmen } \\
\text { Unternehmens- } \\
\text { gruppen }\end{array}$ \\
\hline $\mathbf{1}$ & 203 & 182 & 203 & 182 \\
$\mathbf{3}$ & 31 & 30 & 62 & 60 \\
$\mathbf{4}$ & 5 & 7 & 15 & 21 \\
$\mathbf{5}$ & 5 & 5 & 20 & 20 \\
$\mathbf{6}$ & 4 & 2 & 20 & 10 \\
$\mathbf{7}$ & 1 & 1 & 6 & 6 \\
$\mathbf{1 0}$ & & 1 & & 7 \\
$\mathbf{1 1}$ & 2 & 1 & 20 & 10 \\
$\mathbf{1 2}$ & 1 & 1 & 11 & 11 \\
$\mathbf{1 4}$ & & 1 & & 12 \\
$\mathbf{1 7}$ & 1 & & 14 & 17 \\
$\mathbf{1 9}$ & 1 & 1 & 17 & 38 \\
$\mathbf{3 5}$ & 2 & 2 & 38 & 67 \\
$\mathbf{6 7}$ & 1 & & 35 & $\mathbf{4 6 1}$ \\
\hline Summe & $\mathbf{2 5 7}$ & $\mathbf{2 3 5}$ & $\mathbf{4 6 1}$ & \\
\hline \hline
\end{tabular}

Quelle: $C D G$

Reiht man die Unternehmens(-gruppen) nach der Anzahl der Unternehmenspartner an CD-Labors, dann sieht man, dass der voestalpine Konzern mit seinen Tochterunternehmen absolut dominierend ist. Insgesamt war die Gruppe 67-mal als Unternehmenspartner angeführt, wobei bei manchen CDLabors auch mehrere Unternehmen des Konzerns beteiligt waren. Bereinigt um diese Mehrfachbeteiligungen verschiedener Tochterunternehmen, verbleiben immer noch 40 verschiedene CD-Labors mit einer Unternehmensbeteiligung des voestalpine Konzerns. Von diesen 40 CD-Labors waren in 37 Fällen weitere Unternehmen beteiligt, die sich insgesamt auf 108 Partner summieren. Auch bei den Unternehmensgruppen von Infineon (12/11), Böhler (11/8) und Siemens (6/4) waren bei manchen CD-Labors mehr als ein Unternehmen aus dem Konzern beteiligt. 
Tabelle 25: Top-Unternehmenspartner nach Anzahl der CD-Labors

\begin{tabular}{|c|c|c|c|}
\hline Unternehmenspartner & & ors & \\
\hline voestalpine Edelstahl GmbH & voestalpine & 10 & 67 \\
\hline voestalpine Giesserei Linz GmbH & voestalpine & 1 & \\
\hline voestalpine Grobblech $\mathrm{GmbH}$ & voestalpine & 1 & \\
\hline voestalpine mechatronics VATRON & voestalpine & 1 & \\
\hline voestalpine Schienen $\mathrm{GmbH}$ & voestalpine & 1 & \\
\hline voestalpine Stahl Donawitz GmbH & voestalpine & 14 & \\
\hline voestalpine Stahl GmbH & voestalpine & 35 & \\
\hline voestalpine Tubulars GmbH \& Co KG & voestalpine & 2 & \\
\hline voestalpine Wire Rod Austria GmbH & voestalpine & 2 & \\
\hline Österreichische Industrieholding AG & & 19 & 19 \\
\hline Primetals Technologies Austria GmbH & & 19 & 19 \\
\hline AVL List $\mathrm{GmbH}$ & & 17 & 17 \\
\hline Infineon Technologies AG & Infineon & 1 & 12 \\
\hline Infineon Technologies Austria AG & Infineon & 11 & \\
\hline Böhler Edelstahl GmbH & Böhler & 6 & 11 \\
\hline Böhler Schmiedetechnik GmbH \& Co KG & Böhler & 5 & \\
\hline RHI AG & & 10 & 10 \\
\hline Merck KGaA & Merck & 5 & 7 \\
\hline Merck Sharp \& Dohme GmbH & Merck & 2 & \\
\hline Siemens AG Corporate Research and Technologies & Siemens AG & 1 & 6 \\
\hline Siemens AG Erlangen & Siemens AG & 1 & \\
\hline Siemens AG Österreich & Siemens AG & 2 & \\
\hline Siemens Healthcare Diagnostics $\mathrm{GmbH}$ & Siemens AG & 2 & \\
\hline Lenzing AG & & 5 & 5 \\
\hline Sandoz GmbH & & 5 & 5 \\
\hline ams AG & & 4 & 4 \\
\hline BIOMAY AG & & 4 & 4 \\
\hline OMV AG & & 4 & 4 \\
\hline Plansee SE & & 4 & 4 \\
\hline Veitsch-Radex GmbH & & 4 & 4 \\
\hline ARC Seibersdorf Research GmbH & & 3 & 3 \\
\hline Borealis AG & & 3 & 3 \\
\hline Carl Zeiss Meditec AG & Carl Zeiss & 1 & 3 \\
\hline Carl Zeiss Meditec Inc. & Carl Zeiss & 2 & \\
\hline EPCOS Components OHG & & 3 & 3 \\
\hline FemtoLasers Produktions GmbH & & 3 & 3 \\
\hline Fresenius Kabi Austria GmbH & Fresenius & 2 & 3 \\
\hline Fresenius Medical Care Deutschland GmbH & Fresenius & 1 & \\
\hline KEBA AG & & 3 & 3 \\
\hline
\end{tabular}

Quelle: $C D G$ 
Wie wir bei den CD-Labors gesehen haben, gibt es einen gewissen Unternehmenskern, der bei vielen CD-Labors involviert ist. Betrachtet man die Unternehmenspartner der JR-Zentren, so kann man erkennen, dass nur in 7 von 25 Fällen das jeweilige Unternehmen auch schon Partner eines CDLabors war. Es werden daher durch die JR-Zentren vor allem Unternehmen angesprochen, die noch nicht Teil des Förderprogramm CDG waren.

Tabelle 26: Unternehmenspartner der JR-Zentren, Querverweis CD-Labors

\begin{tabular}{l} 
Unternehmenspartner JR-Zentrum \\
\hline A1 Telekom Austria AG \\
Bluetechnix R\&D GmbH \\
DREI-BANKEN-EDV Gesellschaft m.b.H. \\
Gebrüder Weiss Gesellschaft m.b.H. \\
High Q Laser GmbH \\
IKARUS Security Software GmbH \\
infeo GmbH \\
Infineon Technologies Austria AG \\
Intel Austria GmbH \\
Kapsch TrafficCom AG \\
Lantiq A GmbH \\
LG NEXERA Business Solutions AG \\
LOYTEC electronics GmbH \\
myPEX \\
NXP Semiconductors Austria GmbH \\
Oregano Systems - Design \& Consulting GmbH \\
Österreichische Staatsdruckerei GmbH \\
Salzburg AG für Energie, Verkehr und Telekommunikation \\
Salzburg Wohnbau GmbH \\
SEC Consult Unternehmensberatung GmbH \\
Siemens AG Corporate Research and Technologies \\
Siemens AG Österreich \\
Vorarlberger Kraftwerke AG \\
Vorarlberger Landes- und Hypothekenbank Aktiengesellschaft \\
Vorarlberger Landes-Versicherung V.a.G.
\end{tabular}

Quelle: $C D G$

Wie erwähnt, kommt dem voestalpine Konzern eine sehr große Bedeutung für die CD-Labors zu. Um aber alle Verbindungen der Unternehmen zu den CD-Labors bzw. JR-Zentren aufzeigen zu können, 
wird in der folgenden Abbildung das gesamte Kooperationsnetzwerk der CDG dargestellt. Der Kern um die voestalpine kommt auch bei dieser Betrachtung entsprechend zur Geltung. .

\section{Abbildung 137: Kooperationsnetzwerk der Unternehmen in der CDG}

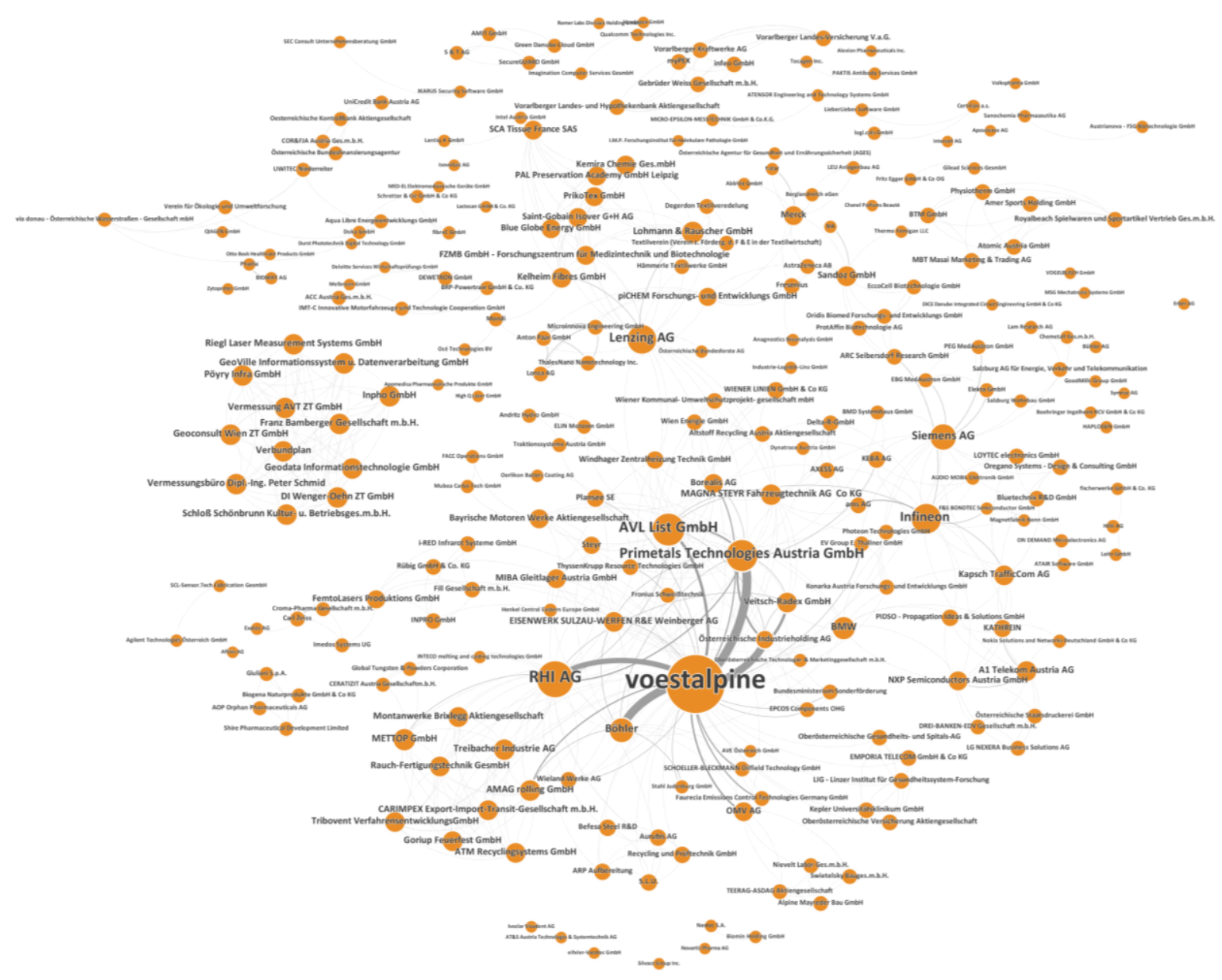

Quelle: Economica/IWI (2017).

Die Unternehmen der voestalpine sind, wie bereits erwähnt, bei 40 verschiedenen CD-Labors Unternehmenspartner und besitzen bei 37 CD-Labors insgesamt 108 weitere Unternehmenspartnerschaften, die sich auf 58 verschiedene Unternehmen zurückführen lassen. Am häufigsten waren Unternehmen der voestalpine gemeinsam mit Primetals Technologies Austria und RHI AG bei CD-Labors engagiert. 
Abbildung 138: Kooperationsnetzwerk der voestalpine in der CDG

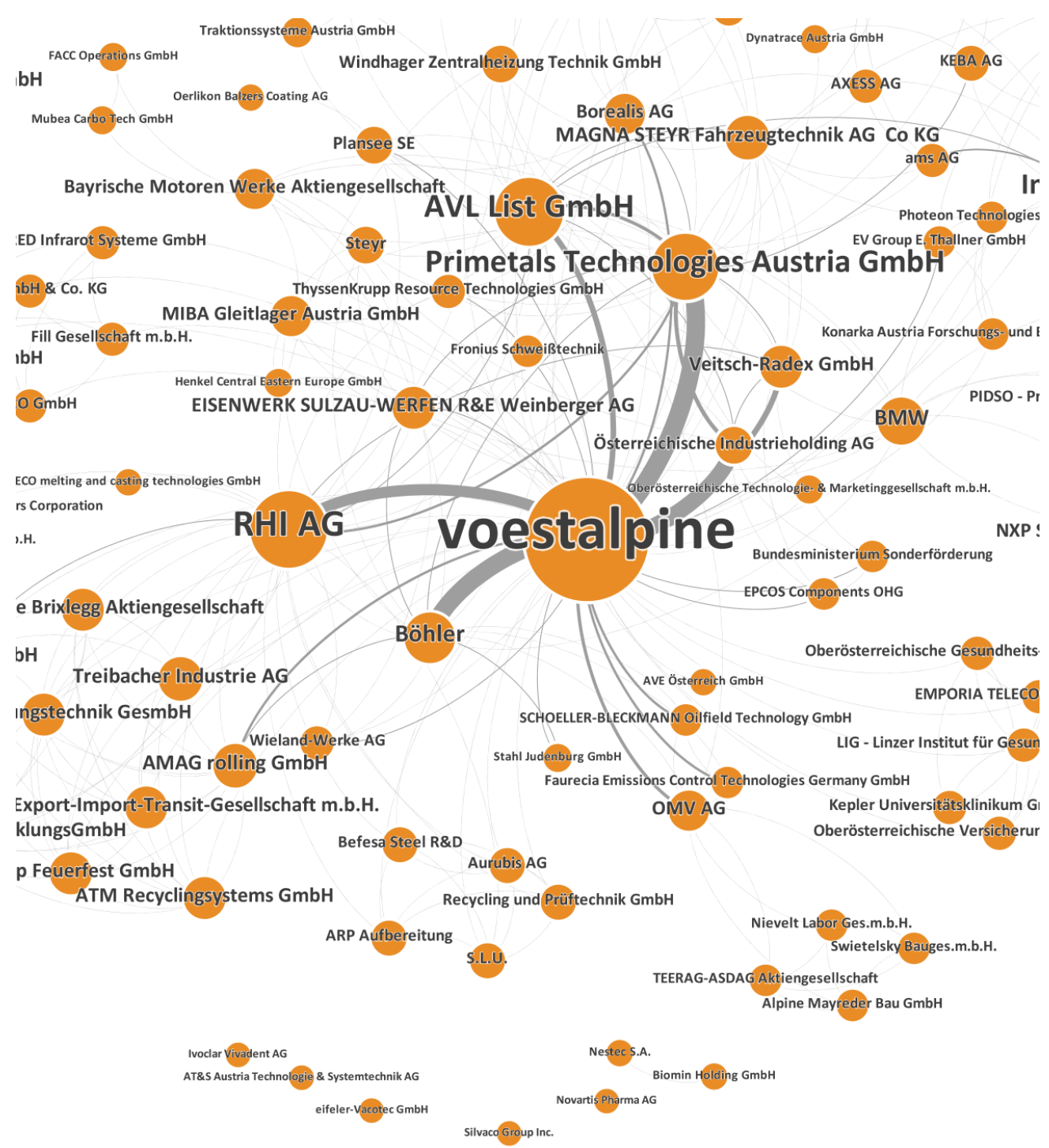

Quelle: Economica/IWI (2017).

\subsubsection{Analyse der Forschungseinrichtung}

Insgesamt gab es bei 7 von 169 CD-Labors zwei Laborleiter, wovon bei 2 CD-Labors jeweils ein Laborleiter von der Montanuniversität Leoben bzw. der Technischen Universität Wien stammte. Bei einem CD-Labor kamen die Laborleiter von der Technischen Universität Graz bzw. der Technischen Universität Wien. Die restlichen CD-Labors mit Doppelleitung waren an der gleichen Universität angesiedelt (2x Universität Linz, Technische Universität Wien, Universität für Bodenkultur Wien). 
Die Technischen Universitäten in Wien und Graz waren diejenigen Universitäten, die die meisten CDLabors durchführten.

Tabelle 27: Verteilung der CD-Labors auf Forschungseinrichtungen

$\begin{array}{lrr}\text { Forschungseinrichtung } & \text { Anzahl }^{47} & \text { Anteil } \\ \text { Technische Universität Wien } & 31,5 & 18,6 \% \\ \text { Technische Universität Graz } & 24 & 14,2 \% \\ \text { Montanuniversität Leoben } & 19 & 11,2 \% \\ \text { Universität Linz } & 18 & 10,7 \% \\ \text { Medizinische Universität Wien } & 17 & 10,1 \% \\ \text { Universität für Bodenkultur Wien } & 16 & 9,5 \% \\ \text { Ausland } & 11 & 6,5 \% \\ \text { Universität Salzburg } & 6 & 3,6 \% \\ \text { Veterinärmedizinische Universität Wien } & 6 & 3,6 \% \\ \text { Universität Innsbruck } & 4 & 2,4 \% \\ \text { Universität Wien } & 4 & 2,4 \% \\ \text { Medizinische Universität Innsbruck } & 3 & 1,8 \% \\ \text { Universität Graz } & 2,5 & 1,5 \% \\ \text { Donau-Universität Krems } & 2 & 1,2 \% \\ \text { Medizinische Universität Graz } & 1 & 0,6 \% \\ \text { Österreichische Akademie der Wissenschaften } & 1 & 0,6 \% \\ \text { Österreichisches Forschungszentrum Seibersdorf GesmbH } & 1 & 0,6 \% \\ \text { Research Center for Non Destructive Testing GmbH } & 1 & 0,6 \% \\ \text { Wirtschaftsuniversität Wien } & 1 & 0,6 \%\end{array}$

Quelle: $C D G$

Folgende Universitäten in Österreich hatten noch nie ein CD-Labor:

- Universität für künstlerische und industrielle Gestaltung Linz

- Universität für angewandte Kunst Wien

- Universität für Musik und darstellende Kunst Graz

- Universität für Musik und darstellende Kunst Wien

- Universität Klagenfurt

- Universität Mozarteum Salzburg

${ }^{47}$ Bei Doppelleitungen wurden die CD-Labors entsprechend aufgeteilt. 
Betrachtet man die folgende Abbildung zur Entwicklung der Anzahl der CD-Labors an den Universitäten, so ist zu erkennen, dass ab 2005 verstärkt auch CD-Labors an Forschungseinrichtungen außerhalb der Spitzengruppe vergeben wurden.

\section{Abbildung 139: Entwicklung der Anzahl der CD-Labors an Universitäten (Spitzengruppe)}

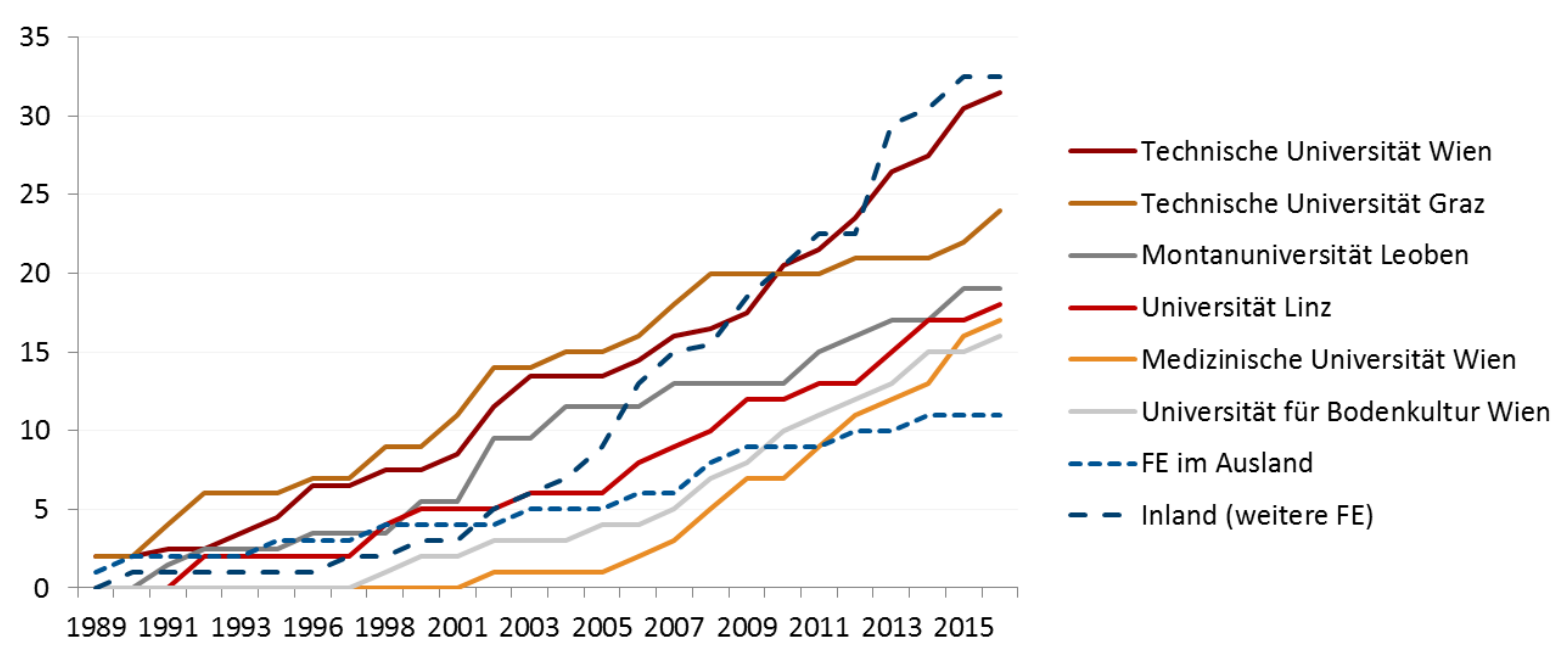

Quelle: Economica/IWI (2017).

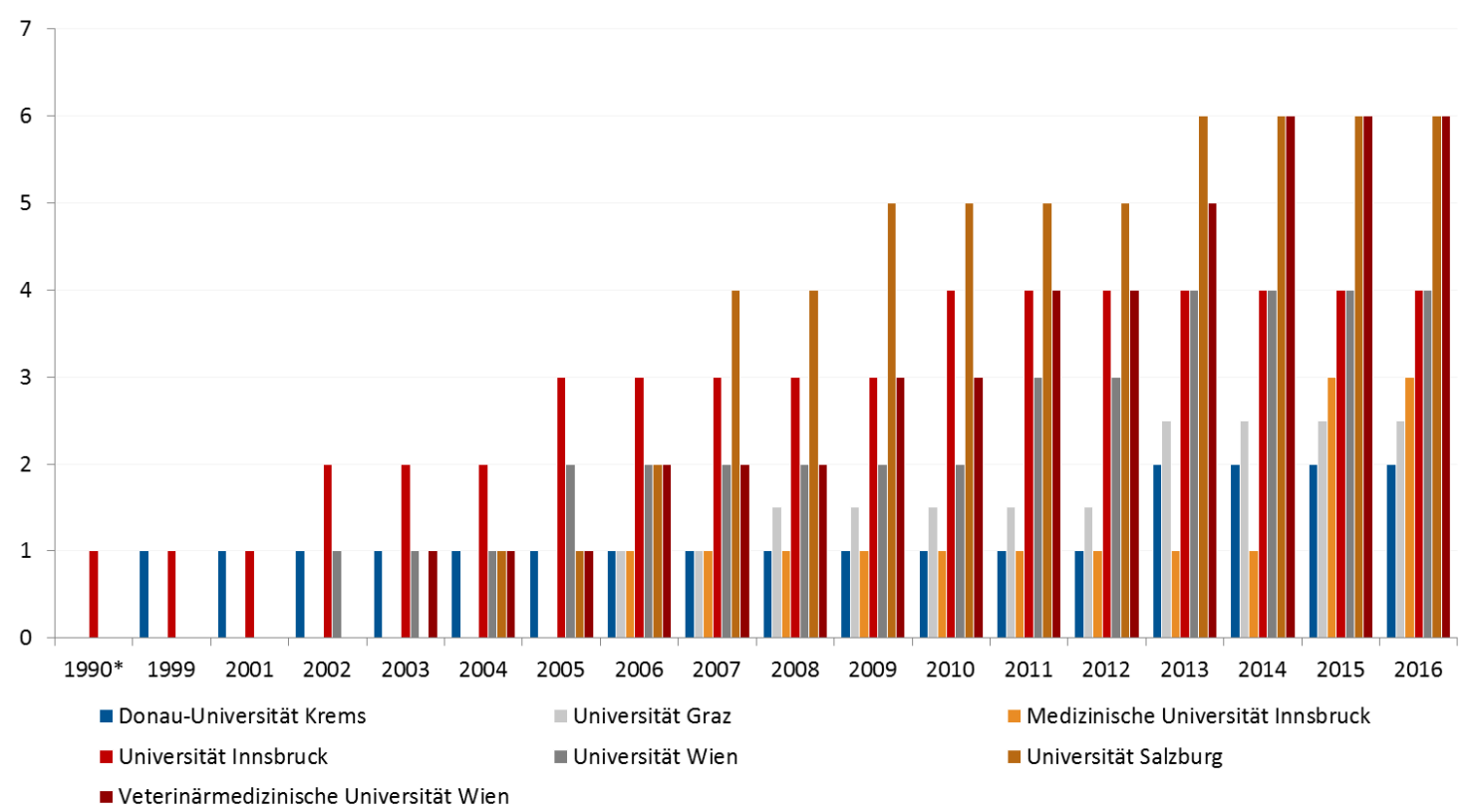

Quelle: Economica/IWI (2017). 
Die fortschreitende verbesserte Verteilung nach Forschungseinrichtungen wird auch durch den Herfindahl-Hirschman-Index $(\mathrm{HHI})^{48}$ über die Konzentration an den österreichischen Forschungseinrichtungen bestätigt.

\section{Abbildung 141: HH-Index der CD-Labors nach Forschungseinrichtungen in Österreich}

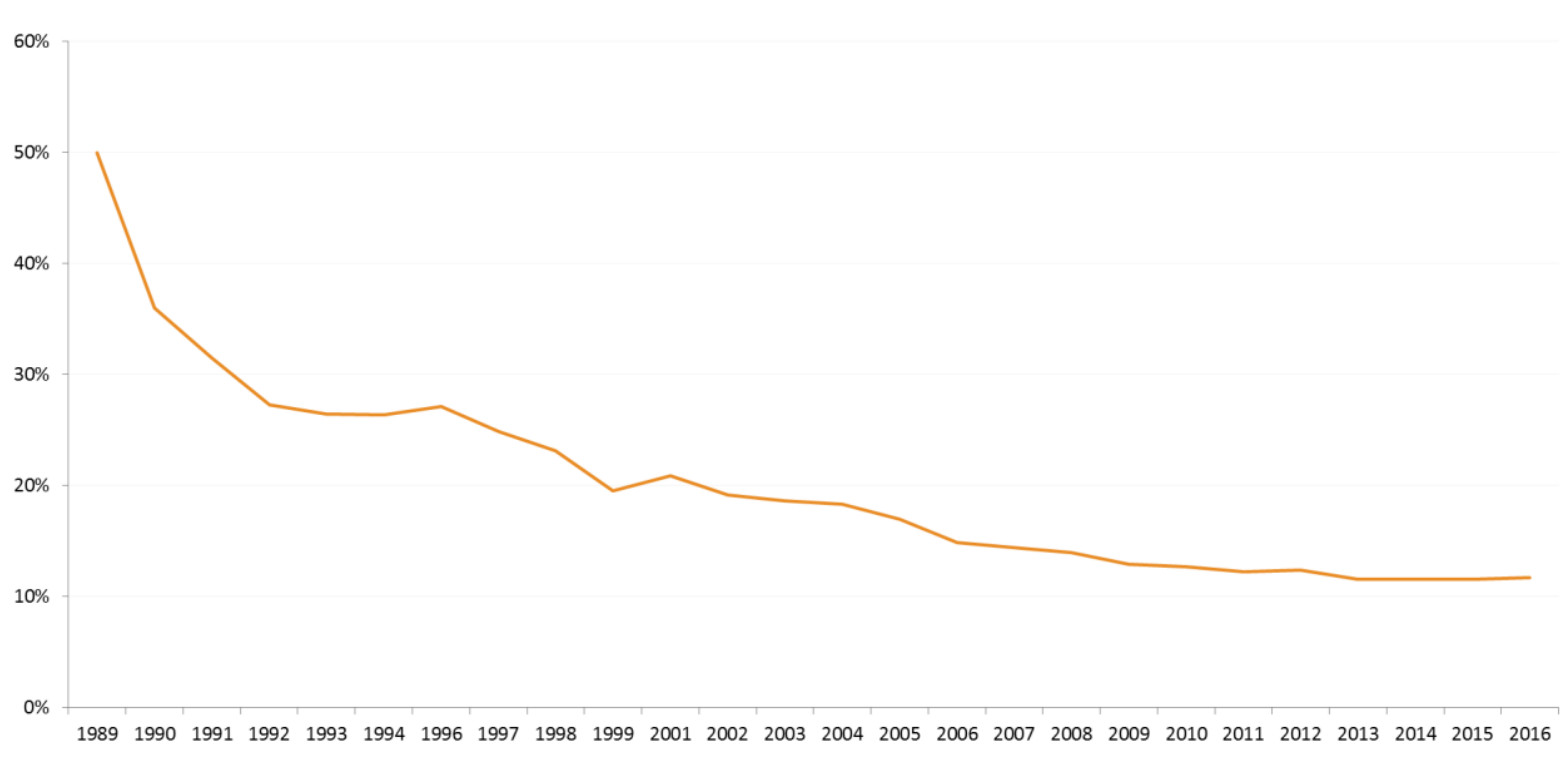

Quelle: Economica/IWI (2017).

Um die unterschiedliche Größe der Forschungseinrichtungen zu berücksichtigen, wurde der GiniKoeffizient bestimmt. Als Variablen wurden dabei die Anzahl der Studierenden sowie die Anzahl der CD-Labors verwendet.

Mit einem Wert von 0,67 ist der Gini-Koeffizient ${ }^{49}$ hier relativ hoch. Dies ist vor allem auf die relativ geringe Zahl von CD-Labors bei einer hohen Zahl von Studierenden an der Universität Wien bzw. der Wirtschaftsuniversität Wien und auf die relativ hohe Zahl von CD-Labors bei einer geringen Zahl von Studierenden an der Montanuniversität Leoben zurückzuführen.

\footnotetext{
${ }^{48}$ Der $\mathrm{HHI}$ ist eine Kennzahl zur Konzentrationsmessung, und berechnet sich aus der Summe aller quadrierten Marktanteile der Wettbewerber eines Marktes. Der Wert ist somit immer kleiner als 1 bzw. 100\%.

49 Der Gini-Koeffizient ist ein statistisches Maß zur Darstellung von Ungleichverteilungen, der aus der Lorenz-Kurve abgeleitet wird. Er nimmt einen Wert zwischen 0 (bei einer gleichmäßigen Verteilung) und 1 (wenn die untersuchte Größe nur auf eine Einheit verteilt ist, $d$. h. bei maximaler Ungleichverteilung) an.
} 
Abbildung 142: Lorenz-Kurve (Anzahl CD-Labors versus Anzahl der Studierenden)

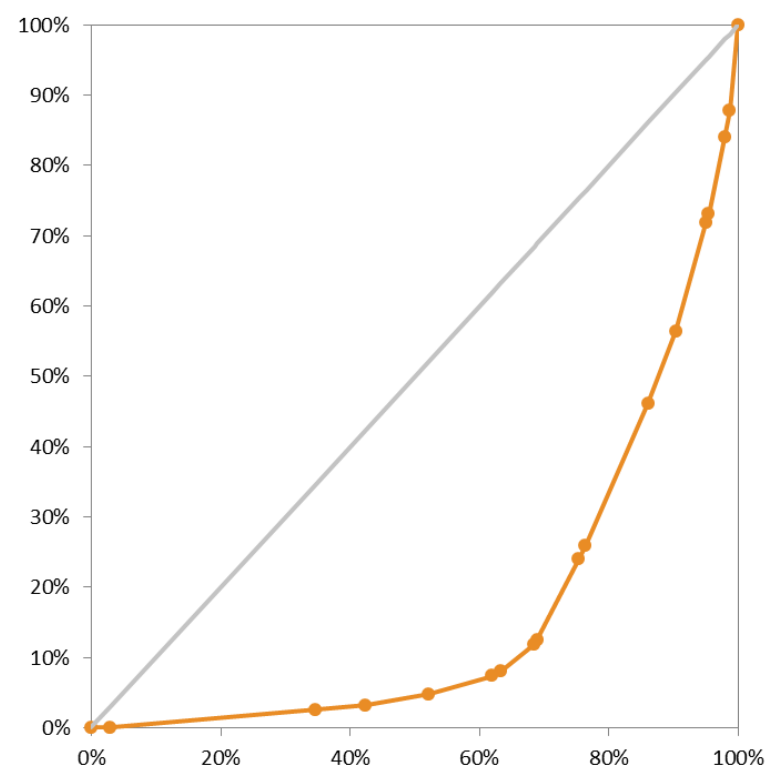

Quelle: Economica/IWI (2017).

Wie bereits erwähnt, befinden sich die Josef Ressel Zentren erst im Aufbau, sodass es auch nicht verwunderlich ist, dass sich nur an 7 von insgesamt 21 Fachhochschulen ${ }^{50}$ (zumindest) ein JRZentrum befindet:

- Fachhochschule Joanneum GmbH

- Fachhochschule Kärnten - Gemeinnützige Privatstiftung

- Fachhochschule Salzburg GmbH

- Fachhochschule Sankt Pölten GmbH

- Fachhochschule Technikum Wien

- Fachhochschule Vorarlberg $\mathrm{GmbH}(2)$

- $\quad$ FH OÖ Studienbetriebs GmbH

Da die derzeitigen F\&E-Kapazitäten an FHs noch sehr unterschiedlich ausgeprägt sind, sind somit auch in naher Zukunft nicht an allen $21 \mathrm{FH}$-Standorten JR-Zentren zu erwarten.

\footnotetext{
${ }^{50}$ http://wissenschaft.bmwfw.gv.at/bmwfw/studium/studieren-in-oesterreich/unis-privatunis-fhs-uebersicht/
} 


\subsection{Finanzierung der Grundlagenforschung in Österreich}

Die Ausgaben für Forschung und Entwicklung betrugen im Jahr 2016 insgesamt rund 10,7 Mrd. Euro, was einer F\&E-Quote von 3,07\% des österreichischen Bruttoinlandsproduktes (BIP) entspricht. ${ }^{51}$ Rund ein Drittel dieser Ausgaben (3,80 Mrd. Euro) werden durch den öffentlichen Sektor (Bund, Bundesländer, sonstige öffentliche Einrichtungen) finanziert. Der Anteil der Grundlagenforschung hat sich dabei in den letzten 10-15 Jahren sehr dynamisch entwickelt. Laut Bundesministerium für Finanzen $(B M F)^{52}$, haben sich von 2002 auf 2013 die Ausgaben für Forschung und Entwicklung mehr als verdoppelt ${ }^{53}$. Im Detail sind sie im Bereich Grundlagenforschung von 819 Mio. Euro im Jahr 2002 auf 1,806 Mrd. Euro im Jahr 2013 angestiegen (+121\%). Zum Vergleich, im Bereich angewandte Forschung gab es einen Anstieg von 1,727 Mrd. Euro im Jahr 2002 auf 3,403 Mrd. Euro im Jahr 2013 (+97\%) und im Bereich experimentelle Entwicklung von 2,051 Mrd. Euro (2002) auf 4,204 Mrd. Euro (2013) (+105\%), wodurch die Grundlagenforschung die höchste Wachstumsrate hatte.

Abbildung 143: Ausgaben für Forschung und Entwicklung, nach Forschungsarten in Mrd. Euro (2002, 2013)

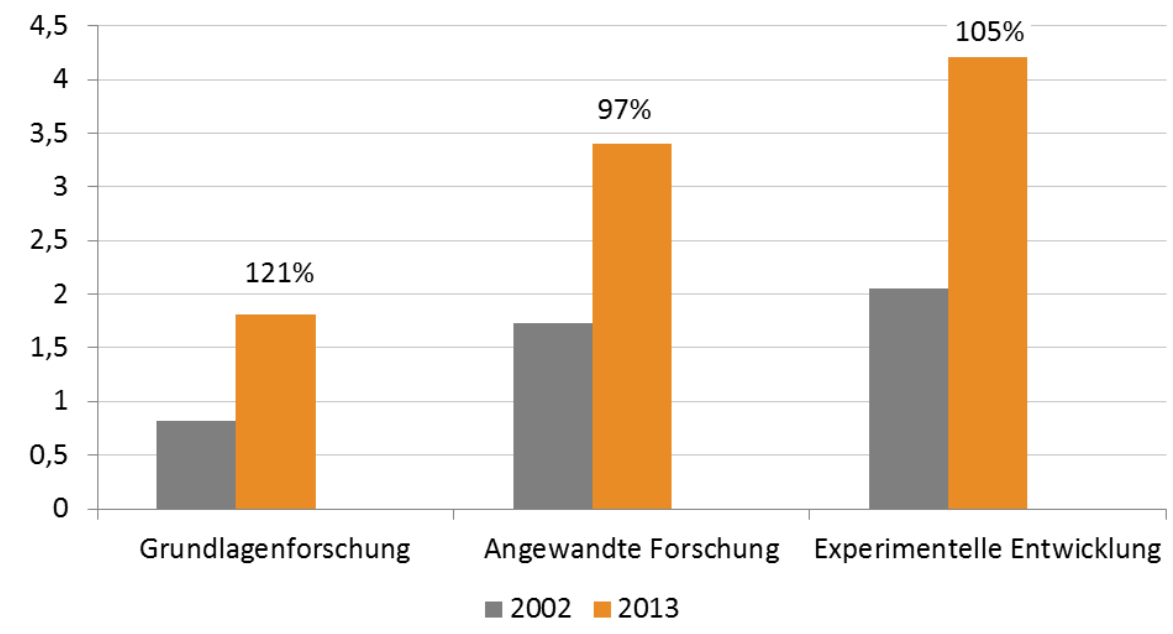

Quelle: Österreichischer Forschungs- und Technologiebericht 2016, BMWFW und BMVIT, Werte 2013: Statistik Austria.

\footnotetext{
${ }^{51}$ Österreichischer Forschungs- und Technologiebericht 2016, BMWFW und BMVIT

${ }^{52}$ F\&E Beilage 2017 des BMF

${ }^{53}$ Beachtet man die Preisentwicklung zwischen 2002 und 2013 mit einer Gesamtinflation von ca. 30 Prozent, dann entspricht dies einem realen Anstieg von über 58 Prozent.
} 
In der folgenden Abbildung werden die Ausgaben für F\&E nach Forschungsarten und Durchführungssektor in Österreich im Jahr 2013 dargestellt. Dem Unternehmenssektor sind demnach insgesamt 72 Prozent (6,78 von 9,41 Mrd. Euro) zuzuordnen, wobei davon nur 6,4 Prozent in die Grundlagenforschung fließen. Insgesamt über alle Durchführungssektoren gesehen sind 19,2 Prozent der F\&E Ausgaben in der Grundlagenforschung zu verorten.

Abbildung 144: F\&E Ausgaben nach Forschungsarten und Durchführungssektor in Österreich, in Mio. Euro, 2013

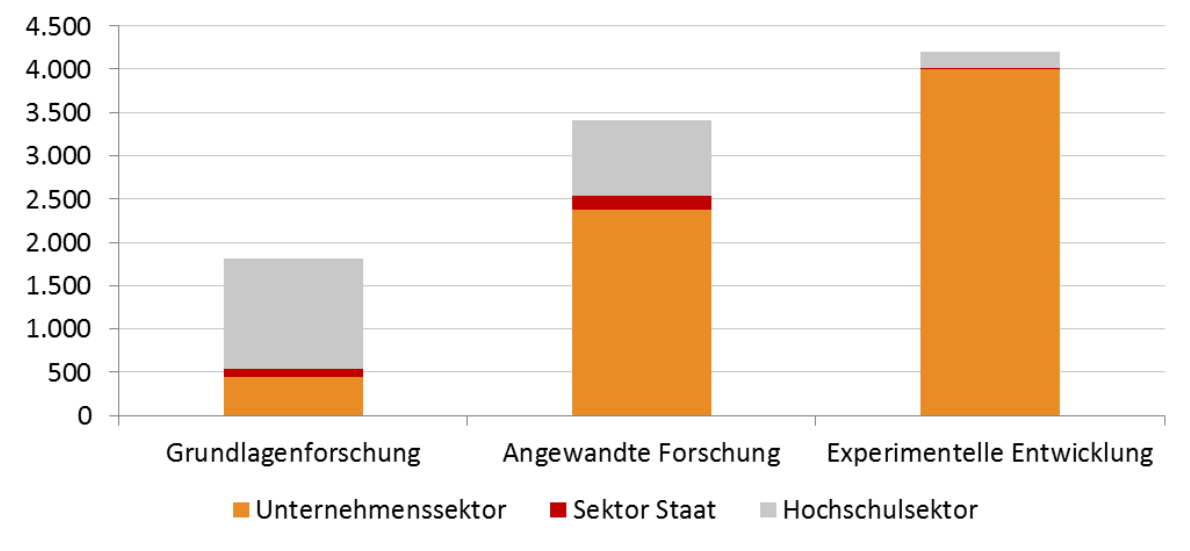

Quelle: Österreichischer Forschungs- und Technologiebericht 2016, BMWFW und BMVIT

Um den Beitrag der Grundlagenforschung der CDG an der gesamten Grundlagenforschung Österreichs zu bestimmen, kann man das Volumen der CDG den gesamten öffentlichen Mitteln für die Grundlagenforschung gegenüberstellen. Im Jahr 2013 wurden mit einem Gesamtbudget von rund 27,5 Mio. Euro 73 CD-Labors und vier JR-Zentren gefördert ${ }^{54}$, das entspricht in etwa $1,5 \%$ der gesamten Ausgaben der Grundlagenforschung bzw. 0,8 \% der gesamten Ausgaben für angewandte Forschung.

Obwohl der Unternehmenssektor F\&E Ausgaben hauptsächlich in den Forschungsarten angewandte Forschung und experimentelle Entwicklung aufweist, gibt es doch einige Wirtschaftszweige, die einen hohen Anteil von Grundlagenforschung an internen F\&E Ausgaben haben (s. Abbildung 145). Während es zu erwarten ist, dass im ÖNCE Abschnitt Forschung und Entwicklung (M72) einige ÖNACE-Klassen mit hohem Anteil der Grundlagenforschung vorkommen, ist vor allem der hohe Anteil in einem Teilbereich (Roheisen, Stahl und Ferrolegierungen; Stahlrohre; Eisen-, Stahlgießerei)

\footnotetext{
54 http://wissenschaft.bmwfw.gv.at/bmwfw/forschung/national/forschung-in-oesterreich/partner-institutionen/christiandoppler-forschungsgesellschaft-cdg/
} 
des ÖNACE-Abschnitts Metallerzeugung und -bearbeitung (C 24) beachtenswert. Kumuliert fallen $38 \%$ der Ausgaben für Grundlagenforschung im Abschnitt Forschung und Entwicklung an. Der ÖNACE-Abschnitt Leder, Lederwaren und Schuhe (C 15) weist zwar auch einen hohen Anteil an Grundlagenforschung an den internen F\&E Ausgaben auf, vereint aber nur 0,1\% der gesamten Ausgaben für Grundlagenforschung. Das gleiche gilt auch für „Forschung und Entwicklung im Bereich Rechts-, Wirtschafts- und Sozialwissenschaften sowie im Bereich Sprach-, Kultur- und Kunstwissenschaften" der nur unwesentlich mehr an den gesamten Ausgaben für Grundlagenforschung aufweist (0,3\%).

Abbildung 145: Top Wirtschaftszweige in Österreich (nach ÖNACE 2008) mit dem höchsten Anteil von Grundlagenforschung an internen F\&E Ausgaben, 2013

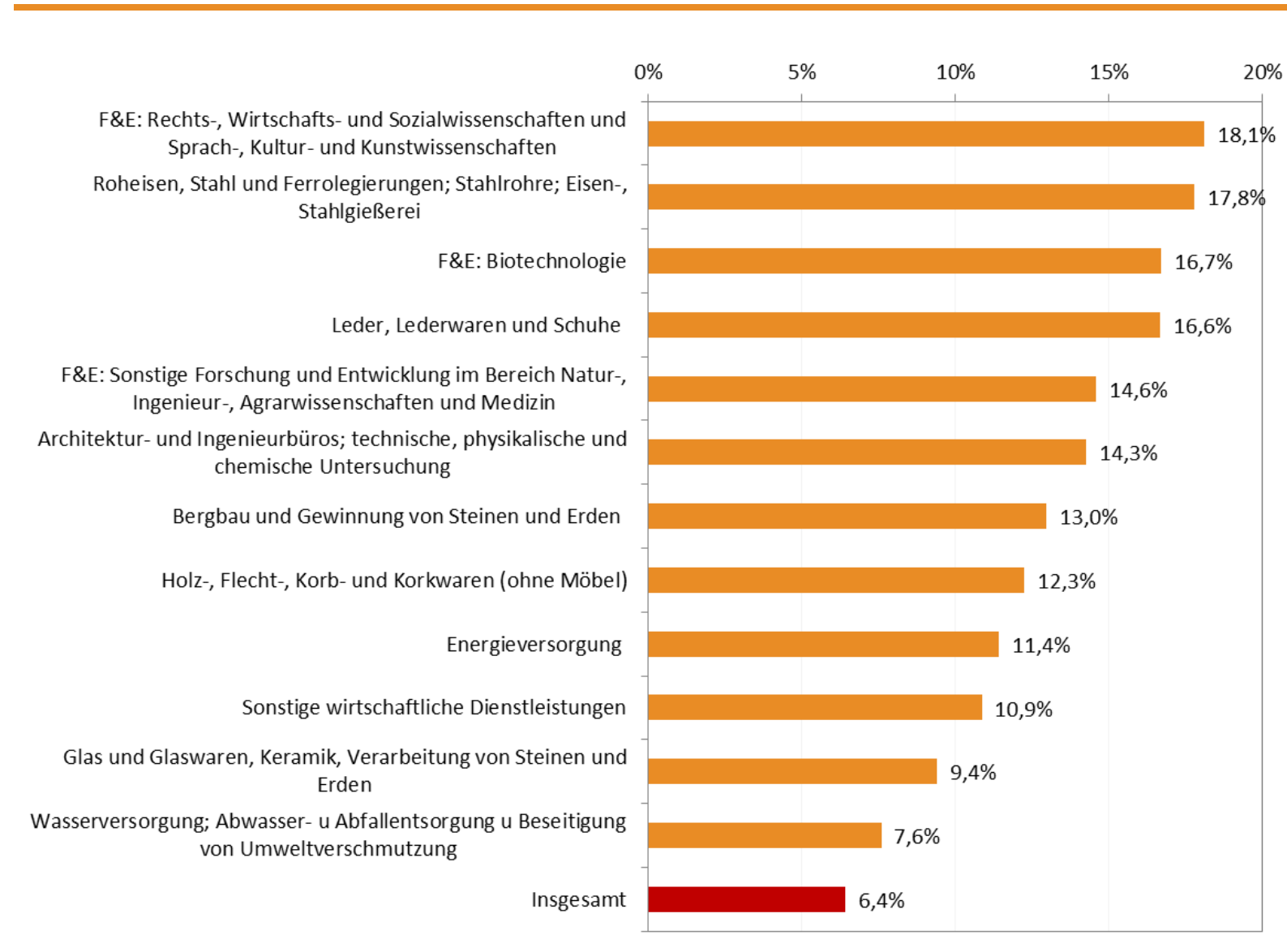

Quelle: Statistik Austria

Besondere Bedeutung kommt der Grundlagenforschung als Standortfaktor im Bereich der „transfer sciences" zu. Das sind jene wissenschaftlichen Gebiete, die eine überdurchschnittlich starke Verbindung zwischen Forschung an akademischen Einrichtungen und in Unternehmen aufweisen 
(wie z.B. die Biotechnologie). Aufgrund dessen finden sich auch die korrespondierenden "Cluster"mäßigen Agglomerationen im Umfeld der Forschungseinrichtungen größtenteils an Standorten mit hoher (Grundlagen-) Forschungsaktivität. Ein österreichisches Beispiel dafür wäre der Biotechnologie Forschungs- und Wirtschaftscluster im Raum Wien ${ }^{55}$.

\subsection{1 Öffentliche Forschungsförderung in Österreich}

Die für die F\&E-Quote relevanten Auszahlungen 2016 im Bundesbudget betrugen 2.682 Mio. Euro. Die Differenz zu den 3,8 Mrd. Euro durch den öffentlichen Sektor ergibt sich erstens dadurch, dass hier die Ausgaben des Bundes, der Bundesländer und sonstiger öffentlicher Einrichtungen enthalten sind. Zweitens errechnet sich der Bundesanteil aus zusätzlichen Ausgabenbereichen, die nur zum Teil direkt aus dem Bundesvoranschlag ableitbar sind. So sind die Mittel der Nationalstiftung für Forschung, Technologie und Entwicklung und die Forschungsprämie ebenfalls zurechenbare Ausgaben. Die folgende Tabelle enthält die wesentlichen Empfänger forschungswirksamer Auszahlungen, gegliedert nach Forschungseinrichtungen (1. die direkt F\&E durchführen; 2. Fördereinrichtungen, die F\&E Dritter fördern).

Tabelle 28: F\&E-Auszahlungen des Bundes nach wesentlichen Empfängern, in Mio. Euro

$\begin{array}{lrr}\text { F\&E-Auszahlungen } & \text { BVA-FV } 2017 & \text { BVA-FV } 2016 \\ \text { Forschungseinrichtungen } & 1.586,0 & 1.555,3 \\ \text { Universitäten inkl. Klinischer Mehraufwand (Bau) } & 44,2 & 42,2 \\ \text { Fachhochschulen } & 22,3 & 22,0 \\ \text { Pädagogische Hochschulen } & 112,2 & 105,0 \\ \text { Österreichische Akademie der Wissenschaften } & 48,8 & 49,8 \\ \text { Austrian Institut of Technology/Austrian Research Centers } & 7,6 & 5,0 \\ \text { Ludwig Boltzmann Gesellschaft } & 53,5 & 53,5 \\ \text { IST-Austria } & 1,6 & 5,5 \\ \text { Med Austron } & 16,0 & 16,7 \\ \text { Höhere Bundeslehranstalten } & & 335,7 \\ \text { Fördereinrichtungen } & 336,5 & 184,2 \\ \text { Forschungsförderungs GmbH } & 176,2 & 22,9 \\ \text { Fonds zur Förderung der wissenschaftlichen Forschung } & 51,5 & \end{array}$

Quelle: F\&E Beilage 2017 des BMF

55 „Stärkefelder im Innovationssystem: Wissenschaftliche Profilbildung und wirtschaftliche Synergien“, AIT (2015) 
Vergleichsweise kann man die konsolidierten Staatsausgaben im Aufgabenbereich Grundlagenforschung (01.4 nach COFOG) (ESVG 2010) heranziehen (s. Abbildung 146). Betrachtet man etwa das Jahr 2013, so wurden insgesamt 1.633 Mio. Euro durch den Staat für die Grundlagenforschung aufgewendet.

Abbildung 146: Staatsausgaben im Bereich Grundlagenforschung, 2012-2015, Österreich, nach ESVG 2010, konsolidiert, in Mio. Euro

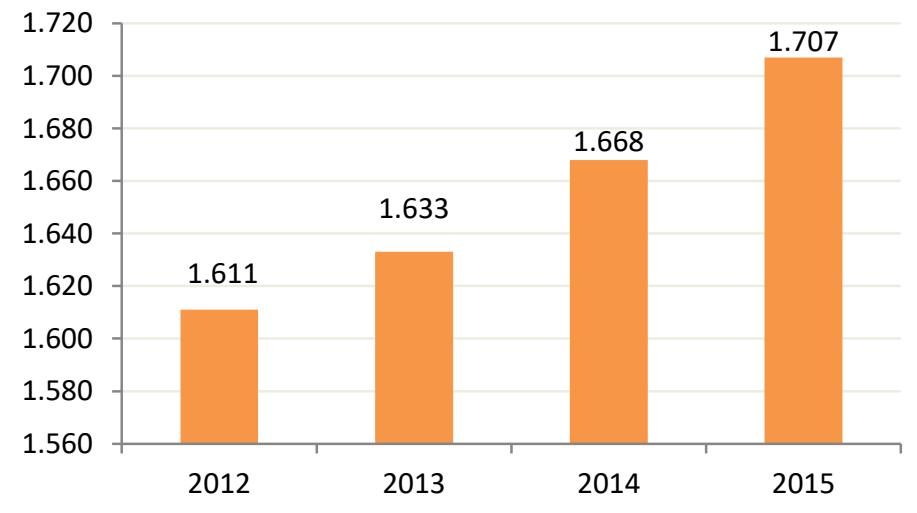

Quelle: Statistik Austria

Neben den Forschungsausgaben des Bundes (laut Bundesvoranschlag) gibt es auch noch die Ausschüttungen der Nationalstiftung für Forschung, Technologie und Entwicklung (FTE-Stiftung), die dem Bund (statistisch) zugerechnet werden. Die zur Verfügung stehenden Mittel entstammen aus Zinserträgen zweckgewidmeter Vermögen und sind daher sehr volatil. Da 2016 nur 18 Mio. Euro ausgeschüttet wurden, werden einmalig insgesamt 100 Mio. Euro aus Mitteln der Stabilitätsabgabe für Kreditinstitute bereitgestellt, wobei die konkrete Aufteilung noch nicht erfolgt ist.

Tabelle 29: Ausschüttungen der FTE-Stiftung, in Mio. Euro

\begin{tabular}{l|rrrrr}
\hline FTE-Stiftung - Begünstigte & 2012 & 2013 & 2014 & 2015 & 2016 \\
FFG & 19,4 & 30,0 & 12,5 & 37,9 & 5,0 \\
FWF & 13,0 & 23,2 & 12,0 & 20,0 & 5,0 \\
Akademie der Wissenschaften & 8,0 & 9,5 & 4,0 & 6,5 & 2,0 \\
\hline Christian Doppler Forschungsgesellschaft & 4,5 & 7,0 & 4,2 & 8,0 & 2,0 \\
Ludwig Boltzmann Gesellschaft & 4,1 & 7,6 & 2,0 & 4,6 & 2,0 \\
AWS & 2,3 & 15,5 & 4,0 & 8,0 & 2,0 \\
Summe & 51,3 & 92,8 & 38,7 & 85,0 & 18,0
\end{tabular}

Quelle: F\&E Beilage 2017 des BMF 
Hinzu kommen 2016 erstmalig noch Mittel des Österreich-Fonds, der ebenfalls von der FTE-Stiftung verwaltet wird.

Tabelle 30: Ausschüttungen des Österreich-Fonds, in Mio. Euro

$\begin{array}{lr} & 2016 \\ \text { FFG } & 11,2 \\ \text { FWF } & 10 \\ \text { Akademie der Wissenschaften } & 3 \\ \text { Christian Doppler Forschungsgesellschaft } & 2,5 \\ \text { Ludwig Boltzmann Gesellschaft } & 2 \\ \text { AWS } & 5 \\ \text { Summe } & 33,7\end{array}$

Quelle: F\&E Beilage 2017 des BMF

\subsubsection{Internationaler Vergleich}

Die Erhöhungen der österreichischen Ausgaben für Forschung und Entwicklung haben auch in internationalen Statistiken einen entsprechenden Niederschlag gefunden. Lag Österreich Mitte der 90er Jahre hinsichtlich der Forschungsquote noch hinter Deutschland, war diese Lücke 10 Jahre später geschlossen. Die Werte der letzten Jahre zeigen sogar, dass sich Österreich von Deutschland etwas absetzen und 2014 zu den EU-Spitzenreitern Schweden und Finnland aufschließen konnte.

\section{Abbildung 147: Ausgaben für Forschung und Entwicklung in Prozent des BIP, 1995 - 2014}

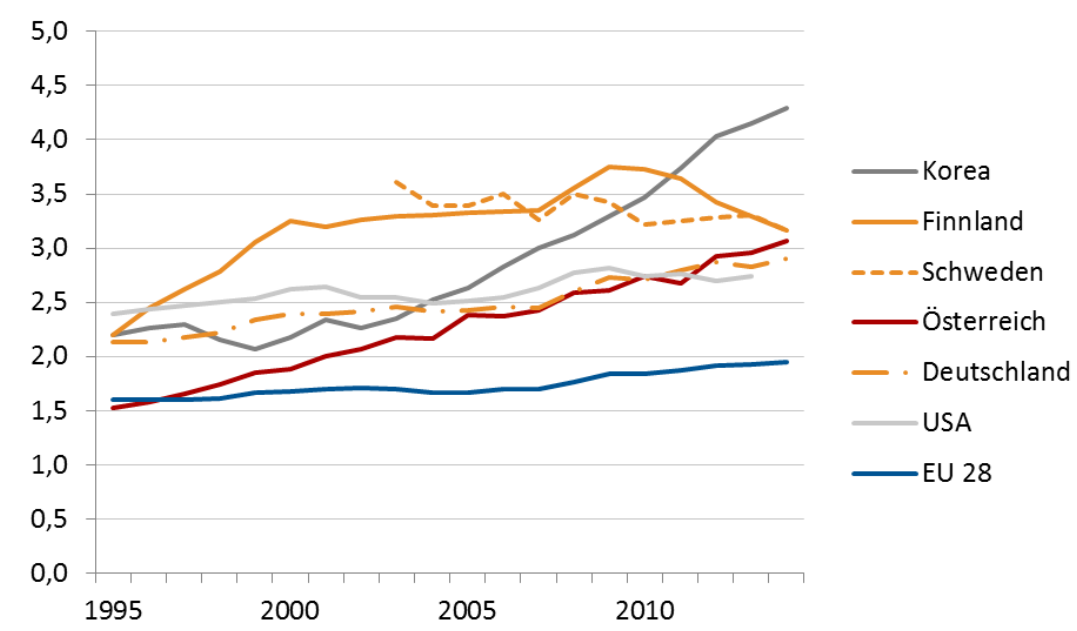


Die folgende Abbildung zeigt die F\&E-Quoten der EU-Länder und von Südkorea für 2015. Hier liegt Österreich in der EU hinter Schweden auf der zweiten Position, noch vor Dänemark, Finnland und Deutschland. Beachtlich ist der Vorsprung von Südkorea, mit einer F\&E-Quote von etwa 4,3 Prozent.

Abbildung 148: Ausgaben für Forschung und Entwicklung, in Prozent des BIP, 2015

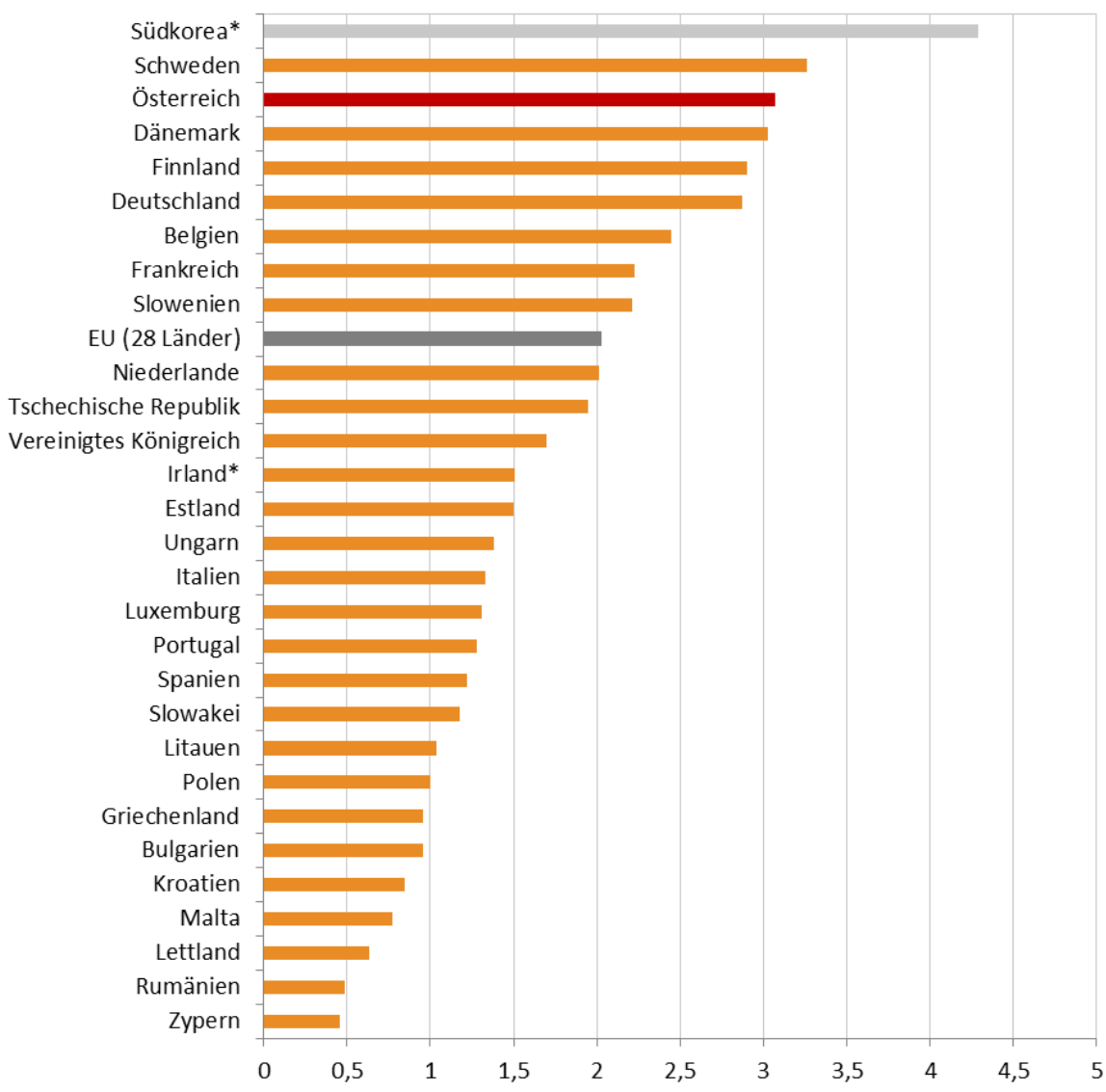

Quelle: Eurostat; * Diese Werte stammen aus dem Jahr 2014.

Betrachtet man die österreichischen Ausgaben für Grundlagenforschung im internationalen Vergleich, so zeigt sich, dass Österreich mit einer Quote von 0,56\% des BIP (2013) ebenfalls zu den führenden Ländern in der EU gehört. Konkret liegt Österreich mit diesem Wert vor Dänemark (2012: 0,52 \%) und Frankreich (2012: 0,54\%). Weltweit gesehen liegen allerdings z.B. Südkorea (2013: 
0,75\%) oder die Schweiz (2012: 0,9\%) ${ }^{56}$ bei den Ausgaben für Grundlagenforschung (in Prozent des BIP) deutlich vor Österreich.

Abbildung 149: Ausgaben für Grundlagenforschung in Prozent des BIP, 2012 bzw. 2013

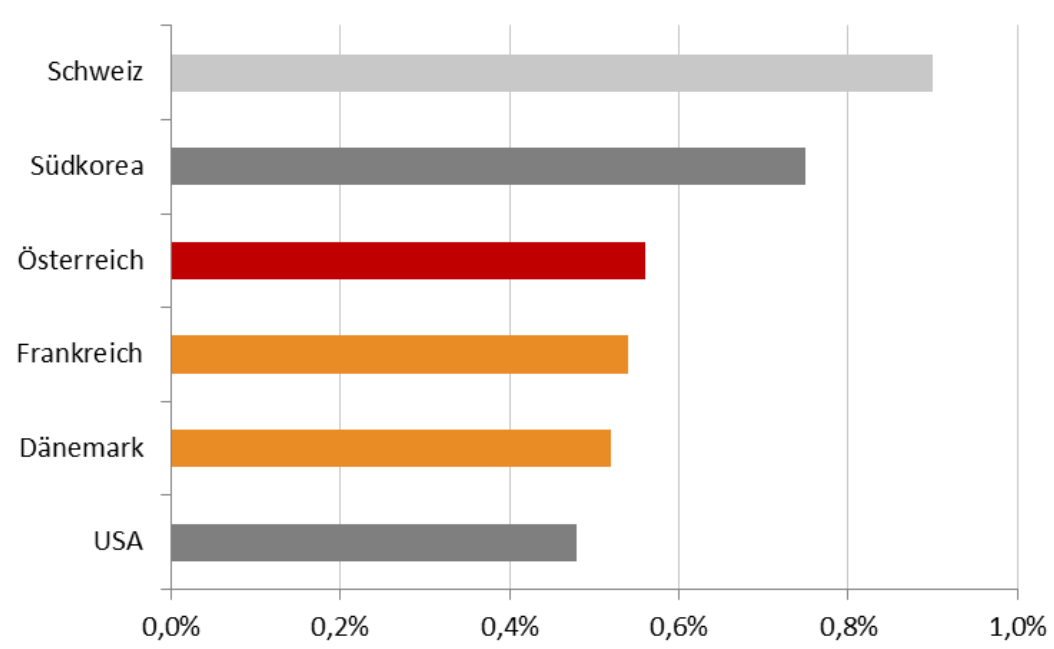

Quelle: Österreichischer Forschungs- und Technologiebericht 2016, BMWFW und BMVIT.

Besonders aufschlussreich ist der Vergleich mit Deutschland. Dort wurden im Jahr 2014 durch Staat, Wirtschaft und Wissenschaft insgesamt rund 84,3 Mrd. Euro für F\&E ausgegeben. Der Unternehmenssektor in Deutschland liefert dabei einen etwas geringen Anteil (67,5\%) als jener in Österreich (70,8\%), wodurch der Staat in Deutschland relativ gesehen mehr zu den innerbetrieblichen F\&E-Ausgaben beisteuert.

Aufgegliedert nach Forschungsarten lässt sich bei den öffentlichen Ausgaben für Forschung und Entwicklung eine klare Priorisierung der Grundlagenforschung in Deutschland feststellen (siehe Abbildung 150). In Deutschland entfallen auf die Grundlagenforschung etwa 58\% der öffentlichen F\&E-Budgetmitte ${ }^{57}$ (12.527 Mio. Euro).

\footnotetext{
${ }^{56}$ Der internationale Vergleich von Ausgaben für Grundlagenforschung ist jedoch nur bedingt möglich, da viele Länder, wie etwa Finnland oder Schweden, in ihren F\&E-Erhebungen nicht nach Forschungsarten unterscheiden.

${ }^{57}$ Hier kann nur der Wert für 2014 berücksichtigt werden, da die Ausgaben für Forschung und Entwicklung nach der Art der Forschungstätigkeit vom Statistischen Bundesamt in Deutschland nur im vierjährigen Rhythmus erhoben werden.
} 
Abbildung 150: Interne Ausgaben für Forschung und Entwicklung der wissenschaftlichen Einrichtungen des öffentlichen Sektors nach Forschungsart in Mio. Euro, Deutschland, 2014

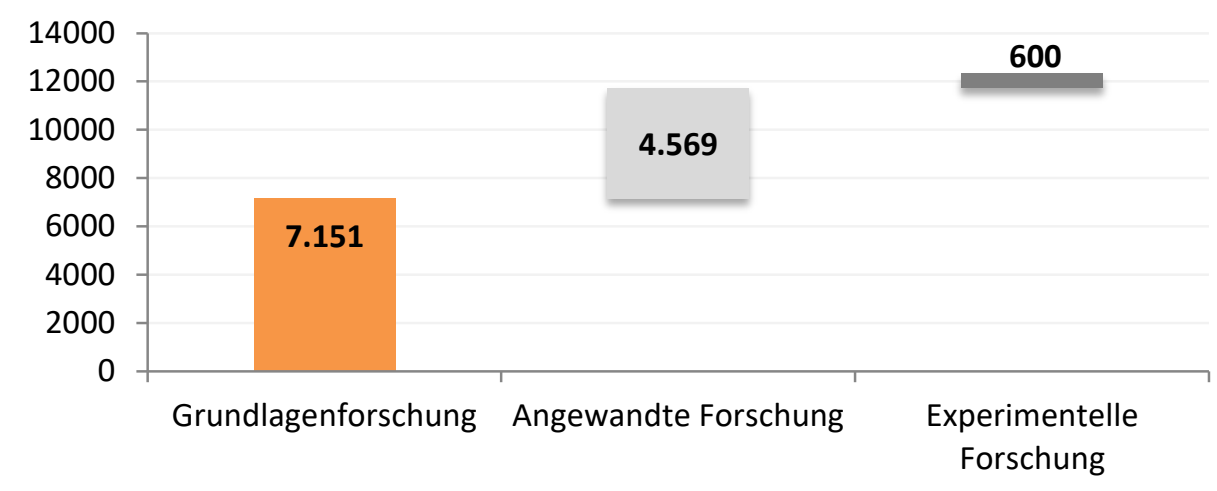

Quelle: Statistisches Bundesamt ${ }^{58}$

\subsubsection{Impakt der Grundlagenforschung}

Als mittelbarer Output der Grundlagenforschung kann u.a. die Highly Cited Researchers List 2016 herangezogen werden, die eingeschränkt auf ehemalige CD-Laborleiter auch die Bedeutung der CDG beschreibt. Bei der Highly Cited Researchers List handelt es sich um eine Liste der weltweit einflussreichsten Wissenschaftler, die von Clarivate Analytics publiziert wird und auf einer Analyse von Web of Science ${ }^{59}$ Daten beruht. Die Liste für 2016 ergibt sich aus den meist-zitierten Artikeln in wissenschaftlichen Fachjournalen im Zeitraum 2004-2014. Als ,meist-zitiert' werden dabei jene Publikationen bezeichnet, die sich unter den Top 1\% der Zitierungen innerhalb ihres Fachbereichs und Publikationsjahres befinden. Von den 20 in Österreich tätigen Forschern, die in der Liste für 2016 aufscheinen, befinden sich zwei, die im Zeitraum 2004-2014 auch als CD-Laborleiter tätig waren. Verglichen mit dem Anteil an Budgetmitteln, die der CDG zur Verfügung stehen, ist das ein sehr gutes Ergebnis.

Einen gewissen Aufschluss über den Wert der Grundlagenforschung lassen auch Innovationsrankings zu, die anhand verschiedener Indikatoren die Innovationsleistung einer Volkswirtschaft erfassen und

\footnotetext{
${ }^{58}$ „Finanzen und Steuern. Ausgaben, Einnahmen und Personal der öffentlichen und öffentlich geförderten Einrichtungen für Wissenschaft, Forschung und Entwicklung" (2016) Fachserie 14 Reihe 3.6, Statistisches Bundesamt, Wiesbaden

${ }^{59}$ Web of Science ist einer der bekanntesten Provider von wissenschaftlichen Online-Zitations- und Literaturdatenbanken.
} 
in einer international vergleichbaren Kennzahl ausdrücken. Für einen EU-weiten Vergleich eignet sich hierbei das European Innovation Scoreboard (EIS) der Europäischen Kommission (s. Abbildung 151).

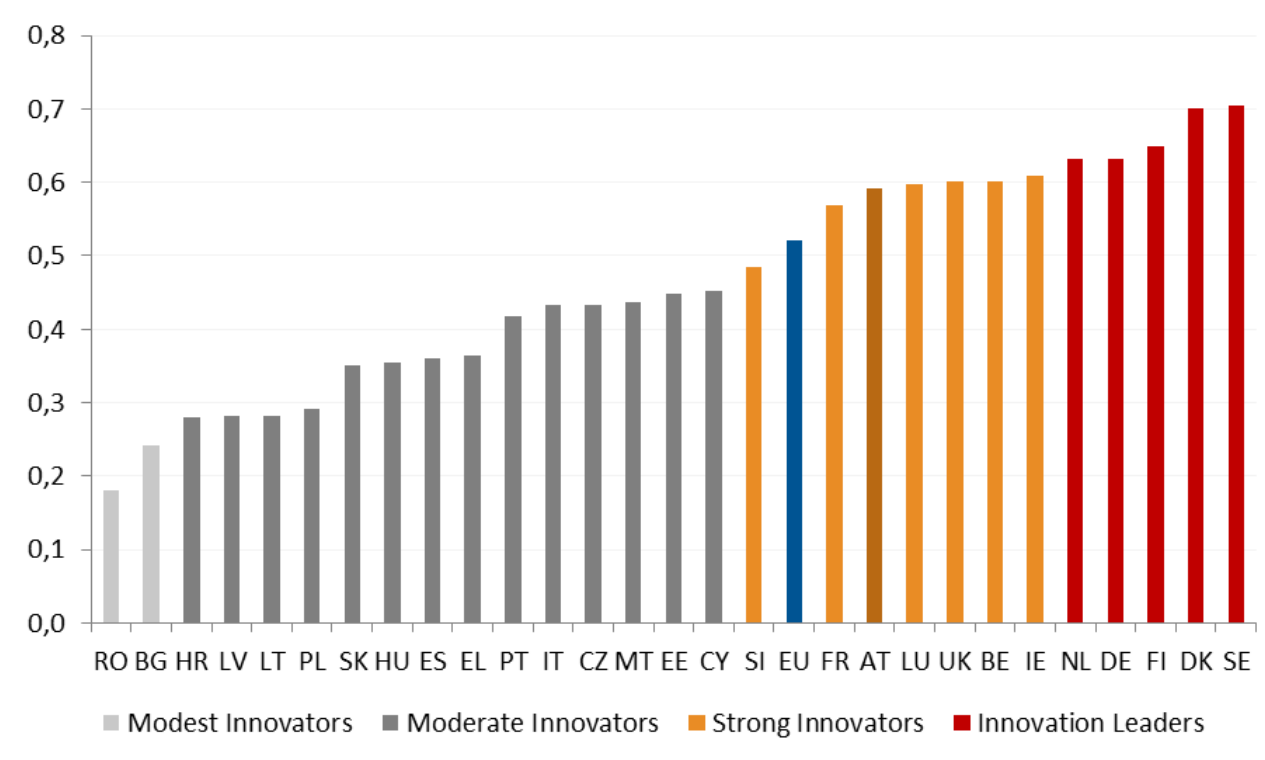

Quelle: Europäische Kommission ${ }^{60}$

Österreich belegte 2016 einen Platz unter den Top 10 Nationen in der EU, und konnte den deutlichen Vorsprung relativ zum EU-Durchschnitt in etwa halten. Nichts desto trotz bleibt ein substantieller Abstand zur Gruppe der ,Innovation Leaders', zu denen Deutschland, die skandinavischen Länder sowie die Niederlande gehören. Die Zielsetzung der Bundesregierung im Rahmen der FTI-Strategie, dass Österreich ebenfalls ein ,Innovation Leader' ${ }^{\prime}$ wird ${ }^{61}$ erscheint daher nur langfristig möglich. Auffallend ist, dass sich in dieser Gruppe vor allem jene Länder - wie z.B. Deutschland - befinden, die ausgezeichnete Grundlagenforschung betreiben.

In der folgenden Abbildung wird der EIS-Innovationsindexwert für die "Strong Innovators" zwischen 2008 und 2015 wiedergegeben, wodurch eine Entwicklung über die Zeit erkennbar ist. Betrachtet man den Beobachtungszeitraum, so hat Österreich den Höchstwert des Innovationsindex aus dem Jahr 2010 seither nicht mehr erreicht. Für die meisten Gruppenmitglieder hat sich der

\footnotetext{
60 „European Innovation Scoreboard 2016 Main Report", Europäische Kommission (2016)

${ }^{61}$ WKO Kommentar Wirtschaftspolitik 2015/15 „Österreich im Innovation Union Scoreboard 2015: Reality-Check auf dem Weg zum Innovation Leader": https://www.wko.at/Content.Node/Interessenvertretung/Standort-und-Innovation/2015-15Innovation-Union-Scoreboard---Oesterreich-nur-noch-E.pdf
} 
Innovationsgrad im Zeitraum 2008-2015 verbessert, wobei die größten jährlichen Zuwächse in Großbritannien (durchschnittliche Wachstumsrate 2008-2015: 2,0\%), Slowenien (1,2\%), Belgien $(0,9 \%)$ und Frankreich $(0,8 \%)$ verzeichnet wurden. Das durchschnittliche jährliche Wachstum Österreichs lag hingegen, mit 0,2\%, unter jenem der EU $(0,7 \%)$ und relativ zum EU-Durchschnitt ist die Innovationsleistung Österreichs im Beobachtungszeitraum gesunken.

Abbildung 152: EIS Innovations-Index in der Gruppe der „Strong Innovators“, 2008-2015

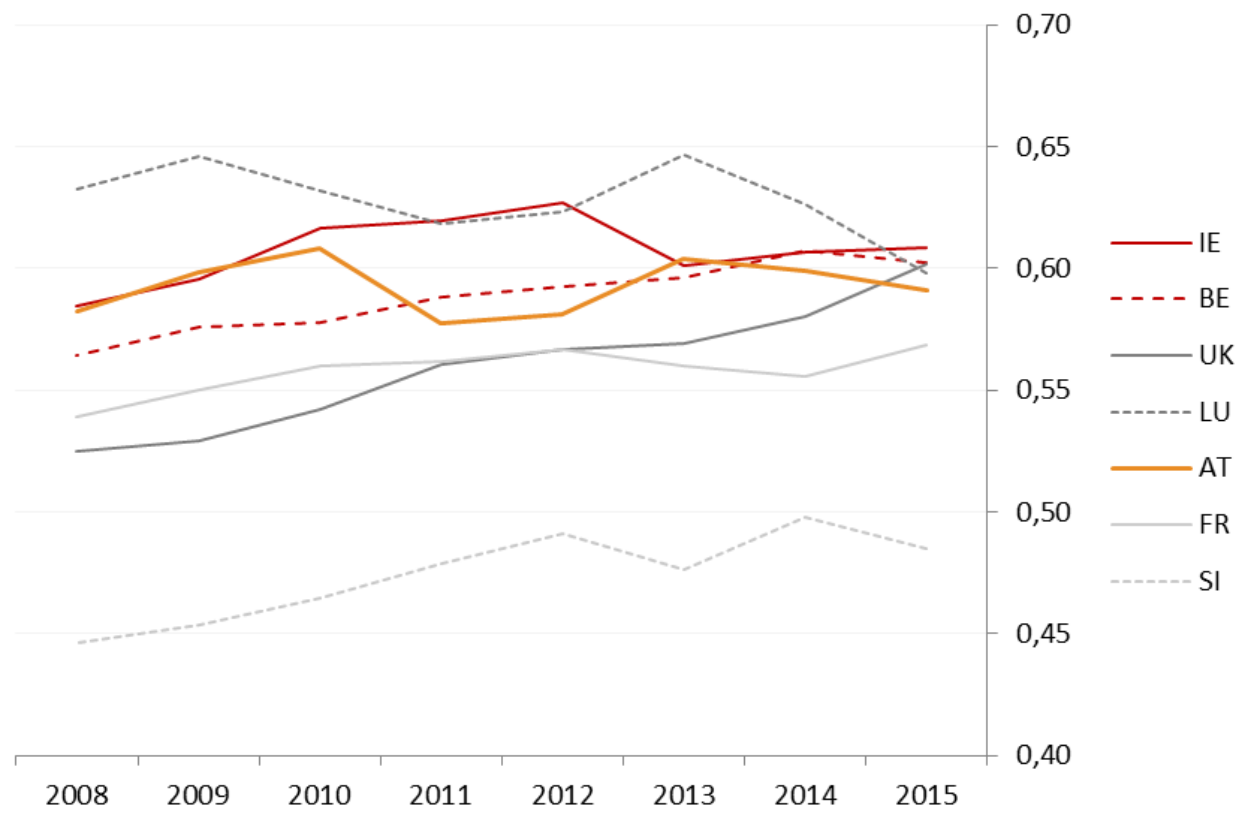

Quelle: Europäische Kommission ${ }^{62}$

FWF-Präsidentin Pascale Ehrenfreund ${ }^{63}$ argumentiert, dass für einen Aufstieg Österreichs in die Gruppe der ,Innovation Leader' eine intensivere Verknüpfung zwischen Grundlagenforschung und angewandter Forschung nötig wäre. Dies ist in Österreich aber insofern erschwert, da Grundlagenforschung vor allem vom Hochschulsektor getragen wird, während im Bereich Angewandte Forschung die Unternehmen den wichtigsten Durchführungssektor stellen. Tendenziell wird in Österreich vor allem in forschungsintensiven Branchen relativ wenig in Grundlagenforschung

\footnotetext{
62 „European Innovation Scoreboard 2016 Main Report“, Europäische Kommission (2016)
} 
investiert. Schibany und Gassler ${ }^{64}$ (2010) sind der Ansicht, dass dies als Zeichen für eine funktionierende „Arbeitsteilung“ zwischen Unternehmen, die sich auf angewandte Forschung spezialisieren, und öffentlicher Forschung, welche die Grundlagenforschung übernimmt, interpretiert werden kann. Interessant bleibt diese Aspekt jedoch im Vergleich zu Deutschland, wo Unternehmen eine bedeutendere Rolle in der Forschungsfinanzierung übernehmen und die Innovationsleistung in weiterer Folge deutlich über der von Österreich liegt.

Hier wäre ein geeigneter Anknüpfungspunkt für die CDG, um die Unternehmensseite und die Hochschulen (noch) näher aufeinander zuzuführen und somit zu einer übergreifenden Forschungsund Innovationsgemeinschaft beizutragen. Langfristige Investitionen in die Grundlagenforschung sind Teil der Voraussetzungen für technologische Entwicklungen und die Wettbewerbsfähigkeit in der Zukunft. Hierfür ist ein ausreichendes Maß an Finanzierung - vor allem im Vergleich mit der in Österreich wesentlich stärker geförderten angewandten Forschung - erforderlich. Falls das Ziel, ein europaweiter "Innovation Leader“ zu werden, erreicht werden soll, ist es notwendig, durch ausreichende Förderung der Grundlagenforschung zur Entstehung von radikalen Innovationen beizutragen sowie den kompetitiven Anteil der Forschungsfinanzierung anzuheben.

\footnotetext{
${ }^{64}$ Andreas Schibany, Helmut Gassler (2010) „Nutzen und Effekte der Grundlagenforschung“, Joanneum Research Graz.
} 


\subsection{Patentanalyse}

In diesem Abschnitt wird eine Patentanalyse präsentiert, mit deren Hilfe vielversprechende Themenbereiche für gezielte Aktivitäten der Christian Doppler Gesellschaft identifiziert werden sollen. Auf Basis von Patentveröffentlichungen wird versucht, die beiden Fragestellungen nach den aktuellen Technologiestärkefeldern Österreichs und nach weltweit hoch dynamischen Technologiefeldern zu beantworten.

Im ersten Teil der Patentanalyse werden die Patentveröffentlichungen der letzten 20 Jahre nach dem Patentklassifizierungsschema CPC (Cooperative Patent Classification) in rund 11.000 Technologiefeldern (=CPC Hauptgruppen) unterteilt, und für jedes Technologiefeld die weltweite Patentanmeldedynamik jener aus Österreich gegenübergestellt. Daraus leiten sich die Technologiestärkefelder Österreichs ab. Jene 15 Technologiefelder mit dem höchsten Patentanteil aus Österreich werden in 10 Themengruppen zusammengefasst und der inter-disziplinäre Charakter aller Patentveröffentlichungen der letzten 10 Jahre (weltweite Datenbasis) auf ihre konvergierenden Technologien („Crossing-Technologies“) untersucht. Aus der relativen Stärke in den Crossing-Technologies, der Industriestruktur und internationalen Wissensnetzwerken der einzelnen Themengruppen leitet sich eine Abschätzung für vielversprechende Aktivitäten der CDG ab.

Im zweiten Teil wurden alle weltweiten Technologiefelder nach ihrer Dynamik gereiht und daraus ungeachtet der Position Österreichs - die am schnellsten wachsenden Themen abgeleitet. Die Top-15 Technologiefelder lassen sich in weiterer Folge in sechs Themengruppen zusammenfassen, die mögliche Bereiche für Forschungsschwerpunkte zukünftiger CD-Labors bzw. JR-Zentren darstellen.

\subsubsection{Identifikation der Technologiestärkefelder Österreichs}

Von den untersuchten Technologiefeldern, die über die CPC Hauptgruppen definiert wurden, zeigen im 5-Jahresintervall zwischen 2012/01 und 2016/12 rund 3.400 mehr als 1.000 Patentfamilien ${ }^{65}$. Die folgende Abbildung zeigt die Verteilung der Patentintensitäten (im Sinne von veröffentlichten Patentfamilien) nach den einzelnen Technologiefeldern. Die weitere Bestimmung und Analyse der Technologiestärkefelder in Österreich beruht auf diesen 3.400 Technologiefeldern.

\footnotetext{
${ }^{65}$ Eine Patentfamilie ist definiert als ein Komplex von Patenten, die in verschiedenen Ländern bzw. nationalen Patentämtern zum Schutz ein und derselben Erfindung registriert werden.
} 


\section{Abbildung 153: Patenthäufigkeiten der Technologiefelder}

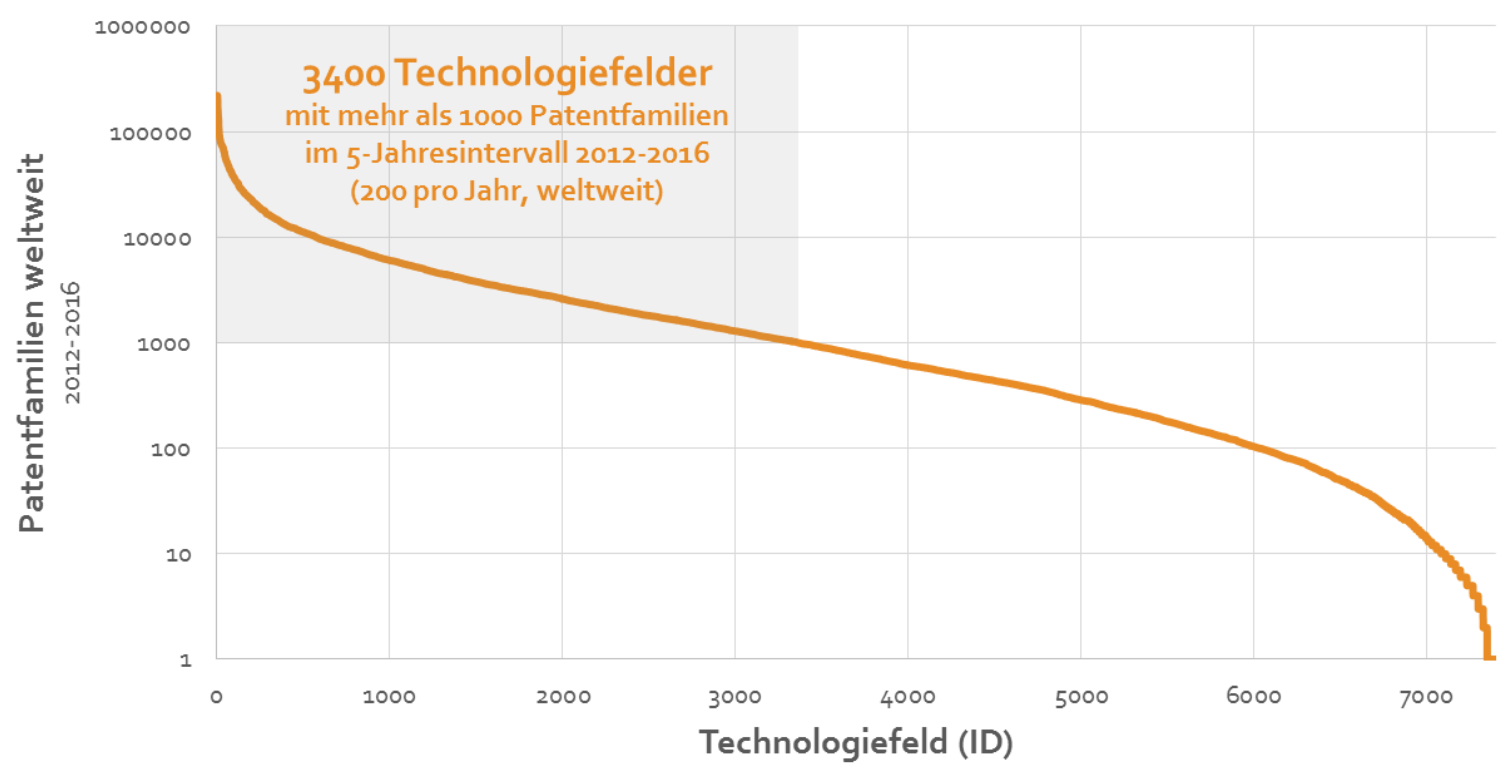

Quelle: Economica (2017).

Die Verteilung des Patentanteils aus Österreich, bezogen auf alle Patentfamilien eines Technologiefeldes („relative Stärke“, $F_{A T} / F_{\text {Wo }}$ ) wurde für die vier Zeitintervalle (i) 1996-2001, (ii) 20022006, (iii) 2007-2011 und (iv) 2012-2016 bestimmt. Es zeigt sich, dass die Zahl der Technologiefelder mit geringer relativer Stärke (Anteil von Patentfamilien aus Österreich kleiner als 2,5\%, bezogen auf alle Patentfamilien weltweit) in den ersten drei Untersuchungsperioden von 3.477 auf 4.326 zunahm, und im jüngsten Intervall 2012-2016 wieder rückläufig war (4.128 Technologiefelder unter 2,5\% Patentanteil aus Österreich). Dies deutet auf eine Trendumkehr hin zu höherer Spezialisierung, was auch durch die Zunahme der Zahl jener Technologiefelder mit höherer relativer Stärke (ab 2,5\% bzw. ab 5\%) unterstützt wird.

Diese Beobachtung wurde sowohl bei der Betrachtung aller Technologiefelder gemacht, als auch bei der gesonderten Betrachtung jener rund 3.400 Technologiefelder mit einem weltweiten Patentoutput von mehr als 1.000 Patentfamilien im 5-Jahresintervall. Auch in dieser Analyse der 3.400 am häufigsten patentierten Technologiefelder (Abbildung 155) ist der Anstieg bei der Anzahl von Patentklassen mit relativer Stärke von über $2.5 \%$ bzw. über $5.0 \%$ bemerkenswert. Dies zeigt die Spezialisierung der österreichischen Innovatoren auf Stärkefelder entgegen dem Trend zwischen 1996 und 2011. 


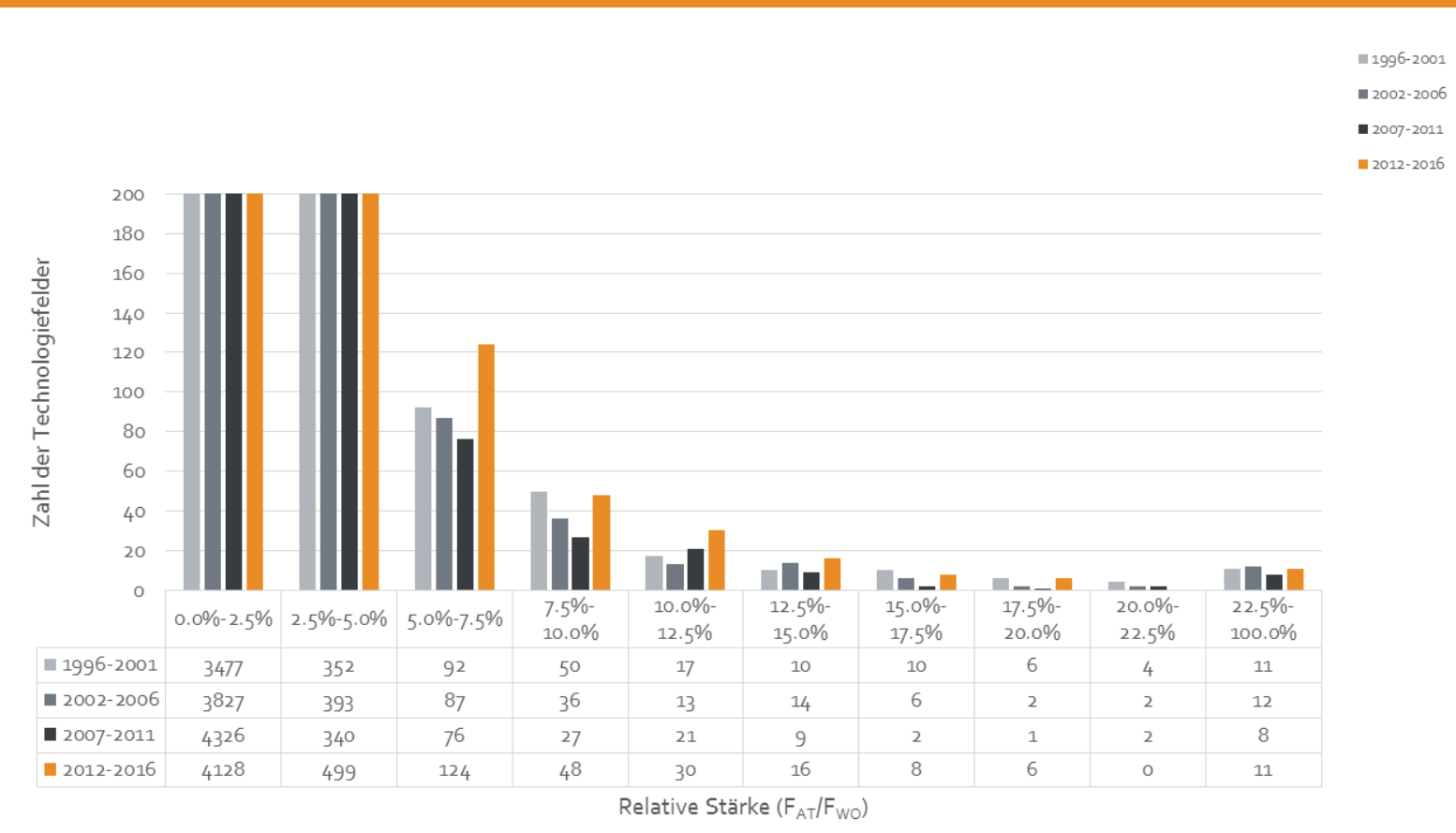

Quelle: Economica (2017).

Abbildung 155: Verteilung der Themen nach relativer Stärke (Top 3.400 Technologiefelder)

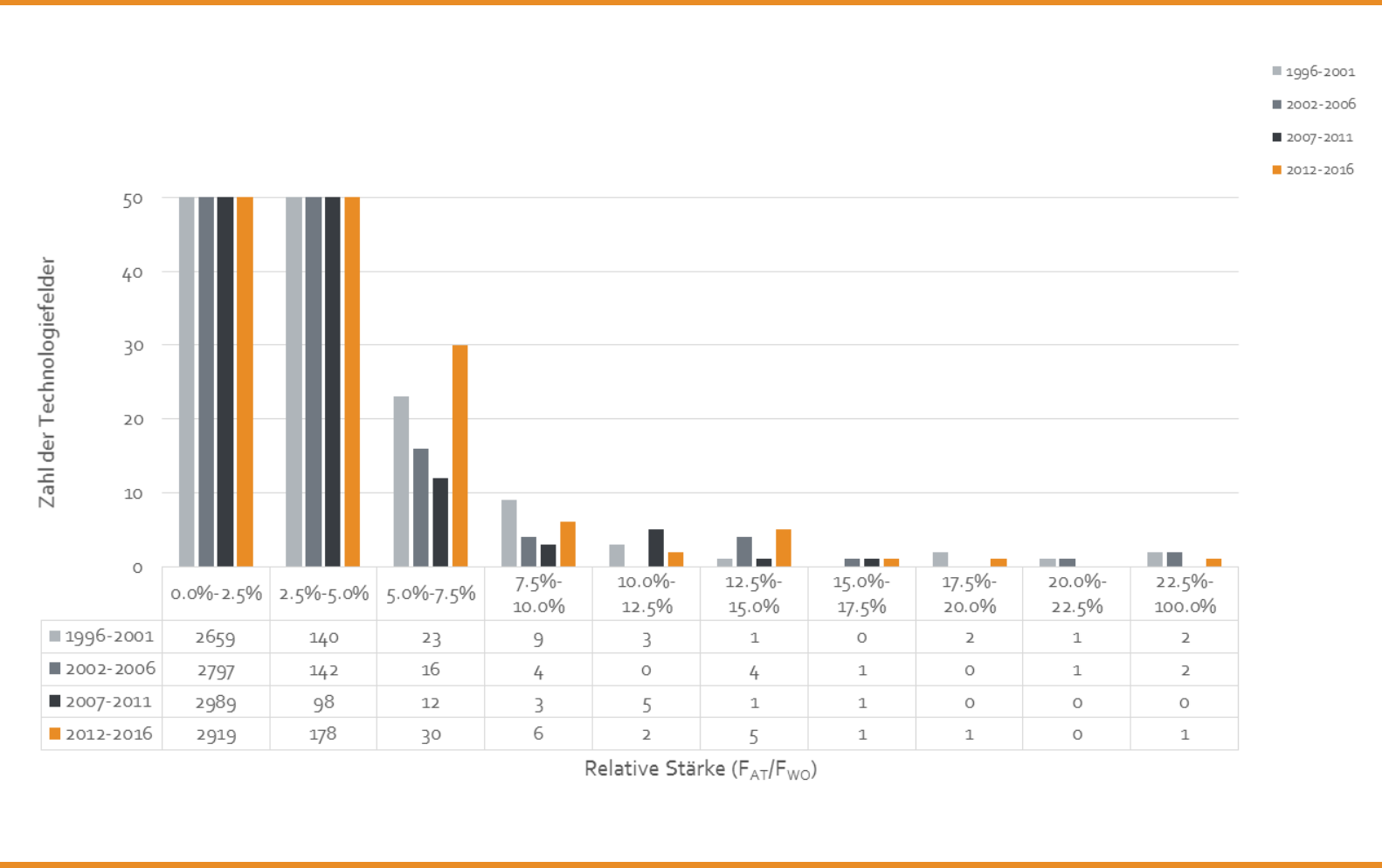

Quelle: Economica (2017). 


\section{Abbildung 156: Relative Themenintensität Österreich}

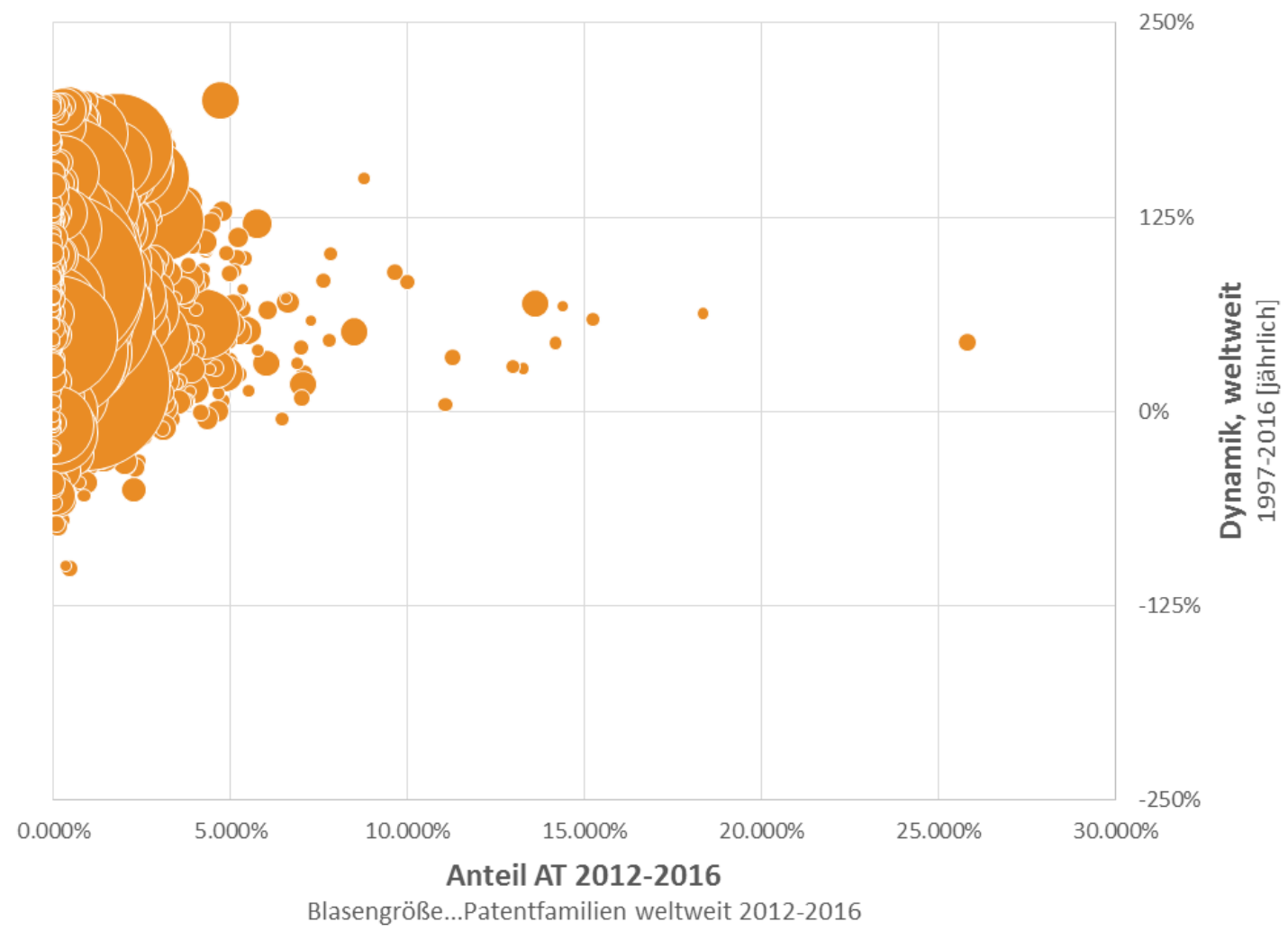

Quelle: Economica (2017).

Die Verteilung der Technologiefelder auf den Achsen Patentanteil und weltweite Dynamik zeigt mögliche Strategien in der Selektion von vielversprechenden Themen. Im rechten Bereich des Technologieportfolios sind jene Technologiefelder eingetragen, die einen besonders hohen Patentanteil aus Österreich haben, im oberen Bereich jene mit hohen weltweiten Wachstumsraten. Die Wachstumsrate wurde dabei als jährliche Steigung der Patentanmeldefrequenz bezogen auf den Mittelwert (relative jährliche Wachstumsrate) bestimmt.

Für die weitere Analyse wurden nun Technologiefelder identifiziert, die einerseits einen hohen Patentanteil aus Österreich aber andererseits gleichzeitig auch eine deutlich positive weltweite Dynamik aufweisen. Aufgrund dieser beiden Kriterien wurden 15 Technologiefelder mit dem höchsten Patentanteil aus Österreich zwischen 2012 und 2016 in Tabelle 31 zusammengefasst, wobei - wie bereits gesagt - nur jene Technologiefelder mit zumindest durchschnittlich jährlich 200 Patentfamilien weltweit betrachtet wurden. Mit 25,8\% Patentanteil weist das Thema „Einzelheiten von Rolltreppen und Fahrsteigen" die höchste relative Stärke auf, gefolgt von Bestandteilen, 
Einzelheiten oder Zubehör für Seilbahnen $(18,4 \%$ Patentanteil aus Österreich) und Sicherheitseinrichtungen für Rolltreppen oder Fahrsteige (15,2\%). Weitere Themen mit Patentanteilen zwischen $11 \%$ und $15 \%$ sind sonstige Eisenbahnfahrzeuge (z.B. Gerüstwagen), Cellulosefasern, Schubladen (für Tische, Schränke o.ä.), Trockenpartie von Papiermaschinen, Stahlerzeugung im Direktverfahren, Handwerkzeuge zum Nageln und Handstempel. Die Themen Gleisbau, Industrieöfen, Sicherheitsvorrichtungen beim spanlosen Metallbearbeiten, Metallbearbeitung und Bäckereiöfen für besondere Backwaren weisen einen Patentanteil aus Österreich zwischen $7,8 \%$ und $10 \%$ auf.

Tabelle 31: Top-15 Technologiestärkefelder Österreichs nach Patentanteil

\begin{tabular}{|c|c|c|c|}
\hline IPC L4 & Description & $\mathrm{F}_{\mathrm{AT}} / \mathrm{F}_{\text {WO }}$ & $\begin{array}{l}\text { Dyn }_{\text {wo }} \\
1996-2016\end{array}$ \\
\hline B66B23 & Aufzüge > Einzelteile von Rolltreppen oder Fahrsteigen & $25.8 \%$ & $44.7 \%$ \\
\hline $\mathrm{B} 61 \mathrm{~B}_{12}$ & $\begin{array}{l}\text { Eisenbahnanlagen > Bestandteile, Einzelheiten oder Zubehör für Seilbahnen und an- } \\
\text { und abkuppelbare Systeme }\end{array}$ & $18.4 \%$ & $63.0 \%$ \\
\hline B66B29 & Aufzüge > Sicherheitseinrichtungen für Rolltreppen oder Fahrsteige & $15.2 \%$ & $59.2 \%$ \\
\hline $\mathrm{B} 61 \mathrm{D}_{15}$ & $\begin{array}{l}\text { Einzelheiten oder Arten des Wagenaufbaus von Eisenbahnfahrzeugen > Sonstige } \\
\text { Eisenbahnfahrzeuge, z.B. Gerüstwagen }\end{array}$ & $14.4 \%$ & $67.8 \%$ \\
\hline $\mathrm{Do1F}_{2}$ & $\begin{array}{l}\text { Chemische Gesichtspunkte bei der Herstellung von künstlichen Fäden, Filamenten, } \\
\text { Fasern, Borsten oder Bändern > Künstliche Monokomponentenfäden oder dgl. aus } \\
\text { Cellulose oder Cellulosederivaten }\end{array}$ & $14.2 \%$ & $43.9 \%$ \\
\hline A47B88 & Tische > Schubladen für Tische, Schränke oder ähnliche Möbel & $13.6 \%$ & $69.8 \%$ \\
\hline $\mathrm{D}_{21} \mathrm{~F}_{5}$ & $\begin{array}{l}\text { Papiermaschinen > Trockenpartie an Maschinen zur fortlaufenden Herstellung von } \\
\text { Papierbahnen }\end{array}$ & $13 \cdot 3 \%$ & $27.8 \%$ \\
\hline $\mathrm{C}_{21 \mathrm{~B} 13}$ & $\begin{array}{l}\text { Gewinnung von Eisen oder Stahl > Gewinnung von Eisenschwamm oder flüssigem Stahl } \\
\text { durch Direktverfahren }\end{array}$ & $13.0 \%$ & $29.0 \%$ \\
\hline $\mathrm{B}_{25} \mathrm{C}_{1}$ & Handwerkzeuge zum Nageln oder Heften > Handwerkzeuge zum Nageln & $11.3 \%$ & $35.0 \%$ \\
\hline $\mathrm{B}_{41 \mathrm{~K} 1}$ & $\begin{array}{l}\text { Stempel > Tragbare handbetätigte Vorrichtungen ohne Mittel zum Unterstützen oder } \\
\text { Festlegen der zu stempelnden Gegenstände, z.B. Handstempel }\end{array}$ & $11.1 \%$ & $4 \cdot 5 \%$ \\
\hline E01B29 & Gleisoberbau > Verlegen, Auswechseln oder Aufnehmen von Gleisen & $10.0 \%$ & $83 \cdot 5 \%$ \\
\hline $\mathrm{F}_{27} \mathrm{~B} 17$ & Industrieöfen, Schachtöfen, Brennöfen, Retorten allgemein > Industrieöfen & $9.6 \%$ & $89.6 \%$ \\
\hline $\mathrm{B}_{21} \mathrm{D}_{55}$ & $\begin{array}{l}\text { Bearbeiten oder Verarbeiten von Blechen, Metallrohren, -stangen oder -profilen ohne } \\
\text { wesentliches Abtragen des Werkstoffs > Sicherheitseinrichtungen zum Schutz der in } \\
\text { dieser Unterklasse behandelten Maschinen oder Vorrichtungen sowie ihrer } \\
\text { Bedienungspersonen }\end{array}$ & $8.8 \%$ & $150.1 \%$ \\
\hline $\mathrm{B}_{21} \mathrm{D}_{5}$ & $\begin{array}{l}\text { Bearbeiten oder Verarbeiten von Blechen, Metallrohren, -stangen oder -profilen ohne } \\
\text { wesentliches Abtragen des Werkstoffs > Biegen von Blechen, z.B. zum Bilden einfacher } \\
\text { Krümmungen }\end{array}$ & $8.5 \%$ & $51.0 \%$ \\
\hline $\mathrm{A} 21 \mathrm{~B} 5$ & Bäckereiöfen > Backgeräte für besondere Backwaren & $7.8 \%$ & $101.7 \%$ \\
\hline
\end{tabular}

Quelle: Economica (2017).

Diese 15 Technologiefelder, in denen Erfinder in Österreich besonders stark patentieren, wurden in 10 Themengruppen zusammengefasst. Diese Themengruppen lauten: Aufzüge, Cellulose, Eisenbahn, Lebensmittel, Metallbearbeitung, Möbel, Papier, Stahl, Stempel und Werkzeug. Wichtig für die Inter- 
pretation der Ergebnisse ist, dass nur jene Patentklassen mit besonders hohem Patentanteil aus Österreich für die Definition der Themengruppen verwendet wurden ${ }^{66}$. Die zehn Themengruppen wurden in weiterer Folge hinsichtlich Industriestruktur, weltweiter Wissensnetzwerke, Dynamik und ihrer Crossing-Technologies untersucht, die detaillierten Auswertungen dazu befinden sich im Anhang.

Tabelle 32: Die 10 Themengruppen der 15 Technologiestärkefelder Österreichs

\begin{tabular}{|c|c|c|}
\hline Themengruppe & IPC L4 & Description \\
\hline Aufzüge & $\mathrm{B} 66 \mathrm{~B} 23$ & 3 Aufzüge > Einzelteile von Rolltreppen oder Fahrsteigen \\
\hline Aufzüge & B66B29 & 9 Aufzüge $>$ Sicherheitseinrichtungen für Rolltreppen oder Fahrsteige \\
\hline Cellulose & D01F2 & $\begin{array}{l}\text { Chemische Gesichtspunkte bei der Herstellung von künstlichen Fäden, Filamenten, Fasern, } \\
\text { Borsten oder Bändern > Künstliche Monokomponentenfäden oder dgl. aus Cellulose oder } \\
\text { Cellulosederivaten }\end{array}$ \\
\hline Eisenbahn & B61B12 & $\begin{array}{l}2 \text { Eisenbahnanlagen > Bestandteile, Einzelheiten oder Zubehör für Seilbahnen und an- und } \\
\text { abkuppelbare Systeme }\end{array}$ \\
\hline Eisenbahn & B61D15 & $\begin{array}{l}5 \text { Einzelheiten oder Arten des Wagenaufbaus von Eisenbahnfahrzeugen > Sonstige } \\
\text { Eisenbahnfahrzeuge, z.B. Gerüstwagen }\end{array}$ \\
\hline Eisenbahn & E01B29 & 9 Gleisoberbau > Verlegen, Auswechseln oder Aufnehmen von Gleisen \\
\hline Lebensmittel & A21B5 & Bäckereiöfen > Backgeräte für besondere Backwaren \\
\hline $\begin{array}{l}\text { Metallbearbeitung } \\
\text { Metallbearbeitung }\end{array}$ & B B21D55 & $\begin{array}{l}5 \text { Bearbeiten oder Verarbeiten von Blechen, Metallrohren, -stangen oder -profilen ohne } \\
\text { wesentliches Abtragen des Werkstoffs > Sicherheitseinrichtungen zum Schutz der in dieser } \\
\text { Bearbeiten oder Verarbeiten von Blechen, Metallrohren, -stangen oder -profilen ohne } \\
\text { wesentliches Abtragen des Werkstoffs > Biegen von Blechen, z.B. zum Bilden einfacher }\end{array}$ \\
\hline Möbel & A47B88 & 8 Tische $>$ Schubladen für Tische, Schränke oder ähnliche Möbel \\
\hline Papier & D21F5 & $\begin{array}{l}\text { Papiermaschinen > Trockenpartie an Maschinen zur fortlaufenden Herstellung von } \\
\text { Papierbahnen }\end{array}$ \\
\hline Stahl & C21B13 & $\begin{array}{l}3 \text { Gewinnung von Eisen oder Stahl > Gewinnung von Eisenschwamm oder flüssigem Stahl durch } \\
\text { Direktverfahren }\end{array}$ \\
\hline Stahl & F27B17 & 7 Industrieöfen, Schachtöfen, Brennöfen, Retorten allgemein > Industrieöfen \\
\hline Stempel & B41K1 & $\begin{array}{l}\text { Stempel > Tragbare handbetätigte Vorrichtungen ohne Mittel zum Unterstützen oder Festlegen } \\
\text { der zu stempelnden Gegenstände, z.B. Handstempel }\end{array}$ \\
\hline Werkzeug & $\mathrm{B} 25 \mathrm{C} 1$ & Handwerkzeuge zum Nageln oder Heften > Handwerkzeuge zum Nageln \\
\hline
\end{tabular}

Quelle: Economica (2017).

\footnotetext{
${ }^{66}$ So umfasst die Themengruppe „Aufzüge“ etwa die beiden Bereiche „Einzelheiten von Rolltreppen oder Fahrsteigen“ (definiert über die Patentklasse B66B23) und Sicherheitseinrichtungen für Rolltreppen oder Fahrsteige (definiert über die Patentklasse B66B29). Weitere Aspekte von Aufzügen werden in der Auswertung nicht betrachtet.
} 
Die 15 Technologiestärkefelder mit dem höchsten Patentanteil (relative Stärke) aus Österreich können anhand der drei Parameter (i) „,relative Stärke“, (ii) Konzentration der Industriestruktur (,Zahl der Unternehmen“) und (iii) der weltweiten Dynamik über den 20-Jahreszeitraum überblicksartig dargestellt werden. In Tabelle 33 werden dabei für die relative Stärke die Symbole "+ (Patentanteil aus Österreich zwischen 5\% und 10\%), "++" (10-15\%), und " ${ }^{+++}$(15-20\%) verwendet. Die Konzentration der Industriestruktur wird durch die Zahl der Unternehmen mit mindestens 3 Patentfamilien von Erfindern in Österreich (Veröffentlichungszeitraum 2012-2016) mit den Symbolen $-(\leq 2)$, +- (3-4) und $+(\geq 5)$ zusammengefasst. Die weltweite Dynamik als dritter Parameter wurde mit + (0-50\% relatives jährliches Wachstum zwischen 1996 und 2016), ++ (50-100\%) und +++ (>100\%) abgekürzt.

Tabelle 33: Zusammenfassung Stärkefelder

$\begin{array}{lccc}\text { Stärkefeld } & \text { Relative Stärke } & \begin{array}{c}\text { Zahl der } \\ \text { Unternehmen }\end{array} & \text { Weltweite Dynamik } \\ \text { Aufzüge } & +++ & +- & + \\ \text { Cellulose } & ++ & +- & + \\ \text { Eisenbahn } & +++ & + & ++ \\ \text { Lebensmittel } & + & - & +++ \\ \text { Metallverarbeitung } & + & +- & +++ \\ \text { Möbel } & ++ & +- & ++ \\ \text { Papier } & ++ & +- & + \\ \text { Stahl } & ++ & + & + \\ \text { Stempel } & ++ & - & + \\ \text { Werkzeug } & ++ & - & +\end{array}$

Quelle: Economica (2017). 


\subsubsection{Weltweite Wachstumsthemen}

Die Verteilung der Technologien auf den beiden Achsen „Patentoutput“ (Patentfamilien, weltweit, 2012-2016) und „Dynamik“ (jährliche relative Änderungsrate der Patentfamilien über den Zeitraum 1997-2016) zeigt eine Vielzahl von Themen mit hohen Wachstumsraten.

Abbildung 157: Weltweite Dynamik und Häufigkeit von 3400 Technologiefeldern

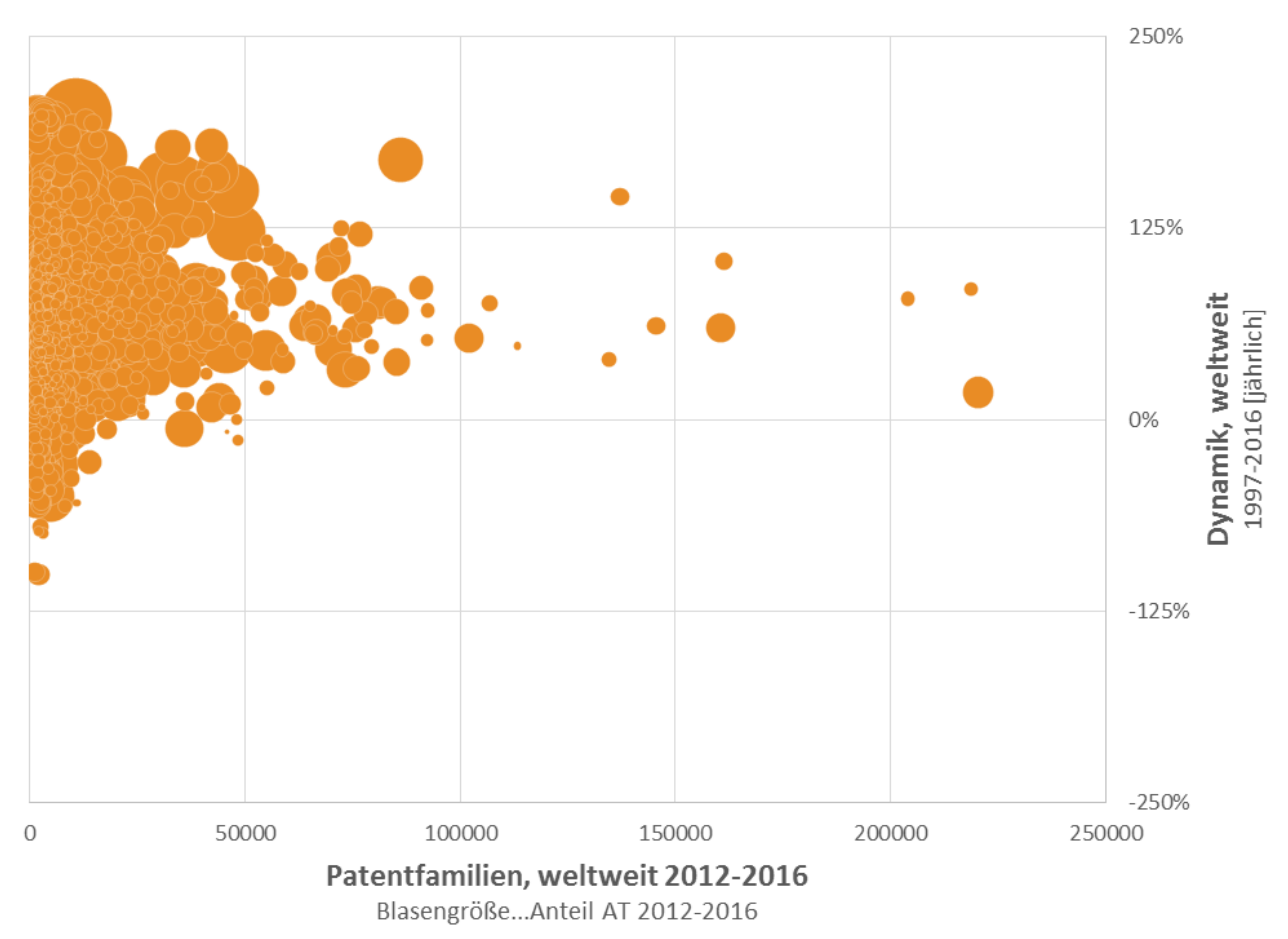

Quelle: Economica (2017).

Die Zahl der Technologiefelder mit mehr als 1.000 Patentfamilien weltweit im 5-Jahresintervall zwischen 2012 und 2016 wurde hinsichtlich ihrer Dynamik (relative jährliche Änderungsrate der Zahl der veröffentlichten Patentfamilien im Zeitraum zwischen 1996 und 2016) untersucht. Etwa 500 Technologiefelder weisen dabei eine jährliche Wachstumsrate von mehr als 100\% auf.

Für die weitere Analyse wurden die hinsichtlich der Dynamik folgende Top-15 Technologiefelder herangezogen: Additive Fertigungsverfahren (B33Y10), Schaltungsanordnungen oder Systeme für die Abgabe oder Verteilung elektrischer Leistung (H02J50), Additive Fertigungsvorrichtungen; Details oder Zubehör von additiven Fertigungsvorrichtungen (B33Y30), Lichterzeugende Elemente von Halbleiterlichtquellen (F21Y115), Lichtquellen unter Verwendung von Halbleiterbauelementen als 
lichterzeugende Elemente, z.B. unter Verwendung von Leuchtdioden [LEDs] oder Lasern (F21K9), Nahrung aus dem Meer; Erzeugnisse aus Fisch; Fischmehl; Roggenersatzprodukte; deren Zubereitung oder Behandlung (A23L17), Nicht schaltbare Kupplungen > Einzelheiten von Stellorganen (F16D125), Instrumente, Geräte oder Zubehör besonders ausgebildet für Chirurgie oder Diagnose, z.B. für die Behandlung von Verrenkungen oder zum Schutz von Wundrändern (A61B90), Computer-unterstützte Chirurgie; Manipulatoren oder Roboter, besonders ausgebildet für die Anwendung in der Chirurgie (A61B34), Futtermittel, besonders ausgebildet für bestimmte Tiere (A23K50), Fleischerzeugnisse; Fleischmehl; Herstellung und Behandlung derselben (A23L13), Nicht schaltbare Kupplungen > Art der Betätigung des Stellorgans (F16D121), aus Getreide hergestellte Produkte; Malzprodukte (A23L7), Obst- oder Gemüseprodukte; deren Zubereitung oder Behandlung (A23L19), Futtermittel (A23K10).

\section{Abbildung 158: Verteilung der weltweiten Patentdynamik von 3.400 Technologiefeldern*}

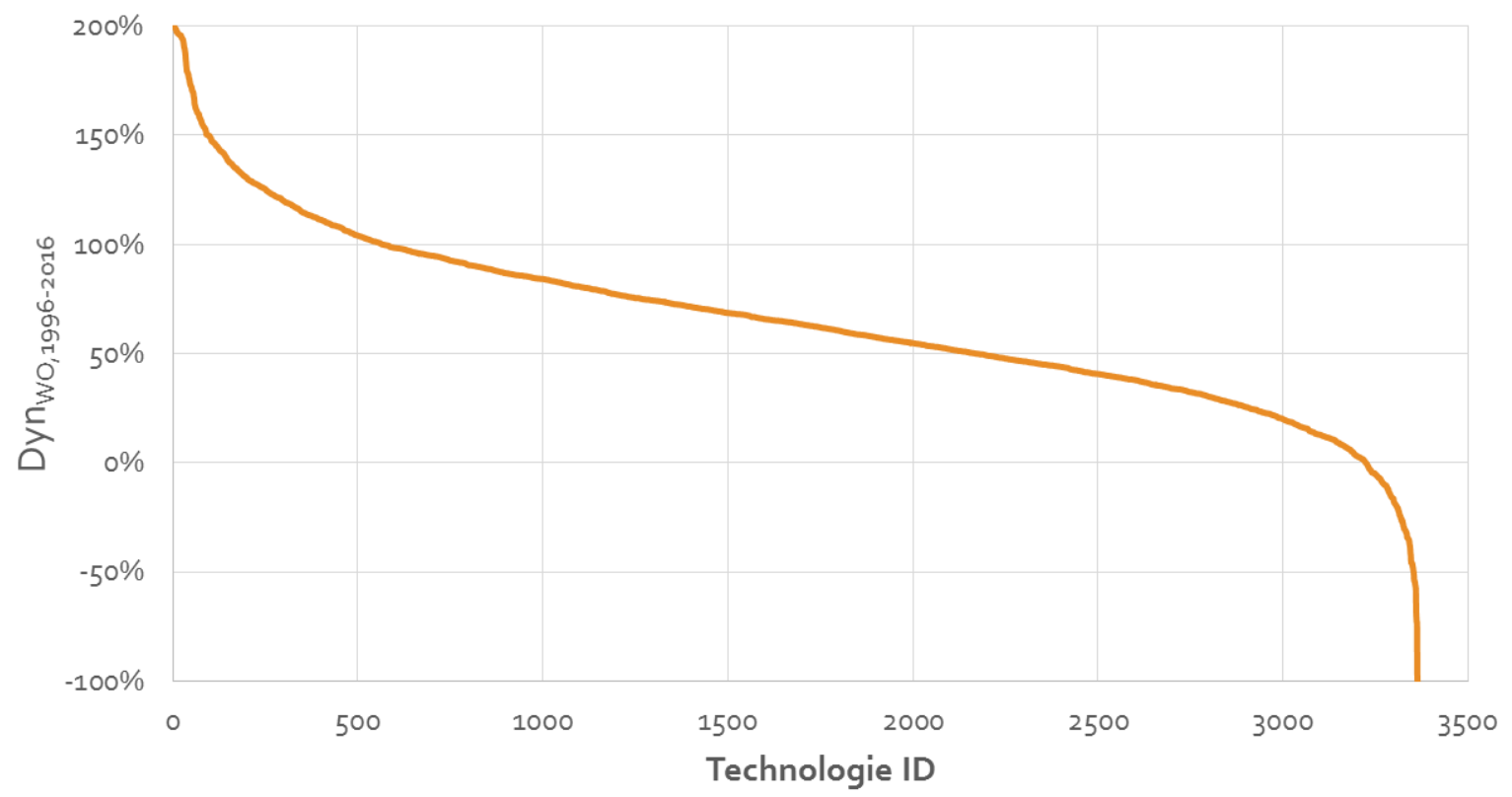

Quelle: Economica (2017). * Technologiefelder mit zumindest 1.000 Patentfamilien zwischen 2012 und 2016 
Tabelle 34: Top-15 Wachstumsthemen weltweit

\begin{tabular}{|c|c|c|c|}
\hline IPC & Description & $\begin{array}{l}\operatorname{Dyn}_{\text {wo, } 1996-} \\
\quad 2016\end{array}$ & $\begin{array}{l}\mathrm{F}_{\mathrm{AT}} / \mathrm{F}_{\mathrm{WO}, 2012-} \\
\quad 2016\end{array}$ \\
\hline B33Y10 & Additive Fertigungsverfahren & $200 \%$ & $1.113 \%$ \\
\hline $\mathrm{H} 02 \mathrm{~J} 50$ & $\begin{array}{l}\text { Schaltungsanordnungen oder Systeme für die Abgabe oder } \\
\text { Verteilung elektrischer Leistung }\end{array}$ & $200 \%$ & $0.474 \%$ \\
\hline B33Y30 & $\begin{array}{l}\text { Additive Fertigungsvorrichtungen; Details oder Zubehör von } \\
\text { additiven Fertigungsvorrichtungen }\end{array}$ & $200 \%$ & $0.938 \%$ \\
\hline F21Y115 & Lichterzeugende Elemente von Halbleiterlichtquellen & $200 \%$ & $4.722 \%$ \\
\hline F21K9 & $\begin{array}{l}\text { Lichtquellen unter Verwendung von Halbleiterbauelementen } \\
\text { als lichterzeugende Elemente, z.B. unter Verwendung von } \\
\text { Leuchtdioden [LEDs] oder Lasern }\end{array}$ & $199 \%$ & $0.760 \%$ \\
\hline A23L17 & $\begin{array}{l}\text { Nahrung aus dem Meer; Erzeugnisse aus Fisch; Fischmehl; } \\
\text { Rogenersatzprodukte; deren Zubereitung oder Behandlung }\end{array}$ & $199 \%$ & $0.000 \%$ \\
\hline F16D125 & Nicht schaltbare Kupplungen > Einzelheiten von Stellorganen & $199 \%$ & $1.515 \%$ \\
\hline A61B90 & $\begin{array}{l}\text { Instrumente, Geräte oder Zubehör besonders ausgebildet für } \\
\text { Chirurgie oder Diagnose, z.B. für die Behandlung von } \\
\text { Verrenkungen oder zum Schutz von Wundrändern }\end{array}$ & $199 \%$ & $0.164 \%$ \\
\hline A61B34 & $\begin{array}{l}\text { Computer-unterstützte Chirurgie; Manipulatoren oder Roboter, } \\
\text { besonders ausgebildet für die Anwendung in der Chirurgie }\end{array}$ & $198 \%$ & $0.000 \%$ \\
\hline A23K50 & Futtermittel, besonders ausgebildet für bestimmte Tiere & $197 \%$ & $0.487 \%$ \\
\hline A23L13 & $\begin{array}{l}\text { Fleischerzeugnisse; Fleischmehl; Herstellung und Behandlung } \\
\text { derselben }\end{array}$ & $197 \%$ & $1.143 \%$ \\
\hline F16D121 & $\begin{array}{l}\text { Nicht schaltbare Kupplungen }>\text { Art der Betätigung des } \\
\text { Stellorgans }\end{array}$ & $197 \%$ & $0.797 \%$ \\
\hline A23L7 & aus Getreide hergestellte Produkte; Malzprodukte & $197 \%$ & $0.000 \%$ \\
\hline A23L19 & $\begin{array}{l}\text { Obst- oder Gemüseprodukte; deren Zubereitung oder } \\
\text { Behandlung }\end{array}$ & $197 \%$ & $0.267 \%$ \\
\hline A23K10 & Futtermittel & $197 \%$ & $1.119 \%$ \\
\hline
\end{tabular}

Quelle: Economica (2017).

Diese weltweit Top-15 Wachstumstechnologien können in die Themengruppen (1) Additive Fertigungsverfahren, (2) Verteilung elektrischer Leistung, (3) nicht-schaltbare Kupplungen, (4) Beleuchtung (LED, OLED, flächenförmige Lichtquellen), (5) Anwendungsspezifische Robotik, z.B. Smart-Health, und (6) Lebensmitteltechnologie gegliedert werden. Für jede Themengruppe wurden im Anhang weitere Analysen inklusive der Unternehmen mit Patenten aus Österreich dargestellt. 
Tabelle 35: Wachstumsthemen weltweit, gruppiert

\begin{tabular}{|c|c|c|}
\hline \multicolumn{3}{|l|}{ Themengruppe } \\
\hline \multirow{2}{*}{$\begin{array}{l}\text { Additive } \\
\text { Manufacturing }\end{array}$} & B33Y10 & Additive Fertigungsverfahren \\
\hline & B33Y30 & $\begin{array}{l}\text { Additive Fertigungsvorrichtungen; Details oder Zubehör von } \\
\text { additiven Fertigungsvorrichtungen }\end{array}$ \\
\hline \multirow[t]{2}{*}{ Chirurgie/Robotik } & A61B90 & $\begin{array}{l}\text { Instrumente, Geräte oder Zubehör besonders ausgebildet für } \\
\text { Chirurgie oder Diagnose, z.B. für die Behandlung von } \\
\text { Verrenkungen oder zum Schutz von Wundrändern }\end{array}$ \\
\hline & A61B34 & $\begin{array}{l}\text { Computer-unterstützte Chirurgie; Manipulatoren oder Roboter, } \\
\text { besonders ausgebildet für die Anwendung in der Chirurgie }\end{array}$ \\
\hline \multirow[t]{6}{*}{$\begin{array}{l}\text { Futter-/ } \\
\text { Lebensmittel }\end{array}$} & A23L17 & $\begin{array}{l}\text { Nahrung aus dem Meer; Erzeugnisse aus Fisch; Fischmehl; } \\
\text { Roggenersatzprodukte; deren Zubereitung oder Behandlung }\end{array}$ \\
\hline & A23K50 & Futtermittel, besonders ausgebildet für bestimmte Tiere \\
\hline & A23L13 & $\begin{array}{l}\text { Fleischerzeugnisse; Fleischmehl; Herstellung und Behandlung } \\
\text { derselben }\end{array}$ \\
\hline & A23L7 & aus Getreide hergestellte Produkte; Malzprodukte \\
\hline & A23L19 & Obst- oder Gemüseprodukte; deren Zubereitung oder Behandlung \\
\hline & A23K10 & Futtermittel \\
\hline \multirow[t]{2}{*}{ Licht } & F21Y115 & Lichterzeugende Elemente von Halbleiterlichtquellen \\
\hline & F21K9 & $\begin{array}{l}\text { Lichtquellen unter Verwendung von Halbleiterbauelementen als } \\
\text { lichterzeugende Elemente, z.B. unter Verwendung von } \\
\text { Leuchtdioden [LEDs] oder Lasern }\end{array}$ \\
\hline \multirow{2}{*}{$\begin{array}{l}\text { nicht-schaltbare } \\
\text { Kupplungen }\end{array}$} & F16D125 & Nicht schaltbare Kupplungen > Einzelheiten von Stellorganen \\
\hline & F16D121 & Nicht schaltbare Kupplungen > Art der Betätigung des Stellorgans \\
\hline $\begin{array}{l}\text { Verteilung elektr. } \\
\text { Leistung }\end{array}$ & $\mathrm{H} 02 \mathrm{~J} 50$ & $\begin{array}{l}\text { Schaltungsanordnungen oder Systeme für die Abgabe oder } \\
\text { Verteilung elektrischer Leistung }\end{array}$ \\
\hline
\end{tabular}

Quelle: Economica (2017).

Dabei zeichnet sich der Bereich Beleuchtung durch eine bestehende hohe relative Stärke aus. Unternehmen aus Österreich in dem Bereich sind beispielsweise in der Automobilzulieferindustrie (ZKW) tätig. Andere Themen, etwa der Bereich Smart-Health, erscheinen aufgrund ausgeprägter Stärken bestimmter Regionen im Bereich Robotik (konkret Oberösterreich) interessante mögliche zukünftige Schwerpunkte zu sein.

Der Bereich der Lebensmittel/Futtermittel wurde bereits im ersten Teil der Analyse anhand der Stärkefelder (dort: Backmaschinen) als rasch wachsend charakterisiert. Unter den Top-15 der weltweit am schnellsten wachsenden Themen finden sich zahlreiche Themen, die der Themengruppe der Lebensmittel zugeordnet werden. 


\subsubsection{Verknüpfung der Patentanalyse mit CD-Labors bzw. JR-Zentren}

In diesem Abschnitt erfolgt eine Verknüpfung zwischen der Patentanalyse und der Erhebung der Unternehmensbeteiligungen an den CD-Labors bzw. JR-Zentren. Sowohl den zehn Technologiestärkefeldern als auch den einzelnen CD-Labors können Unternehmen zugeordnet werden, sodass basierend auf dieser Information eine etwaige Überschneidung identifiziert werden kann.

Abbildung 159: CD-Labors und JR-Zentren in den Technologiestärkefeldern Österreich

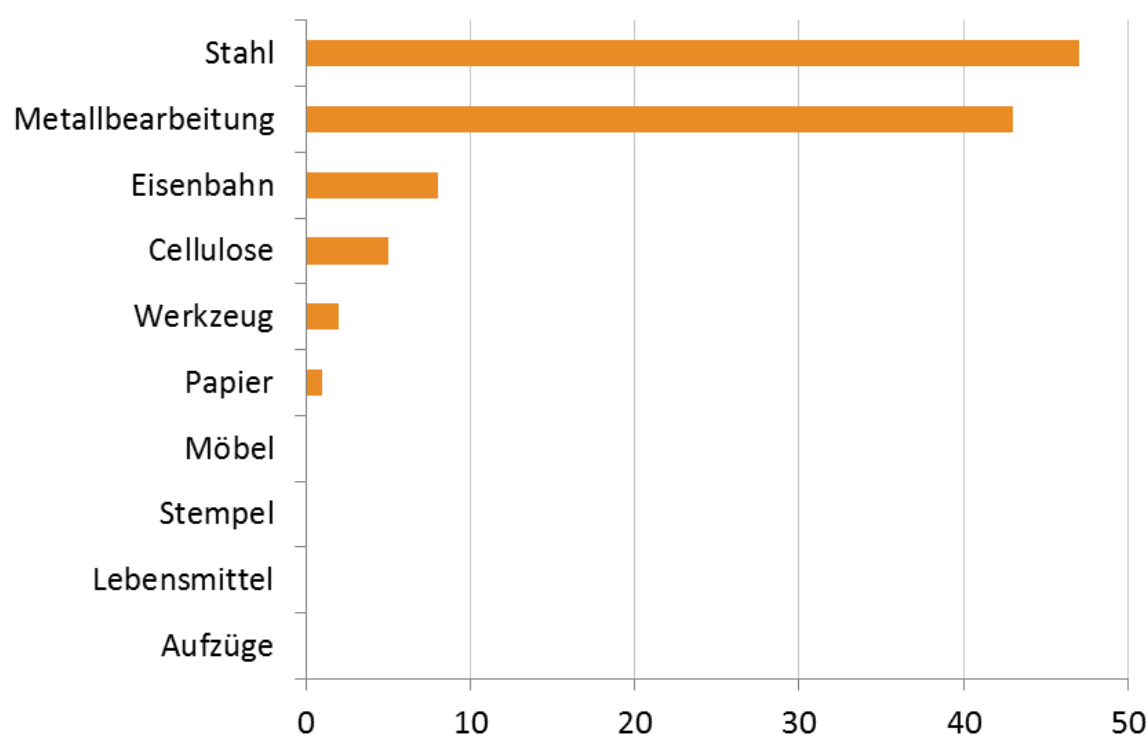

Quelle: Economica/IWI (2017).

Aufgrund der hohen Verknüpfung der voestalpine mit den CD-Labors werden auch die entsprechenden zwei zugeordneten Technologiestärkefelder am häufigsten genannt. Insgesamt waren oder sind Unternehmen aus sechs von zehn österreichischen Technologiestärkefeldern am CDG-Programm involviert. Die Verlinkung zwischen dem CDG-Programmen und den österreichischen Technologiestärkefeldern konstituiert sich dabei durch 11 Unternehmen, die sowohl Unternehmenspartner waren oder sind, und gleichzeitig auch im Besitz von Patenten in den österreichischen Technologiestärkefeldern sind. 
Tabelle 36: Unternehmen mit Patenten in Technologiestärkefeldern und CD-Labors

\begin{tabular}{|c|c|c|}
\hline $\begin{array}{l}\text { CD- } \\
\text { Labors }\end{array}$ & Unternehmen & Technologiefeld \\
\hline \multirow[t]{2}{*}{38} & VOESTALPINE STAHL & Metallbearbeitung \\
\hline & & Stahl \\
\hline 19 & PRIMETALS TECHNOLOGIES & Stahl \\
\hline \multirow[t]{2}{*}{6} & SIEMENS AG & Eisenbahn \\
\hline & & Stahl \\
\hline 5 & LENZING & Cellulose \\
\hline 3 & KEBA AG & Möbel \\
\hline 2 & KELHEIM FIBRES & Cellulose \\
\hline \multirow[t]{2}{*}{2} & HILTI AG & Metallbearbeitung \\
\hline & & Werkzeug \\
\hline 1 & SWIETELSKY BAUGESELLSCHAFT & Eisenbahn \\
\hline 1 & VOESTALPINE SCHIENEN & Eisenbahn \\
\hline 1 & FRITZ EGGER & Papier \\
\hline 1 & IVOCLAR VIVADENT & Stahl \\
\hline
\end{tabular}

Quelle: Economica/IWI (2017).

Darüber hinaus sind bereits fünf von sechs Wachstumsthemen mit österreichischen Unternehmen vertreten, die ein Patent besitzen und Unternehmenspartner im CDG-Programm waren.

Abbildung 160: CD-Labors und JR-Zentren in den Wachstumsthemen

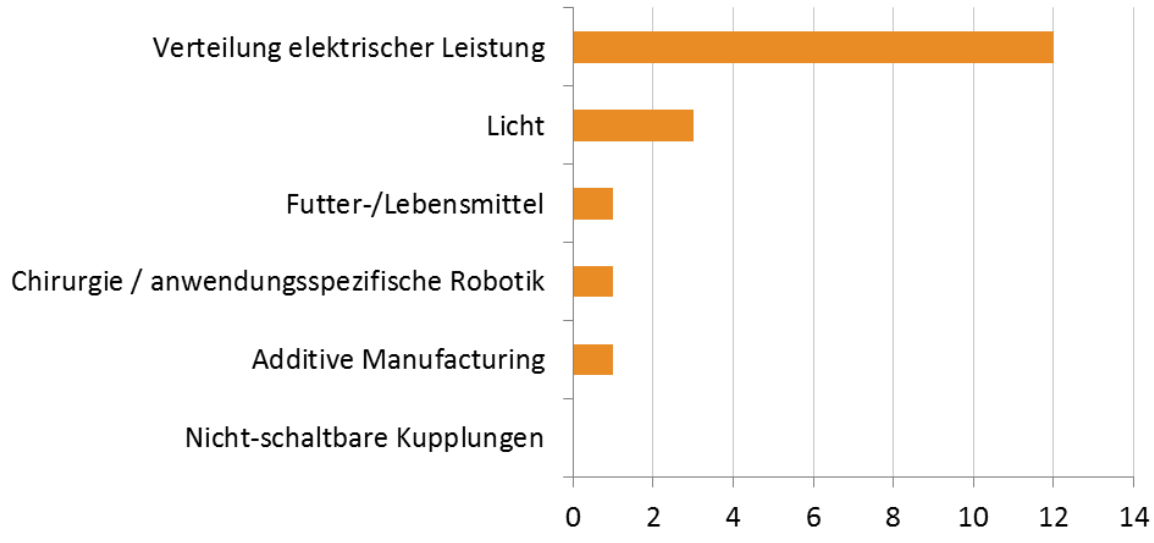

Quelle: Economica/IWI (2017).

Absolut dominierend bei dieser Betrachtung ist Infineon, das in 12 unterschiedlichen CD-Labors bzw. einem JR-Zentrum als Unternehmenspartner fungiert. Daneben gibt es vier weitere österreichische Unternehmen mit Patenten in den Wachstumsthemen und mit CD-Laborbeteiligungen. Hinzu 
kommen über 50 Unternehmen, die zwar Patente aufweisen, aber noch nie Unternehmenspartner waren, und somit einen Pool an potenziellen Unternehmenspartnern für zukünftige CD-Labors bzw. JR-Zentren in den weltweiten Wachstumsthemen darstellen.

Tabelle 37: Unternehmen mit Patenten in Wachstumsthemen und CD-Labors

$\begin{array}{lrll}\text { CD-Labors } & \text { Unternehmen } & \text { Wachstumsthema } \\ 12 & \text { INFINEON TECHNOLOGIES } & \text { Verteilung elektrischer Leistung } \\ 2 & \text { EV GROUP } & \text { Licht } \\ 1 & \text { IVOCLAR VIVADENT } & \text { Additive Manufacturing } \\ & & \text { Licht } \\ 1 & \text { OTTO BOCK HEALTHCARE PROD } & \text { Chirurgie / anwendungsspezifische Robotik } \\ 1 & \text { ERBER AG } & \text { Futter-/Lebensmittel }\end{array}$

Quelle: Economica/IWI (2017).

\subsection{Schlussfolgerungen}

Es gibt bei den CD-Labors einen sehr starken Unternehmenskern, so vereinen rund 10 Prozent der Unternehmen beinahe die Hälfte aller Unternehmensbeteiligungen. Von besonderer Bedeutung ist dabei die voestalpine, die alleine in 40 unterschiedlichen CD-Labors mit 108 weiteren Unternehmenspartnern beteiligt war. Die meisten CD-Labors waren an der Technischen Universität Wien angesiedelt, gefolgt von der Technischen Universität Graz, der Montanuniversität Leoben, der Universität Linz, der Medizinische Universität Wien und der Universität für Bodenkultur Wien. Seit 2005 werden CD-Labors verstärkt auch außerhalb dieser Forschungseinrichtungen eingerichtet.

Die Finanzierung der Grundlagenforschung in Österreich ist seit 2002 überdurchschnittlich angestiegen, wobei diese vor allem im Hochschulsektor stattfindet. Hier existiert mit der CDG ein guter Anknüpfungspunkt, um die Unternehmensseite und die Hochschulen näher zueinander zu führen und somit zu einer übergreifenden Forschungs- und Innovationsgemeinschaft beizutragen. Denn langfristige Investitionen in die Grundlagenforschung sind Teil der Voraussetzungen für technologische Entwicklungen und Wettbewerbsfähigkeit in der Zukunft. Hierfür ist ein ausreichendes Maß an Finanzierung erforderlich. Falls das Ziel, ein europaweiter „Innovation Leader" zu werden, erreicht werden soll, ist es notwendig durch ausreichende Förderung der 
Grundlagenforschung zur Entstehung von radikalen Innovationen beizutragen sowie den kompetitiven Anteil der Forschungsfinanzierung anzuheben.

Die F\&E-Aktivitäten, die in den CD-Labors bzw. JR-Zentren durchgeführt werden sind tendenziell grundlagenorientierter als die firmeninterne F\&E. So gesehen leistet die CDG einen wesentlichen Beitrag zur Grundlagenforschung mit der besonderen Qualität, dass hier Themen bearbeitet werden, die dennoch stark aus der (Unternehmens-)Praxis kommen und Grundlagenforschung sehr anwendungs- und zielorientiert gedacht wird.

Mit Hilfe der Patentanalyse wurde untersucht, inwieweit ein Zusammenhang zwischen den österreichischen Technologiestärkefeldern, das sind Patent-Hauptklassen mit hohem heimischen Anteil, und den CD-Labors bzw. JR-Zentren besteht. Darüber hinaus wurde auch untersucht, ob die weltweiten Wachstumsthemen bereits in das System CDG Eingang gefunden haben.

Die Technologiestärkefelder Österreichs können anhand ihrer relativen Stärke, der Zahl der Unternehmen und der weltweiten Dynamik folgendermaßen gruppiert werden: a) Die Bereiche "Aufzüge“ und „Eisenbahn“ weisen jeweils eine herausragende relative Stärke auf. Dabei ist die weltweite Dynamik positiv, im Fall von Eisenbahn sogar hoch. Die Industriestruktur ist im Thema des Bereichs „Eisenbahn“ breiter (mit höherer Zahl von patentaktiven Unternehmen) als im Fall der Aufzüge. b) Die Stärkefelder in den Bereichen „Cellulose“, „Möbel“, „Papier“ und „Stahl“ weisen eine hohe relative Stärke auf (jedoch geringere als jene in den Bereichen „Aufzüge" und „Eisenbahn“), wobei die Industriestruktur sehr konzentriert ist (geringe Zahl von patentaktiven Unternehmen). Die weltweite Dynamik ist in drei Stärkefeldern positiv, jedoch im untersten Intervall zwischen 0 und $50 \%$ bzw. etwas höher im Fall von Möbel. c) Eine dritte Gruppe von Stärkefeldern ist jene der Bereiche „Lebensmittel“ und „Metallverarbeitung", die sich durch eine geringe relative Stärke jedoch weltweit besonders hohe Dynamik auszeichnen. Die Industriestruktur in den beiden Themen ist im Fall von Lebensmitteln sehr konzentriert, bzw. im Fall von Metallverarbeitung breiter. d) Für die vierte Gruppe, "Stempel" und "Werkzeug" ist die hohe relative Stärke bei einer geringen Zahl von patentaktiven Unternehmen mit Erfindern in Österreich, sowie eine weltweite nur leicht progressive Dynamik charakteristisch.

Die Unternehmen mit Patenten in den zehn österreichischen Technologiestärkefeldern waren / sind in sechs von zehn Fällen (Stahl, Metallbearbeitung, Eisenbahn, Cellulose, Werkzeug und Papier) auch an CD-Labors beteiligt. Dies zeigt einen gewissen Zusammenhang zwischen dem CDG-Programm und den österreichischen Technologiestärkefeldern laut Patentanalyse auf, der auch durch das 
Programmziel technologische Hebelwirkung mit dem Indikator Patente als Output gegeben ist. Das CDG-Programm hat damit auch dazu beigetragen, dass diese Technologiestärkefelder in dieser Form in Österreich aufgebaut werden konnten. Die Verlinkung zwischen dem CDG-Programmen und den österreichischen Technologiestärkefeldern konstituiert sich dabei durch 11 Unternehmen, die sowohl Unternehmenspartner waren oder sind, und gleichzeitig auch im Besitz von Patenten in den österreichischen Technologiestärkefeldern sind.

Grundsätzlich wäre es überlegenswert in den derzeit noch nicht vorkommenden Technologiestärkefeldern CD-Labors bzw. JR-Zentren zu forcieren, denn dadurch könnte einerseits die Vorreiterposition Österreichs in diesen Bereichen abgesichert werden, und andererseits könnte damit innerhalb des CDG-Programms eine technologische Themenverbreiterung vorgenommen werden. Es ist jedoch kritisch zu hinterfragen, ob sich der jeweilige Spezialbereich der Themengruppen Aufzüge (Einzelteile von Rolltreppen oder Fahrsteigen; Sicherheitseinrichtungen für Rolltreppen oder Fahrsteige), Möbel (Tische > Schubladen für Tische, Schränke oder ähnliche Möbel), Stempel (Tragbare handbetätigte Vorrichtungen ohne Mittel zum Unterstützen oder Festlegen der zu stempelnden Gegenstände, z.B. Handstempel) bzw. Lebensmittel (Bäckereiöfen > Backgeräte für besondere Backwaren) sich für ein CD-Labor bzw. ein JR-Zentrum eignet.

Etwas vielversprechender erscheint daher eine Orientierung an weltweit boomenden Themen. Die Top-15 Technologiefelder, gereiht nach deren weltweiten Dynamik, lassen sich in folgende Themengruppen zusammenfassen: (1) Additive Fertigungsverfahren, (2) Verteilung elektrischer Leistung, (3) Nicht-schaltbare Kupplungen, (4) Beleuchtung (LED, OLED, flächenförmige Lichtquellen), (5) Anwendungsspezifische Robotik, z.B. Smart-Health, und (6) Lebensmitteltechnologie.

Obwohl bereits aus fünf der sechs Themengruppen, mit der weltweit höchsten Patentdynamik der Technologiefelder, Unternehmen in Österreich mit Patenten und CD-Labors existieren, ist dieser Zusammenhang jedoch lediglich durch fünf Unternehmen hergestellt. Drei Unternehmen waren dabei einmal und ein Unternehmen war an zwei CD-Labors beteiligt. Einzig Infineon hatte 12 CDLabors aus dem Bereich Verteilung elektrischer Leistung. Da es daneben noch weitere 50 Unternehmen gibt, die Patente in den weltweiten Wachstumsbereichen aufweisen, aber noch nie Unternehmenspartner waren, gibt es hier noch ein großes Potenzial an Unternehmenspartnern für zukünftige Forschungseinrichtungen. 


\section{Synopse}

Die aktuell durchgeführte Evaluierung sowie eine detaillierte Auseinandersetzung mit der Entwicklung des Programms seit der letzten Evaluierung im Jahr 2011 führen im Ergebnis zu einer klaren Empfehlung die Programminitiative zur Förderung der Einrichtung und des Betriebs von Christian Doppler Labors fortzusetzen.

Das Programm der CD-Labors ist (nach wie vor) ein Förderinstrument mit klarem Förderkonzept und -ziel sowie hohem Zielerreichungsgrad und „eigenem Charakter“. Vor allem die Langfristigkeit und die dadurch ermöglichte intensive Kooperation bilden einen starken (Forschungs-)Kern und sorgen für einen nachhaltigen Humankapitalaufbau. Das CD-Labor ist somit „nicht nur ein weiteres Förderprogramm, es formt eine intensiv und fokussiert arbeitende (Forschungs-)Gruppe aus". Als renommiertes, historisch gewachsenes Förderprogramm erzeugt es auch Sichtbarkeit. CD-Labors werden als „Marke“ über die österreichischen Grenzen hinaus wahrgenommen und stärken dadurch auch den heimischen Forschungsstandort.

Einfache Stakeholderstrukturen sowie die zielorientierte und gute Zusammenarbeit der CDG mit dem Programmträger BMWFW führen zu einer nach wie vor hohen Qualität in der Programmgestaltung sowie zu einer äußerst effizienten Programmabwicklung.

Die Zufriedenheit der Fördernehmer (Wissenschaft, Wirtschaft) mit dem Programm ist (nach wie vor) sehr hoch, insbesondere auch mit den (Betreuungs-)Leistungen der CDG. Die bei der letzten Evaluierung (2011) sowie bei der Studie zu Handlungsoptionen für eine weitere Optimierung des CD-Programms (2013) angeregten Empfehlungen wurden von Seiten der Programmverantwortlichen aufgenommen und die von der CDG umgesetzten Maßnahmen vor allem hinsichtlich administrativer Vereinfachungen zeigen positive Wirkung. Dies zeigt auch die Bereitschaft der CDG die an sich schon hohe Qualität des Programms bzw. der Programmabwicklung dennoch weiter zu verbessern.

Für die Partnerunternehmen aus der Wirtschaft hat das CD-Programm nach wie vor ein breites Nutzenspektrum. Dieses reicht von der Bewertung verschiedener Lösungsstrategien (Radarfunktion) über die inkrementelle Verbesserung bestehender Produkte/Prozesse bis hin zur Entwicklung neuer Produktionsverfahren und radikal neuer Produkte. Hohen Nutzen durch die Beteiligung an einem CDLabor sehen die Unternehmen vor allem im Kompetenzaufbau, dem Aufzeigen neuer technologischer Optionen und in der Stärkung ihrer technologischen Problemlösungskompetenz. Positiv ist weiters 
anzumerken, dass verbunden mit dem Wissenstransfer eine Erhöhung der Wettbewerbsfähigkeit gegenüber Konkurrenten verbunden mit einer Stärkung des Unternehmensstandorts einhergeht.

Die Josef Ressel Zentren, als Pilotprojekt bereits 2008 ins Leben gerufen, wurden 2012 inhaltlich und organisatorisch an die CDG angekoppelt. Wesentliche Elemente des CD-Modells wurden dabei übernommen und in adaptierter Form auf das JR-Zentren-Programm übertragen. Ziel war es, die Erfahrungen des CD-Programms aus dem Wissenstransfer zwischen Wissenschaft und Wirtschaft zu nutzen und Synergieeffekte in der Programmabwicklung zu erzielen.

Im Rahmen der Evaluierung konnte eine geringe Zahl an bereits laufenden JR-Zentren betrachtet werden, denen - ähnlich den CD-Labors - ein positiver Evaluierungsbefund ausgestellt werden kann. Die JR-Zentren befinden sich noch in einer frühen Entwicklungsphase, und es bleibt daher abzuwarten, ob zukünftige Evaluierungen Änderungsbedarf beim Programmdesign sehen, oder ob der eigene Charakter, vor allem gegenüber den CD-Labors, bereits mit der derzeitigen Ausgestaltung des Förderprogramms erkennbar sein wird. Dies muss auch im Einklang und unter Berücksichtigung zukünftiger Rahmenbedingungen der Fachhochschulen bzw. des Fachhochschulwesens erfolgen. Eine systemische Relevanz der JR-Zentren im Nationalen Innovationssystem (NIS) wird erst mittelfristig und in den kommenden Jahren zu beurteilen sein.

Zusammenfassend ist die Fortführung der Programminitiative Christian Doppler Labors eindeutig zu befürworten. Das CDG-Förderprogramm überzeugt nach wie vor durch hohe Flexibilität und Anpassungsfähigkeit und weist auch nach Jahrzehnten des Bestehens einen hohen Erfolg im Bereich der Wirtschafts-Wissenschafts-Kooperationsbeziehungen auf.

Auch der noch jungen und seit 2012 in die CDG eingegliederten Programminitiative der Josef Ressel Zentren ist ein grundsätzlich positiver Befund auszustellen. Weitere zukünftige Evaluierungen, bei denen die Erfahrungen bereits abgeschlossener JR-Zentren eingegangen sein werden, können Aufschluss darüber erbringen, ob es einer Nachjustierung des Programmdesigns bedarf, oder ob der „eigene Charakter" des Förderprogramms bereits mit den aktuellen Rahmenbedingungen zum Vorschein tritt. Anzumerken ist hier, dass die Entwicklungsmöglichkeiten der JR-Zentren auch stark von der allgemeinen strukturellen Entwicklung des Fachhochschulwesens in den kommenden Jahren abhängen.

Die Programme der CDG bilden eine institutionalisierte Fördernische, insbesondere die CD-Labors sind durch ihren eigenen Charakter sehr erfolgreich. Die (thematische) Offenheit und Flexibilität des 
Programms - bereits jetzt ein wichtiger Erfolgsfaktor - wird in Zukunft noch bedeutender werden und muss neben dem hohen wissenschaftlichen Qualitätsanspruch weiterhin im Fokus stehen.

Auch im Rahmen dieser Evaluierung muss das hohe persönliche Engagement der Vereinsgremien im Allgemeinen und des CDG-Generalsekretariats im Besonderen hervorgehoben werden. Die Betreuungsleistung ist hoch und bestehende Potentiale bei der Programmumsetzung (insb. hinsichtlich administrativer Vereinfachungen) wurden mit Engagement umgesetzt.

Mittelfristig gilt es, neben der Aufrechterhaltung des hohen Niveaus und der weiteren Feinjustierung, vor allem die Programmschiene der Josef Ressel Zentren strategisch und strukturell harmonischer in das Gesamtsystem CDG einzufügen, ihr einen eigenen Charakter zu geben und bestehende Potentiale stärker abzurufen. 


\section{Tabellenverzeichnis}

Tabelle 1: Operationalisierbare Ziele $\quad 12$

Tabelle 2: Indikatoren zur Prüfung der Zielerreichung 12

Tabelle 3: Übersicht Forschungsthemen (Themencluster) CD-Labors 20

Tabelle 4: Übersicht Forschungsthemen (Themencluster) JR-Zentren 21

Tabelle 5: Verlauf der CD-Labors $\quad 22$

Tabelle 6: Übersicht Abschlussfragebögen CD-Labors 23

Tabelle 7: Anzahl der wissenschaftlichen Mitarbeiter, wissenschaftlichen Arbeiten 23

Tabelle 8: Publikationen im Rahmen des CD-Labors $\quad 24$

Tabelle 9: Konferenzteilnahmen $\quad 25$

Tabelle 10: Anzahl der Preise/Rufe im Rahmen des CD-Labors $\quad 25$

Tabelle 11: Kooperationen mit anderen Forschergruppen im Rahmen des CD-Labors 26

Tabelle 12: Korrelationskoeffizienten $\quad 48$

Tabelle 13: Indikatoren mit Gesamtmittel normiert $\quad 49$

Tabelle 14: Vergleich Evaluierung 2011 und 2016

Tabelle 15: Publikationen der JR-Zentren (2013-2015, absolut und normiert) 57

Tabelle 16: Konferenzaktivitäten der JR-Zentren (2013-15, absolut und normiert) 58

Tabelle 17: Kooperationen der JRZ (2013-15) 59

Tabelle 18: Weiterreichende Effekte $\quad 59$

Tabelle 19: Programmziele und Indikatoren $\quad 64$

Tabelle 20: Beispiel einer Bewertungsmatrix $\quad 65$

Tabelle 21: Bewertungsskala des paarweisen Vergleichs $\quad 65$

Tabelle 22: Rücklauf der Onlineerhebung (Übersicht) 96

Tabelle 23: Konkurrenzsituation bei Partnerunternehmen gleicher Branche (Übersicht) 144

Tabelle 24: Anzahl der Unternehmen, nach Anzahl der CD-Laborbeteiligungen 176

Tabelle 25: Top-Unternehmenspartner nach Anzahl der CD-Labors 177

Tabelle 26: Unternehmenspartner der JR-Zentren, Querverweis CD-Labors 178

Tabelle 27: Verteilung der CD-Labors auf Forschungseinrichtungen 181

Tabelle 28: F\&E-Auszahlungen des Bundes nach wesentlichen Empfängern, in Mio. Euro 188

Tabelle 29: Ausschüttungen der FTE-Stiftung, in Mio. Euro 189

Tabelle 30: Ausschüttungen des Österreich-Fonds, in Mio. Euro 190

Tabelle 31: Top-15 Technologiestärkefelder Österreichs nach Patentanteil 201

Tabelle 32: Die 10 Themengruppen der 15 Technologiestärkefelder Österreichs 202

Tabelle 33: Zusammenfassung Stärkefelder 203

Tabelle 34: Top-15 Wachstumsthemen weltweit 206 
Tabelle 35: Wachstumsthemen weltweit, gruppiert

Tabelle 36: Unternehmen mit Patenten in Technologiestärkefeldern und CD-Labors 209

Tabelle 37: Unternehmen mit Patenten in Wachstumsthemen und CD-Labors 210

Tabelle 38: Top Patentländer im Bereich Aufzüge 245

Tabelle 39: Industriestruktur in Österreich im Bereich Aufzüge $\quad 246$

Tabelle 40: Crossing-Technologies im Bereich Aufzüge und deren relative Stärke 247

$\begin{array}{ll}\text { Tabelle 41: Top Patentländer im Bereich Cellulose } & 248\end{array}$

Tabelle 42: Industriestruktur in Österreich im Bereich Cellulose $\quad 249$

Tabelle 43: Crossing-Technologies im Bereich Cellulose und deren relative Stärke 250

Tabelle 44: Top Patentländer im Bereich Eisenbahn 252

Tabelle 45: Industriestruktur in Österreich im Bereich Eisenbahn 254

Tabelle 46: Crossing-Technologies im Bereich Eisenbahn und deren relative Stärke 255

Tabelle 47: Top Patentländer im Bereich Lebensmittel 257

Tabelle 48: Industriestruktur in Österreich im Bereich Lebensmittel 258

Tabelle 49: Crossing-Technologies im Bereich Lebensmittel und deren relative Stärke 259

Tabelle 50: Top Patentländer im Bereich Metallbearbeitung 260

Tabelle 51: Industriestruktur in Österreich im Bereich Metallbearbeitung 261

Tabelle 52: Crossing-Technologies im Bereich Metallbearbeitung und deren relative Stärke 263

Tabelle 53: Top Patentländer im Bereich Möbel 265

Tabelle 54: Industriestruktur in Österreich im Bereich Möbel 266

Tabelle 55: Crossing-Technologies im Bereich Möbel und deren relative Stärke 267

$\begin{array}{ll}\text { Tabelle 56: Top Patentländer im Bereich Papier } & 268\end{array}$

Tabelle 57: Industriestruktur in Österreich im Bereich Papier 269

Tabelle 58: Crossing-Technologies im Bereich Papier und deren relative Stärke 270

Tabelle 59: Top Patentländer im Bereich Stahl $\quad 272$

Tabelle 60: Industriestruktur in Österreich im Bereich Stahl 273

Tabelle 61: Crossing-Technologies im Bereich Stahl und deren relative Stärke 274

Tabelle 62: Top Patentländer 276

Tabelle 63: Industriestruktur in Österreich im Bereich Stempel 277

Tabelle 64: Crossing-Technologies im Bereich Stempel und deren relative Stärke 278

Tabelle 65: Industriestruktur in Österreich im Bereich Werkzeug 280

Tabelle 66: Top Patentländer im Bereich Werkzeug 280

Tabelle 67: Crossing-Technologies im Bereich Werkzeug und deren relative Stärke 281 


\section{Abbildungsverzeichnis}

Abbildung 1: Wirkungsebenen des Förderprogramms 2

Abbildung 2: Umsetzungskonzept 3

Abbildung 3: Organisationsstruktur der CDG 10

Abbildung 4: Verfahren zur Prüfung und Beurteilung von Anträgen zur Einreichung eines CD-Labors 16

Abbildung 5: Verfahren zur Prüfung und Beurteilung von Anträgen zur Einreichung eines JR-Zentrums 18

Abbildung 6: Fördermittel der CD-Labors in Mio. Euro 28

Abbildung 7: Mittlere Gesamtmittel der CD-Labors, nach Endjahr $\quad 29$

Abbildung 8: Anzahl der wissenschaftlichen Mitarbeiterinnen und Mitarbeiter 30

Abbildung 9: Zusammenhang Fördermittel und Anzahl der wissenschaftlichen Mitarbeiterinnen und Mitarbeiter 31

Abbildung 10: Anzahl der Diplomarbeiten, absolut und in Relation zu den Gesamtmitteln in Mio. Euro 32

Abbildung 11: Anzahl der Dissertationen, absolut und in Relation zu den Gesamtmitteln in Mio. Euro 33

Abbildung 12: Anzahl der Habilitationen, absolut und in Relation zu den Gesamtmitteln in Mio. Euro 34

Abbildung 13: Vorträge, absolut und in Relation zu den Gesamtmitteln in Mio. Euro 35

Abbildung 14: Geladene Vorträge, absolut und in Relation zu den Gesamtmitteln in Mio. Euro 36

Abbildung 15: Poster, absolut und in Relation zu den Gesamtmitteln in Mio. Euro 37

Abbildung 16: Referierte Artikel, absolut und in Relation zu den Gesamtmitteln in Mio. Euro 38

Abbildung 17: Nicht referierte Artikel, absolut und in Relation zu den Gesamtmitteln in Mio. EUR 39

Abbildung 18: Sonstige Publikationen, absolut und in Relation zu den Gesamtmitteln in Mio. Euro 40

Abbildung 19: Zusätzliche Fördermittel in Relation zu den Gesamtmitteln 41

Abbildung 20: Preise und Rufe, absolut und in Relation zu den Gesamtmitteln in Mio. Euro 42

Abbildung 21: Gutachtertätigkeit: Absolute Anzahl der Zeitschriften und in Relation zu den Gesamtmitteln in Mio. Euro

Abbildung 22: Kooperationen neu, absolut und in Relation zu den Gesamtmitteln in Mio. Euro 44

Abbildung 23: Kooperationen vorhanden, absolut und in Relation zu den Gesamtmitteln in Mio. Euro 45

Abbildung 24: Mitarbeiterwechsel in die Wirtschaft, absolut und in Relation zu den Gesamtmitteln in Mio. Euro 46

Abbildung 25: Patente, absolut und in Relation zu den Gesamtmitteln in Mio. Euro 47

Abbildung 26: Indikatoren in Relation zu den Gesamtmitteln (in Mio. Euro) 51

Abbildung 27: Fördermittel laut Budgetplan der JR-Zentren in Mio. Euro 54

Abbildung 28: Kumulierte Fördermittel 2013-15 der JR-Zentren in Mio. Euro 55

Abbildung 29: Unternehmensbeteiligungen der JR-Zentren (2013-15) 55

Abbildung 30: Personal finanziert durch die CDG in den JR-Zentren versus Budgetmittel (2013-15) 56

Abbildung 31: Personal finanziert durch die CDG in den JR-Zentren (in Köpfen, 2013-15) 56 
Abbildung 32: Bewertung der Programmziele: BMWFW, CDG

Abbildung 33: Bewertung der Programmziele: Labor- und Zentrumsleiter 67

Abbildung 34: Bewertung der Programmziele: Unternehmen $\quad 68$

Abbildung 35: Bewertung der Programmziele: Stakeholder $\quad 69$

Abbildung 36: Ausmaß, in denen Erfahrungen in der Forschung bzw. Lehre wirksam geworden sind (falls $\begin{array}{ll}\text { ein Einfluss besteht) } & 70\end{array}$

Abbildung 37: Gesamteindruck von der Qualität der Zusammenarbeit mit der Industrie 71

Abbildung 38: Ausmaß der Forschungsarbeiten nach dem Auslaufen des CD-Labors (Folgeexpertise) 72

Abbildung 39: Gingen aus dem CD-Labor Wissenschaftler hervor, die eigene Forschungsgruppen leiten bzw. geleitet haben?

Abbildung 40: Gemeinsame PR-Aktivitäten des CD-Labors und der Unternehmenspartner 73

Abbildung 41: Wurden aufgrund der Tätigkeiten des CD-Labors die Forschungsaktivitäten des Heimat gebenden Instituts im Themenbereich des CD-Labors nachhaltig (über den Förderzeitraum hinaus) verstärkt? $\quad 74$

Abbildung 42: Einfluss des CD-Labors auf die Universitäre Lehre

Abbildung 43: Generelle Auswirkungen des CD-Labors auf das Drittmittelvolumen des Heimat gebenden Instituts/Departments (auch außerhalb des Themenbereichs des CD-Labors) 75

Abbildung 44: Generelle Auswirkungen des CD-Labors auf die Unternehmenskooperationen 76

Abbildung 45: Zusammenarbeit im CD-Labor 77

Abbildung 46: Praxisnähe der Forschungsarbeit $\quad 77$

Abbildung 47: Produkt- oder Prozessinnovationen $\quad 78$

Abbildung 48: Weiterführende innerbetriebliche Forschungs- und Entwicklungsaktivitäten 78

Abbildung 49: Folgeprojekte mit der Universität Forschungseinrichtung/Fachhochschule 79

Abbildung 50: Zusätzliche Förderungen $\quad 79$

Abbildung 51: Auswirkungen des CD-Labors auf das Unternehmen 80

Abbildung 52: Zufriedenheit mit Aspekten des Programms bzw. der Programmabwicklung 97

Abbildung 53: Zufriedenheit mit Aspekten des Programms bzw. der Programmabwicklung im Zeitverlauf

$(2005,2011,2016) \quad 98$

Abbildung 54: Einschätzung der begleitenden wissenschaftlichen Kontrolle 101

Abbildung 55: Einschätzung der begleitenden wissenschaftlichen Kontrolle im Zeitverlauf $(2011,2016) 101$

Abbildung 56: Bewertung von Anzahl und Zeitpunkt der Zwischenevaluierungen 102

Abbildung 57: Zufriedenheit mit den Leistungen der CDG 103

Abbildung 58: Zufriedenheit mit den Leistungen der CDG im Zeitverlauf (2005, 2011, 2016) 104

Abbildung 59: Motivation zur CD-Laborgründung im Zeitverlauf (2005, 2011, 2016) 105

Abbildung 60: Kontakt zum Unternehmenspartner vor der CD-Laborgründung 105

Abbildung 61: Erwartungen und Ziele bei der CD-Laborgründung und deren Bedeutung 106 
Abbildung 62: Einfluss der Laborleiter/Laborleiterinnen auf die Forschungsprogrammformulierung und auf die Moduldefinition

Abbildung 63: Einfluss der Laborleiter/Laborleiterinnen auf die Forschungsprogrammformulierung und auf die Moduldefinition im Zeitverlauf (2005, 2011, 2016)

Abbildung 64: Inputs des Unternehmenspartners

Abbildung 65: Prozent der Gesamtarbeitszeit an der Universität, die dem CD-Labor gewidmet wird/wurde

Abbildung 66: Nutzung des 30\%igen wissenschaftlichen Forschungsfreiraums im Zeitverlauf (2005, 2011, 2016)

Abbildung 67: Nutzen für das Universitäts-/Forschungsinstitut aus dem CD-Labor

Abbildung 68: COMET/Kompetenzzentrum, Spin-off im Zeitverlauf $(2011,2016)$

Abbildung 69: Werdegang ehemaliger Labormitarbeiter/Labormitarbeiterinnen

Abbildung 70: Durchführung der Forschungsprojekte, auch ohne CD-Labor

Abbildung 71: Wie haben Sie von der internationalen Kooperation profitiert bzw. profitieren Sie davon?

Abbildung 72: Administrativer Aufwand eines CD-Labors im Vergleich zu anderen Förderprogrammen

Abbildung 73: Zufriedenheit mit Aspekten des Programms bzw. der Programmabwicklung

Abbildung 74: Einschätzung der begleitenden wissenschaftlichen Kontrolle

Abbildung 75: Bewertung des Zeitpunktes der Zwischenevaluierung

Abbildung 76: Zufriedenheit mit den Leistungen der CDG

Abbildung 77: Motivation zur JR-Zentrumsgründung

Abbildung 78: Kontakt zum Unternehmenspartner vor der JR-Zentrumsgründung

Abbildung 79: Erwartungen und Ziele bei der JR-Zentrumsgründung und deren Bedeutung

Abbildung 80: Einfluss des Zentrumsleiters/der Zentrumsleiterin auf die Forschungsprogrammformulierung und auf die Moduldefinition

Abbildung 81: Inputs des Unternehmenspartners

Abbildung 82: Prozent der Gesamtarbeitszeit an der Fachhochschule, die dem JR-Zentrum gewidmet wird

Abbildung 83: Nutzen für das FH-Institut aus dem JR-Zentrum

Abbildung 84: Durchführung der Forschungsprojekte, auch ohne JR-Zentrum

Abbildung 85: Sind Erfahrungen aus industrierelevanten Projekten bereits in den akademischen Betrieb des Heimat gebenden Instituts eingeflossen?

Abbildung 86: Bisheriger Gesamteindruck von der Qualität der Zusammenarbeit mit der Industrie

Abbildung 87: Auswirkung des JR-Zentrums auf das Drittmittelvolumen des Heimat gebenden Instituts/Departments

Abbildung 88: Auswirkung des JR-Zentrums generell auf die Unternehmenskooperationen des Heimat gebenden Instituts/Departments 
Abbildung 89: Gibt es Initiativen das JR-Zentrum nach dem Förderzeitraum als eigenständiges Unternehmen/als eigene Einrichtung weiterzuführen?

Abbildung 90: Administrativer Aufwand eines JR-Zentrums im Vergleich zu andern Förderprogrammen

Abbildung 91: Beurteilung der Laufzeit des CD-Labors (von 7 Jahren) im Zeitverlauf $(2005,2011,2016)$

Abbildung 92: Zufriedenheit mit Aspekten des Programms bzw. der Programmabwicklung

Abbildung 93: Zufriedenheit mit Aspekten des Programms bzw. der Programmabwicklung im Zeitverlauf $(2005,2011,2016)$

Abbildung 94: Einschätzung der begleitenden wissenschaftlichen Kontrolle

Abbildung 95: Einschätzung der begleitenden wissenschaftlichen Kontrolle im Zeitverlauf $(2011,2016)$

Abbildung 98: Motivation als Unternehmenspartner zur CD-Laborgründung bzw. zur Beteiligung am CD-

Labor

Abbildung 99: Art der Forschungsaktivitäten im CD-Labor

Abbildung 100: Kontakt zu Wissenschaftlern/Wissenschaftlerinnen vor der CD-Laborgründung im Zeitverlauf $(2011,2016)$

Abbildung 101: Einfluss seitens des Unternehmens auf die Forschungsprogrammformulierung und auf die Moduldefinition

Abbildung 102: Einfluss seitens des Unternehmens auf die Forschungsprogrammformulierung und auf die Moduldefinition im Zeitverlauf $(2005,2011,2016)$

Abbildung 103: Durchführung der Forschungskooperation im CD-Labor im Zeitverlauf $(2005,2011,2016)$

Abbildung 104: Häufigkeit der Kontakte

Abbildung 105: Häufigkeit der Kontakte im Zeitverlauf (2005, 2011, 2016)

Abbildung 106: Bewertung von Aspekten der Zusammenarbeit im CD-Labor

Abbildung 107: Weitere beteiligte Unternehmen am CD-Labor

Abbildung 108: Haben bzw. hatten die Forschungsergebnisse aus der Beteiligung am CD-Labor wirtschaftliche Relevanz für folgende Aspekte?

Abbildung 109: Nutzen für die Unternehmen durch die CD-Laborgründung bzw. -beteiligung

Abbildung 110: Auswirkungen der CD-Laborgründung auf Unternehmen im Zeitverlauf (2005, 2011, 2016)

Abbildung 111: Kosten-Nutzen Relation im Zeitverlauf (2005, 2011, 2016)

Abbildung 112: Weitere Forschungsprojekte im Rahmen eines CD-Labors?

Abbildung 113: Durchführung auch ohne CD-Labor im Zeitverlauf $(2011,2016)$

Abbildung 114: Voraussichtliche Finanzierung des Forschungsprojekts ohne CD-Labor im Zeitverlauf $(2011,2016)$ 
Abbildung 116: Beurteilung der Laufzeit des JR-Zentrums (von 5 Jahren)

Abbildung 117: Zufriedenheit mit Aspekten des Programms bzw. der Programmabwicklung 154

Abbildung 118: Einschätzung der begleitenden wissenschaftlichen Kontrolle 155

Abbildung 119: Zufriedenheit mit den Leistungen der CDG 155

Abbildung 120: Motivation als Unternehmenspartner zur JR-Zentrumsgründung 156

Abbildung 121: Art der Forschungsaktivitäten im JR-Zentrum 156

Abbildung 122: Kontakt zu Wissenschaftlern/Wissenschaftlerinnen vor der JR-Zentrumsgründung 157

Abbildung 123: Einfluss seitens des Unternehmens auf die Forschungsprogrammformulierung und auf $\begin{array}{ll}\text { die Moduldefinition } & 158\end{array}$

$\begin{array}{lr}\text { Abbildung 124: JR-Zentrum eher eigenständig oder kooperativ } & 158\end{array}$

Abbildung 125: Häufigkeit der Kontakte $\quad 159$

Abbildung 126: Bewertung von Aspekten der Zusammenarbeit im JR-Zentrum 159

Abbildung 127: Haben bzw. hatten die Forschungsergebnisse aus der Beteiligung am JR-Zentrum wirtschaftliche Relevanz für folgende Aspekte? 160

Abbildung 128: Nutzen für die Unternehmen durch die JR-Zentrumsgründung bzw. -beteiligung 161

Abbildung 129: Auswirkungen der JR-Zentrumsgründung auf Unternehmen 161

Abbildung 130: Kosten-Nutzen Relation 162

Abbildung 131: Weitere Forschungsprojekte im Rahmen eines JR-Zentrums? 163

Abbildung 132: Durchführung des Forschungsprojekts auch ohne JR-Zentrum? 163

Abbildung 133: Nutzung direkter Forschungsförderprogramme in Zukunft im Unternehmen 164

Abbildung 134: Anzahl der Unternehmen, differenziert nach Anzahl der CD-Laborbeteiligungen 174

Abbildung 135: Anzahl der Unternehmensbeteiligungen, differenziert nach Anzahl der CD$\begin{array}{ll}\text { Laborbeteiligungen } & 174\end{array}$

Abbildung 136: Verteilung der Unternehmen und CD-Laborbeteiligungen 175

Abbildung 137: Kooperationsnetzwerk der Unternehmen in der CDG $\quad 179$

Abbildung 138: Kooperationsnetzwerk der voestalpine in der CDG 180

Abbildung 139: Entwicklung der Anzahl der CD-Labors an Universitäten (Spitzengruppe) 182

Abbildung 140: Entwicklung der Anzahl der CD-Labors an Universitäten mit mehr als 2 CD-Labors 182

Abbildung 141: HH-Index der CD-Labors nach Forschungseinrichtungen in Österreich 183

Abbildung 142: Lorenz-Kurve (Anzahl CD-Labors versus Anzahl der Studierenden) 184

Abbildung 143: Ausgaben für Forschung und Entwicklung, nach Forschungsarten in Mrd. Euro (2002, 2013)

Abbildung 144: F\&E Ausgaben nach Forschungsarten und Durchführungssektor in Österreich, in Mio. Euro, 2013

Abbildung 145: Top Wirtschaftszweige in Österreich (nach ÖNACE 2008) mit dem höchsten Anteil von 
Abbildung 146: Staatsausgaben im Bereich Grundlagenforschung, 2012-2015, Österreich, nach ESVG 2010, konsolidiert, in Mio. Euro

Abbildung 147: Ausgaben für Forschung und Entwicklung in Prozent des BIP, 1995 - 2014

Abbildung 148: Ausgaben für Forschung und Entwicklung, in Prozent des BIP, 2015

Abbildung 149: Ausgaben für Grundlagenforschung in Prozent des BIP, 2012 bzw. 2013

Abbildung 150: Interne Ausgaben für Forschung und Entwicklung der wissenschaftlichen Einrichtungen des öffentlichen Sektors nach Forschungsart in Mio. Euro, Deutschland, 2014

Abbildung 151: European Innovation Scoreboard (EIS) Innovations-Index 2016

Abbildung 152: EIS Innovations-Index in der Gruppe der „Strong Innovators“, 2008-2015

Abbildung 153: Patenthäufigkeiten der Technologiefelder

Abbildung 154: Verteilung der Themen nach relativer Stärke Österreichs

Abbildung 155: Verteilung der Themen nach relativer Stärke (Top 3.400 Technologiefelder)

Abbildung 156: Relative Themenintensität Österreich

Abbildung 157: Weltweite Dynamik und Häufigkeit von 3400 Technologiefeldern

Abbildung 158: Verteilung der weltweiten Patentdynamik von 3.400 Technologiefeldern*

Abbildung 159: CD-Labors und JR-Zentren in den Technologiestärkefeldern Österreich

Abbildung 160: CD-Labors und JR-Zentren in den Wachstumsthemen

Abbildung 161: Weltweite Dynamik im Bereich Aufzüge

Abbildung 162: Internationale Vernetzung im Bereich Aufzüge

Abbildung 163: Crossing-Technologies im Bereich Aufzüge

Abbildung 164: Weltweite Dynamik im Bereich Cellulose

Abbildung 165: Internationale Vernetzung im Bereich Cellulose

Abbildung 166: Crossing-Technologies im Bereich Cellulose

Abbildung 167: Weltweite Dynamik im Bereich Eisenbahn

Abbildung 168: Internationale Vernetzung im Bereich Eisenbahn

Abbildung 169: Crossing-Technologies im Bereich Eisenbahn

Abbildung 170: Weltweite Dynamik im Bereich Lebensmittel

Abbildung 171: Internationale Vernetzung im Bereich Lebensmittel

Abbildung 172: Crossing-Technologies im Bereich Lebensmittel

Abbildung 173: Weltweite Dynamik im Bereich Metallbearbeitung

Abbildung 174: Internationale Vernetzung im Bereich Metallbearbeitung

Abbildung 175: Crossing-Technologies im Bereich Metallbearbeitung

Abbildung 176: Weltweite Dynamik im Bereich Möbel 
Abbildung 180: Internationale Vernetzung im Bereich Papier

Abbildung 183: Internationale Vernetzung im Bereich Stahl

Abbildung 184: Crossing-Technologies im Bereich Stahl

Abbildung 185: Weltweite Dynamik im Bereich Stempel

Abbildung 186: Crossing-Technologies im Bereich Stempel

Abbildung 187: Weltweite Dynamik im Bereich Werkzeug

Abbildung 188: Crossing-Technologies im Bereich Werkzeug

Abbildung 189: Weltweite Dynamik im Additive Fertigungsverfahren

Abbildung 190: Weltweite Dynamik im Bereich „Verteilung elektrischer Leistung“

Abbildung 191: Weltweite Dynamik im Bereich nicht-schaltbare Kupplungen

Abbildung 193: Weltweite Dynamik im Bereich Chirurgie/anwendungsspezifische Robotik 


\section{Quellen}

BMVIT/BMWFJ (2007), Richtlinien zur Förderung der wirtschaftlich-technischen Forschung und Technologieentwicklung (FTE-Richtlinien) gemäß $\S 11 \mathrm{Z} 1$ bis 5 des Forschungs- und Technologieförderungsgesetzes (FTFG) des Bundesministers für Verkehr, Innovation und Technologie vom 19. 11. 2007 (GZ BMVIT-609.986/0011-III/I2/2007) und des Bundesministers für Wirtschaft und Arbeit vom 30. 11. 2007 (GZ BMWA-97.005/0002-C1/9/2007)

BMVIT/BMWFW (2015), RICHTLINIE zur Förderung der wirtschaftlich - technischen Forschung, Technologieentwicklung und Innovation (FTI - Richtlinie 2015), Struktur-FTI-RL, des Bundesministers für Verkehr, Innovation und Technologie (GZ BMVIT-609.986/0011-III/I2/2014) und des Bundesministers für Wissenschaft, Forschung und Wirtschaft (GZ BMWFW-97.005/0003-C1/9/2014) mit Geltung ab 1. 1. 2015

BMWFJ/CDG (2012), Bewertungshandbuch zum Programm Förderung der Einrichtung und des Betriebs von Josef Ressel Zentren, GZ.: BMWFJ-97.700/0001-C1/9/2012, Genehmigt am 13. 1. 2012 vom Bundesministerium für Wirtschaft, Familie und Jugend

BMWFJ/CDG (2013), Bewertungshandbuch zum Programm Förderung der Einrichtung und des Betriebs von Christian Doppler Labors, GZ.: BMWFJ-97.430/0021-C1/9/2013, Genehmigt am 12. 8. 2013 vom Bundesministerium für Wirtschaft, Familie und Jugend

BMWFJ/CDG (2013b), Programmdokument 2014 zur Förderung der Einrichtung und des Betriebs von Christian Doppler Labors, Programmdokument gemäß Punkt 5.1. der FTE-Richtlinien für die Christian Doppler Forschungsgesellschaft, GZ.: BMWFJ-97.430/0026-C1/9/2013, Genehmigt am 20.11.2013

BMWFJ/CDG (2014), Programmdokument 2014 zur Förderung der Einrichtung und des Betriebs von Josef Ressel Zentren, Programmdokument gemäß Punkt 5.1. der FTE-Richtlinien für die Christian Doppler Forschungsgesellschaft, GZ.: BMWFJ-97.700/0001-C1/9/2014, Genehmigt am 23.01.2014

BMWFW/CDG (2015), Leitfaden zur Einrichtung eines Christian Doppler Labors, Fassung vom 17.06.2015

BMWFW/CDG (2015b), Handbuch zum Betrieb eines Christian Doppler Labors, Fassung vom 04.03.2015

BMWFW/CDG (2015c), Leitfaden zur Einrichtung eines Josef Ressel Zentrums, Fassung vom 01.07.2015

BMWFW/CDG (2015d), Handbuch zum Betrieb eines Josef Ressel Zentrums, Fassung vom 24.06.2015

CDG (2014), Informationen zur Mitgliedschaft in der Christian Doppler Forschungsgesellschaft, Fassung vom 23.05.2014

CDG (2015), Statuten des gemeinnützigen Vereins Christian Doppler Forschungsgesellschaft, Fassung vom 18.09.2015

CONVELOP (2010), Evaluierung des Pilotprogramms „Josef Ressel Zentren“, Endbericht, 30.09.2010; http://www.fteval.at/upload/JRZ-Evaluation_Endbericht.pdf

DESSER, H. (2001), Das Werden und Wachsen der Christian Doppler Forschungsgesellschaft, 1988-2000, Wien

ECONOMICA/IWI (2012), Nutzen-, Programm-, und Systemevaluierung der Christian Doppler Forschungsgesellschaft, Wien; http://www.fteval.at/upload/Evaluierung_Christian_Doppler_Forschungsgesellschaft.pdf

ECONOMICA/IWI (2013), Handlungsoptionen für die weitere Optimierung des CD-Programms, Dezember 2013, Wien

JOANNEUM RESEARCH/TECHNOPOLIS/KMU FORSCHUNG AUSTRIA/FRAUNHOFER - ISI (2005), Evaluierung der Christian Doppler Forschungsgesellschaft (CDG), Wien;

http://www.fteval.at/upload/Evaluierung_der_Christian_Doppler_Forschungsgesellschaft_(CDG)._Policy _Paper.pdf 
RECHNUNGSHOF (2007), Christian Doppler Forschungsgesellschaft, Rechnungshofbericht Reihe Bund 2007/11, Vorlage vom 23. August 2007, Wien;

http://www.rechnungshof.gv.at/fileadmin/downloads/2007/berichte/berichte_bund/Bund_2007_11.pd f

RECHNUNGSHOF (2016), Forschungsfinanzierung in Österreich, Rechnungshofbericht Reihe Bund 2016/8, Wien, im Juni 2016;

http://www.rechnungshof.gv.at/fileadmin/downloads/_jahre/2016/berichte/berichte_bund/Bund_201 6_08.pdf

TECHNOPOLIS (2015), Evaluierung der Forschungsförderung für Fachhochschulen in Österreich, Wien

UNTERER, U. (2005), CHRISTIAN DOPPLER FORSCHUNGSGESELLSCHAFT, Frauenfördernde Maßnahmen, Foliensatz, 16.03.2006, http://www.w-fforte.at/fileadmin/Redaktion/Daten/PDF/BMWA_CDG.pdf

\section{Internet}

http://www.bmwfw.gv.at/

https://www.cdg.ac.at/ 


\section{Interviews (Person, Organisation):}

Aussersdorfer, Karin, Mag., voestalpine Stahl GmbH

Brandner, Marco, Dr., Plansee SE

Ferreira-Briza, Fatima, Univ.Prof. Dr.; Universität Salzburg

Horauer, Martin, Prof.(FH) Dipl.-Ing. Dr.; Technikum Wien

Kürnsteiner, Hubert, Dr., Sandoz GmbH

Müllner, Waltraud, DI Dr., A1 Telekom Austria AG

Rossmanith, Peter, Dr.; Veterinärmedizinische Universität Wien

Weber, Viktoria, Univ.Prof. Dr.; Donau-Universität Krems

\section{AHP Bewertungen}

MR Dr. Ulrike Unterer, DDr. Martin Pilch, BMWFW

Univ.Prof. Dr. Reinhart Kögerler, em.o.Univ.Prof. DI Dr. Franz G. Rammerstorfer, CDG

Prof.(FH) DI Mag. Dr. Dominik Engel, FH Salzburg

Assoc.Prof. DI Dr. David Stifter, Universität Linz

DI Leo Arpa (Mondi Uncoated Fine \& Kraft Paper GmbH)

Dr. Schmidl (Aurubis AG), Deutschland 


\section{ANHANG}

\section{Fragebogen CD-LABOR: LABORLEITERIN/-LEITER}

1. Wie zufrieden sind (bzw. waren) Sie mit den folgenden Aspekten des Programms bzw. der Programmabwicklung?

(I) Klarheit des Förderkonzepts und Förderziels

(II) Administrativer Aufwand der Antragsstellung

(III) Angemessenheit der Evaluierungskriterien

(IV) Bearbeitungsdauer der eingereichten Anträge

(V) Administrativer Aufwand während der Laufzeit des CD-Labors

a) sehr zufrieden; b) zufrieden; c) wenig zufrieden; d) unzufrieden

1b. Wenn bei 1(II) und/oder 1(V) c) oder d) dann:

Wo sehen Sie Potential für administrative Vereinfachungen? spielen)

2. Wie beurteilen Sie die Laufzeit des CD-Labors von 7 Jahren?

a) gerade richtig; b) zu kurz; c) zu lang

3. Wie schätzen Sie die begleitende wissenschaftliche Kontrolle ein? Empfehlungen u. Auflagen...

(I) ... bei der 2-Jahresevaluierung

(II) ... bei der 5-Jahresevaluierung.

a) sehr hilfreich; b) hilfreich; c) wenig hilfreich; d) nicht hilfreich; e) bisher noch nicht erfolgt

4. Wie bewerten Sie Anzahl und Zeitpunkt der Zwischenevaluierungen?

(I) Anzahl

a) gerade richtig; b) zu viele; c) zu wenige

(II) Zeitpunkt

a) passend; b) unpassend:___ (Bitte Nennung von Gründen/Veränderungsvorschlägen)

5. Wie zufrieden sind (bzw. waren) Sie mit den folgenden Leistungen der CDG?

(I) Betreuung während der Antragstellung

(II) Betreuung des CD-Labors während der Laufzeit

(III) Öffentlichkeitsarbeit

a) sehr zufrieden; b) zufrieden; c) wenig zufrieden; d) unzufrieden

6. Von wem kam die Motivation zur CD-Laborgründung?

a) Vom Unternehmenspartner

b) Vom wissenschaftlichen Partner

c) Von beiden Partnern

7. Welcher Kontakt bestand zum Unternehmenspartner vor der Laborgründung?

(Mehrfachantworten möglich)

a) Gemeinsame Forschungsprojekte

b) Kontakte über AbsolventInnen 

c) Lose Kontakte
d) Keine Kontakte

8. Welche Erwartungen und Ziele hatten Sie bei der Laborgründung hinsichtlich des CD-Labors und wie bedeutend waren Ihnen diese?

(I) Prestigegewinn

(II) Aufbau eines eigenen Forschungsteams

(III) Intensivierung der Unternehmenskontakte

(IV) Langfristig finanziell abgesichertes Arbeiten an einem Themenkreis

(V) Finanzielle Absicherung der Forschungsarbeit

(VI) Sonstige (eigene Nennungen)

a) sehr wichtig; b) wichtig; c) weniger wichtig; d) unwichtig

9. Wie viel Einfluss hatten Sie als Laborleiter/Laborleiterin auf die

(I) Forschungsprogrammformulierung und

(II) auf die Moduldefinition?
a) sehr hohen Einfluss; b) hohen Einfluss; c) wenig Einfluss

10. In welchem Ausmaß lieferte der Unternehmenspartner Inputs hinsichtlich folgender Aspekte?
(I) Vermittlung von Kontakten
(II) Mitarbeit an Forschungsarbeiten
(III)Bereitstellung zusätzlicher Infrastruktur
(IV) Formulierung des Forschungsprogramms
(V) Feedback zu Forschungsaktivitäten
(VI)Moduldefinition

a) in sehr hohem Ausmaß; b) in hohem Ausmaß; c) in geringem Ausmaß; d) gar nicht

11. Wie viel Prozent Ihrer Gesamtarbeitszeit an der Universität/Forschungseinrichtung widmen (bzw. widmeten) Sie durchschnittlich Ihrem CD-Labor?

$\%$ (Angaben in \% der Gesamtarbeitszeit an der Universität/Forschungseinrichtung)

12. Zu welchem Zweck wird (bzw. wurde) der 30\%ige wissenschaftliche Forschungsfreiraum in Ihrem Labor üblicherweise genutzt? (Mehrfachantworten möglich)
(I) Konferenzen
(II) Publikationen, Veröffentlichungen
(III) Eigenständige Forschung
(IV) Arbeit an Master-, Diplomarbeiten, Dissertationen und Habilitationen
(V) Weiterbildung
(VI) Lehre

13. Ergab sich aus dem CD-Labor folgender Nutzen für das Universitäts-/Forschungsinstitut und wie hoch war dieser?

(I) Attraktivität als Arbeitgeber

(II) Prestigegewinn

(III) Verbesserte Stellung des Instituts

(IV) Attraktivität als Kooperationspartner 
(V) Erhöhung des Praxisbezuges der Lehre

(VI) Akquisition zusätzlicher Forschungsprojekte

a) sehr hoher Nutzen; b) hoher Nutzen; c) geringer Nutzen; d) kein Nutzen; e) noch nicht einschätzbar

Ist Ihr CD-Labor bereits abgeschlossen:

(Ja) Weiter mit Frage 14 (ohne Frage 19)

(Nein) Weiter mit Frage 18

14. Haben die wissenschaftlichen Aktivitäten des CD-Labors (anschließend) zur Gründung eines COMET-Zentrums oder eines anderen Kompetenzzentrums beigetragen?

a) Ja ___ (Nennung des Namens) im Forschungsbereich ___(Nennung)

b) Nein

15. Ist Ihr Institut nunmehr wissenschaftlicher Partner bei einem COMET-Zentrum oder einem anderen Kompetenzzentrum bzw. einer Forschungseinrichtung?

a) Ja, und zwar bei: ___ (Nennung des Namens) im Forschungsbereich (Nennung)

b) Nein

16. Hat sich aus dem damaligen CD-Labor ein Spin-off entwickelt?
a) Ja (Nennung des Namens)
b) Nein

17. Bitte geben Sie im Folgenden eine Einschätzung zum Werdegang Ihrer CDLabormitarbeiterInnen an? Bitte versuchen Sie eine prozentuelle Zuordnung zu folgenden Kategorien, welche in Summe $100 \%$ ergeben.

(I) Wie viele MitarbeiterInnen wechselten zum Unternehmenspartner? $\%$

(II) Wie viele wechselten zu einem anderen Unternehmen? $\%$

(III) Wie viele machten sich selbständig? __ \%

(IV) Wie viele blieben an der Universität/Forschungseinrichtung oder wechselten zu einer anderen Universität/Forschungseinrichtung? $\%$

(V) Wie viele sind nunmehr bei einem COMET-Zentrum angestellt? $\%$

(VI) Wie viele sind nunmehr bei einem anderen CD-Labor angestellt? $\%$

(VII) Werdegang nicht bekannt/Keine Angaben möglich $\%$

18. Hätten Sie die Forschungsprojekte, die Sie im Rahmen des CD-Labors durchführen bzw. durchgeführt haben, auch ohne CD-Labor durchgeführt?
a) Ja, im selben Umfang
b) Ja, aber in geringerem Umfang
c) Ja, aber in deutlich geringerem Umfang
d) Nein, gar nicht.

19. Gibt es Initiativen das CD-Labor nach dem Förderzeitraum als eigenständiges Unternehmen/als eigene Einrichtung weiter zu führen?
a) Ja
b) Nein
c) Noch nicht einschätzbar 
20. Hat/Hatte Ihr CD-Labor einen internationalen Partner im Rahmen des CD-Labors?
a) Ja (Nennung)
Grund/Gründe: (Nennung)
b) Nein

21. Führt/Führte Ihr CD-Labor ein Modul und/oder Forschungsarbeiten an einem ausländischen Standort durch?

a) Ja ___ (Nennung)

a2) Was sind/waren die Gründe (z.B. bereits Kontakt zu ausländischem Partner vorhanden; Know-how in Ö nicht vorhanden)?

b) Nein (Nennung)

22. Wenn Frage $\mathbf{2 0}$ oder $\mathbf{2 1}$ mit ja beantwortet wurde: Wie haben Sie von der internationalen Kooperation profitiert bzw. wie profitieren Sie davon?

(I) Forschungsleistung im Ausland ist kostengünstiger

(II) Neues Know-how durch den ausländischen Partner erworben

(III) Neue Unternehmenskontakte im Ausland erworben

(IV) Sonstiges: (Nennung)

a) trifft stark zu; b) trifft eher zu; c) trifft eher nicht zu; d) trifft nicht zu

23. Wenn Frage $\mathbf{2 0}$ oder $\mathbf{2 1}$ mit ja beantwortet wurde: Planen Sie weitere Industry-ScienceKooperationen im Ausland mit ausländischen Partnern, die sie im Rahmen des CD-Labors kennengelernt haben?

a) Ja

b) Nein

24. Wie beurteilen Sie den administrativen Aufwand eines CD-Labors im Vergleich zu anderen Förderprogrammen? Der administrative Aufwand eines CD-Labors ist im Vergleich zu einem ...

(I) ...FFG-Projekt in Kooperation mit Unternehmen

(II) ...EU-Projekt in Kooperation mit Unternehmen

(III) ...FWF-Projekt

a) höher b) gleich hoch; c) niedriger; d) kein Vergleich möglich

25. Wie hoch ist/war in Summe der administrativen Aufwand des CD-Labors? Bitte geben Sie eine Schätzung der Wochenstunden im Jahresdurchschnitt an.

_(Stunden pro Woche im Jahresdurchschnitt) 


\title{
Fragebogen CD-LABOR: UNTERNEHMENSPARTNER
}

\author{
In wie vielen CD-Labors ist und war Ihr Unternehmen in Summe bisher aktiv: \\ (in einem) Weiter mit Fragebogenentwurf 02 \\ (in mehreren) Weiter mit Fragebogenentwurf 02b
}

\section{Wie beurteilen Sie die Laufzeit des CD-Labors von 7 Jahren?}

a) gerade richtig; b) zu kurz; c) zu lang

2. Wie zufrieden sind (bzw. waren) Sie mit den folgenden Aspekten des Programms bzw. der Programmabwicklung?

(I) Klarheit des Förderkonzepts und Förderziels

(II) Administrativer Aufwand der Antragsstellung

(III) Angemessenheit der Evaluierungskriterien

(IV) Bearbeitungsdauer der eingereichten Anträge

(V) Administrativer Aufwand während der Laufzeit des CD-Labors

a) sehr zufrieden; b) zufrieden; c) wenig zufrieden; d) unzufrieden

2b. Wenn bei 2(II) und/oder 2(V) c) oder d) dann:

Wo sehen Sie Potential für administrative Vereinfachungen? Beispielen)

(Bitte Nennung von

3. Wie schätzen Sie die begleitende wissenschaftliche Kontrolle ein? Empfehlungen u. Auflagen...

(I) ... bei der 2-Jahresevaluierung

(II) ... bei der 5-Jahresevaluierung.

a) sehr hilfreich; b) hilfreich; c) wenig hilfreich; d) nicht hilfreich; e) bisher noch nicht erfolgt

4. Wie zufrieden sind (bzw. waren) Sie mit den folgenden Leistungen der CDG?

(I) Betreuung während der Antragstellung

(II) Betreuung des CD-Labors während der Laufzeit

(III) Öffentlichkeitsarbeit

a) sehr zufrieden; b) zufrieden; c) wenig zufrieden; d) unzufrieden

5. Was war Ihre Motivation als Unternehmenspartner zur CD-Laborgründung bzw. zur Beteiligung am CD-Labor und wie wichtig waren Ihnen die einzelnen Punkte dabei?

(I) Aufbau einer Kooperationsplattform mit anderen Unternehmen

(II) Recruiting: Zugang zu jungen Talenten

(III) Einstieg in neues Forschungsthema

(IV) Eigene anwendungsorientierte Grundlagenforschung zu teuer

(V) Kein internes Personal für eigene anwendungsorientierte Grundlagenforschung

(VI) Auslagerung der Forschungsaktivitäten

(VII) Aufbau einer strategischen Allianz mit der Universität

(VIII) Langfristige Sicherung des Zugangs zu wissenschaftlichem Know-how

a) sehr wichtig; b) wichtig; c) weniger wichtig; d) unwichtig 
6. Welcher Art sind/waren die Forschungsaktivitäten im CD-Labor (im Vergleich zu rein unternehmensintern durchgeführten F\&E Tätigkeiten)?

\begin{tabular}{|l|l|l|l|l|l|r|}
\hline $\begin{array}{l}\text { außerhalb unseres } \\
\text { technologischen Kernbereichs }\end{array}$ & & & & & & $\begin{array}{r}\text { innerhalb unseres } \\
\text { technologischen Kernbereichs }\end{array}$ \\
\hline geringes technologisches Risiko & & & & & & \\
technologisch einfach & & & & & & hohes technologisches Risiko \\
\hline kurzfristig anwendungsorientiert & & & & & & langfristig grundlagenorientiert \\
\hline wenig kooperationsintensiv & & & & & & \\
\hline wissenschaftlich einfach & & & & & & kooperationsintensiv \\
\hline
\end{tabular}

7. Hatte Ihr Unternehmen vor der CD-Laborgründung Kontakte zu Wissenschaftlern/Wissenschaftlerinnen?
a) Gemeinsame Forschungsprojekte
b) Lose Kontakte
c) Keine Kontakte

8. Wie hoch war der Einfluss seitens der Unternehmen auf

(I) die Forschungsprogrammformulierung und

(II) die Moduldefinition?

a) sehr hoher Einfluss; b) hoher Einfluss; c) geringer Einfluss; d) kein Einfluss

9. Arbeitet/Arbeitete das CD-Labor bei der Durchführung der Forschungskooperation mehrheitlich eher eigenständig oder kooperativ an den von den Unternehmen definierten Aufgaben?
a) Eigenständiges Arbeiten
b) Eher eigenständiges Arbeiten
c) Eher kooperatives Arbeiten
d) Kooperatives Arbeiten

10. Wie häufig sind/waren die Kontakte zu Wissenschaftlern/Wissenschaftlerinnen im CD-Labor

(I) mittels Email, Telefon etc. und

(II) bei gemeinsamen Arbeitssitzungen?

a) mehrmals pro Monat; b) mehrmals pro Quartal; c) seltenerer Kontakt; d) kein Kontakt; e) keine Angabe (Laufzeit noch zu kurz)

11. Wie bewerten Sie folgende Aspekte der Zusammenarbeit im CD-Labor?

(I) Termintreue

(II) Arbeitseffizienz 
(III) Absicherung des geistigen Eigentumsrechtes

(IV) Stabilität des Forschungsteams

(V) Verständnis des CD-Labors für Problemstellung

(VI) Wissenschaftliche Qualität der Arbeiten

a) sehr gut; b) gut; c) akzeptabel; d) schlecht; e) keine Angabe (Laufzeit noch zu kurz)

12. Sind abgesehen von Ihrem Unternehmen noch andere Unternehmen (Partnerunternehmen) am CD-Labor beteiligt (gewesen)? Wenn ja, stammen diese aus der gleichen Branche?
a) Ja, Partnerunternehmen aus der gleichen Branche
Anzahl:
b) Ja, Partnerunternehmen aus der Zulieferbranche
Anzahl:
c) Ja, Partnerunternehmen auf der Kundenseite
Anzahl:
d) Nein

13. Wenn es Partnerunternehmen aus der gleichen Branche gibt bzw. gab, mit wie vielen davon besteht bzw. bestand ein Konkurrenzverhältnis?
a) Keine unmittelbare Konkurrenz
b) Zum Teil in unmittelbarer Konkurrenz
c) In unmittelbarer Konkurrenz
Anzahl: $\frac{\text { Anzahl: }}{\text { Anzahl: }}$

14. Haben bzw. hatten die Forschungsergebnisse aus der Beteiligung am CD-Labor wirtschaftliche Relevanz für folgende Aspekte?
a) Hilfe bei Bewertung versch. Lösungsstrategien (Radarfunktion)
b) Für inkrementelle Verbesserung bestehender Produkte/Prozesse anwendbar
c) Für Entwicklung neuer Produktionsverfahren umsetzbar
d) Für Entwicklung radikal neuer Produkte umsetzbar
e) Keine unmittelbare Nutzung, aber vielleicht in Zukunft wichtig
f) Kein Nutzen/Keine wirtschaftliche Relevanz
g) Sonstige wirtschaftliche Relevanz: (eigene Nennung)

15. Wie schätzen Sie den Nutzen für Ihr Unternehmen durch die CD-Laborgründung bzw. beteiligung hinsichtlich folgender Punkte ein?

(I) Prestigegewinn

(II) Zugang zu qualifizierten MitarbeiterInnen

(III) Aufzeigen neuer technologischer Optionen

(IV) Kompetenzaufbau

(V) Abbau der Zugangsbarrieren zur wissenschaftlicher Forschung

(VI) Stärkung für österreichischen F\&E-Standort

(VII) Stärkung der technologischen Problemlösungskompetenz

(VIII) Verbesserung der Kooperationskultur

(IX) Aufbau von Technologieführerschaft

(X) Internationale Wettbewerbsfähigkeit

a) sehr hoher Nutzen; b) hoher Nutzen; c) geringer Nutzen; d) kein Nutzen

16. Welche der folgenden Auswirkungen der CD-Laborgründung auf Unternehmen sehen Sie? (Mehrfachantworten möglich)

a) Gewonnene Kontinuität der F\&E-Aktivitäten 
b) Bessere Umsetzung der F\&E-Aktivitäten

c) Nachhaltige Verbesserung des Kooperationsverhaltens

d) Gestiegene F\&E-Ausgaben

e) Eingehen von mit höherem Risiko behafteten F\&E-Aktivitäten

17. Wie beurteilen Sie die Kosten-Nutzen Relation des bisherigen Engagements im CD-Labor aus Sicht Ihres Unternehmens?

a) Kosten deutlich höher als Nutzen

b) Kosten etwas höher als Nutzen

c) Nutzen entspricht Kosten

d) Nutzen etwas höher als Kosten

e) Nutzen deutlich höher als Kosten

18. Plant Ihr Unternehmen auch in Zukunft Forschungsprojekte im Rahmen eines CD-Labors durchzuführen

a) ja, in gleichem Ausmaß b) ja, in höherem Ausmaß; c) Ja, aber in geringerem Ausmaß; d) nein____(Nennung von Grund/Gründen)

19. Hätten Sie die Forschungsprojekte, die Sie im Rahmen des CD-Labors durchführen bzw. durchgeführt haben, auch ohne CD-Labor durchgeführt?

a) ja, im selben Umfang; b) ja, aber in geringerem Umfang; c) ja, aber in deutlich geringerem Umfang; d) nein, gar nicht

20. Wenn ja, wie hätten Sie die Forschungsprojekte voraussichtlich finanziert?

(Mehrfachnennung möglich)

a) firmenintern

b) über andere Förderprogramme, z.B. (Nennung)

21. Wie wird sich die Nutzung direkter Forschungsförderprogramme (z.B. CDG, FFG, AWS etc.) in Zukunft in Ihrem Unternehmen generell entwickeln?

(I) Mittelfristig (in den kommenden 5 Jahren)

(II) Langfristig (in den kommenden 10 Jahren)

a) stark steigen; b) steigen; c) gleich bleiben; d) zurück gehen (Nennung von

Grund/Gründen); e) stark zurück gehen (Nennung von Grund/Gründen); f) noch nicht einschätzbar 


\section{Fragebogen JR-ZENTRUM: ZENTRUMSLEITERIN/-LEITER}

1. Wie zufrieden sind (bzw. waren) Sie mit den folgenden Aspekten des Programms bzw. der Programmabwicklung?

(I) Klarheit des Förderkonzepts und Förderziels

(II) Administrativer Aufwand der Antragsstellung

(III) Angemessenheit der Evaluierungskriterien

(IV) Bearbeitungsdauer der eingereichten Anträge

(V) Administrativer Aufwand während der Laufzeit des JR-Zentrums

a) sehr zufrieden; b) zufrieden; c) wenig zufrieden; d) unzufrieden

1b. Wenn bei 1 (II) und/oder $1(\mathrm{~V}) \mathrm{c}$ ) oder d) dann:

Wo sehen Sie Potential für administrative Vereinfachungen? Beispielen)

(Bitte Nennung von

2. Wie beurteilen Sie die Laufzeit des JR-Zentrums von $\mathbf{5}$ Jahren?

a) gerade richtig; b) zu kurz; c) zu lang

3. Wie schätzen Sie die begleitende wissenschaftliche Kontrolle ein (Empfehlungen und Auflagen bei der 2-Jahresevaluierung)?

a) sehr hilfreich; b) hilfreich; c) wenig hilfreich; d) nicht hilfreich; e) bisher noch nicht erfolgt

4. Wie bewerten Sie den Zeitpunkt der Zwischenevaluierung?

a) passend; b) unpassend:_____(Bitte Nennung von Gründen/Veränderungsvorschlägen)

5. Wie zufrieden sind Sie mit den folgenden Leistungen der CDG?

(I) Betreuung während der Antragstellung

(II) Betreuung des JR-Zentrums während der Laufzeit

(III) Öffentlichkeitsarbeit

a) sehr zufrieden; b) zufrieden; c) wenig zufrieden; d) unzufrieden

6. Von wem kam die Motivation zur Gründung des JR-Zentrums?
a) Vom Unternehmenspartner
b) Vom wissenschaftlichen Partner
c) Von beiden Partnern

7. Welcher Kontakt bestand zum Unternehmenspartner vor der Zentrumsgründung? (Mehrfachantworten möglich)
a) Gemeinsame Forschungsprojekte
b) Kontakte über AbsolventInnen
c) Lose Kontakte
d) Keine Kontakte

8. Welche Erwartungen und Ziele hatten Sie bei der Zentrumsgründung hinsichtlich des JRZentrums und wie bedeutend waren Ihnen diese?

(I) Prestigegewinn 
(II) Aufbau eines eigenen Forschungsteams

(III) Intensivierung der Unternehmenskontakte

(IV) Langfristig finanziell abgesichertes Arbeiten an einem Themenkreis

(V) Finanzielle Absicherung der Forschungsarbeit

(VI) Sonstige (eigene Nennungen)

a) sehr wichtig; b) wichtig; c) weniger wichtig; d) unwichtig

9. Wie viel Einfluss hatten Sie als Zentrumsleiter/in auf die

(I) Forschungsprogrammformulierung und

(II) auf die Moduldefinition?

a) sehr hohen Einfluss; b) hohen Einfluss; c) wenig Einfluss

10. In welchem Ausmaß lieferte der Unternehmenspartner Inputs hinsichtlich folgender Aspekte?

(I) Vermittlung von Kontakten

(II) Mitarbeit an Forschungsarbeiten

(III)Bereitstellung zusätzlicher Infrastruktur

(IV) Formulierung des Forschungsprogramms

(V) Feedback zu Forschungsaktivitäten

(VI)Moduldefinition

a) in sehr hohem Ausmaß; b) in hohem Ausmaß; c) in geringem Ausmaß; d) gar nicht

11. Wie viel Prozent Ihrer Gesamtarbeitszeit an der Fachhochschule widmen Sie durchschnittlich Ihrem JR-Zentrum?

$\%$ (Angaben in \% der Gesamtarbeitszeit an der Fachhochschule)

12. Ergab sich aus dem JR-Zentrum folgender Nutzen für das FH-Institut und wie hoch war dieser?

(I) Attraktivität als Arbeitgeber

(II) Prestigegewinn

(III) Verbesserte Stellung des Instituts

(IV) Attraktivität als Kooperationspartner

(V) Erhöhung des Praxisbezuges der Lehre

(VI) Akquisition zusätzlicher Forschungsprojekte

a) sehr hoher Nutzen; b) hoher Nutzen; c) geringer Nutzen; d) kein Nutzen; e) noch nicht einschätzbar

13. Hätten Sie die Forschungsprojekte, die Sie im Rahmen des JR-Zentrums durchführen, auch ohne JR-Zentrum durchgeführt?
a) Ja, im selben Umfang
b) Ja, aber in geringerem Umfang
c) Ja, aber in deutlich geringerem Umfang
d) Nein, gar nicht.

14. Hat Ihr JR-Zentrum einen internationalen Partner im Rahmen des JR-Zentrums?

a) Ja. Und zwar (Nennung)

Grund/Gründe: (Nennung) 
b) Nein

15. Führt Ihr JR-Zentrum ein Modul an einem ausländischen Standort durch?
a) Ja, und zwar (Nennung)
a2) Was waren die Gründe für das Modul am ausländischen Standort (z.B. bereits Kontakt zu ausländischem Partner vorhanden, Know-how in Ö nicht vorhanden)? (Nennung)
b) Nein

16. Wenn Frage 14 oder 15 mit ja beantwortet wurde: Wie haben Sie von der internationalen Kooperation profitiert?

(I) Forschungsleistung im Ausland ist kostengünstiger

(II) Neues Know-how durch den ausländischen Partner erworben

(III) Neue Unternehmenskontakte im Ausland erworben

(IV) Sonstiges: (Nennung)

a) trifft stark zu; b) trifft eher zu; c) trifft eher nicht zu; d) trifft nicht zu

17. Wenn Frage $\mathbf{1 4}$ oder $\mathbf{1 5}$ mit ja beantwortet wurde: Planen Sie weitere Industry-ScienceKooperationen im Ausland mit ausländischen Partnern, die sie im Rahmen des JR-Zentrums kennengelernt haben?
a) Ja
b) Nein

18. Sind Erfahrungen aus den industrierelevanten Projekten bereits in den akademischen Betrieb des Heimat gebenden Instituts eingeflossen?

(I) In die Forschung

(II) In die Lehre

a) ja, in großem Ausmaß; b) ja, in mittlerem Ausmaß; c) ja, in geringem Ausmaß d) nein; e) noch nicht einschätzbar

19. Beschreiben Sie Ihren bisherigen Gesamteindruck von der Qualität der Zusammenarbeit mit der Industrie.

(I) Relevanz der industriellen Fragestellung für die Forschungsarbeit des JR-Zentrums

(II) Intellektueller Input seitens der Industrie

(III) Materieller Input seitens der Industrie (nicht finanzielle Eigenleistungen)

(IV) Zusammenarbeit mit den Kontaktpersonen der Industriepartner

(V) Ausmaß an wissenschaftlichem Freiraum

a) sehr gut; b) gut; c) befriedigend; d) genügend; e) nicht genügend; f) noch nicht einschätzbar

20. Wie hat sich das JR-Zentrum bisher auf das Drittmittelvolumen des Heimat gebenden Instituts/Departments ausgewirkt, auch außerhalb des Themenbereichs des JR-Zentrums?

a) stark zunehmend; b) zunehmend; c) gleich bleibend; d) rückläufig; e) noch nicht einschätzbar

21. Wie hat sich das JR-Zentrum generell auf die Unternehmenskooperationen des Heimat gebenden Instituts/Departments ausgewirkt? 
a) stark zunehmend; b) zunehmend; c) gleich bleibend; d) rückläufig; e) noch nicht einschätzbar

22. Gibt es Initiativen das JR-Zentrum nach dem Förderzeitraum als eigenständiges Unternehmen/als eigene Einrichtung weiter zu führen?
a) Ja
b) Nein
c) Noch nicht einschätzbar

23. Wie beurteilen Sie den administrativen Aufwand eines JR-Zentrums im Vergleich zu anderen Förderprogrammen? Der administrative Aufwand eines JR-Zentrums ist im Vergleich zu einem...

(I) ... FFG-Projekt in Kooperation mit Unternehmen

(II) ... EU-Projekt in Kooperation mit Unternehmen

(III) ... FWF-Projekt

a) höher b) gleich hoch; c) niedriger; d) kein Vergleich möglich

24. Wie hoch ist in Summe der administrative Aufwand des JR-Zentrums? Bitte geben Sie eine Schätzung der Wochenstunden im Jahresdurchschnitt an.

(Stunden pro Woche im Jahresdurchschnitt) 


\section{Fragebogen JR-ZENTRUM: UNTERNEHMENSPARTNER}

1. Wie beurteilen Sie die Laufzeit des JR-Zentrums von 5 Jahren?

a) gerade richtig; b) zu kurz; c) zu lang

2. Wie zufrieden sind (bzw. waren) Sie mit den folgenden Aspekten des Programms bzw. der Programmabwicklung?

(I) Klarheit des Förderkonzepts und Förderziels

(II) Administrativer Aufwand der Antragsstellung

(III) Angemessenheit der Evaluierungskriterien

(IV) Bearbeitungsdauer der eingereichten Anträge

(V) Administrativer Aufwand während der Laufzeit des JR-Zentrums

a) sehr zufrieden; b) zufrieden; c) wenig zufrieden; d) unzufrieden

2b. Wenn bei 2(II) und/oder 2(V) c) oder d) dann:

Wo sehen Sie Potential für administrative Vereinfachungen? Beispielen)

(Bitte Nennung von

3. Wie schätzen Sie die begleitende wissenschaftliche Kontrolle ein (Empfehlungen und Auflagen bei der 2-Jahresevaluierung)?

a) sehr hilfreich; b) hilfreich; c) wenig hilfreich; d) nicht hilfreich; e) bisher noch nicht erfolgt

4. Wie zufrieden sind Sie mit den folgenden Leistungen der CDG?

(I) Betreuung während der Antragstellung

(II) Betreuung des JR-Zentrums während der Laufzeit

(III) Öffentlichkeitsarbeit

a) sehr zufrieden; b) zufrieden; c) wenig zufrieden; d) unzufrieden

5. Was war Ihre Motivation als Unternehmenspartner zur Gründung eines JR-Zentrums bzw. zur Beteiligung am JR-Zentrum und wie wichtig waren Ihnen die einzelnen Punkte dabei?

(I) Aufbau einer Kooperationsplattform mit anderen Unternehmen

(II) Recruiting: Zugang zu jungen Talenten

(III) Einstieg in neues Forschungsthema

(IV) Eigene anwendungsorientierte Forschung zu teuer

(V) Kein internes Personal für eigene anwendungsorientierte Forschung

(VI) Auslagerung der Forschungsaktivitäten

(VII) Aufbau einer strategischen Allianz mit der Fachhochschule

(VIII) Langfristige Sicherung des Zugangs zu wissenschaftlichem Know-how

a) sehr wichtig; b) wichtig; c) weniger wichtig; d) unwichtig 
6. Welcher Art waren die Forschungsaktivitäten im JR-Zentrum (im Vergleich zu rein unternehmensintern durchgeführten F\&E Tätigkeiten)?

\begin{tabular}{|l|l|l|l|l|l|r|}
\hline $\begin{array}{l}\text { außerhalb unseres } \\
\text { technologischen Kernbereichs }\end{array}$ & & & & & & $\begin{array}{r}\text { innerhalb unseres } \\
\text { technologischen Kernbereichs }\end{array}$ \\
\hline geringes technologisches Risiko & & & & & & \\
technologisch einfach & & & & & & hohes technologisches Risiko \\
\hline kurzfristig anwendungsorientiert & & & & & & langfristig grundlagenorientiert \\
\hline wenig kooperationsintensiv & & & & & & \\
\hline wissenschaftlich einfach & & & & & & kooperationsintensiv \\
\hline
\end{tabular}

7. Hatte Ihr Unternehmen vor der JR-Zentrumsgründung Kontakte zu Wissenschaftlern/Wissenschaftlerinnen?
a) gemeinsame Forschungsprojekte
b) lose Kontakte
c) keine Kontakte

8. Wie hoch war der Einfluss seitens der Unternehmen auf (I) die Forschungsprogrammformulierung und

(II) die Moduldefinition?

a) sehr hoher Einfluss; b) hoher Einfluss; c) geringer Einfluss; d) kein Einfluss

9. Arbeiten die JR-Zentren bei der Durchführung der Forschungskooperationen mehrheitlich eher eigenständig oder kooperativ an den von den Unternehmen definierten Aufgaben?
a) Eigenständiges Arbeiten
b) Eher eigenständiges Arbeiten
c) Eher kooperatives Arbeiten
d) Kooperatives Arbeiten

10. Wie häufig waren die Kontakte zu Wissenschaftlern/Wissenschaftlerinnen im JR-Zentrum

(I) mittels Email, Telefon etc. und

(II) bei gemeinsamen Arbeitssitzungen?

a) mehrmals pro Monat; b) mehrmals pro Quartal; c) seltenerer Kontakt; d) kein Kontakt; e) keine Angabe (Laufzeit noch zu kurz)

11. Wie bewerten Sie folgende Aspekte der Zusammenarbeit im JR-Zentrum?
(I) Termintreue
(II) Arbeitseffizienz
(III) Absicherung des geistigen Eigentumsrechtes
(IV) Stabilität des Forschungsteams
(V) Verständnis des JR-Zentrums für Problemstellung 
(VI) Wissenschaftliche Qualität der Arbeiten

a) sehr gut; b) gut; c) akzeptabel; d) schlecht; e) keine Angabe (Laufzeit noch zu kurz)

12. Sind abgesehen von Ihrem Unternehmen noch andere Unternehmen (Partnerunternehmen) am JR-Zentrum beteiligt (gewesen)? Wenn ja, stammen diese aus der gleichen Branche?

a) Ja, Partnerunternehmen aus der gleichen Branche Anzahl:

b) Ja, Partnerunternehmen aus der Zulieferbranche Anzahl:

c) Ja, Partnerunternehmen auf der Kundenseite Anzahl:

d) Nein

13. Wenn es Partnerunternehmen aus der gleichen Branche gibt bzw. gab, mit wie vielen davon besteht bzw. bestand ein Konkurrenzverhältnis?
a) keine unmittelbare Konkurrenz
b) zum Teil in unmittelbarer Konkurrenz
c) in unmittelbarer Konkurrenz
Anzahl:
Anzahl:
Anzahl:

14. Haben bzw. hatten die Forschungsergebnisse aus der Beteiligung an den JR-Zentren wirtschaftliche Relevanz für folgende Aspekte?
a) Hilfe bei Bewertung versch. Lösungsstrategien (Radarfunktion)
b) Für inkrementelle Verbesserung bestehender Produkte/Prozesse anwendbar
c) Für Entwicklung neuer Produktionsverfahren umsetzbar
d) Für Entwicklung radikal neuer Produkte umsetzbar
e) Keine unmittelbare Nutzung, aber vielleicht in Zukunft wichtig
f) Kein Nutzen/Keine wirtschaftliche Relevanz
g) Sonstige wirtschaftliche Relevanz: (eigene Nennung)

15. Wie schätzen Sie den Nutzen für Ihr Unternehmen durch die JR-Zentrumsgründung bzw. beteiligung hinsichtlich folgender Punkte ein?

(I) Prestigegewinn

(II) Zugang zu qualifizierten MitarbeiterInnen

(III) Aufzeigen neuer technologischer Optionen

(IV) Kompetenzaufbau

(V) Abbau der Zugangsbarrieren zur wissenschaftlicher Forschung

(VI) Stärkung für österreichischen F\&E-Standort

(VII) Stärkung der technologischen Problemlösungskompetenz

(VIII) Verbesserung der Kooperationskultur

(IX) Aufbau von Technologieführerschaft

(X) Internationale Wettbewerbsfähigkeit

a) sehr hoch; b) hoch; c) gering; d) keiner

16. Welche der folgenden Auswirkungen der JR-Zentrumsgründung auf Unternehmen sehen Sie? (Mehrfachantworten möglich)
a) gewonnene Kontinuität der F\&E-Aktivitäten
b) bessere Umsetzung der F\&E-Aktivitäten
c) nachhaltige Verbesserung des Kooperationsverhaltens
d) gestiegene F\&E-Ausgaben 
e) Eingehen von mit höherem Risiko behafteten F\&E-Aktivitäten

17. Wie beurteilen Sie die Kosten-Nutzen Relation des bisherigen Engagements im JR-Zentrum aus Sicht Ihres Unternehmens?
a) Kosten deutlich höher als Nutzen
b) Kosten etwas höher als Nutzen
c) Nutzen entspricht Kosten
d) Nutzen etwas höher als Kosten
e) Nutzen deutlich höher als Kosten

18. Plant Ihr Unternehmen auch in Zukunft Forschungsprojekte im Rahmen eines JR-Zentrums durchzuführen?

a) ja, in gleichem Ausmaß b) ja, in höherem Ausmaß; c) Ja, aber in geringerem Ausmaß; d) nein____(Nennung von Grund/Gründen)

19. Hätten Sie die Forschungsprojekte, die Sie im Rahmen des JR-Zentrums durchführen bzw. durchgeführt haben, auch ohne CD-Labor durchgeführt?

a) Ja, im selben Umfang; b) Ja, aber in geringerem Umfang; c) Ja, aber in deutlich geringerem Umfang; d) Nein, gar nicht.

20. Wenn ja, wie hätten Sie die Forschungsprojekte voraussichtlich finanziert? (Mehrfachnennung möglich)

a) firmenintern

b) über andere Förderprogramme, z.B. (Nennung)

21. Wie wird sich die Nutzung direkter Forschungsförderprogramme (z.B. CDG, FFG, AWS etc.) in Zukunft in Ihrem Unternehmen generell entwickeln?

(I) Mittelfristig (in den kommenden 5 Jahren)

(II) Langfristig (in den kommenden 10 Jahren)

a) stark steigen; b) steigen; c) gleich bleiben; d) zurück gehen___ (Nennung von Grund/Gründen); e) stark zurück gehen (Nennung von Grund/Gründen); f) noch nicht einschätzbar 


\section{Patentanalyse: Beschreibung der Technologiestärkefelder Österreich}

Es werden die 10 Themengruppen der 15 österreichischen Technologiestärkefelder jeweils im Detail beginnend mit der Dynamik in dem Patentbereich beschrieben. Anschließend werden die Länder mit den meisten Patenten und deren internationales Kooperationsnetzwerk dargestellt. Es folgt eine Auflistung von Unternehmen, bei denen zumindest ein österreichischer Erfinder an der Erstellung eines Patentes beteiligt war. Abgeschlossen wird die Detailbetrachtung der Technologiestärkefelder mit einer Analyse zu den Crossing-Technologies.

\section{Technologiestärkefeld „Aufzüge“}

Die Technologiestärkefelder im Bereich „Aufzüge“ weisen weltweit einen jährlichen Zuwachs von 44\% seit 1997 auf, wobei der Trend auch in den letzten Jahren anhielt.

\section{Abbildung 161: Weltweite Dynamik im Bereich Aufzüge}

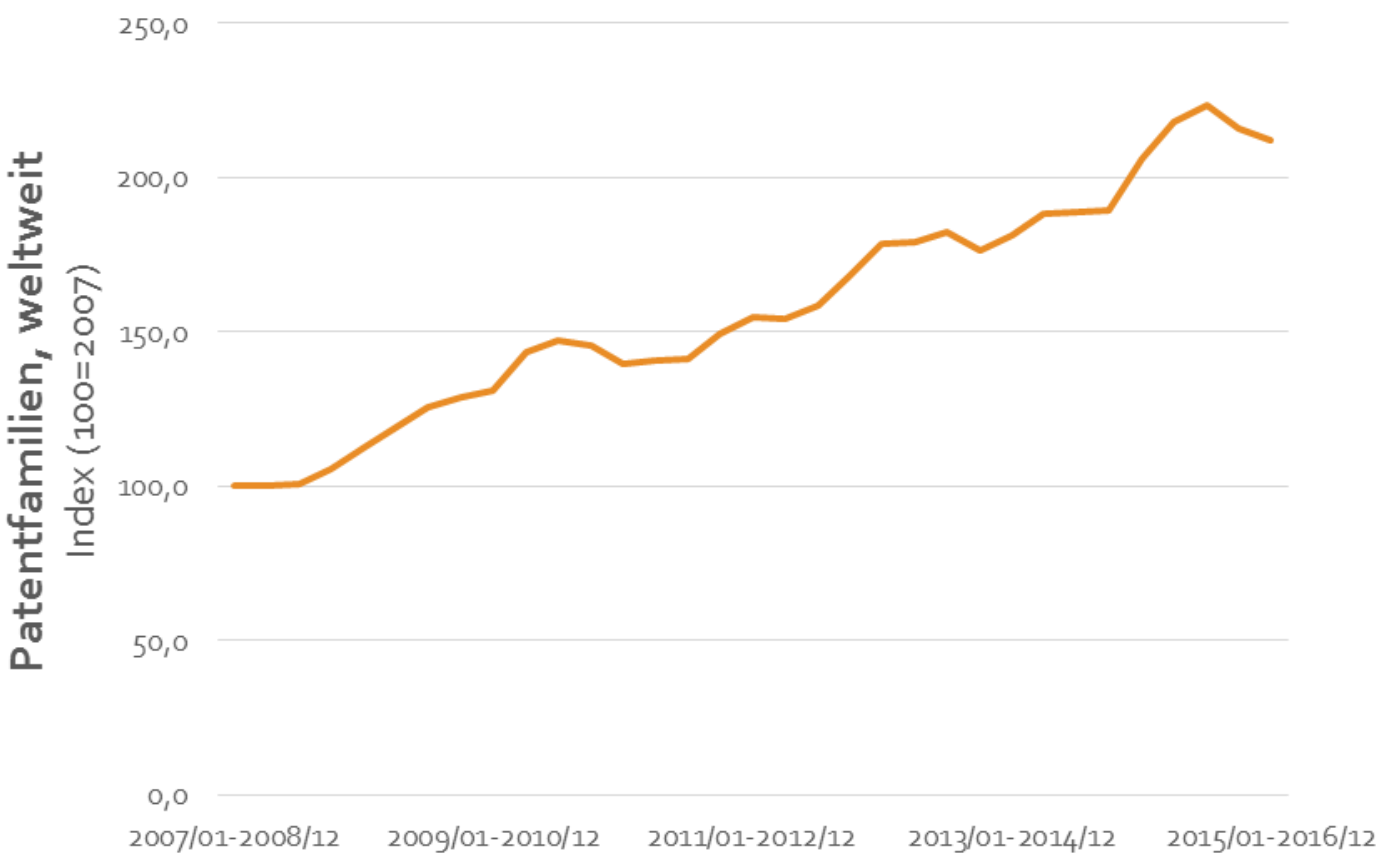

Quelle: Economica (2017).

Im Länder-Ranking nach Patentfamilien liegt Österreich hinter China und Japan auf Rang 3, vor Korea und Deutschland (in absoluten Zahlen). Dabei werden die Patentfamilien durch den Erfindersitz regional zugeordnet und ausgewertet. 
Tabelle 38: Top Patentländer im Bereich Aufzüge

\begin{tabular}{|l|l|l|}
\hline \begin{tabular}{l|l|} 
Länder- \\
kürzel
\end{tabular} & $\begin{array}{l}\text { Patent- } \\
\text { familien }\end{array}$ & Erfinder \\
\hline CN & 284 & 285 \\
\hline JP & 139 & 230 \\
\hline AT & 98 & 63 \\
\hline KR & 96 & 97 \\
\hline DE & 64 & 90 \\
\hline ES & 58 & 64 \\
\hline US & 49 & 73 \\
\hline TW & 24 & 37 \\
\hline FI & 20 & 18 \\
\hline CA & 13 & 15 \\
\hline
\end{tabular}

Quelle: Economica (2017).

Österreich hat auch einen vergleichsweise hohen Grad internationaler Vernetzung in diesem Thema und innoviert mit 6 anderen Ländern (bestimmt durch Ko-Erfinderanalyse der Patentfamilien), womit es hinter den USA (9 Ko-Erfinderländer) den zweiten Rang belegt, gefolgt von Deutschland und China mit jeweils 5 Ko-Erfinderländern. Dies zeigt die gute Einbindung an die internationalen Wissensnetzwerke auf dem Gebiet der Aufzüge (betrachtet wurden die Stärkefelder Einzelteile von Rolltreppen/Fahrsteigen und Sicherheitseinrichtungen). 
Die Unternehmen Inventio und Otis Elevator sind die Patentanmelder mit dem höchsten Patentoutput von Erfindern in Österreich in den letzten 10 Jahren, mit 52 bzw. 35 veröffentlichten Patentfamilien bei Einzelteilen von Rolltreppen/Fahrsteigen und Sicherheitseinrichtungen.

Tabelle 39: Industriestruktur in Österreich im Bereich Aufzüge

\begin{tabular}{lcc|}
\hline Anmelder & Pat.Fam. & Erfinder \\
\hline Gesamt* & $\mathbf{9 8}$ & $\mathbf{6 3}$ \\
\hline INVENTIO AG & 52 & 34 \\
\hline OTIS ELEVATOR & 35 & 18 \\
\hline INNOVA PATENT & 1 & 1 \\
\hline SEMPERIT AG & 3 & 6 \\
\hline MERTL DANIEL & 3 & 2 \\
\hline SCHINDLER ELEVATOR & 1 & 2 \\
\hline MATHEISL MICHAEL & 1 & 3 \\
\hline TUEV OESTERREICH & 1 & 1 \\
\hline SCHUNK WIEN & 1 & 2
\end{tabular}

Quelle: Economica (2017).

*Anmerkung: Manche Erfinder waren für mehrere Unternehmen tätig, daher ist die Erfinderanzahl bei Gesamt kleiner als die Summe der Erfinder in den Unternehmen.

Abbildung 163: Crossing-Technologies im Bereich Aufzüge

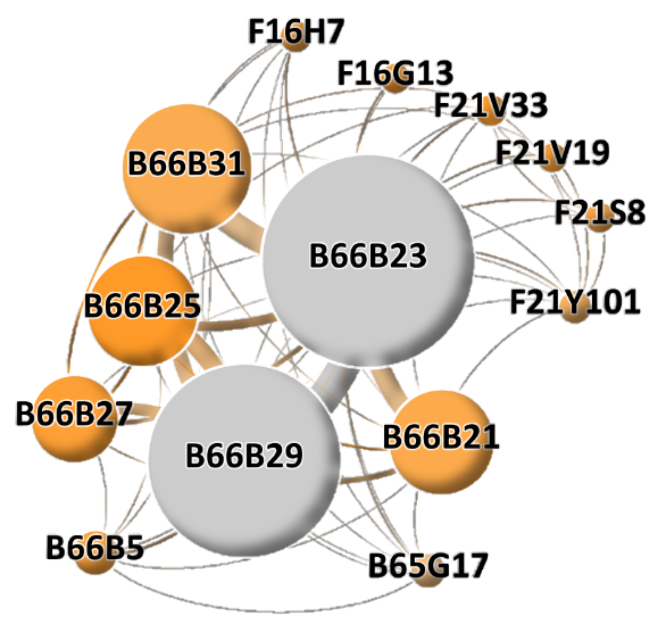

Quelle: Economica (2017). 
Eine Bestimmung der konvergierenden Technologien zeigt Themen von Beleuchtung (punktförmige Lichtquellen, Anordnung bzw. Befestigung von Lichtquellen), Getriebe und Antriebselemente (Riemen/Ketten) als häufige Crossing-Technologies.

Besonders bei Beleuchtung liegt der Patentoutput aus Österreich zwischen 2 und 3\% bezogen auf den weltweiten Patentoutput, sodass sich daraus relevante Doppelstärkefelder ergeben.

Tabelle 40: Crossing-Technologies im Bereich Aufzüge und deren relative Stärke

\begin{tabular}{|l|l|l}
\hline IPC & Beschreibung & $F_{\text {AT }} / F_{\text {wo }}$ \\
\hline B66B31 & $\begin{array}{l}\text { Heben .. Aufzuege .. Zubehoer fuer Rolltreppen oder Fahrsteige, z.B. zum } \\
\text { Sterilisieren oder Reinigen }\end{array}$ & $11,0 \%$ \\
\hline B66B25 & Heben .. Aufzuege .. Steuern und Regeln von Rolltreppen oder Fahrsteigen & $14,3 \%$ \\
\hline B66B21 & Heben .. Aufzuege .. Rolltreppen oder Fahrsteige & $23,0 \%$ \\
\hline B66B27 & Heben .. Aufzuege .. Funktionsanzeige fuer Rolltreppen oder Fahrsteige & $16,7 \%$ \\
\hline B66B5 & $\begin{array}{l}\text { Heben .. Aufzuege .. Anwendung von Ueberwachungs-, Fehlerkorrektur- oder } \\
\text { Sicherheitseinrichtungen fuer Aufzuege }\end{array}$ & $0,8 \%$ \\
\hline B65G17 & $\begin{array}{l}\text { Foerdern .. Transport- oder Lagervorrichtungen, z.B. Foerderer zum Laden oder } \\
\text { Abladen .. Foerderer mit einem endlosen Zugmittel, z.B. einer Kette, das seine }\end{array}$ & $1,8 \%$ \\
\hline F21Y101 & $\begin{array}{l}\text { Bewegung auf eine durchgehende oder im wesentlichen durchgehende } \\
\text { Basttragflaeche ... }\end{array}$ & $1,8 \%$ \\
\hline F21V33 & $\begin{array}{l}\text { Beleuchtung .. Funktionsmerkmale oder Einzelheiten von Leuchten oder } \\
\text { Beleuchtungssystemen .. Bauliche Kombinationen von Leuchten mit anderen } \\
\text { Gegenstaenden, soweit nicht anderweitig vorgesehen }\end{array}$ & $1,3 \%$ \\
\hline F16H7 & $\begin{array}{l}\text { Maschinenelemente oder -einheiten .. Getriebe .. Getriebe zum Uebertragen } \\
\text { einer Drehbewegung durch endlose, biegsame Uebertragungsglieder }\end{array}$ & $1,4 \%$ \\
\hline F16G13 & $\begin{array}{l}\text { Maschinenelemente oder -einheiten .. Riemen, Kabel oder Seile, vorwiegend fuer } \\
\text { Antriebszwecke .. Ketten } \\
\text { Beleuchtung .. Ortsfeste Leuchten oder Beleuchtungssysteme .. Leuchten, die zum } \\
\text { Befestigen bestimmt sind } \\
\text { Beleuchtung .. Funktionsmerkmale oder Einzelheiten von Leuchten oder } \\
\text { Beleuchtungssystemen .. Befestigen von Lichtquellen oder Lampenfassungen }\end{array}$ & $1,3 \%$ \\
\hline
\end{tabular}

Quelle: Economica (2017).

\section{Technologiestärkefeld „Cellulose“}

Der Bereich Cellulose wächst mit 44\% jährlich (20-Jahreszeitraum), wobei die Betrachtung der letzten 10 Jahre ein zyklisches Patentanmeldemuster zeigt. 
Abbildung 164: Weltweite Dynamik im Bereich Cellulose

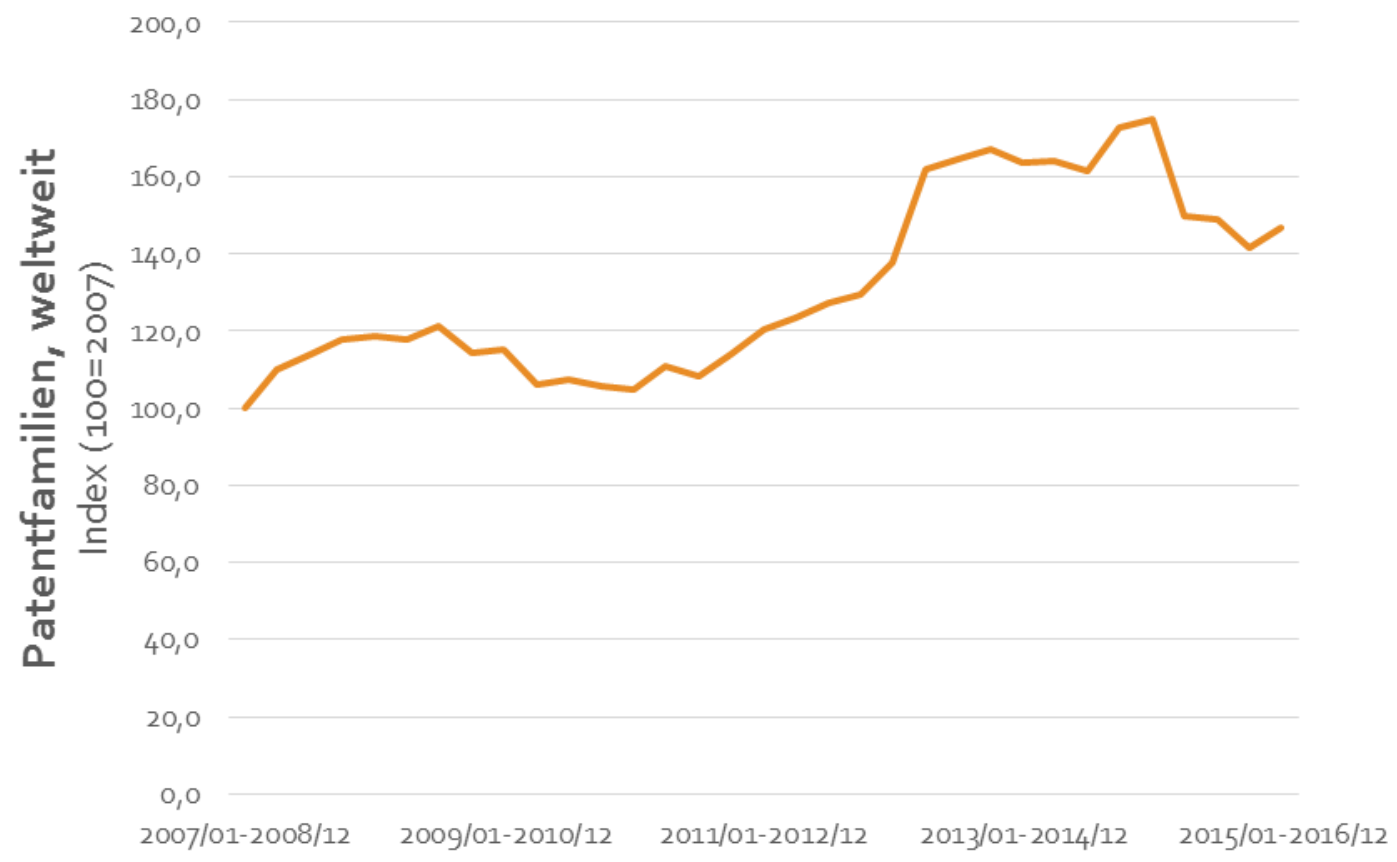

Quelle: Economica (2017).

Österreich liegt hinsichtlich Patent-Output (Patentfamilien von Erfindern in Österreich) weltweit auf Rang 6, hinter China, Korea, USA, Deutschland und Japan, und deutlich vor Taiwan, Großbritannien, Finnland und Frankreich.

Tabelle 41: Top Patentländer im Bereich Cellulose

\begin{tabular}{|l|l|l|}
\hline $\begin{array}{l}\text { Länder- } \\
\text { kürzel }\end{array}$ & $\begin{array}{l}\text { Patent- } \\
\text { familien }\end{array}$ & Erfinder \\
\hline CN & 181 & 349 \\
\hline KR & 137 & 100 \\
\hline US & 91 & 174 \\
\hline DE & 90 & 126 \\
\hline JP & 73 & 158 \\
\hline AT & 63 & 82 \\
\hline TW & 33 & 34 \\
\hline GB & 19 & 23 \\
\hline FI & 17 & 40 \\
\hline FR & 10 & 24 \\
\hline
\end{tabular}

Quelle: Economica (2017). 
Die internationale Vernetzung ist mit 2 Ko-Erfindernationen relativ schwach ausgeprägt. Die USA weisen 13 Ko-Erfinderländer, Deutschland 8, Großbritannien 5 und China 3 auf.

Quelle: Economica (2017).

Die Industriestruktur ist durch die Lenzing AG bestimmt (45 Patentfamilien in der betrachteten Patentklasse). Weiters veröffentlichten Kelheim Fibres, Glanzstoff Bohemia und Helfenberger Immobilien 3 oder mehr Patentfamilien in dem Thema.

Tabelle 42: Industriestruktur in Österreich im Bereich Cellulose

\begin{tabular}{lcc|}
\hline Anmelder & Pat.Fam. & Erfinder \\
\hline Gesamt & $\mathbf{6 3}$ & $\mathbf{8 2}$ \\
\hline CHEMIEFASER LENZING & 45 & 70 \\
\hline KELHEIM FIBRES & 5 & 1 \\
\hline GLANZSTOFF BOHEMIA & 5 & 8 \\
\hline HELFENBERGER IMMOBILIEN & 3 & 1 \\
\hline AUROTEC GMBH & 2 & 2 \\
\hline SCHAEFFLER TECHNOLOGIES & 1 & 1 \\
\hline UNIV INNSBRUCK & 1 & 4 \\
\hline ZIMMER AG & 1 & 3 \\
\hline
\end{tabular}

Quelle: Economica (2017).

Aus den Crossing-Technologies gehen die verknüpften Themen der Weberei und FaserNachbehandlung hervor. In diesen Themen bestehen keine besonderen Technologie-Stärkefelder, 
sodass im Bereich Cellulose zwar eine hohe Technologiekompetenz ersichtlich wird, jedoch keine unmittelbaren Chancen durch weitere Themenvernetzung offensichtlich werden.

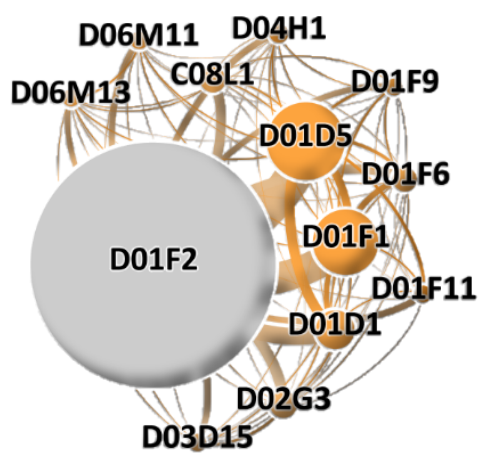

Quelle: Economica (2017).

Tabelle 43: Crossing-Technologies im Bereich Cellulose und deren relative Stärke

\begin{tabular}{|c|c|c|}
\hline ipc & descr & $\begin{array}{r}\mathrm{F}_{\mathrm{AT}} / \mathrm{F}_{\mathrm{WO}}, \\
2012-2016\end{array}$ \\
\hline D01F2 & $\begin{array}{l}\text { Textilien oder flexible Materialien, soweit nicht anderweitig vorgesehen .. } \\
\text { Chemische Gesichtspunkte bei der Herstellung von kuenstlichen Faeden, } \\
\text { Filamenten, Fasern, Borsten oder Baendern .. Kuenstliche } \\
\text { Monokomponentenfaeden oder dgl. aus Cellulose oder Cellulosederivaten }\end{array}$ & $14,20 \%$ \\
\hline D01D5 & $\begin{array}{l}\text { Textilien oder flexible Materialien, soweit nicht anderweitig vorgesehen .. } \\
\text { Mechanische Verfahren oder Vorrichtungen fuer die Herstellung von kuenstlichen } \\
\text { Faeden, Filamenten, Fasern, Borsten oder Baendern .. Bildung von Filamenten, } \\
\text { Spinnfasern oder dgl. }\end{array}$ & $1,20 \%$ \\
\hline D01F1 & $\begin{array}{l}\text { Textilien oder flexible Materialien, soweit nicht anderweitig vorgesehen .. } \\
\text { Chemische Gesichtspunkte bei der Herstellung von kuenstlichen Faeden, } \\
\text { Filamenten, Fasern, Borsten oder Baendern .. Allgemeine Verfahren zur } \\
\text { Herstellung von Chemiefasern oder dgl. }\end{array}$ & $2,20 \%$ \\
\hline D01D1 & $\begin{array}{l}\text { Textilien oder flexible Materialien, soweit nicht anderweitig vorgesehen .. } \\
\text { Mechanische Verfahren oder Vorrichtungen fuer die Herstellung von kuenstlichen } \\
\text { Faeden, Filamenten, Fasern, Borsten oder Baendern .. Behandlung von } \\
\text { fadenbildendem oder aehnlichem Material }\end{array}$ & $1,30 \%$ \\
\hline D02G3 & $\begin{array}{l}\text { Garne .. Kraeuseln oder Texturieren von Fasern, Faeden, Garnen oder Zwirnen .. } \\
\text { Garne oder Zwirne, z.B. Effektgarne }\end{array}$ & $0,80 \%$ \\
\hline C08L1 & $\begin{array}{l}\text { Organische makromolekulare Verbindungen .. Massen auf Basis } \\
\text { makromolekularer Verbindungen .. Massen auf Basis von Polysacchariden oder } \\
\text { ihrer Derivate }\end{array}$ & $1,20 \%$ \\
\hline
\end{tabular}




\begin{tabular}{|c|c|c|}
\hline D01F6 & $\begin{array}{l}\text { Textilien oder flexible Materialien, soweit nicht anderweitig vorgesehen .. } \\
\text { Chemische Gesichtspunkte bei der Herstellung von kuenstlichen Faeden, } \\
\text { Filamenten, Fasern, Borsten oder Baendern .. Kuenstliche } \\
\text { Monokomponentenfaeden oder dgl. aus synthetischen Polymeren }\end{array}$ & $0,70 \%$ \\
\hline $\mathrm{D} 04 \mathrm{H} 1$ & $\begin{array}{l}\text { Flechten .. Herstellen von flaechigem Textilgut, z.B. aus Fasern oder } \\
\text { fadenfoermigem Material .. Formen oder Arten nicht gewebter Stoffe }\end{array}$ & $1,30 \%$ \\
\hline D06M11 & $\begin{array}{l}\text { Behandlung von Textilien oder dgl. .. Behandeln von Fasern, Faeden, Garnen, } \\
\text { Textilgut, Federn oder aus solchen Materialien hergestelltem Fasergut, soweit } \\
\text { nicht anderweitig in Klasse sref ref=D06>sref }>\text { vorgesehen .. Behandeln von } \\
\text { Fasern, Faeden, Garnen, Textilgut oder von aus solchen Materialien hergestelltem } \\
\text { Fasermaterial mit anorganischen Stoffen oder deren Komplexverbindungen }\end{array}$ & $0,40 \%$ \\
\hline D03D15 & $\begin{array}{l}\text { Weberei .. Gewebe .. Gewebe, gekennzeichnet durch das Material oder die } \\
\text { Zusammensetzung des Garnes oder anderer verwendeter Kett- oder } \\
\text { Schusselemente }\end{array}$ & $1,10 \%$ \\
\hline D06M13 & $\begin{array}{l}\text { Behandlung von Textilien oder dgl. .. Behandeln von Fasern, Faeden, Garnen, } \\
\text { Textilgut, Federn oder aus solchen Materialien hergestelltem Fasergut, soweit } \\
\text { nicht anderweitig in Klasse sref ref=D06>sref }>\text { vorgesehen .. Behandeln von } \\
\text { Fasern, Faeden, Garnen, Textilgut oder von aus solchen Materialien hergestelltem } \\
\text { Fasermaterial mit nicht makromolekularen organischen Verbindungen }\end{array}$ & $1,30 \%$ \\
\hline D01F11 & $\begin{array}{l}\text { Textilien oder flexible Materialien, soweit nicht anderweitig vorgesehen .. } \\
\text { Chemische Gesichtspunkte bei der Herstellung von kuenstlichen Faeden, } \\
\text { Filamenten, Fasern, Borsten oder Baendern .. Chemische Nachbehandlung von } \\
\text { Chemiefasern oder dgl. im Zuge der Herstellung }\end{array}$ & $1,80 \%$ \\
\hline
\end{tabular}

Quelle: Economica (2017).

\section{Technologiestärkefeld „Eisenbahn“}

Die unter der Eisenbahn subsummierten Technologien (Seilbahnen, Gerüstwagen, Verlegen von Gleisen) wuchsen in den letzten 20 Jahren mit rund 60\% pro Jahr, wobei auch in den letzten 10 Jahren - jedenfalls bis 2015 - ein kontinuierlicher Zuwachs der veröffentlichten Patentfamilien beobachtet wurde. 
Abbildung 167: Weltweite Dynamik im Bereich Eisenbahn

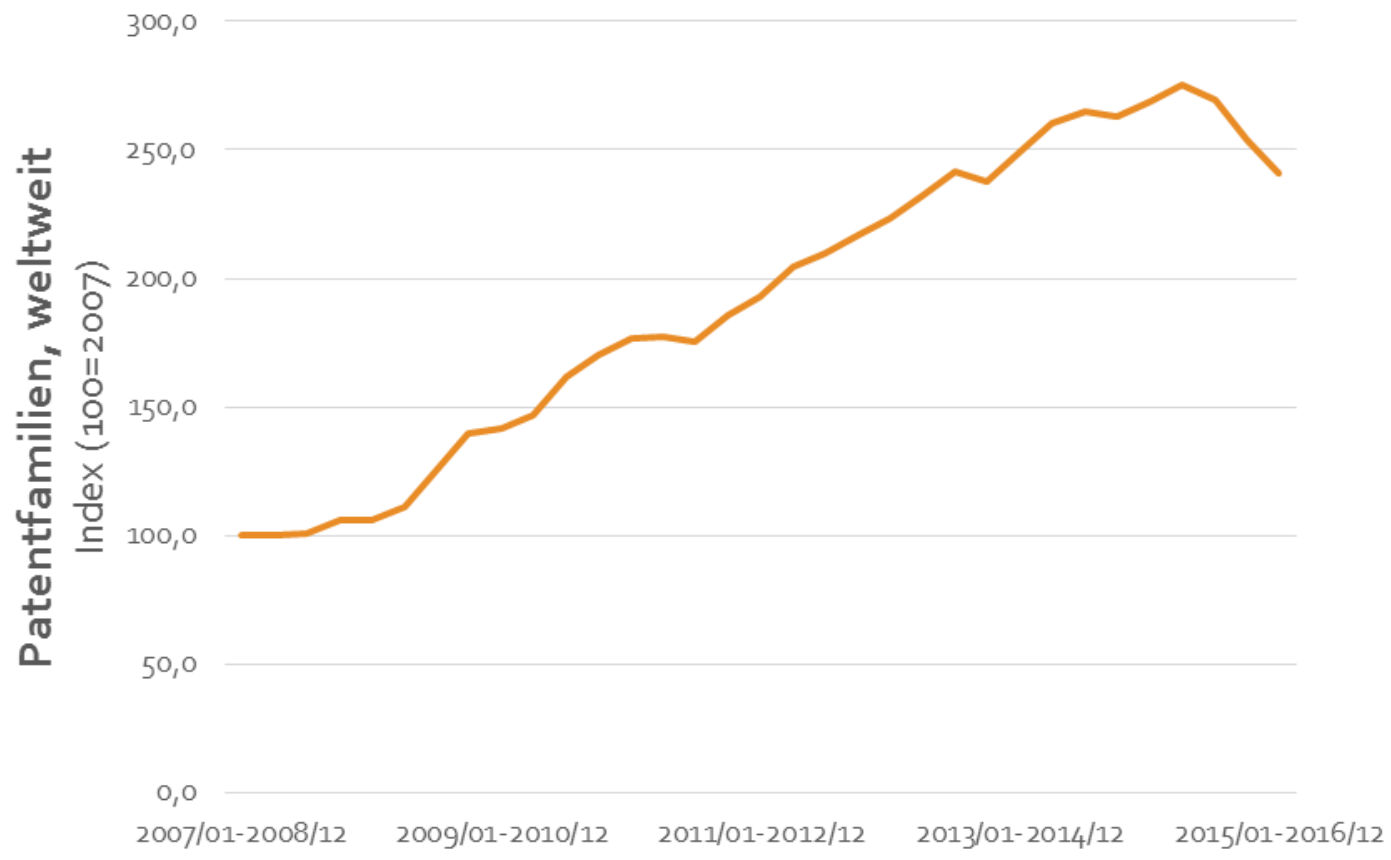

Quelle: Economica (2017).

Österreichs Patentoutput liegt weltweit auf Rang 6 (10-Jahreszeitraum, alle Patentfamilien weltweit) hinter China, USA, Korea, Frankreich und Deutschland.

Tabelle 44: Top Patentländer im Bereich Eisenbahn

\begin{tabular}{|l|l|l|}
\hline $\begin{array}{l}\text { Länder- } \\
\text { kürzel }\end{array}$ & $\begin{array}{l}\text { Patent- } \\
\text { familien }\end{array}$ & Erfinder \\
\hline CN & 302 & 699 \\
\hline US & 215 & 271 \\
\hline KR & 184 & 176 \\
\hline FR & 170 & 181 \\
\hline DE & 161 & 189 \\
\hline AT & 122 & 98 \\
\hline RU & 82 & 144 \\
\hline CH & 63 & 46 \\
\hline ES & 44 & 67 \\
\hline JP & 34 & 52 \\
\hline
\end{tabular}

Quelle: Economica (2017). 
Aus Sicht der Patentdichte (Patente pro Einwohner) wie auch aus Sicht der Erfinderdichte (Erfinder pro Einwohner) liegt Österreich mit deutlichem Abstand auf Rang 1. Dieses Bild ist konsistent mit den Ergebnissen unserer jüngsten Studien, die eine breitere Definition des Themas (alle Bereiche von Eisenbahn umfassend) zu Grunde legten.

Die Struktur der internationalen Wissensnetzwerke ist bietet ein untypisches Bild, da die USA üblicherweise am stärksten international vernetzt - lediglich 2 Ko-Erfindernationen aufweist. Frankreich und Deutschland liegen mit 5 bzw. 6 Ko-Erfindernationen vor Österreich mit 3.

Quelle: Economica (2017).

Die Industriestruktur in den ausgewählten Themen von Eisenbahn umfasst zahlreiche Unternehmen mit zumindest drei Patentfamilien. Innova Patent, Plasser \& Theurer, Siemens, Bombardier, Robel, ÖBB und System 7 führen die Liste der Patentanmelder nach Patentfamilien mit Erfindern in Österreich an. 
Tabelle 45: Industriestruktur in Österreich im Bereich Eisenbahn

\begin{tabular}{lcc|}
\hline AnMElder & Pat.Fam. & Erfinder \\
\hline Gesamt & $\mathbf{1 2 2}$ & $\mathbf{9 8}$ \\
\hline INNOVA PATENT & 53 & 42 \\
\hline PLASSER BAHNBAUMASCH & 20 & 13 \\
\hline SIEMENS AG & 7 & 8 \\
\hline BOMBARDIER TRANSP & 5 & 9 \\
\hline ROBEL BAHNBAUMASCHINEN & 4 & 1 \\
\hline OEBB INFRASTRUKTUR & 3 & 1 \\
\hline SYSTEMT RAILSUPPORT & 5 & 2 \\
\hline SEMPERIT AG & 2 & 2 \\
\hline SWIETELSKY & & \\
BAUGESELLSCHAFT & 2 & 2 \\
\hline SIEMENS TRANSPORTATION & 1 & 1 \\
\hline ZUEBLIN AG & 1 & 1 \\
\hline WOPFNER KURT & 1 & 1 \\
\hline KETTL MARKUS & 1 & 1 \\
\hline OUTDOOR VENTURES & 1 & 1 \\
\hline SKIDATA AG & 1 & 1 \\
\hline RTE TECHNOLOGIE & 1 & 1 \\
\hline HEITKAMP RAIL & 1 & 1 \\
\hline STOFFANELLER WERNER & 1 & 1 \\
\hline DOPPELMAYR FRANCE & 1 & 1 \\
\hline ORTNER CHRISTIAN & 1 & 1 \\
\hline PCC E & 1 & 1 \\
\hline ROLIC INVEST & 1 & 1 \\
\hline ENGEL EDWIN & 1 & 1 \\
\hline WEICHENWERK WÃ-RTH & 1 & 1 \\
\hline NEMETH CHRISTOPHER & 1 & 1 \\
\hline ALPINE EN & 1 & 1 \\
\hline GLS BAU & 1 & 1 \\
\hline SCHNABLER KARL & 1 & 1 \\
\hline VOESTALPINE SCHIENEN & 1 & 1 \\
\hline
\end{tabular}

Quelle: Economica (2017).

Nicht nur die Themen des primären Suchprofils zeigen hohe Patentintensität und relative Stärke, sondern auch nahezu sämtliche Crossing-Technologies. Dies ist ein Anzeichen für die hohe technologische Kompetenz in konvergierenden Technologien. Das Thema Eisenbahn zählt aufgrund der breiten Industriestruktur, der hohen Erfinder- und Patentdichte, wie auch aufgrund der hohen relativen Stärke bei den konvergierenden Technologien zu einer Schlüsseltechnologie. 
Abbildung 169: Crossing-Technologies im Bereich Eisenbahn

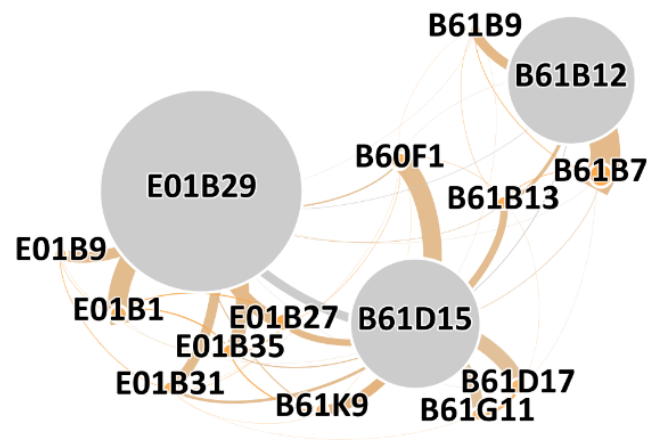

Quelle: Economica (2017).

Tabelle 46: Crossing-Technologies im Bereich Eisenbahn und deren relative Stärke

\begin{tabular}{|c|c|c|}
\hline ірc & descr & $\begin{array}{l}\mathbf{F}_{\mathrm{AT}} / \mathbf{F}_{\mathrm{WO}} \\
2012-2016\end{array}$ \\
\hline E01B29 & Bauwesen .. Gleisoberbau .. Verlegen, Auswechseln oder Aufnehmen von Gleisen & $10,00 \%$ \\
\hline B61D15 & $\begin{array}{l}\text { Eisenbahnen .. Einzelheiten oder Arten des Wagenaufbaus von Eisenbahnfahrzeugen } \\
\text {.. Sonstige Eisenbahnfahrzeuge, z.B. Geruestwagen }\end{array}$ & $14,40 \%$ \\
\hline B61B12 & $\begin{array}{l}\text { Eisenbahnen .. Eisenbahnanlagen .. Bestandteile, Einzelheiten oder Zubehoer fuer } \\
\text { Seilbahnen und an- und abkuppelbare Systeme }\end{array}$ & $18,40 \%$ \\
\hline B61B7 & Eisenbahnen .. Eisenbahnanlagen .. Seilbahnen & $12,70 \%$ \\
\hline E01B27 & $\begin{array}{l}\text { Bauwesen .. Gleisoberbau .. Herstellen, Unterhalten, Erneuern oder Abtragen des } \\
\text { Bettungskoerpers oder des Gleises }\end{array}$ & $25,10 \%$ \\
\hline E01B1 & $\begin{array}{l}\text { Bauwesen .. Gleisoberbau .. Aufbau des Gleisoberbaues fuer Eisen- oder } \\
\text { Straszenbahnen }\end{array}$ & $11,90 \%$ \\
\hline B60F1 & $\begin{array}{l}\text { Transportieren .. Fahrzeuge fuer den Verkehr sowohl auf Schienen als auch auf } \\
\text { Straszen .. Fahrzeuge sowohl fuer Schienen- als auch fuer Straszenverkehr }\end{array}$ & $1,10 \%$ \\
\hline B61D17 & $\begin{array}{l}\text { Eisenbahnen .. Einzelheiten oder Arten des Wagenaufbaus von Eisenbahnfahrzeugen } \\
\text {.. Einzelheiten des Wagenaufbaus von Eisenbahn- oder Straszenbahnfahrzeugen }\end{array}$ & $5,30 \%$ \\
\hline E01B35 & $\begin{array}{l}\text { Bauwesen .. Gleisoberbau .. Anwendung von Messgeraeten oder -vorrichtungen fuer } \\
\text { den Gleisbau }\end{array}$ & $12,40 \%$ \\
\hline B61B13 & Eisenbahnen .. Eisenbahnanlagen .. Andere Eisenbahnanlagen & $0,40 \%$ \\
\hline E01B31 & $\begin{array}{l}\text { Bauwesen .. Gleisoberbau .. Bearbeiten von Schienen, Schwellen, Unterlegplatten } \\
\text { oder dgl. auf der Strecke }\end{array}$ & $11,00 \%$ \\
\hline B61K9 & $\begin{array}{l}\text { Eisenbahnen .. Andere Hilfseinrichtungen fuer Eisenbahnen .. Profillehren fuer } \\
\text { Eisenbahnfahrzeuge }\end{array}$ & $4,10 \%$ \\
\hline E01B9 & Bauwesen .. Gleisoberbau .. Befestigung der Schienen auf den Schwellen oder dgl. & $3,00 \%$ \\
\hline B61G11 & Eisenbahnen .. Kupplungen besonders ausgebildet fuer Eisenbahnfahrzeuge .. Puffer & $3,60 \%$ \\
\hline B61B9 & $\begin{array}{l}\text { Eisenbahnen .. Eisenbahnanlagen .. Straßenbahn- oder Seilbahnanlagen mit starrem } \\
\text { Gleis und Zugseil }\end{array}$ & $12,30 \%$ \\
\hline
\end{tabular}

Quelle: Economica (2017). 


\section{Technologiestärkefeld „Lebensmittel“}

Im Themenbereich Lebensmittel weist Österreich in einigen Technologien - speziell bei Maschinen für Backwaren - hohen Patentoutput auf.

Abbildung 170: Weltweite Dynamik im Bereich Lebensmittel

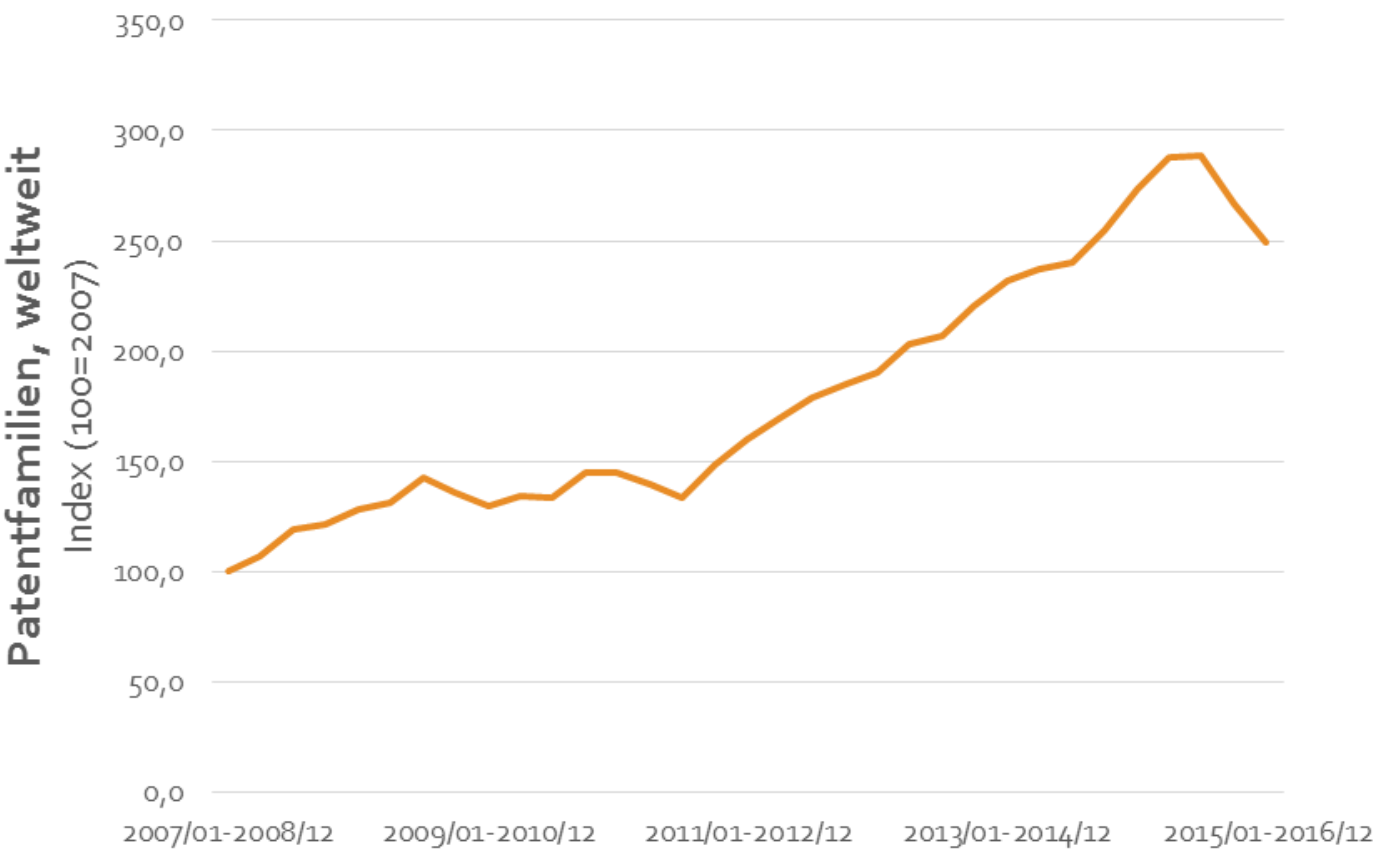

Quelle: Economica (2017).

Österreich liegt im nationalen Ranking auf Rang 7 hinter China, Korea, USA, Taiwan, Deutschland und Frankreich. Dabei zeichnet sich das Thema durch besonders hohe weltweite Dynamik aus. Anders als in den USA, Deutschland, Kanada oder China innovieren Erfinder in Österreich bei diesem Thema nicht gemeinsam mit internationalen Ko-Erfindern. Die USA weist 3 Ko-Erfindernationen auf, Deutschland 2, Kanada und China jeweils eine. 
Tabelle 47: Top Patentländer im Bereich Lebensmittel

\begin{tabular}{|l|l|l|}
\hline \begin{tabular}{l|l} 
Länder- \\
kürzel
\end{tabular} & $\begin{array}{l}\text { Patent- } \\
\text { famillen }\end{array}$ & Erfinder \\
\hline CN & 210 & 228 \\
\hline KR & 115 & 91 \\
\hline US & 73 & 117 \\
\hline TW & 33 & 33 \\
\hline DE & 24 & 35 \\
\hline FR & 21 & 24 \\
\hline AT & 17 & 23 \\
\hline UA & 13 & 10 \\
\hline CA & 8 & 7 \\
\hline RU & 8 & 13 \\
\hline
\end{tabular}

Quelle: Economica (2017).

\section{Abbildung 171: Internationale Vernetzung im Bereich Lebensmittel}

Quelle: Economica (2017).

Generell ist die Industriestruktur in dem untersuchten (engen, jedoch weltweit stark wachsenden) Themenbereich in Österreich sehr konzentriert, mit lediglich einem Unternehmen mit mehr als 2 Patentfamilien (Haas Food). 
Tabelle 48: Industriestruktur in Österreich im Bereich Lebensmittel

\begin{tabular}{lcc|} 
Anmelder & Pat.Fam. & Erfinder \\
Gesamt & $\mathbf{1 7}$ & $\mathbf{2 3}$ \\
HAAS FOOD & 15 & 20 \\
BALIK KURT & 1 & 1 \\
EL BAKRY & 1 & 1
\end{tabular}

Quelle: Economica (2017).

Aus dem Netzwerk der verknüpften Themen, wie es von interdisziplinären Patentfamilien abgeleitet wurde, geht der Zusammenhang mit Möbeln (Küchenausstattung) hervor. Hier könnte gegebenenfalls Vernetzungspotenzial bestehen, da auch im Bereich Möbel regional ausgeprägte Stärkefelder bestehen.

Abbildung 172: Crossing-Technologies im Bereich Lebensmittel

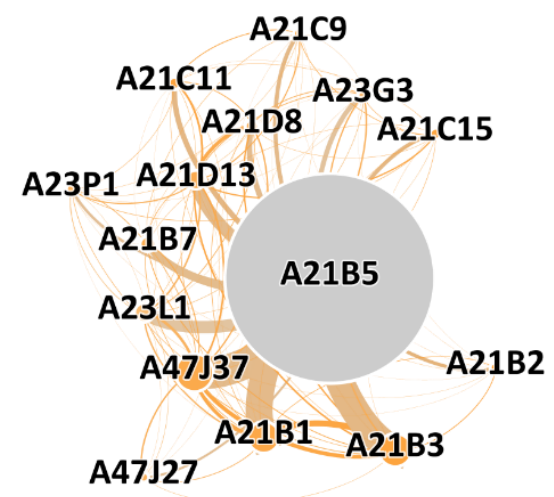

Quelle: Economica (2017). 
Tabelle 49: Crossing-Technologies im Bereich Lebensmittel und deren relative Stärke

\begin{tabular}{|c|c|c|}
\hline ipc & descr & $\begin{array}{r}\mathbf{F}_{\text {AT }} / \mathbf{F}_{\text {WO }} \\
2012-2016\end{array}$ \\
\hline A21B5 & Lebensmittel .. Baeckereioefen .. Backgeraete fuer besondere Backwaren & $7,80 \%$ \\
\hline A47J37 & Moebel .. Kuechenausstattung .. Backen & $0,50 \%$ \\
\hline A21B3 & Lebensmittel .. Baeckereioefen .. Teile oder Zubehoer fuer Baeckereioefen & $2,40 \%$ \\
\hline A21B1 & Lebensmittel .. Baeckereioefen & $1,80 \%$ \\
\hline A21D13 & $\begin{array}{l}\text { Lebensmittel .. Behandeln, z.B. Konservieren von Mehl oder Teig zum Backen, z.B. } \\
\text { durch Zugabe von Substanzen .. Fertige oder halb fertige Baeckereierzeugnisse }\end{array}$ & $0,40 \%$ \\
\hline A23L1 & Lebensmittel .. Lebensmittel oder nichtalkoholische & $0,30 \%$ \\
\hline A21B7 & Lebensmittel .. Baeckereioefen .. Baeckereianlagen & $1,40 \%$ \\
\hline A21D8 & $\begin{array}{l}\text { Lebensmittel .. Behandeln, z.B. Konservieren von Mehl oder Teig zum Backen, z.B. } \\
\text { durch Zugabe von Substanzen .. Verfahren zur Teigbereitung oder zum Backen }\end{array}$ & $0,70 \%$ \\
\hline A21C11 & $\begin{array}{l}\text { Lebensmittel .. Maschinen oder Zubehoer zum Herstellen oder Verarbeiten von } \\
\text { Teigen .. Andere Maschinen zum endgueltigen Formen des Teiges vor dem } \\
\text { Kochen oder Backen }\end{array}$ & $1,90 \%$ \\
\hline A23G3 & Lebensmittel .. Kakao .. Konfekt & $0,50 \%$ \\
\hline A21C15 & $\begin{array}{l}\text { Lebensmittel .. Maschinen oder Zubehoer zum Herstellen oder Verarbeiten von } \\
\text { Teigen .. Vorrichtungen zum Behandeln von Backwaren }\end{array}$ & $9,50 \%$ \\
\hline A21C9 & $\begin{array}{l}\text { Lebensmittel .. Maschinen oder Zubehoer zum Herstellen oder Verarbeiten von } \\
\text { Teigen .. Andere Vorrichtungen zum Behandeln von Teig oder Teigstuecken }\end{array}$ & $4,80 \%$ \\
\hline A21B2 & $\begin{array}{l}\text { Lebensmittel .. Baeckereioefen .. Hochfrequenz- oder infrarotbeheizte } \\
\text { Backgeraete }\end{array}$ & $2,50 \%$ \\
\hline A23P1 & $\begin{array}{l}\text { Lebensmittel .. Formen oder Bearbeiten von Lebensmitteln, soweit nicht } \\
\text { vollstaendig von einer einzelnen anderen Unterklasse umfasst .. Formen oder } \\
\text { Bearbeiten von Lebensmitteln }\end{array}$ & $0,40 \%$ \\
\hline A47J27 & Moebel .. Kuechenausstattung .. Kochen & $0,30 \%$ \\
\hline
\end{tabular}

Quelle: Economica (2017).

\section{Technologiestärkefeld „Metallbearbeitung“}

Die selektierten Themen aus dem Bereich Metallverarbeitung zeigen weltweit besonders hohe Dynamik und Österreich liegt hinsichtlich Patentoutput auf Rang 7 hinter Korea, China, Deutschland, USA, Japan und Taiwan, wobei es einen hohen Grad internationaler Vernetzung aufweist. 
Abbildung 173: Weltweite Dynamik im Bereich Metallbearbeitung

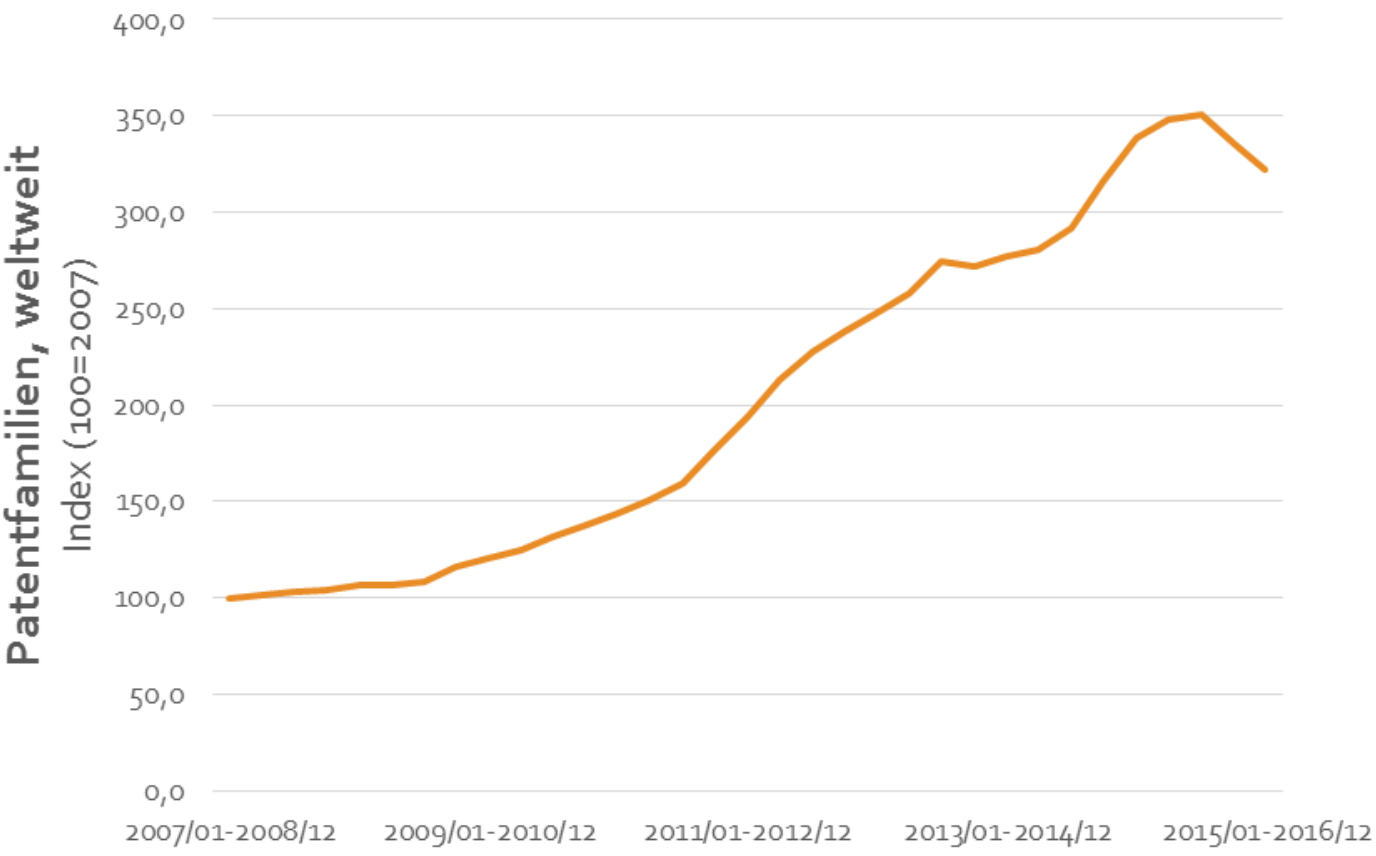

Quelle: Economica (2017).

Tabelle 50: Top Patentländer im Bereich Metallbearbeitung

\begin{tabular}{|l|l|l|}
\hline $\begin{array}{l}\text { Länder- } \\
\text { kürzel }\end{array}$ & $\begin{array}{l}\text { Patent- } \\
\text { familien }\end{array}$ & Erfinder \\
\hline KR & 672 & 354 \\
\hline CN & 478 & 708 \\
\hline DE & 361 & 550 \\
\hline US & 265 & 401 \\
\hline JP & 256 & 416 \\
\hline TW & 78 & 78 \\
\hline AT & $\mathbf{7 1}$ & $\mathbf{8 1}$ \\
\hline FR & 67 & 82 \\
\hline RU & 38 & 60 \\
\hline IT & 35 & 39 \\
\hline
\end{tabular}

Quelle: Economica (2017). 
Die Erfinder in Deutschland und USA innovieren mit jeweils 9 anderen Ko-Erfindernationen, Japan mit 6 und Österreich mit 5, womit es hinsichtlich seiner Gradzentralität auf dem weltweit vierten Rang liegt.

Quelle: Economica (2017).

Aus Sicht der Industriestruktur zeigt sich ein sehr diversifiziertes Bild mit einer breiten Basis von Unternehmen mit Patentoutput. Drei Unternehmen (Trumpf, Welser und Hilti) haben in den letzten 10 Jahren mehr als 3 Patentfamilien zu den selektierten Technologien aus dem Bereich der Metallverarbeitung veröffentlicht.

Tabelle 51: Industriestruktur in Österreich im Bereich Metallbearbeitung

\begin{tabular}{lcc|}
\hline Anmelder & Pat.Fam. & Erfinder \\
\hline Gesamt & $\mathbf{7 1}$ & $\mathbf{8 1}$ \\
\hline TRUMPF MASCHINEN & 38 & 42 \\
\hline WELSER PROFILE & 7 & 7 \\
\hline HILTI AG & 6 & 1 \\
\hline VOESTALPINE KREMS & 2 & 6 \\
\hline SALVAGNINI ITALIA & 2 & 3 \\
REINHARD FRANZE & 2 & 1 \\
\hline STEINER ERWIN & 2 & 1 \\
\hline TRODAT GMBH & 1 & 2 \\
VOESTALPINE STAHL & 1 & 2
\end{tabular}




\begin{tabular}{lll}
\hline KEBA AG & 1 & 1 \\
\hline TROPPMANN HUBERT & 1 & 1 \\
\hline THYSSENKRUPP PRESTA & 1 & 1 \\
\hline MIBA SINTER & 1 & 4 \\
\hline FELDER KG & 1 & 1 \\
\hline KOBE STEEL & 1 & 5 \\
\hline WUKO MASCHB & 1 & 1 \\
\hline FERROBOTICS COMPLIANT & 1 & 2 \\
\hline LIEBHERR HAUSGERAETE & 1 & 1 \\
\hline TRUMER SCHUTZBAUTEN & 1 & 2 \\
\hline CASTOLIN GES & 1 & 2 \\
\hline
\end{tabular}

Quelle: Economica (2017).

Das Themennetzwerk (Crossing-Technologies) liefert keine Hinweise für vielversprechendes weiteres Vernetzungspotenzial.

Abbildung 175: Crossing-Technologies im Bereich Metallbearbeitung

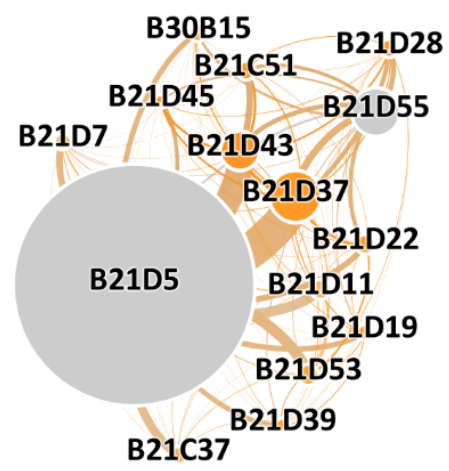

Quelle: Economica (2017). 
Tabelle 52: Crossing-Technologies im Bereich Metallbearbeitung und deren relative Stärke

\begin{tabular}{|l|l|c|}
\hline ipc & descr & $\mathrm{F}_{\text {AT }} / F_{\text {wo, }}$ \\
$2012-2016$
\end{tabular}

Formgebung .. Bearbeiten oder Verarbeiten von Blechen, Metallrohren, -stangen oder -profilen ohne wesentliches Abtragen des Werkstoffs ..

B21D55 Sicherheitseinrichtungen zum Schutz der in dieser Unterklasse behandelten Maschinen oder Vorrichtungen sowie ihrer Bedienungspersonen

Formgebung .. Bearbeiten oder Verarbeiten von Blechen, Metallrohren, -stangen oder -profilen ohne wesentliches Abtragen des Werkstoffs .. Vorrichtungen zum

B21D43 Zufuehren, Ausrichten oder Lagern, speziell fuer oder verbunden mit, bzw. angeordnet an Be- oder Verarbeitungsvorrichtungen fuer Bleche, Metallrohre oder Metallprofile

Formgebung .. Bearbeiten oder Verarbeiten von Blechen, Metallrohren, -stangen

B21D53 oder -profilen ohne wesentliches Abtragen des Werkstoffs .. Herstellen anderer besonders gestalteter Gegenstaende

Formgebung .. Bearbeiten oder Verarbeiten von Blechen, Metallrohren, -stangen

B21D11 oder -profilen ohne wesentliches Abtragen des Werkstoffs .. Biegen von

Halbzeugsorten

Formgebung .. Bearbeiten oder Verarbeiten von Blechen, Metallrohren, -stangen

B21D22 oder - profilen ohne wesentliches Abtragen des Werkstoffs .. Formstanzen

Formgebung .. Bearbeiten oder Verarbeiten von Blechen, Metallrohren, -stangen

B21D28 oder -profilen ohne wesentliches Abtragen des Werkstoffs .. Stanzschneiden

Formgebung .. Herstellen von Blechen, Metalldraht, -stangen, -rohren

Metallprofilen oder Halbzeugen auf andere Weise als durch Walzen .. Mess-,

B21C51 Ueberwachungs-, Anzeige-, Zaehl- oder Markiereinrichtungen, die zur Verwendung beim Herstellvorgang

Formgebung .. Herstellen von Blechen, Metalldraht, -stangen, -rohren Metallprofilen oder Halbzeugen auf andere Weise als durch Walzen .. Herstellen

B21C37 von Blechen, Metallstangen, -draht, -rohren, -profilen oder aehnlichem Halbzeug, soweit nicht anderweitig vorgesehen

B30B15 Pressen .. Pressen allgemein .. Einzelheiten von oder Zubehoer fuer Pressen

Formgebung .. Bearbeiten oder Verarbeiten von Blechen, Metallrohren, -stangen

B21D7 oder -profilen ohne wesentliches Abtragen des Werkstoffs .. Biegen von Stangen, Profilen oder Rohren

Formgebung .. Bearbeiten oder Verarbeiten von Blechen, Metallrohren, -stangen

B21D19 oder -profilen ohne wesentliches Abtragen des Werkstoffs .. Boerdeln oder andere Kantenbearbeitung, z.B. von Rohren 
Formgebung .. Bearbeiten oder Verarbeiten von Blechen, Metallrohren, -stangen oder -profilen ohne wesentliches Abtragen des Werkstoffs .. Verbinden von

B21D39 Gegenstaenden oder Teilen, z.B. Ueberziehen mit Blech auf andere Weise als durch Plattieren

Formgebung .. Bearbeiten oder Verarbeiten von Blechen, Metallrohren, -stangen oder -profilen ohne wesentliches Abtragen des Werkstoffs .. Auswerf- oder

B21D45 Abstreifvorrichtungen in Maschinen oder Werkzeugen, die in dieser Unterklasse behandelt sind

Quelle: Economica (2017).

\section{Technologiestärkefeld „Möbel“}

Die selektierten Technologien aus dem Bereich der Möbel verzeichnen weltweit hohes Wachstum, wobei der Trend in den letzten 10 Jahren anhielt.

\section{Abbildung 176: Weltweite Dynamik im Bereich Möbel}

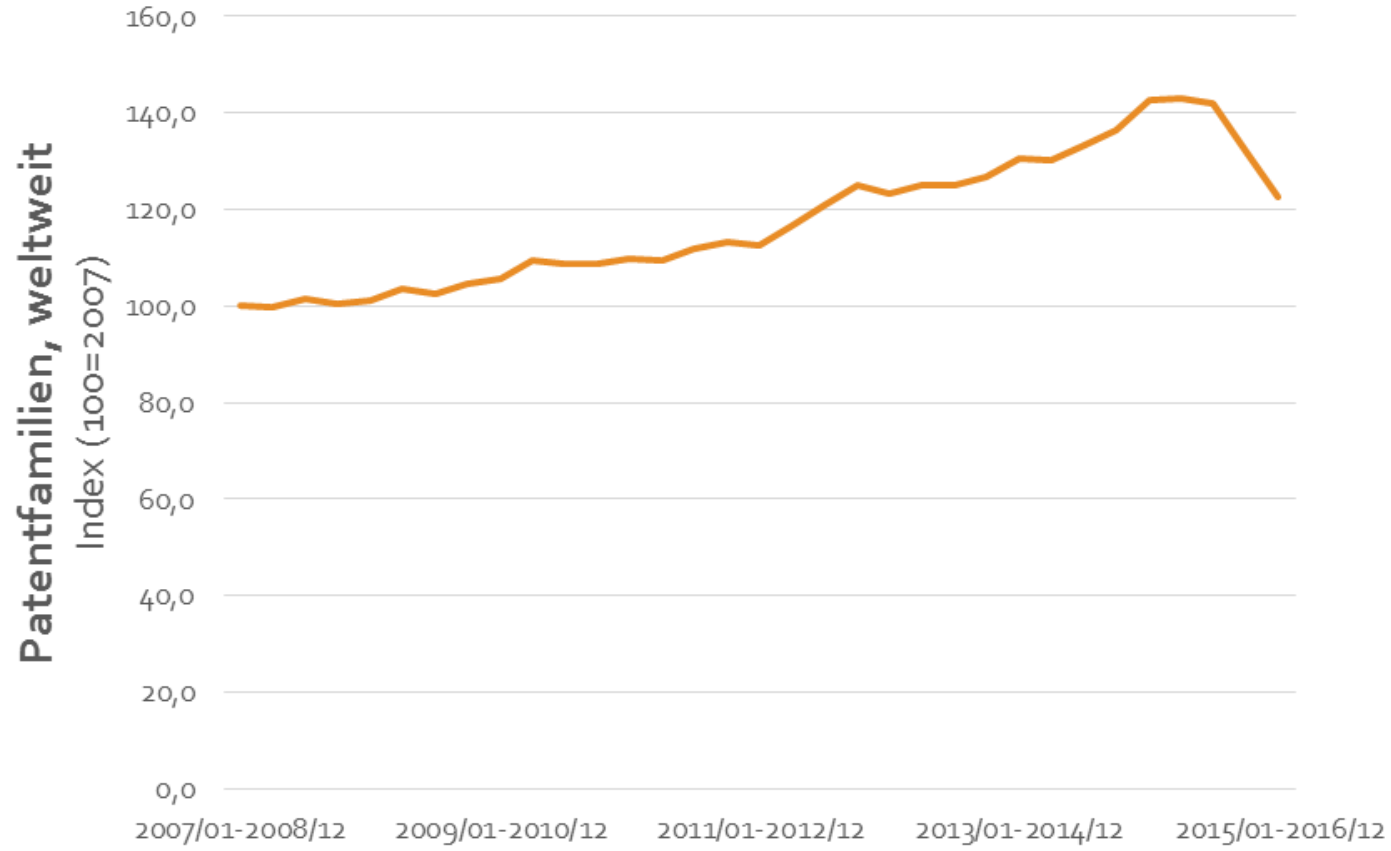

Quelle: Economica (2017).

Österreich liegt hinsichtlich Patentoutput auf Rang 6 hinter Taiwan, China, USA, Deutschland und Korea, wobei es sehr gut in internationale Wissensnetzwerke eingebunden ist. 
Tabelle 53: Top Patentländer im Bereich Möbel

\begin{tabular}{|l|l|l|}
\hline $\begin{array}{l}\text { Länder- } \\
\text { kürzel }\end{array}$ & $\begin{array}{l}\text { Patent- } \\
\text { familien }\end{array}$ & Erfinder \\
\hline TW & 851 & 279 \\
\hline CN & 575 & 403 \\
\hline US & 566 & 849 \\
\hline DE & 480 & 470 \\
\hline KR & 329 & 166 \\
\hline AT & 167 & 110 \\
\hline JP & 91 & 128 \\
\hline TR & 89 & 66 \\
\hline ES & 65 & 53 \\
\hline CA & 45 & 40 \\
\hline
\end{tabular}

Quelle: Economica (2017).

Die Zahl der Ko-Erfindernationen liegt mit 5 hinter USA (13), Deutschland (7) gleichauf mit China (5) und Frankreich (5).

\section{Abbildung 177: Internationale Vernetzung im Bereich Möbel}

Quelle: Economica (2017). 
Lediglich drei Patentanmelder weisen einen Patentoutput von mehr als 4 Patenfamilien in den letzten 10 Jahren auf: Blum, Fulterer und Grass.

Tabelle 54: Industriestruktur in Österreich im Bereich Möbel

\begin{tabular}{lcc|}
\hline Anmelder & Pat.Fam. & Erfinder \\
Gesamt & $\mathbf{1 6 7}$ & $\mathbf{1 1 0}$ \\
BLUM GMBH & 95 & 64 \\
FULTERER GMBH & 28 & 10 \\
\hline GRASS GMBH & 19 & 15 \\
\hline BALIKO KARL & 4 & 1 \\
\hline METZLER GMBH & 2 & 1 \\
\hline KROPF PETER & 2 & 6 \\
\hline GRONBACH FORSCHUNGS & 2 & 2 \\
\hline MICHATEK K & 2 & 1 \\
\hline KEBA AG & 1 & 1 \\
\hline LIEBHERR HAUSGERAETE & 1 & 1 \\
\hline FORM ORANGE & 1 & 1 \\
\hline UNIV WIEN & 1 & 1 \\
\hline KRÜGER ERWIN & 1 & 1 \\
\hline SAMET KALIP & 1 & 1 \\
\hline KUESTER AUTOMOTIVE & 1 & 1 \\
\hline ALFIT AG & 1 & 1 \\
\hline PRENTNER CHRISTIAN & 1 & 2 \\
\hline MIELE & 1 & 1 \\
\hline MERZ MEYER & 1 & 1 \\
\hline GEORG FRACCARO & 1 & 1 \\
\hline ZIMMERMANN MARTIN & 1 & 2 \\
\hline LANG GERTRUDE & 1 & 1 \\
\hline
\end{tabular}

Quelle: Economica (2017).

Thematische Verknüpfungen bestehen in hohem Maß nicht nur mit unmittelbar eng verwandten Themen, wie z.B. Scharnieren oder Kühlschränken, sondern besonders auch mit Bereichen aus der Elektrotechnik (H05K7, Gedruckte Schaltungen), sowie mit Maschinenelementen wie Wellenlager (F16C29). Ein aktueller und wesentlicher Technologietreiber im Bereich der Möbel (und besonders auch in der hier fokussierten Technologie betreffend Schubladen) ist die digitale Transformation, speziell die neuen Anwendungen im Bereich Smart-Logistics, die entsprechende Innovationen erfordern. 
Abbildung 178: Crossing-Technologies im Bereich Möbel

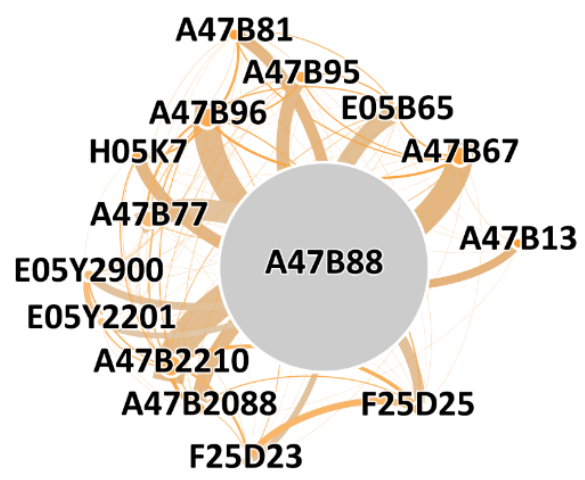

Quelle: Economica (2017).

Tabelle 55: Crossing-Technologies im Bereich Möbel und deren relative Stärke

\begin{tabular}{|c|c|c|}
\hline ipc & descr & $\begin{array}{l}F_{\text {AT }} / F_{\text {WO, }} \\
2012-2016\end{array}$ \\
\hline A47B88 & Moebel .. Tische .. Schubladen fuer Tische, Schraenke oder aehnliche Moebel & $13,60 \%$ \\
\hline A47B96 & Moebel .. Tische .. Einzelheiten von Schraenken, Gestellen oder Regalen & $2,30 \%$ \\
\hline A47B67 & Moebel .. Tische .. Kommoden & $1,60 \%$ \\
\hline A47B77 & Moebel .. Tische .. Kuechenschraenke & $2,00 \%$ \\
\hline E05B65 & Schloesser .. Schloesser fuer besondere Verwendungszwecke & $1,30 \%$ \\
\hline H05K7 & $\begin{array}{l}\text { Elektrotechnik, soweit nicht anderweitig vorgesehen .. Gedruckte Schaltungen .. } \\
\text { Konstruktive Einzelheiten, soweit sie verschiedenen Typen elektrischer Geraete } \\
\text { gemeinsam sind }\end{array}$ & $0,40 \%$ \\
\hline A47B95 & Moebel .. Tische .. Beschlaege fuer Moebel & $5,20 \%$ \\
\hline F25D25 & $\begin{array}{l}\text { Kaelteerzeugung oder Kuehlung .. Kuehlschraenke .. Einbringen, Halterung oder } \\
\text { Entnehmen des Kuehlguts }\end{array}$ & $1,50 \%$ \\
\hline A47B81 & $\begin{array}{l}\text { Moebel .. Tische .. Schraenke, Gestelle oder Regale, die in besonderer Weise fuer } \\
\text { andere Zwecke ausgebildet sind, z.B. zur Aufbewahrung von Gewehren oder Ski }\end{array}$ & $0,80 \%$ \\
\hline A47B13 & Moebel .. Tische .. Einzelheiten von Tischen oder Pulten & $1,20 \%$ \\
\hline F25D23 & $\begin{array}{l}\text { Kaelteerzeugung oder Kuehlung .. Kuehlschraenke .. Allgemeine bauliche } \\
\text { Merkmale }\end{array}$ & $2,10 \%$ \\
\hline E05F1 & $\begin{array}{l}\text { Schloesser .. Vorrichtungen zum Bewegen der Fluegel in die Offen- oder } \\
\text { Schlieszstellung .. Schlieszer oder Oeffner fuer Fluegel, soweit sie nicht an anderer } \\
\text { Stelle in dieser Unterklasse vorgesehen sind }\end{array}$ & $3,80 \%$ \\
\hline F16C29 & $\begin{array}{l}\text { Maschinenelemente oder -einheiten .. Wellen .. Lager fuer nur geradlinig bewegte } \\
\text { Teile }\end{array}$ & $1,40 \%$ \\
\hline E05F5 & $\begin{array}{l}\text { Schloesser .. Vorrichtungen zum Bewegen der Fluegel in die Offen- oder } \\
\text { Schlieszstellung .. Bremsvorrichtungen, z.B. Bewegungsdaempfer }\end{array}$ & $5,10 \%$ \\
\hline A47B63 & $\begin{array}{l}\text { Moebel .. Tische .. Schraenke, Gestelle oder Regale, in besonderer Weise zur } \\
\text { Aufbewahrung von Buechern, Akten, Formularen oder dgl. ausgebildet }\end{array}$ & $0,00 \%$ \\
\hline
\end{tabular}

Quelle: Economica (2017). 


\section{Technologiestärkefeld „Papier“}

Bei den weltweit wachsenden Themen aus dem Bereich Papier, deren Dynamik jedoch in den letzten Jahren stagniert, ist Österreich hinsichtlich Patentoutput hinter Deutschland und Finnland auf Rang 3 und somit in einer Spitzenposition.

\section{Abbildung 179: Weltweite Dynamik im Bereich Papier}

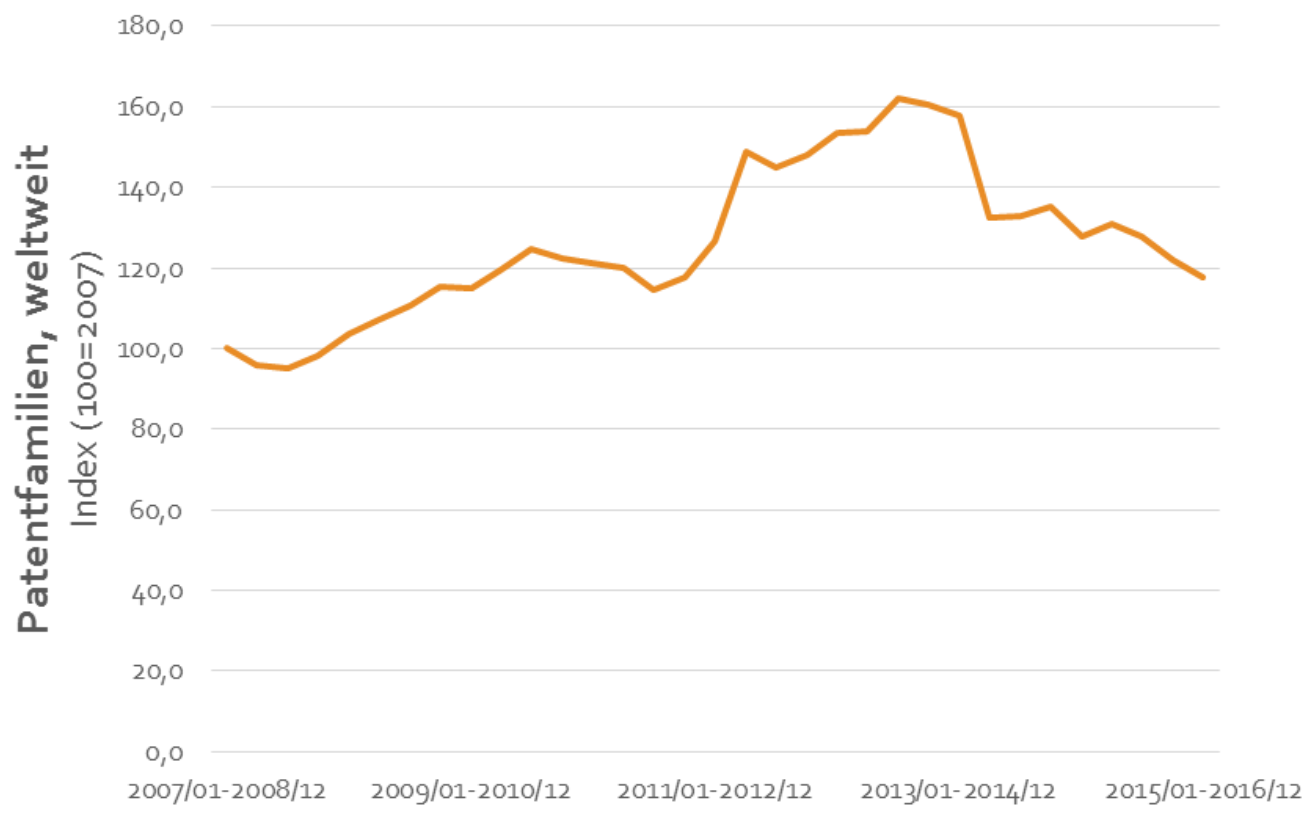

Quelle: Economica (2017).

Tabelle 56: Top Patentländer im Bereich Papier

\begin{tabular}{|l|l|l|}
\hline $\begin{array}{l}\text { Länder- } \\
\text { kürzel }\end{array}$ & $\begin{array}{l}\text { Patent- } \\
\text { familien }\end{array}$ & Erfinder \\
\hline DE & 261 & 303 \\
\hline FI & 149 & 228 \\
\hline AT & 100 & 88 \\
\hline US & 62 & 114 \\
\hline CN & 57 & 106 \\
\hline CA & 28 & 49 \\
\hline BR & 27 & 25 \\
\hline JP & 22 & 33 \\
\hline KR & 18 & 23 \\
\hline UA & 13 & 15 \\
\hline
\end{tabular}

Quelle: Economica (2017). 
Mit 5 Ko-Erfindernationen ist die Gradzentralität hinter Deutschland und USA (jeweils 9 KoErfindernationen) auf dem weltweit dritten Rang. Zwei Unternehmen weisen Patentoutput von über zehn Patentfamilien in den letzten 10 Jahren auf (Voith, Andritz).

Tabelle 57: Industriestruktur in Österreich im Bereich Papier

\begin{tabular}{lcc|}
\hline Anmelder & Pat.Fam. & Erfinder \\
\hline Gesamt & $\mathbf{1 0 0}$ & $\mathbf{8 8}$ \\
\hline VOITH PATENT & 80 & 69 \\
\hline ANDRITZ AG & 12 & 15 \\
\hline ICKINGER GEORG & 5 & 1 \\
\hline DELFORTGROUP AG & 1 & 1 \\
\hline BARTELMUSS KLAUS & 1 & 1 \\
\hline FRITZ EGGER & 1 & 1 \\
\hline
\end{tabular}

Quelle: Economica (2017).

Abbildung 180: Internationale Vernetzung im Bereich Papier

Quelle: Economica (2017).

Im Technologienetzwerk, hier besonders auch aus der Verteilung der Dynamik der verknüpften Themen, drücken sich die Transformations- und Adaptionsprozesse die durch die Digitalisierung erforderlich sind, aus. Während Themen wie Kalander stagnieren, sind Spezialpapiere, aber auch die Vorbereitung von fasrigen Rohstoffen Wachstumsthemen. Anknüpfungspunkte zum Stärkefeld 
„Cellulose" bestehen allein schon aufgrund der Ähnlichkeiten der Beschaffungsmärkte (Rohstoff "Holz").

Abbildung 181: Crossing-Technologies im Bereich Papier

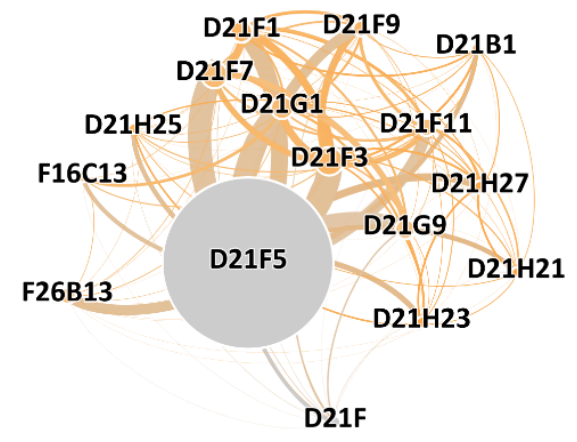

Quelle: Economica (2017).

Tabelle 58: Crossing-Technologies im Bereich Papier und deren relative Stärke

\begin{tabular}{|c|c|c|}
\hline ipc & descr & $\begin{array}{l}\text { Dyn }_{\text {wo, }} \\
1996-2016\end{array}$ \\
\hline D21F5 & $\begin{array}{l}\text { Papier .. Papiermaschinen .. Trockenpartie an Maschinen zur fortlaufenden } \\
\text { Herstellung von Papierbahnen }\end{array}$ & $27,80 \%$ \\
\hline D21F3 & $\begin{array}{l}\text { Papier .. Papiermaschinen .. Presspartie an Maschinen zur fortlaufenden } \\
\text { Herstellung von Papierbahnen }\end{array}$ & $-1,20 \%$ \\
\hline D21F7 & $\begin{array}{l}\text { Papier .. Papiermaschinen .. Andere Einzelheiten an Maschinen zur fortlaufenden } \\
\text { Herstellung von Papierbahnen }\end{array}$ & $31,00 \%$ \\
\hline D21G1 & Papier .. Kalander & $-16,70 \%$ \\
\hline D21F1 & $\begin{array}{l}\text { Papier .. Papiermaschinen .. Nasspartie an Maschinen zur fortlaufenden } \\
\text { Herstellung von Papierbahnen }\end{array}$ & $8,40 \%$ \\
\hline D21G9 & Papier .. Kalander .. Sonstiges Zubehoer fuer Papiermaschinen & $15,40 \%$ \\
\hline D21F11 & $\begin{array}{l}\text { Papier .. Papiermaschinen .. Verfahren zum fortlaufenden Herstellen von Papier, } \\
\text { Karton oder nasser Vliese fuer die Herstellung von Hartfaserplatten auf } \\
\text { Papiermaschinen }\end{array}$ & $27,40 \%$ \\
\hline F26B13 & $\begin{array}{l}\text { Trocknen .. Trocknen von festen Guetern oder Erzeugnissen durch Entfernen von } \\
\text { Fluessigkeit .. Maschinen oder Vorrichtungen zum Trocknen von Geweben, } \\
\text { Faserstoffen, Garnen oder anderen Materialien groszer Laenge mit Foerderung }\end{array}$ & $35,20 \%$ \\
\hline D21F9 & $\begin{array}{l}\text { Papier .. Papiermaschinen .. Vollstaendige Maschinen zur fortlaufenden } \\
\text { Herstellung von Papierbahnen }\end{array}$ & $11,10 \%$ \\
\hline $\mathrm{D} 21 \mathrm{H} 27$ & $\begin{array}{l}\text { Papier .. Faserbreimischungen [Pulpen] .. Spezialpapier, soweit nicht anderweitig } \\
\text { vorgesehen, z.B. im Mehrstufenverfahren hergestellt }\end{array}$ & $34,90 \%$ \\
\hline $\mathrm{D} 21 \mathrm{H} 23$ & $\begin{array}{l}\text { Papier .. Faserbreimischungen [Pulpen] .. Verfahren oder Vorrichtungen, um der } \\
\text { Pulpe oder dem Papier Material zuzusetzen }\end{array}$ & $28,10 \%$ \\
\hline
\end{tabular}


D21H25 Papier .. Faserbreimischungen [Pulpen] .. Nachbehandlung von Papier

D21H21 Papier .. Faserbreimischungen [Pulpen] .. Der Pulpe zugefuegtes nicht faseriges

D21B1 Material, gekennzeichnet durch seine Funktion, Form oder Eigenschaften

\section{Technologiestärkefeld „Stahl“}

Bei den wachsenden Themen im Bereich Stahl liegt Österreich unter den Top-6 weltweit hinsichtlich Patentoutput hinter China, Korea, Japan, USA und Deutschland.

\section{Abbildung 182: Weltweite Dynamik im Bereich Stahl}

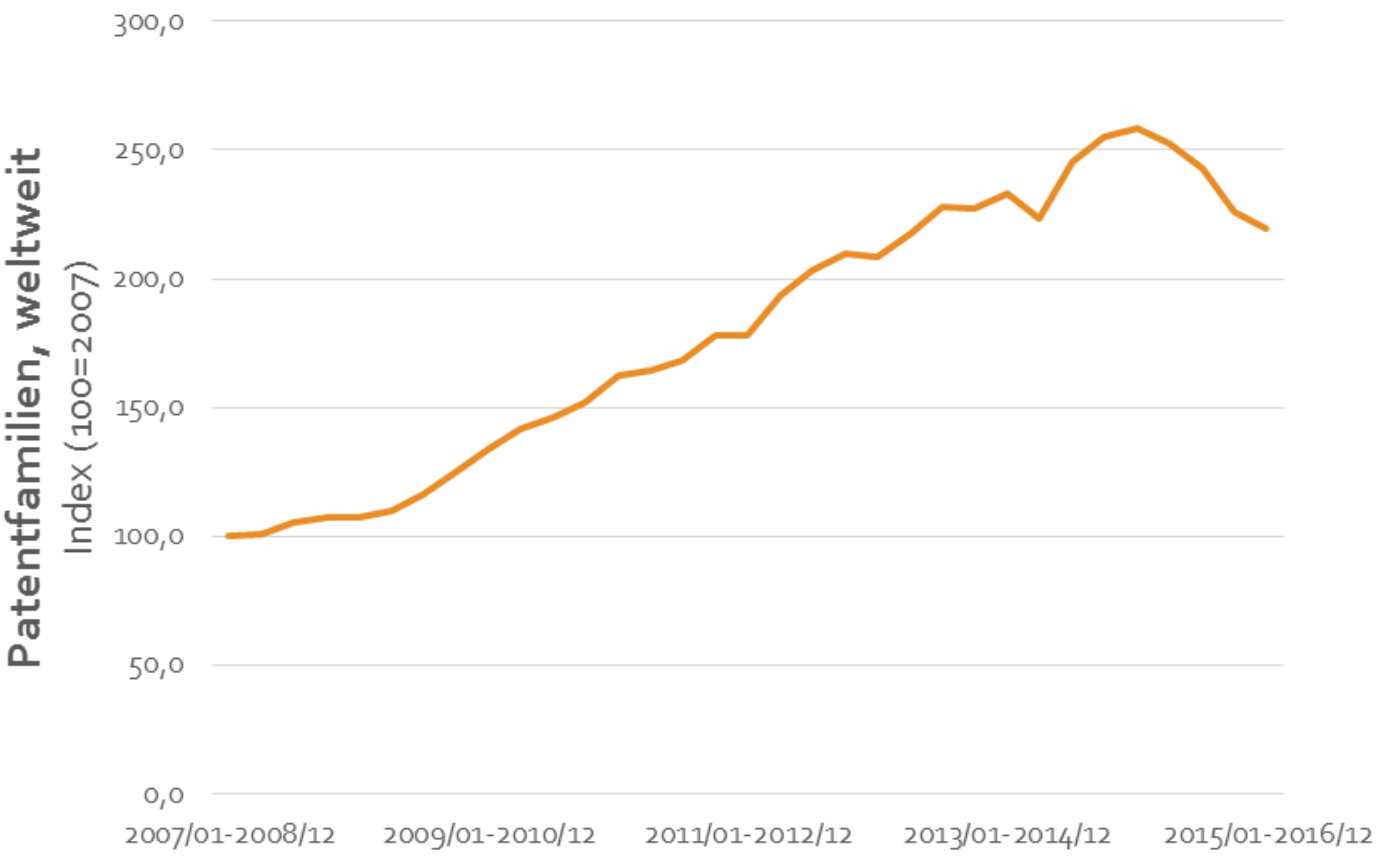

Quelle: Economica (2017). 
Tabelle 59: Top Patentländer im Bereich Stahl

\begin{tabular}{|l|l|l|}
\hline \begin{tabular}{l|l|} 
Länder- \\
kürzel
\end{tabular} & $\begin{array}{l}\text { Patent- } \\
\text { familien }\end{array}$ & Erfinder \\
\hline CN & 288 & 590 \\
\hline KR & 284 & 235 \\
\hline JP & 261 & 513 \\
\hline US & 241 & 421 \\
\hline DE & 157 & 253 \\
\hline AT & 113 & 88 \\
\hline UA & 49 & 84 \\
\hline TW & 38 & 60 \\
\hline RU & 37 & 75 \\
\hline AU & 30 & 26 \\
\hline
\end{tabular}

Quelle: Economica (2017).

Es weist im Ko-Erfindernetzwerk hinter den USA (13 Ko-Erfinderländer), Deutschland (11) und Kanada (6) mit 5 Ko-Erfinderländern die vierthöchste Gradzentralität auf.

\section{Abbildung 183: Internationale Vernetzung im Bereich Stahl}

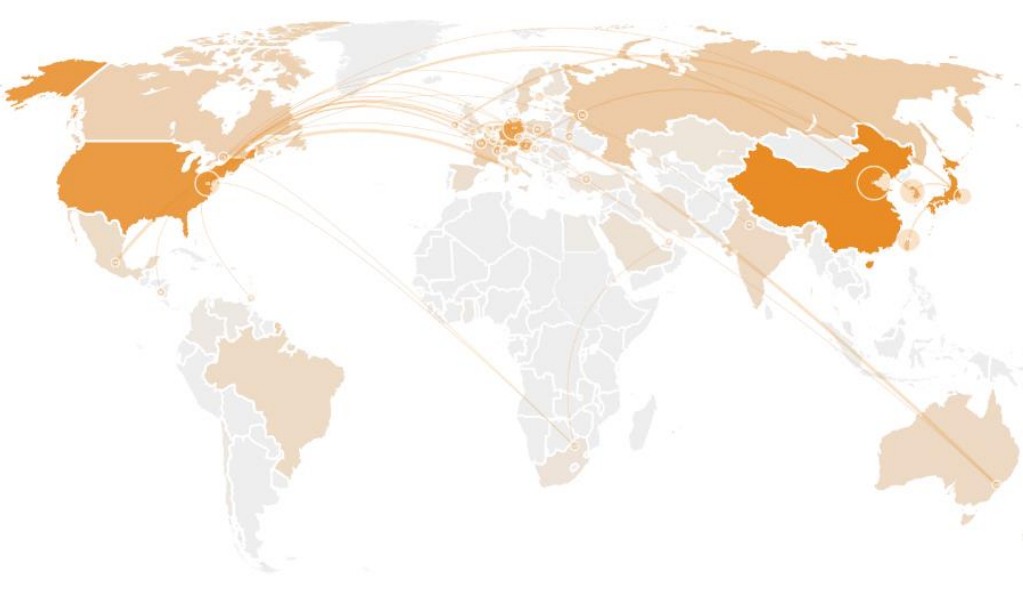

Quelle: Economica (2017).

Die Industriestruktur ist sehr konzentriert, mit drei Unternehmen mit mehr als 3 Patentfamilien in den letzten 10 Jahren (Siemens VAI $\rightarrow$ heute Primetals, Ivoclar Vivadent AG und Voestalpine). 
Tabelle 60: Industriestruktur in Österreich im Bereich Stahl

\begin{tabular}{|c|c|c|}
\hline Anmelder & Pat.Fam. & Erfinder \\
\hline Gesamt & 113 & 88 \\
\hline SIEMENS VAI & 65 & 52 \\
\hline IVOCLAR VIVADENT & 29 & 15 \\
\hline VOEST ALPINE & 4 & 15 \\
\hline VOESTALPINE STAHL & 3 & 5 \\
\hline POSCO & 3 & 6 \\
\hline PATCO ENGINEERING & 2 & 1 \\
\hline AMANN GIRRBACH & 2 & 1 \\
\hline PRIMETALS TECHNOLOGIES & 2 & 7 \\
\hline SIEMENS AG & 1 & 9 \\
\hline ZEHETHOFER KARL & 1 & 1 \\
\hline ANDRITZ MAERZ & 1 & 1 \\
\hline AIR LIQUIDE & 1 & 4 \\
\hline MAERZ OFENBAU & 1 & 1 \\
\hline POLYSIUS AG & 1 & 1 \\
\hline ENGEL AUSTRIA & 1 & 2 \\
\hline RACLAVSKY MILAN & 1 & 1 \\
\hline \multicolumn{3}{|l|}{ LKR } \\
\hline LEICHTMETALLKOMPETENZZENTRUM & 1 & 2 \\
\hline
\end{tabular}

Quelle: Economica (2017).

Das Themennetzwerk zeigt die wachsende Bedeutung von Umwelttechnologien in dem Bereich. Wachstumsthemen im Netzwerk der verknüpften Technologien sind aus dem Bereich der Energierückgewinnung, aber auch in der Vorbereitung von Rohstoffen für den Produktionsprozess. 


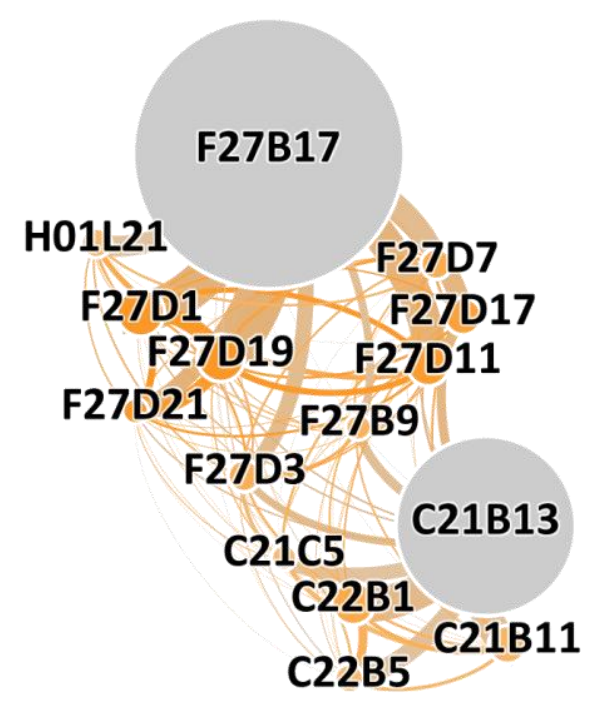

Quelle: Economica (2017).

Tabelle 61: Crossing-Technologies im Bereich Stahl und deren relative Stärke

\begin{tabular}{|c|c|c|}
\hline ipc & descr & $\begin{array}{l}F_{\text {AT }} / F_{W O} \\
2012-2016\end{array}$ \\
\hline F27B17 & $\begin{array}{l}\text { Industrieoefen .. Industrieoefen, Schachtoefen, Brennoefen, Retorten allgemein .. } \\
\text { Industrieoefen, die nicht von einer der Gruppen mref ref=F27B0001000000 } \\
\text { endRef=F27B0015000000>mref }>\text { umfasst sind }\end{array}$ & $9,60 \%$ \\
\hline C21B13 & $\begin{array}{l}\text { Huettenwesen .. Gewinnung von Eisen oder Stahl .. Gewinnung von } \\
\text { Eisenschwamm oder fluessigem Stahl durch Direktverfahren }\end{array}$ & $13,00 \%$ \\
\hline F27D1 & $\begin{array}{l}\text { Industrieoefen .. Einzelheiten oder Zubehoer fuer Industrieoefen, Schachtoefen, } \\
\text { Brennoefen oder Retorten, soweit sie nicht auf eine Ofenart eingeschraenkt sind } \\
\text {.. Auskleidungen }\end{array}$ & $2,50 \%$ \\
\hline F27D19 & $\begin{array}{l}\text { Industrieoefen .. Einzelheiten oder Zubehoer fuer Industrieoefen, Schachtoefen, } \\
\text { Brennoefen oder Retorten, soweit sie nicht auf eine Ofenart eingeschraenkt sind } \\
\text {.. Anordnung von Steuer- oder Regelvorrichtungen [Feuerbruecken] }\end{array}$ & $7,60 \%$ \\
\hline C22B1 & $\begin{array}{l}\text { Metallhuettenwesen .. Gewinnen oder Feinen von Metallen .. Vorbehandlung von } \\
\text { Erzen oder Schrott }\end{array}$ & $2,40 \%$ \\
\hline F27D11 & $\begin{array}{l}\text { Industrieoefen .. Einzelheiten oder Zubehoer fuer Industrieoefen, Schachtoefen, } \\
\text { Brennoefen oder Retorten, soweit sie nicht auf eine Ofenart eingeschraenkt sind } \\
\text {.. Anordnung elektrischer Heizelemente in oder an Oefen }\end{array}$ & $2,50 \%$ \\
\hline C21B11 & $\begin{array}{l}\text { Huettenwesen .. Gewinnung von Eisen oder Stahl .. Roheisengewinnung, auszer in } \\
\text { Hochoefen }\end{array}$ & $9,20 \%$ \\
\hline F27D17 & $\begin{array}{l}\text { Industrieoefen .. Einzelheiten oder Zubehoer fuer Industrieoefen, Schachtoefen, } \\
\text { Brennoefen oder Retorten, soweit sie nicht auf eine Ofenart eingeschraenkt sind } \\
\text {.. Abhitzeverwertung }\end{array}$ & $5,70 \%$ \\
\hline
\end{tabular}


Industrieoefen .. Einzelheiten oder Zubehoer fuer Industrieoefen, Schachtoefen,

F27D3 Brennoefen oder Retorten, soweit sie nicht auf eine Ofenart eingeschraenkt sind .. Beschicken

Industrieoefen .. Einzelheiten oder Zubehoer fuer Industrieoefen, Schachtoefen,

F27D7 Brennoefen oder Retorten, soweit sie nicht auf eine Ofenart eingeschraenkt sind

.. Herstellen, Erhalten oder Umwaelzen der Atmosphaere in Heizraeumen

Grundlegende elektrische Bauteile .. Halbleiterbauelemente .. Verfahren oder

H01L21 Geraete, besonders ausgebildet fuer die Herstellung oder Behandlung von

Halbleiter- oder Festkoerperbauelementen oder Teilen davon

C22B5

Metallhuettenwesen .. Gewinnen oder Feinen von Metallen .. Allgemeine

Verfahren der Reduktion zu Metallen

$3,40 \%$

Industrieoefen .. Einzelheiten oder Zubehoer fuer Industrieoefen, Schachtoefen,

F27D21 Brennoefen oder Retorten, soweit sie nicht auf eine Ofenart eingeschraenkt sind .. Anordnung von Ueberwachungseinrichtungen

Industrieoefen .. Industrieoefen, Schachtoefen, Brennoefen, Retorten allgemein ..

Oefen fuer mechanisch bewegten Einsatz, z.B. Tunneloefen

Huettenwesen .. Weiterverarbeiten von Roheisen, z.B. Feinen, Herstellen von

Schweiszeisen oder Stahl .. Herstellen von Kohlenstoffstahl, z.B. von unlegiertem

C21C5 Stahl mit niedrigem oder mittlerem Kohlenstoffgehalt, oder von Gussstahl

Quelle: Economica (2017).

\section{Technologiestärkefeld „Stempel“}

Das Thema Stempel ist weltweit vor allem von China und Taiwan besetzt, der Patentoutput ist sehr konzentriert auf die ersten beiden Erfinderländer. 
Abbildung 185: Weltweite Dynamik im Bereich Stempel

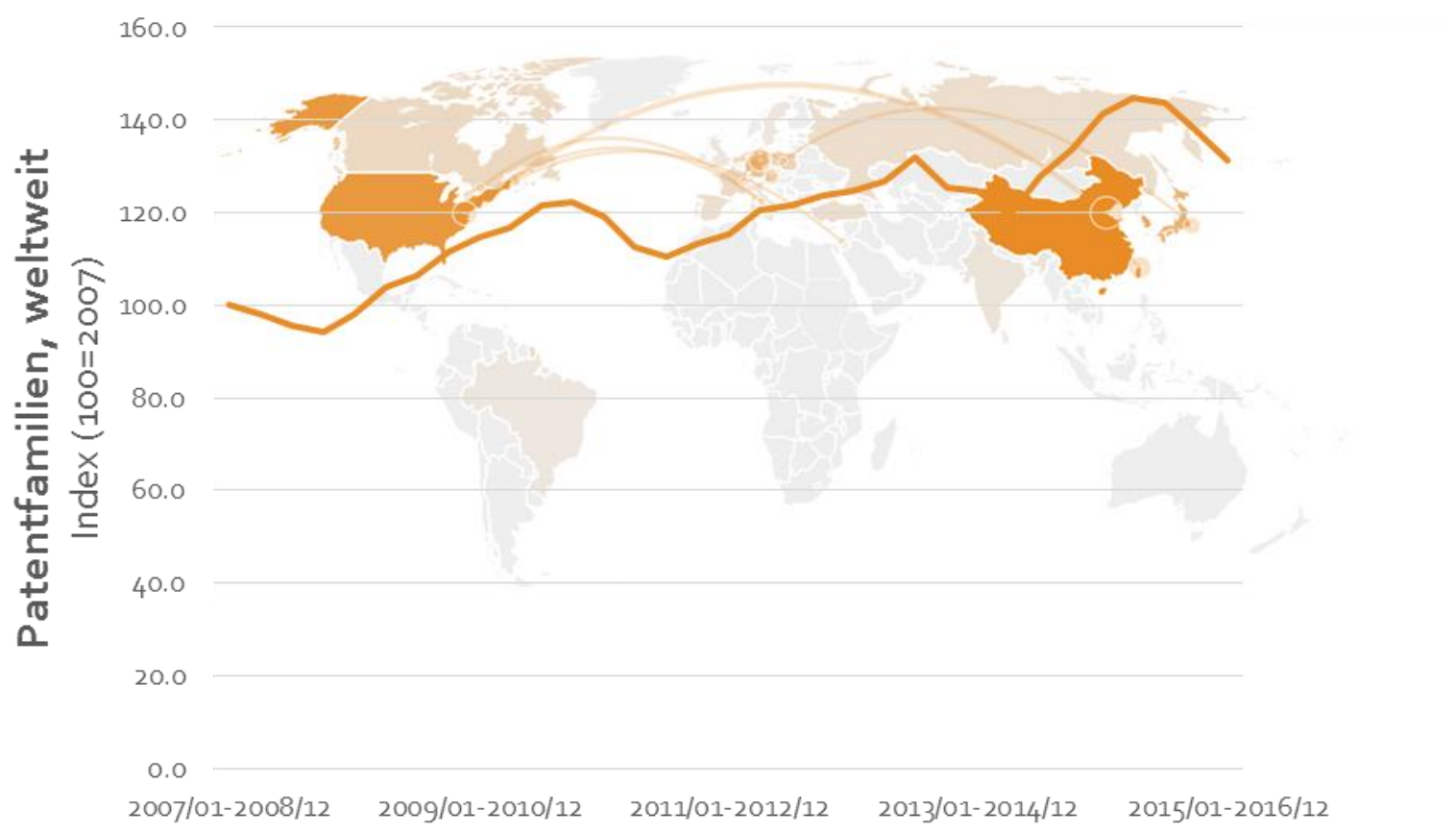

Quelle: Economica (2017).

Österreich liegt auf Rang 6, und die Einbindung in internationale Wissensnetzwerke ist mit einer KoErfindernation vergleichsweise gering.

Tabelle 62: Top Patentländer

\begin{tabular}{|l|l|l|}
\hline $\begin{array}{l}\text { Länder- } \\
\text { kürzel }\end{array}$ & $\begin{array}{l}\text { Patent- } \\
\text { familien }\end{array}$ & Erfinder \\
\hline CN & 234 & 191 \\
\hline TW & 169 & 130 \\
\hline US & 86 & 144 \\
\hline KR & 85 & 94 \\
\hline JP & 49 & 68 \\
\hline AT & $\mathbf{2 3}$ & 17 \\
\hline PL & 23 & 14 \\
\hline DE & 22 & 39 \\
\hline TR & 6 & 5 \\
\hline CA & 5 & 9 \\
\hline
\end{tabular}

Quelle: Economica (2017). 
Die beiden Unternehmen Colop und Trodat weisen Patentoutput von mehr als 3 Patentfamilien im 10-Jahresintervall auf.

Tabelle 63: Industriestruktur in Österreich im Bereich Stempel

\begin{tabular}{lcc|} 
Anmelder & Pat.Fam. & Erfinder \\
\hline Gesamt & $\mathbf{2 3}$ & $\mathbf{1 7}$ \\
COLOP STEMPELERZEUGUNG & 14 & 3 \\
\hline TRODAT GMBH & 7 & 12 \\
TECHNISCHE KANZLEI & 1 & 1 \\
\hline KUEHTREIBER FRANZ & 1 & 1
\end{tabular}

Quelle: Economica (2017).

Potenziale, die sich durch die Digitalisierung ergeben könnten, dürfen nicht außer Acht gelassen werden. Das Themennetzwerk zeigt aktuelle Verknüpfungen mit Themen der Datenverarbeitung sowie auch mit G06K19 (Datenerkennung - Aufzeichnungsträger für Maschinen, bei denen mindestens ein Teil zur Aufnahme von digitalen Markierungen bestimmt ist).

Abbildung 186: Crossing-Technologies im Bereich Stempel

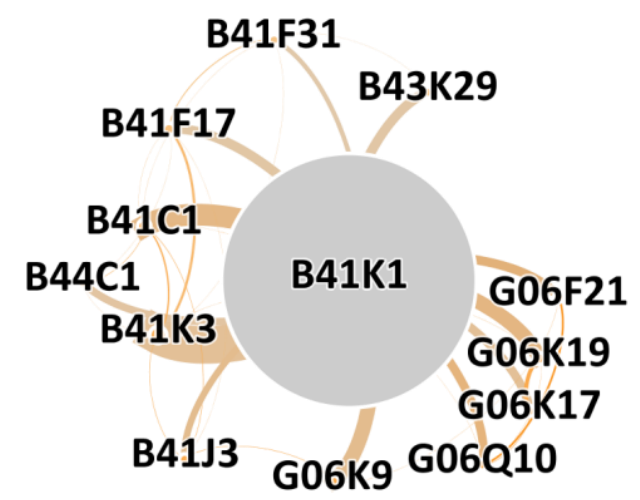

Quelle: Economica (2017). 
Tabelle 64: Crossing-Technologies im Bereich Stempel und deren relative Stärke

\begin{tabular}{|c|c|c|}
\hline ipc & descr & $\begin{array}{r}F_{\text {AT }} / F_{W O} \\
2012-2016\end{array}$ \\
\hline B41K1 & $\begin{array}{l}\text { Drucken .. Stempel .. Tragbare handbetaetigte Vorrichtungen ohne Mittel zum } \\
\text { Unterstuetzen oder Festlegen der zu stempelnden Gegenstaende, z.B. } \\
\text { Handstempel }\end{array}$ & $11,10 \%$ \\
\hline B41K3 & $\begin{array}{l}\text { Drucken .. Stempel .. Vorrichtungen, die zum Unterstuetzen der zu stempelnden } \\
\text { Gegenstaende angebaute Mittel haben }\end{array}$ & $3,90 \%$ \\
\hline B41C1 & $\begin{array}{l}\text { Drucken .. Verfahren zur Herstellung oder Nachbildung von Druckformen .. } \\
\text { Druckformherstellung }\end{array}$ & $0,50 \%$ \\
\hline B41F17 & $\begin{array}{l}\text { Drucken .. Druckmaschinen oder -pressen .. Druckvorrichtungen oder -maschinen } \\
\text { besonderer Bauart oder fuer Sonderzwecke, soweit nicht anderweitig vorgesehen }\end{array}$ & $0,70 \%$ \\
\hline G06K19 & $\begin{array}{l}\text { Datenverarbeitung .. Erkennen von Daten .. Aufzeichnungstraeger fuer } \\
\text { Maschinen, bei denen mindestens ein Teil zur Aufnahme von digitalen } \\
\text { Markierungen bestimmt ist }\end{array}$ & $1,50 \%$ \\
\hline G06K9 & $\begin{array}{l}\text { Datenverarbeitung .. Erkennen von Daten .. Verfahren oder Anordnungen zum } \\
\text { Lesen oder Erkennen gedruckter oder geschriebener Zeichen oder zum Erkennen } \\
\text { von Mustern, z.B. Fingerabdruecken }\end{array}$ & $0,30 \%$ \\
\hline B41J3 & $\begin{array}{l}\text { Drucken .. Schreibmaschinen .. Schreibmaschinen oder Einzeltypen- Drucker oder } \\
\text { Markierungsvorrichtungen, gekennzeichnet durch den Zweck, fuer den sie gebaut } \\
\text { sind }\end{array}$ & $0,50 \%$ \\
\hline B43K29 & $\begin{array}{l}\text { Schreib- oder Zeichengeraete .. Geraete zum Schreiben oder Zeichnen .. Mit } \\
\text { anderen Gegenstaenden kombinierte Schreibgeraete }\end{array}$ & $0,50 \%$ \\
\hline G06F21 & $\begin{array}{l}\text { Datenverarbeitung .. Elektrische digitale Datenverarbeitung .. } \\
\text { Sicherheitseinrichtungen zum Schutz von Rechnern oder Rechnersystemen gegen } \\
\text { unberechtigten Zugriff }\end{array}$ & $0,30 \%$ \\
\hline G06Q10 & $\begin{array}{l}\text { Datenverarbeitung .. Datenverarbeitungssysteme oder -verfahren, besonders } \\
\text { angepasst an verwaltungstechnische, geschaeftliche, finanzielle oder } \\
\text { betriebswirtschaftliche Zwecke, sowie an geschaeftsbezogene Ueberwachungs- } \\
\text { ode .. Verwaltung }\end{array}$ & $0,30 \%$ \\
\hline B41F31 & Drucken .. Druckmaschinen oder -pressen .. Farbwerke oder -vorrichtungen & $0,10 \%$ \\
\hline G06K17 & $\begin{array}{l}\text { Datenverarbeitung .. Erkennen von Daten .. Verfahren oder Anordnungen zur } \\
\text { Zusammenarbeit von Geraeten, die in zwei oder mehr der Hauptgruppen mref } \\
\text { ref=G06K0001000000 endRef=G06K0015000000>mref }>\text { umfasst sind, z.B. } \\
\text { automatische Karteien mit }\end{array}$ & $0,30 \%$ \\
\hline B44C1 & $\begin{array}{l}\text { Dekorationskunst oder -technik .. Verfahren zum Herstellen von Verzierungen .. } \\
\text { Verfahren zum Verzieren von Oberflaechen, soweit nicht anderweitig } \\
\text { ausdruecklich vorgesehen }\end{array}$ & $1,00 \%$ \\
\hline
\end{tabular}

Quelle: Economica (2017). 


\section{Technologiestärkefeld „Werkzeug“}

Ähnlich wie beim Thema aus dem Gebiet „Stempel“ ist die weltweite Dynamik beim selektierten Technologiefeld im Bereich „Werkzeug“ über den 20-Jahreszeitraum wachsend, jedoch in den letzten 10 Jahren rückläufig.

\section{Abbildung 187: Weltweite Dynamik im Bereich Werkzeug}

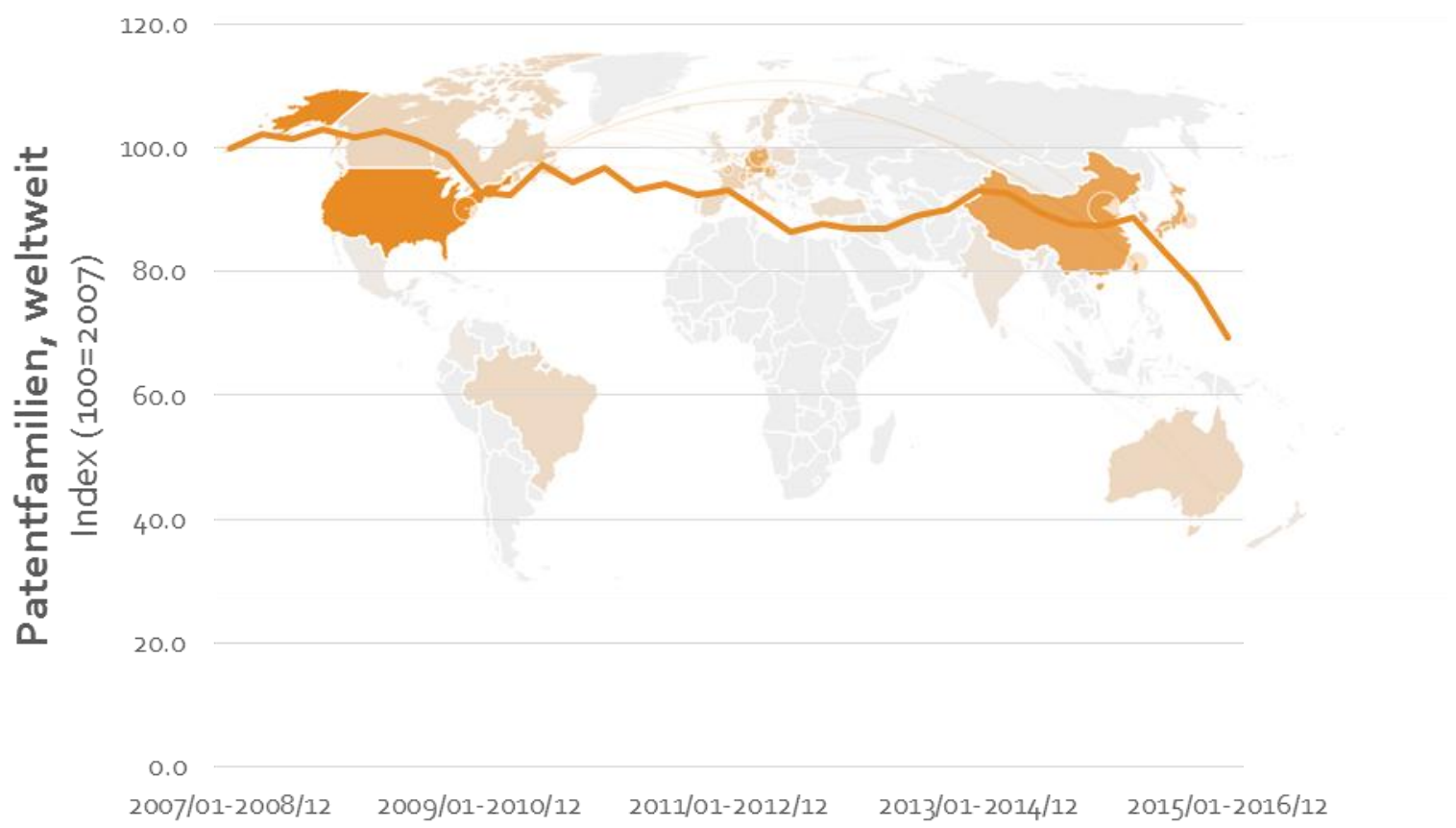

Quelle: Economica (2017).

Das Thema wird von einem Unternehmen (Hilti) mit Erfindern in Österreich so stark besetzt, dass Österreich im weltweiten Ranking des Patentoutputs (10-Jahresintervall) auf Rang 5, hinter Taiwan, USA, China und Japan liegt. 
Tabelle 65: Industriestruktur in Österreich im Bereich Werkzeug

\begin{tabular}{lcc|} 
Anmelder & Pat.Fam. & Erfinder \\
Gesamt & $\mathbf{1 1 0}$ & $\mathbf{2 2}$ \\
\hline HILTI AG & 107 & 21 \\
\hline BLESSING MATTHIAS & 1 & 1 \\
\hline LATSCHBACHER GMBH & 1 & 1 \\
\hline JOHANN HOERTNAGL & 1 & 1 \\
\hline
\end{tabular}

Quelle: Economica (2017).

Tabelle 66: Top Patentländer im Bereich Werkzeug

\begin{tabular}{|l|l|l|}
\hline $\begin{array}{l}\text { Länder- } \\
\text { kürzel }\end{array}$ & $\begin{array}{l}\text { Patent- } \\
\text { familien }\end{array}$ & Erfinder \\
\hline TW & 567 & 214 \\
\hline US & 295 & 341 \\
\hline CN & 253 & 191 \\
\hline JP & 139 & 138 \\
\hline AT & 110 & 22 \\
\hline DE & 102 & 108 \\
\hline CH & 96 & 44 \\
\hline KR & 86 & 95 \\
\hline LI & 47 & 32 \\
\hline FR & 31 & 20 \\
\hline
\end{tabular}

Quelle: Economica (2017).

Bei dem Stärkefeld handelt es sich um Werkzeuge zum Heften oder Nageln, die Analyse der CrossingTechnologies zeigt die Verknüpfungen mit anderen Handwerkzeugen ebenso wie mit den Nägeln und Klammern selbst. Verknüpfungen mit Stärkefeldern aus anderen Bereichen gehen aus den CrossingTechnologies des Stärkefeldes „Werkzeug" nicht hervor. 
Abbildung 188: Crossing-Technologies im Bereich Werkzeug

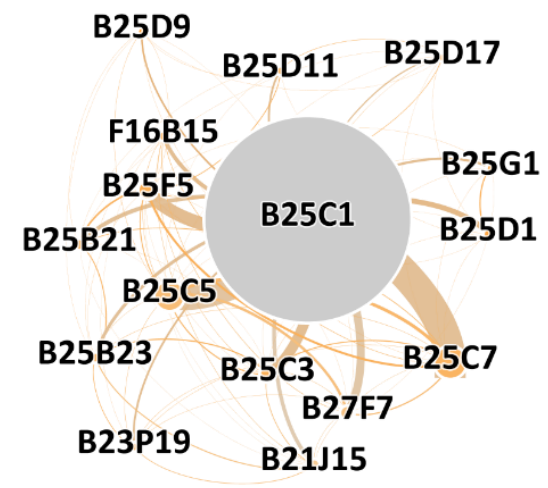

Quelle: Economica (2017).

Tabelle 67: Crossing-Technologies im Bereich Werkzeug und deren relative Stärke

\begin{tabular}{|c|c|c|}
\hline ipc & descr & $\begin{array}{r}F_{\text {AT }} / F_{\text {WO }} \\
2012-2016\end{array}$ \\
\hline B25C1 & $\begin{array}{l}\text { Handwerkzeuge .. Handwerkzeuge zum Nageln oder Heften .. Handwerkzeuge } \\
\text { zum Nageln }\end{array}$ & $11,30 \%$ \\
\hline B25C7 & $\begin{array}{l}\text { Handwerkzeuge .. Handwerkzeuge zum Nageln oder Heften .. Zubehoer fuer } \\
\text { Werkzeuge zum Nageln oder Heften, z.B. Gestelle }\end{array}$ & $2,40 \%$ \\
\hline B25C5 & $\begin{array}{l}\text { Handwerkzeuge .. Handwerkzeuge zum Nageln oder Heften .. Durch Muskelkraft } \\
\text { betriebene tragbare Heftwerkzeuge }\end{array}$ & $4,80 \%$ \\
\hline B25F5 & $\begin{array}{l}\text { Handwerkzeuge .. Kombinierte oder Mehrzweckwerkzeuge, soweit nicht } \\
\text { anderweitig vorgesehen ... Einzelheiten oder Bestandteile von tragbaren } \\
\text { Werkzeugen mit Kraftantrieb, soweit diese nicht auf den durchgefuehrten } \\
\text { Bearbeitungsvorgang bezogen und nicht anderweitig vorgesehen sind }\end{array}$ & $1,10 \%$ \\
\hline B25C3 & $\begin{array}{l}\text { Handwerkzeuge .. Handwerkzeuge zum Nageln oder Heften .. Tragbare } \\
\text { Vorrichtungen zum Halten und Fuehren von Naegeln }\end{array}$ & $0,80 \%$ \\
\hline B27F7 & $\begin{array}{l}\text { Bearbeiten oder Konservieren von Holz oder aehnlichem Werkstoff .. } \\
\text { Gegenstaende mit Schwalbenschwanzverbindung .. Nageln oder Klammern }\end{array}$ & $4,70 \%$ \\
\hline B25D1 & Handwerkzeuge .. Schlagwerkzeuge .. Handhaemmer & $0,00 \%$ \\
\hline B25B21 & $\begin{array}{l}\text { Handwerkzeuge .. Werkzeuge oder Werkbankvorrichtungen zum Befestigen, } \\
\text { Verbinden, Loesen oder Halten, soweit nicht anderweitig vorgesehen .. Tragbare } \\
\text { kraftangetriebene Werkzeuge zum Anziehen oder Loesen von Schrauben oder } \\
\text { Muttern }\end{array}$ & $1,20 \%$ \\
\hline F16B15 & $\begin{array}{l}\text { Maschinenelemente oder -einheiten .. Vorrichtungen zum Befestigen oder } \\
\text { Sichern von Konstruktionselementen oder Maschinenteilen, z.B. Naegel, Bolzen, } \\
\text { Schrauben, Sprengringe, Klemmen, Klammern oder Keile .. Befestigungen ohne } \\
\text { Gewinde }\end{array}$ & $1,90 \%$ \\
\hline
\end{tabular}


Handwerkzeuge .. Werkzeuge oder Werkbankvorrichtungen zum Befestigen, Verbinden, Loesen oder Halten, soweit nicht anderweitig vorgesehen ..

B25B23 Einzelheiten oder Zubehoer der Schraubenschluessel, Mutterschluessel und Schraubendreher

\begin{tabular}{|c|c|c|}
\hline B21J15 & Formgebung .. Schmieden .. Nieten & $1,10 \%$ \\
\hline B25G1 & Handwerkzeuge .. Griffe fuer Handgeraete .. Bauart von Griffen & $1,00 \%$ \\
\hline B23Р19 & $\begin{array}{l}\text { Werkzeugmaschinen .. Sonstige Metallbearbeitung .. Maschinen zum } \\
\text { Zusammensetzen oder Auseinandernehmen von Metallwerkstuecken oder von } \\
\text { Metall- und Nichtmetallwerkstuecken, mit oder ohne Verformen }\end{array}$ & $0,70 \%$ \\
\hline B25D11 & $\begin{array}{l}\text { Handwerkzeuge .. Schlagwerkzeuge .. Tragbare Schlagwerkzeuge mit } \\
\text { elektromotorischem Antrieb }\end{array}$ & $3,10 \%$ \\
\hline B25D9 & $\begin{array}{l}\text { Handwerkzeuge .. Schlagwerkzeuge .. Tragbare durch Druckfluessigkeit oder } \\
\text { Druckluft angetriebene Schlagwerkzeuge, z.B. mit mehreren gleichzeitig } \\
\text { arbeitenden Schlagwerkzeugmeiszeln }\end{array}$ & $1,80 \%$ \\
\hline B25D17 & $\begin{array}{l}\text { Handwerkzeuge .. Schlagwerkzeuge .. Einzelheiten von tragbaren } \\
\text { Schlagwerkzeugen mit Kraftantrieb oder Zubehoer hierfuer }\end{array}$ & $2,10 \%$ \\
\hline
\end{tabular}

Quelle: Economica (2017).

\section{Patentanalyse: Beschreibung der weltweiten Wachstumsthemen Weltweite Wachstumsthemen im Bereich Additive Fertigungsverfahren}

Die Wachstumsthemen im Bereich „Additive Fertigungsverfahren“ zeigten ab 2011 exponentielles Wachstum, wobei ab 2014 eine weitere Beschleunigung der Patentanmeldedynamik beobachtet wurde. Weltweit sind die Erfinder-Hubs in den USA, Deutschland, Japan, Frankreich und Korea. Österreich liegt im Ranking nach Erfindern auf Position 21. Die Patentanmelder in Österreich sind die TU Wien, die Universität Wien, Ivoclar Vivadent, Lithoz, Way to Production und Headlight Analytics. 
Abbildung 189: Weltweite Dynamik im Additive Fertigungsverfahren

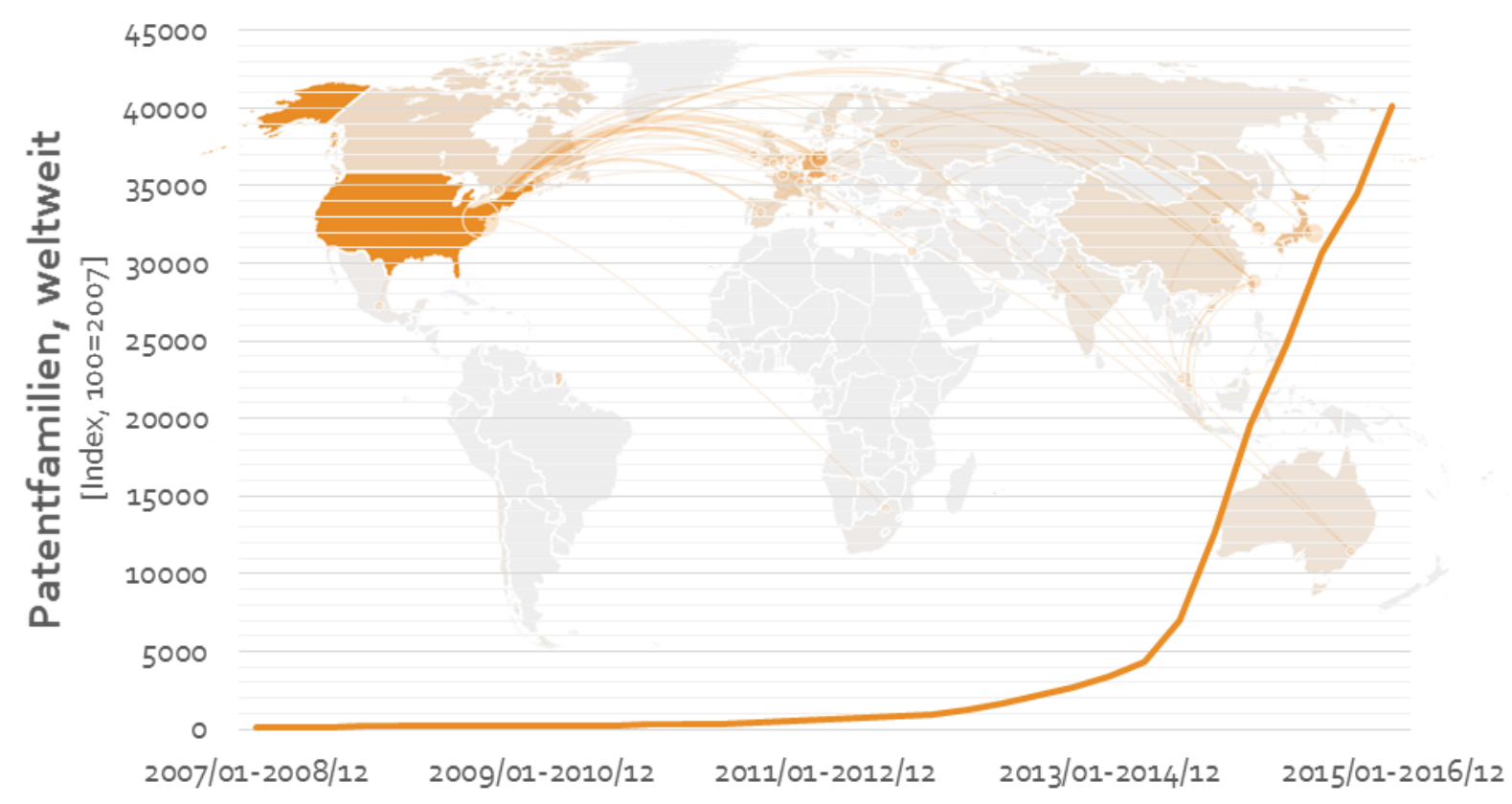

Quelle: Economica (2017).

\section{Weltweite Wachstumsthemen im Bereich Verteilung elektrischer Leistung}

In den besonders rasch wachsenden Themen aus dem Bereich "Verteilung elektrischer Leistung" weisen die Unternehmen MAGNA INTERIORS, FRONIUS INT, BAUMANN HOLDING, INFINEON TECHNOLOGIES und DEUTSCHE TELEKOM Patentfamilien mit Erfindern in Österreich auf. Das Thema ist weltweit über den gesamten 10-Jahreszeitraum wachsend, mit einer signifikanten Beschleunigung ab 2014. Die führenden Erfindernationen sind USA, Japan, Deutschland, Korea und Taiwan. Österreich liegt auf Rang 14 nach Erfindern. 
Abbildung 190: Weltweite Dynamik im Bereich „Verteilung elektrischer Leistung“

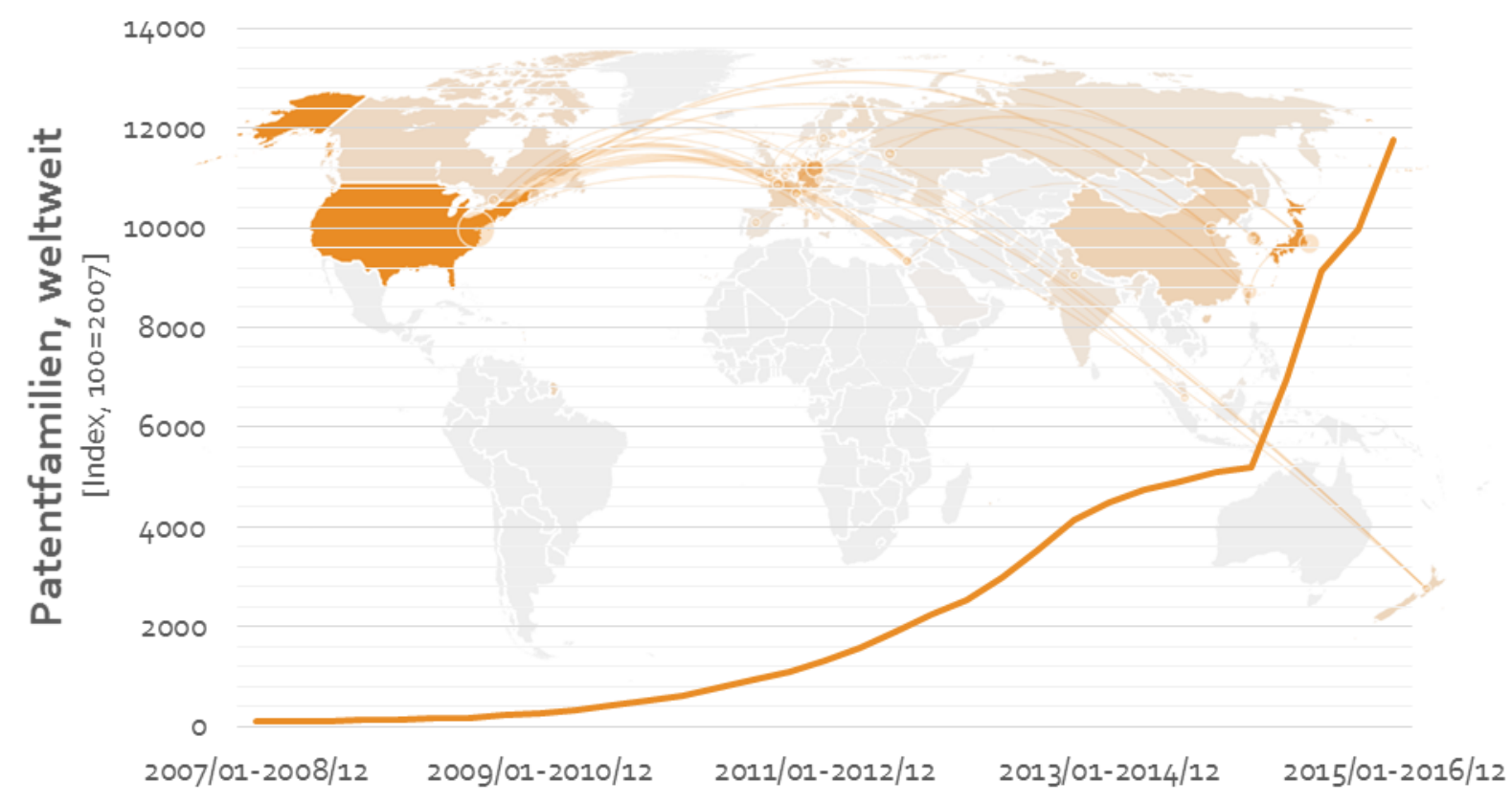

Quelle: Economica (2017).

\section{Weltweite Wachstumsthemen im Bereich nicht-schaltbare Kupplungen}

Die Themen aus dem Bereich „nicht-schaltbare Kupplungen“ zeigten zwischen 2010 und 2012 besonders hohe Wachstumsraten. Seit 2013 verlangsamt sich der Trend, wobei in den letzten beiden Jahren ein leichter Rückgang der Patentanmeldefrequenzen beobachtet wurde. Anmelder mit Erfindern in Österreich sind VE VIENNA ENGINEERING, KNORR BREMSE, MAGNA POWERTRAIN, BODNER, OTIS ELEVATOR, EKONEN TODD und GEN ELECTRIC, wobei Österreich insgesamt nach Erfindern weltweit auf Rang 9 liegt. Angeführt wird das Ranking von Japan, Deutschland, USA, Frankreich und Korea. 
Abbildung 191: Weltweite Dynamik im Bereich nicht-schaltbare Kupplungen

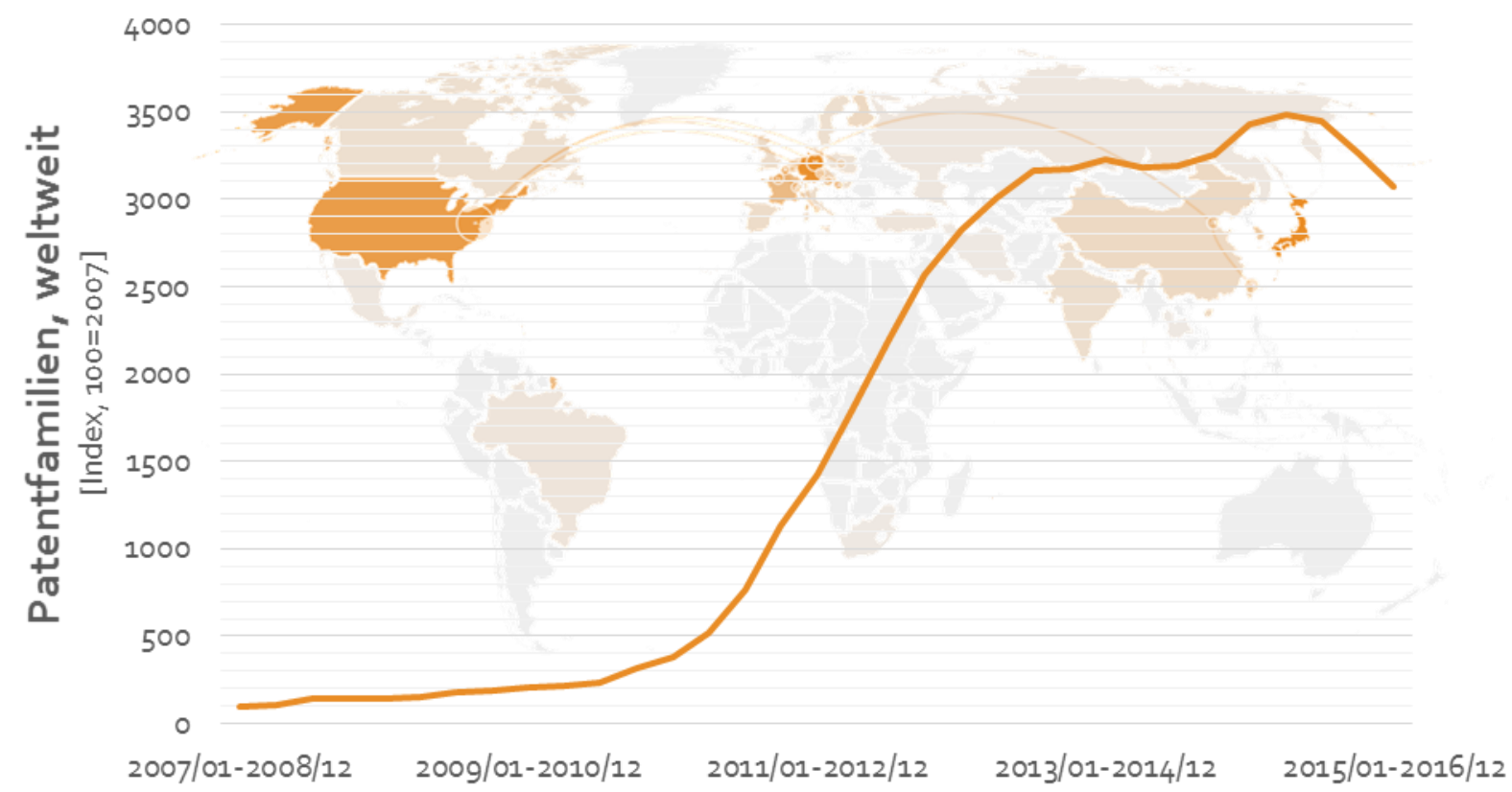

Quelle: Economica (2017).

\section{Weltweite Wachstumsthemen im Bereich Licht}

Die Entwicklung der beiden besonders schnell wachsenden Themen aus dem Bereich "Licht" (Lichtquellen unter Verwendung von Halbleiterbauelementen als lichterzeugende Elemente, z.B. unter Verwendung von Leuchtdioden [LEDs] oder Lasern) zeigt ab 2014 sprunghaften Anstieg, wobei Österreich auf Rang 10 im internationalen Spitzenfeld hinsichtlich der Erfinderzahl liegt. Das Ranking wird von USA, Japan, Deutschland, China und Taiwan angeführt. Die Industriestruktur ist - verglichen mit den anderen untersuchten weltweiten Wachstumsthemen - im Bereich „Licht" sehr breit mit einer Vielzahl von Unternehmen. Den größten Innovationsoutput (gemessen an der Zahl von Patentfamilien bzw. Erfindern) stammt von der Zumtobel Gruppe (Zumtobel, Tridonic), gefolgt von Zizala und Ledon (bis 2012 Teil der Zumtobel Gruppe). 
Abbildung 192: Weltweite Dynamik im Bereich Licht

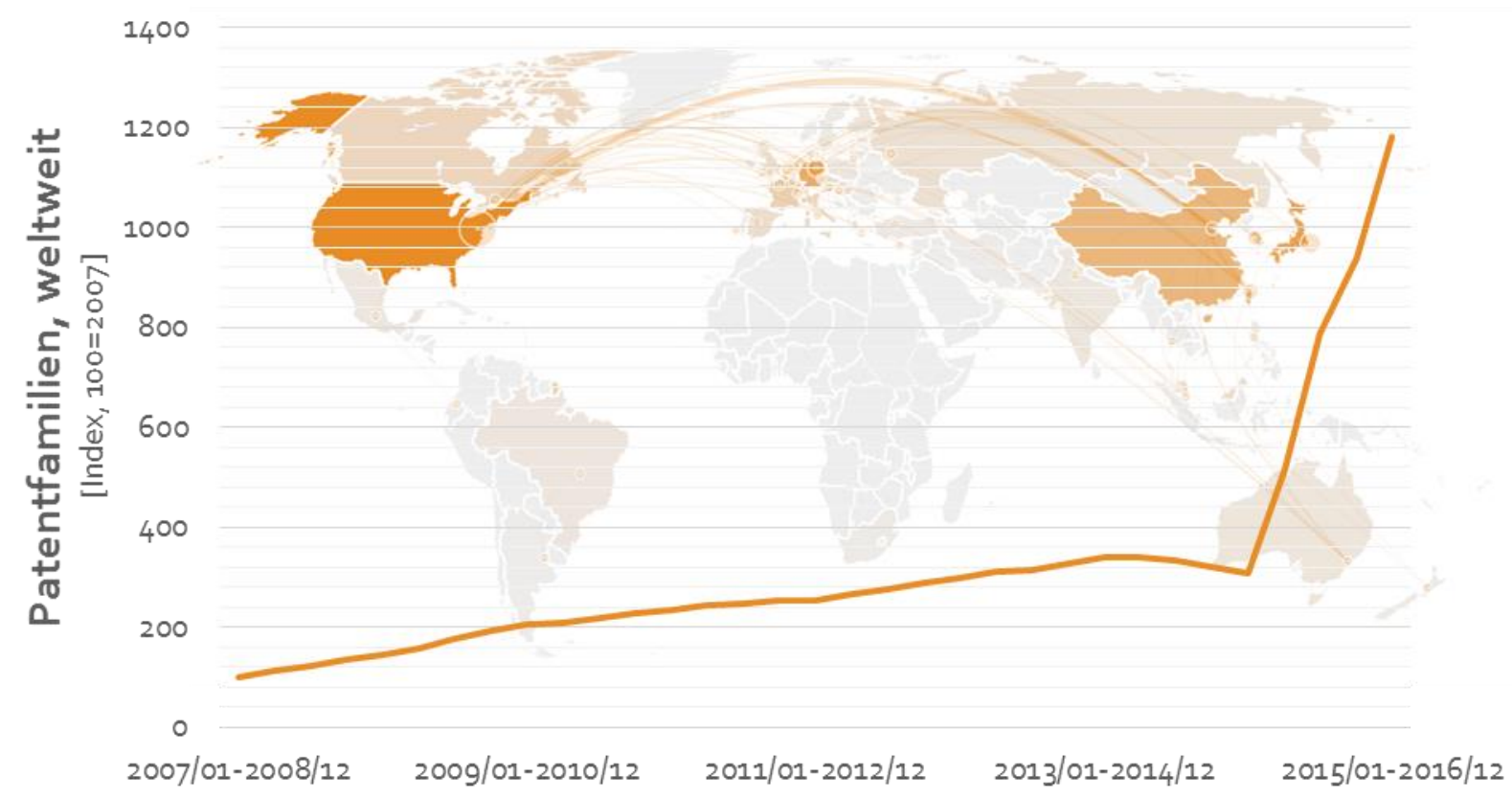

Quelle: Economica (2017).

\section{Weltweite Wachstumsthemen im Bereich Chirurgie/anwendungsspezifische Robotik}

Chirurgie und anwendungsspezifische Robotik ist über den gesamten 10-Jahreszeitraum exponentiell wachsend, mit USA, Deutschland, Japan, Niederlande und Kanada als führende Erfindernationen. Österreich liegt auf Rang 23, mit den Anmeldern W \& H DENTALWERKE BÜRMOOS, MED EL ELEKTROMEDIZINISCHE GERÄTE, AQUESYS INC, OTTO BOCK HEALTHCARE PROD, DEPUY SYNTHES PRODUCTS, BENKER EMANUEL, UNIV INNSBRUCK, HAGL PETER und KARL LEIBINGER. 
Abbildung 193: Weltweite Dynamik im Bereich Chirurgie/anwendungsspezifische Robotik

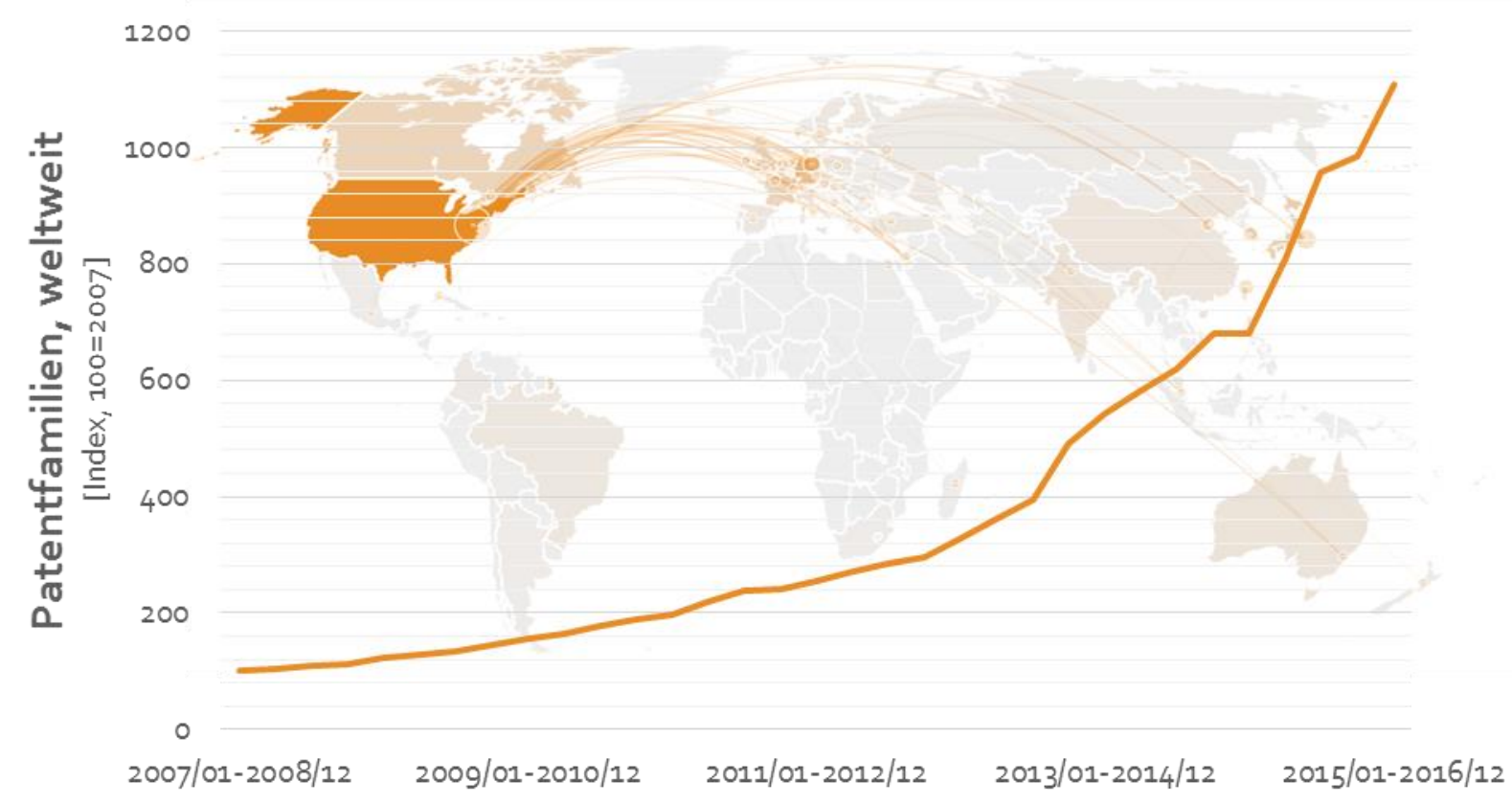

Quelle: Economica (2017).

\section{Weltweite Wachstumsthemen im Bereich Futter- oder Lebensmittel}

Die Wachstumsthemen auf dem Bereich Futter-/Lebensmittel

- Nahrung aus dem Meer; Erzeugnisse aus Fisch; Fischmehl; Roggenersatzprodukte (z.B. Kaviarersatzprodukte); deren Zubereitung oder Behandlung

- Futtermittel, besonders ausgebildet für bestimmte Tiere

- Fleischerzeugnisse / Fleischmehl / Herstellung und Behandlung derselben

- Aus Getreide hergestellte Produkte / Malzprodukte

- Obst- oder Gemüseprodukte; deren Zubereitung oder Behandlung

- Futtermittel

zeigten 2012 eine erste Phase des beschleunigten Wachstums, besonders stark erhöhte sich die Zahl der veröffentlichten Patentfamilien ab 2014. 
Österreich liegt, mit den Patentanmeldern ERBER AG, UNIV GRAZ, HAMA FOODSERVICE, VIRBAC SA, GATTRINGER JOSEF, HAUF REINER und WEICHSELBAUM JOHANN auf Rand 23 im weltweiten Ranking nach Erfindern, das von den USA, Japan, Korea, Russland und Frankreich angeführt wird.

Abbildung 194: Weltweite Dynamik im Bereich Futter- oder Lebensmittel

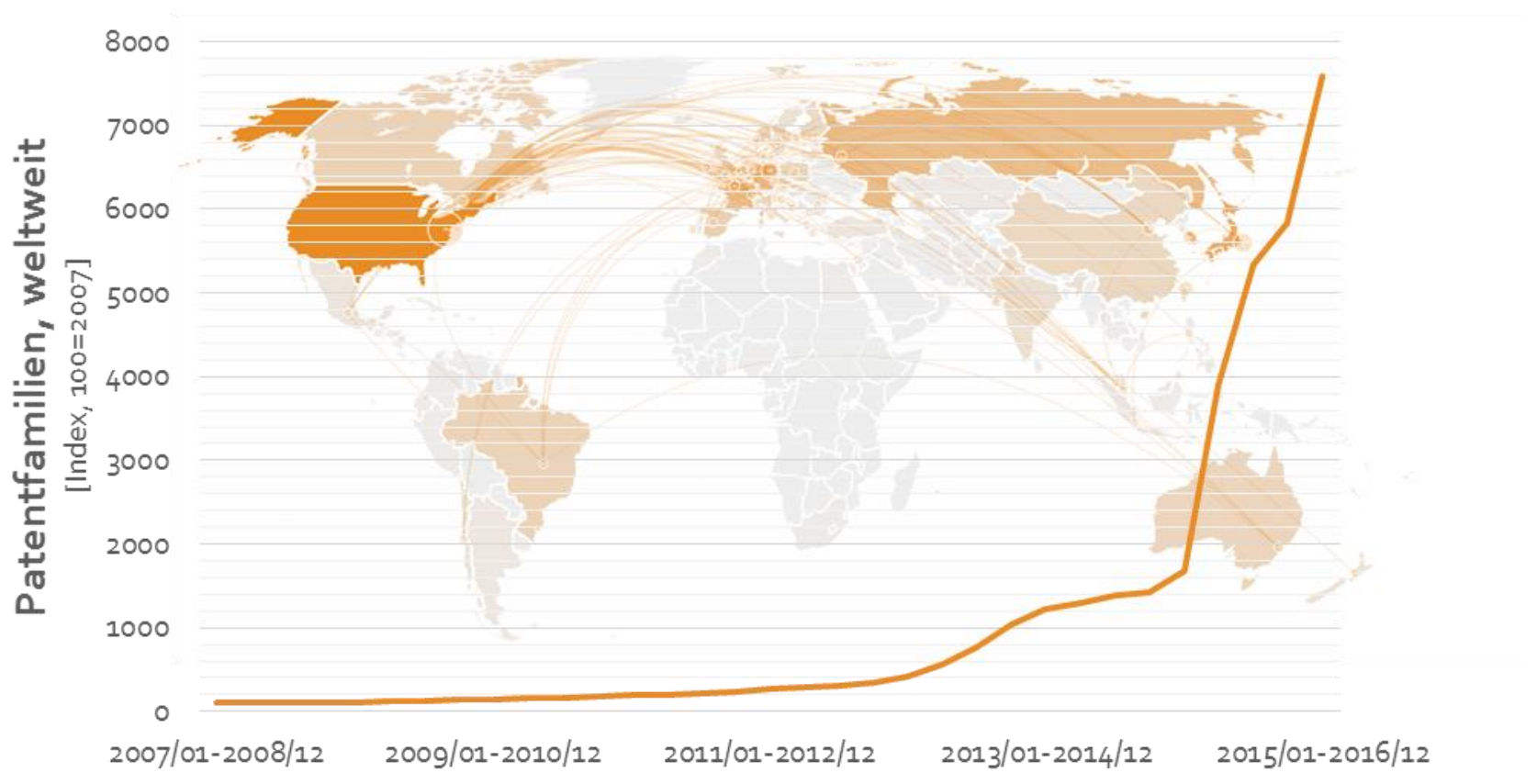

Quelle: Economica (2017). 


\section{Autorlnnen:}

Alt R., Berrer H., Borrmann J., Brunner Ph.,

Dolle B. Helmenstein C., Jöchle J.,Pirker J.,

Pohl, P., Popko, J., Schmidl M., Schneider H.

(C) 2017 Economica

Institut für Wirtschaftsforschung

Liniengasse $50-52$

A-1060 Wien

www.economica.at
IWI

Industriewissenschaftliches Institut

Mittersteig 10/4

1050 Wien

www.iwi.ac.at 